\title{
Application of machine learning and reaction optimization for the iterative improvement of enantioselectivity of cinchona-derived phase transfer catalysts
}

\author{
Katrina W. Lexa ${ }^{1}$, Kevin M. Belyk ${ }^{2 *}$, Jeremy Henle ${ }^{3}$, Bangping Xiang ${ }^{2}$, Robert P. Sheridan ${ }^{4}$, \\ Scott E. Denmark ${ }^{3}$, Rebecca T. Ruck ${ }^{2}$, Edward C. Sherer ${ }^{*}$ \\ ${ }^{1}$ Department of Computational and Structural Chemistry, MRL, Merck \& Co., Inc., Rahway, NJ, 07065, USA. \\ ${ }^{2}$ Department of Process Research \& Development, MRL, Merck \& Co., Inc., Rahway, NJ, 07065, USA. \\ ${ }^{3}$ Roger Adams Laboratory, Department of Chemistry, University of Illinois, Urbana, IL, 61801, USA. \\ ${ }^{4}$ Department of Computational and Structural Chemistry, MRL, Merck \& Co., Inc., Kenilworth, NJ, 07033, USA. \\ * corresponding authors; email: kevin_belyk@merck.com; edward_sherer@merck.com
}

\section{Table of Contents}

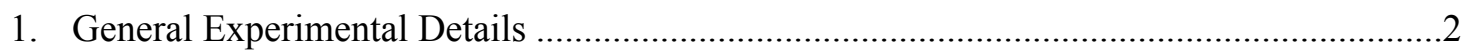

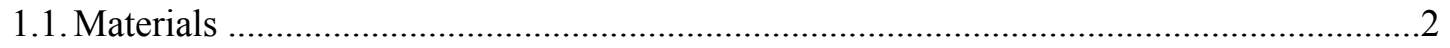

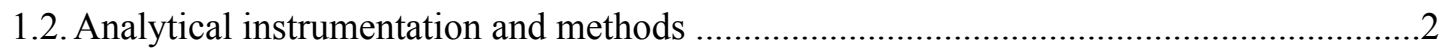

1.2.1. For doubly-quaternized phase-transfer catalysts ...........................................

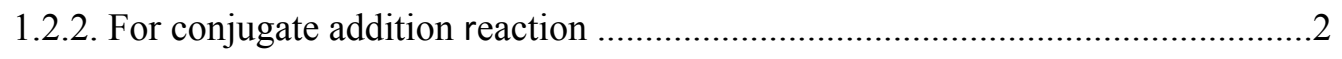

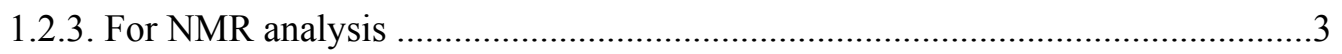

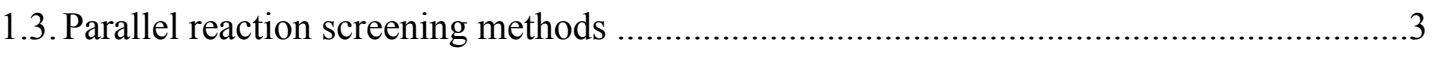

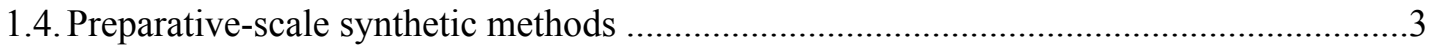

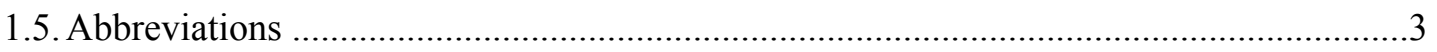

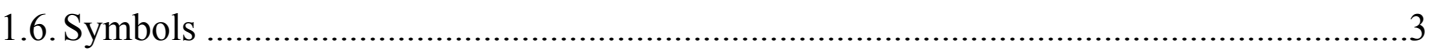

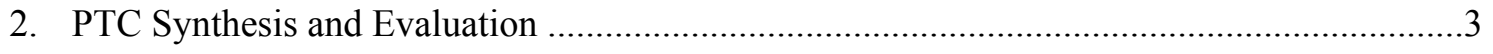

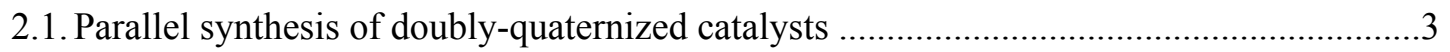

2.2. Parallel evaluation of catalysts in the conjugate addition reaction for rounds $1-5$............4

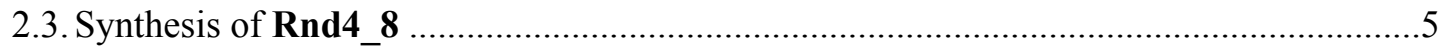

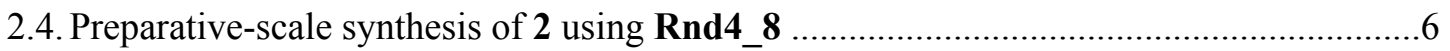

2.5. NMR spectra, UPLC-MS \& LRMS data for the synthesis of Rnd4_8 ...........................7

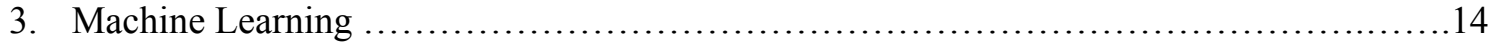

3.1. Background on ML........................................................... 14

3.2. Details of additional ML models explored......................................... 16

3.3. Additional descriptors from the combined RF model.............................. 17

3.4. Table of catalyst names and random forest predictions........................... 17

3.5. Table of all SMILES strings and associated experimental data (selectivity)............29

3.6. Table of all molecular structures...........................................69

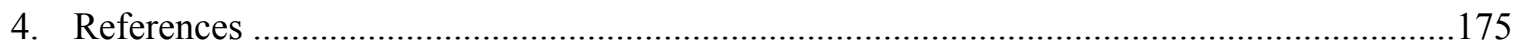




\section{Experimental Section}

\section{General Experimental Details}

All reactions were performed under a nitrogen atmosphere unless otherwise noted. All solvent evaporation (concentration) was done in vacuo using variable vacuum using a rotary evaporator attached to a vacuum controller (ca. $400-10 \mathrm{mmHg}$ ) unless otherwise described. Purification of compounds for characterization, unless otherwise described, were performed on a Teledyne ISCO CombiFlash ${ }^{\circledR} R f 200$.

\subsection{Materials}

All reagents and solvents were purchased from commercial suppliers and used without further purification. Synthesis of guanidine 1 was performed using previously reported procedures. ${ }^{1}$ Previously reported mono-quaternized phase-transfer catalysts used here were synthesized using previously described procedures ${ }^{2}$ or based on references cited therein. Water $(0.03$ micron filtered, Thermo Scientific $^{\mathrm{TM}}$ catalog number W81) was deoxygenated prior to being used for all phase-transfer catalyzed reactions. The acetonitrile and water used to prepare the UPLC mobile phases were purchased from Thermo Scientific ${ }^{\mathrm{TM}}$ : acetonitrile (0.1 micron filtered, catalog number A9561, UHPLC-MS, $\left.1 \mathrm{~L}\right)$ and water (0.03 micron filtered, catalog number W81, UHPLC-MS, $1 \mathrm{~L})$. DMSO- $d_{6}(99.9 \% d$-content, catalog DLM-10TB-10X0.75) $+0.05 \% \mathrm{v} / \mathrm{v}$ TMS, was purchased from Cambridge Isotope Laboratories in sealed ampules and used as received.

\subsection{Analytical Instrumentation and Methods}

\subsubsection{Doubly-Quaternized PTC Reaction Monitoring and LRMS Conditions.}

Instrumentation: All UPLC-MS analysis and low resolution (LR) ESI MS determination was performed on a Waters Acquity UPLC interfaced with a Waters Xevo G2 QTof ESI with the source temperature set to $150{ }^{\circ} \mathrm{C}$ and a cone voltage of $30 \mathrm{~V}$. Sample preparation: Samples with a concentration of about 0.3 $0.4 \mathrm{mg} / \mathrm{mL}$ catalyst were prepared in 2:1 (v:v) MeCN:water. UPLC Column: ACQUITY UPLCTM BEH C18 VanGuard Pre-column (130 A pore size, $1.7 \mu \mathrm{m}$ particle size, $2.1 \mathrm{~mm} \times 5 \mathrm{~mm}$ length; part number

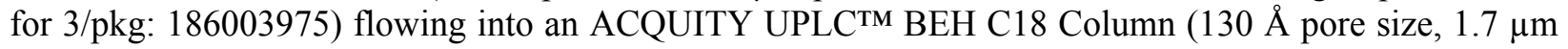
particle size, $2.1 \mathrm{~mm}$ internal diameter $\times 50 \mathrm{~mm}$ length; part number 186002350). Binary eluent system: eluent $\mathrm{A}=$ aqueous mobile phase $\left(3960 \mathrm{~mL} \mathrm{H} \mathrm{H}_{2} \mathrm{O}, 40 \mathrm{~mL}\right.$ pH 3.5 buffer, details below), eluent $\mathrm{B}=(3600$ $\mathrm{mL} \mathrm{MeCN}, 360 \mathrm{~mL} \mathrm{H} \mathrm{H}_{2} \mathrm{O}, 40 \mathrm{~mL} \mathrm{pH} 3.5$ buffer). The $\mathrm{pH} 3.5$ buffer is prepared by dissolving ammonium formate $(12.6 \mathrm{~g}, 0.200 \mathrm{~mol})$ and formic acid $(7.9 \mathrm{~mL}, 9.6 \mathrm{~g}, 0.21 \mathrm{~mol})$ in $1000 \mathrm{~mL}$ water. Gradient conditions: isocratic $60 \% \mathrm{~A}$ at $t=0 \mathrm{~min}$ to $t=2.0 \mathrm{~min}$ then ramp to $0.1 \% \mathrm{~A}$ at $t=2.50 \mathrm{~min}$, followed by a hold at $0.1 \%$ A until $t=3.90 \mathrm{~min}$, and a subsequent change to $60 \% \mathrm{~A}$ at $t=3.91 \mathrm{~min}$ until $t=4.00 \mathrm{~min}$. Flow rate: $0.8 \mathrm{~mL} / \mathrm{min}$; Sample injection volume: $1 \mu \mathrm{L}$; Column temperature: $50{ }^{\circ} \mathrm{C}$; UV detection: 300 $\mathrm{nm}$ (primary), $210 \mathrm{~nm}$ (secondary).

\subsubsection{Chiral RPLC Method for Conjugate Addition Reaction Monitoring.}

All chiral RPLC analysis was performed on an Agilent series 1100 HPLC system. Sample preparation: Samples with a concentration of $0.5 \mathrm{mg} / \mathrm{mL} 1$ or 2 were prepared in 80:20 (v:v) MeCN:water acidified with $1 \mathrm{vol} \%$ TFA to prevent the racemic background cyclization of $\mathbf{1}$ to $\mathbf{2}$ that occurs in non-acidified MeCN:water mixtures. HPLC Column: Chiral Technologies, Inc. ChiralPAK IC-3 immobilized polysaccharide column $(150 \mathrm{~mm} \times 4.6 \mathrm{~mm}$ ID, $3 \mu \mathrm{m}$ particle size; Part No. 83524); Binary eluent system: 
eluent $\mathrm{A}=$ aqueous sodium tetraborate with trifluoroacetic acid $\left(4.0 \mathrm{~g} \mathrm{Na}_{2} \mathrm{~B}_{4} \mathrm{O}_{7} \cdot 10 \mathrm{H}_{2} \mathrm{O}+1.6 \mathrm{~mL}\right.$ TFA in 4.0 L water) eluent B = MeCN; Isocratic conditions: $26 \%$ A for $5.3 \mathrm{~min} ;$ Flow rate: $1.0 \mathrm{~mL} / \mathrm{min}$; Sample injection volume: $5 \mu \mathrm{L}$; Column temperature: $30{ }^{\circ} \mathrm{C}$; $U V$ detection: $280 \mathrm{~nm}$ (primary), $210 \mathrm{~nm}$ (secondary). Retention times: minor enantiomer $(R)-2 t_{r}=4.0 \mathrm{~min}$, guanidine $1 t_{r}=4.3 \mathrm{~min}$, major enantiomer $(S)-2 t_{r}=4.8 \mathrm{~min}$.

\subsubsection{NMR Characterization.}

Proton nuclear magnetic resonance ( ${ }^{1} \mathrm{H}$ NMR) spectra, proton decoupled carbon nuclear magnetic resonance $\left({ }^{13} \mathrm{C}\left\{{ }^{1} \mathrm{H}\right\}\right.$ NMR $)$ spectra, proton coupled fluorine nuclear magnetic resonance $\left({ }^{19} \mathrm{~F} \mathrm{NMR}\right)$, and proton decoupled fluorine nuclear magnetic resonance $\left({ }^{19} \mathrm{~F}\left\{{ }^{1} \mathrm{H}\right\}\right.$ NMR) were recorded at $25{ }^{\circ} \mathrm{C}$ on a Bruker DRX-500 spectrometer using DMSO- $d_{6}+0.05 \% \mathrm{v} / \mathrm{v}$ TMS. Chemical shifts for carbon are reported in parts per million downfield from tetramethylsilane and are referenced to the carbon resonances of the NMR solvent. The residual solvent signal for samples in DMSO- $d_{6}$ was referenced to $2.50 \mathrm{ppm}$ for ${ }^{1} \mathrm{H}$ and $39.52 \mathrm{ppm}$ for ${ }^{13} \mathrm{C}$. Multiplicities are reported using the following abbreviations: $\mathrm{s}=$ singlet, $\mathrm{d}=$ doublet, $\mathrm{t}=$ triplet, $\mathrm{q}=$ quartet, $\mathrm{m}=$ multiplet, $\mathrm{om}=$ overlapped multiplet.

\subsection{Parallel Reaction Screening Methods}

All reactions were performed inside a positive-pressure glovebox under a nitrogen atmosphere using standard high-throughput experimentation techniques. ${ }^{3}$ Synthesis of doubly-quaternized phase-transfer catalysts and their evaluation were performed in parallel using a 96-well plate format. All reactions were carried out in 8x30 shell vials (Analytical Sales \& Services, Inc.; Part No. 884001) using parylene coated stir dowels (V\&P Scientific, Inc.; Part No. VP711D-1) as stir bars. The 8x30 shell vials were sealed in a Para-dox 96-well parallel synthesis/optimization block assembly (Analytical Sales \& Services, Inc.; Part No. 96960). The stirring mechanism used for all parallel experimentation was a heated/cooled tumble stirring module obtained from Symyx Technologies (Unchained Labs). All in vacuo solvent removal and centrifugation from 96-well reaction blocks was performed inside a glovebox under a nitrogen atmosphere using a Genevac ${ }^{\mathrm{TM}}$ centrifugal evaporator.

\subsection{Preparative-Scale Synthetic Methods}

All phase-transfer catalyzed reactions run on $>2.5$ mmole scale substrate were carried using a MettlerToledo Advanced Thermostat EasyMax 102 system. Utilized $50 \mathrm{~mL}$ or $100 \mathrm{~mL}$ EasyMax two-piece glass reactors (EasyMax Part No. 51161255 or 51161620) equipped with an overhead stirrer magnetic drive set (EasyMax Part No. 30213866 or 30113925 ), downward pitched-blade element (EasyMax Part No. 51161549 or 51161549), and thermocouple under a nitrogen atmosphere.

\subsection{Abbreviations}

$\mathrm{DCM}=$ dichloromethane, $\mathrm{DMF}=N, N$-dimethylformamide, $\mathrm{DMSO}=$ dimethylsulfoxide, EtOAc $=$ ethyl acetate, $\mathrm{ESI}=$ electrospray ionization $\left(\mathrm{ESI}^{+}\right.$and $\mathrm{ESI}^{-}$denote positive or negative mode, respectively), $\mathrm{HPLC}=$ high performance liquid chromatography, $\mathrm{HR}=$ high resolution, IPA $=$ 2-propanol, LC $=$ liquid chromatography, $\mathrm{LR}=$ low-resolution, $\mathrm{MeCN}=$ acetonitrile, $\mathrm{MeOH}=$ methanol, $\mathrm{MS}=$ mass spectrometry, PTC $=$ phase-transfer catalyst, $\mathrm{QToF}=$ quadrupole time of flight, $\mathrm{RPLC}=$ reverse phase liquid chromatography, TBME = tert-butyl methyl ether, TFA = trifluoroacetic acid, $\mathrm{THF}=$ tetrahydrofuran, UPLC = ultra-performance liquid chromatography, $\mathrm{v} / \mathrm{v}=$ volume per volume percent.

\subsection{Symbols}

$\delta=$ parts per million $(\mathrm{ppm}), t_{r}=$ retention time.

\section{PTC Synthesis and Evaluation}


2.1 Parallel Synthesis of Doubly-Quaternized PTCs: Synthesis of each bis-quat PTC was performed on $10 \mu \mathrm{mol}$ scale with modification of the previously reported reaction conditions and isolation. ${ }^{4}$ Only PTCs shown to be $>80 \%$ pure by UPLC analysis (at UV $=300 \mathrm{~nm}$ ) were taken forward for evaluation in the conjugate addition reaction. Purity of each bis-quat catalyst was evaluated at $300 \mathrm{~nm}$ to eliminate the UPLC area percent contribution of each alkylating agent, all of which had negligible UV adsorption at this wavelength relative to cinchonidine-derived products. The bis-quat catalysts carried forward were 80$92 \%$ pure with the remaining cinchonidine-derived species consisting of mixtures of unreacted mono-quat and the presumed Hofmann elimination by-product based on UPLC-MS analysis of the reaction mixtures. For a study of the Hofmann elimination decomposition pathway of a doubly-quaternized Cinchonaalkaloid derived PTC. ${ }^{5}$
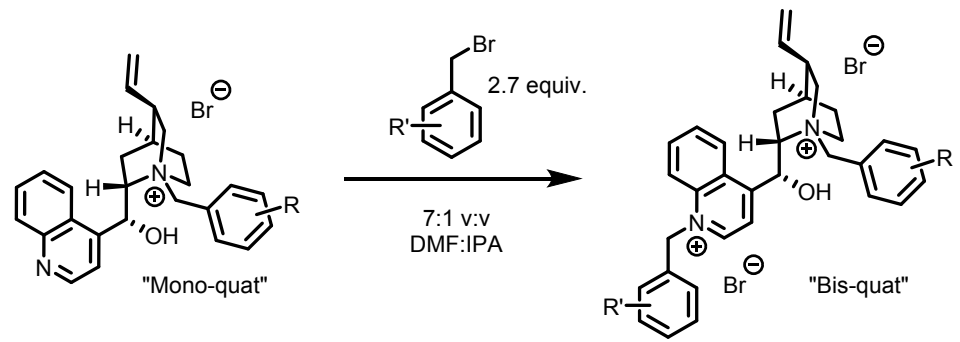

Representative procedure: For a typical synthesis experiment, the benzyl bromide derivative $(27 \mu \mathrm{mol}$, 2.7 equiv.) was added to a mixture of IPA $(10 \mu \mathrm{L})$ and a $0.125 \mathrm{mM}$ mixture of each mono-quat $(10 \mu \mathrm{mol}$, $80 \mu \mathrm{L})$ pre-dispensed to $8 \times 30$ shell vials. The reaction block was sealed and agitated at $400 \mathrm{rpm}$ on a tumble stirrer at $70-73{ }^{\circ} \mathrm{C}(18 \mathrm{~h})$. Cooled reaction vials to room temperature and sampled each mixture for UPLC-MS analysis. Each reaction vial with bis-quat meeting the purity specification was diluted with isopropyl acetate $(90 \mu \mathrm{L})$ followed by $n$-heptane $(180-270 \mu \mathrm{L})$ with agitation. The resulting suspensions (or biphasic mixtures) were agitated (1-2 h) and the entire 96-well plate centrifuged to settle the resulting precipitate (or provide a distinct lower phase containing the bis-quat). The supernatant from each vial was decanted (or upper organic phase separated in the case of a bi-phasic mixture). Each precipitate (or lower organic phase) was diluted with $\mathrm{MeOH}(600 \mu \mathrm{L})$ with agitation and the resulting mixture sampled (17-34 $\mu \mathrm{L}, 0.25-0.5 \mu \mathrm{mol}$ bis-quat) out to new $8 \times 30$ shell vials pre-dosed with toluene $(50 \mu \mathrm{L})$. The solvent was removed in vacuo in preparation for catalyst evaluation.

2.2 Parallel Evaluation of Catalysts in the Conjugate Addition Reaction for Rounds 1-5: Evaluation of the doubly-quaternized catalysts were performed on $2.5 \mu \mathrm{mol}$ scale 1 with modifications to the original conditions $^{1}$ utilizing previously described high-throughput experimentation techniques developed for phase-transfer catalysis. ${ }^{2}$ Due to the dependence of enantioselectivity on agitation rate for this reaction, multiple catalysts from prior rounds of evaluation were included in each subsequent round of new catalyst evaluation to insure that reproducible reaction performance was achieved. An agitation rate of $>400 \mathrm{rpm}$ is necessary to obtain reproducible results. A systematic study of how enantioselectivity for the reaction is impacted by agitation rate can be found in the supporting information of reference 1 . All rounds of evaluation also included the original catalyst, Rnd1_1, ${ }^{1}$ for direct head-to-head comparison with new catalysts.

Representative procedure: In a typical parallel catalyst evaluation experiment, a $25 \mathrm{mM}$ solution of guanidine $1(2.5 \mu \mathrm{mol}, 100 \mu \mathrm{L})$ was dispensed to each of 96 vials pre-dosed with the desired PTCs $(0.25$ $0.5 \mu \mathrm{mol}, 10-20 \mathrm{~mol} \%$ ). Cooled the 96 -well reaction block to $2-4{ }^{\circ} \mathrm{C}$ and added a chilled solution of 113 $\mathrm{mM}$ aqueous $\mathrm{K}_{3} \mathrm{PO}_{4}(3.75 \mu \mathrm{mol}, 33.3 \mu \mathrm{L})$ to each reaction vial without agitation. The reaction block was held at $2-4{ }^{\circ} \mathrm{C}$, sealed and the reactions agitated on a tumble stirrer at $450-550 \mathrm{rpm}(18-20 \mathrm{~h})$. A systematic study of how enantioselectivity for the reaction is impacted by agitation rate can be found in the supporting information of reference ${ }^{1}$. Agitation was stopped, the reactions diluted with chilled toluene, and organic layer sampled for chiral RPLC analysis. 


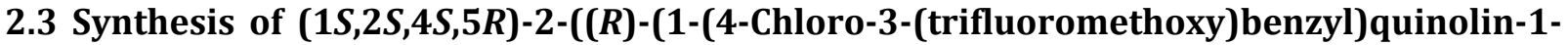 ium-4-yl)(hydroxy)methyl)-1-(3-fluoro-5-(perfluoroethyl)benzyl)-5-vinylquinuclidin-1-ium Bromide (Rnd4_8).}

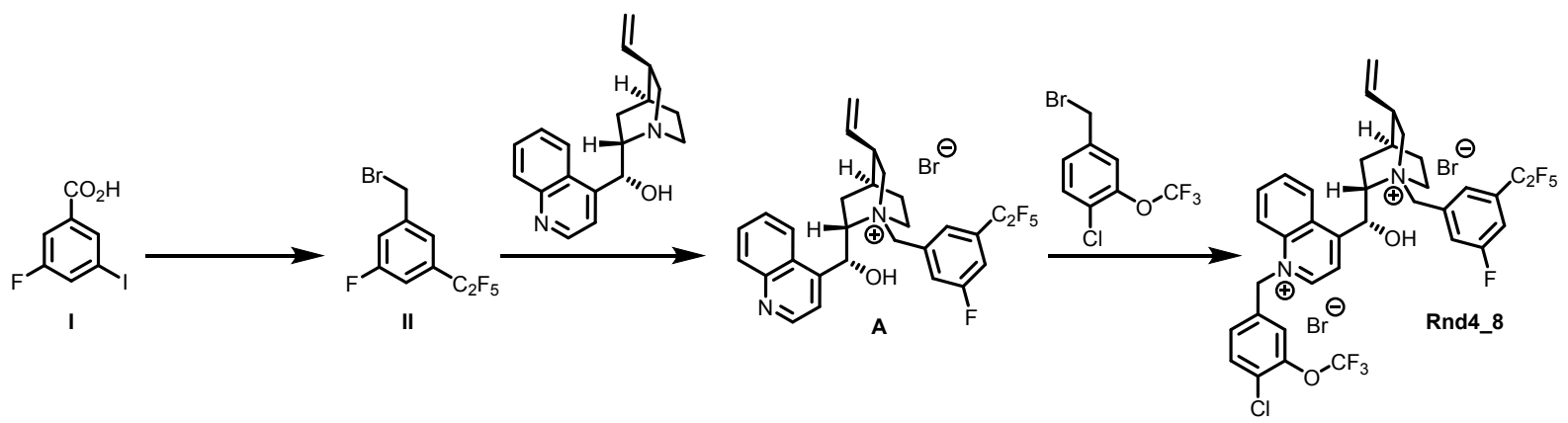

Mono-quaternized catalyst A (Scheme 5) was prepared from commercially available 3-fluoro-5iodobenzoic acid (I) using the following procedure: Thionyl chloride (67.1 g, $564 \mathrm{mmol}, 3.0$ equiv) was added dropwise with stirring at $0{ }^{\circ} \mathrm{C}$ to 3 -fluoro-5-iodobenzoic acid (50 g, $188 \mathrm{mmol}, 1.0$ equiv) in ethanol $(500 \mathrm{~mL})$. The resulting solution was heated to reflux for $1 \mathrm{~h}$. The resulting mixture was concentrated in vacuo to provide ethyl 3-fluoro-5-iodobenzoate (50 g, 90\%) as a white solid. Other perfluoroalkyl aryl mono-quaternized PTCs used in round 5 were all prepared using a similar procedure starting from either I or ethyl 3,5-diiodobenzoate and the desired perfluoroalkylanoate.

Next, potassium tert-butoxide (76 g, $677 \mathrm{mmol}, 4.0$ equiv) was added to copper(I) chloride (33.7 g, $337 \mathrm{mmol}, 2.0$ equiv) in DMF (500 mL) at $25^{\circ} \mathrm{C}$ and then was warmed to $50{ }^{\circ} \mathrm{C}$ whereupon ethyl 2,2,3,3,3-pentafluoropropanoate (65.3 g, $340 \mathrm{mmol}, 2.0$ equiv) was added. After agitation for $50{ }^{\circ} \mathrm{C}$ $(4 \mathrm{~h})$, triethylamine trihydrofluoride $(21.9 \mathrm{~g}, 136 \mathrm{mmol}, 0.80$ equiv) and the ethyl 3-fluoro-5iodobenzoate ( $50 \mathrm{~g}, 170 \mathrm{mmol}, 1.0$ equiv ) were added. The resulting solution was stirred overnight at $85{ }^{\circ} \mathrm{C}$ and then was cooled to room temperature whereupon it was quenched by the addition of water. Following dilution with tert-butyl methyl ether (TBME), the resulting solids were removed by filtration and the resulting solution was extracted with additional TBME. The combined organic layers were concentrated in vacuo to provide ethyl 3-fluoro-5-(pentafluoroethyl)benzoate (28 g, $58 \%$ ) as yellow oil.

Next, a solution of lithium borohydride ( $97.8 \mathrm{~mL}, 196 \mathrm{mmol}, 2.0$ equiv) in THF was added dropwise with stirring to the ethyl 3-fluoro-5-(pentafluoroethyl)benzoate (28 g, $97.8 \mathrm{mmol}, 1.0$ equiv) in THF $(400 \mathrm{~mL})$. The resulting solution was stirred overnight at room temperature then was quenched by the addition of $\mathrm{MeOH}$. The resulting solution was diluted with water and was extracted with $\mathrm{CH}_{2} \mathrm{Cl}_{2}$. The combined organic layers were concentrated in vacuo and the residue purified on silica gel $1: 10$ v:v EtOAc/petroleum ether) to provide of 3-fluoro-5-(perfluoroethyl)phenyl)methanol (20 g, 84\%) as yellow oil.

Next, phosphorous tribromide (24.4 mL, $90.0 \mathrm{mmol}, 1.10$ equiv) was added to (3-fluoro-5(perfluoroethyl) phenyl)methanol (20 g, $81.9 \mathrm{mmol}, 1.0$ equiv) in diethyl ether $(250 \mathrm{~mL})$ with agitation. The resulting solution was stirred overnight at room temperature then was quenched by the addition of water. The resulting solution was extracted with TBME, the organic layers were 
combined and concentrated in vacuo to provide 1-(bromomethyl)-3-fluoro-5(pentafluoroethyl)benzene (II) (18 g, 72\%) as yellow oil.

Crude II (18.8 g, $61.2 \mathrm{mmol}, 1.0$ equiv) was then added to (-)-cinchonidine (16.3 g, $55.4 \mathrm{mmol}, 0.90$ equiv) in toluene $(200 \mathrm{~mL})$ and was stirred at $80{ }^{\circ} \mathrm{C}$ for $2 \mathrm{~h}$. The mixture was cooled to room temperature and the precipitate collected by filtration. The crude product was recrystallized from $\mathrm{MeOH} / \mathrm{TBME}(1: 15 \mathrm{v}: \mathrm{v})$ to provide $(1 S, 2 S, 4 S, 5 R)$-1-(3-fluoro-5-(perfluoroethyl)benzyl)-2-((R)hydroxy(quinolin-4-yl)methyl)-5-vinylquinuclidin-1-ium bromide (A) (20 g, 59\%) as a white solid. ${ }^{1} \mathrm{H}$ NMR $\left(500 \mathrm{MHz}, \mathrm{DMSO}-d_{6}\right) \delta 8.98(\mathrm{~d}, J=4.5 \mathrm{~Hz}, 1 \mathrm{H}), 8.31(\mathrm{~d}, J=8.2 \mathrm{~Hz}, 1 \mathrm{H}), 8.14-8.09(\mathrm{~m}, 2 \mathrm{H})$, $8.06(\mathrm{~s}, 1 \mathrm{H}), 7.91(\mathrm{~d}, J=8.6 \mathrm{~Hz}, 1 \mathrm{H}), 7.85$ (ddd, $J=8.3,6.9,1.1 \mathrm{~Hz}, 1 \mathrm{H}), 7.81$ (d, $J=4.5 \mathrm{~Hz}, 1 \mathrm{H}), 7.76$ (ddd, $J=8.2,6.9,1.2 \mathrm{~Hz}, 1 \mathrm{H}), 6.71(\mathrm{~d}, J=4.6 \mathrm{~Hz}, 1 \mathrm{H}), 6.54(\mathrm{~d}, J=3.7 \mathrm{~Hz}, 1 \mathrm{H}$ ), 5.68 (ddd, $J=17.1,10.6$, $6.4 \mathrm{~Hz}, 1 \mathrm{H}), 5.29$ (dd, $J=63.1,12.4 \mathrm{~Hz}, 2 \mathrm{H}), 5.18(\mathrm{~d}, J=17.3 \mathrm{~Hz}, 1 \mathrm{H}), 4.95(\mathrm{~d}, J=10.5 \mathrm{~Hz}, 1 \mathrm{H}), 4.42-$ $4.31(\mathrm{~m}, 1 \mathrm{H}), 3.91(\mathrm{t}, J=9.0 \mathrm{~Hz}, 1 \mathrm{H}), 3.84(\mathrm{dt}, J=12.3,3.7 \mathrm{~Hz}, 1 \mathrm{H}), 3.43-3.34(\mathrm{~m}, 1 \mathrm{H}), 3.29(\mathrm{dd}, J=$ 11.3, $4.8 \mathrm{~Hz}, 1 \mathrm{H}), 2.66(\mathrm{~s}, 1 \mathrm{H}), 2.18-2.04(\mathrm{~m}, 2 \mathrm{H}), 2.03-1.98(\mathrm{~m}, 1 \mathrm{H}), 1.81(\mathrm{t}, J=9.3 \mathrm{~Hz}, 1 \mathrm{H}), 1.31$ $(\mathrm{dt}, J=12.9,6.4 \mathrm{~Hz}, 1 \mathrm{H}) .{ }^{13} \mathbf{C}\left\{{ }^{1} \mathbf{H}\right\} \mathbf{N M R}\left(126 \mathrm{MHz}, \mathrm{DMSO}-d_{6}\right) \delta 161.92\left(\mathrm{~d}, J_{\mathrm{CF}}=247.8 \mathrm{~Hz}\right), 150.13$, 147.61, 145.00, 138.01, $132.24\left(\mathrm{~d}, J_{\mathrm{CF}}=8.2 \mathrm{~Hz}\right), 129.87,129.46\left(\mathrm{td}, J_{\mathrm{CF}}=24.5,8.3 \mathrm{~Hz}\right), 129.39$, $128.03-127.81(\mathrm{~m}, 1 \mathrm{C}), 127.27,125.26\left(\mathrm{~d}, J_{\mathrm{CF}}=22.3 \mathrm{~Hz}\right), 124.25,123.56,120.07,118.51$ (qt, $J_{\mathrm{CF}}=$ 286, 39.1 Hz), 116.38, $115.74\left(\mathrm{dt}, J_{\mathrm{CF}}=24.8,6.1 \mathrm{~Hz}\right), 112.48\left(\mathrm{tq}, J_{\mathrm{CF}}=254,39.0 \mathrm{~Hz}\right), 68.12,63.98$, $61.08,59.13,50.76,36.93,25.76,24.22,20.92 .{ }^{19}$ F NMR $\left(471 \mathrm{MHz}\right.$, DMSO- $\left.d_{6}\right) \delta-83.80,-109.60$ (dd, $\left.{ }^{3} J_{\mathrm{HF}-o}=8.9,9.0 \mathrm{~Hz}, 1 \mathrm{~F}\right),-112.6$ to -113.9 (om, 2F). ${ }^{19} \mathbf{F}\left\{{ }^{1} \mathbf{H}\right\}$ NMR (471 MHz, DMSO- $\left.d_{6}\right) \delta-83.80$, 109.60, -112.6 to -113.9 (om, 2F). MS (ESI) m/z calcd. for $\mathrm{C}_{28} \mathrm{H}_{27} \mathrm{~F}_{6} \mathrm{~N}_{2} \mathrm{O}^{+}[\mathrm{M}]^{+}$521.2, found 521.2.

Next, a warm solution of A $(2.08 \mathrm{~g}, 3.46 \mathrm{mmol})$ and 4-(bromomethyl)-1-chloro-2(trifluoromethoxy)benzene $(2.28 \mathrm{~g}, 7.88 \mathrm{mmol})$ in a mixture of DMF $(7.0 \mathrm{~mL})$ and $i$-PrOH $(1.0 \mathrm{~mL})$ was heated with agitation to $70^{\circ} \mathrm{C}$ for $6.5 \mathrm{~h}$. The reaction mixture was cooled to $5{ }^{\circ} \mathrm{C}$ and was slowly added to ice-cold ethyl acetate $(100 \mathrm{~mL})$ seeded with $\mathbf{R n d 4} \mathbf{8}$. The resulting white suspension was aged overnight at room temperature, then was filtered, and the filter cake was washed with ethyl acetate $(60 \mathrm{~mL})$. The cake was dried under vacuum to give $\mathbf{R n d 4 \_ 8}$ as a white solid (2.53 g, 95 area \% by RPLC-MS analysis, 81\% yield). Rnd4_8 was used as is without further purification. ${ }^{\mathbf{1}} \mathbf{H}$ NMR $\left(500 \mathrm{MHz}, \mathrm{DMSO}-d_{6}\right) \delta 9.86(\mathrm{~d}, J=6.3 \mathrm{~Hz}, 1 \mathrm{H}), 8.84(\mathrm{~d}, J=8.4 \mathrm{~Hz}, 1 \mathrm{H}), 8.60(\mathrm{~d}, J=9.0 \mathrm{~Hz}, 1 \mathrm{H}), 8.51$ $(\mathrm{d}, J=6.1 \mathrm{~Hz}, 1 \mathrm{H}), 8.33-8.25(\mathrm{~m}, 1 \mathrm{H}), 8.19(\mathrm{~d}, J=8.9 \mathrm{~Hz}, 1 \mathrm{H}), 8.17-8.09(\mathrm{~m}, 2 \mathrm{H}), 7.92(\mathrm{~d}, J=8.6 \mathrm{~Hz}$, $1 \mathrm{H}), 7.86(\mathrm{~s}, 1 \mathrm{H}), 7.74(\mathrm{~d}, J=8.4 \mathrm{~Hz}, 1 \mathrm{H}), 7.46(\mathrm{dd}, J=8.5,2.0 \mathrm{~Hz}, 1 \mathrm{H}), 7.27(\mathrm{~d}, J=5.2 \mathrm{~Hz}, 1 \mathrm{H}), 6.84$ (d, $J=4.6 \mathrm{~Hz}, 1 \mathrm{H}), 6.54-6.43(\mathrm{~m}, 2 \mathrm{H}), 5.67$ (ddd, $J=17.2,10.6,6.5 \mathrm{~Hz}, 1 \mathrm{H}), 5.54-5.39(\mathrm{~m}, 2 \mathrm{H}), 5.23$ $(\mathrm{d}, J=17.3 \mathrm{~Hz}, 1 \mathrm{H}), 4.95(\mathrm{~d}, J=10.5 \mathrm{~Hz}, 1 \mathrm{H}), 4.42(\mathrm{t}, J=10.7 \mathrm{~Hz}, 1 \mathrm{H}), 4.05(\mathrm{t}, J=8.9 \mathrm{~Hz}, 2 \mathrm{H}), 3.41(\mathrm{t}, J$ $=11.5 \mathrm{~Hz}, 1 \mathrm{H}), 3.32(\mathrm{td}, J=11.3,4.7 \mathrm{~Hz}, 1 \mathrm{H}), 2.68(\mathrm{~s}, 1 \mathrm{H}), 2.20-1.98(\mathrm{~m}, 3 \mathrm{H}), 1.84(\mathrm{t}, J=9.3 \mathrm{~Hz}, 1 \mathrm{H})$, $1.49-1.36(\mathrm{~m}, 1 \mathrm{H}) .{ }^{13} \mathrm{C}\left\{{ }^{1} \mathrm{H}\right\}$ NMR $\left(126 \mathrm{MHz}, \mathrm{DMSO}-d_{6}\right) \delta 161.89\left(\mathrm{~d}, J_{\mathrm{CF}}=247.9 \mathrm{~Hz}\right), 158.14,149.85$, 144.18 - 144.06 (m, 1C), 137.99, 137.12, 135.45, 134.91, 132.10 (d, $J_{\mathrm{CF}}=8.2 \mathrm{~Hz}$ ), 131.67, 130.74, $129.49\left(\mathrm{td}, J_{\mathrm{CF}}=24.4,8.2 \mathrm{~Hz}\right), 128.37,128.08-127.85(\mathrm{~m}, 1 \mathrm{C}), 126.79,126.59,126.13,125.27\left(\mathrm{~d}, J_{\mathrm{CF}}\right.$ $=22.3 \mathrm{~Hz}$ ), $123.00,121.72,119.93\left(\mathrm{q}, J_{\mathrm{CF}}=258.6 \mathrm{~Hz}\right), 119.76,118.50$ (qt, $J_{\mathrm{CF}}=286.3,39.1 \mathrm{~Hz}$ ), $116.57,115.82\left(\mathrm{dt}, J_{\mathrm{CF}}=25.0,5.3 \mathrm{~Hz}\right), 112.48\left(\mathrm{tq}, J_{\mathrm{CF}}=254.6,38.2 \mathrm{~Hz}\right), 67.67,64.70,60.79,59.33$, 58.66, 50.77, 36.90, 25.84, 24.25, 21.12. ${ }^{19}$ F NMR (471 MHz, DMSO- $\left.d_{6}\right) \delta-56.96,-83.79,-109.54$ (dd, $\left.{ }^{3} J_{\mathrm{HF}-o}=8.8,8.9 \mathrm{~Hz}, 1 \mathrm{~F}\right),-112.6$ to -113.9 (om, $\left.2 \mathrm{~F}\right) .{ }^{19} \mathbf{F}\left\{{ }^{1} \mathbf{H}\right\} \mathbf{N M R}\left(471 \mathrm{MHz}, \mathrm{DMSO}-d_{6}\right) \delta-56.96$, 83.79, $-109.54,-112.6$ to -113.9 (om, 2F). MS (ESI) m/z calcd. for $\mathrm{C}_{36} \mathrm{H}_{32} \mathrm{ClF}_{9} \mathrm{~N}_{2} \mathrm{O}_{2}{ }^{2+}[\mathrm{M}]^{2+} 365.1$, found 365.0.

2.4 Preparative-Scale Synthesis of Methyl-(S)-2-(8-fluoro-3-(2-methoxy-5-(trifluoro-methyl) phenyl)-2-(4-(3-methoxyphenyl)-piperazin-1-yl)-3,4-dihydro-quinazolin-4-yl) Acetate (2) using Rnd4_8. 
Guanidine 1 (1.51 g, $2.57 \mathrm{mmol}$ ) and 1,3-difluorobenzene (18 mL) were charged into a 50-mL EasyMax vessel equipped with an overhead stirrer (blade height setting $=5$ ). The resulting solution was cooled to about $0{ }^{\circ} \mathrm{C}$ then a chilled solution of $0.43 \mathrm{M}$ aqueous $\mathrm{K}_{3} \mathrm{PO}_{4}(8.95 \mathrm{~mL}, 3.85 \mathrm{mmol}, 1.5$ equiv) was charged without agitation. The resulting biphasic mixture was cooled to $-5{ }^{\circ} \mathrm{C}$ and the agitation rate then increased to 600 RPM. Next, catalyst Rnd4_8 (114 mg, 0.128 mmol, 0.05 equiv) was added all at once as a chilled suspension in 1,3-difluorobenzene $(2.0 \mathrm{~mL})$. An internal reaction temperature of -3 to $-5{ }^{\circ} \mathrm{C}$ was maintained with agitation at $600 \mathrm{RPM}$ until no starting material remained $(2 \mathrm{~h})$. Upon completion, the reaction mixture was warmed to room temperature and the phases were separated. The organic phase was loaded onto a RediSep ${ }^{\circledR}$ solid load silica cartridge, and the product was purified using a $40 \mathrm{~g}$ RediSep Gold ${ }^{\circledR}$ silica cartridge (gradient: 1:9 EtOAc/hexanes to $1: 1 \mathrm{EtOAc} /$ hexanes $)$ to give $(S)-2\left(1.49 \mathrm{~g}, 96 \mathrm{wt} \%\right.$ as determined by ${ }^{1} \mathrm{H}-\mathrm{NMR}$ analysis against 1,3,5-trimethoxybenzene internal standard, $96 \%$ assay yield, $89 \% e e$ ) as an oil. The ${ }^{1} \mathrm{H}$ and ${ }^{13} \mathrm{C}\left\{{ }^{1} \mathrm{H}\right\}$ NMR spectra matched the literature data. ${ }^{33}$
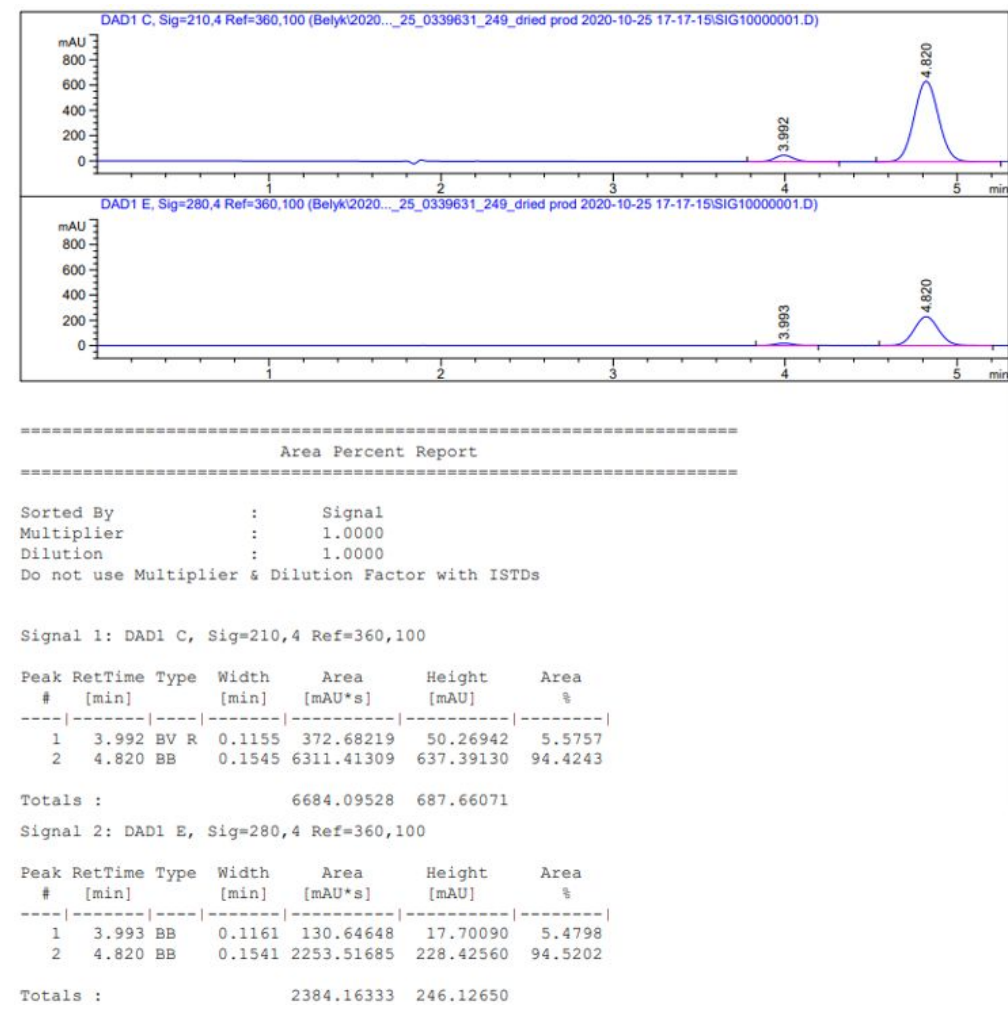
2.5. NMR Spectra and LRMS Data for Mono-quat A and Rnd4_8

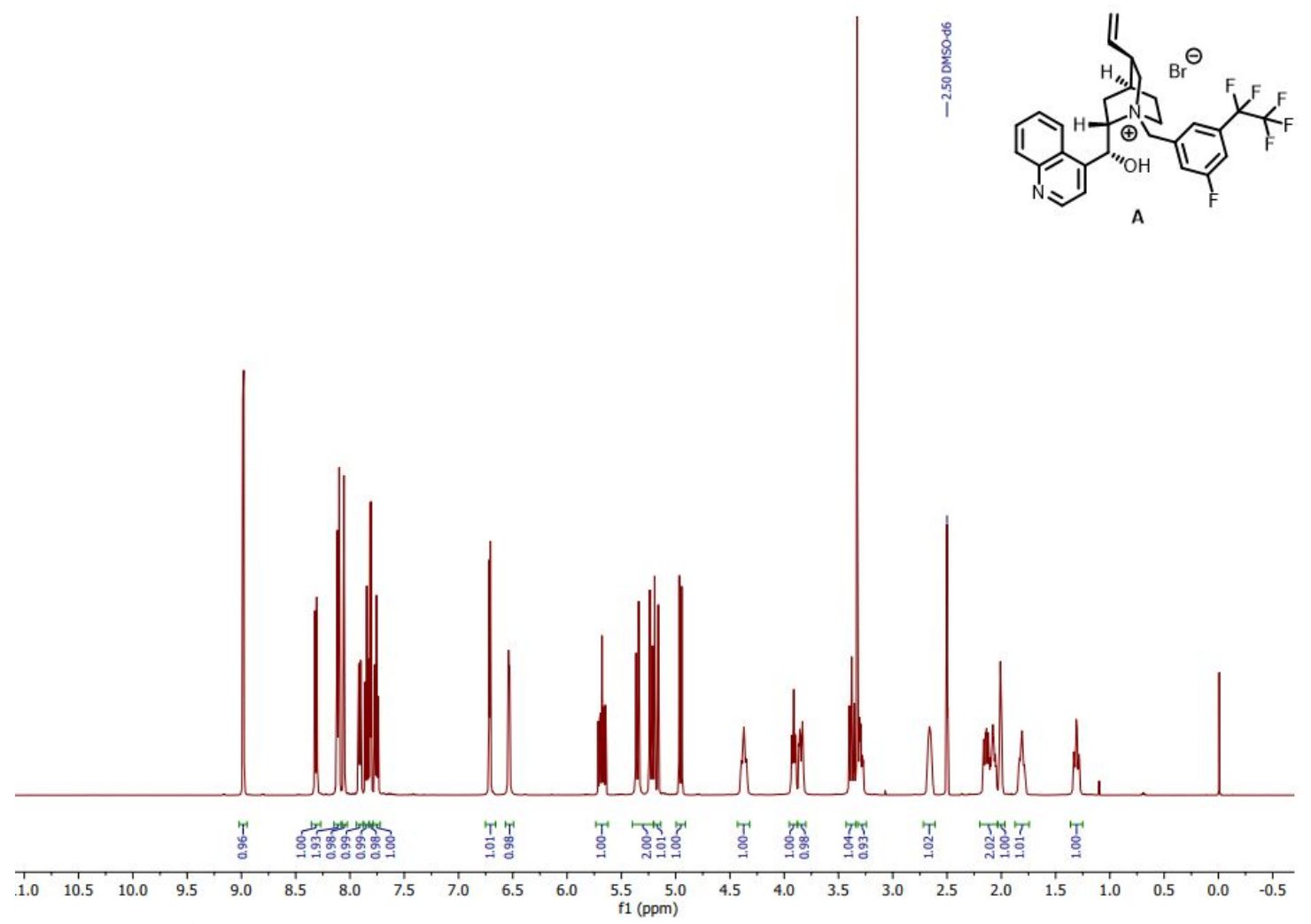

Figure S1. ${ }^{1} \mathrm{H}$ NMR (500 MHz) spectrum of A in DMSO- $d_{6}$. 


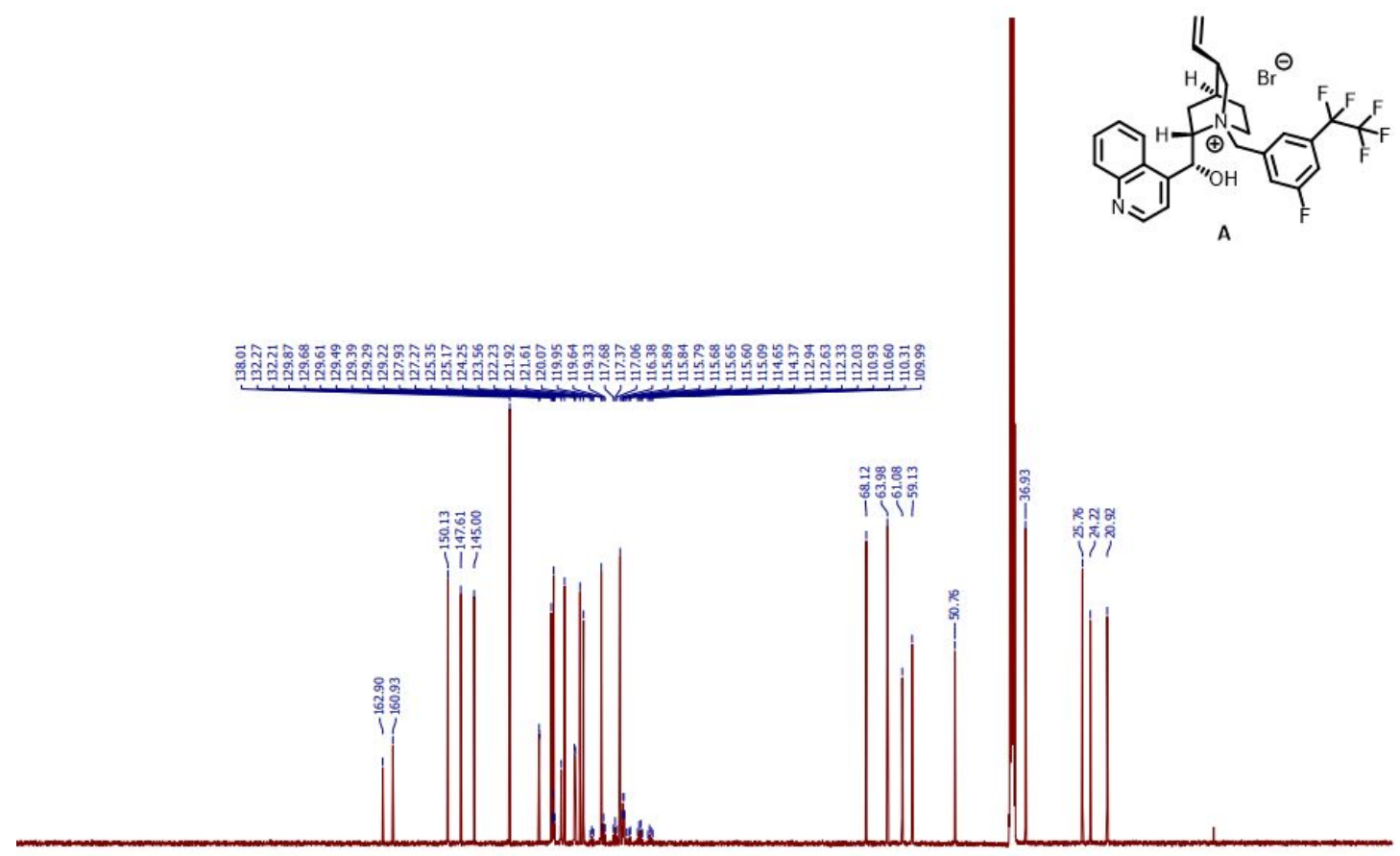

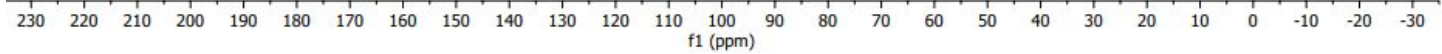

Figure S2. ${ }^{13} \mathrm{C}\left\{{ }^{1} \mathrm{H}\right\}$ NMR (126 MHz) spectrum of $\mathbf{A}$ in DMSO- $d_{6}$.

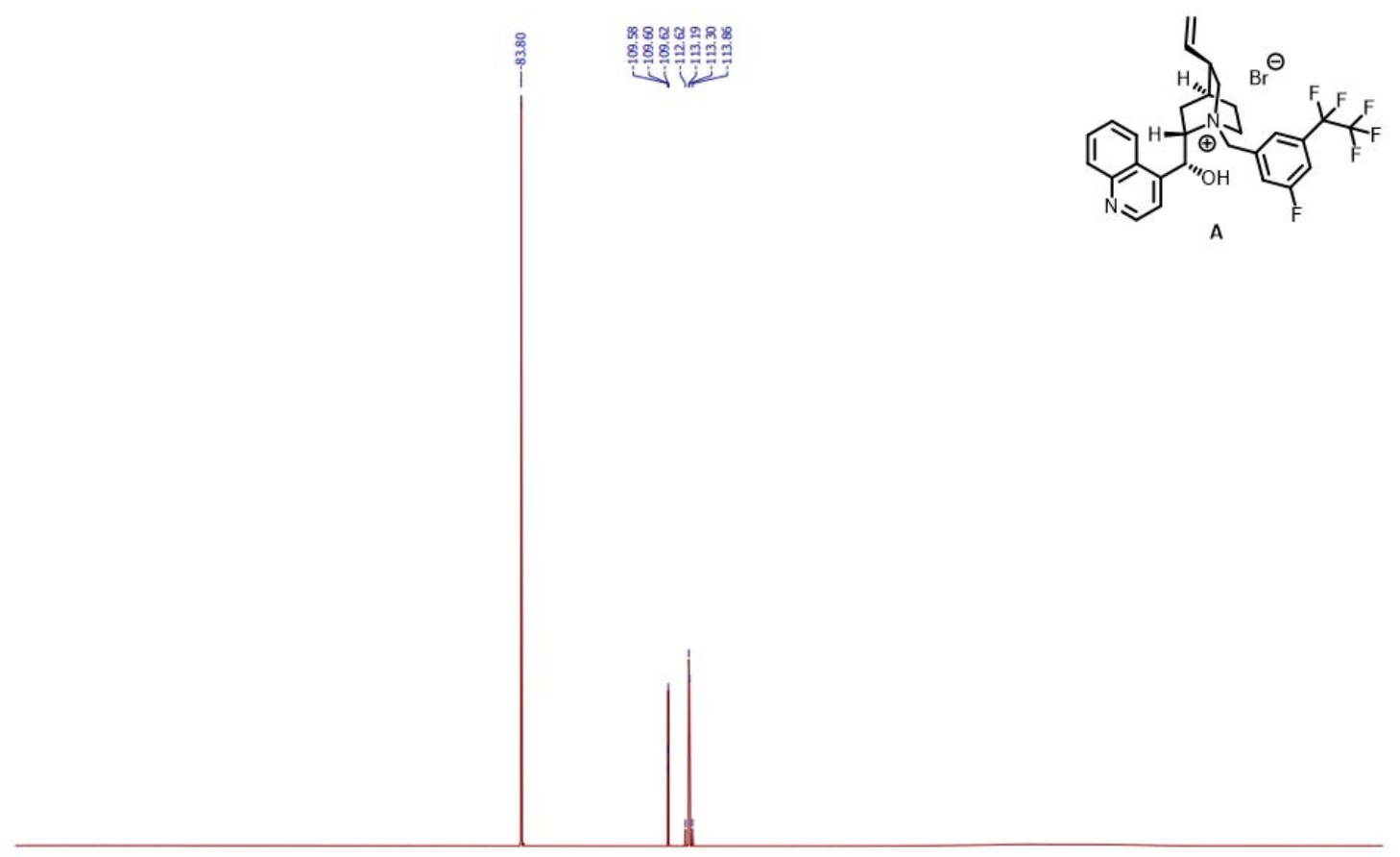

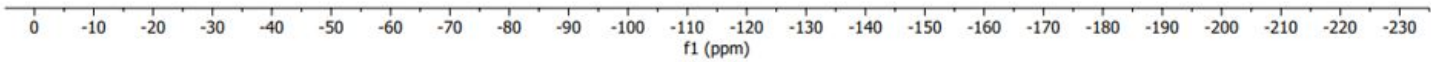

Figure S3. ${ }^{19} \mathrm{~F}$ NMR (471 MHz) spectrum of $\mathbf{A}$ in DMSO- $d_{6}$. 


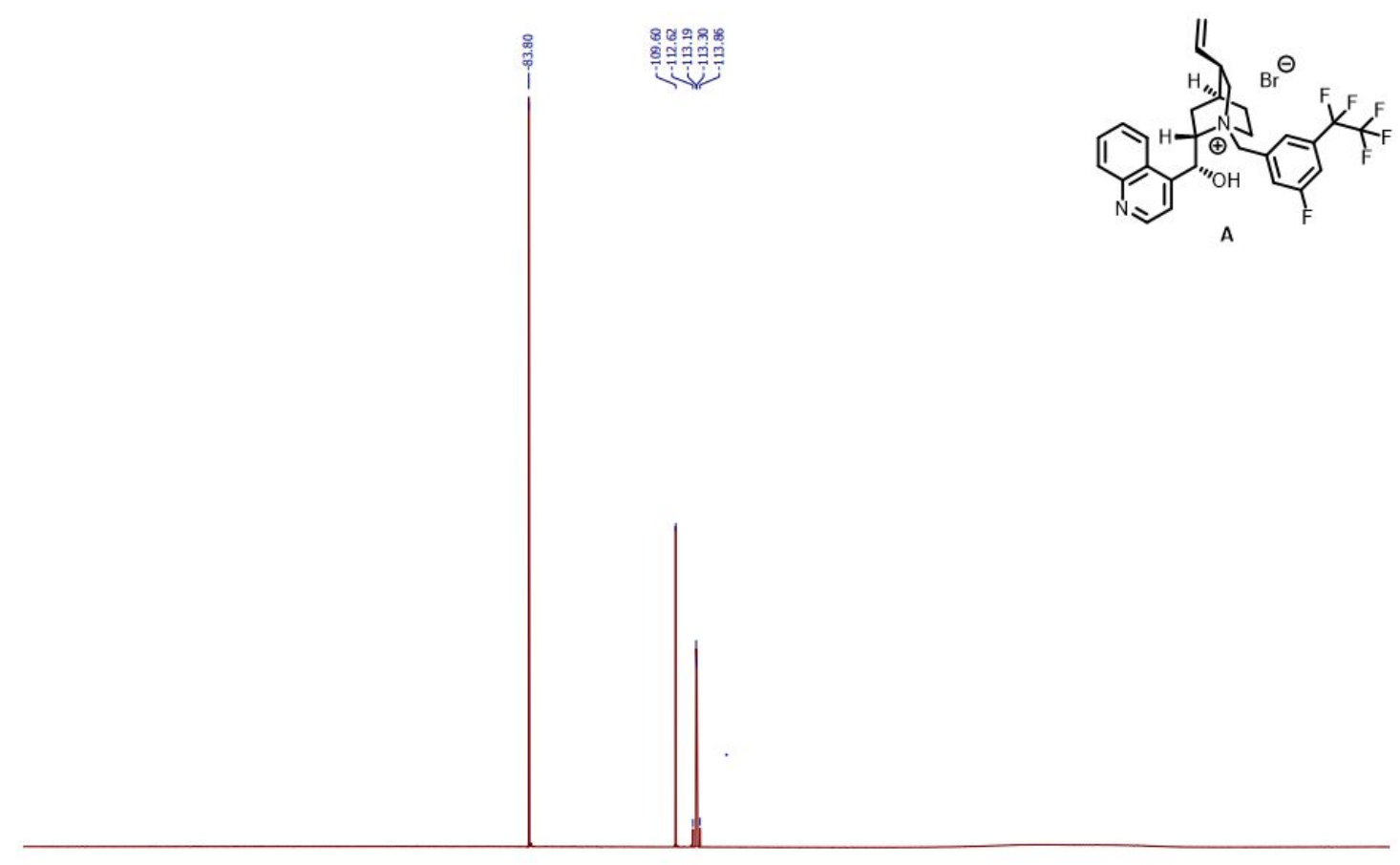

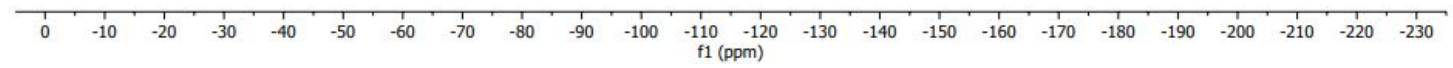

Figure S4. ${ }^{19} \mathrm{~F}\left\{{ }^{1} \mathrm{H}\right\}$ NMR $(471 \mathrm{MHz})$ spectrum of $\mathbf{A}$ in DMSO- $d_{6}$.

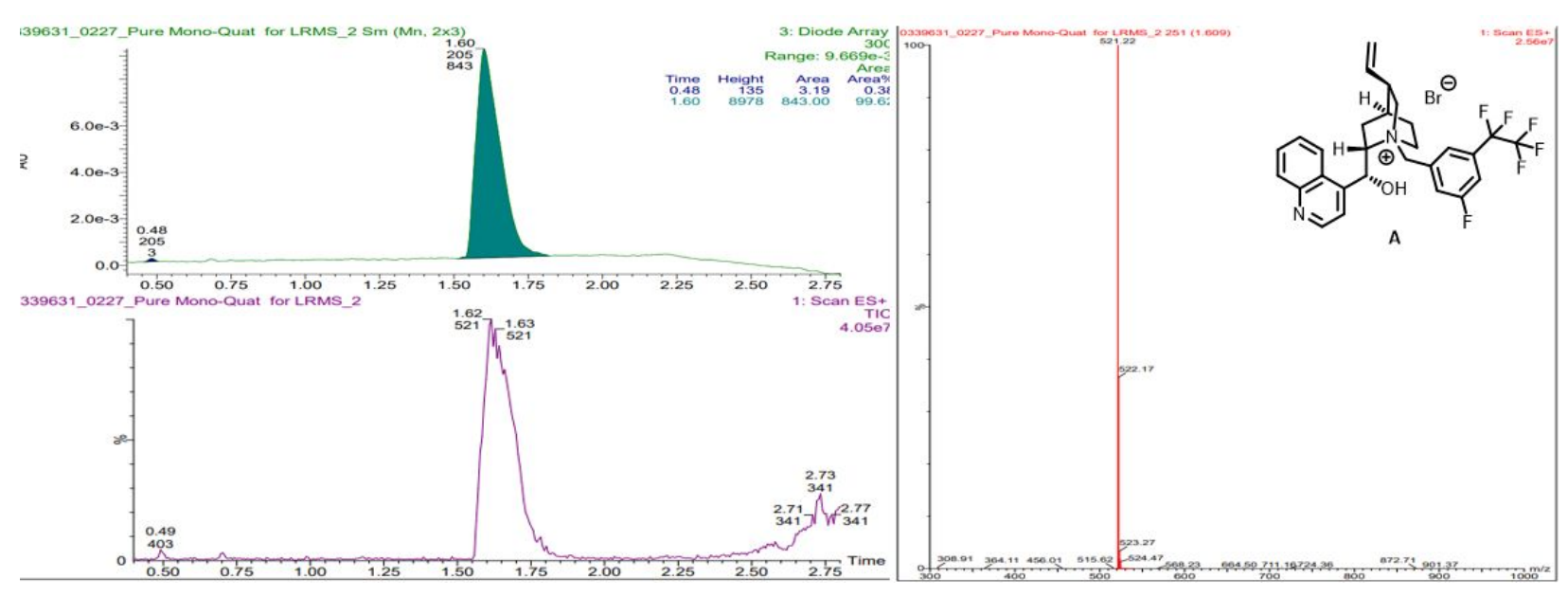

Figure S5. UPLC-MS and Low resolution $\mathrm{ESI}^{+} \mathrm{MS}$ of $\mathbf{A}$. 

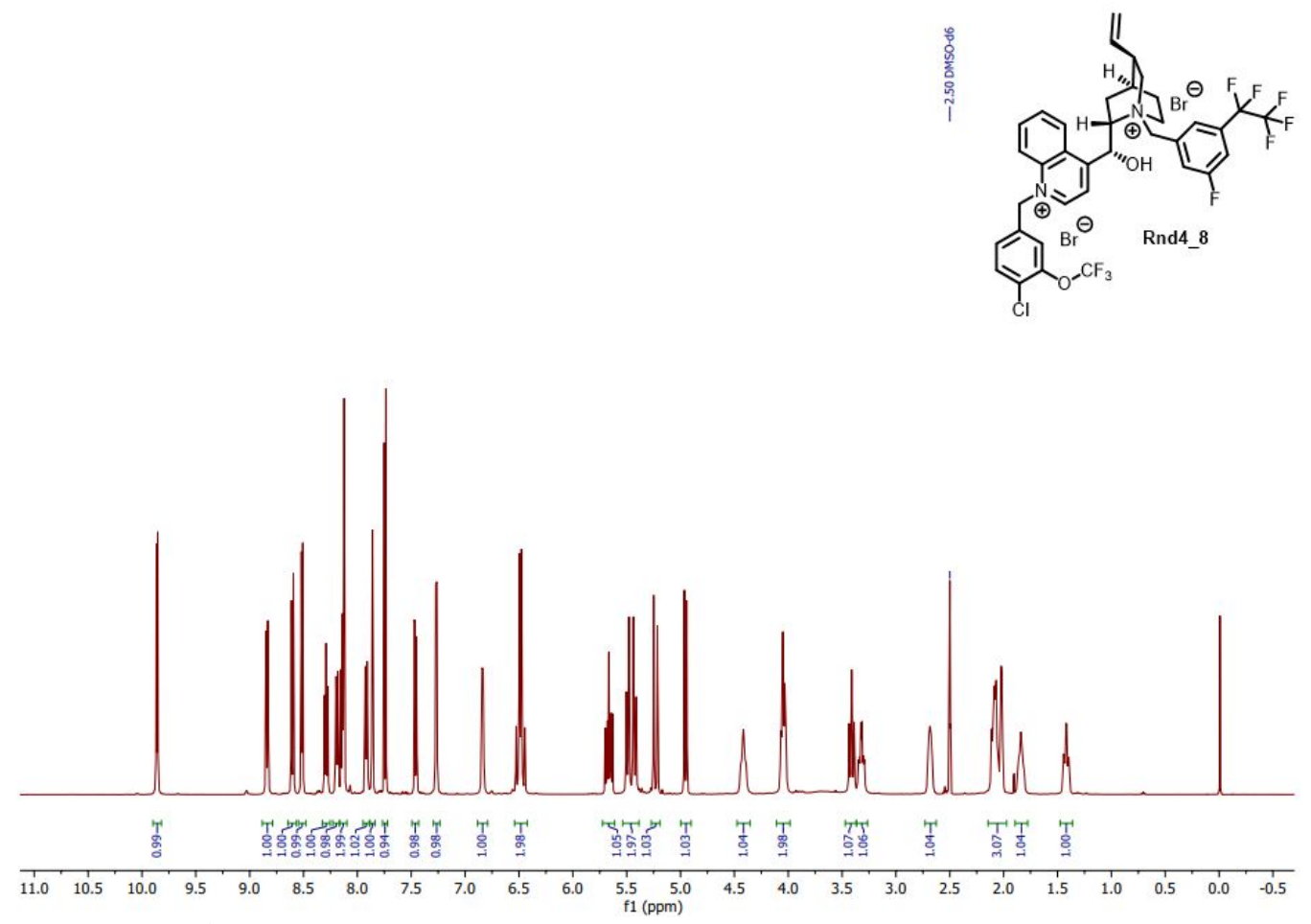

Figure S6. ${ }^{1} \mathrm{H}$ NMR $(500 \mathrm{MHz})$ spectrum of Rnd4_8 in DMSO- $d_{6}$.

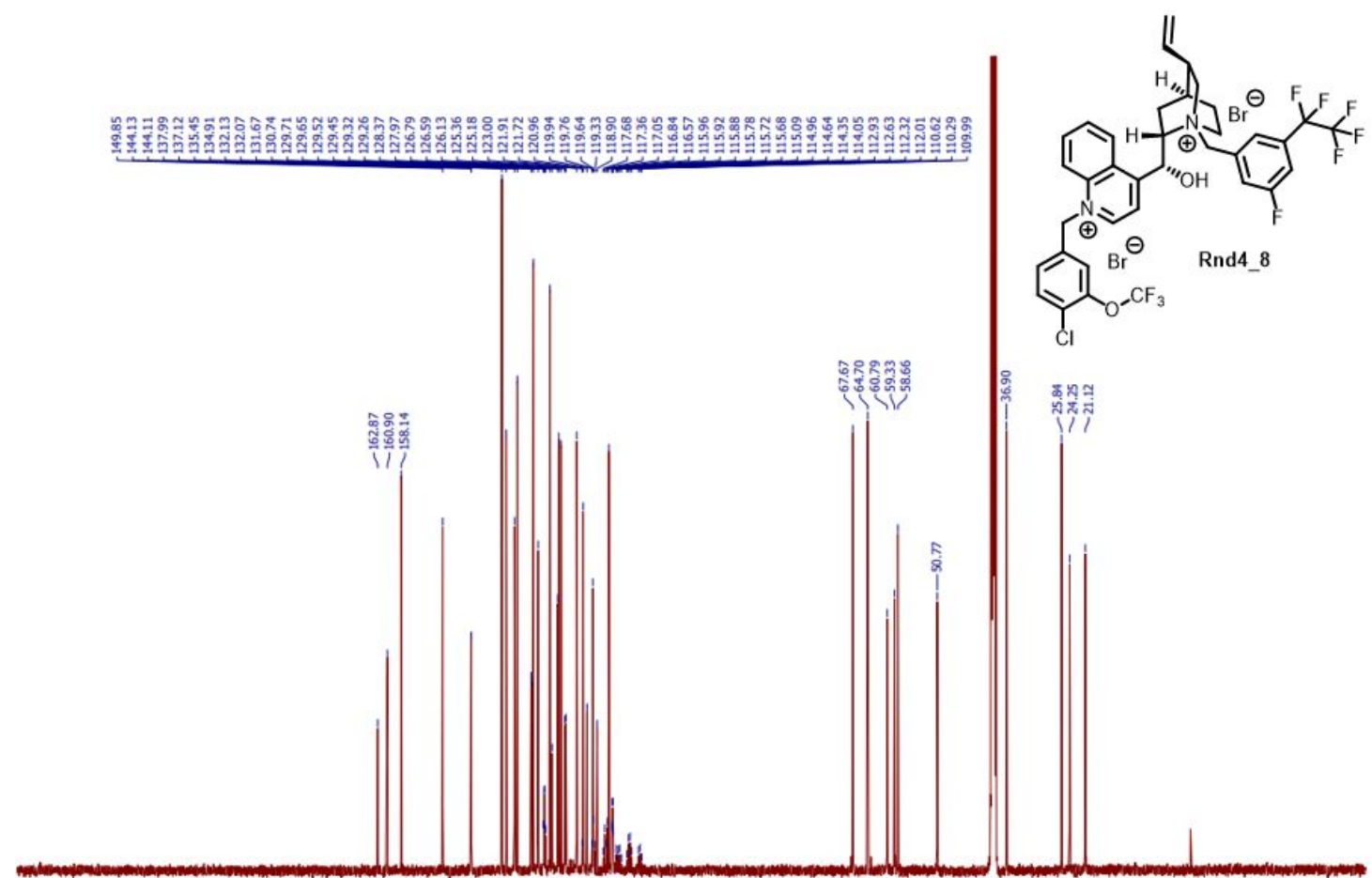

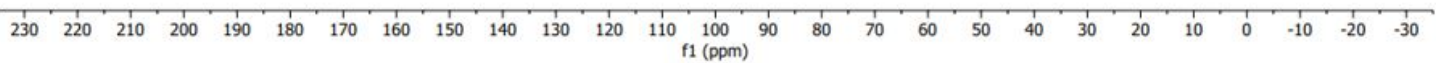

Figure S7. ${ }^{13} \mathrm{C}\left\{{ }^{1} \mathrm{H}\right\}$ NMR $(126 \mathrm{MHz})$ spectrum of Rnd4_8 in DMSO- $d_{6}$. 

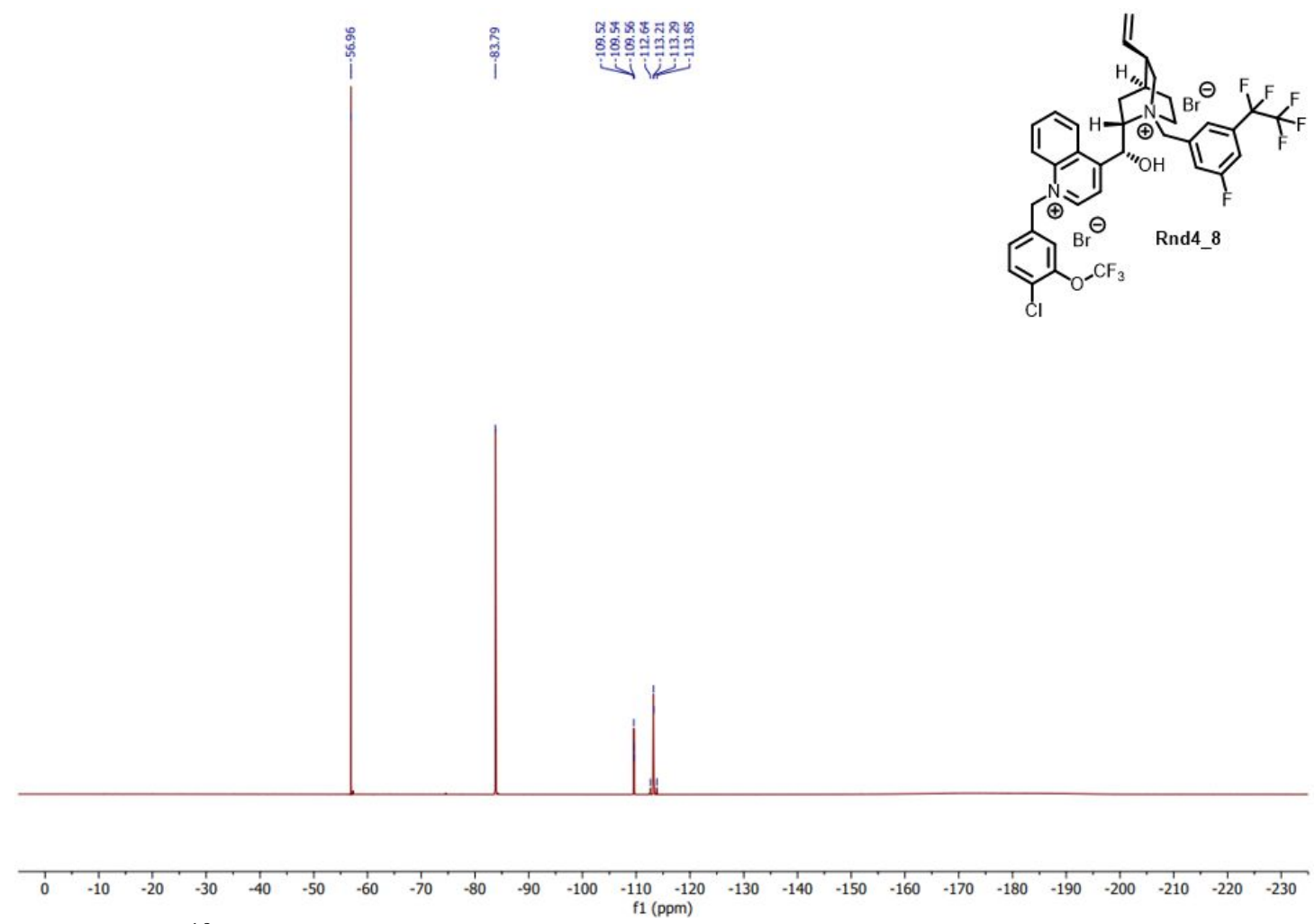

Figure S8. ${ }^{19}$ F NMR (471 MHz) spectrum of Rnd4_8 in DMSO- $d_{6}$. 

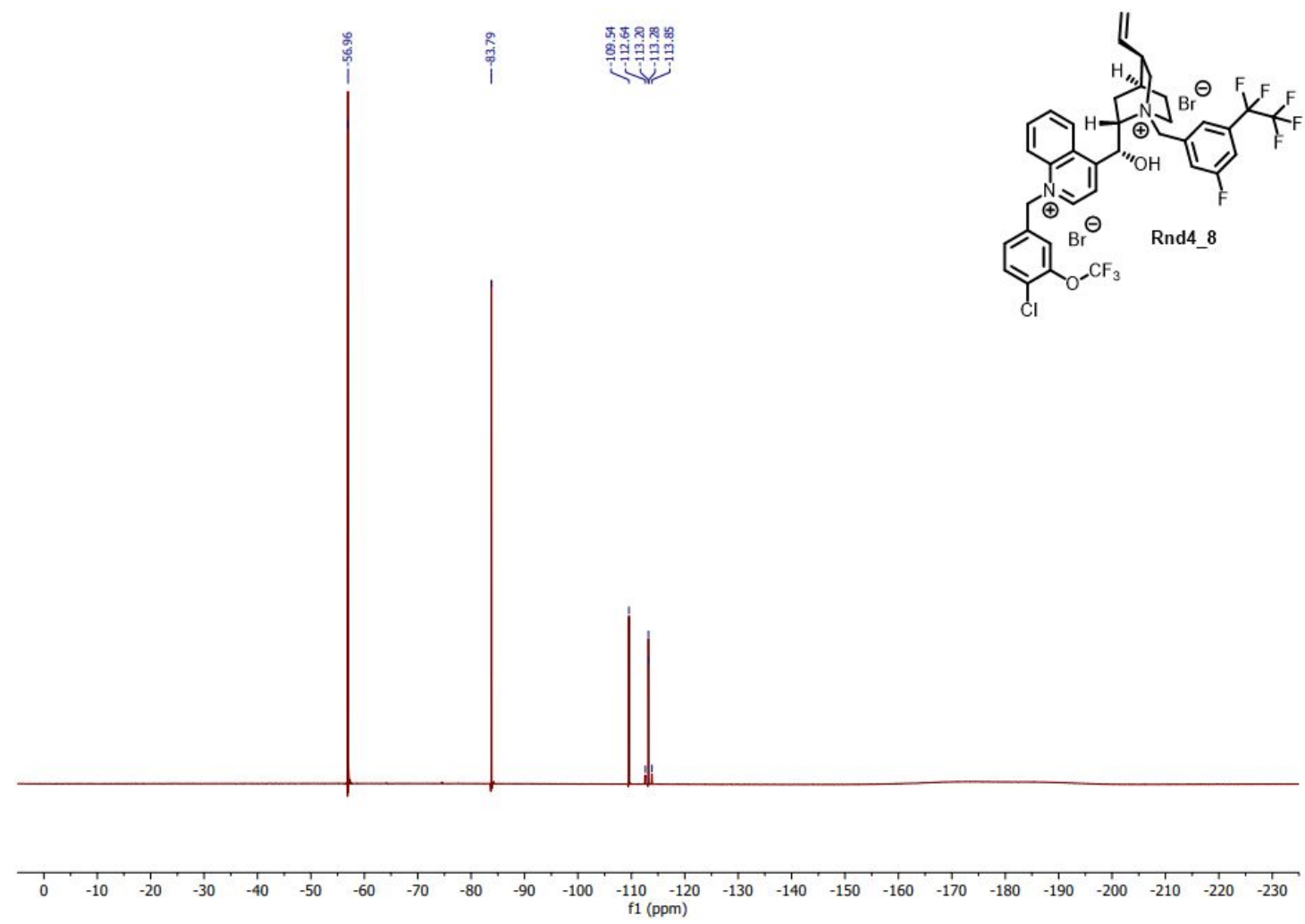

Figure S9. ${ }^{19} \mathrm{~F}\left\{{ }^{1} \mathrm{H}\right\}$ NMR (471 MHz) spectrum of Rnd4_8 in DMSO- $d_{6}$.

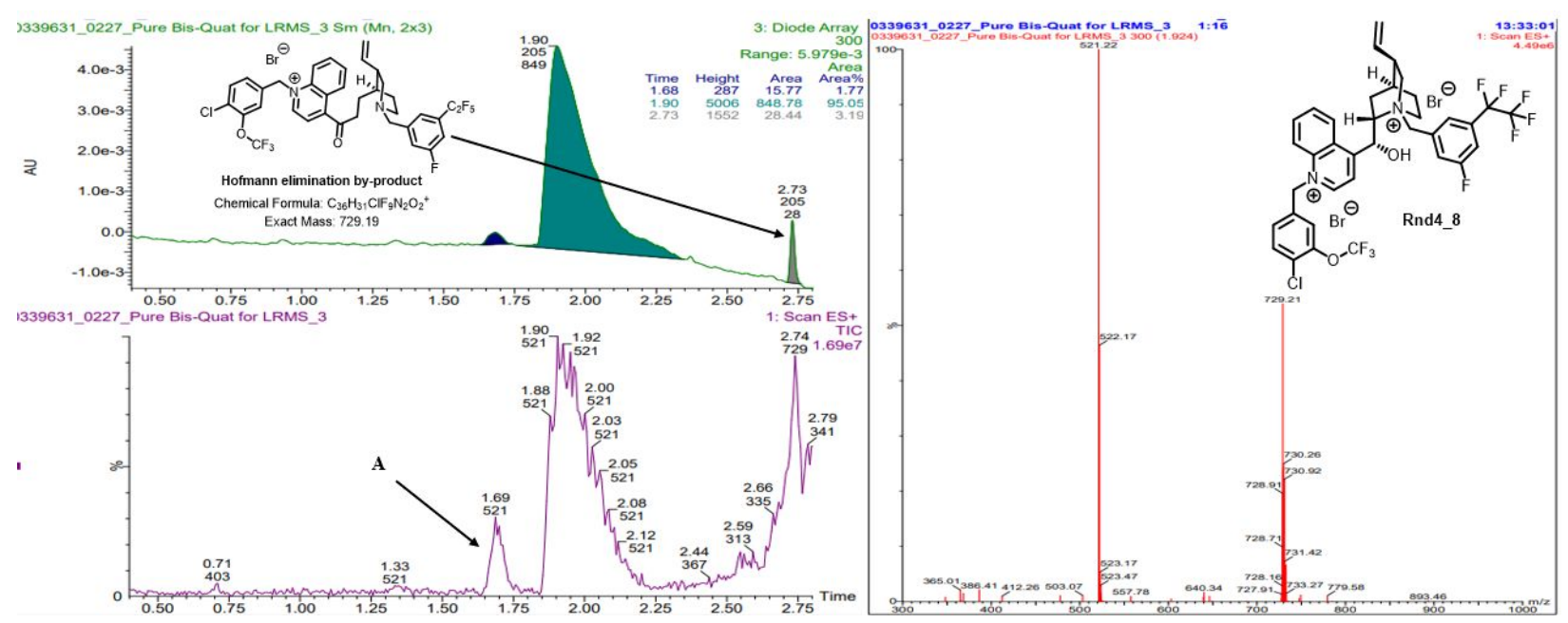

Figure S10. UPLC-MS and Low resolution ESI ${ }^{+}$MS of Rnd4_8. 


\section{Machine Learning}

\subsection{Additional background on machine learning}

Many types of 2D descriptors exist in the literature, with varying degrees of interpretability. In general, these 2D descriptors can be divided into two categories: substructures and computable physical properties. Through collective experience, it has been found internally at MRL that frequently, the most accurate $\mathrm{ML}$ method/descriptor combination is $\mathrm{RF}$ with atom pair ( $\mathrm{AP}^{6}$ ) descriptors (substructure type) combined with $\mathrm{MOE}^{7}$ 2D descriptors (physical properties). A common critique of using descriptors of this type is that they are not necessarily easily interpretable by humans to gain insight into mechanism. Operating with the desire to leverage more chemically intuitive descriptors, the Sigman lab has demonstrated the utility of descriptor sets such as Charton, Sterimol and QM-derived vibrational descriptors. ${ }^{8-13}$ Since we have had continued success with AP and MOE 2D descriptors, we pursued models using those methods here.

The workflow used to construct the 2D ML models is as follows:

1) From Excel of Chemdraw, export and SDF containing molecule names and 2D structures

2) For direct $2 \mathrm{D}$ descriptor calculations, perform no additional work-up of the molecules

a. All RF models presented in the main manuscript are based on 2D descriptors

3) For comparing how $2 \mathrm{D}$ descriptors may differ being calculated from flat molecular structures or from those structures converted to a single representative 3D structure (not necessarily the lowest energy nor an ensemble representation) with hydrogens (e.g., generation with Corina) it is possible to process the SDF through scripts which add hydrogens and perform minimizations. While this was not performed for this study, we routinely add hydrogens with in-house scripts and then minimize with Schrödinger's MacroMode ${ }^{14}$ using the MMFF forcefield.

4) The SDF is passed through in-house scripts which calculate a wide range of molecular descriptors but here are limited to $\mathrm{AP}^{6}, \mathrm{DP}^{15}$, and MOE $2 \mathrm{D}^{7}$.

5) The experimental enantioselectivity values are converted to the $\ln (\mathrm{er})$ where er is the enantiomeric ratio

6) The descriptors are fed into out in-house machine learning platform which runs a wide range of ML methods to determine which modeling method and descriptor combination is best

7) To determine which method is best for a given set of data, an $80 / 20$ split on the training data set where $80 \%$ is used to build the model, and $20 \%$ is used to test the model is performed. This $80 / 20$ split is performed randomly somewhere between 10 and 20 times to arrive at average model statistics which are referred to as X-trial cross-validated, e.g., 10-trial CV predictions.

a. The method which has the best CV-R ${ }^{2}$ is considered to be the best method for constructing the 'full model' from the available training set data.

b. Once we have determined which method is most internally statistically significant, we rebuild the model using all the data and then use that model to make predictions on forward looking molecular designs.

c. At this point we are no longer working under the construct of Train-Test-Validate and instead consider ourselves to have a full model derived from all available training set data and that is used for prospective predictions on compounds the model never saw.

8) Since the majority of the manuscript centers on support vector machine (SVM) and random forest (RF) we limit our detailed description to those two methods

a. When building random forest models we use an in-house version of the RF methodology, however, internal testing indicates the performance of our RF method is equivalent to that of commercially available RF methods in the statistical package $\mathrm{R}(\underline{\mathrm{R}}$ : What is $\mathrm{R}$ ? ( $\mathrm{r}-$ project.org)). 
b. Random Forest methodology

i. R module RandomForest

ii. Number of trees $=100$

iii. $m$ try $=\mathrm{M} / 3$ where $\mathrm{M}$ is the number of unique descriptors

iv. Node size $=5$

v. Model significance is gauged by $\mathrm{Y}$-scrambling the data and if the training set model is not significantly better than the Y-scrambled model, we do not look to prospectively apply the model for design

c. Support vector machine methodology

i. Internal implantation of the open source code: LIBSVM

ii. https://www.csie.ntu.edu.tw/ cjlin/libsvm/

iii. Parameter $\mathrm{C}$ is adjusted by cross-validation of the training set

iv. Kernel type $=$ linear

Descriptor importances are calculated by "permutation". That is, each descriptor k is randomly reassigned among all the molecules, and the RMSE prediction of the "out of bag sample" for the random forest trees is monitored. The most important descriptors are those for which the accuracy of prediction falls the most. We use Y-scrambled datasets to determine what the baseline descriptor importances look like, i.e. when there is no structure-activity.

Explanation of descriptors in addition to what is presented in the main text:

\section{AP (atom pair) descriptors}

The reference is Carhart et al. J. Chem. Inf. Comput. Sci. 1985, 25, 64-73.

Note that the reference handles the nomenclature differently.

Our in-house implementation has a form like C10Br1006.

You can think of it as the following:

Atom type $1=\mathrm{C} 10$

Atom type $2=\mathrm{Br} 10$

Distance between them in bonds 06

Atom type 1 and atom type 2 are in canonical order (usually reverse alphabetical).

The type is further divided: $\mathrm{C}$ element type 1 the number of bonded non hydrogens and 0 the number of pi electrons. The pi electrons $0=\mathrm{sp} 3$ or no double bonds to the atom, $1=\mathrm{sp} 2$ or one double bond to the atom, and $2=\mathrm{sp} 3$ or two double bonds to the atom.

\section{DP descriptors}

These are called "BP” descriptors in Kearsley et al. J. Chem. Inf. Comput. Sci. 1996, 36, 118127.

They look like this: DP1206

Atom type $1=1$

Atom type $2=2$

Distance between them in bonds $=06$

Atom types are:

1 cation

2 anion

3 neutral H-bond donor

4 neutral H-bond acceptor 
5 polar. This can be both donor and acceptor (as in hydroxide) or ambiguous donor/acceptor because of tautomerization as in imidazole.

6. hydrophobic atom

7. none of the above. Carboxy carbon would be an example.

Again the order is canonicalized

\subsection{Details of additional ML models explored}

For the 3D approach, structures were prepared and minimized using with the MMFF94x force field as implemented in MOE. ${ }^{7}$ The descriptor set that was utilized for model generation and catalyst selection were QM-calculated electrostatic molecular interaction field (MIF) and molecular mechanics-calculated steric potential energy MIF descriptors. ${ }^{16,}{ }^{17}$ Additional analyses were performed using GRIND ${ }^{18}$ and ASO+ESPMAX ${ }^{19}$ descriptor sets. Conformational studies were performed using MOE, with LowModeMD sampling of the conformer space. Library alignment (superposition) and Leonard-Jones Potential (L-J) CoMFA models were accomplished using Tripos' SYBYL-X55 3D-QSAR suite. Additional 3D-QSAR models were made using MIF descriptors using inhouse modeling scripts to generate PLS and Multivariate Adaptive Regression Splines (MARS) ${ }^{20}$ models. CV methods used Leave-One-Out (LOO) $\mathrm{R}^{2}$ to determine the predictivity of models.

The CoMFA model (Figure S11) was generated for Round 1 using SYBYL-X ${ }^{21}$, giving a 6-component PLS model with $\mathrm{R}^{2} \mathrm{LOO}=0.50$ and $\mathrm{R}^{2}=0.77$ (self-fit). An interpretation of the model is provided in Figure S1. Initial modeling of the conformational ensembles of Round 1 PTCs indicated greater conformational flexibility of the R1 substituents. It was hypothesized that this variability in PTC substituent overlays might be driving the worse performance of the CoMFA approach. In order to better address the overlay problem, a low energy conformer of Rnd1_1 was complexed with a model fragment of the guanidinate anion of letermovir. The R1 and R2 substituents of the remaining 177 member library were built in virtually in the context of this catalyst-substrate complex. In this new model, an 8-component PLS model based on L-J CoMFA descriptors had improved self-fit predictivity $\mathrm{R}^{2} \mathrm{LOO}=0.46$ and $\mathrm{R}^{2}=0.85$ (self-fit). The improvement in selfconsistency of the model was attributed to focusing the conformational search space on the 177 member substrate complexed set. It was gratifying that a better representation of the chemical environment provided an improvement in statistical significance ( $\mathrm{R}^{2}$ self-fit), and a clear freezing out of R1 conformational space was noted in the overlays. Additional work constraining dihedrals to limit ensemble variability or applying MIF descriptors and MARS models failed to improve the predictivity and are not discussed further.
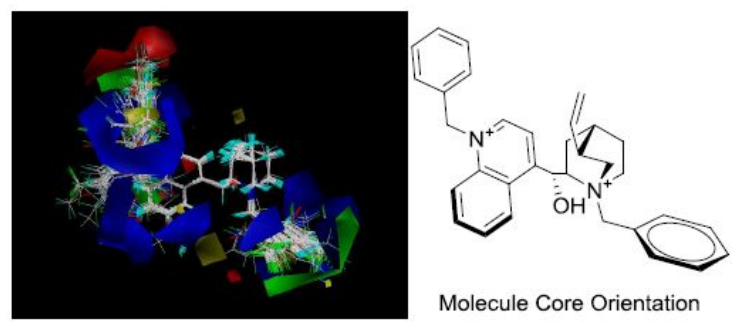

Figure S11: CoMFA model fields generated for Round 1 (blue: increasing positive charge beneficial, red: increasing negative charge beneficial, green: increasing steric bulk beneficial, yellow: decreasing steric bulk beneficial). Right: 
Orientation of the bis-quat catalysts in the CoMFA

field display to the left.

\subsection{Additional descriptors from the combined RF model}

Table S1 provides additional descriptors from the combined random forest model which have weights lower than the +/- 2.9 threshold described in the paper. These descriptors relate to the presence of fluorine content and help to explain why more heavily fluorinated catalysts are predicted in later rounds of optimization.

Table S1: Importance of descriptors from the RF AP/DP ML model (DP descriptors begin 'DP', all others AP)

\begin{tabular}{|c|c|c|c|}
\hline Descriptor & Coefficient & Impact & Interpretation \\
\hline C40C2012 & 2.72 & Increase er & $\begin{array}{l}\mathrm{sp}^{3} \mathrm{C} w / o \text { Hs to } \mathrm{sp}^{3} \mathrm{w} / 2 \mathrm{H}-12 \\
\text { bonds. This is CF2 }\end{array}$ \\
\hline C31C2111 & 2.40 & Increase er & $\begin{array}{l}\mathrm{sp}^{2} \mathrm{Cw} / \mathrm{o} \mathrm{Hs} \text { to } \mathrm{sp}^{2} \mathrm{C} w / 1 \mathrm{H}-11 \\
\text { bonds. This is bis-quat } \mathrm{R} 1 / \mathrm{R} 2 \\
\text { aromatics }\end{array}$ \\
\hline F10C3014 & 1.71 & Increase er & $\begin{array}{l}\mathrm{F} \text { to } \mathrm{sp}^{3} \mathrm{C} w / 1 \mathrm{H}-14 \text { bonds. This } \\
\text { is presence of } \mathrm{CF} 3\end{array}$ \\
\hline F10C2014 & 1.68 & Increase er & $\begin{array}{l}\mathrm{F} \text { to } \mathrm{sp}^{3} \mathrm{Cw} / 2 \mathrm{H}-14 \text { bonds. This } \\
\text { is presence of } \mathrm{CF} 3 \text { to quinuclidine }\end{array}$ \\
\hline O20C3104 & -1.91 & Decrease er & $\begin{array}{l}\text { Ether } 0 \text { to } \mathrm{sp}^{2} \mathrm{C} \text { w/o Hs }-4 \text { bonds. } \\
\text { This is methoxy on the quinoline } \\
\text { and methoxy on R2 }\end{array}$ \\
\hline C31C1002 & -2.54 & Decrease er & $\begin{array}{l}\mathrm{sp}^{2} \mathrm{C} w / \mathrm{o} \mathrm{Hs} \text { to } \mathrm{sp}^{3} \mathrm{C} \mathrm{w} / 3 \mathrm{Hs}-2 \\
\text { bonds. }\end{array}$ \\
\hline
\end{tabular}

3.4 Table of catalyst names and random forest predictions. An indication is made as to what type of prediction the RF values represent, and whether the catalysts was synthesized as a repeat and part of a previous training set.

\begin{tabular}{|c|c|c|c|c|c|}
\hline Name & Repeat? & $\begin{array}{c}\text { RF Prediction } \\
\text { In (er) }\end{array}$ & $\begin{array}{c}\text { Measured } \\
\text { In(er) }\end{array}$ & Dataset & Prediciton Type \\
\hline Rnd1_1 & & 1.58 & 2.14 & Training & Cross validated training set \\
\hline Rnd1_2 & 1.47 & 2.04 & Training & Cross validated training set \\
\hline Rnd1_3 & 1.48 & 1.99 & Training & Cross validated training set \\
\hline Rnd1_4 & 1.52 & 1.99 & Training & Cross validated training set \\
\hline Rnd1_5 & 0.95 & 1.98 & Training & Cross validated training set \\
\hline Rnd1_6 & 1.39 & 1.90 & Training & Cross validated training set \\
\hline Rnd1_7 & 1.41 & 1.89 & Training & Cross validated training set \\
\hline Rnd1_8 & 1.50 & 1.89 & Training & Cross validated training set \\
\hline Rnd1_9 & 1.47 & 1.89 & Training & Cross validated training set \\
\hline Rnd1_10 & 1.42 & 1.88 & Training & Cross validated training set \\
\hline
\end{tabular}




\begin{tabular}{|c|c|c|c|c|}
\hline Rnd1_11 & 1.42 & 1.87 & Training & Cross validated training set \\
\hline Rnd1_12 & 1.63 & 1.87 & Training & Cross validated training set \\
\hline Rnd1_13 & 1.58 & 1.84 & Training & Cross validated training set \\
\hline Rnd1_14 & 1.52 & 1.82 & Training & Cross validated training set \\
\hline Rnd1_15 & 1.28 & 1.82 & Training & Cross validated training set \\
\hline Rnd1_16 & 1.52 & 1.82 & Training & Cross validated training set \\
\hline Rnd1_17 & 1.33 & 1.82 & Training & Cross validated training set \\
\hline Rnd1_18 & 1.34 & 1.80 & Training & Cross validated training set \\
\hline Rnd1_19 & 1.32 & 1.79 & Training & Cross validated training set \\
\hline Rnd1_20 & 1.56 & 1.79 & Training & Cross validated training set \\
\hline Rnd1_21 & 1.03 & 1.79 & Training & Cross validated training set \\
\hline Rnd1_22 & 1.68 & 1.79 & Training & Cross validated training set \\
\hline Rnd1_23 & 1.19 & 1.77 & Training & Cross validated training set \\
\hline Rnd1_24 & 1.28 & 1.77 & Training & Cross validated training set \\
\hline Rnd1_25 & 1.58 & 1.75 & Training & Cross validated training set \\
\hline Rnd1_26 & 1.26 & 1.73 & Training & Cross validated training set \\
\hline Rnd1_27 & 1.43 & 1.73 & Training & Cross validated training set \\
\hline Rnd1_28 & 1.42 & 1.70 & Training & Cross validated training set \\
\hline Rnd1_29 & 1.57 & 1.69 & Training & Cross validated training set \\
\hline Rnd1_30 & 1.64 & 1.69 & Training & Cross validated training set \\
\hline Rnd1_31 & 1.62 & 1.67 & Training & Cross validated training set \\
\hline Rnd1_32 & 1.22 & 1.66 & Training & Cross validated training set \\
\hline Rnd1_33 & 1.00 & 1.62 & Training & Cross validated training set \\
\hline Rnd1_34 & 1.45 & 1.62 & Training & Cross validated training set \\
\hline Rnd1_35 & 1.38 & 1.62 & Training & Cross validated training set \\
\hline Rnd1_36 & 0.88 & 1.62 & Training & Cross validated training set \\
\hline Rnd1_37 & 1.21 & 1.61 & Training & Cross validated training set \\
\hline Rnd1_38 & 1.45 & 1.61 & Training & Cross validated training set \\
\hline Rnd1_39 & 1.30 & 1.61 & Training & Cross validated training set \\
\hline Rnd1_40 & 1.20 & 1.60 & Training & Cross validated training set \\
\hline Rnd1_41 & 1.46 & 1.56 & Training & Cross validated training set \\
\hline Rnd1_42 & 1.69 & 1.56 & Training & Cross validated training set \\
\hline Rnd1_43 & 1.38 & 1.56 & Training & Cross validated training set \\
\hline Rnd1_44 & 1.52 & 1.55 & Training & Cross validated training set \\
\hline Rnd1_45 & 1.63 & 1.55 & Training & Cross validated training set \\
\hline Rnd1_46 & 0.78 & 1.55 & Training & Cross validated training set \\
\hline Rnd1_47 & 1.44 & 1.53 & Training & Cross validated training set \\
\hline Rnd1_48 & 1.25 & 1.52 & Training & Cross validated training set \\
\hline Rnd1_49 & 1.34 & 1.52 & Training & Cross validated training set \\
\hline Rnd1_50 & 1.03 & 1.51 & Training & Cross validated training set \\
\hline Rnd1_51 & 1.32 & 1.51 & Training & Cross validated training set \\
\hline Rnd1_52 & 1.01 & 1.51 & Training & Cross validated training set \\
\hline Rnd1_53 & 1.36 & 1.48 & Training & Cross validated training set \\
\hline Rnd1_54 & 1.14 & 1.47 & Training & Cross validated training set \\
\hline Rnd1_55 & 1.22 & 1.45 & Training & Cross validated training set \\
\hline Rnd1_56 & 1.02 & 1.45 & Training & Cross validated training set \\
\hline Rnd1_57 & 1.08 & 1.45 & Training & Cross validated training set \\
\hline Rnd1_58 & 1.20 & 1.39 & Training & Cross validated training set \\
\hline
\end{tabular}




\begin{tabular}{|c|c|c|c|c|}
\hline Rnd1_59 & 1.63 & 1.39 & Training & Cross validated training set \\
\hline Rnd1_60 & 1.21 & 1.39 & Training & Cross validated training set \\
\hline Rnd1_61 & 0.61 & 1.39 & Training & Cross validated training set \\
\hline Rnd1_62 & 0.58 & 1.36 & Training & Cross validated training set \\
\hline Rnd1_63 & 1.12 & 1.36 & Training & Cross validated training set \\
\hline Rnd1_64 & 1.33 & 1.36 & Training & Cross validated training set \\
\hline Rnd1_65 & 1.10 & 1.36 & Training & Cross validated training set \\
\hline Rnd1_66 & 0.96 & 1.32 & Training & Cross validated training set \\
\hline Rnd1_67 & 1.23 & 1.32 & Training & Cross validated training set \\
\hline Rnd1_68 & 1.41 & 1.30 & Training & Cross validated training set \\
\hline Rnd1_69 & 1.33 & 1.27 & Training & Cross validated training set \\
\hline Rnd1_70 & 1.24 & 1.27 & Training & Cross validated training set \\
\hline Rnd1_71 & 1.51 & 1.25 & Training & Cross validated training set \\
\hline Rnd1_72 & 1.06 & 1.24 & Training & Cross validated training set \\
\hline Rnd1_73 & 0.93 & 1.24 & Training & Cross validated training set \\
\hline Rnd1 74 & 1.28 & 1.23 & Training & Cross validated training set \\
\hline Rnd1 75 & 1.28 & 1.23 & Training & Cross validated training set \\
\hline Rnd1_76 & 1.44 & 1.21 & Training & Cross validated training set \\
\hline Rnd1_77 & 1.53 & 1.18 & Training & Cross validated training set \\
\hline Rnd1_78 & 1.54 & 1.18 & Training & Cross validated training set \\
\hline Rnd1_79 & 0.94 & 1.18 & Training & Cross validated training set \\
\hline Rnd1_80 & 1.44 & 1.18 & Training & Cross validated training set \\
\hline Rnd1_81 & 1.36 & 1.14 & Training & Cross validated training set \\
\hline Rnd1_82 & 0.62 & 1.13 & Training & Cross validated training set \\
\hline Rnd1_83 & 1.46 & 1.13 & Training & Cross validated training set \\
\hline Rnd1_84 & 1.61 & 1.13 & Training & Cross validated training set \\
\hline Rnd1_85 & 1.11 & 1.13 & Training & Cross validated training set \\
\hline Rnd1_86 & 1.18 & 1.13 & Training & Cross validated training set \\
\hline Rnd1_87 & 1.09 & 1.10 & Training & Cross validated training set \\
\hline Rnd1_88 & 1.15 & 1.10 & Training & Cross validated training set \\
\hline Rnd1_89 & 0.77 & 1.10 & Training & Cross validated training set \\
\hline Rnd1_90 & 0.96 & 1.10 & Training & Cross validated training set \\
\hline Rnd1_91 & 1.39 & 1.07 & Training & Cross validated training set \\
\hline Rnd1_92 & 1.06 & 1.07 & Training & Cross validated training set \\
\hline Rnd1_93 & 1.09 & 1.07 & Training & Cross validated training set \\
\hline Rnd1_94 & 1.14 & 0.97 & Training & Cross validated training set \\
\hline Rnd1_95 & 1.03 & 0.90 & Training & Cross validated training set \\
\hline Rnd1_96 & 0.57 & 0.90 & Training & Cross validated training set \\
\hline Rnd1_97 & 1.06 & 0.88 & Training & Cross validated training set \\
\hline Rnd1_98 & 0.50 & 0.85 & Training & Cross validated training set \\
\hline Rnd1_99 & 1.37 & 0.85 & Training & Cross validated training set \\
\hline Rnd1_100 & 1.42 & 0.82 & Training & Cross validated training set \\
\hline Rnd1_101 & 1.15 & 0.82 & Training & Cross validated training set \\
\hline Rnd1_102 & 0.79 & 0.82 & Training & Cross validated training set \\
\hline Rnd1_103 & 0.54 & 0.81 & Training & Cross validated training set \\
\hline Rnd1_104 & 0.35 & 0.78 & Training & Cross validated training set \\
\hline Rnd1_105 & 0.75 & 0.78 & Training & Cross validated training set \\
\hline Rnd1_106 & 1.26 & 0.78 & Training & Cross validated training set \\
\hline
\end{tabular}




\begin{tabular}{|c|c|c|c|c|}
\hline Rnd1_107 & 1.01 & 0.75 & Training & Cross validated training set \\
\hline Rnd1 108 & 0.98 & 0.73 & Training & Cross validated training set \\
\hline Rnd1_109 & 0.36 & 0.73 & Training & Cross validated training set \\
\hline Rnd1_110 & 1.48 & 0.71 & Training & Cross validated training set \\
\hline Rnd1_111 & 0.49 & 0.71 & Training & Cross validated training set \\
\hline Rnd1_112 & 0.32 & 0.71 & Training & Cross validated training set \\
\hline Rnd1_113 & 0.55 & 0.70 & Training & Cross validated training set \\
\hline Rnd1_114 & 0.82 & 0.69 & Training & Cross validated training set \\
\hline Rnd1_115 & 0.82 & 0.66 & Training & Cross validated training set \\
\hline Rnd1_116 & 0.38 & 0.64 & Training & Cross validated training set \\
\hline Rnd1_117 & 1.42 & 0.62 & Training & Cross validated training set \\
\hline Rnd1_118 & 0.81 & 0.62 & Training & Cross validated training set \\
\hline Rnd1_119 & 1.02 & 0.62 & Training & Cross validated training set \\
\hline Rnd1_120 & 0.73 & 0.58 & Training & Cross validated training set \\
\hline Rnd1_121 & 0.65 & 0.55 & Training & Cross validated training set \\
\hline Rnd1_122 & 1.22 & 0.51 & Training & Cross validated training set \\
\hline Rnd1_123 & 0.40 & 0.51 & Training & Cross validated training set \\
\hline Rnd1_124 & 0.64 & 0.51 & Training & Cross validated training set \\
\hline Rnd1_125 & 0.55 & 0.49 & Training & Cross validated training set \\
\hline Rnd1_126 & 0.63 & 0.49 & Training & Cross validated training set \\
\hline Rnd1_127 & 0.52 & 0.48 & Training & Cross validated training set \\
\hline Rnd1_128 & 0.48 & 0.45 & Training & Cross validated training set \\
\hline Rnd1_129 & 0.28 & 0.43 & Training & Cross validated training set \\
\hline Rnd1_130 & 1.36 & 0.41 & Training & Cross validated training set \\
\hline Rnd1_131 & 1.10 & 0.41 & Training & Cross validated training set \\
\hline Rnd1_132 & 1.40 & 0.41 & Training & Cross validated training set \\
\hline Rnd1_133 & 0.94 & 0.41 & Training & Cross validated training set \\
\hline Rnd1_134 & 0.47 & 0.41 & Training & Cross validated training set \\
\hline Rnd1_135 & 0.74 & 0.41 & Training & Cross validated training set \\
\hline Rnd1_136 & 1.24 & 0.41 & Training & Cross validated training set \\
\hline Rnd1_137 & 1.07 & 0.41 & Training & Cross validated training set \\
\hline Rnd1_138 & 0.62 & 0.41 & Training & Cross validated training set \\
\hline Rnd1_139 & 0.48 & 0.41 & Training & Cross validated training set \\
\hline Rnd1_140 & 1.00 & 0.41 & Training & Cross validated training set \\
\hline Rnd1_141 & 1.01 & 0.41 & Training & Cross validated training set \\
\hline Rnd1_142 & 0.77 & 0.41 & Training & Cross validated training set \\
\hline Rnd1_143 & 1.47 & 0.41 & Training & Cross validated training set \\
\hline Rnd1_144 & 1.06 & 0.41 & Training & Cross validated training set \\
\hline Rnd1_145 & 0.72 & 0.41 & Training & Cross validated training set \\
\hline Rnd1_146 & 1.23 & 0.38 & Training & Cross validated training set \\
\hline Rnd1_147 & 1.04 & 0.36 & Training & Cross validated training set \\
\hline Rnd1_148 & 0.60 & 0.36 & Training & Cross validated training set \\
\hline Rnd1_149 & 0.50 & 0.34 & Training & Cross validated training set \\
\hline Rnd1_150 & 0.58 & 0.32 & Training & Cross validated training set \\
\hline Rnd1_151 & 0.40 & 0.28 & Training & Cross validated training set \\
\hline Rnd1_152 & 0.35 & 0.28 & Training & Cross validated training set \\
\hline Rnd1_153 & 0.54 & 0.26 & Training & Cross validated training set \\
\hline Rnd1_154 & 0.37 & 0.26 & Training & Cross validated training set \\
\hline
\end{tabular}




\begin{tabular}{|c|c|c|c|c|}
\hline Rnd1_155 & 1.61 & 0.20 & Training & Cross validated training set \\
\hline Rnd1_156 & 0.34 & 0.20 & Training & Cross validated training set \\
\hline Rnd1_157 & 0.40 & 0.20 & Training & Cross validated training set \\
\hline Rnd1_158 & 0.55 & 0.20 & Training & Cross validated training set \\
\hline Rnd1_159 & 0.41 & 0.18 & Training & Cross validated training set \\
\hline Rnd1_160 & 0.93 & 0.18 & Training & Cross validated training set \\
\hline Rnd1_161 & 0.34 & 0.18 & Training & Cross validated training set \\
\hline Rnd1_162 & 0.42 & 0.18 & Training & Cross validated training set \\
\hline Rnd1_163 & 0.29 & 0.16 & Training & Cross validated training set \\
\hline Rnd1_164 & 0.25 & 0.14 & Training & Cross validated training set \\
\hline Rnd1_165 & 0.26 & 0.12 & Training & Cross validated training set \\
\hline Rnd1_166 & 0.33 & 0.10 & Training & Cross validated training set \\
\hline Rnd1_167 & 0.37 & 0.10 & Training & Cross validated training set \\
\hline Rnd1_168 & 0.30 & 0.10 & Training & Cross validated training set \\
\hline Rnd1_169 & 0.58 & 0.06 & Training & Cross validated training set \\
\hline Rnd1_170 & 0.47 & 0.06 & Training & Cross validated training set \\
\hline Rnd1_171 & 0.29 & 0.06 & Training & Cross validated training set \\
\hline Rnd1_172 & 0.26 & 0.06 & Training & Cross validated training set \\
\hline Rnd1_173 & 0.27 & -0.06 & Training & Cross validated training set \\
\hline Rnd1_174 & 1.34 & -0.14 & Training & Cross validated training set \\
\hline Rnd1_175 & 0.41 & -0.22 & Training & Cross validated training set \\
\hline Rnd1_176 & 0.27 & -0.26 & Training & Cross validated training set \\
\hline Rnd1_177 & 1.29 & -0.34 & Training & Cross validated training set \\
\hline Rnd1a_1 & 0.92 & 1.14 & Prospective & Prospective prediction \\
\hline Rnd1a_2 & 0.89 & 0.44 & Prospective & Prospective prediction \\
\hline Rnd1a 3 & 0.82 & 0.56 & Prospective & Prospective prediction \\
\hline Rnd1a_4 & 0.93 & 1.97 & Prospective & Prospective prediction \\
\hline Rnd1a_5 & 0.93 & 1.60 & Prospective & Prospective prediction \\
\hline Rnd1a_6 & 1.06 & 1.62 & Prospective & Prospective prediction \\
\hline Rnd1a_7 & 1.01 & 1.90 & Prospective & Prospective prediction \\
\hline Rnd1a_8 & 0.67 & 0.88 & Prospective & Prospective prediction \\
\hline Rnd1a_9 & 0.88 & 0.25 & Prospective & Prospective prediction \\
\hline Rnd1a_10 & 1.00 & 1.07 & Prospective & Prospective prediction \\
\hline Rnd1a_11 & 1.26 & 1.43 & Prospective & Prospective prediction \\
\hline Rnd1a_12 & 0.71 & 1.40 & Prospective & Prospective prediction \\
\hline Rnd1a_13 & 0.56 & 0.50 & Prospective & Prospective prediction \\
\hline Rnd1a_14 & 0.73 & 0.51 & Prospective & Prospective prediction \\
\hline Rnd1a_15 & 0.50 & 0.73 & Prospective & Prospective prediction \\
\hline Rnd1a_16 & 0.83 & 0.50 & Prospective & Prospective prediction \\
\hline Rnd1a_17 & 0.47 & 0.86 & Prospective & Prospective prediction \\
\hline Rnd1a_18 & 0.81 & 0.11 & Prospective & Prospective prediction \\
\hline Rnd1a_19 & 0.87 & 0.10 & Prospective & Prospective prediction \\
\hline Rnd1a_20 & 0.71 & 0.00 & Prospective & Prospective prediction \\
\hline Rnd2_1 & 0.91 & 1.68 & Prospective & Prospective prediction \\
\hline Rnd2_2 & 1.08 & 1.49 & Prospective & Prospective prediction \\
\hline Rnd2_3 & 1.74 & 1.60 & Prospective & Prospective prediction \\
\hline Rnd2_4 & 1.66 & 1.47 & Prospective & Prospective prediction \\
\hline Rnd2_5 & 1.79 & 2.11 & Prospective & Prospective prediction \\
\hline
\end{tabular}




\begin{tabular}{|c|c|c|c|c|}
\hline Rnd2_6 & 1.83 & 1.58 & Prospective & Prospective prediction \\
\hline Rnd2_7 & 1.26 & 1.67 & Prospective & Prospective prediction \\
\hline Rnd2_8 & 0.97 & 1.50 & Prospective & Prospective prediction \\
\hline Rnd2_9 & 1.11 & 1.49 & Prospective & Prospective prediction \\
\hline Rnd2_10 & 1.17 & 1.32 & Prospective & Prospective prediction \\
\hline Rnd2_11 & 1.69 & 1.92 & Prospective & Prospective prediction \\
\hline Rnd2_12 & 1.60 & 1.60 & Prospective & Prospective prediction \\
\hline Rnd2_13 & 1.68 & 2.21 & Prospective & Prospective prediction \\
\hline Rnd2_14 & 1.79 & 1.97 & Prospective & Prospective prediction \\
\hline Rnd2_15 & 1.40 & 1.19 & Prospective & Prospective prediction \\
\hline Rnd2_16 & 1.14 & 1.14 & Prospective & Prospective prediction \\
\hline Rnd2_17 & 1.21 & 1.55 & Prospective & Prospective prediction \\
\hline Rnd2_18 & 1.20 & 1.29 & Prospective & Prospective prediction \\
\hline Rnd2_19 & 1.75 & 2.03 & Prospective & Prospective prediction \\
\hline Rnd2_20 & 1.69 & 1.79 & Prospective & Prospective prediction \\
\hline Rnd2_21 & 1.77 & 2.28 & Prospective & Prospective prediction \\
\hline Rnd2_22 & 1.79 & 1.88 & Prospective & Prospective prediction \\
\hline Rnd2_23 & 1.39 & 1.67 & Prospective & Prospective prediction \\
\hline Rnd2_24 & 1.18 & 1.13 & Prospective & Prospective prediction \\
\hline Rnd2_25 & 1.05 & 1.47 & Prospective & Prospective prediction \\
\hline Rnd2_26 & 1.15 & 1.43 & Prospective & Prospective prediction \\
\hline Rnd2_27 & 1.61 & 1.55 & Prospective & Prospective prediction \\
\hline Rnd2_28 & 1.58 & 1.35 & Prospective & Prospective prediction \\
\hline Rnd2_29 & 1.63 & 2.00 & Prospective & Prospective prediction \\
\hline Rnd2_30 & 1.66 & 1.69 & Prospective & Prospective prediction \\
\hline Rnd2_31 & 1.36 & 1.63 & Prospective & Prospective prediction \\
\hline Rnd2_32 & 1.11 & 1.31 & Prospective & Prospective prediction \\
\hline Rnd2_33 & 0.87 & 1.21 & Prospective & Prospective prediction \\
\hline Rnd2_34 & 1.00 & 0.47 & Prospective & Prospective prediction \\
\hline Rnd2_35 & 1.51 & 1.45 & Prospective & Prospective prediction \\
\hline Rnd2_36 & 1.48 & 1.35 & Prospective & Prospective prediction \\
\hline Rnd2_37 & 1.47 & 1.79 & Prospective & Prospective prediction \\
\hline Rnd2_38 & 1.68 & 1.52 & Prospective & Prospective prediction \\
\hline Rnd2_39 & 1.16 & 0.95 & Prospective & Prospective prediction \\
\hline Rnd2_40 & 1.00 & 1.12 & Prospective & Prospective prediction \\
\hline Rnd2_41 & 1.00 & 1.49 & Prospective & Prospective prediction \\
\hline Rnd2_42 & 1.16 & 1.40 & Prospective & Prospective prediction \\
\hline Rnd2_43 & 1.68 & 1.63 & Prospective & Prospective prediction \\
\hline Rnd2_44 & 1.64 & 1.43 & Prospective & Prospective prediction \\
\hline Rnd2_45 & 1.63 & 2.10 & Prospective & Prospective prediction \\
\hline Rnd2_46 & 1.75 & 1.70 & Prospective & Prospective prediction \\
\hline Rnd2_47 & 1.33 & 1.64 & Prospective & Prospective prediction \\
\hline Rnd2_48 & 1.07 & 1.27 & Prospective & Prospective prediction \\
\hline Rnd2_49 & 0.93 & 1.29 & Prospective & Prospective prediction \\
\hline Rnd2_50 & 1.04 & 1.35 & Prospective & Prospective prediction \\
\hline Rnd2_51 & 1.71 & 1.76 & Prospective & Prospective prediction \\
\hline Rnd2_52 & 1.67 & 1.75 & Prospective & Prospective prediction \\
\hline Rnd2_53 & 1.69 & 1.96 & Prospective & Prospective prediction \\
\hline
\end{tabular}




\begin{tabular}{|c|c|c|c|c|c|}
\hline Rnd2_54 & & 1.74 & 1.81 & Prospective & Prospective prediction \\
\hline Rnd2_55 & & 1.24 & 1.39 & Prospective & Prospective prediction \\
\hline Rnd2_56 & & 1.12 & 1.29 & Prospective & Prospective prediction \\
\hline Rnd2_57 & & 1.00 & 1.40 & Prospective & Prospective prediction \\
\hline Rnd2_58 & & 1.13 & 1.21 & Prospective & Prospective prediction \\
\hline Rnd2_59 & & 1.58 & 1.47 & Prospective & Prospective prediction \\
\hline Rnd2_60 & & 1.56 & 1.46 & Prospective & Prospective prediction \\
\hline Rnd2_61 & & 1.54 & 1.98 & Prospective & Prospective prediction \\
\hline Rnd2_62 & & 1.67 & 1.52 & Prospective & Prospective prediction \\
\hline Rnd2_63 & & 1.23 & 1.61 & Prospective & Prospective prediction \\
\hline Rnd2_64 & & 1.11 & 1.07 & Prospective & Prospective prediction \\
\hline Rnd2_65 & & 1.04 & 1.52 & Prospective & Prospective prediction \\
\hline Rnd2_66 & & 1.19 & 1.36 & Prospective & Prospective prediction \\
\hline Rnd2_67 & & 1.72 & 1.50 & Prospective & Prospective prediction \\
\hline Rnd2_68 & & 1.65 & 1.59 & Prospective & Prospective prediction \\
\hline Rnd2_69 & & 1.65 & 1.77 & Prospective & Prospective prediction \\
\hline Rnd2_70 & & 1.76 & 1.68 & Prospective & Prospective prediction \\
\hline Rnd2_71 & & 1.37 & 1.47 & Prospective & Prospective prediction \\
\hline Rnd2_72 & & 1.05 & 1.30 & Prospective & Prospective prediction \\
\hline Rnd2_73 & & 0.74 & 0.60 & Prospective & Prospective prediction \\
\hline Rnd2 74 & & 0.83 & 0.47 & Prospective & Prospective prediction \\
\hline Rnd2_75 & & 1.34 & 1.45 & Prospective & Prospective prediction \\
\hline Rnd2_76 & & 1.22 & 1.47 & Prospective & Prospective prediction \\
\hline Rnd2_77 & & 1.20 & 1.20 & Prospective & Prospective prediction \\
\hline Rnd2_78 & & 1.48 & 1.30 & Prospective & Prospective prediction \\
\hline Rnd2_79 & & 0.96 & 0.93 & Prospective & Prospective prediction \\
\hline Rnd2_80 & & 0.92 & 0.84 & Prospective & Prospective prediction \\
\hline Rnd3_1 & Rnd2_1 & 0.96 & 1.63 & Repeat & Previous round training set \\
\hline Rnd3_2 & & 1.96 & 2.12 & Prospective & Prospective prediction \\
\hline Rnd3_3 & Rnd2_3 & 1.76 & 1.42 & Repeat & Previous round training set \\
\hline Rnd3_4 & Rnd2_4 & 1.68 & 1.37 & Repeat & Previous round training set \\
\hline Rnd3_5 & Rnd2_5 & 1.80 & 2.07 & Repeat & Previous round training set \\
\hline Rnd3_6 & Rnd2_6 & 1.81 & 1.33 & Repeat & Previous round training set \\
\hline Rnd3_7 & & 1.86 & 1.91 & Prospective & Prospective prediction \\
\hline Rnd3_8 & Rnd2_8 & 1.04 & 1.40 & Repeat & Previous round training set \\
\hline Rnd3_9 & Rnd2_25 & 1.04 & 1.43 & Repeat & Previous round training set \\
\hline Rnd3_10 & & 1.84 & 1.96 & Prospective & Prospective prediction \\
\hline Rnd3_11 & Rnd2_27 & 1.71 & 1.53 & Repeat & Previous round training set \\
\hline Rnd3_12 & Rnd2_28 & 1.65 & 1.35 & Repeat & Previous round training set \\
\hline Rnd3_13 & Rnd2_29 & 1.75 & 1.98 & Repeat & Previous round training set \\
\hline Rnd3_14 & Rnd2_30 & 1.65 & 1.50 & Repeat & Previous round training set \\
\hline Rnd3_15 & & 1.63 & 1.73 & Prospective & Prospective prediction \\
\hline Rnd3_16 & Rnd2_32 & 1.11 & 1.23 & Repeat & Previous round training set \\
\hline Rnd3_17 & & 1.14 & 1.56 & Prospective & Prospective prediction \\
\hline Rnd3_18 & & 1.97 & 2.18 & Prospective & Prospective prediction \\
\hline Rnd3_19 & & 1.76 & 1.79 & Prospective & Prospective prediction \\
\hline Rnd3_20 & & 1.71 & 1.71 & Prospective & Prospective prediction \\
\hline Rnd3_21 & & 1.81 & 2.29 & Prospective & Prospective prediction \\
\hline
\end{tabular}




\begin{tabular}{|c|c|c|c|c|c|}
\hline Rnd3_22 & & 1.83 & 1.87 & Prospective & Prospective prediction \\
\hline Rnd3_23 & & 1.62 & 1.74 & Prospective & Prospective prediction \\
\hline Rnd3_24 & & 1.11 & 1.03 & Prospective & Prospective prediction \\
\hline Rnd3_25 & Rnd2_41 & 1.01 & 1.46 & Repeat & Previous round training set \\
\hline Rnd3_26 & & 1.83 & 2.03 & Prospective & Prospective prediction \\
\hline Rnd3_27 & Rnd2_43 & 1.74 & 1.48 & Repeat & Previous round training set \\
\hline Rnd3_28 & Rnd2_44 & 1.67 & 1.35 & Repeat & Previous round training set \\
\hline Rnd3_29 & Rnd2_45 & 1.74 & 2.09 & Repeat & Previous round training set \\
\hline Rnd3_30 & Rnd2_46 & 1.69 & 1.59 & Repeat & Previous round training set \\
\hline Rnd3_31 & & 1.58 & 1.74 & Prospective & Prospective prediction \\
\hline Rnd3_32 & Rnd2_48 & 1.07 & 1.21 & Repeat & Previous round training set \\
\hline Rnd3_33 & Rnd2_49 & 1.01 & 1.19 & Repeat & Previous round training set \\
\hline Rnd3_34 & & 1.85 & 1.85 & Prospective & Prospective prediction \\
\hline Rnd3_35 & Rnd2_51 & 1.74 & 1.77 & Repeat & Previous round training set \\
\hline Rnd3_36 & Rnd2_52 & 1.67 & 1.73 & Repeat & Previous round training set \\
\hline Rnd3_37 & Rnd2_53 & 1.75 & 1.92 & Repeat & Previous round training set \\
\hline Rnd3_38 & Rnd2_54 & 1.75 & 1.79 & Repeat & Previous round training set \\
\hline Rnd3_39 & & 1.49 & 1.50 & Prospective & Prospective prediction \\
\hline Rnd3_40 & Rnd2_56 & 1.10 & 1.27 & Repeat & Previous round training set \\
\hline Rnd3_41 & Rnd2_57 & 0.83 & 1.29 & Repeat & Previous round training set \\
\hline Rnd3_42 & & 1.71 & 1.79 & Prospective & Prospective prediction \\
\hline Rnd3_43 & Rnd2_59 & 1.61 & 1.46 & Repeat & Previous round training set \\
\hline Rnd3_44 & Rnd2_60 & 1.59 & 1.33 & Repeat & Previous round training set \\
\hline Rnd3_45 & Rnd2_61 & 1.58 & 1.90 & Repeat & Previous round training set \\
\hline Rnd3_46 & Rnd2_62 & 1.69 & 1.59 & Repeat & Previous round training set \\
\hline Rnd3 47 & & 1.31 & 1.60 & Prospective & Prospective prediction \\
\hline Rnd3_48 & Rnd2_64 & 1.06 & 1.03 & Repeat & Previous round training set \\
\hline Rnd3_49 & Rnd2_65 & 1.00 & 1.45 & Repeat & Previous round training set \\
\hline Rnd3_50 & & 1.80 & 1.87 & Prospective & Prospective prediction \\
\hline Rnd3_51 & Rnd2_67 & 1.77 & 1.51 & Repeat & Previous round training set \\
\hline Rnd3_52 & Rnd2_68 & 1.69 & 1.44 & Repeat & Previous round training set \\
\hline Rnd3_53 & Rnd2_69 & 1.75 & 1.75 & Repeat & Previous round training set \\
\hline Rnd3_54 & Rnd2_70 & 1.72 & 1.68 & Repeat & Previous round training set \\
\hline Rnd3_55 & & 1.60 & 1.69 & Prospective & Prospective prediction \\
\hline Rnd3_56 & Rnd2_72 & 1.08 & 1.28 & Repeat & Previous round training set \\
\hline Rnd3_57 & & 0.17 & 0.88 & Prospective & Prospective prediction \\
\hline Rnd3_58 & & 0.78 & 1.39 & Prospective & Prospective prediction \\
\hline Rnd3_59 & & 1.23 & 1.24 & Prospective & Prospective prediction \\
\hline Rnd3_60 & & 1.26 & 1.27 & Prospective & Prospective prediction \\
\hline Rnd3_61 & & 1.06 & 1.57 & Prospective & Prospective prediction \\
\hline Rnd3_62 & & 1.10 & 1.40 & Prospective & Prospective prediction \\
\hline Rnd3_63 & & 0.60 & 0.99 & Prospective & Prospective prediction \\
\hline Rnd3_64 & & 0.77 & 0.76 & Prospective & Prospective prediction \\
\hline Rnd3_65 & & 0.65 & 1.41 & Prospective & Prospective prediction \\
\hline Rnd3_66 & & 1.44 & 2.00 & Prospective & Prospective prediction \\
\hline Rnd3_67 & & 1.61 & 1.84 & Prospective & Prospective prediction \\
\hline Rnd3_68 & & 1.57 & 1.66 & Prospective & Prospective prediction \\
\hline Rnd3_69 & & 1.50 & 2.09 & Prospective & Prospective prediction \\
\hline
\end{tabular}




\begin{tabular}{|c|c|c|c|c|c|}
\hline Rnd3_70 & & 1.71 & 1.89 & Prospective & Prospective prediction \\
\hline Rnd3_71 & & 1.13 & 1.66 & Prospective & Prospective prediction \\
\hline Rnd3_72 & & 1.03 & 1.20 & Prospective & Prospective prediction \\
\hline Rnd3_73 & Rnd2_9 & 1.07 & 1.33 & Repeat & Previous round training set \\
\hline Rnd3_74 & & 1.94 & 2.09 & Prospective & Prospective prediction \\
\hline Rnd3_75 & Rnd2_11 & 1.81 & 1.94 & Repeat & Previous round training set \\
\hline Rnd3_76 & Rnd2_12 & 1.73 & 1.67 & Repeat & Previous round training set \\
\hline Rnd3_77 & Rnd2_13 & 1.83 & 2.23 & Repeat & Previous round training set \\
\hline Rnd3_78 & Rnd2_14 & 1.88 & 2.02 & Repeat & Previous round training set \\
\hline Rnd3_79 & & 1.54 & 1.66 & Prospective & Prospective prediction \\
\hline Rnd3_80 & Rnd2_16 & 1.12 & 1.10 & Repeat & Previous round training set \\
\hline Rnd3_81 & & 1.13 & 1.54 & Prospective & Prospective prediction \\
\hline Rnd3_82 & & 2.02 & 2.15 & Prospective & Prospective prediction \\
\hline Rnd3_83 & Rnd2_19 & 1.76 & 2.06 & Repeat & Previous round training set \\
\hline Rnd3_84 & Rnd2_20 & 1.72 & 1.79 & Repeat & Previous round training set \\
\hline Rnd3_85 & Rnd2_21 & 1.81 & 2.27 & Repeat & Previous round training set \\
\hline Rnd3_86 & Rnd2_22 & 1.81 & 1.89 & Repeat & Previous round training set \\
\hline Rnd3_87 & & 1.62 & 1.76 & Prospective & Prospective prediction \\
\hline Rnd3_88 & Rnd2_24 & 1.15 & 1.05 & Repeat & Previous round training set \\
\hline Rnd4_1 & & 1.61 & 1.54 & Prospective & Prospective prediction \\
\hline Rnd4_2 & & 1.80 & 1.87 & Prospective & Prospective prediction \\
\hline Rnd4_3 & & 1.69 & 1.78 & Prospective & Prospective prediction \\
\hline Rnd4_4 & & 1.04 & 1.26 & Prospective & Prospective prediction \\
\hline Rnd4_5 & & 1.28 & 1.61 & Prospective & Prospective prediction \\
\hline Rnd4_6 & & 1.67 & 1.63 & Prospective & Prospective prediction \\
\hline Rnd4_7 & & 1.85 & 1.57 & Prospective & Prospective prediction \\
\hline Rnd4_8 & & 2.30 & 1.67 & Prospective & Prospective prediction \\
\hline Rnd4_9 & & 2.16 & 1.54 & Prospective & Prospective prediction \\
\hline Rnd4_10 & & 1.59 & 1.22 & Prospective & Prospective prediction \\
\hline Rnd4_11 & & 2.02 & 1.62 & Prospective & Prospective prediction \\
\hline Rnd4_12 & & 2.06 & 1.68 & Prospective & Prospective prediction \\
\hline Rnd4_13 & Rnd3_20 & 1.70 & 1.66 & Repeat & Previous round training set \\
\hline Rnd4_14 & Rnd3_21 & 2.28 & 2.01 & Repeat & Previous round training set \\
\hline Rnd4_15 & Rnd3_18 & 2.18 & 1.94 & Repeat & Previous round training set \\
\hline Rnd4_16 & & 1.70 & 1.31 & Prospective & Prospective prediction \\
\hline Rnd4_17 & Rnd3_19 & 1.83 & 1.78 & Repeat & Previous round training set \\
\hline Rnd4_18 & Rnd3_22 & 1.94 & 1.83 & Repeat & Previous round training set \\
\hline Rnd4_19 & Rnd2_12 & 1.68 & 1.64 & Repeat & Previous round training set \\
\hline Rnd4_20 & Rnd2_13 & 2.20 & 2.11 & Repeat & Previous round training set \\
\hline Rnd4_21 & Rnd3_74 & 2.03 & 1.93 & Repeat & Previous round training set \\
\hline Rnd4_22 & & 1.56 & 1.39 & Prospective & Prospective prediction \\
\hline Rnd4_23 & Rnd2_11 & 1.93 & 1.85 & Repeat & Previous round training set \\
\hline Rnd4_24 & Rnd2_14 & 2.00 & 1.96 & Repeat & Previous round training set \\
\hline Rnd4_25 & & 1.62 & 1.53 & Prospective & Prospective prediction \\
\hline Rnd4_26 & & 1.94 & 1.86 & Prospective & Prospective prediction \\
\hline Rnd4_27 & & 1.84 & 1.84 & Prospective & Prospective prediction \\
\hline Rnd4_28 & & 1.37 & 1.31 & Prospective & Prospective prediction \\
\hline Rnd4_29 & & 1.68 & 1.61 & Prospective & Prospective prediction \\
\hline
\end{tabular}




\begin{tabular}{|c|c|c|c|c|}
\hline Rnd4_30 & 1.74 & 1.69 & Prospective & Prospective prediction \\
\hline Rnd4_31 & 1.78 & 1.48 & Prospective & Prospective prediction \\
\hline Rnd4_32 & 1.89 & 1.76 & Prospective & Prospective prediction \\
\hline Rnd4_33 & 1.58 & 1.68 & Prospective & Prospective prediction \\
\hline Rnd4_34 & 1.51 & 1.08 & Prospective & Prospective prediction \\
\hline Rnd4_35 & 1.76 & 1.55 & Prospective & Prospective prediction \\
\hline Rnd4_36 & 1.64 & 1.57 & Prospective & Prospective prediction \\
\hline Rnd4_37 & 1.75 & 1.57 & Prospective & Prospective prediction \\
\hline Rnd4_38 & 2.33 & 1.91 & Prospective & Prospective prediction \\
\hline Rnd4_39 & 2.21 & 1.97 & Prospective & Prospective prediction \\
\hline Rnd4_40 & 1.56 & 1.36 & Prospective & Prospective prediction \\
\hline Rnd4_41 & 1.96 & 1.66 & Prospective & Prospective prediction \\
\hline Rnd4_42 & 2.05 & 1.74 & Prospective & Prospective prediction \\
\hline Rnd4_43 & 1.77 & 1.41 & Prospective & Prospective prediction \\
\hline Rnd4_44 & 1.82 & 1.17 & Prospective & Prospective prediction \\
\hline Rnd4_45 & 1.14 & 0.94 & Prospective & Prospective prediction \\
\hline Rnd4_46 & 1.15 & 0.69 & Prospective & Prospective prediction \\
\hline Rnd4_47 & 1.72 & 1.40 & Prospective & Prospective prediction \\
\hline Rnd4_48 & 1.40 & 1.22 & Prospective & Prospective prediction \\
\hline Rnd4_49 & 1.60 & 1.25 & Prospective & Prospective prediction \\
\hline Rnd4_50 & 1.42 & 1.20 & Prospective & Prospective prediction \\
\hline Rnd4_51 & 1.22 & 1.00 & Prospective & Prospective prediction \\
\hline Rnd4_52 & 1.28 & 0.81 & Prospective & Prospective prediction \\
\hline Rnd4_53 & 1.72 & 1.27 & Prospective & Prospective prediction \\
\hline Rnd4_54 & 1.74 & 1.20 & Prospective & Prospective prediction \\
\hline Rnd4_55 & 1.09 & 1.11 & Prospective & Prospective prediction \\
\hline Rnd4 56 & 1.09 & 1.08 & Prospective & Prospective prediction \\
\hline Rnd4_57 & 0.93 & 0.93 & Prospective & Prospective prediction \\
\hline Rnd4_58 & 0.67 & 0.89 & Prospective & Prospective prediction \\
\hline Rnd4_59 & 1.26 & 1.11 & Prospective & Prospective prediction \\
\hline Rnd4_60 & 1.09 & 1.01 & Prospective & Prospective prediction \\
\hline Rnd4_61 & 0.99 & 1.17 & Prospective & Prospective prediction \\
\hline Rnd4_62 & 1.06 & 1.17 & Prospective & Prospective prediction \\
\hline Rnd4_63 & 0.79 & 1.06 & Prospective & Prospective prediction \\
\hline Rnd4_64 & 0.74 & 0.95 & Prospective & Prospective prediction \\
\hline Rnd4_65 & 1.06 & 1.17 & Prospective & Prospective prediction \\
\hline Rnd4_66 & 1.01 & 1.11 & Prospective & Prospective prediction \\
\hline Rnd4_67 & 1.15 & 1.28 & Prospective & Prospective prediction \\
\hline Rnd4_68 & 1.23 & 1.24 & Prospective & Prospective prediction \\
\hline Rnd4 69 & 0.96 & 1.05 & Prospective & Prospective prediction \\
\hline Rnd4_70 & 1.03 & 0.86 & Prospective & Prospective prediction \\
\hline Rnd4_71 & 1.27 & 1.32 & Prospective & Prospective prediction \\
\hline Rnd4_72 & 1.22 & 1.25 & Prospective & Prospective prediction \\
\hline Rnd4_73 & 0.86 & 1.11 & Prospective & Prospective prediction \\
\hline Rnd4_74 & 0.85 & 1.10 & Prospective & Prospective prediction \\
\hline Rnd4_75 & 0.74 & 0.98 & Prospective & Prospective prediction \\
\hline Rnd4_76 & 0.71 & 0.84 & Prospective & Prospective prediction \\
\hline Rnd4_77 & 0.88 & 1.10 & Prospective & Prospective prediction \\
\hline
\end{tabular}




\begin{tabular}{|c|c|c|c|c|}
\hline Rnd4_78 & 0.92 & 1.04 & Prospective & Prospective prediction \\
\hline Rnd5_1 & 2.25 & 2.12 & Prospective & Prospective prediction \\
\hline Rnd5_2 & 2.23 & 2.03 & Prospective & Prospective prediction \\
\hline Rnd5_3 & 2.22 & 2.11 & Prospective & Prospective prediction \\
\hline Rnd5 4 & 2.21 & 2.06 & Prospective & Prospective prediction \\
\hline Rnd5_5 & 2.20 & 2.00 & Prospective & Prospective prediction \\
\hline Rnd5_6 & 2.18 & 2.10 & Prospective & Prospective prediction \\
\hline Rnd5 7 & 2.16 & 2.17 & Prospective & Prospective prediction \\
\hline Rnd5_8 & 2.06 & 1.97 & Prospective & Prospective prediction \\
\hline Rnd5_9 & 2.06 & 2.10 & Prospective & Prospective prediction \\
\hline Rnd5 10 & 2.04 & 2.00 & Prospective & Prospective prediction \\
\hline Rnd5_11 & 2.03 & 2.08 & Prospective & Prospective prediction \\
\hline Rnd5_12 & 2.03 & 2.02 & Prospective & Prospective prediction \\
\hline Rnd5 13 & 2.02 & 2.04 & Prospective & Prospective prediction \\
\hline Rnd5_14 & 2.01 & 2.04 & Prospective & Prospective prediction \\
\hline Rnd5 15 & 2.01 & 1.93 & Prospective & Prospective prediction \\
\hline Rnd5_16 & 1.97 & 1.93 & Prospective & Prospective prediction \\
\hline Rnd5 17 & 1.96 & 2.21 & Prospective & Prospective prediction \\
\hline Rnd5_18 & 1.94 & 1.96 & Prospective & Prospective prediction \\
\hline Rnd5_19 & 1.93 & 2.13 & Prospective & Prospective prediction \\
\hline Rnd5_20 & 1.92 & 1.95 & Prospective & Prospective prediction \\
\hline Rnd5_21 & 1.92 & 1.90 & Prospective & Prospective prediction \\
\hline Rnd5_22 & 1.90 & 1.92 & Prospective & Prospective prediction \\
\hline Rnd5_23 & 1.90 & 1.89 & Prospective & Prospective prediction \\
\hline Rnd5 24 & 1.87 & 1.91 & Prospective & Prospective prediction \\
\hline Rnd5_25 & 1.87 & 1.94 & Prospective & Prospective prediction \\
\hline Rnd5_26 & 1.86 & 1.97 & Prospective & Prospective prediction \\
\hline Rnd5_27 & 1.83 & 2.03 & Prospective & Prospective prediction \\
\hline Rnd5_28 & 1.82 & 1.98 & Prospective & Prospective prediction \\
\hline Rnd5_29 & 1.81 & 2.11 & Prospective & Prospective prediction \\
\hline Rnd5_30 & 1.80 & 1.90 & Prospective & Prospective prediction \\
\hline Rnd5_31 & 1.79 & 2.04 & Prospective & Prospective prediction \\
\hline Rnd5_32 & 1.79 & 1.89 & Prospective & Prospective prediction \\
\hline Rnd5_33 & 1.78 & 2.02 & Prospective & Prospective prediction \\
\hline Rnd5_34 & 1.77 & 1.87 & Prospective & Prospective prediction \\
\hline Rnd5_35 & 1.76 & 1.85 & Prospective & Prospective prediction \\
\hline Rnd5_36 & 1.74 & 1.85 & Prospective & Prospective prediction \\
\hline Rnd5_37 & 1.70 & 1.81 & Prospective & Prospective prediction \\
\hline Rnd5_38 & 1.68 & 1.87 & Prospective & Prospective prediction \\
\hline Rnd5_39 & 1.67 & 2.00 & Prospective & Prospective prediction \\
\hline Rnd5_40 & 1.66 & 1.80 & Prospective & Prospective prediction \\
\hline Rnd5_41 & 1.66 & 1.90 & Prospective & Prospective prediction \\
\hline Rnd5_42 & 1.65 & 1.92 & Prospective & Prospective prediction \\
\hline Rnd5_43 & 1.65 & 1.16 & Prospective & Prospective prediction \\
\hline Rnd5 44 & 1.65 & 2.14 & Prospective & Prospective prediction \\
\hline Rnd5_45 & 1.64 & 1.78 & Prospective & Prospective prediction \\
\hline Rnd5_46 & 1.63 & 2.03 & Prospective & Prospective prediction \\
\hline Rnd5_47 & 1.62 & 1.80 & Prospective & Prospective prediction \\
\hline
\end{tabular}




\begin{tabular}{|c|c|c|c|c|}
\hline Rnd5_48 & 1.62 & 1.85 & Prospective & Prospective prediction \\
\hline Rnd5_49 & 1.62 & 2.03 & Prospective & Prospective prediction \\
\hline Rnd5_50 & 1.62 & 1.18 & Prospective & Prospective prediction \\
\hline Rnd5_51 & 1.59 & 1.90 & Prospective & Prospective prediction \\
\hline Rnd5 52 & 1.56 & 1.75 & Prospective & Prospective prediction \\
\hline Rnd5_53 & 1.53 & 1.85 & Prospective & Prospective prediction \\
\hline Rnd5_54 & 1.52 & 1.83 & Prospective & Prospective prediction \\
\hline Rnd5_55 & 1.52 & 1.85 & Prospective & Prospective prediction \\
\hline Rnd5_56 & 1.50 & 1.88 & Prospective & Prospective prediction \\
\hline Rnd5_57 & 1.49 & 1.46 & Prospective & Prospective prediction \\
\hline Rnd5_58 & 1.49 & 1.20 & Prospective & Prospective prediction \\
\hline Rnd5_59 & 1.47 & 1.79 & Prospective & Prospective prediction \\
\hline Rnd5 60 & 1.46 & 1.88 & Prospective & Prospective prediction \\
\hline Rnd5_61 & 1.45 & 1.19 & Prospective & Prospective prediction \\
\hline Rnd5_62 & 1.45 & 1.72 & Prospective & Prospective prediction \\
\hline Rnd5_63 & 1.41 & 1.90 & Prospective & Prospective prediction \\
\hline Rnd5_64 & 1.41 & 1.87 & Prospective & Prospective prediction \\
\hline Rnd5_65 & 1.41 & 1.93 & Prospective & Prospective prediction \\
\hline Rnd5_66 & 1.39 & 1.80 & Prospective & Prospective prediction \\
\hline Rnd5_67 & 1.34 & 1.82 & Prospective & Prospective prediction \\
\hline Rnd5_68 & 1.34 & 1.86 & Prospective & Prospective prediction \\
\hline Rnd5_69 & 1.32 & 1.33 & Prospective & Prospective prediction \\
\hline Rnd5_70 & 1.31 & 1.51 & Prospective & Prospective prediction \\
\hline Rnd5 71 & 1.26 & 1.82 & Prospective & Prospective prediction \\
\hline Rnd5_72 & 1.25 & 1.82 & Prospective & Prospective prediction \\
\hline Rnd5 73 & 1.24 & 1.74 & Prospective & Prospective prediction \\
\hline Rnd5 74 & 1.23 & 1.89 & Prospective & Prospective prediction \\
\hline Rnd5 75 & 1.23 & 1.84 & Prospective & Prospective prediction \\
\hline Rnd5_76 & 1.22 & 1.90 & Prospective & Prospective prediction \\
\hline Rnd5_77 & 1.22 & 1.88 & Prospective & Prospective prediction \\
\hline Rnd5_78 & 1.17 & 1.87 & Prospective & Prospective prediction \\
\hline Rnd5_79 & 1.07 & 1.72 & Prospective & Prospective prediction \\
\hline Rnd5_80 & 1.06 & 1.74 & Prospective & Prospective prediction \\
\hline Rnd5_81 & 1.05 & 1.48 & Prospective & Prospective prediction \\
\hline Rnd5_82 & 1.02 & 1.34 & Prospective & Prospective prediction \\
\hline Rnd5_83 & 1.01 & 1.72 & Prospective & Prospective prediction \\
\hline Rnd5_84 & 0.99 & 1.39 & Prospective & Prospective prediction \\
\hline Rnd5_85 & 0.77 & 1.85 & Prospective & Prospective prediction \\
\hline
\end{tabular}




\subsection{Table of all SMILES strings and associated experimental data (selectivity)}

\begin{tabular}{|c|c|c|c|c|}
\hline Name & $\begin{array}{l}\text { Exp. } \\
\operatorname{Ln}(e r)\end{array}$ & Ratio & Exp.ee & Smiles \\
\hline Rnd1_1 & 2.14 & 8.52 & 79.00 & $\begin{array}{l}{[n+](C c 1 c c(C(F)(F) F) c c(C(F)(F) F) c 1)(c c c 2[C @ H]([C @] 3([N+] 4(C[C @ @ H]([C @ @]]([H])(C C 4) C 3) C=} \\
c) c c 5 c c(C(F)(F) F) c c(F) c 5)[H]) O[H]) c(\operatorname{cccc} 6) c 26\end{array}$ \\
\hline Rnd1_2 & 2.04 & 7.70 & 77.00 & $\begin{array}{l}{[n+](C c 1 c c(C(F)(F) F) c c c 1)(c c c 2[C @ H]([C @] 3([N+] 4(C[C @ @ @ H]([C @ @]([H])(C C 4) C 3) C=C) C c 5 c c l} \\
C(F)(F) F) c c(C(F)(F) F) c 5)[H]) O[H]) c(c c c c 6) c 26\end{array}$ \\
\hline Rnd1_3 & 1.99 & 7.33 & 76.00 & $\begin{array}{l}{[n+](\mathrm{Cc} 1 \mathrm{cc}(\mathrm{C}(\mathrm{F})(\mathrm{F}) \mathrm{F}) \mathrm{cc}(\mathrm{C}(\mathrm{F})(\mathrm{F}) \mathrm{F}) \mathrm{c} 1)(\mathrm{ccc} 2[\mathrm{C} @ \mathrm{H}]([\mathrm{C} @] 3([\mathrm{~N}+] 4(\mathrm{C}[\mathrm{C} @ @ \mathrm{H}]([\mathrm{C} @ @]]([\mathrm{H}])(\mathrm{CC} 4) \mathrm{C} 3) \mathrm{C}=} \\
\mathrm{C}) \mathrm{Cc} 5 \mathrm{c}(\mathrm{F}) \mathrm{cc}(\mathrm{Br}) \mathrm{cc5})[\mathrm{H}]) \mathrm{O}[\mathrm{H}]) \mathrm{c}(\mathrm{cccc} 6) \mathrm{c} 26\end{array}$ \\
\hline Rnd1_4 & 1.99 & 7.33 & 76.00 & $\begin{array}{l}{[n+](C c 1 c(C(F)(F) F) c c c(C(F)(F) F) c 1)(\operatorname{ccc} 2[C @ H]([C @] 3([N+] 4(C[C @ @ H]([C @ @]([H])(C C 4) C 3) C=} \\
C) C c 5 c c(C(F)(F) F) c c(F) c 5)[H]) O[H]) c(\operatorname{cccc} 6) c 26\end{array}$ \\
\hline Rnd1_5 & 1.98 & 7.23 & 75.70 & $\begin{array}{l}{[n+](C c 1 c(O C) c c([N+](=O)[O-} \\
]) c c 1)(\operatorname{ccc} 2[C @ H]([C @] 3([N+] 4(C[C @ @ H]([C @ @]([H])(C C 4) C 3) C=C) C c 5 c c(C(F)(F) F) c c(C(F)(F) F) \\
c 5)[H]) O[H]) c(c c c c 6) c 26\end{array}$ \\
\hline Rnd1_6 & 1.90 & 6.69 & 74.00 & $\begin{array}{l}{[n+](\mathrm{Cc} 1 \mathrm{cc}(\mathrm{C}(\mathrm{F})(\mathrm{F}) \mathrm{F}) \mathrm{cc}(\mathrm{C}(\mathrm{F})(\mathrm{F}) \mathrm{F}) \mathrm{c} 1)(\mathrm{ccc} 2[\mathrm{C} @ \mathrm{H}]([\mathrm{C} @] 3([\mathrm{~N}+] 4(\mathrm{C}[\mathrm{C} @ @ \mathrm{H}]([\mathrm{C} @ @]([\mathrm{H}])(\mathrm{CC} 4) \mathrm{C} 3) \mathrm{C}=} \\
\mathrm{C}) \mathrm{Cc} 5 \mathrm{ccc}(\mathrm{Br}) \mathrm{cc} 5 \mathrm{Cl})[\mathrm{H}]) \mathrm{O}[\mathrm{H}]) \mathrm{c}(\mathrm{cccc} 6) \mathrm{c} 26\end{array}$ \\
\hline Rnd1_7 & 1.89 & 6.60 & 73.70 & $\begin{array}{l}{[n+](C c 1 c(C(F)(F) F) c c(C(F)(F) F) c c 1)(c c c 2[C @ H]([C @] 3([N+] 4(C[C @ @ H]([C @ @]]([H])(C C 4) C 3) C=} \\
c) c c 5 c c(C(F)(F) F) c c(C(F)(F) F) c 5)[H]) O[H]) c(c c c c 6) c 26\end{array}$ \\
\hline Rnd1_8 & 1.89 & 6.60 & 73.70 & $\begin{array}{l}{[n+](C c 1 c c(C(F)(F) F) c c(C(F)(F) F) c 1)(c c c 2[C @ H]([C @] 3([N+] 4(C[C @ @ H]([C @ @]]([H])(C C 4) C 3) C=} \\
C) \operatorname{cc5} c c(C(F)(F) F) c c(C(F)(F) F) c 5)[H]) O[H]) c(c c c c 6) c 26\end{array}$ \\
\hline Rnd1_9 & 1.89 & 6.60 & 73.70 & $\begin{array}{l}{[n+](C c 1 c(C(F)(F) F) c c c(C(F)(F) F) c 1)(\operatorname{ccc} 2[C @ H]([C @] 3([N+] 4(C[C @ @ H]([C @ @]([H])(C C 4) C 3) C=} \\
C) \operatorname{cc5} 5 c(C(F)(F) F)(c c 5)[H]) O[H]) c(\operatorname{cccc} 6) c 26\end{array}$ \\
\hline Rnd1_10 & 1.88 & 6.55 & 73.50 & $\begin{array}{l}{[\mathrm{n}+](\mathrm{Cc} 1 \mathrm{cc}(\mathrm{C}(\mathrm{F})(\mathrm{F}) \mathrm{F}) \mathrm{cc}(\mathrm{C}(\mathrm{F})(\mathrm{F}) \mathrm{F}) \mathrm{c} 1)(\mathrm{ccc} 2[\mathrm{C} @ \mathrm{H}]([\mathrm{C} @] 3([\mathrm{~N}+] 4(\mathrm{C}[\mathrm{C} @ @ \mathrm{H}]([\mathrm{C} @ @]([\mathrm{H}])(\mathrm{CC} 4) \mathrm{C} 3) \mathrm{C}=} \\
\mathrm{C}) \mathrm{Cc} 5 \operatorname{ccc}(\mathrm{F}) \operatorname{cc} 5 \mathrm{Cl})[\mathrm{H}]) \mathrm{O}[\mathrm{H}]) \mathrm{c}(\mathrm{cccc} 6) \mathrm{c} 26\end{array}$ \\
\hline
\end{tabular}




\begin{tabular}{|c|c|c|c|c|}
\hline Rnd1_11 & 1.87 & 6.49 & 73.30 & $\begin{array}{l}{[n+](C c 1 c c(C(F)(F) F) c c(C(F)(F) F) c 1)(\operatorname{ccc} 2[C @ H]([C @] 3([N+] 4(C[C @ @ H]([C @ @]([H])(C C 4) C 3) C=} \\
c) c c 5 c c(C(F)(F) F) \operatorname{ccc} 5 C l)[H]) O[H]) c(c c c c 6) c 26\end{array}$ \\
\hline Rnd1_12 & 1.87 & 6.46 & 73.20 & $\begin{array}{l}{[n+](C c 1 c c c(F) c(C(F)(F) F) c 1)(c c c 2[C @ H]([C @] 3([N+] 4(C[C @ @ H]([C @ @]]([H])(C C 4) C 3) C=C) C c 5 c} \\
c(C(F)(F) F) c c(C(F)(F) F) c 5)[H]) O[H]) c(c c c c 6) c 26\end{array}$ \\
\hline Rnd1_13 & 1.84 & 6.27 & 72.50 & $\begin{array}{l}{[n+](\mathrm{Cc} 1 \mathrm{cc}(\mathrm{Cl}) \mathrm{cc}(\mathrm{C}(\mathrm{F})(\mathrm{F}) \mathrm{F}) \mathrm{c1})(\mathrm{ccc} 2[\mathrm{C} @ \mathrm{H}]([\mathrm{C} @] 3([\mathrm{~N}+] 4(\mathrm{C}[\mathrm{C} @ @ \mathrm{H}]([\mathrm{C} @ @]([\mathrm{H}])(\mathrm{CC} 4) \mathrm{C} 3) \mathrm{C}=\mathrm{C}) \mathrm{Cc} 5} \\
\mathrm{cc}(\mathrm{C}(\mathrm{F})(\mathrm{F}) \mathrm{F}) \mathrm{cc}(\mathrm{F}) \mathrm{c})[\mathrm{H}]) \mathrm{H}] \mathrm{O}[\mathrm{H}]) \mathrm{c}(\mathrm{cccc} 6) \mathrm{c} 26\end{array}$ \\
\hline Rnd1_14 & 1.82 & 6.14 & 72.00 & $\begin{array}{l}{[n+](C c 1 c(B r) c c c(C(F)(F) F) c 1)(\operatorname{ccc} 2[C @ H]([C @] 3([N+] 4(C[C @ @ H]([C @ @]([H])(C C 4) C 3) C=C) C c 5} \\
c c(C(F)(F) F) c c(C(F)(F) F) c 5)[H]) O[H]) c(c c c c 6) c 26\end{array}$ \\
\hline Rnd1_15 & 1.82 & 6.14 & 72.00 & $\begin{array}{l}{[n+](\mathrm{Cc} 1 \mathrm{cc}(\mathrm{OC}) \mathrm{cc}(\mathrm{C}(\mathrm{F})(\mathrm{F}) \mathrm{F}) \mathrm{c} 1)(\mathrm{ccc} 2[\mathrm{C} @ \mathrm{H}]([\mathrm{C} @] 3([\mathrm{~N}+] 4(\mathrm{C}[\mathrm{C} @ @ \mathrm{H}]([\mathrm{C} @ @]([\mathrm{H}])(\mathrm{CC} 4) \mathrm{C} 3) \mathrm{C}=\mathrm{C}) \mathrm{Cc}} \\
5 \mathrm{cc}(\mathrm{C}(\mathrm{F})(\mathrm{F}) \mathrm{F}) \mathrm{cc}(\mathrm{C}(\mathrm{F})(\mathrm{F}) \mathrm{F}) \mathrm{c} 5)[\mathrm{H}]) \mathrm{O}[\mathrm{H}]) \mathrm{c}(\mathrm{cccc} 6) \mathrm{c} 26\end{array}$ \\
\hline Rnd1_16 & 1.82 & 6.14 & 72.00 & $\begin{array}{l}{[\mathrm{n}+](\mathrm{Cc} 1 \mathrm{c}(\mathrm{Br}) \mathrm{ccc}(\mathrm{C}(\mathrm{F})(\mathrm{F}) \mathrm{F}) \mathrm{c} 1)(\mathrm{ccc} 2[\mathrm{C} @ \mathrm{H}]([\mathrm{C} @] 3([\mathrm{~N}+] 4(\mathrm{C}[\mathrm{C} @ @ \mathrm{H}]([\mathrm{C} @ @]([\mathrm{H}])(\mathrm{CC} 4) \mathrm{C} 3) \mathrm{C}=\mathrm{C}) \mathrm{Cc} 5} \\
\mathrm{cc}(\mathrm{C}(\mathrm{F})(\mathrm{F}) \mathrm{F}) \mathrm{cc}(\mathrm{F}) \mathrm{c5})[\mathrm{H}]) \mathrm{O}[\mathrm{H}]) \mathrm{c}(\mathrm{cccc} 6) \mathrm{c} 26\end{array}$ \\
\hline Rnd1_17 & 1.82 & 6.14 & 72.00 & $\begin{array}{l}{[n+](\mathrm{Cc} 1 \mathrm{cc}(\mathrm{Cl}) \mathrm{cc}(\mathrm{C}(\mathrm{F})(\mathrm{F}) \mathrm{F}) \mathrm{c} 1)(\mathrm{ccc} 2[\mathrm{C} @ \mathrm{H}]([\mathrm{C} @] 3([\mathrm{~N}+] 4(\mathrm{C}[\mathrm{C} @ @ \mathrm{H}]([\mathrm{C} @ @]([\mathrm{H}])(\mathrm{CC} 4) \mathrm{C} 3) \mathrm{C}=\mathrm{C}) \mathrm{Cc} 5} \\
\mathrm{c}(\mathrm{F}) \mathrm{cc}(\mathrm{Br}) \mathrm{cc5})[\mathrm{H}]) \mathrm{O}[\mathrm{H}]) \mathrm{c}(\mathrm{cccc} 6) \mathrm{c} 26\end{array}$ \\
\hline Rnd1_18 & 1.80 & 6.07 & 71.70 & $\begin{array}{l}{[n+](\mathrm{Cc} 1 \mathrm{cc}(\mathrm{C}(\mathrm{F})(\mathrm{F}) \mathrm{F}) \mathrm{cc}(\mathrm{C}(\mathrm{F})(\mathrm{F}) \mathrm{F}) \mathrm{c} 1)(\mathrm{ccc} 2[\mathrm{C} @ \mathrm{H}]([\mathrm{C} @] 3([\mathrm{~N}+] 4(\mathrm{C}[\mathrm{C} @ @ \mathrm{H}]([\mathrm{C} @ @]([\mathrm{H}])(\mathrm{CC} 4) \mathrm{C} 3) \mathrm{C}=} \\
\mathrm{C}) \mathrm{Cc} 5 \mathrm{ccc}(\mathrm{Cl}) \mathrm{cc} 5 \mathrm{~F})[\mathrm{H}]) \mathrm{O}[\mathrm{H}]) \mathrm{c}(\mathrm{cccc} 6) \mathrm{c} 26\end{array}$ \\
\hline Rnd1_19 & 1.79 & 5.99 & 71.40 & $\begin{array}{l}{[n+](C c 1 c(B r) c c c(C(F)(F) F) c 1)(\operatorname{ccc} 2[C @ H]([C @] 3([N+] 4(C[C @ @ H]([C @ @]([H])(C C 4) C 3) C=C) C c 5} \\
\operatorname{cc}(C(F)(F) F)(c c c 5)[H]) O[H]) c(\operatorname{cccc}) c 26\end{array}$ \\
\hline Rnd1_20 & 1.79 & 5.99 & 71.40 & $\begin{array}{l}{[n+](C c 1 c c(F) c c(C(F)(F) F) c 1)(\operatorname{ccc} 2[C @ H]([C @] 3([N+] 4(C[C @ @ H]([C @ @]]([H])(C C 4) C 3) C=C) C c 5 c} \\
c(C(F)(F) F) c c(F) c 5)[H]) O[H]) c(c c c c 6) c 26\end{array}$ \\
\hline Rnd1_21 & 1.79 & 5.97 & 71.30 & $\begin{array}{l}{[\mathrm{n}+](\mathrm{Cc} 1 \mathrm{ccc}(\mathrm{CHN}) \mathrm{c}(\mathrm{Br}) \mathrm{c} 1)(\mathrm{ccc} 2[\mathrm{C} @ \mathrm{H}]([\mathrm{C} @] 3([\mathrm{~N}+] 4(\mathrm{C}[\mathrm{C} @ @ \mathrm{H}]([\mathrm{C} @ @]([\mathrm{H}])(\mathrm{CC} 4) \mathrm{C} 3) \mathrm{C}=\mathrm{C}) \mathrm{Cc} 5 \mathrm{cc}(} \\
\mathrm{C}(\mathrm{F})(\mathrm{F}) \mathrm{F}) \mathrm{cc}(\mathrm{C}(\mathrm{F})(\mathrm{F}) \mathrm{F}) \mathrm{c} 5)[\mathrm{H}]) \mathrm{O}[\mathrm{H}]) \mathrm{c}(\mathrm{cccc} 6) \mathrm{c} 26\end{array}$ \\
\hline Rnd1_22 & 1.79 & 5.97 & 71.30 & $\begin{array}{l}{[n+](\mathrm{Cc} 1 \mathrm{ccc}(\mathrm{Cl}) \mathrm{c}(\mathrm{C}(\mathrm{F})(\mathrm{F}) \mathrm{F}) \mathrm{c} 1)(\mathrm{ccc} 2[\mathrm{C} @ \mathrm{H}]([\mathrm{C} @] 3([\mathrm{~N}+] 4(\mathrm{C}[\mathrm{C} @ @ \mathrm{H}]([\mathrm{C} @ @]([\mathrm{H}])(\mathrm{CC} 4) \mathrm{C} 3) \mathrm{C}=\mathrm{C}) \mathrm{Cc} 5} \\
\operatorname{cc}(\mathrm{C}(\mathrm{F})(\mathrm{F}) \mathrm{F}) \mathrm{cc}(\mathrm{C}(\mathrm{F})(\mathrm{F}) \mathrm{F}) \mathrm{c} 5)[\mathrm{H}]) \mathrm{O}[\mathrm{H}]) \mathrm{c}(\mathrm{cccc} 6) \mathrm{c} 26\end{array}$ \\
\hline
\end{tabular}




\begin{tabular}{|c|c|c|c|c|}
\hline Rnd1_23 & 1.77 & 5.90 & 71.00 & $\begin{array}{l}{[n+](C c 1 c(F) c c c(O C(F)(F) F) c 1)(c c c 2[C @ H]([C @] 3([N+] 4(C[C @ @ H]([C @ @]([H])(C C 4) C 3) C=C) C c} \\
5 c c(C(F)(F) F) c c(C(F)(F) F) c 5)[H]) O[H]) c(c c c c 6) c 26\end{array}$ \\
\hline Rnd1_24 & 1.77 & 5.90 & 71.00 & $\begin{array}{l}{[n+](\mathrm{Cc} 1 \mathrm{ccc}(\mathrm{Cl}) \mathrm{c}(\mathrm{C}(\mathrm{F})(\mathrm{F}) \mathrm{F}) \mathrm{c} 1)(\mathrm{ccc} 2[\mathrm{C} @ \mathrm{H}]([\mathrm{C} @] 3([\mathrm{~N}+] 4(\mathrm{C}[\mathrm{C} @ @ \mathrm{H}]([\mathrm{C} @ @]([\mathrm{H}])(\mathrm{CC} 4) \mathrm{C} 3) \mathrm{C}=\mathrm{C}) \mathrm{Cc} 5} \\
\mathrm{c}(\mathrm{F}) \mathrm{cc}(\mathrm{Br}) \mathrm{cc5})[\mathrm{H}]) \mathrm{O}[\mathrm{H}]) \mathrm{c}(\mathrm{cccc} 6) \mathrm{c} 26\end{array}$ \\
\hline Rnd1_25 & 1.75 & 5.73 & 70.30 & $\begin{array}{l}{[n+](\mathrm{Cc} 1 \mathrm{cc}(\mathrm{Br}) \mathrm{cc}(\mathrm{C}(\mathrm{F})(\mathrm{F}) \mathrm{F}) \mathrm{c} 1)(\mathrm{ccc} 2[\mathrm{C} @ \mathrm{H}]([\mathrm{C} @] 3([\mathrm{~N}+] 4(\mathrm{C}[\mathrm{C} @ @ \mathrm{H}]([\mathrm{C} @ @]([\mathrm{H}])(\mathrm{CC} 4) \mathrm{C} 3) \mathrm{C}=\mathrm{C}) \mathrm{Cc5}} \\
\mathrm{cc}(\mathrm{C}(\mathrm{F})(\mathrm{F}) \mathrm{F}) \mathrm{cc}(\mathrm{C}(\mathrm{F})(\mathrm{F}) \mathrm{F}) \mathrm{c} 5)[\mathrm{H}]) \mathrm{O}[\mathrm{H}]) \mathrm{c}(\mathrm{cccc} 6) \mathrm{c} 26\end{array}$ \\
\hline Rnd1_26 & 1.73 & 5.67 & 70.00 & $\begin{array}{l}{[n+](\mathrm{Cc} 1 \mathrm{cc}(\mathrm{C}(\mathrm{F})(\mathrm{F}) \mathrm{F}) \mathrm{cc}(\mathrm{C}(\mathrm{F})(\mathrm{F}) \mathrm{F}) \mathrm{c} 1)(\mathrm{ccc} 2[\mathrm{C} @ \mathrm{H}]([\mathrm{C} @] 3([\mathrm{~N}+] 4(\mathrm{C}[\mathrm{C} @ @ \mathrm{H}]([\mathrm{C} @ @]([\mathrm{H}])(\mathrm{CC} 4) \mathrm{C} 3) \mathrm{C}=} \\
\mathrm{C}) \mathrm{Cc} 5 \mathrm{cc}(\mathrm{F}) \mathrm{ccc} 5 \mathrm{Br})[\mathrm{H}]) \mathrm{O}[\mathrm{H}]) \mathrm{c}(\mathrm{cccc}) \mathrm{c} 26\end{array}$ \\
\hline Rnd1_27 & 1.73 & 5.67 & 70.00 & $\begin{array}{l}{[n+](\mathrm{Cc} 1 \mathrm{cc}(\mathrm{C}(\mathrm{F})(\mathrm{F}) \mathrm{F}) \mathrm{cc}(\mathrm{C}(\mathrm{F})(\mathrm{F}) \mathrm{F}) \mathrm{c} 1)(\mathrm{ccc} 2[\mathrm{C} @ \mathrm{H}]([\mathrm{C} @] 3([\mathrm{~N}+] 4(\mathrm{C}[\mathrm{C} @ @ \mathrm{H}]([\mathrm{C} @ @]([\mathrm{H}])(\mathrm{CC} 4) \mathrm{C} 3) \mathrm{C}=} \\
\mathrm{C}) \mathrm{Cc} 5 \mathrm{ccc}(\mathrm{C}(\mathrm{F})(\mathrm{F}) \mathrm{F}) \mathrm{cc} 5 \mathrm{~F})[\mathrm{H}]) \mathrm{O}[\mathrm{H}]) \mathrm{c}(\mathrm{cccc} 6) \mathrm{c} 26\end{array}$ \\
\hline Rnd1_28 & 1.70 & 5.45 & 69.00 & $\begin{array}{l}{[n+](\mathrm{Cc} 1 \mathrm{cc}(\mathrm{F}) \mathrm{c}(\mathrm{F}) \mathrm{c}(\mathrm{F}) \mathrm{c} 1)(\mathrm{ccc} 2[\mathrm{C} @ \mathrm{H}]([\mathrm{C} @] 3([\mathrm{~N}+] 4(\mathrm{C}[\mathrm{C} @ @ \mathrm{H}]([\mathrm{C} @ @]([\mathrm{H}])(\mathrm{CC} 4) \mathrm{C} 3) \mathrm{C}=\mathrm{C}) \mathrm{Cc} 5 \mathrm{cc}(\mathrm{Cl}} \\
\mathrm{F})(\mathrm{F}) \mathrm{F}) \mathrm{cc}(\mathrm{C}(\mathrm{F})(\mathrm{F}) \mathrm{F}) \mathrm{c} 5)[\mathrm{H}]) \mathrm{O}[\mathrm{H}]) \mathrm{c}(\mathrm{cccc} 6) \mathrm{c} 26\end{array}$ \\
\hline Rnd1_29 & 1.69 & 5.41 & 68.80 & $\begin{array}{l}{[n+](\mathrm{Cc} 1 \mathrm{ccc}(\mathrm{C}(\mathrm{F})(\mathrm{F}) \mathrm{F}) \mathrm{c}(\mathrm{Cl}) \mathrm{c} 1)(\mathrm{ccc} 2[\mathrm{C} @ \mathrm{H}]([\mathrm{C} @] 3([\mathrm{~N}+] 4(\mathrm{C}[\mathrm{C} @ @ \mathrm{H}]([\mathrm{C} @ @]([\mathrm{H}])(\mathrm{CC} 4) \mathrm{C} 3) \mathrm{C}=\mathrm{C}) \mathrm{Cc} 5} \\
\mathrm{cc}(\mathrm{C}(\mathrm{F})(\mathrm{F}) \mathrm{F}) \mathrm{cc}(\mathrm{C}(\mathrm{F})(\mathrm{F}) \mathrm{F}) \mathrm{c} 5)[\mathrm{H}]) \mathrm{O}[\mathrm{H}]) \mathrm{c}(\mathrm{cccc} 6) \mathrm{c} 26\end{array}$ \\
\hline Rnd1_30 & 1.69 & 5.41 & 68.80 & $\begin{array}{l}{[n+](\mathrm{Cc} 1 \mathrm{cc}(\mathrm{F}) \mathrm{cc}(\mathrm{C}(\mathrm{F})(\mathrm{F}) \mathrm{F}) \mathrm{c} 1)(\mathrm{ccc} 2[\mathrm{C} @ \mathrm{H}]([\mathrm{C} @] 3([\mathrm{~N}+] 4(\mathrm{C}[\mathrm{C} @ @ \mathrm{H}]([\mathrm{C} @ @]([\mathrm{H}])(\mathrm{CC} 4) \mathrm{C} 3) \mathrm{C}=\mathrm{C}) \mathrm{Cc} 5 \mathrm{c}} \\
\mathrm{c}(\mathrm{C}(\mathrm{F})(\mathrm{F}) \mathrm{F}) \mathrm{cc}(\mathrm{C}(\mathrm{F})(\mathrm{F}) \mathrm{F}) \mathrm{c5})[\mathrm{H}]) \mathrm{O}[\mathrm{H}]) \mathrm{c}(\mathrm{cccc}) \mathrm{c} 26\end{array}$ \\
\hline Rnd1_31 & 1.67 & 5.33 & 68.40 & $\begin{array}{l}{[n+](\mathrm{Cc} 1 \mathrm{cc}(\mathrm{C}(\mathrm{F})(\mathrm{F}) \mathrm{F}) \mathrm{cc}(\mathrm{C}(\mathrm{F})(\mathrm{F}) \mathrm{F}) \mathrm{c1})(\mathrm{ccc} 2[\mathrm{C} @ \mathrm{H}]([\mathrm{C} @] 3([\mathrm{~N}+] 4(\mathrm{C}[\mathrm{C} @ @ \mathrm{H}]([\mathrm{C} @ @]([\mathrm{H}])(\mathrm{CC} 4) \mathrm{C} 3) \mathrm{C}=} \\
\mathrm{C}) \mathrm{Cc} 5 \mathrm{ccc}(\mathrm{Cl}) \mathrm{c}(\mathrm{C}(\mathrm{F})(\mathrm{F}) \mathrm{F}) \mathrm{c} 5)(\mathrm{H}]) \mathrm{O}[\mathrm{H}]) \mathrm{c}(\mathrm{cccc} 6) \mathrm{c} 26\end{array}$ \\
\hline Rnd1_32 & 1.66 & 5.25 & 68.00 & $\begin{array}{l}{[\mathrm{n}+](\mathrm{Cc} 1 \mathrm{cccc}(\mathrm{C}(\mathrm{F})(\mathrm{F}) \mathrm{F}) \mathrm{c} 1)(\mathrm{ccc} 2[\mathrm{C} @ \mathrm{H}]([\mathrm{C} @] 3([\mathrm{~N}+] 4(\mathrm{C}[\mathrm{C} @ @ \mathrm{H}]([\mathrm{C} @ @]([\mathrm{H}])(\mathrm{CC} 4) \mathrm{C} 3) \mathrm{C}=\mathrm{C}) \mathrm{Cc} 5 \mathrm{c}(\mathrm{F})} \\
\mathrm{cc}(\mathrm{Br}) \mathrm{cc5})[\mathrm{H}]) \mathrm{O}[\mathrm{H}]) \mathrm{c}(\mathrm{cccc} 6) \mathrm{c} 26\end{array}$ \\
\hline Rnd1_33 & 1.62 & 5.06 & 67.00 & $\begin{array}{l}{[\mathrm{n}+](\mathrm{Cc} 1 \mathrm{c}(\mathrm{Br}) \mathrm{ccc}(\mathrm{C}(\mathrm{F})(\mathrm{F}) \mathrm{F}) \mathrm{c} 1)(\mathrm{ccc} 2[\mathrm{C} @ \mathrm{H}]([\mathrm{C} @] 3([\mathrm{~N}+] 4(\mathrm{C}[\mathrm{C} @ @ \mathrm{H}]([\mathrm{C} @ @]([\mathrm{H}])(\mathrm{CC} 4) \mathrm{C} 3) \mathrm{C}=\mathrm{C}) \mathrm{Cc} 5} \\
\mathrm{c}(\mathrm{F}) \mathrm{cc}(\mathrm{Br}) \mathrm{cc} 5)[\mathrm{H}]) \mathrm{O}[\mathrm{H}]) \mathrm{c}(\mathrm{cccc} 6) \mathrm{c} 26\end{array}$ \\
\hline Rnd1_34 & 1.62 & 5.06 & 67.00 & $\begin{array}{l}{[n+](\mathrm{Cc} 1 \mathrm{ccc}(\mathrm{C}(\mathrm{F})(\mathrm{F}) \mathrm{F}) \mathrm{cc} 1)(\mathrm{ccc} 2[\mathrm{C} @ \mathrm{H}]([\mathrm{C} @] 3([\mathrm{~N}+] 4(\mathrm{C}[\mathrm{C} @ @ \mathrm{H}]([\mathrm{C} @ @]([\mathrm{H}])(\mathrm{CC} 4) \mathrm{C} 3) \mathrm{C}=\mathrm{C}) \mathrm{Cc} 5 \mathrm{cc}(} \\
\mathrm{C}(\mathrm{F})(\mathrm{F}) \mathrm{F}) \mathrm{cc}(\mathrm{C}(\mathrm{F})(\mathrm{F}) \mathrm{F}) \mathrm{c} 5)[\mathrm{H}]) \mathrm{O}[\mathrm{H}]) \mathrm{c}(\mathrm{cccc} 6) \mathrm{c} 26\end{array}$ \\
\hline
\end{tabular}




\begin{tabular}{|c|c|c|c|c|}
\hline Rnd1_35 & 1.62 & 5.06 & 67.00 & $\begin{array}{l}{[n+](C c 1 c c c c(C(F)(F) F) c 1)(c c c 2[C @ H]([C @] 3([N+] 4(C[C @ @ H]([C @ @]([H])(C C 4) C 3) C=C) C c 5 c c(} \\
C(F)(F) F)(c c c 5)[H]) O[H]) c(\operatorname{cccc} 6) c 26\end{array}$ \\
\hline Rnd1_36 & 1.62 & 5.06 & 67.00 & $\begin{array}{l}{[n+](\mathrm{Cc} 1 \mathrm{cc}(\mathrm{C}) \mathrm{cc}(\mathrm{C}) \mathrm{c} 1)(\mathrm{ccc} 2[\mathrm{C} @ \mathrm{H}]([\mathrm{C} @] 3([\mathrm{~N}+] 4(\mathrm{C}[\mathrm{C} @ @ \mathrm{H}]([\mathrm{C} @ @]([\mathrm{H}])(\mathrm{CC} 4) \mathrm{C} 3) \mathrm{C}=\mathrm{C}) \mathrm{Cc} 5 \mathrm{cc}(\mathrm{F}) \mathrm{cc}} \\
\mathrm{c5})[\mathrm{H}]) \mathrm{O}[\mathrm{H}]) \mathrm{c}(\mathrm{cccc} 6) \mathrm{c} 26\end{array}$ \\
\hline Rnd1_37 & 1.61 & 5.02 & 66.80 & $\begin{array}{l}{[n+](C c 1 c(B r) c c c(O C(F)(F) F) c 1)(c c c 2[C @ H]([C @] 3([N+] 4(C[C @ @ H]([C @ @]]([H])(C C 4) C 3) C=C) C c} \\
5 c c(C(F)(F) F) c c(C(F)(F) F) c 5)[H]) O[H]) c(c c c c 6) c 26\end{array}$ \\
\hline Rnd1_38 & 1.61 & 5.02 & 66.80 & $\begin{array}{l}{[n+]((\mathrm{Cc} 1 \mathrm{ccc}(\mathrm{Cl}) \mathrm{c}(\mathrm{C}(\mathrm{F})(\mathrm{F}) \mathrm{F}) \mathrm{c} 1)(\mathrm{ccc} 2[\mathrm{C} @ \mathrm{H}]([\mathrm{C} @] 3([\mathrm{~N}+] 4(\mathrm{C}[\mathrm{C} @ @ \mathrm{H}]([\mathrm{C} @ @]([\mathrm{H}])(\mathrm{CC} 4) \mathrm{C} 3) \mathrm{C}=\mathrm{C}) \mathrm{Cc} 5} \\
\mathrm{cc}(\mathrm{C}(\mathrm{F})(\mathrm{F}) \mathrm{F}) \mathrm{ccc5})[\mathrm{H}]) \mathrm{O}[\mathrm{H}]) \mathrm{c}(\mathrm{cccc} 6) \mathrm{c} 26\end{array}$ \\
\hline Rnd1_39 & 1.61 & 4.99 & 66.60 & $\begin{array}{l}{[n+](C c 1 c c(C(F)(F) F) c c(C(F)(F) F) c 1)(\operatorname{ccc} 2[C @ H]([C @] 3([N+] 4(C[C @ @ H]([C @ @]([H])(C C 4) C 3) C=} \\
C) C c 5 c c(F) \operatorname{ccc} 5 F)[H]) O[H]) c(c c c c 6) c 26\end{array}$ \\
\hline Rnd1_40 & 1.60 & 4.97 & 66.50 & $\begin{array}{l}{[n+](\mathrm{Cc} 1 \mathrm{c}(\mathrm{C}(\mathrm{F})(\mathrm{F}) \mathrm{F}) \mathrm{ccc}(\mathrm{C}(\mathrm{F})(\mathrm{F}) \mathrm{F}) \mathrm{c} 1)(\mathrm{ccc} 2[\mathrm{C} @ \mathrm{H}]([\mathrm{C} @] 3([\mathrm{~N}+] 4(\mathrm{C}[\mathrm{C} @ @ \mathrm{H}]([\mathrm{C} @ @]([\mathrm{H}])(\mathrm{CC} 4) \mathrm{C} 3) \mathrm{C}=} \\
\mathrm{C}) \mathrm{Cc} 5 \mathrm{c}(\mathrm{F}) \mathrm{cc}(\mathrm{Br}) \mathrm{cc} 5)[\mathrm{H}]) \mathrm{O}[\mathrm{H}]) \mathrm{c}(\mathrm{cccc} 6) \mathrm{c} 26\end{array}$ \\
\hline Rnd1_41 & 1.56 & 4.78 & 65.40 & $\begin{array}{l}{[n+](C c 1 c c(C(F)(F) F) c c(C(F)(F) F) c 1)(c c c 2[C @ H]([C @] 3([N+] 4(C[C @ @ H]([C @ @]([H])(C C 4) C 3) C=} \\
C) \operatorname{Cc} 5 \operatorname{ccc}(F) \operatorname{cc} 5 F)[H]) O[H]) c(c c c c 6) c 26\end{array}$ \\
\hline Rnd1_42 & 1.56 & 4.76 & 65.30 & $\begin{array}{l}{[n+](\mathrm{Cc} 1 \mathrm{cc}(\mathrm{Cl}) \mathrm{cc}(\mathrm{C}(\mathrm{F})(\mathrm{F}) \mathrm{F}) \mathrm{c} 1)(\mathrm{ccc} 2[\mathrm{C} @ \mathrm{H}]([\mathrm{C} @] 3([\mathrm{~N}+] 4(\mathrm{C}[\mathrm{C} @ @ \mathrm{H}]([\mathrm{C} @ @]([\mathrm{H}])(\mathrm{CC} 4) \mathrm{C} 3) \mathrm{C}=\mathrm{C}) \mathrm{Cc} 5} \\
\mathrm{cc}(\mathrm{C}(\mathrm{F})(\mathrm{F}) \mathrm{F}) \mathrm{cc}(\mathrm{C}(\mathrm{F})(\mathrm{F}) \mathrm{F}) \mathrm{c} 5)[\mathrm{H}]) \mathrm{O}[\mathrm{H}]) \mathrm{c}(\mathrm{cccc} 6) \mathrm{c} 26\end{array}$ \\
\hline Rnd1_43 & 1.56 & 4.76 & 65.30 & $\begin{array}{l}{[n+](C c 1 c c(F) c c(F) c 1)(c c c 2[C @ H]([C @] 3([N+] 4(C[C @ @ @ H]([C @ @]([H])(C C 4) C 3) C=C) C c 5 c c(C(F)(} \\
F) F) c c(C(F)(F) F) c 5)[H]) O[H]) c(c c c c 6) c 26\end{array}$ \\
\hline Rnd1_44 & 1.55 & 4.71 & 65.00 & $\begin{array}{l}{[n+](\mathrm{Cc} 1 \mathrm{c}(\mathrm{SC}(\mathrm{F})(\mathrm{F}) \mathrm{F}) \mathrm{cccc} 1)(\mathrm{ccc} 2[\mathrm{C} @ \mathrm{H}]([\mathrm{C} @] 3([\mathrm{~N}+] 4(\mathrm{C}[\mathrm{C} @ @ \mathrm{H}]([\mathrm{C} @ @]]([\mathrm{H}])(\mathrm{CC} 4) \mathrm{C} 3) \mathrm{C}=\mathrm{C}) \mathrm{Cc} 5 \mathrm{cc}(} \\
\mathrm{C}(\mathrm{F})(\mathrm{F}) \mathrm{F}) \mathrm{cc}(\mathrm{C}(\mathrm{F})(\mathrm{F}) \mathrm{F}) \mathrm{c} 5)[\mathrm{H}]) \mathrm{O}[\mathrm{H}]) \mathrm{c}(\mathrm{cccc} 6) \mathrm{c} 26\end{array}$ \\
\hline Rnd1_45 & 1.55 & 4.71 & 65.00 & $\begin{array}{l}{[n+](\mathrm{Cc} 1 \mathrm{cc}(\mathrm{C}(\mathrm{F})(\mathrm{F}) \mathrm{F}) \mathrm{cc}(\mathrm{C}(\mathrm{F})(\mathrm{F}) \mathrm{F}) \mathrm{c} 1)(\mathrm{ccc} 2[\mathrm{C} @ \mathrm{H}]([\mathrm{C} @] 3([\mathrm{~N}+] 4(\mathrm{C}[\mathrm{C} @ @ \mathrm{H}]([\mathrm{C} @ @]([\mathrm{H}])(\mathrm{CC} 4) \mathrm{C} 3) \mathrm{C}=} \\
\mathrm{C}) \mathrm{Cc} 5 \mathrm{cc}(\mathrm{F}) \mathrm{c}(\mathrm{C}(\mathrm{F})(\mathrm{F}) \mathrm{F}) \mathrm{cc} 5)[\mathrm{H}]) \mathrm{O}[\mathrm{H}]) \mathrm{c}(\mathrm{cccc} 6) \mathrm{c} 26\end{array}$ \\
\hline Rnd1_46 & 1.55 & 4.71 & 65.00 & $\begin{array}{l}{[\mathrm{n}+](\mathrm{Cc} 1 \mathrm{cc}(\mathrm{OC}) \mathrm{cc}(\mathrm{C}(\mathrm{F})(\mathrm{F}) \mathrm{F}) \mathrm{c1})(\mathrm{ccc} 2[\mathrm{C} @ \mathrm{H}]([\mathrm{C} @] 3([\mathrm{~N}+] 4(\mathrm{C}[\mathrm{C} @ @ \mathrm{H}]([\mathrm{C} @ @]([\mathrm{H}])(\mathrm{CC} 4) \mathrm{C} 3) \mathrm{C}=\mathrm{C}) \mathrm{Cc}} \\
5 \mathrm{c}(\mathrm{F}) \mathrm{cc}(\mathrm{Br}) \mathrm{cc} 5)[\mathrm{H}]) \mathrm{O}[\mathrm{H}]) \mathrm{c}(\mathrm{cccc} 6) \mathrm{c} 26\end{array}$ \\
\hline
\end{tabular}




\begin{tabular}{|c|c|c|c|c|}
\hline Rnd1_47 & 1.53 & 4.60 & 64.30 & $\begin{array}{l}{[n+](C c 1 c c(C(F)(F) F) c c(C(F)(F) F) c 1)(c c c 2[C @ H]([C @] 3([N+] 4(C[C @ @ H]([C @ @]([H])(C C 4) C 3) C=} \\
C) C c 5 c c(F) c c(F) c 5)[H]) O[H]) c(c c c c 6) c 26\end{array}$ \\
\hline Rnd1_48 & 1.52 & 4.56 & 64.00 & $\begin{array}{l}{[n+](C c 1 c(C(F)(F) F) c c c c 1)(c c c 2[C @ H]([C @] 3([N+] 4(C[C @ @ H]([C @ @]([H])(C C 4) C 3) C=C) C c 5 c c(} \\
C(F)(F) F) c c(C(F)(F) F) c 5)[H]) O[H]) c(c c c c 6) c 26\end{array}$ \\
\hline Rnd1_49 & 1.52 & 4.56 & 64.00 & $\begin{array}{l}{[n+](C c 1 c c(C(F)(F) F) c c(C(F)(F) F) c 1)(\operatorname{ccc} 2[C @ H]([C @] 3([N+] 4(C[C @ @ H]([C @ @]([H])(C C 4) C 3) C=} \\
C) C c 5 c(F) c(F) c c(F) c 5)[H]) O[H]) c(c c c c 6) c 26\end{array}$ \\
\hline Rnd1_50 & 1.51 & 4.52 & 63.80 & $\begin{array}{l}{[\mathrm{n}+](\mathrm{Cc} 1 \mathrm{c}([\mathrm{N}+](=\mathrm{O})[\mathrm{O}-]) \mathrm{cc}([\mathrm{N}+](=\mathrm{O})[\mathrm{O}-} \\
]) \mathrm{cc} 1)(\mathrm{ccc} 2[\mathrm{C} @ \mathrm{H}]([\mathrm{C} @] 3([\mathrm{~N}+] 4(\mathrm{C}[\mathrm{C} @ @ \mathrm{H}]([\mathrm{C} @ @]([\mathrm{H}])(\mathrm{CC} 4) \mathrm{C} 3) \mathrm{C}=\mathrm{C}) \mathrm{Cc} 5 \mathrm{cc}(\mathrm{C}(\mathrm{F})(\mathrm{F}) \mathrm{F}) \mathrm{cc}(\mathrm{C}(\mathrm{F})(\mathrm{F}) \mathrm{F}) \\
\mathrm{c5})[\mathrm{H}]) \mathrm{O}[\mathrm{H}]) \mathrm{c}(\mathrm{cccc} 6) \mathrm{c} 26\end{array}$ \\
\hline Rnd1_51 & 1.51 & 4.52 & 63.80 & $\begin{array}{l}{[\mathrm{n}+](\mathrm{Cc} 1 \mathrm{cc}(\mathrm{C}(\mathrm{F})(\mathrm{F}) \mathrm{F}) \mathrm{cc}(\mathrm{C}(\mathrm{F})(\mathrm{F}) \mathrm{F}) \mathrm{c} 1)(\mathrm{ccc} 2[\mathrm{C} @ \mathrm{H}]([\mathrm{C} @] 3([\mathrm{~N}+] 4(\mathrm{C}[\mathrm{C} @ @ \mathrm{H}]([\mathrm{C} @ @]([\mathrm{H}])(\mathrm{CC} 4) \mathrm{C} 3) \mathrm{C}=} \\
\mathrm{C}) \mathrm{Cc} 5 \mathrm{cc}(\mathrm{Cl}) \operatorname{ccc} 5 \mathrm{Cl})[\mathrm{H}]) \mathrm{O}[\mathrm{H}]) \mathrm{c}(\mathrm{cccc} 6) \mathrm{c} 26\end{array}$ \\
\hline Rnd1_52 & 1.51 & 4.51 & 63.70 & $\begin{array}{l}{[n+](\operatorname{Cc} 1 c c(C(F)(F) F) c c(C(F)(F) F) c 1)(\operatorname{ccc} 2[C @ H]([C @] 3([N+] 4(C[C @ @ H]([C @ @]([H])(C C 4) C 3) C=} \\
C) \operatorname{Cc} 5 \operatorname{ccccc} 5 C l)[H]) O[H]) c(\operatorname{cccc} 6) c 26\end{array}$ \\
\hline Rnd1_53 & 1.48 & 4.41 & 63.00 & $\begin{array}{l}{[n+](\mathrm{Cc} 1 \mathrm{cc}(\mathrm{F}) \mathrm{cc}(\mathrm{C}(\mathrm{F})(\mathrm{F}) \mathrm{F}) \mathrm{c} 1)(\mathrm{ccc} 2[\mathrm{C} @ \mathrm{H}]([\mathrm{C} @] 3([\mathrm{~N}+] 4(\mathrm{C}[\mathrm{C} @ @ \mathrm{H}]([\mathrm{C} @ @]([\mathrm{H}])(\mathrm{CC} 4) \mathrm{C} 3) \mathrm{C}=\mathrm{C}) \mathrm{Cc} 5 \mathrm{c}} \\
(\mathrm{F}) \mathrm{cc}(\mathrm{Br}) \mathrm{cc} 5)[\mathrm{H}]) \mathrm{O}[\mathrm{H}]) \mathrm{c}(\mathrm{cccc} 6) \mathrm{c} 26\end{array}$ \\
\hline Rnd1_54 & 1.47 & 4.33 & 62.50 & $\begin{array}{l}{[n+](\mathrm{Cc} 1 \mathrm{cc}(\mathrm{Br}) \mathrm{cc}(\mathrm{Br}) \mathrm{c} 1)(\mathrm{ccc} 2[\mathrm{C} @ \mathrm{H}]([\mathrm{C} @] 3([\mathrm{~N}+] 4(\mathrm{C}[\mathrm{C} @ @ \mathrm{H}]([\mathrm{C} @ @]([\mathrm{H}])(\mathrm{CC} 4) \mathrm{C} 3) \mathrm{C}=\mathrm{C}) \mathrm{Cc} 5 \mathrm{cc}(\mathrm{C}(} \\
\mathrm{F})(\mathrm{F}) \mathrm{F}) \mathrm{cc}(\mathrm{C}(\mathrm{F})(\mathrm{F}) \mathrm{F}) \mathrm{c5})[\mathrm{H}]) \mathrm{O}[\mathrm{H}]) \mathrm{c}(\mathrm{cccc}) \mathrm{c} 26\end{array}$ \\
\hline Rnd1_55 & 1.45 & 4.26 & 62.00 & $\begin{array}{l}{[n+](\mathrm{Cc} 1 \mathrm{c}(\mathrm{Br}) \mathrm{ccc}(\mathrm{Cl}) \mathrm{c} 1)(\mathrm{ccc} 2[\mathrm{C} @ \mathrm{H}]([\mathrm{C} @] 3([\mathrm{~N}+] 4(\mathrm{C}[\mathrm{C} @ @ \mathrm{H}]([\mathrm{C} @ @]([\mathrm{H}])(\mathrm{CC} 4) \mathrm{C} 3) \mathrm{C}=\mathrm{C}) \mathrm{Cc} 5 \mathrm{cc}(\mathrm{C}(\mathrm{F}} \\
)(\mathrm{F}) \mathrm{F}) \mathrm{cc}(\mathrm{C}(\mathrm{F})(\mathrm{F}) \mathrm{F}) \mathrm{c5})[\mathrm{H}]) \mathrm{O}[\mathrm{H}]) \mathrm{c}(\mathrm{cccc} 6) \mathrm{c} 26\end{array}$ \\
\hline Rnd1_56 & 1.45 & 4.26 & 62.00 & $\begin{array}{l}{[n+]((\mathrm{Cc} 1 \mathrm{cc}([\mathrm{N}+](=\mathrm{O})[\mathrm{O}-} \\
]) \mathrm{ccc} 1)(\mathrm{ccc} 2[\mathrm{C} @ \mathrm{H}]([\mathrm{C} @] 3([\mathrm{~N}+] 4(\mathrm{C}[\mathrm{C} @ @ \mathrm{H}]([\mathrm{C} @ @]([\mathrm{H}])(\mathrm{CC} 4) \mathrm{C} 3) \mathrm{C}=\mathrm{C}) \mathrm{Cc} 5 \mathrm{cc}(\mathrm{C}(\mathrm{F})(\mathrm{F}) \mathrm{F}) \mathrm{cc}(\mathrm{C}(\mathrm{F})(\mathrm{F}) \mathrm{F} \\
) \mathrm{c5})[\mathrm{H}]) \mathrm{O}[\mathrm{H}]) \mathrm{c}(\mathrm{cccc} 6) \mathrm{c} 26\end{array}$ \\
\hline Rnd1_57 & 1.45 & 4.26 & 62.00 & $\begin{array}{l}{[\mathrm{n}+](\mathrm{Cc} 1 \mathrm{cc}(\mathrm{OC}) \mathrm{cc}(\mathrm{C}(\mathrm{F})(\mathrm{F}) \mathrm{F}) \mathrm{c} 1)(\mathrm{ccc} 2[\mathrm{C} @ \mathrm{H}]([\mathrm{C} @] 3([\mathrm{~N}+] 4(\mathrm{C}[\mathrm{C} @ @ \mathrm{H}]([\mathrm{C} @ @]([\mathrm{H}])(\mathrm{CC} 4) \mathrm{C} 3) \mathrm{C}=\mathrm{C}) \mathrm{Cc}} \\
5 \mathrm{cc}(\mathrm{C}(\mathrm{F})(\mathrm{F}) \mathrm{F}) \mathrm{ccc5})[\mathrm{H}]) \mathrm{O}[\mathrm{H}]) \mathrm{c}(\mathrm{cccc} 6) \mathrm{c} 26\end{array}$ \\
\hline Rnd1_58 & 1.39 & 4.00 & 60.00 & $\begin{array}{l}{[\mathrm{n}+](\mathrm{Cc} 1 \mathrm{ccc}(\mathrm{I}) \mathrm{cc} 1)(\mathrm{ccc} 2[\mathrm{C} @ \mathrm{H}]([\mathrm{C} @] 3([\mathrm{~N}+] 4(\mathrm{C}[\mathrm{C} @ @ \mathrm{H}]([\mathrm{C} @ @]([\mathrm{H}])(\mathrm{CC} 4) \mathrm{C} 3) \mathrm{C}=\mathrm{C}) \mathrm{Cc} 5 \mathrm{cc}(\mathrm{C}(\mathrm{F})(\mathrm{F}) \mathrm{F}} \\
) \operatorname{cc}(\mathrm{C}(\mathrm{F})(\mathrm{F}) \mathrm{F}) \mathrm{c5})[\mathrm{H}]) \mathrm{O}[\mathrm{H}]) \mathrm{c}(\mathrm{cccc}) \mathrm{c} 26\end{array}$ \\
\hline
\end{tabular}




\begin{tabular}{|c|c|c|c|c|}
\hline Rnd1_59 & 1.39 & 4.00 & 60.00 & $\begin{array}{l}{[n+](C c 1 c c(C(F)(F) F) c c(C(F)(F) F) c 1)(c c c 2[C @ H]([C @] 3([N+] 4(C[C @ @ H]([C @ @]([H])(C C 4) C 3) C=} \\
C) C c 5 c c(C(F)(F) F) c(F) c c 5)[H]) O[H]) c(c c c c 6) c 26\end{array}$ \\
\hline Rnd1_60 & 1.39 & 4.00 & 60.00 & $\begin{array}{l}{[n+](\mathrm{Cc} 1 \mathrm{ccc}([\mathrm{N}+](=\mathrm{O})[\mathrm{O}-} \\
]) c(\mathrm{~F}) \mathrm{c} 1)(\mathrm{ccc} 2[\mathrm{C} @ \mathrm{H}]([\mathrm{C} @ 3) 3([\mathrm{~N}+] 4(\mathrm{C}[\mathrm{C} @ @ \mathrm{H}]([\mathrm{C} @ @]([\mathrm{H}])(\mathrm{CC} 4) \mathrm{C} 3) \mathrm{C}=\mathrm{C}) \mathrm{Cc} 5 \mathrm{cc}(\mathrm{C}(\mathrm{F})(\mathrm{F}) \mathrm{F}) \mathrm{cc}(\mathrm{C}(\mathrm{F})(\mathrm{F}) \\
\mathrm{F}) \mathrm{c5})[\mathrm{H}]) \mathrm{O}[\mathrm{H}]) \mathrm{c}(\mathrm{cccc} 6) \mathrm{c} 26\end{array}$ \\
\hline Rnd1_61 & 1.39 & 4.00 & 60.00 & $\begin{array}{l}{[\mathrm{n}+](\mathrm{Cc} 1 \mathrm{c}(\mathrm{Br}) \mathrm{ccc}(\mathrm{OC}) \mathrm{c} 1)(\mathrm{ccc} 2[\mathrm{C} @ \mathrm{H}]([\mathrm{C} @] 3([\mathrm{~N}+] 4(\mathrm{C}[\mathrm{C} @ @ \mathrm{H}]([\mathrm{C} @ @]([\mathrm{H}])(\mathrm{CC} 4) \mathrm{C} 3) \mathrm{C}=\mathrm{C}) \mathrm{Cc} 5 \mathrm{cc}(\mathrm{O}} \\
\mathrm{C}(\mathrm{F})(\mathrm{F}) \mathrm{F}) \mathrm{ccc})[\mathrm{H}]) \mathrm{O}[\mathrm{H}]) \mathrm{c}(\mathrm{cccc}) \mathrm{c} 26\end{array}$ \\
\hline Rnd1_62 & 1.36 & 3.88 & 59.00 & $\begin{array}{l}{[\mathrm{n}+](\mathrm{Cc} 1 \mathrm{c}(\mathrm{F}) \mathrm{cc}(\mathrm{Br}) \mathrm{cc} 1)(\mathrm{ccc} 2[\mathrm{C} @ \mathrm{H}]([\mathrm{C} @] 3([\mathrm{~N}+] 4(\mathrm{C}[\mathrm{C} @ @ \mathrm{H}]([\mathrm{C} @ @]([\mathrm{H}])(\mathrm{CC} 4) \mathrm{C} 3) \mathrm{C}=\mathrm{C}) \mathrm{Cc} 5 \mathrm{c}(\mathrm{F}) \mathrm{cc}(} \\
\mathrm{Br}) \mathrm{cc} 5)[\mathrm{H}]) \mathrm{O}[\mathrm{H}]) \mathrm{c}(\mathrm{cccc} 6) \mathrm{c} 26\end{array}$ \\
\hline Rnd1_63 & 1.36 & 3.88 & 59.00 & $\begin{array}{l}{[\mathrm{n}+](\mathrm{Cc} 1 \mathrm{ccc}([\mathrm{N}+](=\mathrm{O})[\mathrm{O}-} \\
]) \mathrm{cc} 1)(\mathrm{ccc} 2[\mathrm{C} @ \mathrm{H}]([\mathrm{C} @] 3([\mathrm{~N}+] 4(\mathrm{C}[\mathrm{C} @ @ \mathrm{H}]([\mathrm{C} @ @]([\mathrm{H}])(\mathrm{CC} 4) \mathrm{C} 3) \mathrm{C}=\mathrm{C}) \mathrm{Cc} 5 \mathrm{cc}(\mathrm{C}(\mathrm{F})(\mathrm{F}) \mathrm{F}) \mathrm{cc}(\mathrm{C}(\mathrm{F})(\mathrm{F}) \mathrm{F}) \\
\mathrm{c5})[\mathrm{H}]) \mathrm{O}[\mathrm{H}]) \mathrm{c}(\mathrm{cccc} 6) \mathrm{c} 26\end{array}$ \\
\hline Rnd1_64 & 1.36 & 3.88 & 59.00 & $\begin{array}{l}{[n+](\mathrm{Cc} 1 \mathrm{cc}(\mathrm{C}(\mathrm{F})(\mathrm{F}) \mathrm{F}) \mathrm{cc}(\mathrm{C}(\mathrm{F})(\mathrm{F}) \mathrm{F}) \mathrm{c} 1)(\mathrm{ccc} 2[\mathrm{C} @ \mathrm{H}]([\mathrm{C} @] 3([\mathrm{~N}+] 4(\mathrm{C}[\mathrm{C} @ @ \mathrm{H}]([\mathrm{C} @ @]([\mathrm{H}])(\mathrm{CC} 4) \mathrm{C} 3) \mathrm{C}=} \\
\mathrm{C}) \mathrm{Cc} 5 \mathrm{cc}(\mathrm{Cl}) \operatorname{ccc} 5)[\mathrm{H}]) \mathrm{O}[\mathrm{H}]) \mathrm{c}(\mathrm{cccc} 6) \mathrm{c} 26\end{array}$ \\
\hline Rnd1_65 & 1.36 & 3.88 & 59.00 & $\begin{array}{l}{[\mathrm{n}+](\mathrm{Cc} 1 \mathrm{ccc}(\mathrm{CHN}) \mathrm{cc} 1)(\mathrm{ccc} 2[\mathrm{C} @ \mathrm{H}]([\mathrm{C} @] 3([\mathrm{~N}+] 4(\mathrm{C}[\mathrm{C} @ @ \mathrm{H}]([\mathrm{C} @ @]([\mathrm{H}])(\mathrm{CC} 4) \mathrm{C} 3) \mathrm{C}=\mathrm{C}) \mathrm{Cc} 5 \mathrm{cc}(\mathrm{C}(\mathrm{F})(} \\
\mathrm{F}) \mathrm{F}) \mathrm{cc}(\mathrm{C}(\mathrm{F})(\mathrm{F}) \mathrm{F}) \mathrm{c} 5)[\mathrm{H}]) \mathrm{O}[\mathrm{H}]) \mathrm{c}(\mathrm{cccc} 6) \mathrm{c} 26\end{array}$ \\
\hline Rnd1_66 & 1.32 & 3.76 & 58.00 & $\begin{array}{l}{[n+](\mathrm{Cc} 1 \mathrm{c}(\mathrm{Br}) \mathrm{ccc}(\mathrm{OC}) \mathrm{c} 1)(\mathrm{ccc} 2[\mathrm{C} @ \mathrm{H}]([\mathrm{C} @] 3([\mathrm{~N}+] 4(\mathrm{C}[\mathrm{C} @ @ \mathrm{H}]([\mathrm{C} @ @]([\mathrm{H}])(\mathrm{CC} 4) \mathrm{C} 3) \mathrm{C}=\mathrm{C}) \mathrm{Cc} 5 \mathrm{cc}(\mathrm{Cl}} \\
\mathrm{F})(\mathrm{F}) \mathrm{F}) \mathrm{cc}(\mathrm{C}(\mathrm{F})(\mathrm{F}) \mathrm{F}) \mathrm{c5})[\mathrm{H}]) \mathrm{O}[\mathrm{H}]) \mathrm{c}(\mathrm{cccc}) \mathrm{c} 26\end{array}$ \\
\hline Rnd1_67 & 1.32 & 3.73 & 57.70 & $\begin{array}{l}{[\mathrm{n}+](\mathrm{Cc} 1 \mathrm{c}([\mathrm{N}+](=\mathrm{C})[\mathrm{O}-} \\
]) \mathrm{ccc}(\mathrm{C}(\mathrm{F})(\mathrm{F}) \mathrm{F}) \mathrm{c} 1)(\mathrm{ccc} 2[\mathrm{C} @ \mathrm{H}]([\mathrm{C} @] 3([\mathrm{~N}+] 4(\mathrm{C}[\mathrm{C} @ @ \mathrm{H}]([\mathrm{C} @ @]([\mathrm{H}])(\mathrm{CC} 4) \mathrm{C} 3) \mathrm{C}=\mathrm{C}) \mathrm{Cc} 5 \mathrm{cc}(\mathrm{C}(\mathrm{F})(\mathrm{F}) \mathrm{F}) \\
\mathrm{cc}(\mathrm{C}(\mathrm{F})(\mathrm{F}) \mathrm{F}) \mathrm{c} 5)[\mathrm{H}]) \mathrm{O}[\mathrm{H}]) \mathrm{c}(\mathrm{cccc} 6) \mathrm{c} 26\end{array}$ \\
\hline Rnd1_68 & 1.30 & 3.65 & 57.00 & $\begin{array}{l}{[n+](C c 1 c(C(F)(F) F) c c c(F) c 1)(c c c 2[C @ H]([C @] 3([N+] 4(C[C @ @ H]([C @ @]]([H])(C C 4) C 3) C=C) C c 5 c} \\
c(C(F)(F) F) c c(C(F)(F) F) c 5)[H]) O[H]) c(c c c c 6) c 26\end{array}$ \\
\hline Rnd1_69 & 1.27 & 3.55 & 56.00 & $\begin{array}{l}{[n+](C c 1 c c(C(F)(F) F) c c(C(F)(F) F) c 1)(\operatorname{ccc} 2[C @ H]([C @] 3([N+] 4(C[C @ @ H]([C @ @]([H])(C C 4) C 3) C=} \\
C) \operatorname{cc} 5 c c(O C(F)(F) F) c c c 5)[H]) O[H]) c(c c c c 6) c 26\end{array}$ \\
\hline Rnd1_70 & 1.27 & 3.55 & 56.00 & $\begin{array}{l}{[\mathrm{n}+](\mathrm{Cc} 1 \mathrm{cc}(\mathrm{F}) \mathrm{ccc} 1)(\operatorname{ccc} 2[\mathrm{C} @ \mathrm{H}]([\mathrm{C} @] 3([\mathrm{~N}+] 4(\mathrm{C}[\mathrm{C} @ @ \mathrm{H}]([\mathrm{C} @ @]([\mathrm{H}])(\mathrm{CC} 4) \mathrm{C} 3) \mathrm{C}=\mathrm{C}) \mathrm{Cc} 5 \mathrm{cc}(\mathrm{C}(\mathrm{F})(\mathrm{F}) \mathrm{F}} \\
) \operatorname{cc}(\mathrm{C}(\mathrm{F})(\mathrm{F}) \mathrm{F}) \mathrm{c5})[\mathrm{H}]) \mathrm{O}[\mathrm{H}]) \mathrm{c}(\mathrm{cccc}) \mathrm{c} 26\end{array}$ \\
\hline
\end{tabular}




\begin{tabular}{|c|c|c|c|c|}
\hline Rnd1_71 & 1.25 & 3.49 & 55.50 & $\begin{array}{l}{[n+](C c 1 c c(C(F)(F) F) c c(C(F)(F) F) c 1)(\operatorname{ccc} 2[C @ H]([C @] 3([N+] 4(C[C @ @ H]([C @ @]([H])(C C 4) C 3) C=} \\
C) C c 5 c c(F) c(F) c c 5)[H]) O[H]) c(c c c c 6) c 26\end{array}$ \\
\hline Rnd1_72 & 1.24 & 3.44 & 55.00 & $\begin{array}{l}{[n+](C c 1 c([N+](=O)[O-} \\
]) c c(B r) c c 1)(\operatorname{ccc} 2[C @ H]([C @] 3([N+] 4(C[C @ @ H]([C @ @]([H])(C C 4) C 3) C=C) C c 5 c c(C(F)(F) F) c c(C(F \\
)(F) F) c 5)[H]) O[H]) c(c c c c 6) c 26\end{array}$ \\
\hline Rnd1_73 & 1.24 & 3.44 & 55.00 & $\begin{array}{l}{[n+](C c 1 c(C \# N) c c c c 1)(\operatorname{ccc} 2[\mathrm{C} @ H]([C @] 3([\mathrm{~N}+] 4(\mathrm{C}[\mathrm{C} @ @ H]([\mathrm{C} @ @]([\mathrm{H}])(\mathrm{CC} 4) \mathrm{C} 3) \mathrm{C}=\mathrm{C}) \mathrm{Cc} 5 \mathrm{cc}(\mathrm{C}(\mathrm{F})(} \\
\mathrm{F}) \mathrm{F}) \mathrm{cc}(\mathrm{C}(\mathrm{F})(\mathrm{F}) \mathrm{F}) \mathrm{c} 5)[\mathrm{H}]) \mathrm{O}[\mathrm{H}]) \mathrm{c}(\mathrm{cccc} 6) \mathrm{c} 26\end{array}$ \\
\hline Rnd1_74 & 1.23 & 3.42 & 54.70 & $\begin{array}{l}{[n+](\mathrm{Cc} 1 \mathrm{c}(\mathrm{Br}) \mathrm{ccc}(\mathrm{F}) \mathrm{c} 1)(\mathrm{ccc} 2[\mathrm{C} @ \mathrm{H}]([\mathrm{C} @] 3([\mathrm{~N}+] 4(\mathrm{C}[\mathrm{C} @ @ \mathrm{H}]([\mathrm{C} @ @]([\mathrm{H}])(\mathrm{CC} 4) \mathrm{C} 3) \mathrm{C}=\mathrm{C}) \mathrm{Cc} 5 \mathrm{cc}(\mathrm{C}(\mathrm{F})} \\
(\mathrm{F}) \mathrm{F}) \mathrm{cc}(\mathrm{C}(\mathrm{F})(\mathrm{F}) \mathrm{F}) \mathrm{c5})[\mathrm{H}]) \mathrm{O}[\mathrm{H}]) \mathrm{c}(\mathrm{cccc} 6) \mathrm{c} 26\end{array}$ \\
\hline Rnd1_75 & 1.23 & 3.41 & 54.60 & $\begin{array}{l}{[n+](C c 1 c c(C(F)(F) F) c c(C(F)(F) F) c 1)(c c c 2[C @ H]([C @] 3([N+] 4(C[C @ @ H]([C @ @]([H])(C C 4) C 3) C=} \\
C) \operatorname{cc5} 5 c(B r) \operatorname{ccc} 5)[H]) O[H]) c(c c c c 6) c 26\end{array}$ \\
\hline Rnd1_76 & 1.21 & 3.34 & 53.90 & $\begin{array}{l}{[n+](\mathrm{Cc} 1 c(\mathrm{C}(\mathrm{F})(\mathrm{F}) \mathrm{F}) \mathrm{cc}(\mathrm{F}) \mathrm{cc} 1)(\mathrm{ccc} 2[\mathrm{C} @ \mathrm{H}]([\mathrm{C} @] 3([\mathrm{~N}+] 4(\mathrm{C}[\mathrm{C} @ @ \mathrm{H}]([\mathrm{C} @ @]([\mathrm{H}])(\mathrm{CC} 4) \mathrm{C} 3) \mathrm{C}=\mathrm{C}) \mathrm{Cc} 5 \mathrm{c}} \\
\mathrm{c}(\mathrm{C}(\mathrm{F})(\mathrm{F}) \mathrm{F}) \mathrm{cc}(\mathrm{C}(\mathrm{F})(\mathrm{F}) \mathrm{F}) \mathrm{c} 5)[\mathrm{H}]) \mathrm{O}[\mathrm{H}]) \mathrm{c}(\mathrm{cccc} 6) \mathrm{c} 26\end{array}$ \\
\hline Rnd1_77 & 1.18 & 3.26 & 53.00 & $\begin{array}{l}{[n+](\mathrm{Cc} 1 \mathrm{cc}(\mathrm{C}(\mathrm{F})(\mathrm{F}) \mathrm{F}) \mathrm{cc}(\mathrm{C}(\mathrm{F})(\mathrm{F}) \mathrm{F}) \mathrm{c} 1)(\mathrm{ccc} 2[\mathrm{C} @ \mathrm{H}]([\mathrm{C} @] 3([\mathrm{~N}+] 4(\mathrm{C}[\mathrm{C} @ @ \mathrm{H}]([\mathrm{C} @ @]([\mathrm{H}])(\mathrm{CC} 4) \mathrm{C} 3) \mathrm{C}=} \\
\mathrm{C}) \mathrm{Cc} 5 \mathrm{cl}(\mathrm{Cl}) \mathrm{c}(\mathrm{C}(\mathrm{F})(\mathrm{F}) \mathrm{F}) \mathrm{ccc} 5)[\mathrm{H}]) \mathrm{O}[\mathrm{H}]) \mathrm{c}(\mathrm{cccc} 6) \mathrm{c} 26\end{array}$ \\
\hline Rnd1 78 & 1.18 & 3.26 & 53.00 & $\begin{array}{l}{[n+](C c 1 c c(C(F)(F) F) c c(C(F)(F) F) c 1)(c c c 2[C @ H]([C @] 3([N+] 4(C[C @ @ H]([C @ @]([H])(C C 4) C 3) C=} \\
C) C c 5 c(F) c(F) c(F) c c 5)[H]) O[H]) c(c c c c 6) c 26\end{array}$ \\
\hline Rnd1_79 & 1.18 & 3.26 & 53.00 & $\begin{array}{l}{[\mathrm{n}+](\mathrm{Cc} 1 \mathrm{c}([\mathrm{N}+](=\mathrm{C})[\mathrm{O}-} \\
]) \mathrm{cccc} 1)(\mathrm{ccc} 2[\mathrm{C} @ \mathrm{H}]([\mathrm{C} @] 3([\mathrm{~N}+] 4(\mathrm{C}[\mathrm{C} @ @ \mathrm{H}]([\mathrm{C} @ @]([\mathrm{H}])(\mathrm{CC} 4) \mathrm{C} 3) \mathrm{C}=\mathrm{C}) \mathrm{Cc} 5 \mathrm{cc}(\mathrm{C}(\mathrm{F})(\mathrm{F}) \mathrm{F}) \mathrm{cc}(\mathrm{C}(\mathrm{F})(\mathrm{F}) \\
\mathrm{F}) \mathrm{c5})[\mathrm{H}]) \mathrm{O}[\mathrm{H}]) \mathrm{c}(\mathrm{cccc} 6) \mathrm{c} 26\end{array}$ \\
\hline Rnd1__80 & 1.18 & 3.26 & 53.00 & $\begin{array}{l}{[n+](\mathrm{Cc} 1 \mathrm{cc}(\mathrm{Cl}) \mathrm{cc}(\mathrm{C}(\mathrm{F})(\mathrm{F}) \mathrm{F}) \mathrm{c1})(\mathrm{ccc} 2[\mathrm{C} @ \mathrm{H}]([\mathrm{C} @] 3([\mathrm{~N}+] 4(\mathrm{C}[\mathrm{C} @ @ \mathrm{H}]([\mathrm{C} @ @]([\mathrm{H}])(\mathrm{CC} 4) \mathrm{C} 3) \mathrm{C}=\mathrm{C}) \mathrm{Cc} 5} \\
\mathrm{cc}(\mathrm{C}(\mathrm{F})(\mathrm{F}) \mathrm{F}) \mathrm{ccc5})[\mathrm{H}]) \mathrm{O}[\mathrm{H}]) \mathrm{c}(\mathrm{cccc} 6) \mathrm{c} 26\end{array}$ \\
\hline Rnd1_81 & 1.14 & 3.14 & 51.70 & $\begin{array}{l}{[n+](\mathrm{Cc} 1 \mathrm{c}(\mathrm{Br}) \mathrm{cc}(\mathrm{F}) \mathrm{c}(\mathrm{F}) \mathrm{c} 1)(\mathrm{ccc} 2[\mathrm{C} @ \mathrm{H}]([\mathrm{C} @] 3([\mathrm{~N}+] 4(\mathrm{C}[\mathrm{C} @ @ \mathrm{H}]([\mathrm{C} @ @]]([\mathrm{H}])(\mathrm{CC} 4) \mathrm{C} 3) \mathrm{C}=\mathrm{C}) \mathrm{Cc} 5 \mathrm{cc}(\mathrm{C}} \\
(\mathrm{F})(\mathrm{F}) \mathrm{F}) \mathrm{cc}(\mathrm{C}(\mathrm{F})(\mathrm{F}) \mathrm{F}) \mathrm{c} 5)[\mathrm{H}]) \mathrm{O}[\mathrm{H}]) \mathrm{c}(\mathrm{cccc} 6) \mathrm{c} 26\end{array}$ \\
\hline Rnd1_82 & 1.13 & 3.11 & 51.30 & $\begin{array}{l}{[n+](\mathrm{Cc} 1 \mathrm{c}(\mathrm{Br}) \mathrm{ccc}(\mathrm{OC}) \mathrm{c1})(\mathrm{ccc} 2[\mathrm{C} @ \mathrm{H}]([\mathrm{C} @] 3([\mathrm{~N}+] 4(\mathrm{C}[\mathrm{C} @ @ \mathrm{H}]([\mathrm{C} @ @]([\mathrm{H}])(\mathrm{CC} 4) \mathrm{C} 3) \mathrm{C}=\mathrm{C}) \mathrm{Cc} 5 \mathrm{cc}(\mathrm{Cl}} \\
\mathrm{F})(\mathrm{F}) \mathrm{F}) \mathrm{ccc} 5)[\mathrm{H}]) \mathrm{O}[\mathrm{H}]) \mathrm{c}(\mathrm{cccc} 6) \mathrm{c} 26\end{array}$ \\
\hline
\end{tabular}




\begin{tabular}{|c|c|c|c|c|}
\hline Rnd1_83 & 1.13 & 3.11 & 51.30 & $\begin{array}{l}{[n+]((\mathrm{Cc} 1 \mathrm{cc}(\mathrm{F}) \mathrm{cc}(\mathrm{C}(\mathrm{F})(\mathrm{F}) \mathrm{F}) \mathrm{c} 1)(\mathrm{ccc} 2[\mathrm{C} @ \mathrm{H}]([\mathrm{C} @] 3([\mathrm{~N}+] 4(\mathrm{C}[\mathrm{C} @ @ \mathrm{H}]([\mathrm{C} @ @]([\mathrm{H}])(\mathrm{CC} 4) \mathrm{C} 3) \mathrm{C}=\mathrm{C}) \mathrm{Cc} 5 \mathrm{c}} \\
\mathrm{c}(\mathrm{C}(\mathrm{F})(\mathrm{F}) \mathrm{F}) \mathrm{ccc} 5)[\mathrm{H}]) \mathrm{O}[\mathrm{H}]) \mathrm{c}(\mathrm{cccc} 6) \mathrm{c} 26\end{array}$ \\
\hline Rnd1_84 & 1.13 & 3.10 & 51.20 & $\begin{array}{l}{[n+](C c 1 c c(C(F)(F) F) c c(C(F)(F) F) c 1)(\operatorname{ccc} 2[C @ H]([C @] 3([N+] 4(C[C @ @ H]([C @ @]([H])(C C 4) C 3) C=} \\
C) C c 5 c c(C(F)(F) F) c c c 5)[H]) O[H]) c(\operatorname{cccc}) c 26\end{array}$ \\
\hline Rnd1_85 & 1.13 & 3.08 & 51.00 & $\begin{array}{l}{[n+](C c 1 c c([N+](=O)[O-]) c c([N+](=O)[O-} \\
]) c 1)(\operatorname{ccc} 2[C @ H]([C @] 3([N+] 4(C[C @ @ H]([C @ @]([H])(C C 4) C 3) C=C) C c 5 c c(C(F)(F) F) c c(C(F)(F) F) c \\
5)[H]) O[H]) c(c c c c 6) c 26\end{array}$ \\
\hline Rnd1_86 & 1.13 & 3.08 & 51.00 & $\begin{array}{l}{[\mathrm{n}+](\mathrm{Cc} 1 \mathrm{ccc}(\mathrm{Br}) \mathrm{cc} 1)(\mathrm{ccc} 2[\mathrm{C} @ \mathrm{H}]([\mathrm{C} @] 3([\mathrm{~N}+] 4(\mathrm{C}[\mathrm{C} @ @ \mathrm{H}]([\mathrm{C} @ @]([\mathrm{H}])(\mathrm{CC} 4) \mathrm{C} 3) \mathrm{C}=\mathrm{C}) \mathrm{Cc} 5 \mathrm{cc}(\mathrm{C}(\mathrm{F})(\mathrm{F})} \\
\mathrm{F}) \mathrm{cc}(\mathrm{C}(\mathrm{F})(\mathrm{F}) \mathrm{F}) \mathrm{c5})[\mathrm{H}]) \mathrm{O}[\mathrm{H}]) \mathrm{c}(\mathrm{cccc} 6) \mathrm{c} 26\end{array}$ \\
\hline Rnd1_87 & 1.10 & 3.00 & 50.00 & $\begin{array}{l}{[\mathrm{n}+](\mathrm{Cc} 1 \mathrm{cc}(\mathrm{Br}) \mathrm{cc}(\mathrm{C}(\mathrm{F})(\mathrm{F}) \mathrm{F}) \mathrm{c} 1)(\mathrm{ccc} 2[\mathrm{C} @ \mathrm{H}]([\mathrm{C} @] 3([\mathrm{~N}+] 4(\mathrm{C}[\mathrm{C} @ @ \mathrm{H}]([\mathrm{C} @ @]([\mathrm{H}])(\mathrm{CC} 4) \mathrm{C} 3) \mathrm{C}=\mathrm{C}) \mathrm{Cc} 5} \\
\mathrm{cc}(\mathrm{Br}) \mathrm{cc}(\mathrm{C}(\mathrm{F})(\mathrm{F}) \mathrm{F}) \mathrm{c} 5)[\mathrm{H}]) \mathrm{O}[\mathrm{H}]) \mathrm{c}(\mathrm{ccc}(\mathrm{OC}) \mathrm{c} 6) \mathrm{c} 26\end{array}$ \\
\hline Rnd1_88 & 1.10 & 3.00 & 50.00 & $\begin{array}{l}{[n+](C c 1 c c(C(F)(F) F) c c(C(F)(F) F) c 1)(\operatorname{ccc} 2[C @ H]([C @] 3([N+] 4(C[C @ @ H]([C @ @]([H])(C C 4) C 3) C=} \\
c) \operatorname{cc} 5 c c(C(F)(F) F) c c(C(F)(F) F) c 5)[H]) O[H]) c(c c c(O C) c 6) c 26\end{array}$ \\
\hline Rnd1_89 & 1.10 & 3.00 & 50.00 & $\begin{array}{l}{[\mathrm{n}+](\mathrm{Cc} 1 \mathrm{c}(\mathrm{Br}) \mathrm{ccc}(\mathrm{OC}) \mathrm{c} 1)(\mathrm{ccc} 2[\mathrm{C} @ \mathrm{H}]([\mathrm{C} @] 3([\mathrm{~N}+] 4(\mathrm{C}[\mathrm{C} @ @ \mathrm{H}]([\mathrm{C} @ @]([\mathrm{H}])(\mathrm{CC} 4) \mathrm{C} 3) \mathrm{C}=\mathrm{C}) \mathrm{Cc} 5 \mathrm{cc}(\mathrm{Cl}} \\
\mathrm{F})(\mathrm{F}) \mathrm{F}) \mathrm{c}(\mathrm{Cl}) \mathrm{cc} 5)[\mathrm{H}]) \mathrm{O}[\mathrm{H}]) \mathrm{c}(\mathrm{cccc} 6) \mathrm{c} 26\end{array}$ \\
\hline Rnd1_90 & 1.10 & 3.00 & 50.00 & $\begin{array}{l}{[n+](\mathrm{Cc} 1 \mathrm{c}(\mathrm{Cl}) \mathrm{cccc} 1)(\mathrm{ccc} 2[\mathrm{C} @ \mathrm{H}]([\mathrm{C} @] 3([\mathrm{~N}+] 4(\mathrm{C}[\mathrm{C} @ @ \mathrm{H}]([\mathrm{C} @ @]([\mathrm{H}])(\mathrm{CC} 4) \mathrm{C} 3) \mathrm{C}=\mathrm{C}) \mathrm{Cc} 5 \mathrm{cc}(\mathrm{C}(\mathrm{F})(\mathrm{F})} \\
\mathrm{F}) \mathrm{cc}(\mathrm{C}(\mathrm{F})(\mathrm{F}) \mathrm{F}) \mathrm{c} 5)[\mathrm{H}]) \mathrm{O}[\mathrm{H}]) \mathrm{c}(\mathrm{cccc} 6) \mathrm{c} 26\end{array}$ \\
\hline Rnd1_91 & 1.07 & 2.92 & 49.00 & $\begin{array}{l}{[n+](C c 1 c(O C(F)(F) F) c c c(F) c 1)(c c c 2[C @ H]([C @] 3([N+] 4(C[C @ @ H]([C @ @]([H])(C C 4) C 3) C=C) C c} \\
5 c c(C(F)(F) F) c c(C(F)(F) F) c 5)[H]) O[H]) c(c c c c 6) c 26\end{array}$ \\
\hline Rnd1__92 & 1.07 & 2.92 & 49.00 & $\begin{array}{l}{[n+](C c 1 c([N+](=O)[O-} \\
]) c c(F) c c 1)(\operatorname{ccc} 2[C @ H]([C @] 3([N+] 4(C[C @ @ H]([C @ @])([H])(C C 4) C 3) C=C) C c 5 c c(C(F)(F) F) c c(C(F) \\
(F) F) c 5)[H]) O[H]) c(c c c c 6) c 26\end{array}$ \\
\hline Rnd1_93 & 1.07 & 2.91 & 48.80 & $\begin{array}{l}{[n+](C c 1 c c(C(F)(F) F) c c(C(F)(F) F) c 1)(\operatorname{ccc} 2[C @ H]([C @] 3([N+] 4(C[C @ @ H]([C @ @]([H])(C C 4) C 3) C=} \\
C) C c 5 c(F) c c c c 5)[H]) O[H]) c(c c c c 6) c 26\end{array}$ \\
\hline Rnd1_94 & 0.97 & 2.64 & 45.00 & $\begin{array}{l}{[n+](\mathrm{Cc} 1 \mathrm{cc}(\mathrm{F}) \mathrm{cc}(\mathrm{C}(\mathrm{F})(\mathrm{F}) \mathrm{F}) \mathrm{c} 1)(\mathrm{ccc} 2[\mathrm{C} @ \mathrm{H}]([\mathrm{C} @] 3([\mathrm{~N}+] 4(\mathrm{C}[\mathrm{C} @ @ \mathrm{H}]([\mathrm{C} @ @]([\mathrm{H}])(\mathrm{CC} 4) \mathrm{C} 3) \mathrm{C}=\mathrm{C}) \mathrm{Cc} 5 \mathrm{c}} \\
\mathrm{c}(\mathrm{F}) \mathrm{cc}(\mathrm{C}(\mathrm{F})(\mathrm{F}) \mathrm{F}) \mathrm{c} 5)[\mathrm{H}]) \mathrm{O}[\mathrm{H}]) \mathrm{c}(\mathrm{ccc}(\mathrm{OC}) \mathrm{c} 6) \mathrm{c} 26\end{array}$ \\
\hline
\end{tabular}




\begin{tabular}{|c|c|c|c|c|}
\hline Rnd1_95 & 0.90 & 2.45 & 42.00 & $\begin{array}{l}{[\mathrm{n}+](\mathrm{Cc} 1 \mathrm{c}(\mathrm{OC}) \mathrm{ccc}(\mathrm{Br}) \mathrm{c1})(\mathrm{ccc} 2[\mathrm{C} @ \mathrm{H}]([\mathrm{C} @] 3([\mathrm{~N}+] 4(\mathrm{C}[\mathrm{C} @ @ \mathrm{H}]([\mathrm{C} @ @]([\mathrm{H}])(\mathrm{CC} 4) \mathrm{C3}) \mathrm{C}=\mathrm{C}) \mathrm{Cc} 5 \mathrm{cc}(\mathrm{Cl}} \\
\mathrm{F})(\mathrm{F}) \mathrm{F}) \mathrm{cc}(\mathrm{C}(\mathrm{F})(\mathrm{F}) \mathrm{F}) \mathrm{c5})[\mathrm{H}]) \mathrm{O}[\mathrm{H}]) \mathrm{c}(\mathrm{cccc} 6) \mathrm{c} 26\end{array}$ \\
\hline Rnd1_96 & 0.90 & 2.45 & 42.00 & $\begin{array}{l}{[\mathrm{n}+](\mathrm{Cc} 1 \mathrm{c}(\mathrm{Br}) \mathrm{ccc}(\mathrm{OC}) \mathrm{c1})(\mathrm{ccc} 2[\mathrm{C} @ \mathrm{H}]([\mathrm{C} @] 3([\mathrm{~N}+] 4(\mathrm{C}[\mathrm{C} @ @ \mathrm{H}]([\mathrm{C} @ @]([\mathrm{H}])(\mathrm{CC} 4) \mathrm{C} 3) \mathrm{C}=\mathrm{C}) \mathrm{Cc} 5 \mathrm{cccc}(} \\
\mathrm{Cl}) \mathrm{c5})[\mathrm{H}]) \mathrm{O}[\mathrm{H}]) \mathrm{c}(\mathrm{cccc} 6) \mathrm{c} 26\end{array}$ \\
\hline Rnd1_97 & 0.88 & 2.41 & 41.30 & $\begin{array}{l}{[n+](C c 1 c c(C(F)(F) F) c c(C(F)(F) F) c 1)(\operatorname{ccc} 2[C @ H]([C @] 3([N+] 4(C[C @ @ H]([C @ @]([H])(C C 4) C 3) C=} \\
c) c c 5 c c([N+](=O)[O-]) c c c 5)[H]) O[H]) c(c c c c 6) c 26\end{array}$ \\
\hline Rnd1_98 & 0.85 & 2.33 & 40.00 & $\begin{array}{l}{[\mathrm{n}+](\mathrm{Cc} 1 \mathrm{c}(\mathrm{Br}) \mathrm{ccc}(\mathrm{OC}) \mathrm{c} 1)(\mathrm{ccc} 2[\mathrm{C} @ \mathrm{H}]([\mathrm{C} @] 3([\mathrm{~N}+] 4(\mathrm{C}[\mathrm{C} @ @ \mathrm{H}]([\mathrm{C} @ @]([\mathrm{H}])(\mathrm{CC} 4) \mathrm{C} 3) \mathrm{C}=\mathrm{C}) \mathrm{Cc} 5 \mathrm{cc}(\mathrm{Cl}} \\
\mathrm{F})(\mathrm{F}) \mathrm{F}) \mathrm{ccc} 5 \mathrm{Cl})[\mathrm{H}]) \mathrm{O}[\mathrm{H}]) \mathrm{c}(\mathrm{cccc} 6) \mathrm{c} 26\end{array}$ \\
\hline Rnd1_99 & 0.85 & 2.33 & 40.00 & $\begin{array}{l}{[n+](C c 1 c c(C(F)(F) F) c c(C(F)(F) F) c 1)(c c c 2[C @ H]([C @] 3([N+] 4(C[C @ @ H]([C @ @]([H])(C C 4) C 3) C=} \\
C) C c 5 c(F) c(F) c c c 5)[H]) O[H]) c(c c c c 6) c 26\end{array}$ \\
\hline Rnd1_100 & 0.82 & 2.28 & 39.00 & $\begin{array}{l}{[n+](\operatorname{Cc} 1 c c(C(F)(F) F) c c(C(F)(F) F) c 1)(\operatorname{ccc} 2[C @ H]([C @] 3([N+] 4(C[C @ @ H]([C @ @]([H])(C C 4) C 3) C=} \\
C) \operatorname{Cc} 5 \operatorname{cc}(F) \operatorname{ccc} 5 C(F)(F) F)[H]) O[H]) c(\operatorname{cccc} 6) c 26\end{array}$ \\
\hline Rnd1_101 & 0.82 & 2.28 & 39.00 & $\begin{array}{l}{[n+](C c 1 c c c(F) c(C(F)(F) F) c 1)(\operatorname{ccc} 2[C @ H]([C @] 3([N+] 4(C[C @ @ H]([C @ @]([H])(C C 4) C 3) C=C) C c 5 c} \\
c(C(F)(F) F) c(F) c c 5)[H]) O[H]) c(\operatorname{ccc}(\mathrm{OC}) \mathrm{c} 6) c 26\end{array}$ \\
\hline Rnd1_102 & 0.82 & 2.28 & 39.00 & $\begin{array}{l}{[\mathrm{n}+](\mathrm{Cc} 1 \mathrm{c}(\mathrm{Br}) \mathrm{ccc}(\mathrm{OC}) \mathrm{c1})(\mathrm{ccc} 2[\mathrm{C} @ \mathrm{H}]([\mathrm{C} @] 3([\mathrm{~N}+] 4(\mathrm{C}[\mathrm{C} @ @ \mathrm{H}]([\mathrm{C} @ @]([\mathrm{H}])(\mathrm{CC} 4) \mathrm{C} 3) \mathrm{C}=\mathrm{C}) \mathrm{Cc} 5 \mathrm{cc}(\mathrm{Cl}} \\
\mathrm{F})(\mathrm{F}) \mathrm{F}) \mathrm{cc}(\mathrm{F}) \mathrm{c} 5)[\mathrm{H}]) \mathrm{O}[\mathrm{H}]) \mathrm{c}(\mathrm{cccc} 6) \mathrm{c} 26\end{array}$ \\
\hline Rnd1_103 & 0.81 & 2.25 & 38.50 & $\begin{array}{l}{[\mathrm{n}+](\mathrm{Cc} 1 \mathrm{c}(\mathrm{Br}) \mathrm{ccc}(\mathrm{OC}) \mathrm{c1})(\mathrm{ccc} 2[\mathrm{C} @ \mathrm{H}]([\mathrm{C} @] 3([\mathrm{~N}+] 4(\mathrm{C}[\mathrm{C} @ @ \mathrm{H}]([\mathrm{C} @ @]([\mathrm{H}])(\mathrm{CC} 4) \mathrm{C} 3) \mathrm{C}=\mathrm{C}) \mathrm{Cc} 5 \mathrm{cc}(\mathrm{Br}} \\
) \mathrm{ccc} 5)[\mathrm{H}]) \mathrm{O}[\mathrm{H}]) \mathrm{c}(\mathrm{cccc} 6) \mathrm{c} 26\end{array}$ \\
\hline Rnd1_104 & 0.78 & 2.17 & 37.00 & $\begin{array}{l}{[\mathrm{n}+](\mathrm{Cc} 1 \mathrm{c}(\mathrm{Br}) \mathrm{ccc}(\mathrm{OC}) \mathrm{c} 1)(\mathrm{ccc} 2[\mathrm{C} @ \mathrm{H}]([\mathrm{C} @] 3([\mathrm{~N}+] 4(\mathrm{C}[\mathrm{C} @ @ \mathrm{H}]([\mathrm{C} @ @]([\mathrm{H}])(\mathrm{CC} 4) \mathrm{C} 3) \mathrm{C}=\mathrm{C}) \mathrm{Cc} 5 \mathrm{c}(\mathrm{F}) \mathrm{c}} \\
\mathrm{c}(\mathrm{Br}) \mathrm{cc} 5)[\mathrm{H}]) \mathrm{O}[\mathrm{H}]) \mathrm{c}(\mathrm{cccc} 6) \mathrm{c} 26\end{array}$ \\
\hline Rnd1_105 & 0.78 & 2.17 & 37.00 & $\begin{array}{l}{[\mathrm{n}+](\mathrm{Cc} 1 \mathrm{c}(\mathrm{Br}) \mathrm{ccc}(\mathrm{OC}) \mathrm{c} 1)(\mathrm{ccc} 2[\mathrm{C} @ \mathrm{H}]([\mathrm{C} @] 3([\mathrm{~N}+] 4(\mathrm{C}[\mathrm{C} @ @ \mathrm{H}]([\mathrm{C} @ @]([\mathrm{H}])(\mathrm{CC} 4) \mathrm{C} 3) \mathrm{C}=\mathrm{C}) \mathrm{Cc} 5 \mathrm{ccc}(} \\
\mathrm{C}(\mathrm{F})(\mathrm{F}) \mathrm{F}) \mathrm{c}(\mathrm{F}) \mathrm{c} 5)[\mathrm{H}]) \mathrm{O}[\mathrm{H}]) \mathrm{c}(\mathrm{cccc} 6) \mathrm{c} 26\end{array}$ \\
\hline Rnd1_106 & 0.78 & 2.17 & 37.00 & $\begin{array}{l}{[n+](C c 1 c c(C(F)(F) F) c c(C(F)(F) F) c 1)(\operatorname{ccc} 2[C @ H]([C @] 3([N+] 4(C[C @ @ H]([C @ @]([H])(C C 4) C 3) C=} \\
C) \operatorname{cc5cc}(C \# N) \operatorname{ccc} 5)[H]) O[H]) c(\operatorname{cccc} 6) c 26\end{array}$ \\
\hline
\end{tabular}




\begin{tabular}{|c|c|c|c|c|}
\hline Rnd1_107 & 0.75 & 2.13 & 36.00 & $\begin{array}{l}{[n+](C c 1 c c c c(C(F)(F) F) c 1)(c c c 2[C @ H]([C @] 3([N+] 4(C[C @ @ H]([C @ @]([H])(C C 4) C 3) C=C) C c 5 c c(} \\
C(F)(F) F)(c c c 5)[H]) O[H]) c(\operatorname{ccc}(\mathrm{OC}) c 6) c 26\end{array}$ \\
\hline Rnd1_108 & 0.73 & 2.08 & 35.00 & $\begin{array}{l}{[n+](\mathrm{Cc} 1 \mathrm{cc}(\mathrm{OC}) \mathrm{cc}(\mathrm{C}(\mathrm{F})(\mathrm{F}) \mathrm{F}) \mathrm{c1})(\mathrm{ccc} 2[\mathrm{C} @ \mathrm{H}]([\mathrm{C} @] 3([\mathrm{~N}+] 4(\mathrm{C}[\mathrm{C} @ @ \mathrm{H}]([\mathrm{C} @ @]([\mathrm{H}])(\mathrm{CC} 4) \mathrm{C} 3) \mathrm{C}=\mathrm{C}) \mathrm{Cc}} \\
5 \mathrm{cc}(\mathrm{C}(\mathrm{F})(\mathrm{F}) \mathrm{F}) \mathrm{cc}(\mathrm{OC}) \mathrm{c5})[\mathrm{H}]) \mathrm{O}[\mathrm{H}]) \mathrm{c}(\mathrm{ccc}(\mathrm{OC}) \mathrm{c} 6) \mathrm{c} 26\end{array}$ \\
\hline Rnd1_109 & 0.73 & 2.08 & 35.00 & $\begin{array}{l}{[\mathrm{n}+](\mathrm{Cc} 1 \mathrm{c}(\mathrm{Br}) \mathrm{ccc}(\mathrm{OC}) \mathrm{c1})(\mathrm{ccc} 2[\mathrm{C} @ \mathrm{H}]([\mathrm{C} @] 3([\mathrm{~N}+] 4(\mathrm{C}[\mathrm{C} @ @ \mathrm{H}]([\mathrm{C} @ @]([\mathrm{H}])(\mathrm{CC} 4) \mathrm{C} 3) \mathrm{C}=\mathrm{C}) \mathrm{Cc} 5 \mathrm{cc}(\mathrm{F})} \\
\operatorname{ccc} 5 \mathrm{Br})[\mathrm{H}]) \mathrm{O}[\mathrm{H}]) \mathrm{c}(\mathrm{cccc} 6) \mathrm{c} 26\end{array}$ \\
\hline Rnd1_110 & 0.71 & 2.03 & 34.00 & $\begin{array}{l}{[n+](C c 1 c c(C(F)(F) F) c c(C(F)(F) F) c 1)(\operatorname{ccc} 2[C @ H]([C @] 3([N+] 4(C[C @ @ H]([C @ @]([H])(C C 4) C 3) C=} \\
C) \operatorname{cc5} 5 c(\operatorname{cccc} 6) \operatorname{c6cc5Br})[H]) O[H]) c(\operatorname{cccc} 7) c 27\end{array}$ \\
\hline Rnd1_111 & 0.71 & 2.03 & 34.00 & $\begin{array}{l}{[\mathrm{n}+](\mathrm{Cc} 1 \mathrm{c}(\mathrm{Br}) \mathrm{ccc}(\mathrm{OC}) \mathrm{c} 1)(\mathrm{ccc} 2[\mathrm{C} @ \mathrm{H}]([\mathrm{C} @] 3([\mathrm{~N}+] 4(\mathrm{C}[\mathrm{C} @ @ \mathrm{H}]([\mathrm{C} @ @]([\mathrm{H}])(\mathrm{CC} 4) \mathrm{C} 3) \mathrm{C}=\mathrm{C}) \mathrm{Cc} 5 \mathrm{ccc}(} \\
\mathrm{C}(\mathrm{F})(\mathrm{F}) \mathrm{F}) \mathrm{cc} 5 \mathrm{~F})[\mathrm{H}]) \mathrm{O}[\mathrm{H}]) \mathrm{c}(\mathrm{cccc} 6) \mathrm{c} 26\end{array}$ \\
\hline Rnd1_112 & 0.71 & 2.03 & 34.00 & $\begin{array}{l}{[\mathrm{n}+](\mathrm{Cc} 1 \mathrm{c}(\mathrm{Br}) \mathrm{ccc}(\mathrm{OC}) \mathrm{c} 1)(\mathrm{ccc} 2[\mathrm{C} @ \mathrm{H}]([\mathrm{C} @] 3([\mathrm{~N}+] 4(\mathrm{C}[\mathrm{C} @ @ \mathrm{H}]([\mathrm{C} @ @]([\mathrm{H}])(\mathrm{CC} 4) \mathrm{C} 3) \mathrm{C}=\mathrm{C}) \mathrm{Cc} 5 \mathrm{c}(\mathrm{F}) \mathrm{c}} \\
\mathrm{c}(\mathrm{Cl}) \mathrm{cc} 5)[\mathrm{H}]) \mathrm{O}[\mathrm{H}]) \mathrm{c}(\mathrm{cccc} 6) \mathrm{c} 26\end{array}$ \\
\hline Rnd1_113 & 0.70 & 2.01 & 33.50 & $\begin{array}{l}{[\mathrm{n}+](\mathrm{Cc} 1 \mathrm{c}(\mathrm{Br}) \mathrm{ccc}(\mathrm{OC}) \mathrm{c1})(\mathrm{ccc} 2[\mathrm{C} @ \mathrm{H}]([\mathrm{C} @] 3([\mathrm{~N}+] 4(\mathrm{C}[\mathrm{C} @ @ \mathrm{H}]([\mathrm{C} @ @]([\mathrm{H}])(\mathrm{CC} 4) \mathrm{C} 3) \mathrm{C}=\mathrm{C}) \mathrm{Cc} 5 \mathrm{cc}(\mathrm{Cl}} \\
\mathrm{F})(\mathrm{F}) \mathrm{F}) \mathrm{ccc} 5 \mathrm{~F})[\mathrm{H}]) \mathrm{O}[\mathrm{H}]) \mathrm{c}(\mathrm{cccc} 6) \mathrm{c} 26\end{array}$ \\
\hline Rnd1_114 & 0.69 & 1.99 & 33.00 & $\begin{array}{l}{[n+](\mathrm{Cc} 1 \mathrm{ccc}(\mathrm{C}(\mathrm{F})(\mathrm{F}) \mathrm{F}) \mathrm{cc} 1)(\mathrm{ccc} 2[\mathrm{C} @ \mathrm{H}]([\mathrm{C} @] 3([\mathrm{~N}+] 4(\mathrm{C}[\mathrm{C} @ @ \mathrm{H}]([\mathrm{C} @ @]([\mathrm{H}])(\mathrm{CC} 4) \mathrm{C} 3) \mathrm{C}=\mathrm{C}) \mathrm{Cc} 5 \mathrm{ccc}(} \\
\mathrm{C}(\mathrm{F})(\mathrm{F}) \mathrm{F}) \mathrm{cc} 5)[\mathrm{H}]) \mathrm{O}[\mathrm{H}]) \mathrm{c}(\mathrm{ccc}(\mathrm{OC}) \mathrm{c} 6) \mathrm{c} 26\end{array}$ \\
\hline Rnd1_115 & 0.66 & 1.94 & 32.00 & $\begin{array}{l}{[n+](\mathrm{Cc} 1 \mathrm{c}(\mathrm{Br}) \mathrm{ccc}(\mathrm{OC}) \mathrm{c} 1)(\mathrm{ccc} 2[\mathrm{C} @ \mathrm{H}]([\mathrm{C} @] 3([\mathrm{~N}+] 4(\mathrm{C}[\mathrm{C} @ @ \mathrm{H}]([\mathrm{C} @ @]([\mathrm{H}])(\mathrm{CC} 4) \mathrm{C} 3) \mathrm{C}=\mathrm{C}) \mathrm{Cc} 5 \mathrm{ccc}(\mathrm{F}} \\
) \mathrm{c}(\mathrm{C}(\mathrm{F})(\mathrm{F}) \mathrm{F}) \mathrm{c} 5)[\mathrm{H}]) \mathrm{O}[\mathrm{H}]) \mathrm{c}(\mathrm{cccc} 6) \mathrm{c} 26\end{array}$ \\
\hline Rnd1_116 & 0.64 & 1.90 & 31.00 & $\begin{array}{l}{[\mathrm{n}+](\mathrm{Cc} 1 \mathrm{c}(\mathrm{Br}) \mathrm{ccc}(\mathrm{OC}) \mathrm{c} 1)(\mathrm{ccc} 2[\mathrm{C} @ \mathrm{H}]([\mathrm{C} @] 3([\mathrm{~N}+] 4(\mathrm{C}[\mathrm{C} @ @ \mathrm{H}]([\mathrm{C} @ @]([\mathrm{H}])(\mathrm{CC} 4) \mathrm{C} 3) \mathrm{C}=\mathrm{C}) \mathrm{Cc} 5 \mathrm{cc}(\mathrm{Cl}} \\
) \mathrm{ccc} 5 \mathrm{Cl})[\mathrm{H}]) \mathrm{O}[\mathrm{H}]) \mathrm{c}(\mathrm{cccc} 6) \mathrm{c} 26\end{array}$ \\
\hline Rnd1_117 & 0.62 & 1.86 & 30.00 & $\begin{array}{l}{[n+](\mathrm{Cc} 1 \mathrm{c}(\mathrm{OC}(\mathrm{F})(\mathrm{F}) \mathrm{F}) \mathrm{cc}(\mathrm{F}) \mathrm{cc} 1)(\mathrm{ccc} 2[\mathrm{C} @ \mathrm{H}]([\mathrm{C} @] 3([\mathrm{~N}+] 4(\mathrm{C}[\mathrm{C} @ @ \mathrm{H}]([\mathrm{C} @ @]([\mathrm{H}])(\mathrm{CC} 4) \mathrm{C} 3) \mathrm{C}=\mathrm{C}) \mathrm{Cc}} \\
5 \mathrm{cc}(\mathrm{C}(\mathrm{F})(\mathrm{F}) \mathrm{F}) \mathrm{cc}(\mathrm{C}(\mathrm{F})(\mathrm{F}) \mathrm{F}) \mathrm{c} 5)[\mathrm{H}]) \mathrm{O}[\mathrm{H}]) \mathrm{c}(\mathrm{cccc} 6) \mathrm{c} 26\end{array}$ \\
\hline Rnd1_118 & 0.62 & 1.86 & 30.00 & $\begin{array}{l}{[n+](\mathrm{Cc} 1 \mathrm{c}(\mathrm{OC}) \operatorname{ccc}(\mathrm{C}(\mathrm{F})(\mathrm{F}) \mathrm{F}) \mathrm{c1})(\mathrm{ccc} 2[\mathrm{C} @ \mathrm{H}]([\mathrm{C} @] 3([\mathrm{~N}+] 4(\mathrm{C}[\mathrm{C} @ @ \mathrm{H}]([\mathrm{C} @ @]([\mathrm{H}])(\mathrm{CC} 4) \mathrm{C} 3) \mathrm{C}=\mathrm{C}) \mathrm{Cc}} \\
5 \mathrm{cc}(\mathrm{C}(\mathrm{F})(\mathrm{F}) \mathrm{F}) \mathrm{ccc} 5 \mathrm{OC})[\mathrm{H}]) \mathrm{O}[\mathrm{H}]) \mathrm{c}(\mathrm{ccc}(\mathrm{OC}) \mathrm{c} 6) \mathrm{c} 26\end{array}$ \\
\hline
\end{tabular}




\begin{tabular}{|c|c|c|c|c|}
\hline Rnd1_119 & 0.62 & 1.86 & 30.00 & $\begin{array}{l}{[n+](\mathrm{Cc} 1 c \mathrm{cc}(\mathrm{OC}) \mathrm{cc}(\mathrm{OC}) \mathrm{c1})(\mathrm{ccc} 2[\mathrm{C} @ \mathrm{H}]([\mathrm{C} @] 3([\mathrm{~N}+] 4(\mathrm{C}[\mathrm{C} @ @ \mathrm{H}]([\mathrm{C} @ @]]([\mathrm{H}])(\mathrm{CC} 4) \mathrm{C} 3) \mathrm{C}=\mathrm{C}) \mathrm{Cc} 5 \mathrm{cc}(\mathrm{C}} \\
(\mathrm{F})(\mathrm{F}) \mathrm{F}) \mathrm{cc}(\mathrm{C}(\mathrm{F})(\mathrm{F}) \mathrm{F}) \mathrm{c} 5)[\mathrm{H}]) \mathrm{O}[\mathrm{H}]) \mathrm{c}(\mathrm{cccc} 6) \mathrm{c} 26\end{array}$ \\
\hline Rnd1_120 & 0.58 & 1.78 & 28.00 & $\begin{array}{l}{[\mathrm{n}+](\mathrm{Cc} 1 \mathrm{ccc}(\mathrm{C}(\mathrm{C})(\mathrm{C}) \mathrm{C}) \mathrm{cc} 1)(\mathrm{ccc} 2[\mathrm{C} @ \mathrm{H}]([\mathrm{C} @] 3([\mathrm{~N}+] 4(\mathrm{C}[\mathrm{C} @ @ \mathrm{H}]([\mathrm{C} @ @]([\mathrm{H}])(\mathrm{CC} 4) \mathrm{C} 3) \mathrm{C}=\mathrm{C}) \mathrm{Cc} 5 \mathrm{ccc}} \\
(\mathrm{C}(\mathrm{C})(\mathrm{C}) \mathrm{C}) \mathrm{cc} 5)[\mathrm{H}]) \mathrm{O}[\mathrm{H}]) \mathrm{c}(\mathrm{ccc}(\mathrm{OC}) \mathrm{c} 6) \mathrm{c} 26\end{array}$ \\
\hline Rnd1_121 & 0.55 & 1.74 & 27.00 & $\begin{array}{l}{[\mathrm{n}+](\mathrm{Cc} 1 \mathrm{c}(\mathrm{F}) \mathrm{ccc}(\mathrm{OC}(\mathrm{F})(\mathrm{F}) \mathrm{F}) \mathrm{c} 1)(\mathrm{ccc} 2[\mathrm{C} @ \mathrm{H}]([\mathrm{C} @] 3([\mathrm{~N}+] 4(\mathrm{C}[\mathrm{C} @ @ \mathrm{H}]([\mathrm{C} @ @]([\mathrm{H}])(\mathrm{CC} 4) \mathrm{C} 3) \mathrm{C}=\mathrm{C}) \mathrm{Cc}} \\
5 \mathrm{cc}(\mathrm{OC}(\mathrm{F})(\mathrm{F}) \mathrm{F}) \operatorname{ccc} \mathrm{cc} F)[\mathrm{H}]) \mathrm{O}[\mathrm{H}]) \mathrm{c}(\mathrm{ccc}(\mathrm{OC}) \mathrm{c} 6) \mathrm{c} 26\end{array}$ \\
\hline Rnd1_122 & 0.51 & 1.67 & 25.00 & $\begin{array}{l}{[n+](C c 1 c c(C(F)(F) F) c c(C(F)(F) F) c 1)(\operatorname{ccc} 2[C @ H]([C @] 3([N+] 4(C[C @ @ H]([C @ @]([H])(C C 4) C 3) C=} \\
C) \operatorname{cc5} 5 \operatorname{ccc}(C(F)(F) F) c c 5[N+](=O)[O-])[H]) O[H]) c(\operatorname{cccc} 6) c 26\end{array}$ \\
\hline Rnd1_123 & 0.51 & 1.67 & 25.00 & $\begin{array}{l}{[\mathrm{n}+](\mathrm{Cc} 1 \mathrm{c}(\mathrm{Br}) \mathrm{ccc}(\mathrm{OC}) \mathrm{c} 1)(\mathrm{ccc} 2[\mathrm{C} @ \mathrm{H}]([\mathrm{C} @] 3([\mathrm{~N}+] 4(\mathrm{C}[\mathrm{C} @ @ \mathrm{H}]([\mathrm{C} @ @]([\mathrm{H}])(\mathrm{CC} 4) \mathrm{C} 3) \mathrm{C}=\mathrm{C}) \mathrm{Cc} 5 \mathrm{cc}(\mathrm{Br}} \\
) \mathrm{ccc} 5 \mathrm{~F})[\mathrm{H}]) \mathrm{O}[\mathrm{H}]) \mathrm{c}(\mathrm{cccc} 6) \mathrm{c} 26\end{array}$ \\
\hline Rnd1_124 & 0.51 & 1.67 & 25.00 & $\begin{array}{l}{[n+](\mathrm{Cc} 1 \mathrm{c}(\mathrm{F}) \mathrm{c}(\mathrm{F}) \mathrm{c}(\mathrm{F}) \mathrm{cc} 1)(\mathrm{ccc} 2[\mathrm{C} @ \mathrm{H}]([\mathrm{C} @] 3([\mathrm{~N}+] 4(\mathrm{C}[\mathrm{C} @ @ \mathrm{H}]([\mathrm{C} @ @]([\mathrm{H}])(\mathrm{CC} 4) \mathrm{C} 3) \mathrm{C}=\mathrm{C}) \mathrm{Cc} 5 \mathrm{ccc}(\mathrm{F}} \\
) \mathrm{c}(\mathrm{F}) \mathrm{c} 5 \mathrm{~F})[\mathrm{H}]) \mathrm{O}[\mathrm{H}]) \mathrm{c}(\mathrm{ccc}(\mathrm{OC}) \mathrm{c} 6) \mathrm{c} 26\end{array}$ \\
\hline Rnd1_125 & 0.49 & 1.63 & 24.00 & $\begin{array}{l}{[\mathrm{n}+](\mathrm{Cc} 1 \mathrm{c}(\mathrm{Br}) \mathrm{ccc}(\mathrm{OC}) \mathrm{c1})(\mathrm{ccc} 2[\mathrm{C} @ \mathrm{H}]([\mathrm{C} @] 3([\mathrm{~N}+] 4(\mathrm{C}[\mathrm{C} @ @ \mathrm{H}]([\mathrm{C} @ @]([\mathrm{H}])(\mathrm{CC} 4) \mathrm{C} 3) \mathrm{C}=\mathrm{C}) \mathrm{Cc} 5 \mathrm{cc}([} \\
\mathrm{N}+](=\mathrm{O})[\mathrm{O}-]) \operatorname{ccc} 5)[\mathrm{H}]) \mathrm{O}[\mathrm{H}]) \mathrm{c}(\mathrm{cccc} 6) \mathrm{c} 26\end{array}$ \\
\hline Rnd1_126 & 0.49 & 1.63 & 24.00 & $\begin{array}{l}{[\mathrm{n}+](\mathrm{Cc} 1 \mathrm{c}(\mathrm{Br}) \mathrm{ccc}(\mathrm{OC}) \mathrm{c} 1)(\mathrm{ccc} 2[\mathrm{C} @ \mathrm{H}]([\mathrm{C} @] 3([\mathrm{~N}+] 4(\mathrm{C}[\mathrm{C} @ @ \mathrm{H}]([\mathrm{C} @ @]([\mathrm{H}])(\mathrm{CC} 4) \mathrm{C} 3) \mathrm{C}=\mathrm{C}) \mathrm{Cc} 5 \mathrm{cc}(\mathrm{F})} \\
\mathrm{cc}(\mathrm{F}) \mathrm{c} 5)[\mathrm{H}]) \mathrm{O}[\mathrm{H}]) \mathrm{c}(\mathrm{cccc} 6) \mathrm{c} 26\end{array}$ \\
\hline Rnd1_127 & 0.48 & 1.61 & 23.50 & $\begin{array}{l}{[\mathrm{n}+](\mathrm{Cc} 1 \mathrm{c}(\mathrm{Br}) \mathrm{ccc}(\mathrm{OC}) \mathrm{c} 1)(\mathrm{ccc} 2[\mathrm{C} @ \mathrm{H}]([\mathrm{C} @] 3([\mathrm{~N}+] 4(\mathrm{C}[\mathrm{C} @ @ \mathrm{H}]([\mathrm{C} @ @]([\mathrm{H}])(\mathrm{CC} 4) \mathrm{C} 3) \mathrm{C}=\mathrm{C}) \mathrm{Cc} 5 \mathrm{cc}(\mathrm{F})} \\
\mathrm{ccc5})[\mathrm{H}]) \mathrm{O}[\mathrm{H}]) \mathrm{c}(\mathrm{cccc}) \mathrm{c} 26\end{array}$ \\
\hline Rnd1_128 & 0.45 & 1.56 & 22.00 & $\begin{array}{l}{[\mathrm{n}+](\mathrm{Cc} 1 \mathrm{c}(\mathrm{Br}) \mathrm{ccc}([\mathrm{N}+](=\mathrm{O})[\mathrm{O}-} \\
]) \mathrm{c} 1)(\mathrm{ccc} 2[\mathrm{C} @ \mathrm{H}]([\mathrm{C} @] 3([\mathrm{~N}+] 4(\mathrm{C}[\mathrm{C} @ @ \mathrm{H}]([\mathrm{C} @ @]([\mathrm{H}])(\mathrm{CC} 4) \mathrm{C} 3) \mathrm{C}=\mathrm{C}) \mathrm{Cc} 5 \mathrm{cc}([\mathrm{N}+](=\mathrm{O})[\mathrm{O}- \\
]) \mathrm{ccc} 5 \mathrm{Br})[\mathrm{H}]) \mathrm{O}[\mathrm{H}]) \mathrm{c}(\mathrm{ccc}(\mathrm{OC}) \mathrm{c} 6) \mathrm{c} 26\end{array}$ \\
\hline Rnd1_129 & 0.43 & 1.53 & 21.00 & $\begin{array}{l}{[\mathrm{n}+](\mathrm{Cc} 1 \mathrm{c}(\mathrm{Br}) \mathrm{ccc}(\mathrm{OC}) \mathrm{c} 1)(\mathrm{ccc} 2[\mathrm{C} @ \mathrm{H}]([\mathrm{C} @] 3([\mathrm{~N}+] 4(\mathrm{C}[\mathrm{C} @ @ \mathrm{H}]([\mathrm{C} @ @]([\mathrm{H}])(\mathrm{CC} 4) \mathrm{C} 3) \mathrm{C}=\mathrm{C}) \mathrm{Cc} 5 \mathrm{ccc}(\mathrm{F}} \\
) \mathrm{cc} 5 \mathrm{Cl})[\mathrm{H}]) \mathrm{O}[\mathrm{H}]) \mathrm{c}(\mathrm{cccc} 6) \mathrm{c} 26\end{array}$ \\
\hline Rnd1_130 & 1.89 & 6.60 & 73.70 & $\begin{array}{l}{[n+](\mathrm{Cc} 1 \mathrm{cc}(\mathrm{OC}(\mathrm{F})(\mathrm{F}) \mathrm{F}) \mathrm{ccc} 1)(\mathrm{ccc} 2[\mathrm{C} @ \mathrm{H}]([\mathrm{C} @] 3([\mathrm{~N}+] 4(\mathrm{C}[\mathrm{C} @ @ \mathrm{H}]([\mathrm{C} @ @]([\mathrm{H}])(\mathrm{CC} 4) \mathrm{C} 3) \mathrm{C}=\mathrm{C}) \mathrm{Cc} 5 \mathrm{cc}} \\
(\mathrm{C}(\mathrm{F})(\mathrm{F}) \mathrm{F}) \mathrm{cc}(\mathrm{C}(\mathrm{F})(\mathrm{F}) \mathrm{F}) \mathrm{c5})[\mathrm{H}]) \mathrm{O}[\mathrm{H}]) \mathrm{c}(\mathrm{cccc}) \mathrm{c} 26\end{array}$ \\
\hline
\end{tabular}




\begin{tabular}{|c|c|c|c|c|}
\hline Rnd1_131 & 0.41 & 1.50 & 20.00 & $\begin{array}{l}{[\mathrm{n}+](\mathrm{Cc} 1 \mathrm{c}(\mathrm{Br}) \mathrm{cccc} 1)(\mathrm{ccc} 2[\mathrm{C} @ \mathrm{H}]([\mathrm{C} @] 3([\mathrm{~N}+] 4(\mathrm{C}[\mathrm{C} @ @ \mathrm{H}]([\mathrm{C} @ @]([\mathrm{H}])(\mathrm{CC} 4) \mathrm{C} 3) \mathrm{C}=\mathrm{C}) \mathrm{Cc} 5 \mathrm{cc}(\mathrm{C}(\mathrm{F})(\mathrm{F})} \\
\mathrm{F}) \mathrm{cc}(\mathrm{C}(\mathrm{F})(\mathrm{F}) \mathrm{F}) \mathrm{c5})[\mathrm{H}]) \mathrm{O}[\mathrm{H}]) \mathrm{c}(\mathrm{cccc} 6) \mathrm{c} 26\end{array}$ \\
\hline Rnd1_132 & 0.41 & 1.50 & 20.00 & $\begin{array}{l}{[\mathrm{n}+](\mathrm{Cc} 1 \mathrm{cc}(\mathrm{Br}) \mathrm{ccc} 1)(\mathrm{ccc} 2[\mathrm{C} @ \mathrm{H}]([\mathrm{C} @] 3([\mathrm{~N}+] 4(\mathrm{C}[\mathrm{C} @ @ \mathrm{H}]([\mathrm{C} @ @]([\mathrm{H}])(\mathrm{CC} 4) \mathrm{C} 3) \mathrm{C}=\mathrm{C}) \mathrm{Cc} 5 \mathrm{cc}(\mathrm{C}(\mathrm{F})(\mathrm{F})} \\
\mathrm{F}) \mathrm{cc}(\mathrm{C}(\mathrm{F})(\mathrm{F}) \mathrm{F}) \mathrm{c5})[\mathrm{H}]) \mathrm{O}[\mathrm{H}]) \mathrm{c}(\mathrm{cccc} 6) \mathrm{c} 26\end{array}$ \\
\hline Rnd1_133 & 0.41 & 1.50 & 20.00 & $\begin{array}{l}{[n+](C c 1 c([N+](=O)[O-} \\
]) c c(O C) c c 1)(c c c 2[C @ H]([C @] 3([N+] 4(C[C @ @ H]([C @ @]([H])(C C 4) C 3) C=C) C c 5 c c(C(F)(F) F) c c(C l \\
F)(F) F) c 5)[H]) O[H]) c(c c c c 6) c 26\end{array}$ \\
\hline Rnd1_134 & 0.41 & 1.50 & 20.00 & 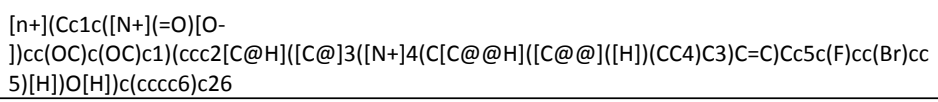 \\
\hline Rnd1_135 & 0.41 & 1.50 & 20.00 & $\begin{array}{l}{[\mathrm{n}+](\mathrm{Cc} 1 \mathrm{c}([\mathrm{N}+])(=\mathrm{O})[\mathrm{O}-} \\
]) \mathrm{cc}(\mathrm{OC}) \mathrm{c}(\mathrm{OC}) \mathrm{c1})(\mathrm{ccc} 2[\mathrm{C} @ \mathrm{H}]([\mathrm{C} @] 3([\mathrm{~N}+] 4(\mathrm{C}[\mathrm{C} @ @ \mathrm{H}]([\mathrm{C} @ @]([\mathrm{H}])(\mathrm{CC} 4) \mathrm{C} 3) \mathrm{C}=\mathrm{C}) \mathrm{Cc} 5 \mathrm{cc}(\mathrm{C}(\mathrm{F})(\mathrm{F}) \mathrm{F}) \mathrm{c} \\
\mathrm{c}(\mathrm{F}) \mathrm{c5})[\mathrm{H}]) \mathrm{O}[\mathrm{H}]) \mathrm{c}(\mathrm{cccc} 6) \mathrm{c} 26\end{array}$ \\
\hline Rnd1_136 & 0.41 & 1.50 & 20.00 & $\begin{array}{l}{[\mathrm{n}+](\mathrm{Cc} 1 \mathrm{cc}(\mathrm{OC}) \mathrm{cc}(\mathrm{C}(\mathrm{F})(\mathrm{F}) \mathrm{F}) \mathrm{c} 1)(\mathrm{ccc} 2[\mathrm{C} @ \mathrm{H}]([\mathrm{C} @] 3([\mathrm{~N}+] 4(\mathrm{C}[\mathrm{C} @ @ \mathrm{H}]([\mathrm{C} @ @]([\mathrm{H}])(\mathrm{CC} 4) \mathrm{C} 3) \mathrm{C}=\mathrm{C}) \mathrm{Cc}} \\
5 \mathrm{cc}(\mathrm{C}(\mathrm{F})(\mathrm{F}) \mathrm{F}) \mathrm{cc}(\mathrm{F}) \mathrm{c} 5)[\mathrm{H}]) \mathrm{O}[\mathrm{H}]) \mathrm{c}(\mathrm{cccc} 6) \mathrm{c} 26\end{array}$ \\
\hline Rnd1_137 & 0.41 & 1.50 & 20.00 & $\begin{array}{l}{[n+](\mathrm{Cc} 1 \mathrm{c}(\mathrm{F}) \mathrm{cc}(\mathrm{Br}) \mathrm{cc} 1)(\mathrm{ccc} 2[\mathrm{C} @ \mathrm{H}]([\mathrm{C} @] 3([\mathrm{~N}+] 4(\mathrm{C}[\mathrm{C} @ @ \mathrm{H}]([\mathrm{C} @ @]([\mathrm{H}])(\mathrm{CC} 4) \mathrm{C} 3) \mathrm{C}=\mathrm{C}) \mathrm{Cc} 5 \mathrm{cc}(\mathrm{C}(\mathrm{F})} \\
(\mathrm{F}) \mathrm{F}) \mathrm{cc}(\mathrm{F}) \mathrm{c5})[\mathrm{H}]) \mathrm{O}[\mathrm{H}]) \mathrm{c}(\mathrm{cccc} 6) \mathrm{c} 26\end{array}$ \\
\hline Rnd1_138 & 0.41 & 1.50 & 20.00 & $\begin{array}{l}{[n+](C c 1 c([N+](=O)[O-} \\
]) c c(O C) c(O C) c 1)(c c c 2[C @ H]([C @] 3([N+] 4(C[C @ @ H]([C @ @]([H])(C C 4) C 3) C=C) C c 5 c c(C(F)(F) F) c \\
c c 5)[H]) O[H]) c(c c c c 6) c 26\end{array}$ \\
\hline Rnd1_139 & 0.41 & 1.50 & 20.00 & $\begin{array}{l}{[\mathrm{n}+](\mathrm{Cc} 1 \mathrm{c}(\mathrm{OC}) \mathrm{ccc}([\mathrm{N}+](=\mathrm{O})[\mathrm{O}-} \\
]) \mathrm{c} 1)(\mathrm{ccc} 2[\mathrm{C} @ \mathrm{H}]([\mathrm{C} @] 3([\mathrm{~N}+] 4(\mathrm{C}[\mathrm{C} @ @ \mathrm{H}]([\mathrm{C} @ @]([\mathrm{H}])(\mathrm{CC} 4) \mathrm{C} 3) \mathrm{C}=\mathrm{C}) \mathrm{Cc} 5 \mathrm{c}(\mathrm{F}) \mathrm{cc}(\mathrm{Br}) \mathrm{cc} 5)[\mathrm{H}]) \mathrm{O}[\mathrm{H}]) \mathrm{c} \\
\text { (cccc6)c26 }\end{array}$ \\
\hline Rnd1_140 & 0.41 & 1.50 & 20.00 & $\begin{array}{l}{[n+](C c 1 c c(O C) c c c 1)(c c c 2[C @ H]([C @] 3([N+] 4(C[C @ @ H]([C @ @]([H])(C C 4) C 3) C=C) C c 5 c c(C(F)(F} \\
) F) c c(C(F)(F) F) c 5)[H]) O[H]) c(c c c c 6) c 26\end{array}$ \\
\hline Rnd1_141 & 0.41 & 1.50 & 20.00 & $\begin{array}{l}{[n+](\mathrm{Cc} 1 \mathrm{c}(\mathrm{F}) \mathrm{cc}(\mathrm{Br}) \mathrm{cc} 1)(\mathrm{ccc} 2[\mathrm{C} @ \mathrm{H}]([\mathrm{C} @] 3([\mathrm{~N}+] 4(\mathrm{C}[\mathrm{C} @ @ \mathrm{H}]([\mathrm{C} @ @]([\mathrm{H}])(\mathrm{CC} 4) \mathrm{C} 3) \mathrm{C}=\mathrm{C}) \mathrm{Cc} 5 \mathrm{cc}(\mathrm{C}(\mathrm{F})} \\
(\mathrm{F}) \mathrm{F}) \mathrm{ccc} 5)[\mathrm{H}]) \mathrm{O}[\mathrm{H}]) \mathrm{c}(\mathrm{cccc} 6) \mathrm{c} 26\end{array}$ \\
\hline Rnd1_142 & 0.41 & 1.50 & 20.00 & $\begin{array}{l}{[n+](C c 1 c(O C) c c c([N+](=O)[O-} \\
]) c 1)(\operatorname{ccc} 2[C @ H]([C @] 3([N+] 4(C[C @ @ H]([C @ @]([H])(C C 4) C 3) C=C) C c 5 c c(C(F)(F) F) c c(F) c 5)[H]) \\
O[H]) c(c c c c 6) c 26\end{array}$ \\
\hline
\end{tabular}




\begin{tabular}{|c|c|c|c|c|}
\hline Rnd1_143 & 2.09 & 8.09 & 78.00 & $\begin{array}{l}{[n+](C c 1 c c c c(C(F)(F) F) c 1)(c c c 2[C @ H]([C @] 3([N+] 4(C[C @ @ H]([C @ @]([H])(C C 4) C 3) C=C) C c 5 c c(} \\
C(F)(F) F) c c(F) c 5)[H]) O[H]) c(c c c c 6) c 26\end{array}$ \\
\hline Rnd1_144 & 0.41 & 1.50 & 20.00 & $\begin{array}{l}{[n+](C c 1 c(F) c c c c 1)(\operatorname{ccc} 2[C @ H]([C @] 3([N+] 4(C[C @ @ H]([C @ @]([H])(C C 4) C 3) C=C) C c 5 c c(C(F)(F) F} \\
) c c(C(F)(F) F) c 5)[H]) O[H]) c(c c c c 6) c 26\end{array}$ \\
\hline Rnd1_145 & 0.41 & 1.50 & 20.00 & $\begin{array}{l}{[n+]((\mathrm{Cc} 1 \mathrm{c}(\mathrm{OC}) \mathrm{ccc}([\mathrm{N}+](=\mathrm{O})[\mathrm{O}-} \\
]) \mathrm{c} 1)(\mathrm{ccc} 2[\mathrm{C} @ \mathrm{H}]([\mathrm{C} @] 3([\mathrm{~N}+] 4(\mathrm{C}[\mathrm{C} @ @ \mathrm{H}]([\mathrm{C} @ @]([\mathrm{H}])(\mathrm{CC} 4) \mathrm{C} 3) \mathrm{C}=\mathrm{C}) \mathrm{Cc} 5 \mathrm{cc}(\mathrm{C}(\mathrm{F})(\mathrm{F}) \mathrm{F}) \mathrm{ccc} 5)[\mathrm{H}]) \mathrm{O}[\mathrm{H} \\
]) \mathrm{c}(\mathrm{cccc} 6) \mathrm{c} 26\end{array}$ \\
\hline Rnd1_146 & 0.38 & 1.47 & 19.00 & $\begin{array}{l}{[\mathrm{n}+](\mathrm{Cc} 1 \mathrm{c} 2 \mathrm{c}(\mathrm{CCCN} 2) \mathrm{cc}(\mathrm{Br}) \mathrm{c1})(\mathrm{ccc} 3[\mathrm{C} @ \mathrm{H}]([\mathrm{C} @] 4([\mathrm{~N}+] 5(\mathrm{C}[\mathrm{C} @ @ \mathrm{H}]([\mathrm{C} @ @]]([\mathrm{H}])(\mathrm{CC} 5) \mathrm{C} 4) \mathrm{C}=\mathrm{C}) \mathrm{Cc} 6} \\
\mathrm{cc}(\mathrm{C}(\mathrm{F})(\mathrm{F}) \mathrm{F}) \mathrm{cc}(\mathrm{C}(\mathrm{F})(\mathrm{F}) \mathrm{F}) \mathrm{c} 6)[\mathrm{H}]) \mathrm{O}[\mathrm{H}]) \mathrm{c}(\mathrm{cccc} 7) \mathrm{c} 37\end{array}$ \\
\hline Rnd1_147 & 0.36 & 1.44 & 18.00 & $\begin{array}{l}{[n+](\mathrm{Cc} 1 \mathrm{c}(\mathrm{C}(\mathrm{F})(\mathrm{F}) \mathrm{F}) \mathrm{ccc}(\mathrm{C}(\mathrm{F})(\mathrm{F}) \mathrm{F}) \mathrm{c} 1)(\mathrm{ccc} 2[\mathrm{C} @ \mathrm{H}]([\mathrm{C} @] 3([\mathrm{~N}+] 4(\mathrm{C}[\mathrm{C} @ @ \mathrm{H}]([\mathrm{C} @ @]([\mathrm{H}])(\mathrm{CC} 4) \mathrm{C} 3) \mathrm{C}=} \\
\mathrm{C}) \mathrm{Cc} 5 \mathrm{cc}(\mathrm{C}(\mathrm{F})(\mathrm{F}) \mathrm{F}) \mathrm{ccc} 5 \mathrm{C}(\mathrm{F})(\mathrm{F}) \mathrm{F})[\mathrm{H}]) \mathrm{O}[\mathrm{H}]) \mathrm{c}(\mathrm{ccc}(\mathrm{OC}) \mathrm{c6}) \mathrm{c} 26\end{array}$ \\
\hline Rnd1_148 & 0.36 & 1.44 & 18.00 & $\begin{array}{l}{[n+](\mathrm{Cc} 1 \mathrm{c}(\mathrm{C}(\mathrm{F})(\mathrm{F}) \mathrm{F}) \mathrm{cccc} 1 \mathrm{Cl})(\mathrm{ccc} 2[\mathrm{C} @ \mathrm{H}]([\mathrm{C} @] 3([\mathrm{~N}+] 4(\mathrm{C}[\mathrm{C} @ @ \mathrm{H}]([\mathrm{C} @ @]([\mathrm{H}])(\mathrm{CC} 4) \mathrm{C} 3) \mathrm{C}=\mathrm{C}) \mathrm{Cc} 5 \mathrm{cl}} \\
\mathrm{Cl}) \operatorname{cccc} 5 \mathrm{C}(\mathrm{F})(\mathrm{F}) \mathrm{F})[\mathrm{H}]) \mathrm{O}[\mathrm{H}]) \mathrm{c}(\mathrm{ccc}(\mathrm{OC}) \mathrm{c} 6) \mathrm{c} 26\end{array}$ \\
\hline Rnd1_149 & 0.34 & 1.41 & 17.00 & $\begin{array}{l}{[\mathrm{n}+](\mathrm{Cc} 1 \mathrm{c}(\mathrm{Br}) \mathrm{ccc}(\mathrm{OC}) \mathrm{c} 1)(\mathrm{ccc} 2[\mathrm{C} @ \mathrm{H}]([\mathrm{C} @] 3([\mathrm{~N}+] 4(\mathrm{C}[\mathrm{C} @ @ \mathrm{H}]([\mathrm{C} @ @]([\mathrm{H}])(\mathrm{CC} 4) \mathrm{C} 3) \mathrm{C}=\mathrm{C}) \mathrm{Cc} 5 \mathrm{cc}(\mathrm{O}} \\
\mathrm{C}) \mathrm{ccc} 5 \mathrm{Br})[\mathrm{H}]) \mathrm{O}[\mathrm{H}]) \mathrm{c}(\mathrm{ccc}(\mathrm{OC}) \mathrm{c6}) \mathrm{c} 26\end{array}$ \\
\hline Rnd1_150 & 0.32 & 1.38 & 16.00 & $\begin{array}{l}{[n+](\mathrm{Cc} 1 \mathrm{c}(\mathrm{Br}) \mathrm{ccc}(\mathrm{OC}) \mathrm{c1})(\mathrm{ccc} 2[\mathrm{C} @ \mathrm{H}]([\mathrm{C} @] 3([\mathrm{~N}+] 4(\mathrm{C}[\mathrm{C} @ @ \mathrm{H}]([\mathrm{C} @ @]([\mathrm{H}])(\mathrm{CC} 4) \mathrm{C} 3) \mathrm{C}=\mathrm{C}) \mathrm{Cc} 5 \mathrm{cccc}(} \\
\mathrm{C}(\mathrm{F})(\mathrm{F}) \mathrm{F}) \mathrm{c} 5 \mathrm{~F})[\mathrm{H}]) \mathrm{O}[\mathrm{H}]) \mathrm{c}(\mathrm{cccc} 6) \mathrm{c} 26\end{array}$ \\
\hline Rnd1_151 & 0.28 & 1.33 & 14.00 & $\begin{array}{l}{[\mathrm{n}+](\mathrm{Cc} 1 \mathrm{c}(\mathrm{Br}) \mathrm{ccc}(\mathrm{F}) \mathrm{c1})(\mathrm{ccc} 2[\mathrm{C} @ \mathrm{H}]([\mathrm{C} @] 3([\mathrm{~N}+] 4(\mathrm{C}[\mathrm{C} @ @ \mathrm{H}]([\mathrm{C} @ @]([\mathrm{H}])(\mathrm{CC} 4) \mathrm{C} 3) \mathrm{C}=\mathrm{C}) \mathrm{Cc} 5 \mathrm{ccc}(\mathrm{F}) \mathrm{c}} \\
\mathrm{c5Br})[\mathrm{H}]) \mathrm{O}[\mathrm{H}]) \mathrm{c}(\mathrm{ccc}(\mathrm{OC}) \mathrm{c} 6) \mathrm{c} 26\end{array}$ \\
\hline Rnd1_152 & 0.28 & 1.33 & 14.00 & $\begin{array}{l}{[\mathrm{n}+](\mathrm{Cc} 1 \mathrm{c}(\mathrm{Br}) \mathrm{ccc}(\mathrm{OC}) \mathrm{c} 1)(\mathrm{ccc} 2[\mathrm{C} @ \mathrm{H}]([\mathrm{C} @] 3([\mathrm{~N}+] 4(\mathrm{C}[\mathrm{C} @ @ \mathrm{H}]([\mathrm{C} @ @]([\mathrm{H}])(\mathrm{CC} 4) \mathrm{C} 3) \mathrm{C}=\mathrm{C}) \mathrm{Cc} 5 \mathrm{ccc}(\mathrm{F}} \\
) \mathrm{cc5} 5)[\mathrm{H}]) \mathrm{O}[\mathrm{H}]) \mathrm{c}(\mathrm{cccc} 6) \mathrm{c} 26\end{array}$ \\
\hline Rnd1_153 & 0.26 & 1.30 & 13.00 & $\begin{array}{l}{[\mathrm{n}+](\mathrm{Cc} 1 \mathrm{c}([\mathrm{N}+](=\mathrm{O})[\mathrm{O}-} \\
]) \mathrm{cc}(\mathrm{OC}) \mathrm{c}(\mathrm{OC}) \mathrm{c1})(\mathrm{ccc} 2[\mathrm{C} @ \mathrm{H}]([\mathrm{C} @] 3([\mathrm{~N}+] 4(\mathrm{C}[\mathrm{C} @ @ \mathrm{H}]([\mathrm{C} @ @]([\mathrm{H}])(\mathrm{CC} 4) \mathrm{C} 3) \mathrm{C}=\mathrm{C}) \mathrm{Cc} 5 \mathrm{cc}(\mathrm{OC}) \mathrm{c}(\mathrm{OC}) \\
\mathrm{cc} 5 \mathrm{Br})[\mathrm{H}]) \mathrm{O}[\mathrm{H}]) \mathrm{c}(\mathrm{ccc}(\mathrm{OC}) \mathrm{c} 6) \mathrm{c} 26\end{array}$ \\
\hline Rnd1_154 & 0.26 & 1.30 & 13.00 & $\begin{array}{l}{[\mathrm{n}+](\mathrm{Cc} 1 \mathrm{c}(\mathrm{Br}) \mathrm{ccc}(\mathrm{OC}) \mathrm{c1})(\mathrm{ccc} 2[\mathrm{C} @ \mathrm{H}]([\mathrm{C} @] 3([\mathrm{~N}+] 4(\mathrm{C}[\mathrm{C} @ @ \mathrm{H}]([\mathrm{C} @ @]([\mathrm{H}])(\mathrm{CC} 4) \mathrm{C} 3) \mathrm{C}=\mathrm{C}) \mathrm{Cc} 5 \mathrm{c}(\mathrm{F}) \mathrm{c}} \\
(\mathrm{F}) \mathrm{ccc} 5)[\mathrm{H}]) \mathrm{O}[\mathrm{H}]) \mathrm{c}(\mathrm{cccc} 6) \mathrm{c} 26\end{array}$ \\
\hline
\end{tabular}




\begin{tabular}{|c|c|c|c|c|}
\hline Rnd1_155 & 2.15 & 8.57 & 79.10 & $\begin{array}{l}{[n+](\mathrm{Cc} 1 \mathrm{ccc}(\mathrm{Cl}) \mathrm{c}(\mathrm{C}(\mathrm{F})(\mathrm{F}) \mathrm{F}) \mathrm{c1})(\mathrm{ccc} 2[\mathrm{C} @ \mathrm{H}]([\mathrm{C} @] 3([\mathrm{~N}+] 4(\mathrm{C}[\mathrm{C} @ @ \mathrm{H}]([\mathrm{C} @ @]([\mathrm{H}])(\mathrm{CC} 4) \mathrm{C} 3) \mathrm{C}=\mathrm{C}) \mathrm{Cc} 5} \\
\mathrm{cc}(\mathrm{C}(\mathrm{F})(\mathrm{F}) \mathrm{F}) \mathrm{cc}(\mathrm{F}) \mathrm{c5})[\mathrm{H}]) \mathrm{O}[\mathrm{H}]) \mathrm{c}(\mathrm{cccc} 6) \mathrm{c} 26\end{array}$ \\
\hline Rnd1_156 & 0.20 & 1.22 & 10.00 & $\begin{array}{l}{[\mathrm{n}+](\mathrm{Cc} 1 \mathrm{c}(\mathrm{Br}) \mathrm{cccc} 1)(\mathrm{ccc} 2[\mathrm{C} @ \mathrm{H}]([\mathrm{C} @] 3([\mathrm{~N}+] 4(\mathrm{C}[\mathrm{C} @ @ \mathrm{H}]([\mathrm{C} @ @]([\mathrm{H}])(\mathrm{CC} 4) \mathrm{C} 3) \mathrm{C}=\mathrm{C}) \mathrm{Cc} 5 \mathrm{ccccc} 5 \mathrm{Br})} \\
[\mathrm{H}]) \mathrm{O}[\mathrm{H}]) \mathrm{c}(\mathrm{ccc}(\mathrm{OC}) \mathrm{c} 6) \mathrm{c} 26\end{array}$ \\
\hline Rnd1_157 & 0.20 & 1.22 & 10.00 & $\begin{array}{l}{[\mathrm{n}+](\mathrm{Cc} 1 \mathrm{c}(\mathrm{Br}) \mathrm{ccc}(\mathrm{OC}) \mathrm{c} 1)(\mathrm{ccc} 2[\mathrm{C} @ \mathrm{H}]([\mathrm{C} @] 3([\mathrm{~N}+] 4(\mathrm{C}[\mathrm{C} @ @ \mathrm{H}]([\mathrm{C} @ @]([\mathrm{H}])(\mathrm{CC} 4) \mathrm{C} 3) \mathrm{C}=\mathrm{C}) \mathrm{Cc} 5 \mathrm{cc}(\mathrm{F})} \\
\mathrm{ccc} 5 \mathrm{~F})[\mathrm{H}]) \mathrm{O}[\mathrm{H}]) \mathrm{c}(\mathrm{cccc} 6) \mathrm{c} 26\end{array}$ \\
\hline Rnd1_158 & 0.20 & 1.22 & 10.00 & $\begin{array}{l}{[\mathrm{n}+](\mathrm{Cc} 1 \mathrm{c}(\mathrm{Br}) \mathrm{ccc}(\mathrm{OC}) \mathrm{c1})(\mathrm{ccc} 2[\mathrm{C} @ \mathrm{H}]([\mathrm{C} @] 3([\mathrm{~N}+] 4(\mathrm{C}[\mathrm{C} @ @ \mathrm{H}]([\mathrm{C} @ @]([\mathrm{H}])(\mathrm{CC} 4) \mathrm{C} 3) \mathrm{C}=\mathrm{C}) \mathrm{Cc} 5 \mathrm{cc}(\mathrm{C}} \\
\# \mathrm{~N}) \mathrm{ccc})[\mathrm{H}]) \mathrm{O}[\mathrm{H}]) \mathrm{c}(\mathrm{cccc} 6) \mathrm{c} 26\end{array}$ \\
\hline Rnd1_159 & 0.18 & 1.20 & 9.00 & $\begin{array}{l}{[\mathrm{n}+](\mathrm{Cc} 1 \mathrm{c}(\mathrm{Br}) \mathrm{ccc}(\mathrm{OC}) \mathrm{c} 1)(\mathrm{ccc} 2[\mathrm{C} @ \mathrm{H}]([\mathrm{C} @] 3([\mathrm{~N}+] 4(\mathrm{C}[\mathrm{C} @ @ \mathrm{H}]([\mathrm{C} @ @]([\mathrm{H}])(\mathrm{CC} 4) \mathrm{C} 3) \mathrm{C}=\mathrm{C}) \mathrm{Cc} 5 \mathrm{cccc}} \\
\mathrm{c5OC}(\mathrm{F})(\mathrm{F}) \mathrm{F})[\mathrm{H}]) \mathrm{O}[\mathrm{H}]) \mathrm{c}(\mathrm{cccc} 6) \mathrm{c} 26\end{array}$ \\
\hline Rnd1_160 & 0.18 & 1.20 & 9.00 & $\begin{array}{l}{[\mathrm{n}+](\mathrm{Cc} 1 \mathrm{cc}(\mathrm{C}(\mathrm{F})(\mathrm{F}) \mathrm{F}) \mathrm{cc}(\mathrm{C}(\mathrm{F})(\mathrm{F}) \mathrm{F}) \mathrm{c} 1)(\mathrm{ccc} 2[\mathrm{C} @ \mathrm{H}]([\mathrm{C} @] 3([\mathrm{~N}+] 4(\mathrm{C}[\mathrm{C} @ @ \mathrm{H}]([\mathrm{C} @ @]([\mathrm{H}])(\mathrm{CC} 4) \mathrm{C} 3) \mathrm{C}=} \\
\mathrm{C}) \mathrm{Cc} 5 \mathrm{c}([\mathrm{N}+](=\mathrm{O})[\mathrm{O}-]) \operatorname{cccc5})[\mathrm{H}]) \mathrm{O}[\mathrm{H}]) \mathrm{c}(\mathrm{cccc} 6) \mathrm{c} 26\end{array}$ \\
\hline Rnd1_161 & 0.18 & 1.20 & 9.00 & $\begin{array}{l}{[\mathrm{n}+](\mathrm{Cc} 1 \mathrm{c}([\mathrm{N}+](=\mathrm{O})[\mathrm{O}-} \\
]) \mathrm{cccc} 1)(\mathrm{ccc} 2[\mathrm{C} @ \mathrm{H}]([\mathrm{C} @] 3([\mathrm{~N}+] 4(\mathrm{C}[\mathrm{C} @ @ \mathrm{H}]([\mathrm{C} @ @]([\mathrm{H}])(\mathrm{CC} 4) \mathrm{C} 3) \mathrm{C}=\mathrm{C}) \mathrm{Cc} 5 \mathrm{ccccc} 5[\mathrm{~N}+](=\mathrm{O})[\mathrm{O}- \\
])[\mathrm{H}]) \mathrm{O}[\mathrm{H}]) \mathrm{c}(\mathrm{ccc}(\mathrm{OC}) \mathrm{c} 6) \mathrm{c} 26\end{array}$ \\
\hline Rnd1_162 & 0.18 & 1.20 & 9.00 & $\begin{array}{l}{[n+](\mathrm{Cc} 1 \mathrm{c}(\mathrm{F}) \operatorname{cccc} 1 \mathrm{~F})(\mathrm{ccc} 2[\mathrm{C} @ \mathrm{H}]([\mathrm{C} @] 3([\mathrm{~N}+] 4(\mathrm{C}[\mathrm{C} @ @ \mathrm{H}]([\mathrm{C} @ @]([\mathrm{H}])(\mathrm{CC} 4) \mathrm{C} 3) \mathrm{C}=\mathrm{C}) \mathrm{Cc} 5 \mathrm{c}(\mathrm{F}) \mathrm{cccc} 5} \\
\mathrm{F})[\mathrm{H}]) \mathrm{O}[\mathrm{H}]) \mathrm{c}(\mathrm{ccc}(\mathrm{OC}) \mathrm{c6}) \mathrm{c} 26\end{array}$ \\
\hline Rnd1_163 & 0.16 & 1.17 & 8.00 & $\begin{array}{l}{[\mathrm{n}+](\mathrm{Cc} 1 \mathrm{c}(\mathrm{I}) \mathrm{cccc} 1)(\mathrm{ccc} 2[\mathrm{C} @ \mathrm{H}]([\mathrm{C} @] 3([\mathrm{~N}+] 4(\mathrm{C}[\mathrm{C} @ @ \mathrm{H}]([\mathrm{C} @ @]([\mathrm{H}])(\mathrm{CC} 4) \mathrm{C} 3) \mathrm{C}=\mathrm{C}) \mathrm{Cc} 5 \mathrm{ccccc} 5 \mathrm{I})[\mathrm{H}]} \\
\mathrm{J} \mathrm{O}[\mathrm{H}]) \mathrm{c}(\mathrm{ccc}(\mathrm{OC}) \mathrm{c} 6) \mathrm{c} 26\end{array}$ \\
\hline Rnd1_164 & 0.14 & 1.15 & 7.00 & $\begin{array}{l}{[\mathrm{n}+](\mathrm{Cc} 1 \mathrm{c}(\mathrm{Br}) \mathrm{ccc}(\mathrm{OC}) \mathrm{c1})(\mathrm{ccc} 2[\mathrm{C} @ \mathrm{H}]([\mathrm{C} @] 3([\mathrm{~N}+] 4(\mathrm{C}[\mathrm{C} @ @ \mathrm{H}]([\mathrm{C} @ @]([\mathrm{H}])(\mathrm{CC} 4) \mathrm{C} 3) \mathrm{C}=\mathrm{C}) \mathrm{Cc} 5 \mathrm{cccc}} \\
\mathrm{c5CHN})[\mathrm{H}]))[\mathrm{H}]) \mathrm{c}(\mathrm{cccc} 6) \mathrm{c} 26\end{array}$ \\
\hline Rnd1_165 & 0.12 & 1.13 & 6.00 & $\begin{array}{l}{[\mathrm{n}+](\mathrm{Cc} 1 \mathrm{c}(\mathrm{Br}) \mathrm{ccc}(\mathrm{OC}) \mathrm{c} 1)(\mathrm{ccc} 2[\mathrm{C} @ \mathrm{H}]([\mathrm{C} @] 3([\mathrm{~N}+] 4(\mathrm{C}[\mathrm{C} @ @ \mathrm{H}]([\mathrm{C} @ @]([\mathrm{H}])(\mathrm{CC} 4) \mathrm{C} 3) \mathrm{C}=\mathrm{C}) \mathrm{Cc} 5 \mathrm{cccc}} \\
\mathrm{c5Cl})[\mathrm{H}]) \mathrm{O}[\mathrm{H}]) \mathrm{c}(\mathrm{cccc} 6) \mathrm{c} 26\end{array}$ \\
\hline Rnd1_166 & 0.10 & 1.11 & 5.00 & $\begin{array}{l}{[n+](\mathrm{Cc} 1 \mathrm{c}(\mathrm{Br}) \operatorname{ccc}(\mathrm{OC}) \mathrm{c} 1)(\mathrm{ccc} 2[\mathrm{C} @ \mathrm{H}]([\mathrm{C} @] 3([\mathrm{~N}+] 4(\mathrm{C}[\mathrm{C} @ @ \mathrm{H}]([\mathrm{C} @ @]([\mathrm{H}])(\mathrm{CC} 4) \mathrm{C} 3) \mathrm{C}=\mathrm{C}) \mathrm{Cc} 5 \mathrm{cc}(\mathrm{F})} \\
\operatorname{ccc} \mathrm{C}(\mathrm{F})(\mathrm{F}) \mathrm{F})[\mathrm{H}]) \mathrm{O}[\mathrm{H}]) \mathrm{c}(\mathrm{cccc} 6) \mathrm{c} 26\end{array}$ \\
\hline
\end{tabular}




\begin{tabular}{|c|c|c|c|c|}
\hline Rnd1_167 & 0.10 & 1.11 & 5.00 & $\begin{array}{l}{[\mathrm{n}+](\mathrm{Cc} 1 \mathrm{c}(\mathrm{Cl}) \mathrm{cccc} 1)(\mathrm{ccc} 2[\mathrm{C} @ \mathrm{H}]([\mathrm{C} @] 3([\mathrm{~N}+] 4(\mathrm{C}[\mathrm{C} @ @ \mathrm{H}]([\mathrm{C} @ @]([\mathrm{H}])(\mathrm{CC} 4) \mathrm{C} 3) \mathrm{C}=\mathrm{C}) \mathrm{Cc} 5 \mathrm{ccccc} 5 \mathrm{Cl})[} \\
\mathrm{H}]) \mathrm{O}[\mathrm{H}]) \mathrm{c}(\mathrm{ccc}(\mathrm{OC}) \mathrm{c} 6) \mathrm{c} 26\end{array}$ \\
\hline Rnd1_168 & 0.10 & 1.11 & 5.00 & $\begin{array}{l}{[\mathrm{n}+](\mathrm{Cc} 1 \mathrm{c}(\mathrm{CHN}) \mathrm{cccc} 1)(\mathrm{ccc} 2[\mathrm{C} @ \mathrm{H}]([\mathrm{C} @] 3([\mathrm{~N}+] 4(\mathrm{C}[\mathrm{C} @ @ \mathrm{H}]([\mathrm{C} @ @]([\mathrm{H}])(\mathrm{CC} 4) \mathrm{C} 3) \mathrm{C}=\mathrm{C}) \mathrm{Cc} 5 \mathrm{ccccc} 5} \\
\mathrm{CHN})[\mathrm{H}]) \mathrm{O}[\mathrm{H}]) \mathrm{c}(\mathrm{ccc}(\mathrm{OC}) \mathrm{c} 6) \mathrm{c} 26\end{array}$ \\
\hline Rnd1_169 & 0.06 & 1.06 & 3.00 & $\begin{array}{l}{[n+](\mathrm{Cc} 1 \mathrm{c}(\mathrm{Br}) \operatorname{ccc}(\mathrm{OC}) \mathrm{c} 1)(\mathrm{ccc} 2[\mathrm{C} @ \mathrm{H}]([\mathrm{C} @] 3([\mathrm{~N}+] 4(\mathrm{C}[\mathrm{C} @ @ \mathrm{H}]([\mathrm{C} @ @]([\mathrm{H}])(\mathrm{CC} 4) \mathrm{C} 3) \mathrm{C}=\mathrm{C}) \mathrm{Cc} 5 \mathrm{ccc}(} \\
\mathrm{C}(\mathrm{F})(\mathrm{F}) \mathrm{F}) \mathrm{cc} 5 \mathrm{C}(\mathrm{F})(\mathrm{F}) \mathrm{F})[\mathrm{H}]) \mathrm{O}[\mathrm{H}]) \mathrm{c}(\mathrm{cccc} 6) \mathrm{c} 26\end{array}$ \\
\hline Rnd1_170 & 0.06 & 1.06 & 3.00 & $\begin{array}{l}{[\mathrm{n}+](\mathrm{Cc} 1 \mathrm{c}(\mathrm{Br}) \mathrm{ccc}(\mathrm{OC}) \mathrm{c} 1)(\mathrm{ccc} 2[\mathrm{C} @ \mathrm{H}]([\mathrm{C} @] 3([\mathrm{~N}+] 4(\mathrm{C}[\mathrm{C} @ @ \mathrm{H}]([\mathrm{C} @ @]([\mathrm{H}])(\mathrm{CC} 4) \mathrm{C} 3) \mathrm{C}=\mathrm{C}) \mathrm{Cc} 5 \mathrm{ccc}(} \\
\mathrm{C}(\mathrm{F})(\mathrm{F}) \mathrm{F}) \mathrm{cc5}[\mathrm{N}+](=\mathrm{O})[\mathrm{O}-])[\mathrm{H}]) \mathrm{O}[\mathrm{H}]) \mathrm{c}(\mathrm{cccc} 6) \mathrm{c} 26\end{array}$ \\
\hline Rnd1_171 & 0.06 & 1.06 & 3.00 & $\begin{array}{l}{[n+](\mathrm{Cc} 1 \mathrm{c}(\mathrm{Br}) \mathrm{ccc}(\mathrm{OC}) \mathrm{c1})(\mathrm{ccc} 2[\mathrm{C} @ \mathrm{H}]([\mathrm{C} @] 3([\mathrm{~N}+] 4(\mathrm{Cc} 5 \mathrm{c}(\mathrm{F}) \mathrm{cccc} 5 \mathrm{Cl}) \mathrm{CC}[\mathrm{C} @]([\mathrm{H}])([\mathrm{C} @ @ \mathrm{H}](\mathrm{C}=\mathrm{C}) \mathrm{C}} \\
4) \mathrm{C})[\mathrm{H}]) \mathrm{O}[\mathrm{H}]) \mathrm{c}(\mathrm{cccc} 6) \mathrm{c} 26\end{array}$ \\
\hline Rnd1_172 & 0.06 & 1.06 & 3.00 & $\begin{array}{l}{[\mathrm{n}+](\mathrm{Cc} 1 \mathrm{c}(\mathrm{Br}) \mathrm{ccc}(\mathrm{OC}) \mathrm{c} 1)(\mathrm{ccc} 2[\mathrm{C} @ \mathrm{H}]([\mathrm{C} @] 3([\mathrm{~N}+] 4(\mathrm{C}[\mathrm{C} @ @ \mathrm{H}]([\mathrm{C} @ @]([\mathrm{H}])(\mathrm{CC} 4) \mathrm{C} 3) \mathrm{C}=\mathrm{C}) \mathrm{Cc} 5 \mathrm{c}(\mathrm{F}) \mathrm{c}} \\
\mathrm{ccc5})[\mathrm{H}]) \mathrm{O}[\mathrm{H}]) \mathrm{c}(\mathrm{cccc} 6) \mathrm{c} 26\end{array}$ \\
\hline Rnd1_173 & -0.06 & 0.94 & -3.00 & $\begin{array}{l}{[\mathrm{n}+](\mathrm{Cc} 1 \mathrm{c}(\mathrm{Br}) \mathrm{ccc}(\mathrm{OC}) \mathrm{c1})(\mathrm{ccc} 2[\mathrm{C} @ \mathrm{H}]([\mathrm{C} @] 3([\mathrm{~N}+] 4(\mathrm{C}[\mathrm{C} @ @ \mathrm{H}]([\mathrm{C} @ @]([\mathrm{H}])(\mathrm{CC} 4) \mathrm{C} 3) \mathrm{C}=\mathrm{C}) \mathrm{Cc} 5 \mathrm{c}([\mathrm{N}} \\
+](=\mathrm{O})[\mathrm{O}-]) \mathrm{cccc} 5)[\mathrm{H}]) \mathrm{O}[\mathrm{H}]) \mathrm{c}(\mathrm{cccc} 6) \mathrm{c} 26\end{array}$ \\
\hline Rnd1_174 & -0.14 & 0.87 & -7.00 & $\begin{array}{l}{[n+](C c 1 c c(C(F)(F) F) c c(C(F)(F) F) c 1)(\operatorname{ccc} 2[C @ H]([C @] 3([N+] 4(C[C @ @ H]([C @ @]([H])(C C 4) C 3) C=} \\
C) \operatorname{Cc} 5 c(C(F)(F) F) \operatorname{cccc5})[H]) O[H]) c(\operatorname{cccc} 6) c 26\end{array}$ \\
\hline Rnd1_175 & -0.22 & 0.80 & -11.00 & $\begin{array}{l}{[\mathrm{n}+](\mathrm{Cc} 1 \mathrm{c}(\mathrm{Br}) \mathrm{ccc}(\mathrm{OC}) \mathrm{c} 1)(\mathrm{ccc} 2[\mathrm{C} @ \mathrm{H}]([\mathrm{C} @] 3([\mathrm{~N}+] 4(\mathrm{C}[\mathrm{C} @ @ \mathrm{H}]([\mathrm{C} @ @]([\mathrm{H}])(\mathrm{CC} 4) \mathrm{C} 3) \mathrm{C}=\mathrm{C}) \mathrm{Cc} 5 \mathrm{ccc}(\mathrm{F}} \\
) \mathrm{cc} 5 \mathrm{C}(\mathrm{F})(\mathrm{F}) \mathrm{F})[\mathrm{H}]) \mathrm{O}[\mathrm{H}]) \mathrm{c}(\mathrm{cccc} 6) \mathrm{c} 26\end{array}$ \\
\hline Rnd1_176 & -0.26 & 0.77 & -13.00 & $\begin{array}{l}{[n+](\mathrm{Cc} 1 \mathrm{c}(\mathrm{Br}) \mathrm{ccc}(\mathrm{OC}) \mathrm{c} 1)(\mathrm{ccc} 2[\mathrm{C} @ \mathrm{H}]([\mathrm{C} @] 3([\mathrm{~N}+] 4(\mathrm{C}[\mathrm{C} @ @ \mathrm{H}]([\mathrm{C} @ @]([\mathrm{H}])(\mathrm{CC} 4) \mathrm{C} 3) \mathrm{C}=\mathrm{C}) \mathrm{Cc} 5 \mathrm{c}(\mathrm{C}(\mathrm{F}} \\
)(\mathrm{F}) \mathrm{F}) \mathrm{cccc} 5)[\mathrm{H}]) \mathrm{O}[\mathrm{H}]) \mathrm{c}(\mathrm{cccc} 6) \mathrm{c} 26\end{array}$ \\
\hline Rnd1_177 & -0.34 & 0.71 & -17.00 & $\begin{array}{l}{[n+](C c 1 c c(C(F)(F) F) c c(C(F)(F) F) c 1)(\operatorname{ccc} 2[C @ H]([C @] 3([N+] 4(C[C @ @ H]([C @ @]([H])(C C 4) C 3) C=} \\
c) C c 5 c(F) \operatorname{cccc5Cl})[H]) O[H]) c(c c c c 6) c 26\end{array}$ \\
\hline Rnd1a_1 & 1.14 & 3.13 & 51.57 & $\begin{array}{l}\mathrm{C1}([\mathrm{C} @ @ \mathrm{OH}](\mathrm{c} 2 \mathrm{cc}[\mathrm{n}+](\mathrm{Cc} 3 \operatorname{ccc}([\mathrm{N}+]([\mathrm{O}- \\
])=\mathrm{O}) \mathrm{c}(\mathrm{F}) \mathrm{c} 3) \mathrm{c}(\mathrm{cccc} 4) \mathrm{c} 24) \mathrm{O}) \mathrm{cc}(\mathrm{C}(\mathrm{C}=\mathrm{C}) \mathrm{C}[\mathrm{N}+] 15 \mathrm{Cc} 6 \mathrm{cc}([\mathrm{N}+]([\mathrm{O}-])=\mathrm{O}) \mathrm{cc}([\mathrm{N}+]([\mathrm{O}-])=0) \mathrm{c} 6) \mathrm{cC5}\end{array}$ \\
\hline
\end{tabular}




\begin{tabular}{|c|c|c|c|c|}
\hline Rnd1a_2 & 0.44 & 1.56 & 21.73 & $\begin{array}{l}\mathrm{C} 1([\mathrm{C} @ @ H](\mathrm{c} 2 \mathrm{cc}[\mathrm{n}+](\mathrm{Cc} 3 \operatorname{cccc}(\mathrm{CHN}) \mathrm{c3}) \mathrm{c}(\mathrm{cccc} 4) \mathrm{c} 24) \mathrm{O}) \mathrm{CC}(\mathrm{C}(\mathrm{C}=\mathrm{C}) \mathrm{C}[\mathrm{N}+] 15 \mathrm{Cc} 6 \mathrm{cc}([\mathrm{N}+]([\mathrm{O}- \\
])=\mathrm{O}) \mathrm{ccc} 6 \mathrm{~F}) \mathrm{CC5}\end{array}$ \\
\hline Rnd1a_3 & 0.56 & 1.75 & 27.25 & $\begin{array}{l}\mathrm{C} 1([\mathrm{C} @ @ H](\mathrm{c} 2 \mathrm{cc}[\mathrm{n}+](\mathrm{Cc} 3 \mathrm{oc}([\mathrm{N}+]([\mathrm{O}- \\
])=\mathrm{O}) \mathrm{cc} 3) \mathrm{c}(\mathrm{cccc} 4) \operatorname{co} 24) \mathrm{O}) \mathrm{CC}(\mathrm{C}(\mathrm{C}=\mathrm{C}) \mathrm{C}[\mathrm{N}+] 15 \mathrm{Cc} 6 \mathrm{ccc}([\mathrm{N}+]([\mathrm{O}-])=\mathrm{O}) \operatorname{cc} 6 \mathrm{Br}) \mathrm{CC} 5\end{array}$ \\
\hline Rnd1a_4 & 1.97 & 7.18 & 75.54 & $\begin{array}{l}\mathrm{C} 1([\mathrm{C} @ @ \mathrm{H}](\mathrm{c} 2 \mathrm{cc}[\mathrm{n}+](\mathrm{Cc} 3 \mathrm{c}(\mathrm{OC}) \mathrm{ccc}(\mathrm{C}(\mathrm{F})(\mathrm{F}) \mathrm{F}) \mathrm{c} 3) \mathrm{c}(\mathrm{cccc} 4) \mathrm{c} 24) \mathrm{O}) \mathrm{CC}(\mathrm{C}(\mathrm{C}=\mathrm{C}) \mathrm{C}[\mathrm{N}+] 15 \mathrm{Cc} 6 \mathrm{ccc}(\mathrm{F}) \mathrm{c}(\mathrm{OC}( \\
\mathrm{F})(\mathrm{F}) \mathrm{F}) \mathrm{c} 6) \mathrm{CC} 5\end{array}$ \\
\hline Rnd1a_5 & 1.60 & 4.95 & 66.40 & $\begin{array}{l}\mathrm{C} 1([\mathrm{C} @ @ H](\mathrm{c} 2 \mathrm{cc}[\mathrm{n}+](\mathrm{Cc} 3 \mathrm{ccc}(\mathrm{C}(\mathrm{F})(\mathrm{F}) \mathrm{F}) \mathrm{cc} 3 \mathrm{~F}) \mathrm{c}(\mathrm{cccc} 4) \mathrm{c} 24) \mathrm{O}) \mathrm{CC}(\mathrm{C}(\mathrm{C}=\mathrm{C}) \mathrm{C}[\mathrm{N}+] 15 \mathrm{Cc} 6 \mathrm{ccc}(\mathrm{F}) \mathrm{c}(\mathrm{OC}(\mathrm{F})( \\
\mathrm{F}) \mathrm{F}) \mathrm{c} 6) \mathrm{CC} 5\end{array}$ \\
\hline Rnd1a_6 & 1.62 & 5.06 & 66.99 & $\begin{array}{l}\mathrm{C} 1([\mathrm{C} @ @ \mathrm{H}](\mathrm{c} 2 \mathrm{cc}[\mathrm{n}+](\mathrm{Cc} 3 \mathrm{c}(\mathrm{Cl}) \mathrm{ccc}(\mathrm{C}(\mathrm{F})(\mathrm{F}) \mathrm{F}) \mathrm{c} 3 \mathrm{Cl}) \mathrm{c}(\mathrm{cccc} 4) \mathrm{c} 24) \mathrm{O}) \mathrm{CC}(\mathrm{C}(\mathrm{C}=\mathrm{C}) \mathrm{C}[\mathrm{N}+] 15 \mathrm{Cc} 6 \mathrm{ccc}(\mathrm{F}) \mathrm{c}(\mathrm{OC} \\
(\mathrm{F})(\mathrm{F}) \mathrm{F}) \mathrm{c} 6) \mathrm{CC} 5\end{array}$ \\
\hline Rnd1a_7 & 1.90 & 6.67 & 73.91 & $\begin{array}{l}\mathrm{C} 1([\mathrm{C} @ @ \mathrm{H}](\mathrm{c} 2 \mathrm{cc}[\mathrm{n}+](\mathrm{Cc} 3 \mathrm{cccc}(\mathrm{OC}(\mathrm{F}) \mathrm{F}) \mathrm{c} 3) \mathrm{c}(\mathrm{cccc} 4) \mathrm{c} 24) \mathrm{O}) \mathrm{CC}(\mathrm{C}(\mathrm{C}=\mathrm{C}) \mathrm{C}[\mathrm{N}+] 15 \mathrm{Cc} 6 \mathrm{ccc}(\mathrm{F}) \mathrm{c}(\mathrm{OC}(\mathrm{F})(\mathrm{F}) \\
\mathrm{F}) \mathrm{c6}) \mathrm{CC} 5\end{array}$ \\
\hline Rnd1a_8 & 0.88 & 2.40 & 41.24 & $\begin{array}{l}\mathrm{C} 1([\mathrm{C} @ @ H](\mathrm{c} 2 \mathrm{cc}[\mathrm{n}+](\mathrm{Cc} 3 \mathrm{c}(\mathrm{OC}) \mathrm{cc}([\mathrm{N}+]([\mathrm{O}- \\
])=\mathrm{O}) \mathrm{cc} 3) \mathrm{c}(\mathrm{cccc} 4) \mathrm{c} 24) \mathrm{O}) \mathrm{cc}(\mathrm{C}(\mathrm{C}=\mathrm{C}) \mathrm{C}[\mathrm{N}+] 15 \mathrm{Cc} 6 \mathrm{cc}(\mathrm{Cl}) \mathrm{c}(\mathrm{OC}) \mathrm{c}(\mathrm{F}) \mathrm{c} 6) \mathrm{CC} 5\end{array}$ \\
\hline Rnd1a_9 & 0.25 & 1.28 & 12.33 & $\begin{array}{l}\mathrm{C} 1([\mathrm{C} @ @ H](\mathrm{c} 2 \mathrm{cc}[\mathrm{n}+](\mathrm{Cc} 3 \mathrm{c}(\mathrm{F}) \mathrm{c}(\mathrm{F}) \mathrm{c}(\mathrm{OC}(\mathrm{F})(\mathrm{F}) \mathrm{F}) \mathrm{c}(\mathrm{F}) \mathrm{c} 3 \mathrm{~F}) \mathrm{c}(\mathrm{cccc} 4) \mathrm{c} 24) \mathrm{O}) \mathrm{cC}(\mathrm{C}(\mathrm{C}=\mathrm{C}) \mathrm{C}[\mathrm{N}+] 15 \mathrm{Cc} 6 \mathrm{ccc}( \\
\mathrm{CHN}) \mathrm{cc6}) \mathrm{CC} 5\end{array}$ \\
\hline Rnd1a_10 & 1.07 & 2.90 & 48.77 & $\begin{array}{l}\mathrm{C} 1([\mathrm{C} @ @ \mathrm{H}](\mathrm{c} 2 \mathrm{cc}[\mathrm{n}+](\mathrm{Cc} 3 \mathrm{c}(\mathrm{OC}) \mathrm{cc}([\mathrm{N}+]([\mathrm{O}- \\
])=\mathrm{O}) \mathrm{cc} 3) \mathrm{c}(\mathrm{cccc} 4) \mathrm{c} 24) \mathrm{O}) \mathrm{CC}(\mathrm{C}(\mathrm{C}=\mathrm{C}) \mathrm{C}[\mathrm{N}+] 15 \mathrm{Cc} 6 \mathrm{ccc}(\mathrm{Cl}) \mathrm{c}(\mathrm{SC}(\mathrm{F})(\mathrm{F}) \mathrm{F}) \mathrm{c} 6) \mathrm{CC} 5\end{array}$ \\
\hline Rnd1a_11 & 1.43 & 4.19 & 61.47 & $\begin{array}{l}\mathrm{C} 1([\mathrm{C} @ @ H](\mathrm{c} 2 \mathrm{cc}[\mathrm{n}+](\mathrm{Cc} 3 \mathrm{c}(\mathrm{F}) \mathrm{c}(\mathrm{C}(\mathrm{F})(\mathrm{F}) \mathrm{F}) \mathrm{ccc} 3) \mathrm{c}(\mathrm{cccc} 4) \mathrm{c} 24) \mathrm{O}) \mathrm{CC}(\mathrm{C}(\mathrm{C}=\mathrm{C}) \mathrm{C}[\mathrm{N}+] 15 \mathrm{Cc} 6 \mathrm{ccc}(\mathrm{Cl}) \mathrm{c}(\mathrm{SC}(\mathrm{F} \\
)(\mathrm{F}) \mathrm{F}) \mathrm{F} 6) \mathrm{CC} 5\end{array}$ \\
\hline Rnd1a_12 & 1.40 & 4.04 & 60.32 & $\begin{array}{l}\mathrm{C} 1([\mathrm{C} @ @ H](\mathrm{c} 2 \mathrm{cc}[\mathrm{n}+](\mathrm{Cc} 3 \mathrm{c}(\mathrm{F}) \mathrm{cc}(\mathrm{OCCCC}) \mathrm{cc} 3 \mathrm{~F}) \mathrm{c}(\mathrm{cccc} 4) \mathrm{c} 24) 0) \mathrm{CC}(\mathrm{C}(\mathrm{C}=\mathrm{C}) \mathrm{C}[\mathrm{N}+] 15 \mathrm{Cc} 6 \mathrm{ccc}(\mathrm{S}(\mathrm{F})(\mathrm{F})(\mathrm{F} \\
)(\mathrm{F}) \mathrm{F}) \mathrm{cc} 6 \mathrm{~F}) \mathrm{CC} 5\end{array}$ \\
\hline Rnd1a_13 & 0.50 & 1.65 & 24.61 & $\begin{array}{l}\mathrm{C1}([\mathrm{C} @ @ \mathrm{H}](\mathrm{c} 2 \mathrm{cc}[\mathrm{n}+](\mathrm{Cc} 30 \mathrm{oc}([\mathrm{N}+]([\mathrm{O}- \\
])=\mathrm{O}) \mathrm{cc3}) \mathrm{c}(\mathrm{cccc} 4) \mathrm{c} 24) \mathrm{O}) \mathrm{CC}(\mathrm{C}(\mathrm{C}=\mathrm{C}) \mathrm{C}[\mathrm{N}+] 15 \mathrm{Cc} 6 \mathrm{c}(\mathrm{F}) \mathrm{cccc} 6 \mathrm{~F}) \mathrm{CC} 5\end{array}$ \\
\hline
\end{tabular}




\begin{tabular}{|c|c|c|c|c|}
\hline Rnd1a_14 & 0.51 & 1.66 & 24.78 & $\begin{array}{l}\mathrm{C} 1([\mathrm{C} @ @ H](\mathrm{c} 2 \mathrm{cc}[\mathrm{n}+](\mathrm{Cc} 3 \mathrm{c}(\mathrm{F}) \mathrm{c}(\mathrm{F}) \mathrm{c}(\mathrm{F}) \mathrm{c}(\mathrm{F}) \mathrm{c} 3 \mathrm{~F}) \mathrm{c}(\mathrm{cccc} 4) \mathrm{c} 24) \mathrm{O}) \mathrm{CC}(\mathrm{C}(\mathrm{C}=\mathrm{C}) \mathrm{C}[\mathrm{N}+] 15 \mathrm{Cc} 6 \mathrm{c}(\mathrm{F}) \mathrm{c}(\mathrm{F}) \mathrm{cc}(\mathrm{F}) \mathrm{c} \\
6 \mathrm{~F}) \mathrm{CC5}\end{array}$ \\
\hline Rnd1a_15 & 0.73 & 2.07 & 34.96 & $\begin{array}{l}\mathrm{C} 1([\mathrm{C} @ @ H](\mathrm{c} 2 \mathrm{cc}[\mathrm{n}+](\mathrm{Cc} 3 \mathrm{ccc}([\mathrm{N}+]([\mathrm{O}-])=\mathrm{O}) \mathrm{cc} 3[\mathrm{~N}+]([\mathrm{O}- \\
])=\mathrm{O}) \mathrm{c}(\mathrm{cccc} 4) \mathrm{c} 24) \mathrm{O}) \mathrm{CC}(\mathrm{C}(\mathrm{C}=\mathrm{C}) \mathrm{C}[\mathrm{N}+] 15 \mathrm{Cc} 6 \mathrm{c}(\mathrm{F}) \mathrm{c}(\mathrm{F}) \mathrm{c}(\mathrm{OC}) \mathrm{c}(\mathrm{F}) \mathrm{c} 6 \mathrm{~F}) \mathrm{cC5}\end{array}$ \\
\hline Rnd1a_16 & 0.50 & 1.65 & 24.43 & $\begin{array}{l}\mathrm{C} 1([\mathrm{C} @ @ H](\mathrm{c} 2 \mathrm{cc}[\mathrm{n}+](\mathrm{Cc} 3 \mathrm{c}(\mathrm{OC}(\mathrm{F})(\mathrm{F}) \mathrm{F}) \mathrm{cc}(\mathrm{Cl}) \mathrm{cc} 3) \mathrm{c}(\mathrm{cccc} 4) \mathrm{c} 24) \mathrm{O}) \mathrm{CC}(\mathrm{C}(\mathrm{C}=\mathrm{C}) \mathrm{C}[\mathrm{N}+] 15 \mathrm{Cc} 6 \mathrm{ccc}(\mathrm{C}(\mathrm{F})(\mathrm{F}) \\
\mathrm{F}) \mathrm{cc6C}(\mathrm{F})(\mathrm{F}) \mathrm{F}) \mathrm{CC} 5\end{array}$ \\
\hline Rnd1a_17 & 0.86 & 2.35 & 40.34 & $\begin{array}{l}\mathrm{C} 1([\mathrm{C} @ @ H](\mathrm{c} 2 \mathrm{cc}[\mathrm{n}+](\mathrm{Cc} 3 \mathrm{cc}(\mathrm{S}(\mathrm{F})(\mathrm{F})(\mathrm{F})(\mathrm{F}) \mathrm{F}) \mathrm{ccc} 30 \mathrm{OC}) \mathrm{c}(\mathrm{cccc} 4) \mathrm{c} 24) \mathrm{O}) \mathrm{CC}(\mathrm{C}(\mathrm{C}=\mathrm{C}) \mathrm{C}[\mathrm{N}+] 15 \mathrm{cc} 6 \mathrm{ccc}(\mathrm{C}(\mathrm{F} \\
)(\mathrm{F}) \mathrm{F}) \mathrm{cc} 6 \mathrm{C}(\mathrm{F})(\mathrm{F}) \mathrm{F}) \mathrm{CC} 5\end{array}$ \\
\hline Rnd1a_18 & 0.11 & 1.12 & 5.67 & $\begin{array}{l}\mathrm{C} 1([\mathrm{C} @ @ \mathrm{H}](\mathrm{c} 2 \mathrm{ccc}[\mathrm{n}+](\mathrm{Cc} 3 \circ \mathrm{oc}([\mathrm{N}+]([\mathrm{O}- \\
])=\mathrm{O}) \mathrm{cc} 3) \mathrm{c}(\mathrm{cccc} 4) \operatorname{c2} 24) \mathrm{O}) \mathrm{cC}(\mathrm{C}(\mathrm{C}=\mathrm{C}) \mathrm{C}[\mathrm{N}+] 15 \mathrm{Cc} 6 \mathrm{ccc}(\mathrm{C}(\mathrm{F})(\mathrm{F}) \mathrm{F}) \operatorname{cc} 6 \mathrm{C}(\mathrm{F})(\mathrm{F}) \mathrm{F}) \mathrm{CC} 5\end{array}$ \\
\hline Rnd1a_19 & 0.10 & 1.10 & 4.87 & $\begin{array}{l}\mathrm{C} 1([\mathrm{C} @ @ \mathrm{H}](\mathrm{c} 2 \mathrm{cc}[\mathrm{n}+](\mathrm{Cc} 3 \mathrm{c}(\mathrm{CHN}) \mathrm{ccc}(\mathrm{F}) \mathrm{c} 3) \mathrm{c}(\mathrm{cccc} 4) \mathrm{c} 24) \mathrm{O}) \mathrm{CC}(\mathrm{C}(\mathrm{C}=\mathrm{C}) \mathrm{C}[\mathrm{N}+] 15 \mathrm{Cc} 6 \mathrm{ccc}(\mathrm{C}(\mathrm{F})(\mathrm{F}) \mathrm{F}) \mathrm{cc} 6 \\
\mathrm{C}(\mathrm{F})(\mathrm{F}) \mathrm{F}) \mathrm{CC} 5\end{array}$ \\
\hline Rnd1a_20 & 0.00 & 1.00 & -0.08 & $\begin{array}{l}\mathrm{C} 1([\mathrm{C} @ @ H](\mathrm{c} 2 \mathrm{cc}[\mathrm{n}+](\mathrm{Cc} 3 \mathrm{c}(\mathrm{OC}) \mathrm{cc}([\mathrm{N}+]([\mathrm{O}- \\
])=\mathrm{O}) \mathrm{cc} 3) \mathrm{c}(\mathrm{cccc} 4) \mathrm{c} 24) \mathrm{O}) \mathrm{CC}(\mathrm{C}(\mathrm{C}=\mathrm{C}) \mathrm{C}[\mathrm{N}+] 15 \mathrm{Cc} 6 \mathrm{ccc}(\mathrm{F}) \mathrm{cc} 6 \mathrm{C}(\mathrm{F})(\mathrm{F}) \mathrm{F}) \mathrm{cC} 5\end{array}$ \\
\hline Rnd2_1 & 1.68 & 5.35 & 68.50 & $\begin{array}{l}{[n+] 1(c 2 c(c([C @ @ H](O)[C @ H] 3[N @ @+](C c 4 c(C(F) F) c c c c 4)(C C 5) C[C @ H](C=C)[C @ @ H] 5 C 3) c c 1} \\
) \operatorname{cccc} 2) \operatorname{cc} 6 c c(C(F)(F) F) c c(C(F)(F) F) c 6\end{array}$ \\
\hline Rnd2_2 & 1.49 & 4.43 & 63.20 & $\begin{array}{l}{[n+] 1(c 2 c(c([C @ @ H](O)[C @ H] 3[N @ @+](C c 4 c(C(F)(F) F) c c c(C(F)(F) F) c 4)(C C 5) C[C @ H](C=C)[C @} \\
@ H] 5 C 3) c c 1) \operatorname{cccc} 2) C c 6 c c(C(F)(F) F) c c(C(F)(F) F) c 6\end{array}$ \\
\hline Rnd2_3 & 1.60 & 4.97 & 66.50 & $\begin{array}{l}{[\mathrm{n}+] 1(\mathrm{c} 2 \mathrm{c}(\mathrm{c}([\mathrm{C} @ @ \mathrm{H}](\mathrm{O})[\mathrm{C} @ \mathrm{H}] 3[\mathrm{~N} @ @+](\mathrm{Cc} 4 \mathrm{cc}(\mathrm{C}(\mathrm{F})(\mathrm{F}) \mathrm{F}) \mathrm{cc}(\mathrm{C}(\mathrm{F})(\mathrm{F}) \mathrm{C}(\mathrm{F})(\mathrm{F}) \mathrm{F}) \mathrm{c} 4)(\mathrm{CC} 5) \mathrm{C}[\mathrm{C} @ \mathrm{H}](\mathrm{C}=} \\
\mathrm{C})[\mathrm{C} @ @ \mathrm{H}] 5 \mathrm{C} 3) \mathrm{cc} 1) \mathrm{cccc} 2) \mathrm{Cc} 6 \mathrm{cc}(\mathrm{C}(\mathrm{F})(\mathrm{F}) \mathrm{F}) \mathrm{cc}(\mathrm{C}(\mathrm{F})(\mathrm{F}) \mathrm{F}) \mathrm{c6}\end{array}$ \\
\hline Rnd2_4 & 1.47 & 4.35 & 62.60 & $\begin{array}{l}{[\mathrm{n}+] 1(\mathrm{c} 2 \mathrm{c}(\mathrm{c}([\mathrm{C} @ @ \mathrm{H}](\mathrm{O})[\mathrm{C} @ \mathrm{H}] 3[\mathrm{~N} @ @+](\mathrm{Cc} 4 \mathrm{cc}(\mathrm{C}(\mathrm{F})(\mathrm{F}) \mathrm{C}(\mathrm{F})(\mathrm{F}) \mathrm{F}) \mathrm{cc}(\mathrm{C}(\mathrm{F})(\mathrm{F}) \mathrm{C}(\mathrm{F})(\mathrm{F}) \mathrm{F}) \mathrm{c} 4)(\mathrm{CC} 5) \mathrm{C}[\mathrm{C}} \\
@ \mathrm{H}](\mathrm{C}=\mathrm{C})[\mathrm{C} @ @ \mathrm{H}] 5 \mathrm{C} 3) \mathrm{cc} 1) \mathrm{cccc}) \mathrm{cc6cc}(\mathrm{C}(\mathrm{F})(\mathrm{F}) \mathrm{F}) \mathrm{cc}(\mathrm{C}(\mathrm{F})(\mathrm{F}) \mathrm{F}) \mathrm{c6}\end{array}$ \\
\hline Rnd2_5 & 2.11 & 8.26 & 78.40 & $\begin{array}{l}{[n+] 1(c 2 c(c([C @ @ H](O)[C @ H] 3[N @ @+](C c 4 c c(F) c c(C(F)(F) C(F)(F) F) c 4)(C C 5) C[C @ H](C=C)[C @} \\
@ H] 5 C 3) c c 1) \operatorname{ccc} 2)(c c 6 c c(C(F)(F) F) c c(C(F)(F) F) c 6\end{array}$ \\
\hline
\end{tabular}




\begin{tabular}{|c|c|c|c|c|}
\hline Rnd2_6 & 1.58 & 4.87 & 65.90 & $\begin{array}{l}{[n+] 1(\mathrm{c} 2 \mathrm{c}(\mathrm{c}([\mathrm{C} @ @ \mathrm{C}](\mathrm{O})[\mathrm{C} @ \mathrm{H}] 3[\mathrm{~N} @ @+](\mathrm{Cc} 4 \mathrm{cc}(\mathrm{C}(\mathrm{F})(\mathrm{F}) \mathrm{F}) \mathrm{cc}(\mathrm{C}(\mathrm{F})(\mathrm{F}) \mathrm{F}) \mathrm{c} 4)(\mathrm{CC} 5) \mathrm{C}[\mathrm{C} @ \mathrm{H}](\mathrm{C}=\mathrm{C})[\mathrm{C} @} \\
@ \mathrm{H}] 5 \mathrm{C} 3) \mathrm{cc} 1) \mathrm{cccc}) \mathrm{Cc} 6 \mathrm{cc}(\mathrm{C}(\mathrm{F})(\mathrm{F}) \mathrm{F}) \mathrm{cc}(\mathrm{C}(\mathrm{F})(\mathrm{F}) \mathrm{F}) \mathrm{c6}\end{array}$ \\
\hline Rnd2_7 & 1.67 & 5.29 & 68.20 & $\begin{array}{l}{[\mathrm{n}+] 1(\mathrm{c} 2 \mathrm{c}(\mathrm{c}([\mathrm{C} @ @ \mathrm{H}](\mathrm{O})[\mathrm{C} @ \mathrm{H}] 3[\mathrm{~N} @ @+](\mathrm{Cc} 4 \mathrm{c}(\mathrm{F}) \mathrm{c}(\mathrm{F}) \mathrm{c}(\mathrm{F}) \mathrm{c}(\mathrm{F}) \mathrm{c4})(\mathrm{CC} 5) \mathrm{C}[\mathrm{C} @ \mathrm{H}](\mathrm{C}=\mathrm{C})[\mathrm{C} @ @ \mathrm{H}] 5 \mathrm{C} 3} \\
) \mathrm{cc} 1) \mathrm{cccc} 2) \mathrm{cc} 6 \mathrm{cc}(\mathrm{C}(\mathrm{F})(\mathrm{F}) \mathrm{F}) \mathrm{cc}(\mathrm{C}(\mathrm{F})(\mathrm{F}) \mathrm{F}) \mathrm{c6}\end{array}$ \\
\hline Rnd2_8 & 1.50 & 4.49 & 63.60 & $\begin{array}{l}{[n+] 1(\mathrm{c} 2 \mathrm{c}(\mathrm{c}([\mathrm{C} @ @ \mathrm{H}](\mathrm{O})[\mathrm{C} @ \mathrm{H}] 3[\mathrm{~N} @ @+](\mathrm{Cc} 4 \mathrm{cc}(\mathrm{C}=\mathrm{O}) \mathrm{c} 5 \mathrm{ccccc} 5) \mathrm{ccc} 4)(\mathrm{CC} 6) \mathrm{C}[\mathrm{C} @ \mathrm{H}](\mathrm{C}=\mathrm{C})[\mathrm{C} @ @} \\
\mathrm{H}] 6 \mathrm{C} 3) \mathrm{cc}) \mathrm{cccc} 2) \mathrm{Cc} 7 \mathrm{cc}(\mathrm{C}(\mathrm{F})(\mathrm{F}) \mathrm{F}) \mathrm{cc}(\mathrm{C}(\mathrm{F})(\mathrm{F}) \mathrm{F}) \mathrm{c} 7\end{array}$ \\
\hline Rnd2_9 & 1.49 & 4.43 & 63.20 & $\begin{array}{l}{[\mathrm{n}+] 1(\mathrm{c} 2 \mathrm{c}(\mathrm{c}([\mathrm{C} @ @ \mathrm{H}](\mathrm{O})[\mathrm{C} @ \mathrm{H}] 3[\mathrm{~N} @ @+](\mathrm{Cc} 4 \mathrm{c}(\mathrm{C}(\mathrm{F}) \mathrm{F}) \mathrm{cccc} 4)(\mathrm{CC} 5) \mathrm{C}[\mathrm{C} @ \mathrm{H}](\mathrm{C}=\mathrm{C})[\mathrm{C} @ @ \mathrm{H}] 5 \mathrm{C} 3) \mathrm{cc} 1} \\
) \mathrm{cccc} 2) \mathrm{Cc} 6 \mathrm{cc}(\mathrm{C}(\mathrm{F})(\mathrm{F}) \mathrm{F}) \mathrm{ccc} 6\end{array}$ \\
\hline Rnd2_10 & 1.32 & 3.75 & 57.90 & $\begin{array}{l}{[n+] 1(\mathrm{c} 2 \mathrm{c}(\mathrm{c}([\mathrm{C} @ @ \mathrm{H}](\mathrm{O})[\mathrm{C} @ \mathrm{H}] 3[\mathrm{~N} @ @+](\mathrm{Cc} 4 \mathrm{c}(\mathrm{C}(\mathrm{F})(\mathrm{F}) \mathrm{F}) \mathrm{ccc}(\mathrm{C}(\mathrm{F})(\mathrm{F}) \mathrm{F}) \mathrm{c4})(\mathrm{CC} 5) \mathrm{C}[\mathrm{C} @ \mathrm{H}](\mathrm{C}=\mathrm{C})[\mathrm{C} @} \\
@ \mathrm{H}] 5 \mathrm{C} 3) \mathrm{cc} 1) \mathrm{cccc}) \mathrm{Cc} 6 \mathrm{cc}(\mathrm{C}(\mathrm{F})(\mathrm{F}) \mathrm{F}) \mathrm{ccc} 6\end{array}$ \\
\hline Rnd2_11 & 1.92 & 6.81 & 74.40 & $\begin{array}{l}{[n+] 1(\mathrm{c} 2 \mathrm{c}(\mathrm{c}([\mathrm{C} @ @ \mathrm{H}](\mathrm{O})[\mathrm{C} @ \mathrm{H}] 3[\mathrm{~N} @ @ @+](\mathrm{Cc} 4 \mathrm{cc}(\mathrm{C}(\mathrm{F})(\mathrm{F}) \mathrm{F}) \mathrm{cc}(\mathrm{C}(\mathrm{F})(\mathrm{F}) \mathrm{C}(\mathrm{F})(\mathrm{F}) \mathrm{F}) \mathrm{c} 4)(\mathrm{CC} 5) \mathrm{C}[\mathrm{C} @ \mathrm{H}](\mathrm{C}=} \\
\mathrm{c})[\mathrm{C} @ @ \mathrm{H}] 5 \mathrm{C} 3) \mathrm{cc} 1) \mathrm{cccc} 2) \mathrm{Cc} 6 \mathrm{cc}(\mathrm{C}(\mathrm{F})(\mathrm{F}) \mathrm{F}) \mathrm{ccc} 6\end{array}$ \\
\hline Rnd2_12 & 1.60 & 4.97 & 66.50 & $\begin{array}{l}{[n+] 1(c 2 c(c([C @ @ H](O)[C @ H] 3[N @ @+](C c 4 c c(C(F)(F) C(F)(F) F) c c(C(F)(F) C(F)(F) F) c 4)(C C 5) C[C} \\
@ H](C=C)[C @ @ H] 5 C 3) c c 1) c c c c 2) C c 6 c c(C(F)(F) F) c c c 6\end{array}$ \\
\hline Rnd2_13 & 2.21 & 9.15 & 80.30 & $\begin{array}{l}{[n+] 1(\mathrm{c} 2 \mathrm{c}(\mathrm{c}([\mathrm{C} @ @ \mathrm{H}](\mathrm{O})[\mathrm{C} @ \mathrm{H}] 3[\mathrm{~N} @ @+](\mathrm{Cc} 4 \mathrm{cc}(\mathrm{F}) \mathrm{cc}(\mathrm{C}(\mathrm{F})(\mathrm{F}) \mathrm{C}(\mathrm{F})(\mathrm{F}) \mathrm{F}) \mathrm{c} 4)(\mathrm{CC} 5) \mathrm{C}[\mathrm{C} @ \mathrm{H}](\mathrm{C}=\mathrm{C})[\mathrm{C} @} \\
@ \mathrm{H}] 5 \mathrm{C} 3) \mathrm{cc} 1) \mathrm{cccc}) \mathrm{Cc} 6 \mathrm{cc}(\mathrm{C}(\mathrm{F})(\mathrm{F}) \mathrm{F}) \mathrm{ccc} 6\end{array}$ \\
\hline Rnd2_14 & 1.97 & 7.16 & 75.50 & $\begin{array}{l}{[n+] 1(\mathrm{c} 2 \mathrm{c}(\mathrm{c}([\mathrm{C} @ @ \mathrm{H}](\mathrm{O})[\mathrm{C} @ \mathrm{H}] 3[\mathrm{~N} @ @+](\mathrm{Cc} 4 \mathrm{cc}(\mathrm{C}(\mathrm{F})(\mathrm{F}) \mathrm{F}) \mathrm{cc}(\mathrm{C}(\mathrm{F})(\mathrm{F}) \mathrm{F}) \mathrm{c} 4)(\mathrm{CC} 5) \mathrm{C}[\mathrm{C} @ \mathrm{H}](\mathrm{C}=\mathrm{C})[\mathrm{C} @} \\
@ \mathrm{H}] 5 \mathrm{C} 3) \mathrm{cc} 1) \mathrm{cccc}) \mathrm{Cc} 6 \mathrm{cc}(\mathrm{C}(\mathrm{F})(\mathrm{F}) \mathrm{F}) \mathrm{ccc} 6\end{array}$ \\
\hline Rnd2_15 & 1.19 & 3.29 & 53.40 & $\begin{array}{l}{[\mathrm{n}+] 1(\mathrm{c} 2 \mathrm{c}(\mathrm{c}([\mathrm{C} @ @ \mathrm{H}](\mathrm{O})[\mathrm{C} @ \mathrm{H}] 3[\mathrm{~N} @ @ @+](\mathrm{Cc} 4 \mathrm{c}(\mathrm{F}) \mathrm{c}(\mathrm{F}) \mathrm{c}(\mathrm{F}) \mathrm{c}(\mathrm{F}) \mathrm{c} 4)(\mathrm{CC} 5) \mathrm{C}[\mathrm{C} @ \mathrm{H}](\mathrm{C}=\mathrm{C})[\mathrm{C} @ @ \mathrm{H}] 5 \mathrm{C} 3} \\
\mathrm{ccc} 1) \mathrm{cccc} 2) \mathrm{Cc} 6 \mathrm{cc}(\mathrm{C}(\mathrm{F})(\mathrm{F}) \mathrm{F}) \mathrm{ccc} 6\end{array}$ \\
\hline Rnd2_16 & 1.14 & 3.14 & 51.70 & $\begin{array}{l}{[n+] 1(\mathrm{c} 2 \mathrm{c}(\mathrm{c}([\mathrm{C} @ @ \mathrm{H}](\mathrm{O})[\mathrm{C} @ \mathrm{H}] 3[\mathrm{~N} @ @+](\mathrm{Cc} 4 \mathrm{cc}(\mathrm{C}=\mathrm{O}) \mathrm{c} 5 \mathrm{ccccc} 5) \mathrm{ccc} 4)(\mathrm{CC} 6) \mathrm{C}[\mathrm{C} @ \mathrm{H}](\mathrm{C}=\mathrm{C})[\mathrm{C} @ @} \\
\mathrm{H}] 6 \mathrm{C} 3) \mathrm{cc} 1) \mathrm{cccc} 2) \mathrm{Cc} 7 \mathrm{cc}(\mathrm{C}(\mathrm{F})(\mathrm{F}) \mathrm{F}) \mathrm{ccc} 7\end{array}$ \\
\hline Rnd2_17 & 1.55 & 4.73 & 65.10 & $\begin{array}{l}{[\mathrm{n}+] 1(\mathrm{c} 2 \mathrm{c}(\mathrm{c}([\mathrm{C} @ @ \mathrm{H}](\mathrm{O})[\mathrm{C} @ \mathrm{H}] 3[\mathrm{~N} @ @+](\mathrm{Cc} 4 \mathrm{c}(\mathrm{C}(\mathrm{F}) \mathrm{F}) \mathrm{cccc} 4)(\mathrm{CC} 5) \mathrm{C}[\mathrm{C} @ \mathrm{H}](\mathrm{C}=\mathrm{C})[\mathrm{C} @ @ \mathrm{H}] 5 \mathrm{C} 3) \mathrm{cc} 1} \\
) \mathrm{cccc} 2) \mathrm{cc} 6 \mathrm{cc}(\mathrm{c}(\mathrm{Cl}) \mathrm{cc} 6) \mathrm{C}(\mathrm{F})(\mathrm{F}) \mathrm{F}\end{array}$ \\
\hline
\end{tabular}




\begin{tabular}{|c|c|c|c|c|}
\hline Rnd2_18 & 1.29 & 3.62 & 56.70 & $\begin{array}{l}{[n+] 1(\mathrm{c} 2 \mathrm{c}(\mathrm{c}([\mathrm{C} @ @ \mathrm{H}](\mathrm{O})[\mathrm{C} @ \mathrm{H}] 3[\mathrm{~N} @ @+](\mathrm{Cc} 4 \mathrm{c}(\mathrm{C}(\mathrm{F})(\mathrm{F}) \mathrm{F}) \mathrm{ccc}(\mathrm{C}(\mathrm{F})(\mathrm{F}) \mathrm{F}) \mathrm{c} 4)(\mathrm{CC} 5) \mathrm{C}[\mathrm{C} @ \mathrm{H}](\mathrm{C}=\mathrm{C})[\mathrm{C} @} \\
@ \mathrm{H}] 5 \mathrm{C} 3) \mathrm{cc} 1) \mathrm{cccc}) \mathrm{Cc6cc}(\mathrm{c}(\mathrm{Cl}) \mathrm{cc} 6) \mathrm{C}(\mathrm{F})(\mathrm{F}) \mathrm{F}\end{array}$ \\
\hline Rnd2_19 & 2.03 & 7.58 & 76.70 & $\begin{array}{l}{[\mathrm{n}+] 1(\mathrm{c} 2 \mathrm{c}(\mathrm{c}([\mathrm{C} @ @ \mathrm{H}](\mathrm{O})[\mathrm{C} @ \mathrm{H}] 3[\mathrm{~N} @ @ @+](\mathrm{Cc} 4 \mathrm{cc}(\mathrm{C}(\mathrm{F})(\mathrm{F}) \mathrm{F}) \mathrm{cc}(\mathrm{C}(\mathrm{F})(\mathrm{F}) \mathrm{C}(\mathrm{F})(\mathrm{F}) \mathrm{F}) \mathrm{c} 4)(\mathrm{CC} 5) \mathrm{C}[\mathrm{C} @ \mathrm{H}](\mathrm{C}=} \\
\mathrm{C})[\mathrm{C} @ @ \mathrm{H}] 5 \mathrm{C} 3) \mathrm{cc} 1) \mathrm{cccc} 2) \mathrm{Cc} 6 \mathrm{cc}(\mathrm{c}(\mathrm{Cl}) \mathrm{cc} 6) \mathrm{C}(\mathrm{F})(\mathrm{F}) \mathrm{F}\end{array}$ \\
\hline Rnd2_20 & 1.79 & 6.02 & 71.50 & $\begin{array}{l}{[n+] 1(c 2 c(c([C @ @ H](O)[C @ H] 3[N @ @+](C c 4 c c(C(F)(F) C(F)(F) F) c c(C(F)(F) C(F)(F) F) c 4)(C C 5) C[C} \\
@ H](C=C)[C @ @ H] 5 C 3) c c 1) c c c c 2) C c 6 c c(c(C l) c c 6) C(F)(F) F\end{array}$ \\
\hline Rnd2_21 & 2.28 & 9.81 & 81.50 & $\begin{array}{l}{[n+] 1(c 2 c(c([C @ @ H](O)[C @ H] 3[N @ @+](C c 4 c c(F) c c(C(F)(F) C(F)(F) F) c 4)(C C 5) C[C @ H](C=C)[C @} \\
@ H] 5 C 3) c c 1) c c c c 2) C c 6 c c(c(C l) c c 6) C(F)(F) F\end{array}$ \\
\hline Rnd2_22 & 1.88 & 6.55 & 73.50 & $\begin{array}{l}{[n+] 1(c 2 c(c([C @ @ H](O)[C @ H] 3[N @ @+](C c 4 c c(C(F)(F) F) c c(C(F)(F) F) c 4)(C C 5) C[C @ H](C=C)[C @} \\
@ H] 5 C 3) c c 1) c c c c 2) C c 6 c c(c(C l) c c 6) C(F)(F) F\end{array}$ \\
\hline Rnd2_23 & 1.67 & 5.29 & 68.20 & $\begin{array}{l}{[\mathrm{n}+] 1(\mathrm{c} 2 \mathrm{c}(\mathrm{c}([\mathrm{C} @ @ \mathrm{H}](\mathrm{O})[\mathrm{C} @ \mathrm{H}] 3[\mathrm{~N} @ @+](\mathrm{Cc} 4 \mathrm{c}(\mathrm{F}) \mathrm{c}(\mathrm{F}) \mathrm{c}(\mathrm{F}) \mathrm{c}(\mathrm{F}) \mathrm{c} 4)(\mathrm{CC} 5) \mathrm{C}[\mathrm{C} @ \mathrm{H}](\mathrm{C}=\mathrm{C})[\mathrm{C} @ @ \mathrm{H}] 5 \mathrm{C} 3} \\
) \mathrm{cc} 1) \mathrm{cccc} 2) \mathrm{cc6cc}(\mathrm{c}(\mathrm{Cl}) \mathrm{cc} 6) \mathrm{C}(\mathrm{F})(\mathrm{F}) \mathrm{F}\end{array}$ \\
\hline Rnd2__24 & 1.13 & 3.11 & 51.30 & $\begin{array}{l}{[n+] 1(\mathrm{c} 2 \mathrm{c}(\mathrm{c}([\mathrm{C} @ @ \mathrm{H}](\mathrm{O})[\mathrm{C} @ \mathrm{H}] 3[\mathrm{~N} @ @+](\mathrm{Cc} 4 \mathrm{cc}(\mathrm{C}=\mathrm{O}=\mathrm{C}) \mathrm{c} 5 \mathrm{ccccc5}) \mathrm{ccc} 4)(\mathrm{CC} 6) \mathrm{C}[\mathrm{C} @ \mathrm{H}](\mathrm{C}=\mathrm{C})[\mathrm{C} @ @} \\
\mathrm{H}] 6 \mathrm{C} 3) \mathrm{cc} 1) \mathrm{cccc} 2) \mathrm{Cc} 7 \mathrm{cc}(\mathrm{c}(\mathrm{Cl}) \mathrm{cc} 7) \mathrm{C}(\mathrm{F})(\mathrm{F}) \mathrm{F}\end{array}$ \\
\hline Rnd2_25 & 1.47 & 4.33 & 62.50 & $\begin{array}{l}{[\mathrm{n}+] 1(\mathrm{c} 2 \mathrm{c}(\mathrm{c}([\mathrm{C} @ @ \mathrm{H}](\mathrm{O})[\mathrm{C} @ \mathrm{H}] 3[\mathrm{~N} @ @+](\mathrm{Cc} 4 \mathrm{c}(\mathrm{C}(\mathrm{F}) \mathrm{F}) \mathrm{cccc} 4)(\mathrm{CC} 5) \mathrm{C}[\mathrm{C} @ \mathrm{H}](\mathrm{C}=\mathrm{C})[\mathrm{C} @ @ \mathrm{H}] 5 \mathrm{C} 3) \mathrm{cc} 1} \\
\operatorname{ccccc} 2) \mathrm{Cc} 6 \mathrm{cc}(\mathrm{Cl}) \mathrm{cc}(\mathrm{C}(\mathrm{F})(\mathrm{F}) \mathrm{F}) \mathrm{c6}\end{array}$ \\
\hline Rnd2_26 & 1.43 & 4.18 & 61.40 & $\begin{array}{l}{[\mathrm{n}+] 1(\mathrm{c} 2 \mathrm{c}(\mathrm{c}([\mathrm{C} @ @ \mathrm{H}](\mathrm{O})[\mathrm{C} @ \mathrm{H}] 3[\mathrm{~N} @ @+](\mathrm{Cc} 4 \mathrm{c}(\mathrm{C}(\mathrm{F})(\mathrm{F}) \mathrm{F}) \mathrm{ccc}(\mathrm{C}(\mathrm{F})(\mathrm{F}) \mathrm{F}) \mathrm{c} 4)(\mathrm{CC} 5) \mathrm{C}[\mathrm{C} @ \mathrm{H}](\mathrm{C}=\mathrm{C})[\mathrm{C} @} \\
@ \mathrm{CH}] 5 \mathrm{C} 3) \mathrm{cc} 1) \mathrm{cccc} 2) \mathrm{Ccc6cc}(\mathrm{Cl}) \mathrm{cc}(\mathrm{C}(\mathrm{F})(\mathrm{F}) \mathrm{F}) \mathrm{c6}\end{array}$ \\
\hline Rnd2_27 & 1.55 & 4.70 & 64.90 & $\begin{array}{l}{[\mathrm{n}+] 1(\mathrm{c} 2 \mathrm{c}(\mathrm{c}([\mathrm{C} @ @ \mathrm{H}](\mathrm{O})[\mathrm{C} @ \mathrm{H}] 3[\mathrm{~N} @ @ @+](\mathrm{Cc} 4 \mathrm{cc}(\mathrm{C}(\mathrm{F})(\mathrm{F}) \mathrm{F}) \mathrm{cc}(\mathrm{C}(\mathrm{F})(\mathrm{F}) \mathrm{C}(\mathrm{F})(\mathrm{F}) \mathrm{F}) \mathrm{c} 4)(\mathrm{CC} 5) \mathrm{C}[\mathrm{C} @ \mathrm{H}](\mathrm{C}=} \\
\mathrm{C})[\mathrm{C} @ @ \mathrm{H}] 5 \mathrm{C} 3) \mathrm{cc} 1) \mathrm{cccc} 2) \mathrm{Cc} 6 \mathrm{cc}(\mathrm{Cl}) \mathrm{cc}(\mathrm{C}(\mathrm{F})(\mathrm{F}) \mathrm{F}) \mathrm{c6}\end{array}$ \\
\hline Rnd2_28 & 1.35 & 3.87 & 58.90 & $\begin{array}{l}{[n+] 1(c 2 c(c([C @ @ H](O)[C @ H] 3[N @ @+](C c 4 c c(C(F)(F) C(F)(F) F) c c(C(F)(F) C(F)(F) F) c 4)(C C 5) C[C} \\
@ H](C=C)[C @ @ H] 5 C 3) c c 1) c c c c 2) C c 6 c c(C l) c c(C(F)(F) F) c 6\end{array}$ \\
\hline Rnd2_29 & 2.00 & 7.40 & 76.20 & $\begin{array}{l}{[n+] 1(\mathrm{c} 2 \mathrm{c}(\mathrm{c}([\mathrm{C} @ @ \mathrm{H}](\mathrm{O})[\mathrm{C} @ \mathrm{H}] 3[\mathrm{~N} @ @+](\mathrm{Cc} 4 \mathrm{cc}(\mathrm{F}) \mathrm{cc}(\mathrm{C}(\mathrm{F})(\mathrm{F}) \mathrm{C}(\mathrm{F})(\mathrm{F}) \mathrm{F}) \mathrm{c4})(\mathrm{CC} 5) \mathrm{C}[\mathrm{C} @ \mathrm{H}](\mathrm{C}=\mathrm{C})[\mathrm{C} @} \\
@ \mathrm{H}] 5 \mathrm{C} 3) \mathrm{cc} 1) \mathrm{cccc}) \mathrm{Cc} 6 \mathrm{cc}(\mathrm{Cl}) \mathrm{cc}(\mathrm{C}(\mathrm{F})(\mathrm{F}) \mathrm{F}) \mathrm{c6}\end{array}$ \\
\hline
\end{tabular}




\begin{tabular}{|c|c|c|c|c|}
\hline Rnd2_30 & 1.69 & 5.41 & 68.80 & $\begin{array}{l}{[n+] 1(\mathrm{c} 2 c(\mathrm{c}([\mathrm{C} @ @ \mathrm{H}](\mathrm{O})[\mathrm{C} @ \mathrm{H}] 3[\mathrm{~N} @ @ @+](\mathrm{Cc} 4 \mathrm{cc}(\mathrm{C}(\mathrm{F})(\mathrm{F}) \mathrm{F}) \mathrm{cc}(\mathrm{C}(\mathrm{F})(\mathrm{F}) \mathrm{F}) \mathrm{c} 4)(\mathrm{CC} 5) \mathrm{C}[\mathrm{C} @ \mathrm{H}](\mathrm{C}=\mathrm{C})[\mathrm{C} @} \\
@ \mathrm{H}] 5 \mathrm{C} 3) \mathrm{cc} 1) \mathrm{cccc} 2) \mathrm{Cc6cc}(\mathrm{Cl}) \mathrm{cc}(\mathrm{C}(\mathrm{F})(\mathrm{F}) \mathrm{F}) \mathrm{c6}\end{array}$ \\
\hline Rnd2_31 & 1.63 & 5.12 & 67.30 & $\begin{array}{l}{[n+] 1(\mathrm{c} 2 \mathrm{c}(\mathrm{c}([\mathrm{C} @ @ H](\mathrm{O})[\mathrm{C} @ \mathrm{H}] 3[\mathrm{~N} @ @ @+](\mathrm{Cc} 4 \mathrm{c}(\mathrm{F}) \mathrm{c}(\mathrm{F}) \mathrm{c}(\mathrm{F}) \mathrm{c}(\mathrm{F}) \mathrm{c} 4)(\mathrm{CC} 5) \mathrm{C}[\mathrm{C} @ \mathrm{H}](\mathrm{C}=\mathrm{C})[\mathrm{C} @ @ \mathrm{H}] 5 \mathrm{C} 3} \\
\operatorname{cc1}) \mathrm{cccc} 2) \mathrm{Cc} 6 \mathrm{cc}(\mathrm{Cl}) \mathrm{cc}(\mathrm{C}(\mathrm{F})(\mathrm{F}) \mathrm{F}) \mathrm{c} 6\end{array}$ \\
\hline Rnd2_32 & 1.31 & 3.71 & 57.50 & $\begin{array}{l}{[\mathrm{n}+] 1(\mathrm{c} 2 \mathrm{c}(\mathrm{c}([\mathrm{C} @ @ \mathrm{H}](\mathrm{O})[\mathrm{C} @ \mathrm{H}] 3[\mathrm{~N} @ @+](\mathrm{Cc} 4 \mathrm{cc}(\mathrm{C}(=\mathrm{O}) \mathrm{c} 5 \mathrm{ccccc5}) \mathrm{ccc} 4)(\mathrm{CC} 6) \mathrm{C}[\mathrm{C} @ \mathrm{H}](\mathrm{C}=\mathrm{C})[\mathrm{C} @ @} \\
\mathrm{H}] 6 \mathrm{C} 3) \mathrm{cc} 1) \mathrm{cccc} 2) \mathrm{Cc} 7 \mathrm{cc}(\mathrm{Cl}) \mathrm{cc}(\mathrm{C}(\mathrm{F})(\mathrm{F}) \mathrm{F}) \mathrm{c} 7\end{array}$ \\
\hline Rnd2_33 & 1.21 & 3.36 & 54.10 & $\begin{array}{l}{[n+] 1(\mathrm{c} 2 \mathrm{c}(\mathrm{c}([\mathrm{C} @ @ \mathrm{H}](\mathrm{O})[\mathrm{C} @ \mathrm{H}] 3[\mathrm{~N} @ @+](\mathrm{Cc} 4 \mathrm{c}(\mathrm{C}(\mathrm{F}) \mathrm{F}) \mathrm{cccc} 4)(\mathrm{CC} 5) \mathrm{C}[\mathrm{C} @ \mathrm{H}](\mathrm{C}=\mathrm{C})[\mathrm{C} @ @ \mathrm{H}] 5 \mathrm{C} 3) \mathrm{cc} 1} \\
\operatorname{cccc} 2) \mathrm{Cc} 6 \mathrm{c}(\mathrm{C}(\mathrm{F})(\mathrm{F}) \mathrm{F}) \mathrm{cc}(\mathrm{C}(\mathrm{F})(\mathrm{F}) \mathrm{F}) \mathrm{ccc} 6\end{array}$ \\
\hline Rnd2_34 & 0.47 & 1.60 & 23.10 & $\begin{array}{l}{[n+] 1(\mathrm{c} 2 \mathrm{c}(\mathrm{c}([\mathrm{C} @ @ \mathrm{H}](\mathrm{O})[\mathrm{C} @ \mathrm{H}] 3[\mathrm{~N} @ @+](\mathrm{Cc} 4 \mathrm{c}(\mathrm{C}(\mathrm{F})(\mathrm{F}) \mathrm{F}) \mathrm{ccc}(\mathrm{C}(\mathrm{F})(\mathrm{F}) \mathrm{F}) \mathrm{c} 4)(\mathrm{CC} 5) \mathrm{C}[\mathrm{C} @ \mathrm{H}](\mathrm{C}=\mathrm{C})[\mathrm{C} @} \\
@ \mathrm{H}] 5 \mathrm{C} 3) \mathrm{cc} 1) \mathrm{cccc} 2) \mathrm{Cccc}(\mathrm{C}(\mathrm{F})(\mathrm{F}) \mathrm{F}) \mathrm{cc}(\mathrm{C}(\mathrm{F})(\mathrm{F}) \mathrm{F}) \mathrm{cc} 6\end{array}$ \\
\hline Rnd2_35 & 1.45 & 4.28 & 62.10 & $\begin{array}{l}{[\mathrm{n}+] 1(\mathrm{c} 2 \mathrm{c}(\mathrm{c}([\mathrm{C} @ @ \mathrm{H}](\mathrm{O})[\mathrm{C} @ \mathrm{H}] 3[\mathrm{~N} @ @ @](\mathrm{Cc} 4 \mathrm{cc}(\mathrm{C}(\mathrm{F})(\mathrm{F}) \mathrm{F}) \mathrm{cc}(\mathrm{C}(\mathrm{F})(\mathrm{F}) \mathrm{C}(\mathrm{F})(\mathrm{F}) \mathrm{F}) \mathrm{c} 4)(\mathrm{CC} 5) \mathrm{C}[\mathrm{C} @ \mathrm{H}](\mathrm{C}=} \\
\mathrm{C})[\mathrm{C} @ @ \mathrm{H}] 5 \mathrm{C} 3) \mathrm{cc} 1) \mathrm{cccc} 2) \mathrm{Cc} 6 \mathrm{c}(\mathrm{C}(\mathrm{F})(\mathrm{F}) \mathrm{F}) \mathrm{cc}(\mathrm{C}(\mathrm{F})(\mathrm{F}) \mathrm{F}) \mathrm{cc} 6\end{array}$ \\
\hline Rnd2_36 & 1.35 & 3.87 & 58.90 & $\begin{array}{l}{[n+] 1(\mathrm{c} 2 \mathrm{c}(\mathrm{c}([\mathrm{C} @ @ H](\mathrm{O})[\mathrm{C} @ \mathrm{H}] 3[\mathrm{~N} @ @+](\mathrm{Cc} 4 \mathrm{cc}(\mathrm{C}(\mathrm{F})(\mathrm{F}) \mathrm{C}(\mathrm{F})(\mathrm{F}) \mathrm{F}) \mathrm{cc}(\mathrm{C}(\mathrm{F})(\mathrm{F}) \mathrm{C}(\mathrm{F})(\mathrm{F}) \mathrm{F}) \mathrm{c} 4)(\mathrm{CC} 5) \mathrm{C}[\mathrm{C}} \\
@ \mathrm{H}](\mathrm{C}=\mathrm{C})[\mathrm{C} @ @ \mathrm{H}] 5 \mathrm{C} 3) \mathrm{cc} 1) \mathrm{cccc} 2) \mathrm{Cc} 6 \mathrm{c}(\mathrm{C}(\mathrm{F})(\mathrm{F}) \mathrm{F}) \mathrm{cc}(\mathrm{C}(\mathrm{F})(\mathrm{F}) \mathrm{F}) \mathrm{cc} 6\end{array}$ \\
\hline Rnd2_37 & 1.79 & 6.02 & 71.50 & $\begin{array}{l}{[\mathrm{n}+] 1(\mathrm{c} 2 \mathrm{c}(\mathrm{c}([\mathrm{C} @ @ \mathrm{H}](\mathrm{O})[\mathrm{C} @ H] 3[\mathrm{~N} @ @+](\mathrm{Cc} 4 \mathrm{cc}(\mathrm{F}) \mathrm{cc}(\mathrm{C}(\mathrm{F})(\mathrm{F}) \mathrm{C}(\mathrm{F})(\mathrm{F}) \mathrm{F}) \mathrm{c} 4)(\mathrm{CC} 5) \mathrm{C}[\mathrm{C} @ \mathrm{H}](\mathrm{C}=\mathrm{C})[\mathrm{C} @} \\
@ \mathrm{H}] 5 \mathrm{C} 3) \mathrm{cc} 1) \mathrm{cccc} 2) \mathrm{Cccc}(\mathrm{C}(\mathrm{F})(\mathrm{F}) \mathrm{F}) \mathrm{cc}(\mathrm{C}(\mathrm{F})(\mathrm{F}) \mathrm{F}) \mathrm{cc} 6\end{array}$ \\
\hline Rnd2_38 & 1.52 & 4.57 & 64.10 & $\begin{array}{l}{[\mathrm{n}+] 1(\mathrm{c} 2 \mathrm{c}(\mathrm{c}([\mathrm{C} @ @ \mathrm{H}](\mathrm{O})[\mathrm{C} @ \mathrm{H}] 3[\mathrm{~N} @ @ @+](\mathrm{Cc} 4 \mathrm{cc}(\mathrm{C}(\mathrm{F})(\mathrm{F}) \mathrm{F}) \mathrm{cc}(\mathrm{C}(\mathrm{F})(\mathrm{F}) \mathrm{F}) \mathrm{c} 4)(\mathrm{CC} 5) \mathrm{C}[\mathrm{C} @ \mathrm{H}](\mathrm{C}=\mathrm{C})[\mathrm{C} @} \\
@ \mathrm{~B}] 5 \mathrm{C} 3) \mathrm{cc} 1) \mathrm{cccc} 2) \mathrm{Cc} 6 \mathrm{c}(\mathrm{C}(\mathrm{F})(\mathrm{F}) \mathrm{F}) \mathrm{cc}(\mathrm{C}(\mathrm{F})(\mathrm{F}) \mathrm{F}) \mathrm{cc6}\end{array}$ \\
\hline Rnd2_39 & 0.95 & 2.59 & 44.30 & $\begin{array}{l}{[\mathrm{n}+] 1(\mathrm{c} 2 \mathrm{c}(\mathrm{c}([\mathrm{C} @ @ H](\mathrm{O})[\mathrm{C} @ \mathrm{H}] 3[\mathrm{~N} @ @+](\mathrm{Cc} 4 \mathrm{c}(\mathrm{F}) \mathrm{c}(\mathrm{F}) \mathrm{c}(\mathrm{F}) \mathrm{c}(\mathrm{F}) \mathrm{c} 4)(\mathrm{CC} 5) \mathrm{C}[\mathrm{C} @ \mathrm{H}](\mathrm{C}=\mathrm{C})[\mathrm{C} @ @ \mathrm{H}] 5 \mathrm{C3}} \\
\operatorname{cc1}) \mathrm{cccc} 2) \mathrm{cc6c}(\mathrm{C}(\mathrm{F})(\mathrm{F}) \mathrm{F}) \mathrm{cc}(\mathrm{C}(\mathrm{F})(\mathrm{F}) \mathrm{F}) \mathrm{cc} 6\end{array}$ \\
\hline Rnd2_40 & 1.12 & 3.07 & 50.90 & $\begin{array}{l}{[\mathrm{n}+] 1(\mathrm{c} 2 \mathrm{c}(\mathrm{c}([\mathrm{C} @ @ \mathrm{H}](\mathrm{O})[\mathrm{C} @ \mathrm{H}] 3[\mathrm{~N} @ @+](\mathrm{Cc} 4 \mathrm{cc}(\mathrm{C}(=\mathrm{O}) \mathrm{c} 5 \mathrm{ccccc} 5) \mathrm{ccc} 4)(\mathrm{CC} 6) \mathrm{C}[\mathrm{C} @ \mathrm{H}](\mathrm{C}=\mathrm{C})[\mathrm{C} @ @} \\
\mathrm{H}] 6 \mathrm{C} 3) \mathrm{cc} 1) \mathrm{cccc}(\mathrm{ccc}) \mathrm{c}(\mathrm{C}(\mathrm{F})(\mathrm{F}) \mathrm{F}) \mathrm{cc}(\mathrm{C}(\mathrm{F})(\mathrm{F}) \mathrm{F}) \mathrm{cc} 7\end{array}$ \\
\hline Rnd2 41 & 1.49 & 4.42 & 63.10 & $\begin{array}{l}{[n+] 1(\mathrm{c} 2 \mathrm{c}(\mathrm{c}([\mathrm{C} @ @ \mathrm{H}](\mathrm{O})[\mathrm{C} @ \mathrm{H}] 3[\mathrm{~N} @ @+](\mathrm{Cc} 4 \mathrm{c}(\mathrm{C}(\mathrm{F}) \mathrm{F}) \mathrm{cccc} 4)(\mathrm{CC} 5) \mathrm{C}[\mathrm{C} @ \mathrm{H}](\mathrm{C}=\mathrm{C})[\mathrm{C} @ @ \mathrm{H}] 5 \mathrm{C} 3) \mathrm{cc} 1} \\
\operatorname{cccc} 2) \mathrm{Cc} 6 \mathrm{cc}(\mathrm{Br}) \mathrm{cc}(\mathrm{C}(\mathrm{F})(\mathrm{F}) \mathrm{F}) \mathrm{c6}\end{array}$ \\
\hline
\end{tabular}




\begin{tabular}{|c|c|c|c|c|}
\hline Rnd2_42 & 1.40 & 4.04 & 60.30 & $\begin{array}{l}{[n+] 1(\mathrm{c} 2 \mathrm{c}(\mathrm{c}([\mathrm{C} @ @ \mathrm{H}](\mathrm{O})[\mathrm{C} @ \mathrm{H}] 3[\mathrm{~N} @ @+](\mathrm{Cc} 4 \mathrm{c}(\mathrm{C}(\mathrm{F})(\mathrm{F}) \mathrm{F}) \mathrm{ccc}(\mathrm{C}(\mathrm{F})(\mathrm{F}) \mathrm{F}) \mathrm{c} 4)(\mathrm{CC} 5) \mathrm{C}[\mathrm{C} @ \mathrm{H}](\mathrm{C}=\mathrm{C})[\mathrm{C} @} \\
@ \mathrm{H}] 5 \mathrm{C} 3) \mathrm{cc} 1) \mathrm{cccc} 2) \mathrm{Cc} 6 \mathrm{cc}(\mathrm{Br}) \mathrm{cc}(\mathrm{C}(\mathrm{F})(\mathrm{F}) \mathrm{F}) \mathrm{c6}\end{array}$ \\
\hline Rnd2_43 & 1.63 & 5.12 & 67.30 & $\begin{array}{l}{[\mathrm{n}+] 1(\mathrm{c} 2 \mathrm{c}(\mathrm{c}([\mathrm{C} @ @ \mathrm{H}](\mathrm{O})[\mathrm{C} @ \mathrm{H}] 3[\mathrm{~N} @ @+](\mathrm{Cc} 4 \mathrm{cc}(\mathrm{C}(\mathrm{F})(\mathrm{F}) \mathrm{F}) \mathrm{cc}(\mathrm{C}(\mathrm{F})(\mathrm{F}) \mathrm{C}(\mathrm{F})(\mathrm{F}) \mathrm{F}) \mathrm{c} 4)(\mathrm{CC}) \mathrm{C}[\mathrm{C} @ \mathrm{H}](\mathrm{C}=} \\
\mathrm{C})[\mathrm{C} @ @] 5 \mathrm{C} 3) \mathrm{cc} 1) \mathrm{cccc} 2) \mathrm{Cc} 6 \mathrm{cc}(\mathrm{Br}) \mathrm{cc}(\mathrm{C}(\mathrm{F})(\mathrm{F}) \mathrm{F}) \mathrm{c6}\end{array}$ \\
\hline Rnd2_44 & 1.43 & 4.18 & 61.40 & $\begin{array}{l}{[n+] 1(\mathrm{c} 2 \mathrm{c}(\mathrm{c}([\mathrm{C} @ @ \mathrm{H}](\mathrm{O})[\mathrm{C} @ \mathrm{H}] 3[\mathrm{~N} @ @+](\mathrm{Cc} 4 \mathrm{cc}(\mathrm{C}(\mathrm{F})(\mathrm{F}) \mathrm{C}(\mathrm{F})(\mathrm{F}) \mathrm{F}) \mathrm{cc}(\mathrm{C}(\mathrm{F})(\mathrm{F}) \mathrm{C}(\mathrm{F})(\mathrm{F}) \mathrm{F}) \mathrm{c4})(\mathrm{CC} 5) \mathrm{C}[\mathrm{C}} \\
@ \mathrm{H}](\mathrm{C}=\mathrm{C})[\mathrm{C} @ @ \mathrm{H}] 5 \mathrm{C} 3) \mathrm{cc} 1) \mathrm{cccc}) \mathrm{Cc} 6 \mathrm{cc}(\mathrm{Br}) \mathrm{cc}(\mathrm{C}(\mathrm{F})(\mathrm{F}) \mathrm{F}) \mathrm{c6}\end{array}$ \\
\hline Rnd2_45 & 2.10 & 8.13 & 78.10 & $\begin{array}{l}{[n+] 1(\mathrm{c} 2 \mathrm{c}(\mathrm{c}([\mathrm{C} @ @ \mathrm{H}](\mathrm{O})[\mathrm{C} @ \mathrm{H}] 3[\mathrm{~N} @ @+](\mathrm{Cc} 4 \mathrm{cc}(\mathrm{F}) \mathrm{cc}(\mathrm{C}(\mathrm{F})(\mathrm{F}) \mathrm{C}(\mathrm{F})(\mathrm{F}) \mathrm{F}) \mathrm{c4})(\mathrm{CC} 5) \mathrm{C}[\mathrm{C} @ \mathrm{H}](\mathrm{C}=\mathrm{C})[\mathrm{C} @} \\
@ \mathrm{H}] 5 \mathrm{C} 3) \mathrm{cc} 1) \mathrm{cccc} 2) \mathrm{Cc} 6 \mathrm{cc}(\mathrm{Br}) \mathrm{cc}(\mathrm{C}(\mathrm{F})(\mathrm{F}) \mathrm{F}) \mathrm{c} 6\end{array}$ \\
\hline Rnd2_46 & 1.70 & 5.49 & 69.20 & $\begin{array}{l}{[n+] 1(c 2 c(c([C @ @ H](O)[C @ H] 3[N @ @+](C c 4 c c(C(F)(F) F) c c(C(F)(F) F) c 4)(C C 5) C[C @ H](C=C)[C @} \\
@ H] 5 C 3) c c 1) c c c c 2)(c c 6 c c(B r) c c(C(F)(F) F) c 6\end{array}$ \\
\hline Rnd2_47 & 1.64 & 5.13 & 67.40 & $\begin{array}{l}{[n+] 1(\mathrm{c} 2 \mathrm{c}(\mathrm{c}([\mathrm{C} @ @ \mathrm{H}](\mathrm{O})[\mathrm{C} @ \mathrm{H}] 3[\mathrm{~N} @ @+](\mathrm{Cc} 4 \mathrm{c}(\mathrm{F}) \mathrm{c}(\mathrm{F}) \mathrm{c}(\mathrm{F}) \mathrm{c}(\mathrm{F}) \mathrm{c4})(\mathrm{CC} 5) \mathrm{C}[\mathrm{C} @ \mathrm{H}](\mathrm{C}=\mathrm{C})[\mathrm{C} @ @ \mathrm{H}] 5 \mathrm{C} 3} \\
) \mathrm{cc1}) \mathrm{cccc}) \mathrm{cc6cc}(\mathrm{Br}) \mathrm{cc}(\mathrm{C}(\mathrm{F})(\mathrm{F}) \mathrm{F}) \mathrm{c} 6\end{array}$ \\
\hline Rnd2_48 & 1.27 & 3.58 & 56.30 & $\begin{array}{l}{[n+] 1(\mathrm{c} 2 \mathrm{c}(\mathrm{c}([\mathrm{C} @ @ \mathrm{H}](\mathrm{O})[\mathrm{C} @ \mathrm{H}] 3[\mathrm{~N} @ @+](\mathrm{Cc} 4 \mathrm{cc}(\mathrm{C}=\mathrm{O}) \mathrm{c} 5 \mathrm{ccccc}) \mathrm{ccc} 4)(\mathrm{CC} 6) \mathrm{C}[\mathrm{C} @ \mathrm{H}](\mathrm{C}=\mathrm{C})[\mathrm{C} @ @} \\
\mathrm{H}] 6 \mathrm{C} 3) \mathrm{cc} 1) \mathrm{cccc} 2) \mathrm{Cc} 7 \mathrm{cc}(\mathrm{Br}) \mathrm{cc}(\mathrm{C}(\mathrm{F})(\mathrm{F}) \mathrm{F}) \mathrm{c} 7\end{array}$ \\
\hline Rnd2_49 & 1.29 & 3.63 & 56.80 & $\begin{array}{l}{[\mathrm{n}+] 1(\mathrm{c} 2 \mathrm{c}(\mathrm{c}([\mathrm{C} @ @ \mathrm{H}](\mathrm{O})[\mathrm{C} @ \mathrm{H}] 3[\mathrm{~N} @ @+](\mathrm{Cc} 4 \mathrm{c}(\mathrm{C}(\mathrm{F}) \mathrm{F}) \mathrm{cccc} 4)(\mathrm{CC} 5) \mathrm{C}[\mathrm{C} @ \mathrm{H}](\mathrm{C}=\mathrm{C})[\mathrm{C} @ @ \mathrm{H}] 5 \mathrm{C} 3) \mathrm{cc1}} \\
) \mathrm{ccc} 2 \mathrm{C}) \mathrm{cc6c}(\mathrm{Br}) \mathrm{ccc}(\mathrm{C}(\mathrm{F})(\mathrm{F}) \mathrm{F}) \mathrm{c6}\end{array}$ \\
\hline Rnd2_50 & 1.35 & 3.87 & 58.90 & $\begin{array}{l}{[n+] 1(\mathrm{c} 2 \mathrm{c}(\mathrm{c}([\mathrm{C} @ @ \mathrm{H}](\mathrm{O})[\mathrm{C} @ \mathrm{H}] 3[\mathrm{~N} @ @+](\mathrm{Cc} 4 \mathrm{c}(\mathrm{C}(\mathrm{F})(\mathrm{F}) \mathrm{F}) \mathrm{ccc}(\mathrm{C}(\mathrm{F})(\mathrm{F}) \mathrm{F}) \mathrm{c} 4)(\mathrm{CC} 5) \mathrm{C}[\mathrm{C} @ \mathrm{H}](\mathrm{C}=\mathrm{C})[\mathrm{C} @} \\
@ \mathrm{H}] 5 \mathrm{C} 3) \mathrm{cc} 1) \mathrm{cccc}) \mathrm{Cc} 6 \mathrm{c}(\mathrm{Br}) \mathrm{ccc}(\mathrm{C}(\mathrm{F})(\mathrm{F}) \mathrm{F}) \mathrm{c6}\end{array}$ \\
\hline Rnd2_51 & 1.76 & 5.83 & 70.70 & $\begin{array}{l}{[n+] 1(\mathrm{c} 2 \mathrm{c}(\mathrm{c}([\mathrm{C} @ @ \mathrm{H}](\mathrm{O})[\mathrm{C} @ \mathrm{H}] 3[\mathrm{~N} @ @+](\mathrm{Cc} 4 \mathrm{cc}(\mathrm{C}(\mathrm{F})(\mathrm{F}) \mathrm{F}) \mathrm{cc}(\mathrm{C}(\mathrm{F})(\mathrm{F}) \mathrm{C}(\mathrm{F})(\mathrm{F}) \mathrm{F}) \mathrm{c} 4)(\mathrm{CC} 5) \mathrm{C}[\mathrm{C} @ \mathrm{H}](\mathrm{C}=} \\
\mathrm{C})[\mathrm{C} @ @ \mathrm{H}] 5 \mathrm{C} 3) \mathrm{cc} 1) \mathrm{cccc} 2) \mathrm{Cc} 6 \mathrm{c}(\mathrm{Br}) \mathrm{ccc}(\mathrm{C}(\mathrm{F})(\mathrm{F}) \mathrm{F}) \mathrm{c6}\end{array}$ \\
\hline Rnd2_52 & 1.75 & 5.73 & 70.30 & $\begin{array}{l}{[n+] 1(\mathrm{c} 2 \mathrm{c}(\mathrm{c}([\mathrm{C} @ @ \mathrm{H}](\mathrm{O})[\mathrm{C} @ \mathrm{H}] 3[\mathrm{~N} @ @ @+](\mathrm{Cc} 4 \mathrm{cc}(\mathrm{C}(\mathrm{F})(\mathrm{F}) \mathrm{C}(\mathrm{F})(\mathrm{F}) \mathrm{F}) \mathrm{cc}(\mathrm{C}(\mathrm{F})(\mathrm{F}) \mathrm{C}(\mathrm{F})(\mathrm{F}) \mathrm{F}) \mathrm{c} 4)(\mathrm{CC} 5) \mathrm{C}[\mathrm{C}} \\
@ \mathrm{H}](\mathrm{C}=\mathrm{C})[\mathrm{C} @ @ \mathrm{H}] 5 \mathrm{C} 3) \mathrm{cc1}) \mathrm{cccc}) \mathrm{Cc} 6 \mathrm{c}(\mathrm{Br}) \mathrm{ccc}(\mathrm{C}(\mathrm{F})(\mathrm{F}) \mathrm{F}) \mathrm{c} 6\end{array}$ \\
\hline Rnd2_53 & 1.96 & 7.10 & 75.30 & $\begin{array}{l}{[n+] 1(\mathrm{c} 2 \mathrm{c}(\mathrm{c}([\mathrm{C} @ @ \mathrm{H}](\mathrm{O})[\mathrm{C} @ \mathrm{H}] 3[\mathrm{~N} @ @+](\mathrm{Cc} 4 \mathrm{cc}(\mathrm{F}) \mathrm{cc}(\mathrm{C}(\mathrm{F})(\mathrm{F}) \mathrm{C}(\mathrm{F})(\mathrm{F}) \mathrm{F}) \mathrm{c4})(\mathrm{CC} 5) \mathrm{C}[\mathrm{C} @ \mathrm{H}](\mathrm{C}=\mathrm{C})[\mathrm{C} @} \\
@ \mathrm{H}] 5 \mathrm{C} 3) \mathrm{cc} 1) \mathrm{cccc}) \mathrm{Cc} 6 \mathrm{c}(\mathrm{Br}) \mathrm{ccc}(\mathrm{C}(\mathrm{F})(\mathrm{F}) \mathrm{F}) \mathrm{c6}\end{array}$ \\
\hline
\end{tabular}




\begin{tabular}{|c|c|c|c|c|}
\hline Rnd2_54 & 1.81 & 6.12 & 71.90 & $\begin{array}{l}{[n+] 1(c 2 c(c([C @ @ H](O)[C @ H] 3[N @ @+](C c 4 c c(C(F)(F) F) c c(C(F)(F) F) c 4)(C C 5) C[C @ H](C=C)[C @} \\
@ H] 5 C 3) c c 1) c c c c 2) C c 6 c(B r) c c c(C(F)(F) F) c 6\end{array}$ \\
\hline Rnd2_55 & 1.39 & 4.03 & 60.20 & $\begin{array}{l}{[n+] 1(\mathrm{c} 2 \mathrm{c}(\mathrm{c}([\mathrm{C} @ @ \mathrm{H}](\mathrm{O})[\mathrm{C} @ \mathrm{H}] 3[\mathrm{~N} @ @+](\mathrm{Cc} 4 \mathrm{c}(\mathrm{F}) \mathrm{c}(\mathrm{F}) \mathrm{c}(\mathrm{F}) \mathrm{c}(\mathrm{F}) \mathrm{c} 4)(\mathrm{CC} 5) \mathrm{C}[\mathrm{C} @ \mathrm{H}](\mathrm{C}=\mathrm{C})[\mathrm{C} @ @ \mathrm{H}] 5 \mathrm{C} 3} \\
) \mathrm{cc} 1) \mathrm{cccc} 2) \mathrm{cc6c}(\mathrm{Br}) \mathrm{ccc}(\mathrm{C}(\mathrm{F})(\mathrm{F}) \mathrm{F}) \mathrm{c6}\end{array}$ \\
\hline Rnd2_56 & 1.29 & 3.62 & 56.70 & $\begin{array}{l}{[n+] 1(c 2 c(c([C @ @ H](O)[C @ H] 3[N @ @+](C c 4 c c(C=O) c 5 c c c c c 5) c c c 4)(C C 6) C[C @ H](C=C)[C @ @ ~} \\
H] 6 C 3) c c 1) c c c c 2) C c 7 c(B r) c c c(C(F)(F) F) c 7\end{array}$ \\
\hline Rnd2_57 & 1.40 & 4.06 & 60.50 & $\begin{array}{l}{[n+] 1(\mathrm{c} 2 \mathrm{c}(\mathrm{c}([\mathrm{C} @ @ \mathrm{H}](\mathrm{O})[\mathrm{C} @ \mathrm{H}] 3[\mathrm{~N} @ @+](\mathrm{Cc} 4 \mathrm{c}(\mathrm{C}(\mathrm{F}) \mathrm{F}) \mathrm{cccc} 4)(\mathrm{CC} 5) \mathrm{C}[\mathrm{C} @ \mathrm{H}](\mathrm{C}=\mathrm{C})[\mathrm{C} @ @ \mathrm{H}] 5 \mathrm{C} 3) \mathrm{cc} 1} \\
) \mathrm{cccc} 2) \mathrm{Cc} 6 \mathrm{cc}(\mathrm{Cl}) \mathrm{c}(\mathrm{C}(\mathrm{F})(\mathrm{F}) \mathrm{F}) \mathrm{cc6}\end{array}$ \\
\hline Rnd2_58 & 1.21 & 3.35 & 54.00 & $\begin{array}{l}{[n+] 1(\mathrm{c} 2 \mathrm{c}(\mathrm{c}([\mathrm{C} @ @ \mathrm{H}](\mathrm{O})[\mathrm{C} @ \mathrm{H}] 3[\mathrm{~N} @ @+](\mathrm{Cc} 4 \mathrm{c}(\mathrm{C}(\mathrm{F})(\mathrm{F}) \mathrm{F}) \mathrm{ccc}(\mathrm{C}(\mathrm{F})(\mathrm{F}) \mathrm{F}) \mathrm{c} 4)(\mathrm{CC} 5) \mathrm{C}[\mathrm{C} @ \mathrm{H}](\mathrm{C}=\mathrm{C})[\mathrm{C} @} \\
@ \mathrm{H}] 5 \mathrm{C} 3) \mathrm{cc} 1) \mathrm{cccc} 2) \mathrm{Cc} 6 \mathrm{cc}(\mathrm{Cl}) \mathrm{c}(\mathrm{C}(\mathrm{F})(\mathrm{F}) \mathrm{F}) \mathrm{cc} 6\end{array}$ \\
\hline Rnd2_59 & 1.47 & 4.35 & 62.60 & $\begin{array}{l}{[\mathrm{n}+] 1(\mathrm{c} 2 \mathrm{c}(\mathrm{c}([\mathrm{C} @ @ \mathrm{H}](\mathrm{O})[\mathrm{C} @ \mathrm{H}] 3[\mathrm{~N} @ @+](\mathrm{Cc} 4 \mathrm{cc}(\mathrm{C}(\mathrm{F})(\mathrm{F}) \mathrm{F}) \mathrm{cc}(\mathrm{C}(\mathrm{F})(\mathrm{F}) \mathrm{C}(\mathrm{F})(\mathrm{F}) \mathrm{F}) \mathrm{c} 4)(\mathrm{CC}) \mathrm{C}[\mathrm{C} @ \mathrm{H}](\mathrm{C}=} \\
\mathrm{C})[\mathrm{C} @ @ \mathrm{H}] 5 \mathrm{C} 3) \mathrm{cc} 1) \mathrm{cccc} 2) \mathrm{Cc} 6 \mathrm{cc}(\mathrm{Cl}) \mathrm{c}(\mathrm{C}(\mathrm{F})(\mathrm{F}) \mathrm{F}) \mathrm{ccc} 6\end{array}$ \\
\hline Rnd2_60 & 1.46 & 4.29 & 62.20 & $\begin{array}{l}{[n+] 1(c 2 c(c([C @ @ H](O)[C @ H] 3[N @ @+](C c 4 c c(C(F)(F) C(F)(F) F) c c(C(F)(F) C(F)(F) F) c 4)(C C 5) C[C} \\
@ H](C=C)[C @ @ H] 5 C 3) c c 1) c c c c 2) C c 6 c c(C l) c(C(F)(F) F) c c 6\end{array}$ \\
\hline Rnd2_61 & 1.98 & 7.26 & 75.80 & $\begin{array}{l}{[n+] 1(\mathrm{c} 2 \mathrm{c}(\mathrm{c}([\mathrm{C} @ @ \mathrm{H}](\mathrm{O})[\mathrm{C} @ \mathrm{H}] 3[\mathrm{~N} @ @+](\mathrm{Cc} 4 \mathrm{cc}(\mathrm{F}) \mathrm{cc}(\mathrm{C}(\mathrm{F})(\mathrm{F}) \mathrm{C}(\mathrm{F})(\mathrm{F}) \mathrm{F}) \mathrm{c} 4)(\mathrm{CC} 5) \mathrm{C}[\mathrm{C} @ \mathrm{H}](\mathrm{C}=\mathrm{C})[\mathrm{C} @} \\
@ \mathrm{H}] 5 \mathrm{C} 3) \mathrm{cc} 1) \mathrm{cccc} 2) \mathrm{Cc} 6 \mathrm{cc}(\mathrm{Cl}) \mathrm{c}(\mathrm{C}(\mathrm{F})(\mathrm{F}) \mathrm{F}) \mathrm{cc} 6\end{array}$ \\
\hline Rnd2_62 & 1.52 & 4.56 & 64.00 & $\begin{array}{l}{[n+] 1(\mathrm{c} 2 \mathrm{c}(\mathrm{c}([\mathrm{C} @ @ \mathrm{H}](\mathrm{O})[\mathrm{C} @ \mathrm{H}] 3[\mathrm{~N} @ @++](\mathrm{Cc} 4 \mathrm{cc}(\mathrm{C}(\mathrm{F})(\mathrm{F}) \mathrm{F}) \mathrm{cc}(\mathrm{C}(\mathrm{F})(\mathrm{F}) \mathrm{F}) \mathrm{c4})(\mathrm{CC} 5) \mathrm{C}[\mathrm{C} @ \mathrm{H}](\mathrm{C}=\mathrm{C})[\mathrm{C} @} \\
@ \mathrm{H}] 5 \mathrm{C} 3) \mathrm{cc} 1) \mathrm{cccc}) \mathrm{Cc6}(\mathrm{cc}(\mathrm{Cl}) \mathrm{c}(\mathrm{C}(\mathrm{F})(\mathrm{F}) \mathrm{F}) \mathrm{cc6}\end{array}$ \\
\hline Rnd2_63 & 1.61 & 5.01 & 66.70 & $\begin{array}{l}{[n+] 1(\mathrm{c} 2 \mathrm{c}(\mathrm{c}([\mathrm{C} @ @ \mathrm{H}](\mathrm{O})[\mathrm{C} @ \mathrm{H}] 3[\mathrm{~N} @ @+](\mathrm{Cc} 4 \mathrm{c}(\mathrm{F}) \mathrm{c}(\mathrm{F}) \mathrm{c}(\mathrm{F}) \mathrm{c}(\mathrm{F}) \mathrm{c} 4)(\mathrm{CC} 5) \mathrm{C}[\mathrm{C} @ \mathrm{H}](\mathrm{C}=\mathrm{C})[\mathrm{C} @ @ \mathrm{H}] 5 \mathrm{C} 3} \\
) \mathrm{cc} 1) \mathrm{cccc} 2) \mathrm{cc6cc}(\mathrm{Cl}) \mathrm{c}(\mathrm{C}(\mathrm{F})(\mathrm{F}) \mathrm{F}) \mathrm{cc6}\end{array}$ \\
\hline Rnd2_64 & 1.07 & 2.91 & 48.90 & $\begin{array}{l}{[n+] 1(\mathrm{c} 2 \mathrm{c}(\mathrm{c}([\mathrm{C} @ @ \mathrm{H}](\mathrm{O})[\mathrm{C} @ \mathrm{H}] 3[\mathrm{~N} @ @+](\mathrm{Cc} 4 \mathrm{cc}(\mathrm{C}(=\mathrm{O}) \mathrm{c5} 5 \mathrm{ccccc} 5) \mathrm{ccc} 4)(\mathrm{CC} 6) \mathrm{C}[\mathrm{C} @ \mathrm{H}](\mathrm{C}=\mathrm{C})[\mathrm{C} @ @} \\
\mathrm{H}] 6 \mathrm{C} 3) \mathrm{cc} 1) \mathrm{cccc} 2) \mathrm{Cc} 7 \mathrm{cc}(\mathrm{Cl}) \mathrm{c}(\mathrm{C}(\mathrm{F})(\mathrm{F}) \mathrm{F}) \mathrm{cc} 7\end{array}$ \\
\hline Rnd2_65 & 1.52 & 4.56 & 64.00 & $\begin{array}{l}{[n+] 1(\mathrm{c} 2 \mathrm{c}(\mathrm{c}([\mathrm{C} @ @ \mathrm{H}](\mathrm{O})[\mathrm{C} @ \mathrm{H}] 3[\mathrm{~N} @ @+](\mathrm{Cc} 4 \mathrm{c}(\mathrm{C}(\mathrm{F}) \mathrm{F}) \mathrm{cccc} 4)(\mathrm{CC} 5) \mathrm{C}[\mathrm{C} @ \mathrm{H}](\mathrm{C}=\mathrm{C})[\mathrm{C} @ @ \mathrm{H}] 5 \mathrm{C} 3) \mathrm{cc} 1} \\
) \operatorname{cccc} 2) \mathrm{cc} 6 \mathrm{cc}(\mathrm{F}) \mathrm{cc}(\mathrm{C}(\mathrm{F})(\mathrm{F}) \mathrm{F}) \mathrm{c} 6\end{array}$ \\
\hline
\end{tabular}




\begin{tabular}{|c|c|c|c|c|}
\hline Rnd2_66 & 1.36 & 3.88 & 59.00 & $\begin{array}{l}{[n+] 1(c 2 c(c([C @ @ H](O)[C @ H] 3[N @ @+](C c 4 c(C(F)(F) F) c c c(C(F)(F) F) c 4)(C C 5) C[C @ H](C=C)[C @} \\
@ H] 5 C 3) c c 1) c c c c 2) C c 6 c c(F) c c(C(F)(F) F) c 6\end{array}$ \\
\hline Rnd2_67 & 1.50 & 4.49 & 63.60 & $\begin{array}{l}{[n+] 1(\mathrm{c} 2 \mathrm{c}(\mathrm{c}([\mathrm{C} @ @ \mathrm{H}](\mathrm{O})[\mathrm{C} @ \mathrm{H}] 3[\mathrm{~N} @ @ @+](\mathrm{Cc} 4 \mathrm{cc}(\mathrm{C}(\mathrm{F})(\mathrm{F}) \mathrm{F}) \mathrm{cc}(\mathrm{C}(\mathrm{F})(\mathrm{F}) \mathrm{C}(\mathrm{F})(\mathrm{F}) \mathrm{F}) \mathrm{c} 4)(\mathrm{CC} 5) \mathrm{C}[\mathrm{C} @ \mathrm{H}](\mathrm{C}=} \\
\mathrm{C})[\mathrm{C} @ @ \mathrm{H}] 5 \mathrm{C} 3) \mathrm{cc} 1) \mathrm{cccc} 2) \mathrm{Cc} 6 \mathrm{cc}(\mathrm{F}) \mathrm{cc}(\mathrm{C}(\mathrm{F})(\mathrm{F}) \mathrm{F}) \mathrm{c6}\end{array}$ \\
\hline Rnd2_68 & 1.59 & 4.90 & 66.10 & $\begin{array}{l}{[n+] 1(c 2 c(c([C @ @ H](O)[C @ H] 3[N @ @+](C c 4 c c(C(F)(F) C(F)(F) F) c c(C(F)(F) C(F)(F) F) c 4)(C C 5) C[C} \\
@ H](C=C)[C @ @ H] 5 C 3) c c 1) c c c c 2) c c 6 c c(F) c c(C(F)(F) F) c 6\end{array}$ \\
\hline Rnd2_69 & 1.77 & 5.90 & 71.00 & $\begin{array}{l}{[n+] 1(c 2 c(c([C @ @ H](O)[C @ H] 3[N @ @+](C c 4 c c(F) c c(C(F)(F) C(F)(F) F) c 4)(C C 5) C[C @ H](C=C)[C @} \\
@ H] 5 C 3) c c 1) c c c c 2) C c 6 c c(F) c c(C(F)(F) F) c 6\end{array}$ \\
\hline Rnd2_70 & 1.68 & 5.37 & 68.60 & $\begin{array}{l}{[n+] 1(c 2 c(c([C @ @ H](O)[C @ H] 3[N @ @+](C c 4 c c(C(F)(F) F) c c(C(F)(F) F) c 4)(C C 5) C[C @ H](C=C)[C @} \\
@ H] 5 C 3) c c 1) c c c c 2) C c 6 c c(F) c c(C(F)(F) F) c 6\end{array}$ \\
\hline Rnd2_71 & 1.47 & 4.36 & 62.70 & $\begin{array}{l}{[n+] 1(\mathrm{c} 2 \mathrm{c}(\mathrm{c}([\mathrm{C} @ @ \mathrm{H}](\mathrm{O})[\mathrm{C} @ \mathrm{H}] 3[\mathrm{~N} @ @+](\mathrm{Cc} 4 \mathrm{c}(\mathrm{F}) \mathrm{c}(\mathrm{F}) \mathrm{c}(\mathrm{F}) \mathrm{c}(\mathrm{F}) \mathrm{c4})(\mathrm{CC} 5) \mathrm{C}[\mathrm{C} @ \mathrm{H}](\mathrm{C}=\mathrm{C})[\mathrm{C} @ @ \mathrm{H}] 5 \mathrm{C} 3} \\
) \mathrm{cc} 1) \mathrm{cccc} 2) \mathrm{cc6cc}(\mathrm{F}) \mathrm{cc}(\mathrm{C}(\mathrm{F})(\mathrm{F}) \mathrm{F}) \mathrm{c6}\end{array}$ \\
\hline Rnd2__72 & 1.30 & 3.68 & 57.30 & $\begin{array}{l}{[n+] 1(c 2 c(c([C @ @ H](O)[C @ H] 3[N @ @+](C c 4 c c(C=O) c 5 c c c c c 5) c c c 4)(C C 6) C[C @ H](C=C)[C @ @ ~} \\
H] 6 C 3) c c 1) c c c c 2) C c 7 c c(F) c c(C(F)(F) F) c 7\end{array}$ \\
\hline Rnd2_73 & 0.60 & 1.82 & 29.00 & $\begin{array}{l}{[\mathrm{n}+] 1(\mathrm{c} 2 \mathrm{c}(\mathrm{c}([\mathrm{C} @ @ \mathrm{H}](\mathrm{O})[\mathrm{C} @ \mathrm{H}] 3[\mathrm{~N} @ @+](\mathrm{Cc} 4 \mathrm{c}(\mathrm{C}(\mathrm{F}) \mathrm{F}) \mathrm{cccc} 4)(\mathrm{CC} 5) \mathrm{C}[\mathrm{C} @ \mathrm{H}](\mathrm{C}=\mathrm{C})[\mathrm{C} @ @ \mathrm{H}] 5 \mathrm{C} 3) \mathrm{cc} 1} \\
) \mathrm{cccc} 2) \mathrm{cc} 6 \mathrm{cc}(\mathrm{Br}) \mathrm{c}(\mathrm{CHN}) \mathrm{cc} 6\end{array}$ \\
\hline Rnd2_74 & 0.47 & 1.61 & 23.30 & $\begin{array}{l}{[n+] 1(\mathrm{c} 2 \mathrm{c}(\mathrm{c}([\mathrm{C} @ @ \mathrm{H}](\mathrm{O})[\mathrm{C} @ \mathrm{H}] 3[\mathrm{~N} @ @+](\mathrm{Cc} 4 \mathrm{c}(\mathrm{C}(\mathrm{F})(\mathrm{F}) \mathrm{F}) \mathrm{ccc}(\mathrm{C}(\mathrm{F})(\mathrm{F}) \mathrm{F}) \mathrm{c4})(\mathrm{CC} 5) \mathrm{C}[\mathrm{C} @ \mathrm{H}](\mathrm{C}=\mathrm{C})[\mathrm{C} @} \\
@ \mathrm{H}] 5 \mathrm{C} 3) \mathrm{cc} 1) \mathrm{cccc}) \mathrm{Cc} 6 \mathrm{cc}(\mathrm{Br}) \mathrm{c}(\mathrm{CHN}) \mathrm{ccc}\end{array}$ \\
\hline Rnd2_75 & 1.45 & 4.26 & 62.00 & $\begin{array}{l}{[\mathrm{n}+] 1(\mathrm{c} 2 \mathrm{c}(\mathrm{c}([\mathrm{C} @ @ \mathrm{H}](\mathrm{O})[\mathrm{C} @ \mathrm{H}] 3[\mathrm{~N} @ @+](\mathrm{Cc} 4 \mathrm{cc}(\mathrm{C}(\mathrm{F})(\mathrm{F}) \mathrm{F}) \mathrm{cc}(\mathrm{C}(\mathrm{F})(\mathrm{F}) \mathrm{C}(\mathrm{F})(\mathrm{F}) \mathrm{F}) \mathrm{c} 4)(\mathrm{CC} 5) \mathrm{C}[\mathrm{C} @ \mathrm{H}](\mathrm{C}=} \\
\mathrm{C})[\mathrm{C} @ @ \mathrm{H}] 5 \mathrm{C} 3) \mathrm{cc} 1) \mathrm{cccc} 2) \mathrm{Cc} 6 \mathrm{cc}(\mathrm{Br}) \mathrm{c}(\mathrm{CHN}) \mathrm{cc6}\end{array}$ \\
\hline Rnd2_76 & 1.47 & 4.36 & 62.70 & $\begin{array}{l}{[n+] 1(c 2 c(c([C @ @ H](O)[C @ H] 3[N @ @+](C c 4 c c(C(F)(F) C(F)(F) F) c c(C(F)(F) C(F)(F) F) c 4)(C C 5) C[C} \\
@ H](C=C)[C @ @ H] 5 C 3) c c 1) c c c c 2) C c 6 c c(B r) c(C \# N) c c 6\end{array}$ \\
\hline Rnd2_77 & 1.20 & 3.32 & 53.70 & $\begin{array}{l}{[n+] 1(\mathrm{c} 2 \mathrm{c}(\mathrm{c}([\mathrm{C} @ @ \mathrm{H}](\mathrm{O})[\mathrm{C} @ \mathrm{H}] 3[\mathrm{~N} @ @+](\mathrm{Cc} 4 \mathrm{cc}(\mathrm{F}) \mathrm{cc}(\mathrm{C}(\mathrm{F})(\mathrm{F}) \mathrm{C}(\mathrm{F})(\mathrm{F}) \mathrm{F}) \mathrm{c4})(\mathrm{CC} 5) \mathrm{C}[\mathrm{C} @ \mathrm{H}](\mathrm{C}=\mathrm{C})[\mathrm{C} @} \\
@ \mathrm{H}] 5 \mathrm{C} 3) \mathrm{cc} 1) \mathrm{cccc}) \mathrm{Cc} 6 \mathrm{cc}(\mathrm{Br}) \mathrm{c}(\mathrm{CHN}) \mathrm{cc6}\end{array}$ \\
\hline
\end{tabular}




\begin{tabular}{|c|c|c|c|c|}
\hline Rnd2_78 & 1.30 & 3.65 & 57.00 & $\begin{array}{l}{[n+] 1(c 2 c(c([C @ @ H](O)[C @ H] 3[N @ @+](C c 4 c c(C(F)(F) F) c c(C(F)(F) F) c 4)(C C 5) C[C @ H](C=C)[C @} \\
@ H] 5 C 3) c c 1) c c c c 2) C c 6 c c(B r) c(C \# N) c c 6\end{array}$ \\
\hline Rnd2_79 & 0.93 & 2.53 & 43.40 & $\begin{array}{l}{[n+] 1(\mathrm{c} 2 \mathrm{c}(\mathrm{c}([\mathrm{C} @ @ \mathrm{H}](\mathrm{O})[\mathrm{C} @ \mathrm{H}] 3[\mathrm{~N} @ @+](\mathrm{Cc} 4 \mathrm{c}(\mathrm{F}) \mathrm{c}(\mathrm{F}) \mathrm{c}(\mathrm{F}) \mathrm{c}(\mathrm{F}) \mathrm{c} 4)(\mathrm{CC} 5) \mathrm{C}[\mathrm{C} @ \mathrm{H}](\mathrm{C}=\mathrm{C})[\mathrm{C} @ @ \mathrm{H}] 5 \mathrm{C} 3} \\
) \mathrm{cc} 1) \mathrm{cccc} 2) \mathrm{cc6cc}(\mathrm{Br}) \mathrm{c}(\mathrm{CHN}) \mathrm{cc6}\end{array}$ \\
\hline Rnd2_80 & 0.84 & 2.32 & 39.80 & $\begin{array}{l}{[n+] 1(\mathrm{c} 2 \mathrm{c}(\mathrm{c}([\mathrm{C} @ @ \mathrm{H}](\mathrm{O})[\mathrm{C} @ \mathrm{H}] 3[\mathrm{~N} @ @+](\mathrm{Cc} 4 \mathrm{cc}(\mathrm{C}(=\mathrm{O}) \mathrm{c5} \mathrm{ccccc5}) \mathrm{ccc} 4)(\mathrm{CC} 6) \mathrm{C}[\mathrm{C} @ \mathrm{H}](\mathrm{C}=\mathrm{C})[\mathrm{C} @ @} \\
\mathrm{H}] 6 \mathrm{C} 3) \mathrm{cc} 1) \mathrm{cccc} 2) \mathrm{Cc} 7 \mathrm{cc}(\mathrm{Br}) \mathrm{c}(\mathrm{CHN}) \mathrm{cc} 7\end{array}$ \\
\hline Rnd3_2 & 2.12 & 8.30 & 78.50 & $\begin{array}{l}{[n+] 1(c 2 c(c([C @ @ H](O)[C @ H] 3[N @ @+](C c 4 c c(F) c c(C(F)(F) F) c 4)(C C 5) C[C @ H](C=C)[C @ @ H] 5 C} \\
3) c c 1) c c c c 2)(c c 6 c c(C(F)(F) F) c c(C(F)(F) F) c 6\end{array}$ \\
\hline Rnd3_7 & 1.91 & 6.78 & 74.30 & $\begin{array}{l}{[\mathrm{n}+] 1(\mathrm{c} 2 \mathrm{c}(\mathrm{c}([\mathrm{C} @ @ \mathrm{H}](\mathrm{O})[\mathrm{C} @ \mathrm{H}] 3[\mathrm{~N} @ @+](\mathrm{Cc} 4 \mathrm{c}(\mathrm{Cl}) \mathrm{cc}(\mathrm{Br}) \mathrm{cc} 4)(\mathrm{CC} 5) \mathrm{C}[\mathrm{C} @ \mathrm{H}](\mathrm{C}=\mathrm{C})[\mathrm{C} @ @ \mathrm{H}] 5 \mathrm{C} 3) \mathrm{cc}} \\
\text { 1)cccc2)Cc6cc(C(F)(F)F)cc(C(F)(F)F)c6 }\end{array}$ \\
\hline Rnd3_10 & 1.96 & 7.13 & 75.40 & $\begin{array}{l}{[\mathrm{n}+] 1(\mathrm{c} 2 \mathrm{c}(\mathrm{c}([\mathrm{C} @ @ \mathrm{H}](\mathrm{O})[\mathrm{C} @ \mathrm{H}] 3[\mathrm{~N} @ @+](\mathrm{Cc} 4 \mathrm{cc}(\mathrm{F}) \mathrm{cc}(\mathrm{C}(\mathrm{F})(\mathrm{F}) \mathrm{F}) \mathrm{c4})(\mathrm{CC} 5) \mathrm{C}[\mathrm{C} @ \mathrm{H}](\mathrm{C}=\mathrm{C})[\mathrm{C} @ @ \mathrm{H}] 5 \mathrm{C}} \\
3) \mathrm{cc} 1) \mathrm{cccc} 2) \mathrm{Cc} 6 \mathrm{cc}(\mathrm{Cl}) \mathrm{cc}(\mathrm{C}(\mathrm{F})(\mathrm{F}) \mathrm{F}) \mathrm{c6}\end{array}$ \\
\hline Rnd3_15 & 1.73 & 5.64 & 69.90 & 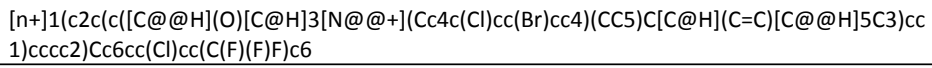 \\
\hline Rnd3_17 & 1.56 & 4.76 & 65.30 & $\begin{array}{l}{[\mathrm{n}+] 1(\mathrm{c} 2 \mathrm{c}(\mathrm{c}([\mathrm{C} @ @ \mathrm{H}](\mathrm{O})[\mathrm{C} @ \mathrm{H}] 3[\mathrm{~N} @ @+](\mathrm{Cc} 4 \mathrm{c}(\mathrm{C}(\mathrm{F}) \mathrm{F}) \mathrm{cccc} 4)(\mathrm{CC} 5) \mathrm{C}[\mathrm{C} @ \mathrm{H}](\mathrm{C}=\mathrm{C})[\mathrm{C} @ @ \mathrm{H}] 5 \mathrm{C} 3) \mathrm{cc} 1} \\
\operatorname{lcccc} 2) \mathrm{Cc} 6 \mathrm{cc}(\mathrm{C}(\mathrm{F})(\mathrm{F}) \mathrm{F}) \mathrm{c}(\mathrm{F}) \mathrm{ccc}\end{array}$ \\
\hline Rnd3_18 & 2.18 & 8.85 & 79.70 & $\begin{array}{l}{[n+] 1(\mathrm{c} 2 \mathrm{c}(\mathrm{c}([\mathrm{C} @ @ \mathrm{H}](\mathrm{O})[\mathrm{C} @ \mathrm{H}] 3[\mathrm{~N} @ @+](\mathrm{Cc} 4 \mathrm{cc}(\mathrm{F}) \mathrm{cc}(\mathrm{C}(\mathrm{F})(\mathrm{F}) \mathrm{F}) \mathrm{c4})(\mathrm{CC}) \mathrm{C}[\mathrm{C} @ \mathrm{H}](\mathrm{C}=\mathrm{C})[\mathrm{C} @ @ \mathrm{H}] 5 \mathrm{C}} \\
3) \mathrm{cc} 1) \mathrm{cccc} 2) \mathrm{Cc} 6 \mathrm{cc}(\mathrm{C}(\mathrm{F})(\mathrm{F}) \mathrm{F}) \mathrm{c}(\mathrm{F}) \mathrm{cc} 6\end{array}$ \\
\hline Rnd3_19 & 1.79 & 6.02 & 71.50 & $\begin{array}{l}{[\mathrm{n}+] 1(\mathrm{c} 2 \mathrm{c}(\mathrm{c}([\mathrm{C} @ @ \mathrm{H}](\mathrm{O})[\mathrm{C} @ \mathrm{H}] 3[\mathrm{~N} @ @ @+](\mathrm{Cc} 4 \mathrm{cc}(\mathrm{C}(\mathrm{F})(\mathrm{F}) \mathrm{F}) \mathrm{cc}(\mathrm{C}(\mathrm{F})(\mathrm{F}) \mathrm{C}(\mathrm{F})(\mathrm{F}) \mathrm{F}) \mathrm{c} 4)(\mathrm{CC} 5) \mathrm{C}[\mathrm{C} @ \mathrm{H}](\mathrm{C}=} \\
\mathrm{C})[\mathrm{C} @ @] 5 \mathrm{C} 3) \mathrm{cc} 1) \mathrm{cccc} 2) \mathrm{cc6cc}(\mathrm{C}(\mathrm{F})(\mathrm{F}) \mathrm{F}) \mathrm{c}(\mathrm{F}) \mathrm{cc} 6\end{array}$ \\
\hline Rnd3_20 & 1.71 & 5.51 & 69.30 & $\begin{array}{l}{[n+] 1(c 2 c(c([C @ @ H](O)[C @ H] 3[N @ @+](C c 4 c c(C(F)(F) C(F)(F) F) c c(C(F)(F) C(F)(F) F) c 4)(C C 5) C[C} \\
@ H](C=C)[C @ @ H] 5 C 3) c c 1) c c c c 2) C c 6 c c(C(F)(F) F) c(F) c c 6\end{array}$ \\
\hline Rnd3_21 & 2.29 & 9.87 & 81.60 & $\begin{array}{l}{[n+] 1(\mathrm{c} 2 \mathrm{c}(\mathrm{c}([\mathrm{C} @ @ \mathrm{H}](\mathrm{O})[\mathrm{C} @ \mathrm{H}] 3[\mathrm{~N} @ @+](\mathrm{Cc} 4 \mathrm{cc}(\mathrm{F}) \mathrm{cc}(\mathrm{C}(\mathrm{F})(\mathrm{F}) \mathrm{C}(\mathrm{F})(\mathrm{F}) \mathrm{F}) \mathrm{c4})(\mathrm{CC} 5) \mathrm{C}[\mathrm{C} @ \mathrm{H}](\mathrm{C}=\mathrm{C})[\mathrm{C} @} \\
@ \mathrm{H}] 5 \mathrm{C} 3) \mathrm{cc} 1) \mathrm{cccc}) \mathrm{Cc} 6 \mathrm{cc}(\mathrm{C}(\mathrm{F})(\mathrm{F}) \mathrm{F}) \mathrm{c}(\mathrm{F}) \mathrm{cc} 6\end{array}$ \\
\hline
\end{tabular}




\begin{tabular}{|c|c|c|c|c|}
\hline Rnd3_22 & 1.87 & 6.46 & 73.20 & $\begin{array}{l}{[n+] 1(c 2 c(c)([C @ @ H](O)[C @ H] 3[N @ @+](C c 4 c c(C(F)(F) F) c c(C(F)(F) F) c 4)(C C 5) C[C @ H](C=C)[C @} \\
@ H] 5 C 3) c c 1) c c c c 2) C c 6 c c(C(F)(F) F) c(F) c c 6\end{array}$ \\
\hline Rnd3_23 & 1.74 & 5.69 & 70.10 & $\begin{array}{l}{[n+] 1(\mathrm{c} 2 \mathrm{c}(\mathrm{c}([\mathrm{C} @ @ \mathrm{H}](\mathrm{O})[\mathrm{C} @ \mathrm{H}] 3[\mathrm{~N} @ @+](\mathrm{Cc} 4 \mathrm{c}(\mathrm{Cl}) \mathrm{cc}(\mathrm{Br}) \mathrm{cc} 4)(\mathrm{CC} 5) \mathrm{C}[\mathrm{C} @ \mathrm{H}](\mathrm{C}=\mathrm{C})[\mathrm{C} @ @ \mathrm{H}] 5 \mathrm{C} 3) \mathrm{cc}} \\
\text { 1)cccc2)Cc6cc(C(F)(F)F)c(F)cc6 }\end{array}$ \\
\hline Rnd3_24 & 1.03 & 2.80 & 47.30 & $\begin{array}{l}{[n+] 1(c 2 c(c([C @ @ H](O)[C @ H] 3[N @ @+](C c 4 c c(C(=)) c 5 c c c c c 5) c c c 4)(C C 6) C[C @ H](C=C)[C @ @ ~} \\
H] 6 C 3) c c 1) c c c c 2) \operatorname{cc} 7 c c(C(F)(F) F) c(F) c c 7\end{array}$ \\
\hline Rnd3_26 & 2.03 & 7.62 & 76.80 & $\begin{array}{l}{[n+] 1(c 2 c(c([C @ @ H](O)[C @ H] 3[N @ @+](C c 4 c c(F) c c(C(F)(F) F) c 4)(C C 5) C[C @ H](C=C)[C @ @ H] 5 C} \\
\text { 3)cc1)cccc2)Cc6cc(Br)cc(C(F)(F)F)c6 }\end{array}$ \\
\hline Rnd3_31 & 1.74 & 5.71 & 70.20 & 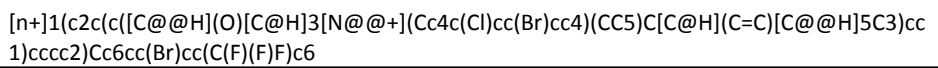 \\
\hline Rnd3_34 & 1.85 & 6.35 & 72.80 & $\begin{array}{l}{[n+] 1(c 2 c(c([C @ @ H](O)[C @ H] 3[N @ @+](C c 4 c c(F) c c(C(F)(F) F) c 4)(C C 5) C[C @ H](C=C)[C @ @ H] 5 C} \\
3) \operatorname{cc} 1) \operatorname{cccc} 2) \operatorname{cc} 6 c(B r) c c c(C(F)(F) F) c 6\end{array}$ \\
\hline Rnd3_39 & 1.50 & 4.49 & 63.60 & 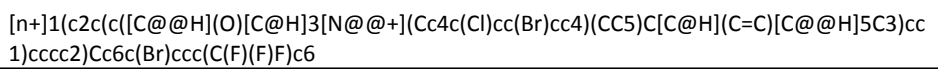 \\
\hline Rnd3_42 & 1.79 & 5.99 & 71.40 & $\begin{array}{l}{[n+] 1(c 2 c(c([C @ @ H](O)[C @ H] 3[N @ @+](C c 4 c c(F) c c(C(F)(F) F) c 4)(C C 5) C[C @ H](C=C)[C @ @ H] 5 C} \\
3) c c 1) c c c c 2) C c 6 c c(C l) c(C(F)(F) F) c c 6\end{array}$ \\
\hline Rnd3_47 & 1.60 & 4.97 & 66.50 & $\begin{array}{l}{[n+] 1(\mathrm{c} 2 \mathrm{c}(\mathrm{c}([\mathrm{C} @ @ \mathrm{H}](\mathrm{O})[\mathrm{C} @ \mathrm{H}] 3[\mathrm{~N} @ @+](\mathrm{Cc} 4 \mathrm{c}(\mathrm{Cl}) \mathrm{cc}(\mathrm{Br}) \mathrm{cc} 4)(\mathrm{CC} 5) \mathrm{C}[\mathrm{C} @ \mathrm{H}](\mathrm{C}=\mathrm{C})[\mathrm{C} @ @ \mathrm{H}] 5 \mathrm{C} 3) \mathrm{cc}} \\
1) \mathrm{cccc} 2) \mathrm{Ccc} 6 \mathrm{cc}(\mathrm{Cl}) \mathrm{c}(\mathrm{C}(\mathrm{F})(\mathrm{F}) \mathrm{F}) \mathrm{cc} 6\end{array}$ \\
\hline Rnd3_50 & 1.87 & 6.49 & 73.30 & $\begin{array}{l}{[n+] 1(c 2 c(c([C @ @ H](O)[C @ H] 3[N @ @+](C c 4 c c(F) c c(C(F)(F) F) c 4)(C C 5) C[C @ H](C=C)[C @ @ H] 5 C} \\
3) c c 1) c c c c 2) C c 6 c c(F) c c(C(F)(F) F) c 6\end{array}$ \\
\hline Rnd3_55 & 1.69 & 5.43 & 68.90 & $\begin{array}{l}{[\mathrm{n}+] 1(\mathrm{c} 2 \mathrm{c}(\mathrm{c}([\mathrm{C} @ @ \mathrm{H}](\mathrm{O})[\mathrm{C} @ \mathrm{H}] 3[\mathrm{~N} @ @+](\mathrm{Cc} 4 \mathrm{c}(\mathrm{Cl}) \mathrm{cc}(\mathrm{Br}) \mathrm{cc} 4)(\mathrm{CC} 5) \mathrm{C}[\mathrm{C} @ \mathrm{H}](\mathrm{C}=\mathrm{C})[\mathrm{C} @ @ \mathrm{H}] 5 \mathrm{C} 3) \mathrm{cc}} \\
\text { 1)cccc2)cc6cc(F)cc(C(F)(F)F)c6 }\end{array}$ \\
\hline Rnd3_57 & 0.88 & 2.41 & 41.30 & $\begin{array}{l}{[\mathrm{n}+] 1(\mathrm{c} 2 \mathrm{c}(\mathrm{c}([\mathrm{C} @ @ \mathrm{H}](\mathrm{O}) \mathrm{C} 3[\mathrm{~N}+](\mathrm{Cc} 4 \mathrm{c}(\mathrm{C}(\mathrm{F}) \mathrm{F}) \mathrm{cccc} 4)(\mathrm{CC} 5) \mathrm{C}[\mathrm{C} @ \mathrm{H}](\mathrm{C}=\mathrm{C}) \mathrm{C} 5 \mathrm{C} 3) \mathrm{cc} 1) \mathrm{cccc} 2) \mathrm{Cc} 6 \mathrm{c}(\mathrm{Br}) \mathrm{cc}} \\
\mathrm{c}(\mathrm{OC}) \mathrm{c} 6\end{array}$ \\
\hline
\end{tabular}




\begin{tabular}{|c|c|c|c|c|}
\hline Rnd3_58 & 1.39 & 4.03 & 60.20 & $\begin{array}{l}{[n+] 1(\mathrm{c} 2 \mathrm{c}(\mathrm{c}([\mathrm{C} @ @ \mathrm{H}](\mathrm{O}) \mathrm{C} 3[\mathrm{~N}+](\mathrm{Cc} 4 \mathrm{cc}(\mathrm{F}) \mathrm{cc}(\mathrm{C}(\mathrm{F})(\mathrm{F}) \mathrm{F}) \mathrm{c} 4)(\mathrm{CC} 5) \mathrm{C}[\mathrm{C} @ \mathrm{H}](\mathrm{C}=\mathrm{C}) \mathrm{C} 5 \mathrm{C} 3) \mathrm{cc} 1) \mathrm{cccc} 2) \mathrm{Cc} 6 \mathrm{c}} \\
(\mathrm{Br}) \mathrm{ccc}(\mathrm{OC}) \mathrm{c} 6\end{array}$ \\
\hline Rnd3_59 & 1.24 & 3.44 & 55.00 & 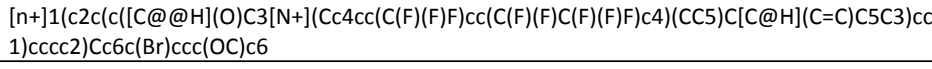 \\
\hline Rnd3_60 & 1.27 & 3.56 & 56.10 & $\begin{array}{l}{[n+] 1(\mathrm{c} 2 \mathrm{c}(\mathrm{c}([\mathrm{C} @ @ \mathrm{H}](\mathrm{O}) \mathrm{C} 3[\mathrm{~N}+](\mathrm{Cc} 4 \mathrm{cc}(\mathrm{C}(\mathrm{F})(\mathrm{F}) \mathrm{C}(\mathrm{F})(\mathrm{F}) \mathrm{F}) \mathrm{cc}(\mathrm{C}(\mathrm{F})(\mathrm{F}) \mathrm{C}(\mathrm{F})(\mathrm{F}) \mathrm{F}) \mathrm{c} 4)(\mathrm{CC} 5) \mathrm{C}[\mathrm{C} @ \mathrm{H}](\mathrm{C}=\mathrm{C}) \mathrm{C}} \\
5 \mathrm{C} 3) \mathrm{cc} 1) \mathrm{cccc} 2) \mathrm{Cc} 6 \mathrm{c}(\mathrm{Br}) \mathrm{ccc}(\mathrm{OC}) \mathrm{c} 6\end{array}$ \\
\hline Rnd3_61 & 1.57 & 4.80 & 65.50 & $\begin{array}{l}{[n+] 1(\mathrm{c} 2 \mathrm{c}(\mathrm{c}([\mathrm{C} @ @ \mathrm{H}](\mathrm{O}) \mathrm{C} 3[\mathrm{~N}+](\mathrm{Cc} 4 \mathrm{cc}(\mathrm{F}) \mathrm{cc}(\mathrm{C}(\mathrm{F})(\mathrm{F}) \mathrm{C}(\mathrm{F})(\mathrm{F}) \mathrm{F}) \mathrm{c4})(\mathrm{CC} 5) \mathrm{C}[\mathrm{C} @ \mathrm{H}](\mathrm{C}=\mathrm{C}) \mathrm{C5C} 3) \mathrm{cc} 1) \mathrm{cccc}} \\
2) \mathrm{Cc} 6 \mathrm{c}(\mathrm{Br}) \mathrm{ccc}(\mathrm{OC}) \mathrm{c6}\end{array}$ \\
\hline Rnd3_62 & 1.40 & 4.06 & 60.50 & $\begin{array}{l}{[\mathrm{n}+] 1(\mathrm{c} 2 \mathrm{c}(\mathrm{c}([\mathrm{C} @ @ \mathrm{H}](\mathrm{O}) \mathrm{C} 3[\mathrm{~N}+](\mathrm{Cc} 4 \mathrm{cc}(\mathrm{C}(\mathrm{F})(\mathrm{F}) \mathrm{F}) \mathrm{cc}(\mathrm{C}(\mathrm{F})(\mathrm{F}) \mathrm{F}) \mathrm{c} 4)(\mathrm{CC} 5) \mathrm{C}[\mathrm{C} @ \mathrm{H}](\mathrm{C}=\mathrm{C}) \mathrm{C5} 53) \mathrm{cc} 1) \mathrm{cccc}} \\
2) \mathrm{Cc} 6 \mathrm{c}(\mathrm{Br}) \mathrm{ccc}(\mathrm{OC}) \mathrm{c6}\end{array}$ \\
\hline Rnd3_63 & 0.99 & 2.69 & 45.80 & $\begin{array}{l}{[n+] 1(\mathrm{c} 2 \mathrm{c}(\mathrm{c}([\mathrm{C} @ @ \mathrm{H}](\mathrm{O}) \mathrm{C3}[\mathrm{N}+](\mathrm{Cc} 4 \mathrm{c}(\mathrm{Cl}) \mathrm{cc}(\mathrm{Br}) \mathrm{cc} 4)(\mathrm{CC} 5) \mathrm{C}[\mathrm{C} @ \mathrm{H}](\mathrm{C}=\mathrm{C}) \mathrm{C5} \mathrm{C} 3) \mathrm{cc} 1) \mathrm{cccc} 2) \mathrm{Cc} 6 \mathrm{c}(\mathrm{Br}) \mathrm{c}} \\
\mathrm{cc}(\mathrm{OC}) \mathrm{c6}\end{array}$ \\
\hline Rnd3_64 & 0.76 & 2.13 & 36.10 & $\begin{array}{l}{[\mathrm{n}+] 1(\mathrm{c} 2 \mathrm{c}(\mathrm{c}([\mathrm{C} @ @ \mathrm{H}](\mathrm{O}) \mathrm{C3}[\mathrm{N}+](\mathrm{Cc} 4 \mathrm{cc}(\mathrm{C}(=\mathrm{O}) \mathrm{c5} 5 \mathrm{ccccc5}) \mathrm{ccc} 4)(\mathrm{CC} 6) \mathrm{C}[\mathrm{C} @ \mathrm{H}](\mathrm{C}=\mathrm{C}) \mathrm{C} 6 \mathrm{C} 3) \mathrm{cc} 1) \mathrm{cccc} 2)} \\
\mathrm{Cc} 7 \mathrm{c}(\mathrm{Br}) \mathrm{ccc}(\mathrm{OC}) \mathrm{c} 7\end{array}$ \\
\hline Rnd3_65 & 1.41 & 4.08 & 60.60 & $\begin{array}{l}{[\mathrm{n}+] 1(\mathrm{c} 2 \mathrm{c}(\mathrm{c}([\mathrm{C} @ @ \mathrm{H}](\mathrm{O})[\mathrm{C} @ \mathrm{H}] 3[\mathrm{~N} @ @+](\mathrm{Cc} 4 \mathrm{c}(\mathrm{C}(\mathrm{F}) \mathrm{F}) \mathrm{cccc} 4)(\mathrm{CC} 5) \mathrm{C}[\mathrm{C} @ \mathrm{H}](\mathrm{C}=\mathrm{C})[\mathrm{C} @ @ \mathrm{H}] 5 \mathrm{C} 3) \mathrm{cc} 1} \\
) \mathrm{cccc} 2) \mathrm{Cc} 6 \mathrm{cc}(\mathrm{OC}(\mathrm{F})(\mathrm{F}) \mathrm{F}) \mathrm{ccc} 6\end{array}$ \\
\hline Rnd3_66 & 2.00 & 7.40 & 76.20 & $\begin{array}{l}{[n+] 1(\mathrm{c} 2 \mathrm{c}(\mathrm{c}([\mathrm{C} @ @ \mathrm{H}](\mathrm{O})[\mathrm{C} @ \mathrm{H}] 3[\mathrm{~N} @ @+](\mathrm{Cc} 4 \mathrm{cc}(\mathrm{F}) \mathrm{cc}(\mathrm{C}(\mathrm{F})(\mathrm{F}) \mathrm{F}) \mathrm{c4})(\mathrm{CC} 5) \mathrm{C}[\mathrm{C} @ \mathrm{H}](\mathrm{C}=\mathrm{C})[\mathrm{C} @ @ \mathrm{H}] 5 \mathrm{C}} \\
3) \mathrm{cc}) \mathrm{cccc} 2) \mathrm{cc6cc}(\mathrm{OC}(\mathrm{F})(\mathrm{F}) \mathrm{F}) \mathrm{ccc} 6\end{array}$ \\
\hline Rnd3_67 & 1.84 & 6.27 & 72.50 & $\begin{array}{l}{[\mathrm{n}+] 1(\mathrm{c} 2 \mathrm{c}(\mathrm{c}([\mathrm{C} @ @ \mathrm{H}](\mathrm{O})[\mathrm{C} @ \mathrm{H}] 3[\mathrm{~N} @ @+](\mathrm{Cc} 4 \mathrm{cc}(\mathrm{C}(\mathrm{F})(\mathrm{F}) \mathrm{F}) \mathrm{cc}(\mathrm{C}(\mathrm{F})(\mathrm{F}) \mathrm{C}(\mathrm{F})(\mathrm{F}) \mathrm{F}) \mathrm{c} 4)(\mathrm{CC} 5) \mathrm{C}[\mathrm{C} @ \mathrm{H}](\mathrm{C}=} \\
\mathrm{C})[\mathrm{C} @ @ \mathrm{H}] 5 \mathrm{C} 3) \mathrm{cc} 1) \mathrm{cccc} 2) \mathrm{Cc} 6 \mathrm{cc}(\mathrm{OC}(\mathrm{F})(\mathrm{F}) \mathrm{F}) \mathrm{ccc} 6\end{array}$ \\
\hline Rnd3_68 & 1.66 & 5.25 & 68.00 & $\begin{array}{l}{[n+] 1(c 2 c(c([C @ @ H](O)[C @ H] 3[N @ @+](C c 4 c c(C(F)(F) C(F)(F) F) c c(C(F)(F) C(F)(F) F) c 4)(C C 5) C[C} \\
@ H](C=C)[C @ @ H] 5 C 3) c c 1) c c c c 2) C c 6 c c(O C(F)(F) F) c c c 6\end{array}$ \\
\hline Rnd3_69 & 2.09 & 8.05 & 77.90 & $\begin{array}{l}{[n+] 1(\mathrm{c} 2 \mathrm{c}(\mathrm{c}([\mathrm{C} @ @ \mathrm{H}](\mathrm{O})[\mathrm{C} @ \mathrm{H}] 3[\mathrm{~N} @ @+](\mathrm{Cc} 4 \mathrm{cc}(\mathrm{F}) \mathrm{cc}(\mathrm{C}(\mathrm{F})(\mathrm{F}) \mathrm{C}(\mathrm{F})(\mathrm{F}) \mathrm{F}) \mathrm{c} 4)(\mathrm{CC} 5) \mathrm{C}[\mathrm{C} @ \mathrm{H}](\mathrm{C}=\mathrm{C})[\mathrm{C} @} \\
@ \mathrm{H}] 5 \mathrm{C} 3) \mathrm{cc} 1) \mathrm{cccc}) \mathrm{Cc} 6 \mathrm{cc}(\mathrm{OC}(\mathrm{F})(\mathrm{F}) \mathrm{F}) \mathrm{ccc} 6\end{array}$ \\
\hline
\end{tabular}




\begin{tabular}{|c|c|c|c|c|}
\hline Rnd3_70 & 1.89 & 6.60 & 73.70 & $\begin{array}{l}{[n+] 1(c 2 c(c([C @ @ H](O)[C @ H] 3[N @ @+](C c 4 c c(C(F)(F) F) c c(C(F)(F) F) c 4)(C C 5) C[C @ H](C=C)[C @} \\
@ H] 5 C 3) c c 1) c c c c 2) C c 6 c c(O C(F)(F) F) c c c 6\end{array}$ \\
\hline Rnd3_71 & 1.66 & 5.25 & 68.00 & 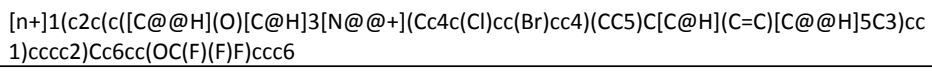 \\
\hline Rnd3_72 & 1.20 & 3.32 & 53.70 & $\begin{array}{l}{[\mathrm{n}+] 1(\mathrm{c} 2 \mathrm{c}(\mathrm{c}([\mathrm{C} @ @ \mathrm{H}](\mathrm{O})[\mathrm{C} @ \mathrm{H}] 3[\mathrm{~N} @ @+](\mathrm{Cc} 4 \mathrm{cc}(\mathrm{C}=\mathrm{O}=\mathrm{C} 5 \mathrm{cccccc} 5) \mathrm{ccc} 4)(\mathrm{CC} 6) \mathrm{C}[\mathrm{C} @ \mathrm{H}](\mathrm{C}=\mathrm{C})[\mathrm{C} @ @} \\
\mathrm{H}] 6 \mathrm{C} 3) \mathrm{cc} 1) \mathrm{cccc}) \mathrm{Cc} 7 \mathrm{cc}(\mathrm{OC}(\mathrm{F})(\mathrm{F}) \mathrm{F}) \mathrm{ccc} 7\end{array}$ \\
\hline Rnd3_74 & 2.09 & 8.09 & 78.00 & $\begin{array}{l}{[n+] 1(\mathrm{c} 2 \mathrm{c}(\mathrm{c}([\mathrm{C} @ @ \mathrm{H}](\mathrm{O})[\mathrm{C} @ \mathrm{H}] 3[\mathrm{~N} @ @+](\mathrm{Cc} 4 \mathrm{cc}(\mathrm{F}) \mathrm{cc}(\mathrm{C}(\mathrm{F})(\mathrm{F}) \mathrm{F}) \mathrm{c4})(\mathrm{CC}) \mathrm{C}[\mathrm{C} @ \mathrm{H}](\mathrm{C}=\mathrm{C})[\mathrm{C} @ @ \mathrm{H}] 5 \mathrm{C}} \\
3) \mathrm{cc}) \mathrm{cccc} 2) \mathrm{cc6cc}(\mathrm{C}(\mathrm{F})(\mathrm{F}) \mathrm{F}) \mathrm{ccc} 6\end{array}$ \\
\hline Rnd3_79 & 1.66 & 5.25 & 68.00 & $\begin{array}{l}{[\mathrm{n}+] 1(\mathrm{c} 2 \mathrm{c}(\mathrm{c}([\mathrm{C} @ @ \mathrm{H}](\mathrm{O})[\mathrm{C} @ \mathrm{H}] 3[\mathrm{~N} @ @+](\mathrm{Cc} 4 \mathrm{c}(\mathrm{Cl}) \mathrm{cc}(\mathrm{Br}) \mathrm{cc} 4)(\mathrm{CC} 5) \mathrm{C}[\mathrm{C} @ \mathrm{H}](\mathrm{C}=\mathrm{C})[\mathrm{C} @ @ \mathrm{H}] 5 \mathrm{C} 3) \mathrm{cc}} \\
\text { 1)cccc2)Cc6cc(C(F)(F)F)ccc6 }\end{array}$ \\
\hline Rnd3_81 & 1.54 & 4.68 & 64.80 & $\begin{array}{l}{[\mathrm{n}+] 1(\mathrm{c} 2 \mathrm{c}(\mathrm{c}([\mathrm{C} @ @ \mathrm{H}](\mathrm{O})[\mathrm{C} @ \mathrm{H}] 3[\mathrm{~N} @ @+](\mathrm{Cc} 4 \mathrm{c}(\mathrm{C}(\mathrm{F}) \mathrm{F}) \mathrm{cccc} 4)(\mathrm{CC} 5) \mathrm{C}[\mathrm{C} @ \mathrm{H}](\mathrm{C}=\mathrm{C})[\mathrm{C} @ @ \mathrm{H}] 5 \mathrm{C} 3) \mathrm{cc} 1} \\
) \mathrm{cccc} 2) \mathrm{Cc} 6 \mathrm{cc}(\mathrm{c}(\mathrm{Cl}) \mathrm{cc} 6) \mathrm{C}(\mathrm{F})(\mathrm{F}) \mathrm{F}\end{array}$ \\
\hline Rnd3_82 & 2.15 & 8.57 & 79.10 & $\begin{array}{l}{[n+] 1(\mathrm{c} 2 \mathrm{c}(\mathrm{c}([\mathrm{C} @ @ \mathrm{H}](\mathrm{O})[\mathrm{C} @ \mathrm{H}] 3[\mathrm{~N} @ @+](\mathrm{Cc} 4 \mathrm{cc}(\mathrm{F}) \mathrm{cc}(\mathrm{C}(\mathrm{F})(\mathrm{F}) \mathrm{F}) \mathrm{c} 4)(\mathrm{CC}) \mathrm{C}[\mathrm{C} @ \mathrm{H}](\mathrm{C}=\mathrm{C})[\mathrm{C} @ @ \mathrm{H}] 5 \mathrm{C}} \\
3) \mathrm{cc} 1) \mathrm{cccc} 2) \mathrm{Cc} 6 \mathrm{cc}(\mathrm{c}(\mathrm{Cl}) \mathrm{cc} 6) \mathrm{C}(\mathrm{F})(\mathrm{F}) \mathrm{F}\end{array}$ \\
\hline Rnd3_87 & 1.76 & 5.83 & 70.70 & $\begin{array}{l}{[\mathrm{n}+] 1(\mathrm{c} 2 \mathrm{c}(\mathrm{c}([\mathrm{C} @ @ \mathrm{H}](\mathrm{O})[\mathrm{C} @ \mathrm{H}] 3[\mathrm{~N} @ @+](\mathrm{Cc} 4 \mathrm{c}(\mathrm{Cl}) \mathrm{cc}(\mathrm{Br}) \mathrm{cc} 4)(\mathrm{CC} 5) \mathrm{C}[\mathrm{C} @ \mathrm{H}](\mathrm{C}=\mathrm{C})[\mathrm{C} @ @ \mathrm{H}] 5 \mathrm{C} 3) \mathrm{cc}} \\
\text { 1)cccc2)Cc6cc(c(Cl)cc6)C(F)(F)F }\end{array}$ \\
\hline Rnd4_1 & 1.61 & 5.01 & 66.75 & $\begin{array}{l}{[n+] 1(c 2 c(c([C @ @ H](O)[C @ H] 3[N @ @+](C c 4 c c(C(F)(F) C(F)(F) F) c c(C(F)(F) C(F)(F) F) c 4)(C C 5) C[C} \\
@ H](C=C)[C @ @ H] 5 C 3) c c 1) c c c c 2) C c 6 c(S C(F)(F) F) c c c c 6\end{array}$ \\
\hline Rnd4_2 & 1.80 & 6.03 & 71.54 & $\begin{array}{l}{[n+] 1(\mathrm{c} 2 \mathrm{c}(\mathrm{c}([\mathrm{C} @ @ \mathrm{H}](\mathrm{O})[\mathrm{C} @ \mathrm{H}] 3[\mathrm{~N} @ @+](\mathrm{Cc} 4 \mathrm{cc}(\mathrm{F}) \mathrm{cc}(\mathrm{C}(\mathrm{F})(\mathrm{F}) \mathrm{C}(\mathrm{F})(\mathrm{F}) \mathrm{F}) \mathrm{c} 4)(\mathrm{CC} 5) \mathrm{C}[\mathrm{C} @ \mathrm{H}](\mathrm{C}=\mathrm{C})[\mathrm{C} @} \\
@ \mathrm{H}] 5 \mathrm{C} 3) \mathrm{cc} 1) \mathrm{cccc}) \mathrm{cc6c}(\mathrm{SC}(\mathrm{F})(\mathrm{F}) \mathrm{F}) \mathrm{cccc6}\end{array}$ \\
\hline Rnd4_3 & 1.69 & 5.44 & 68.94 & $\begin{array}{l}{[\mathrm{n}+] 1(\mathrm{c} 2 \mathrm{c}(\mathrm{c}([\mathrm{C} @ @ \mathrm{H}](\mathrm{O})[\mathrm{C} @ \mathrm{H}] 3[\mathrm{~N} @ @+](\mathrm{Cc} 4 \mathrm{cc}(\mathrm{F}) \mathrm{cc}(\mathrm{C}(\mathrm{F})(\mathrm{F}) \mathrm{F}) \mathrm{c4})(\mathrm{CC}) \mathrm{C}[\mathrm{C} @ \mathrm{H}](\mathrm{C}=\mathrm{C})[\mathrm{C} @ @ \mathrm{H}] 5 \mathrm{C}} \\
3) \mathrm{cc} 1) \mathrm{cccc} 2) \mathrm{cc6c}(\mathrm{SC}(\mathrm{F})(\mathrm{F}) \mathrm{F}) \mathrm{cccc} 6\end{array}$ \\
\hline Rnd4_4 & 1.04 & 2.84 & 47.90 & $\begin{array}{l}{[n+] 1(\mathrm{c} 2 \mathrm{c}(\mathrm{c}([\mathrm{C} @ @ \mathrm{H}](\mathrm{O})[\mathrm{C} @ \mathrm{H}] 3[\mathrm{~N} @ @+](\mathrm{Cc} 4 \mathrm{c}(\mathrm{C}(\mathrm{F}) \mathrm{F}) \mathrm{ccc}(\mathrm{C}(\mathrm{F})(\mathrm{F}) \mathrm{F}) \mathrm{c} 4)(\mathrm{CC} 5) \mathrm{C}[\mathrm{C} @ \mathrm{H}](\mathrm{C}=\mathrm{C})[\mathrm{C} @ @} \\
\mathrm{H}] 5 \mathrm{C} 3) \mathrm{cc} 1) \mathrm{cccc} 2) \mathrm{Cccc}(\mathrm{SC}(\mathrm{F})(\mathrm{F}) \mathrm{F}) \mathrm{cccc} 6\end{array}$ \\
\hline
\end{tabular}




\begin{tabular}{|c|c|c|c|c|}
\hline Rnd4_5 & 1.28 & 3.61 & 56.66 & $\begin{array}{l}{[n+] 1(\mathrm{c} 2 \mathrm{c}(\mathrm{c}([\mathrm{C} @ @ \mathrm{H}](\mathrm{O})[\mathrm{C} @ \mathrm{H}] 3[\mathrm{~N} @ @+](\mathrm{Cc} 4 \mathrm{cc}(\mathrm{C}(\mathrm{F})(\mathrm{F}) \mathrm{F}) \mathrm{cc}(\mathrm{C}(\mathrm{F})(\mathrm{F}) \mathrm{C}(\mathrm{F})(\mathrm{F}) \mathrm{F}) \mathrm{C} 4)(\mathrm{CC} 5) \mathrm{C}[\mathrm{C} @ \mathrm{H}](\mathrm{C}=} \\
\mathrm{C})[\mathrm{C} @ @ \mathrm{H}] 5 \mathrm{C} 3) \mathrm{cc} 1) \mathrm{cccc} 2) \mathrm{Cc} 6 \mathrm{c}(\mathrm{SC}(\mathrm{F})(\mathrm{F}) \mathrm{F}) \mathrm{cccc} 6\end{array}$ \\
\hline Rnd4_6 & 1.67 & 5.30 & 68.26 & $\begin{array}{l}{[n+] 1(\mathrm{c} 2 \mathrm{c}(\mathrm{c}([\mathrm{C} @ @ \mathrm{H}](\mathrm{O})[\mathrm{C} @ \mathrm{H}] 3[\mathrm{~N} @ @+](\mathrm{Cc} 4 \mathrm{cc}(\mathrm{C}(\mathrm{F})(\mathrm{F}) \mathrm{F}) \mathrm{cc}(\mathrm{C}(\mathrm{F})(\mathrm{F}) \mathrm{F}) \mathrm{c} 4)(\mathrm{CC} 5) \mathrm{C}[\mathrm{C} @ \mathrm{H}](\mathrm{C}=\mathrm{C})[\mathrm{C} @} \\
@ \mathrm{H}] 5 \mathrm{C} 3) \mathrm{cc} 1) \mathrm{cccc}) \mathrm{Cc6c}(\mathrm{SC}(\mathrm{F})(\mathrm{F}) \mathrm{F}) \mathrm{cccc6}\end{array}$ \\
\hline Rnd4_7 & 1.85 & 6.33 & 72.71 & $\begin{array}{l}{[n+] 1(c 2 c(c([C @ @ H](O)[C @ H] 3[N @ @+](C c 4 c c(C(F)(F) C(F)(F) F) c c(C(F)(F) C(F)(F) F) c 4)(C C 5) C[C} \\
@ H](C=C)[C @ @ H] 5 C 3) c c 1) c c c c 2) C c 6 c c(O C(F)(F) F) c(C l) c c 6\end{array}$ \\
\hline Rnd4_8 & 2.30 & 9.99 & 81.80 & $\begin{array}{l}{[n+] 1(c 2 c(c([C @ @ H](O)[C @ H] 3[N @ @+](C c 4 c c(F) c c(C(F)(F) C(F)(F) F) c 4)(C C 5) C[C @ H](C=C)[C @} \\
@ H] 5 C 3) c c 1) c c c c 2) C c 6 c c(O C(F)(F) F) c(C l) c c 6\end{array}$ \\
\hline Rnd4_9 & 2.16 & 8.63 & 79.24 & 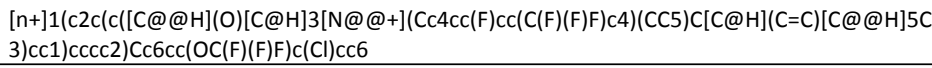 \\
\hline Rnd4_10 & 1.59 & 4.89 & 66.02 & $\begin{array}{l}{[n+] 1(\mathrm{c} 2 \mathrm{c}(\mathrm{c}([\mathrm{C} @ @ \mathrm{H}](\mathrm{O})[\mathrm{C} @ \mathrm{H}] 3[\mathrm{~N} @ @+](\mathrm{Cc} 4 \mathrm{c}(\mathrm{C}(\mathrm{F}) \mathrm{F}) \mathrm{ccc}(\mathrm{C}(\mathrm{F})(\mathrm{F}) \mathrm{F}) \mathrm{c} 4)(\mathrm{CC} 5) \mathrm{C}[\mathrm{C} @ \mathrm{H}](\mathrm{C}=\mathrm{C})[\mathrm{C} @ @} \\
\mathrm{H}] 5 \mathrm{C} 3) \mathrm{cc} 1) \mathrm{cccc} 2) \mathrm{Ccc6cc}(\mathrm{OC}(\mathrm{F})(\mathrm{F}) \mathrm{F}) \mathrm{c}(\mathrm{Cl}) \mathrm{ccc} 6\end{array}$ \\
\hline Rnd4_11 & 2.02 & 7.55 & 76.61 & $\begin{array}{l}{[n+] 1(\mathrm{c} 2 \mathrm{c}(\mathrm{c}([\mathrm{C} @ @ \mathrm{H}](\mathrm{O})[\mathrm{C} @ \mathrm{H}] 3[\mathrm{~N} @ @+](\mathrm{Cc} 4 \mathrm{cc}(\mathrm{C}(\mathrm{F})(\mathrm{F}) \mathrm{F}) \mathrm{cc}(\mathrm{C}(\mathrm{F})(\mathrm{F}) \mathrm{C}(\mathrm{F})(\mathrm{F}) \mathrm{F}) \mathrm{C} 4)(\mathrm{CC} 5) \mathrm{C}[\mathrm{C} @ \mathrm{H}](\mathrm{C}=} \\
\mathrm{C})[\mathrm{C} @ @ \mathrm{H}] 5 \mathrm{C} 3) \mathrm{cc} 1) \mathrm{cccc} 2) \mathrm{Cc} 6 \mathrm{cc}(\mathrm{OC}(\mathrm{F})(\mathrm{F}) \mathrm{F}) \mathrm{c}(\mathrm{Cl}) \mathrm{cc} 6\end{array}$ \\
\hline Rnd4_12 & 2.06 & 7.84 & 77.38 & $\begin{array}{l}{[n+] 1(\mathrm{c} 2 \mathrm{c}(\mathrm{c}([\mathrm{C} @ @ \mathrm{H}](\mathrm{O})[\mathrm{C} @ \mathrm{H}] 3[\mathrm{~N} @ @+](\mathrm{Cc} 4 \mathrm{cc}(\mathrm{C}(\mathrm{F})(\mathrm{F}) \mathrm{F}) \mathrm{cc}(\mathrm{C}(\mathrm{F})(\mathrm{F}) \mathrm{F}) \mathrm{c} 4)(\mathrm{CC} 5) \mathrm{C}[\mathrm{C} @ \mathrm{H}](\mathrm{C}=\mathrm{C})[\mathrm{C} @} \\
@ \mathrm{H}] 5 \mathrm{C} 3) \mathrm{cc} 1) \mathrm{cccc}) \mathrm{Cc} 6 \mathrm{cc}(\mathrm{OC}(\mathrm{F})(\mathrm{F}) \mathrm{F}) \mathrm{c}(\mathrm{Cl}) \mathrm{cc} 6\end{array}$ \\
\hline Rnd4_16 & 1.70 & 5.48 & 69.16 & $\begin{array}{l}{[\mathrm{n}+] 1(\mathrm{c} 2 \mathrm{c}(\mathrm{c}([\mathrm{C} @ @ H](\mathrm{O})[\mathrm{C} @ H] 3[\mathrm{~N} @ @+](\mathrm{Cc} 4 \mathrm{c}(\mathrm{C}(\mathrm{F}) \mathrm{F}) \mathrm{ccc}(\mathrm{C}(\mathrm{F})(\mathrm{F}) \mathrm{F}) \mathrm{c} 4)(\mathrm{CC} 5) \mathrm{C}[\mathrm{C} @ \mathrm{H}](\mathrm{C}=\mathrm{C})[\mathrm{C} @ @} \\
\mathrm{H}] 5 \mathrm{C} 3) \mathrm{cc} 1) \mathrm{cccc} 2) \mathrm{Cc} 6 \mathrm{cc}(\mathrm{C}(\mathrm{F})(\mathrm{F}) \mathrm{F}) \mathrm{c}(\mathrm{F}) \mathrm{cc6}\end{array}$ \\
\hline Rnd4_22 & 1.56 & 4.78 & 65.37 & $\begin{array}{l}{[n+] 1(c 2 c(c([C @ @ H](O)[C @ H] 3[N @ @+](C c 4 c(C(F) F) c c c(C(F)(F) F) c 4)(C C 5) C[C @ H](C=C)[C @ @ ~} \\
H] 5 C 3) c c 1) \operatorname{cccc} 2) \operatorname{Cc6cc}(C(F)(F) F) c c c 6\end{array}$ \\
\hline Rnd4_25 & 1.62 & 5.03 & 66.83 & $\begin{array}{l}{[n+] 1(c 2 c(c([C @ @ H](O)[C @ H] 3[N @ @+](C c 4 c c(C(F)(F) C(F)(F) F) c c(C(F)(F) C(F)(F) F) c 4)(C C 5) C[C} \\
@ H](C=C)[C @ @ H] 5 C 3) c c 1) c c c c 2) C c 6 c c(S C(F)(F) F) c(C l) c c 6\end{array}$ \\
\hline Rnd4_26 & 1.94 & 6.93 & 74.78 & $\begin{array}{l}{[n+] 1(\mathrm{c} 2 \mathrm{c}(\mathrm{c}([\mathrm{C} @ @ \mathrm{H}](\mathrm{O})[\mathrm{C} @ \mathrm{H}] 3[\mathrm{~N} @ @+](\mathrm{Cc} 4 \mathrm{cc}(\mathrm{F}) \mathrm{cc}(\mathrm{C}(\mathrm{F})(\mathrm{F}) \mathrm{C}(\mathrm{F})(\mathrm{F}) \mathrm{F}) \mathrm{c4})(\mathrm{CC} 5) \mathrm{C}[\mathrm{C} @ \mathrm{H}](\mathrm{C}=\mathrm{C})[\mathrm{C} @} \\
@ \mathrm{H}] 5 \mathrm{C} 3) \mathrm{cc} 1) \mathrm{cccc}) \mathrm{Cc} 6 \mathrm{cc}(\mathrm{SC}(\mathrm{F})(\mathrm{F}) \mathrm{F}) \mathrm{c}(\mathrm{Cl}) \mathrm{cc} 6\end{array}$ \\
\hline
\end{tabular}




\begin{tabular}{|c|c|c|c|c|}
\hline Rnd4__27 & 1.84 & 6.32 & 72.67 & $\begin{array}{l}{[n+] 1(\mathrm{c} 2 \mathrm{c}(\mathrm{c}([\mathrm{C} @ @ \mathrm{H}](\mathrm{O})[\mathrm{C} @ \mathrm{H}] 3[\mathrm{~N} @ @+](\mathrm{Cc} 4 \mathrm{cc}(\mathrm{F}) \mathrm{cc}(\mathrm{C}(\mathrm{F})(\mathrm{F}) \mathrm{F}) \mathrm{c4})(\mathrm{CC}) \mathrm{C}[\mathrm{C} @ \mathrm{H}](\mathrm{C}=\mathrm{C})[\mathrm{C} @ @ \mathrm{H}] 5 \mathrm{C}} \\
3) \mathrm{cc} 1) \mathrm{cccc} 2) \mathrm{Cc6cc}(\mathrm{SC}(\mathrm{F})(\mathrm{F}) \mathrm{F}) \mathrm{c}(\mathrm{Cl}) \mathrm{cc} 6\end{array}$ \\
\hline Rnd4_28 & 1.37 & 3.94 & 59.50 & $\begin{array}{l}{[n+] 1(\mathrm{c} 2 \mathrm{c}(\mathrm{c}([\mathrm{C} @ @ \mathrm{H}](\mathrm{O})[\mathrm{C} @ \mathrm{H}] 3[\mathrm{~N} @ @ @+](\mathrm{Cc} 4 \mathrm{c}(\mathrm{C}(\mathrm{F}) \mathrm{F}) \mathrm{ccc}(\mathrm{C}(\mathrm{F})(\mathrm{F}) \mathrm{F}) \mathrm{c4})(\mathrm{CC} 5) \mathrm{C}[\mathrm{C} @ \mathrm{H}](\mathrm{C}=\mathrm{C})[\mathrm{C} @ @} \\
\mathrm{H}] 5 \mathrm{C} 3) \mathrm{cc}) \mathrm{cccc}) \mathrm{Cc} 6 \mathrm{cc}(\mathrm{SC}(\mathrm{F})(\mathrm{F}) \mathrm{F}) \mathrm{c}(\mathrm{Cl}) \mathrm{cc6}\end{array}$ \\
\hline Rnd4_29 & 1.68 & 5.39 & 68.69 & $\begin{array}{l}{[\mathrm{n}+] 1(\mathrm{c} 2 \mathrm{c}(\mathrm{c}([\mathrm{C} @ @ \mathrm{H}](\mathrm{O})[\mathrm{C} @ \mathrm{H}] 3[\mathrm{~N} @ @ @+](\mathrm{Cc} 4 \mathrm{cc}(\mathrm{C}(\mathrm{F})(\mathrm{F}) \mathrm{F}) \mathrm{cc}(\mathrm{C}(\mathrm{F})(\mathrm{F}) \mathrm{C}(\mathrm{F})(\mathrm{F}) \mathrm{F}) \mathrm{c} 4)(\mathrm{CC}) \mathrm{C}[\mathrm{C} @ \mathrm{H}](\mathrm{C}=} \\
\mathrm{C})[\mathrm{C} @ @ \mathrm{H}] 5 \mathrm{C} 3) \mathrm{cc} 1) \mathrm{cccc} 2) \mathrm{Cc} 6 \mathrm{cc}(\mathrm{SC}(\mathrm{F})(\mathrm{F}) \mathrm{F}) \mathrm{c}(\mathrm{Cl}) \mathrm{cc} 6\end{array}$ \\
\hline Rnd4__30 & 1.74 & 5.69 & 70.09 & $\begin{array}{l}{[n+] 1(c 2 c(c([C @ @ H](O)[C @ H] 3[N @ @+](C c 4 c c(C(F)(F) F) c c(C(F)(F) F) c 4)(C C 5) C[C @ H](C=C)[C @} \\
@ H] 5 C 3) c c 1) c c c c 2) C c 6 c c(S C(F)(F) F) c(C l) c c 6\end{array}$ \\
\hline Rnd4_31 & 1.78 & 5.94 & 71.17 & $\begin{array}{l}{[n+] 1(c 2 c(c([C @ @ H](O)[C @ H] 3[N @ @+](C c 4 c c(C(F)(F) C(F)(F) F) c c(C(F)(F) C(F)(F) F) c 4)(C C 5) C[C} \\
@ H](C=C)[C @ @ H] 5 C 3) c c 1) c c c 2) C c 6 c(F) c(C(F)(F) F) c(F) c c 6\end{array}$ \\
\hline Rnd4_32 & 1.89 & 6.60 & 73.67 & $\begin{array}{l}{[n+] 1(c 2 c(c([C @ @ H](O)[C @ H] 3[N @ @+](C c 4 c c(F) c c(C(F)(F) C(F)(F) F) c 4)(C C 5) C[C @ H](C=C)[C @} \\
@ H] 5 C 3) c c 1) c c c c 2) C c 6 c(F) c(C(F)(F) F) c(F) c c 6\end{array}$ \\
\hline Rnd4_33 & 1.58 & 4.84 & 65.77 & $\begin{array}{l}{[n+] 1(c 2 c(c([C @ @ H](O)[C @ H] 3[N @ @+](C c 4 c c(F) c c(C(F)(F) F) c 4)(C C 5) C[C @ H](C=C)[C @ @ ~} \\
3) c c 1) c c c c 2) C c 6 c(F) c(C(F)(F) F) c(F) c c 6\end{array}$ \\
\hline Rnd4_34 & 1.51 & 4.52 & 63.78 & $\begin{array}{l}{[n+] 1(\mathrm{c} 2 \mathrm{c}(\mathrm{c}([\mathrm{C} @ @ \mathrm{H}](\mathrm{O})[\mathrm{C} @ \mathrm{H}] 3[\mathrm{~N} @ @+](\mathrm{Cc} 4 \mathrm{c}(\mathrm{C}(\mathrm{F}) \mathrm{F}) \mathrm{ccc}(\mathrm{C}(\mathrm{F})(\mathrm{F}) \mathrm{F}) \mathrm{c} 4)(\mathrm{CC} 5) \mathrm{C}[\mathrm{C} @ \mathrm{H}](\mathrm{C}=\mathrm{C})[\mathrm{C} @ @} \\
\mathrm{H}] 5 \mathrm{C} 3) \mathrm{cc} 1) \mathrm{cccc} 2) \mathrm{Cc} 6 \mathrm{c}(\mathrm{F}) \mathrm{c}(\mathrm{C}(\mathrm{F})(\mathrm{F}) \mathrm{F}) \mathrm{c}(\mathrm{F}) \mathrm{cc} 6\end{array}$ \\
\hline Rnd4_35 & 1.76 & 5.82 & 70.66 & $\begin{array}{l}{[\mathrm{n}+] 1(\mathrm{c} 2 \mathrm{c}(\mathrm{c}([\mathrm{C} @ @ H](\mathrm{O})[\mathrm{C} @ \mathrm{H}] 3[\mathrm{~N} @ @ @+](\mathrm{Cc} 4 \mathrm{cc}(\mathrm{C}(\mathrm{F})(\mathrm{F}) \mathrm{F}) \mathrm{cc}(\mathrm{C}(\mathrm{F})(\mathrm{F}) \mathrm{C}(\mathrm{F})(\mathrm{F}) \mathrm{F}) \mathrm{c4})(\mathrm{CC} 5) \mathrm{C}[\mathrm{C} @ \mathrm{H}](\mathrm{C}=} \\
\mathrm{C})[\mathrm{C} @ @ \mathrm{H}] 5 \mathrm{C} 3) \mathrm{cc} 1) \mathrm{cccc} 2) \mathrm{Cc} 6 \mathrm{c}(\mathrm{F}) \mathrm{c}(\mathrm{C}(\mathrm{F})(\mathrm{F}) \mathrm{F}) \mathrm{c}(\mathrm{F}) \mathrm{cc6}\end{array}$ \\
\hline Rnd4_36 & 1.64 & 5.16 & 67.53 & $\begin{array}{l}{[n+] 1(c 2 c(c([C @ @ H](O)[C @ H] 3[N @ @+](C c 4 c c(C(F)(F) F) c c(C(F)(F) F) c 4)(C C 5) C[C @ H](C=C)[C @} \\
@ H] 5 C 3) c c 1) c c c c 2) C c 6 c(F) c(C(F)(F) F) c(F) c c 6\end{array}$ \\
\hline Rnd4_37 & 1.75 & 5.77 & 70.44 & $\begin{array}{l}{[n+] 1(c 2 c(c([C @ @ H](O)[C @ H] 3[N @ @+](C c 4 c c(C(F)(F) C(F)(F) F) c c(C(F)(F) C(F)(F) F) c 4)(C C 5) C[C} \\
@ H](C=C)[C @ @ H] 5 C 3) c c 1) c c c c 2) C c 6 c c(C(F)(F) C(F)(F) F) c c c 6\end{array}$ \\
\hline Rnd4 38 & 2.33 & 10.28 & 82.26 & $\begin{array}{l}{[n+] 1(\mathrm{c} 2 \mathrm{c}(\mathrm{c}([\mathrm{C} @ @ \mathrm{H}](\mathrm{O})[\mathrm{C} @ \mathrm{H}] 3[\mathrm{~N} @ @+](\mathrm{Cc} 4 \mathrm{cc}(\mathrm{F}) \mathrm{cc}(\mathrm{C}(\mathrm{F})(\mathrm{F}) \mathrm{C}(\mathrm{F})(\mathrm{F}) \mathrm{F}) \mathrm{c} 4)(\mathrm{CC} 5) \mathrm{C}[\mathrm{C} @ \mathrm{H}](\mathrm{C}=\mathrm{C})[\mathrm{C} @} \\
@ \mathrm{H}] 5 \mathrm{C} 3) \mathrm{cc} 1) \mathrm{cccc} 2) \mathrm{Cc} 6 \mathrm{cc}(\mathrm{C}(\mathrm{F})(\mathrm{F}) \mathrm{C}(\mathrm{F})(\mathrm{F}) \mathrm{F}) \mathrm{ccc} 6\end{array}$ \\
\hline
\end{tabular}




\begin{tabular}{|c|c|c|c|c|}
\hline Rnd4_39 & 2.21 & 9.11 & 80.22 & $\begin{array}{l}{[n+] 1(c 2 c(c)([C @ @ H](O)[C @ H] 3[N @ @+](C c 4 c c(F) c c(C(F)(F) F) c 4)(C C 5) C[C @ H](C=C)[C @ @ H] 5 C} \\
3) c c 1) c c c c 2) C c 6 c c(C(F)(F) C(F)(F) F) c c c 6\end{array}$ \\
\hline Rnd4_40 & 1.56 & 4.76 & 65.29 & $\begin{array}{l}{[n+] 1(c 2 c(c([C @ @ H](O)[C @ H] 3[N @ @+](C c 4 c(C(F) F) c c c(C(F)(F) F) c 4)(C C 5) C[C @ H](C=C)[C @ @ ~} \\
H] 5 C 3) c c 1) \operatorname{cccc} 2) \operatorname{Cc} 6 c c(C(F)(F) C(F)(F) F) c c c 6\end{array}$ \\
\hline Rnd4_41 & 1.96 & 7.09 & 75.28 & $\begin{array}{l}{[n+] 1(c 2 c(c([C @ @ H](O)[C @ H] 3[N @ @+](C c 4 c c(C(F)(F) F) c c(C(F)(F) C(F)(F) F) c 4)(C C 5) C[C @ H](C=} \\
C)[C @ @ H] 5 C 3) c c 1) \operatorname{cccc} 2) C c 6 c c(C(F)(F) C(F)(F) F) c c c 6\end{array}$ \\
\hline Rnd4_42 & 2.05 & 7.78 & 77.23 & $\begin{array}{l}{[n+] 1(c 2 c(c([C @ @ H](O)[C @ H] 3[N @ @+](C c 4 c c(C(F)(F) F) c c(C(F)(F) F) c 4)(C C 5) C[C @ H](C=C)[C @} \\
@ H] 5 C 3) c c 1) c c c c 2) C c 6 c c(C(F)(F) C(F)(F) F) c c c 6\end{array}$ \\
\hline Rnd4_43 & 1.77 & 5.88 & 70.92 & $\begin{array}{l}{[n+] 1(c 2 c(c([C @ @ H](O)[C @ H] 3[N @ @+](C c 4 c c(C(F)(F) C(F)(F) F) c c(C(F)(F) C(F)(F) F) c 4)(C C 5) C[C} \\
@ H](C=C)[C @ @ H] 5 C 3) c c 1) c c c 2) C c 6 c c(O S(=O)(=O) C) c(C l) c c 6\end{array}$ \\
\hline Rnd4_44 & 1.82 & 6.19 & 72.20 & $\begin{array}{l}{[n+] 1(\mathrm{c} 2 \mathrm{c}(\mathrm{c}([\mathrm{C} @ @ \mathrm{H}](\mathrm{O})[\mathrm{C} @ \mathrm{H}] 3[\mathrm{~N} @ @+](\mathrm{Cc} 4 \mathrm{cc}(\mathrm{F}) \mathrm{cc}(\mathrm{C}(\mathrm{F})(\mathrm{F}) \mathrm{C}(\mathrm{F})(\mathrm{F}) \mathrm{F}) \mathrm{c4})(\mathrm{CC} 5) \mathrm{C}[\mathrm{C} @ \mathrm{H}](\mathrm{C}=\mathrm{C})[\mathrm{C} @} \\
@ \mathrm{H}] 5 \mathrm{C} 3) \mathrm{cc} 1) \mathrm{cccc}) \mathrm{Cc} 6 \mathrm{cc}(\mathrm{OS}(=\mathrm{O})(=\mathrm{O}) \mathrm{C}) \mathrm{c}(\mathrm{Cl}) \mathrm{cc} 6\end{array}$ \\
\hline Rnd4_45 & 1.14 & 3.13 & 51.60 & $\begin{array}{l}{[n+] 1(\mathrm{c} 2 \mathrm{c}(\mathrm{c}([\mathrm{C} @ @ \mathrm{H}](\mathrm{O})[\mathrm{C} @ \mathrm{H}] 3[\mathrm{~N} @ @+](\mathrm{Cc} 4 \mathrm{cc}(\mathrm{F}) \mathrm{cc}(\mathrm{C}(\mathrm{F})(\mathrm{F}) \mathrm{F}) \mathrm{c4})(\mathrm{CC} 5) \mathrm{C}[\mathrm{C} @ \mathrm{H}](\mathrm{C}=\mathrm{C})[\mathrm{C} @ @ \mathrm{H}] 5 \mathrm{C}} \\
3) \mathrm{cc} 1) \mathrm{cccc} 2) \mathrm{Cc} 6 \mathrm{cc}(\mathrm{OS}(=\mathrm{O})(=\mathrm{O}) \mathrm{C}) \mathrm{c}(\mathrm{Cl}) \mathrm{ccc}\end{array}$ \\
\hline Rnd4_46 & 1.15 & 3.16 & 51.89 & $\begin{array}{l}{[n+] 1(\mathrm{c} 2 \mathrm{c}(\mathrm{c}([\mathrm{C} @ @ \mathrm{H}](\mathrm{O})[\mathrm{C} @ \mathrm{H}] 3[\mathrm{~N} @ @+](\mathrm{Cc} 4 \mathrm{c}(\mathrm{C}(\mathrm{F}) \mathrm{F}) \mathrm{ccc}(\mathrm{C}(\mathrm{F})(\mathrm{F}) \mathrm{F}) \mathrm{c} 4)(\mathrm{CC} 5) \mathrm{C}[\mathrm{C} @ \mathrm{H}](\mathrm{C}=\mathrm{C})[\mathrm{C} @ @} \\
\mathrm{H}] 5 \mathrm{C} 3) \mathrm{cc} 1) \mathrm{cccc} 2) \mathrm{Cc} 6 \mathrm{cc}(\mathrm{OS}(=\mathrm{O})(=\mathrm{O}) \mathrm{C}) \mathrm{c}(\mathrm{Cl}) \mathrm{cc} 6\end{array}$ \\
\hline Rnd4__47 & 1.72 & 5.58 & 69.61 & $\begin{array}{l}{[\mathrm{n}+] 1(\mathrm{c} 2 \mathrm{c}(\mathrm{c}([\mathrm{C} @ @ \mathrm{H}](\mathrm{O})[\mathrm{C} @ \mathrm{H}] 3[\mathrm{~N} @ @+](\mathrm{Cc} 4 \mathrm{cc}(\mathrm{C}(\mathrm{F})(\mathrm{F}) \mathrm{F}) \mathrm{cc}(\mathrm{C}(\mathrm{F})(\mathrm{F}) \mathrm{C}(\mathrm{F})(\mathrm{F}) \mathrm{F}) \mathrm{c4})(\mathrm{CC} 5) \mathrm{C}[\mathrm{C} @ \mathrm{H}](\mathrm{C}=} \\
\mathrm{C})[\mathrm{C} @ @ \mathrm{H}] 5 \mathrm{C} 3) \mathrm{cc} 1) \mathrm{cccc} 2) \mathrm{Cc} 6 \mathrm{cc}(\mathrm{OS}(=\mathrm{O})(=\mathrm{O}) \mathrm{C}) \mathrm{c}(\mathrm{Cl}) \mathrm{cc6}\end{array}$ \\
\hline Rnd4__48 & 1.40 & 4.04 & 60.36 & $\begin{array}{l}{[n+] 1(c 2 c(c([C @ @ H](O)[C @ H] 3[N @ @+](C c 4 c c(C(F)(F) F) c c(C(F)(F) F) c 4)(C C 5) C[C @ H](C=C)[C @} \\
@ H] 5 C 3) c c 1) c c c c 2) C c 6 c c(O S(=O)(=O) C) c(C l) c c 6\end{array}$ \\
\hline Rnd4_49 & 1.60 & 4.97 & 66.48 & $\begin{array}{l}{[n+] 1(c 2 c(c([C @ @ H](O)[C @ H] 3[N @ @+](C c 4 c c(C(F)(F) C(F)(F) F) c c(C(F)(F) C(F)(F) F) c 4)(C C 5) C[C} \\
@ H](C=C)[C @ @ H] 5 C 3) c c 1) c c c c 2) C c 6 c c c(S(=O)(=O) N 7 C[C @ @ H](O) C C 7) c c 6\end{array}$ \\
\hline Rnd4_50 & 1.42 & 4.14 & 61.09 & $\begin{array}{l}{[\mathrm{n}+] 1(\mathrm{c} 2 \mathrm{c}(\mathrm{c}([\mathrm{C} @ @ \mathrm{H}](\mathrm{O})[\mathrm{C} @ \mathrm{H}] 3[\mathrm{~N} @ @+](\mathrm{Cc} 4 \mathrm{cc}(\mathrm{F}) \mathrm{cc}(\mathrm{C}(\mathrm{F})(\mathrm{F}) \mathrm{C}(\mathrm{F})(\mathrm{F}) \mathrm{F}) \mathrm{c4})(\mathrm{CC} 5) \mathrm{C}[\mathrm{C} @ \mathrm{H}](\mathrm{C}=\mathrm{C})[\mathrm{C} @} \\
@ \mathrm{H}] 5 \mathrm{C} 3) \mathrm{cc} 1) \mathrm{cccc}) \mathrm{Cc} 6 \mathrm{ccc}(\mathrm{S}=\mathrm{O})(=\mathrm{O}) \mathrm{N} 7 \mathrm{C}[\mathrm{C} @ @ \mathrm{H}](\mathrm{O}) \mathrm{cC} 7) \mathrm{cc} 6\end{array}$ \\
\hline
\end{tabular}




\begin{tabular}{|c|c|c|c|c|}
\hline Rnd4_51 & 1.22 & 3.40 & 54.55 & $\begin{array}{l}{[n+] 1(\mathrm{c} 2 \mathrm{c}(\mathrm{c}([\mathrm{C} @ @ \mathrm{H}](\mathrm{O})[\mathrm{C} @ \mathrm{H}] 3[\mathrm{~N} @ @+](\mathrm{Cc} 4 \mathrm{cc}(\mathrm{F}) \mathrm{cc}(\mathrm{C}(\mathrm{F})(\mathrm{F}) \mathrm{F}) \mathrm{c4})(\mathrm{CC}) \mathrm{C}[\mathrm{C} @ \mathrm{H}](\mathrm{C}=\mathrm{C})[\mathrm{C} @ @ \mathrm{H}] 5 \mathrm{C}} \\
3) \mathrm{cc} 1) \mathrm{cccc} 2) \mathrm{Cc} 6 \mathrm{ccc}(\mathrm{S}(=\mathrm{O})(=\mathrm{O}) \mathrm{N} 7 \mathrm{C}[\mathrm{C} @ @ \mathrm{H}](\mathrm{O}) \mathrm{CC} 7) \mathrm{cc} 6\end{array}$ \\
\hline Rnd4_52 & 1.28 & 3.58 & 56.34 & $\begin{array}{l}{[n+] 1(c 2 c(c([C @ @ H](O)[C @ H] 3[N @ @+](C c 4 c(C(F) F) c c c(C(F)(F) F) c 4)(C C 5) C[C @ H](C=C)[C @ @ ~} \\
H] 5 C 3) c c 1) c c c c 2) C c 6 c c c(S(=O)(=O) N 7 C[C @ @ H](O) C C 7) c c 6\end{array}$ \\
\hline Rnd4_53 & 1.72 & 5.60 & 69.69 & $\begin{array}{l}{[n+] 1(c 2 c(c([C @ @ H](O)[C @ H] 3[N @ @+](C c 4 c c(C(F)(F) F) c c(C(F)(F) C(F)(F) F) c 4)(C C 5) C[C @ H](C=} \\
C)[C @ @ H] 5 C 3) c c 1) c c c c 2) C c 6 c c c(S(=O)(=O) N 7 C[C @ @ H](O) C C 7) c c 6\end{array}$ \\
\hline Rnd4_54 & 1.74 & 5.70 & 70.13 & $\begin{array}{l}{[n+] 1(c 2 c(c([C @ @ H](O)[C @ H] 3[N @ @+](C c 4 c c(C(F)(F) F) c c(C(F)(F) F) c 4)(C C 5) C[C @ H](C=C)[C @} \\
@ H] 5 C 3) c c 1) c c c c 2) C c 6 c c c(S(=O)(=O) N 7 C[C @ @ H](O) C C 7) c c 6\end{array}$ \\
\hline Rnd4_55 & 1.09 & 2.97 & 49.65 & 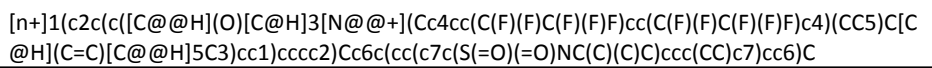 \\
\hline Rnd4_56 & 1.09 & 2.97 & 49.57 & $\begin{array}{l}{[n+] 1(\mathrm{c} 2 \mathrm{c}(\mathrm{c}([\mathrm{C} @ @ \mathrm{H}](\mathrm{O})[\mathrm{C} @ \mathrm{H}] 3[\mathrm{~N} @ @+](\mathrm{Cc} 4 \mathrm{cc}(\mathrm{F}) \mathrm{cc}(\mathrm{C}(\mathrm{F})(\mathrm{F}) \mathrm{C}(\mathrm{F})(\mathrm{F}) \mathrm{F}) \mathrm{c} 4)(\mathrm{CC} 5) \mathrm{C}[\mathrm{C} @ \mathrm{H}](\mathrm{C}=\mathrm{C})[\mathrm{C} @} \\
@ \mathrm{H}] 5 \mathrm{C} 3) \mathrm{cc} 1) \mathrm{cccc} 2) \mathrm{Cc} 6 \mathrm{c}(\mathrm{cc}(\mathrm{c} 7 \mathrm{c}(\mathrm{S}(=\mathrm{O})(=\mathrm{O}) \mathrm{NC}(\mathrm{C})(\mathrm{C}) \mathrm{C}) \mathrm{ccc}(\mathrm{CC}) \mathrm{c7}) \mathrm{cc} 6) \mathrm{C}\end{array}$ \\
\hline Rnd4_57 & 0.93 & 2.54 & 43.46 & 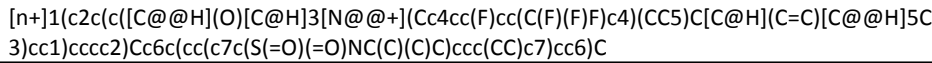 \\
\hline Rnd4_58 & 0.67 & 1.96 & 32.37 & $\begin{array}{l}{[\mathrm{n}+] 1(\mathrm{c} 2 \mathrm{c}(\mathrm{c}([\mathrm{C} @ @ \mathrm{H}](\mathrm{O})[\mathrm{C} @ \mathrm{H}] 3[\mathrm{~N} @ @+](\mathrm{Cc} 4 \mathrm{c}(\mathrm{C}(\mathrm{F}) \mathrm{F}) \mathrm{ccc}(\mathrm{C}(\mathrm{F})(\mathrm{F}) \mathrm{F}) \mathrm{c} 4)(\mathrm{CC} 5) \mathrm{C}[\mathrm{C} @ \mathrm{H}](\mathrm{C}=\mathrm{C})[\mathrm{C} @ @} \\
\mathrm{H}] 5 \mathrm{C} 3) \mathrm{cc} 1) \mathrm{cccc}(\mathrm{ccc}) \mathrm{Cc}(\mathrm{cc}(\mathrm{c} 7 \mathrm{c}(\mathrm{S}(=\mathrm{O})(=\mathrm{O}) \mathrm{NC}(\mathrm{C})(\mathrm{C}) \mathrm{C}) \mathrm{ccc}(\mathrm{CC}) \mathrm{c} 7) \mathrm{cc} 6) \mathrm{C}\end{array}$ \\
\hline Rnd4_59 & 1.26 & 3.52 & 55.76 & $\begin{array}{l}{[\mathrm{n}+] 1(\mathrm{c} 2 \mathrm{c}(\mathrm{c}([\mathrm{C} @ @ \mathrm{C}](\mathrm{O})[\mathrm{C} @ \mathrm{H}] 3[\mathrm{~N} @ @+](\mathrm{Cc} 4 \mathrm{cc}(\mathrm{C}(\mathrm{F})(\mathrm{F}) \mathrm{F}) \mathrm{cc}(\mathrm{C}(\mathrm{F})(\mathrm{F}) \mathrm{C}(\mathrm{F})(\mathrm{F}) \mathrm{F}) \mathrm{c} 4)(\mathrm{CC}) \mathrm{C}[\mathrm{C} @ \mathrm{H}](\mathrm{C}=} \\
\mathrm{C})[\mathrm{C} @ @] 5 \mathrm{C} 3) \mathrm{cc} 1) \mathrm{cccc} 2) \mathrm{Cc} 6 \mathrm{c}(\mathrm{cc}(\mathrm{c} 7 \mathrm{c}(\mathrm{S}(=\mathrm{O})(=\mathrm{O}) \mathrm{NC}(\mathrm{C})(\mathrm{C}) \mathrm{C}) \mathrm{ccc}(\mathrm{CC}) \mathrm{c} 7) \mathrm{ccc}) \mathrm{C}\end{array}$ \\
\hline Rnd4_60 & 1.09 & 2.99 & 49.83 & $\begin{array}{l}{[n+] 1(c 2 c(c([C @ @ H](O)[C @ H] 3[N @ @+](C c 4 c c(C(F)(F) F) c c(C(F)(F) F) c 4)(C C 5) C[C @ H](C=C)[C @} \\
@ H] 5 C 3) c c 1) c c c c 2) C c 6 c(c c(c 7 c(S(=O)(=O) N C(C)(C) C) c c c(C C) c 7) c c 6) C\end{array}$ \\
\hline Rnd4_61 & 0.99 & 2.69 & 45.86 & $\begin{array}{l}{[n+] 1(c 2 c(c([C @ @ H](O)[C @ H] 3[N @ @+](C c 4 c c(C(F)(F) C(F)(F) F) c c(C(F)(F) C(F)(F) F) c 4)(C C 5) C[C} \\
@ H](C=C)[C @ @ H] 5 C 3) c c 1) c c c c 2) C c 6 c c c(c 7 c(S(=O)(=O) N C(C)(C) C) c c c c 7) c c 6\end{array}$ \\
\hline Rnd4_62 & 1.06 & 2.88 & 48.45 & $\begin{array}{l}{[\mathrm{n}+] 1(\mathrm{c} 2 \mathrm{c}(\mathrm{c}([\mathrm{C} @ @ \mathrm{H}](\mathrm{O})[\mathrm{C} @ \mathrm{H}] 3[\mathrm{~N} @ @+](\mathrm{Cc} 4 \mathrm{cc}(\mathrm{F}) \mathrm{cc}(\mathrm{C}(\mathrm{F})(\mathrm{F}) \mathrm{C}(\mathrm{F})(\mathrm{F}) \mathrm{F}) \mathrm{c} 4)(\mathrm{CC} 5) \mathrm{C}[\mathrm{C} @ \mathrm{H}](\mathrm{C}=\mathrm{C})[\mathrm{C} @} \\
@ \mathrm{H}] 5 \mathrm{C} 3) \mathrm{cc} 1) \mathrm{cccc}) \mathrm{Cc} 6 \mathrm{ccc}(\mathrm{c} 7 \mathrm{c}(\mathrm{S}(=\mathrm{O})(=\mathrm{O}) \mathrm{NC}(\mathrm{C})(\mathrm{C}) \mathrm{C}) \mathrm{cccc} 7) \mathrm{cc6}\end{array}$ \\
\hline
\end{tabular}




\begin{tabular}{|c|c|c|c|c|}
\hline Rnd4_63 & 0.79 & 2.21 & 37.71 & $\begin{array}{l}{[\mathrm{n}+] 1(\mathrm{c} 2 \mathrm{c}(\mathrm{c}([\mathrm{C} @ @ \mathrm{H}](\mathrm{O})[\mathrm{C} @ \mathrm{H}] 3[\mathrm{~N} @ @+](\mathrm{Cc} 4 \mathrm{cc}(\mathrm{F}) \mathrm{cc}(\mathrm{C}(\mathrm{F})(\mathrm{F}) \mathrm{F}) \mathrm{c4})(\mathrm{CC}) \mathrm{C}[\mathrm{C} @ \mathrm{H}](\mathrm{C}=\mathrm{C})[\mathrm{C} @ @ \mathrm{H}] 5 \mathrm{C}} \\
3) \mathrm{cc} 1) \operatorname{cccc} 2) \mathrm{cc} 6 \mathrm{ccc}(\mathrm{c} 7 \mathrm{c}(\mathrm{S}(=\mathrm{O})(=\mathrm{O}) \mathrm{NC}(\mathrm{C})(\mathrm{C}) \mathrm{C}) \mathrm{cccc} 7) \mathrm{cc} 6\end{array}$ \\
\hline Rnd4_64 & 0.74 & 2.09 & 35.29 & $\begin{array}{l}{[n+] 1(\mathrm{c} 2 \mathrm{c}(\mathrm{c}([\mathrm{C} @ @ \mathrm{H}](\mathrm{O})[\mathrm{C} @ \mathrm{H}] 3[\mathrm{~N} @ @+](\mathrm{Cc} 4 \mathrm{c}(\mathrm{C}(\mathrm{F}) \mathrm{F}) \mathrm{ccc}(\mathrm{C}(\mathrm{F})(\mathrm{F}) \mathrm{F}) \mathrm{c} 4)(\mathrm{CC} 5) \mathrm{C}[\mathrm{C} @ \mathrm{H}](\mathrm{C}=\mathrm{C})[\mathrm{C} @ @} \\
\mathrm{H}] 5 \mathrm{C} 3) \mathrm{cc} 1) \mathrm{cccc} 2) \mathrm{Cc} 6 \mathrm{ccc}(\mathrm{c} 7 \mathrm{c}(\mathrm{S}(=\mathrm{O})(=\mathrm{O}) \mathrm{NC}(\mathrm{C})(\mathrm{C}) \mathrm{C}) \mathrm{cccc} 7) \mathrm{cc} 6\end{array}$ \\
\hline Rnd4_65 & 1.06 & 2.87 & 48.38 & $\begin{array}{l}{[\mathrm{n}+] 1(\mathrm{c} 2 \mathrm{c}(\mathrm{c}([\mathrm{C} @ @ \mathrm{H}](\mathrm{O})[\mathrm{C} @ \mathrm{H}] 3[\mathrm{~N} @ @+](\mathrm{Cc} 4 \mathrm{cc}(\mathrm{C}(\mathrm{F})(\mathrm{F}) \mathrm{F}) \mathrm{cc}(\mathrm{C}(\mathrm{F})(\mathrm{F}) \mathrm{C}(\mathrm{F})(\mathrm{F}) \mathrm{F}) \mathrm{c} 4)(\mathrm{CC}) \mathrm{C}[\mathrm{C} @ \mathrm{H}](\mathrm{C}=} \\
\mathrm{C})[\mathrm{C} @ @ \mathrm{H}] 5 \mathrm{C} 3) \mathrm{cc} 1) \mathrm{cccc} 2) \mathrm{Cc} 6 \mathrm{ccc}(\mathrm{c} 7 \mathrm{c}(\mathrm{S}(=\mathrm{O})(=\mathrm{O}) \mathrm{NC}(\mathrm{C})(\mathrm{C}) \mathrm{C}) \mathrm{cccc}) \mathrm{ccc}\end{array}$ \\
\hline Rnd4_66 & 1.01 & 2.75 & 46.63 & 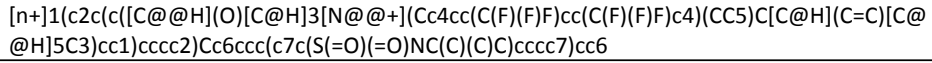 \\
\hline Rnd4_67 & 1.15 & 3.15 & 51.84 & $\begin{array}{l}{[n+] 1(\mathrm{c} 2 \mathrm{c}(\mathrm{c}([\mathrm{C} @ @ \mathrm{H}](\mathrm{O})[\mathrm{C} @ \mathrm{H}] 3[\mathrm{~N} @ @+](\mathrm{Cc} 4 \mathrm{cc}(\mathrm{C}(\mathrm{F})(\mathrm{F}) \mathrm{C}(\mathrm{F})(\mathrm{F}) \mathrm{F}) \mathrm{cc}(\mathrm{C}(\mathrm{F})(\mathrm{F}) \mathrm{C}(\mathrm{F})(\mathrm{F}) \mathrm{F}) \mathrm{c} 4)(\mathrm{CC} 5) \mathrm{C}[\mathrm{C}} \\
@ \mathrm{H}](\mathrm{C}=\mathrm{C})[\mathrm{C} @ \mathrm{H}] 5 \mathrm{C} 3) \mathrm{cc} 1) \mathrm{cccc} 2) \mathrm{cc} 6 \mathrm{ccc}(\mathrm{S}(=\mathrm{O})(=\mathrm{O}) \mathrm{N}(\mathrm{CCC}) \mathrm{ccC}) \mathrm{cc} 6\end{array}$ \\
\hline Rnd4_68 & 1.23 & 3.42 & 54.73 & $\begin{array}{l}{[n+] 1(\mathrm{c} 2 \mathrm{c}(\mathrm{c}([\mathrm{C} @ @ \mathrm{H}](\mathrm{O})[\mathrm{C} @ \mathrm{H}] 3[\mathrm{~N} @ @++](\mathrm{Cc} 4 \mathrm{cc}(\mathrm{F}) \mathrm{cc}(\mathrm{C}(\mathrm{F})(\mathrm{F}) \mathrm{C}(\mathrm{F})(\mathrm{F}) \mathrm{F}) \mathrm{c} 4)(\mathrm{CC} 5) \mathrm{C}[\mathrm{C} @ \mathrm{H}](\mathrm{C}=\mathrm{C})[\mathrm{C} @} \\
@ \mathrm{H}] 5 \mathrm{C} 3) \mathrm{cc} 1) \mathrm{cccc}) \mathrm{Cc} 6 \mathrm{ccc}(\mathrm{S}=\mathrm{O})(=\mathrm{O}) \mathrm{N}(\mathrm{CCC}) \mathrm{ccC}) \mathrm{cc6}\end{array}$ \\
\hline Rnd4_69 & 0.96 & 2.60 & 44.44 & $\begin{array}{l}{[\mathrm{n}+] 1(\mathrm{c} 2 \mathrm{c}(\mathrm{c}([\mathrm{C} @ @ \mathrm{H}](\mathrm{O})[\mathrm{C} @ \mathrm{H}] 3[\mathrm{~N} @ @+](\mathrm{Cc} 4 \mathrm{cc}(\mathrm{F}) \mathrm{cc}(\mathrm{C}(\mathrm{F})(\mathrm{F}) \mathrm{F}) \mathrm{c4})(\mathrm{CC}) \mathrm{C}[\mathrm{C} @ \mathrm{H}](\mathrm{C}=\mathrm{C})[\mathrm{C} @ @ \mathrm{H}] 5 \mathrm{C}} \\
3) \mathrm{cc} 1) \mathrm{cccc} 2) \mathrm{Cc} 6 \mathrm{ccc}(\mathrm{S}(=\mathrm{O})(=\mathrm{O}) \mathrm{N}(\mathrm{CCC}) \mathrm{CCC}) \mathrm{cc} 6\end{array}$ \\
\hline Rnd4_70 & 1.03 & 2.79 & 47.24 & $\begin{array}{l}{[\mathrm{n}+] 1(\mathrm{c} 2 \mathrm{c}(\mathrm{c}([\mathrm{C} @ @ \mathrm{H}](\mathrm{O})[\mathrm{C} @ \mathrm{H}] 3[\mathrm{~N} @ @+](\mathrm{Cc} 4 \mathrm{c}(\mathrm{C}(\mathrm{F}) \mathrm{F}) \mathrm{ccc}(\mathrm{C}(\mathrm{F})(\mathrm{F}) \mathrm{F}) \mathrm{c4})(\mathrm{CC} 5) \mathrm{C}[\mathrm{C} @ \mathrm{H}](\mathrm{C}=\mathrm{C})[\mathrm{C} @ @)} \\
\mathrm{H}] 5 \mathrm{C} 3) \mathrm{cc} 1) \mathrm{cccc} 2) \mathrm{Cc} 6 \mathrm{cccc}(\mathrm{S}(=\mathrm{O})(=) \mathrm{N}(\mathrm{CCC}) \mathrm{CCC}) \mathrm{cc} 6\end{array}$ \\
\hline Rnd4_71 & 1.27 & 3.56 & 56.14 & $\begin{array}{l}{[\mathrm{n}+] 1(\mathrm{c} 2 \mathrm{c}(\mathrm{c}([\mathrm{C} @ @ \mathrm{H}](\mathrm{O})[\mathrm{C} @ \mathrm{H}] 3[\mathrm{~N} @ @+](\mathrm{Cc} 4 \mathrm{cc}(\mathrm{C}(\mathrm{F})(\mathrm{F}) \mathrm{F}) \mathrm{cc}(\mathrm{C}(\mathrm{F})(\mathrm{F}) \mathrm{C}(\mathrm{F})(\mathrm{F}) \mathrm{F}) \mathrm{c} 4)(\mathrm{CC}) \mathrm{C}[\mathrm{C} @ \mathrm{H}](\mathrm{C}=} \\
\mathrm{C})[\mathrm{C} @ @] 5 \mathrm{C} 3) \mathrm{cc} 1) \mathrm{cccc} 2) \mathrm{Cc} 6 \mathrm{ccc}(\mathrm{S}(=\mathrm{O})(=\mathrm{O}) \mathrm{N}(\mathrm{CCC}) \mathrm{CCC}) \mathrm{ccc} 6\end{array}$ \\
\hline Rnd4_72 & 1.22 & 3.39 & 54.40 & $\begin{array}{l}{[\mathrm{n}+] 1(\mathrm{c} 2 \mathrm{c}(\mathrm{c}([\mathrm{C} @ @ \mathrm{H}](\mathrm{O})[\mathrm{C} @ \mathrm{H}] 3[\mathrm{~N} @ @ @+](\mathrm{Cc} 4 \mathrm{cc}(\mathrm{C}(\mathrm{F})(\mathrm{F}) \mathrm{F}) \mathrm{cc}(\mathrm{C}(\mathrm{F})(\mathrm{F}) \mathrm{F}) \mathrm{c4})(\mathrm{CC} 5) \mathrm{C}[\mathrm{C} @ \mathrm{H}](\mathrm{C}=\mathrm{C})[\mathrm{C} @} \\
@ \mathrm{H}] 5 \mathrm{C} 3) \mathrm{cc} 1) \mathrm{cccc} 2) \mathrm{Cc} 6 \mathrm{ccc}(\mathrm{S}=\mathrm{=})(=\mathrm{O}) \mathrm{N}(\mathrm{CCC}) \mathrm{CcC}) \mathrm{cc} 6\end{array}$ \\
\hline Rnd4_73 & 0.86 & 2.36 & 40.49 & $\begin{array}{l}{[n+] 1(c 2 c(c([C @ @ H](O)[C @ H] 3[N @ @+](C c 4 c c(C(F)(F) C(F)(F) F) c c(C(F)(F) C(F)(F) F) c 4)(C C 5) C[C} \\
@ H](C=C)[C @ @ H] 5 C 3) c c 1) c c c 2) \operatorname{cc} 6 c(F) c c(c 7 c(S(=O)(=O) N C(C)(C) C) c c c c 7) c c 6\end{array}$ \\
\hline Rnd4_74 & 0.85 & 2.35 & 40.29 & $\begin{array}{l}{[n+] 1(c 2 c(c([C @ @ H](O)[C @ H] 3[N @ @+](C c 4 c c(F) c c(C(F)(F) C(F)(F) F) c 4)(C C 5) C[C @ H](C=C)[C @} \\
@ H] 5 C 3) c c 1) c c c c 2)(c 6 c(F) c c(c 7 c(S(=O)(=O) N C(C)(C) C) c c c c 7) c c 6\end{array}$ \\
\hline
\end{tabular}




\begin{tabular}{|c|c|c|c|c|}
\hline Rnd4_75 & 0.74 & 2.09 & 35.25 & $\begin{array}{l}{[n+] 1(c 2 c(c([C @ @ H](O)[C @ H] 3[N @ @+](C c 4 c c(F) c c(C(F)(F) F) c 4)(C C 5) C[C @ H](C=C)[C @ @ H] 5 C} \\
\text { 3)cc1)cccc2)Cc6c(F)cc(c7c(S(=O)(=O)Nc(C)(C)C)cccc7)cc6 }\end{array}$ \\
\hline Rnd4_76 & 0.71 & 2.03 & 33.96 & $\begin{array}{l}{[n+] 1(\mathrm{c} 2 \mathrm{c}(\mathrm{c}([\mathrm{C} @ @ \mathrm{H}](\mathrm{O})[\mathrm{C} @ \mathrm{H}] 3[\mathrm{~N} @ @+](\mathrm{Cc} 4 \mathrm{c}(\mathrm{C}(\mathrm{F}) \mathrm{F}) \mathrm{ccc}(\mathrm{C}(\mathrm{F})(\mathrm{F}) \mathrm{F}) \mathrm{c4})(\mathrm{CC} 5) \mathrm{C}[\mathrm{C} @ \mathrm{H}](\mathrm{C}=\mathrm{C})[\mathrm{C} @ @} \\
\mathrm{H}] 5 \mathrm{C} 3) \mathrm{cc} 1) \mathrm{cccc} 2) \mathrm{Cc} 6 \mathrm{c}(\mathrm{F}) \mathrm{cc}(\mathrm{c} 7 \mathrm{c}(\mathrm{S}(=\mathrm{O})(=\mathrm{O}) \mathrm{NC}(\mathrm{C})(\mathrm{C}) \mathrm{C}) \mathrm{cccc} 7) \mathrm{ccc}\end{array}$ \\
\hline Rnd4_77 & 0.88 & 2.42 & 41.52 & $\begin{array}{l}{[\mathrm{n}+] 1(\mathrm{c} 2 \mathrm{c}(\mathrm{c}([\mathrm{C} @ @ \mathrm{H}](\mathrm{O})[\mathrm{C} @ \mathrm{H}] 3[\mathrm{~N} @ @ @+](\mathrm{Cc} 4 \mathrm{cc}(\mathrm{C}(\mathrm{F})(\mathrm{F}) \mathrm{F}) \mathrm{cc}(\mathrm{C}(\mathrm{F})(\mathrm{F}) \mathrm{C}(\mathrm{F})(\mathrm{F}) \mathrm{F}) \mathrm{c4})(\mathrm{CC}) \mathrm{C}[\mathrm{C} @ \mathrm{H}](\mathrm{C}=} \\
\mathrm{C})[\mathrm{C} @ @ \mathrm{H}] 5 \mathrm{C} 3) \mathrm{cc} 1) \mathrm{cccc} 2) \mathrm{Cc} 6 \mathrm{c}(\mathrm{F}) \mathrm{cc}(\mathrm{c} 7 \mathrm{c}(\mathrm{S}(=\mathrm{O})(=\mathrm{O}) \mathrm{NC}(\mathrm{C})(\mathrm{C}) \mathrm{C}) \mathrm{cccc} 7) \mathrm{ccc}\end{array}$ \\
\hline Rnd4_78 & 0.92 & 2.52 & 43.15 & $\begin{array}{l}{[n+] 1(c 2 c(c([C @ @ H](O)[C @ H] 3[N @ @+](C c 4 c c(C(F)(F) F) c c(C(F)(F) F) c 4)(C C 5) C[C @ H](C=C)[C @} \\
@ H] 5 C 3) c c 1) c c c c 2) C c 6 c(F) c c(c 7 c(S(=O)(=O) N C(C)(C) C) c c c c 7) c c 6\end{array}$ \\
\hline Rnd5_1 & 2.25 & 9.48 & 80.91 & $\begin{array}{l}{[\mathrm{C} @ @] 1([\mathrm{H}])(\mathrm{CC}(\mathrm{CC} 2)[\mathrm{C} @ @ \mathrm{H}](\mathrm{C}=\mathrm{C}) \mathrm{C}[\mathrm{N}+] 12 \mathrm{Cc} 3 \mathrm{cc}(\mathrm{C}(\mathrm{C}(\mathrm{C}(\mathrm{F})(\mathrm{F}) \mathrm{F})(\mathrm{F}) \mathrm{F})(\mathrm{F}) \mathrm{F}) \mathrm{cc}(\mathrm{F}) \mathrm{c} 3)[\mathrm{C} @ \mathrm{H}](\mathrm{O}) \mathrm{c} 4 \mathrm{cc}} \\
{[\mathrm{n}+](\mathrm{Cc} 5 \mathrm{cc}(\mathrm{C}(\mathrm{F})(\mathrm{C}(\mathrm{F})(\mathrm{F}) \mathrm{F}) \mathrm{F}) \mathrm{ccc} 5) \mathrm{c}(\mathrm{cccc} 6) \mathrm{c} 46}\end{array}$ \\
\hline Rnd5_2 & 2.23 & 9.31 & 80.60 & $\begin{array}{l}{[\mathrm{C} @ @] 1([\mathrm{H}])(\mathrm{CC}(\mathrm{CC} 2)[\mathrm{C} @ @ \mathrm{H}](\mathrm{C}=\mathrm{C}) \mathrm{C}[\mathrm{N}+] 12 \mathrm{Cc} 3 \mathrm{cc}(\mathrm{C}(\mathrm{C}(\mathrm{C}(\mathrm{F})(\mathrm{F}) \mathrm{F})(\mathrm{F}) \mathrm{F})(\mathrm{F}) \mathrm{F}) \mathrm{cc}(\mathrm{F}) \mathrm{c} 3)[\mathrm{C} @ \mathrm{H}](\mathrm{O}) \mathrm{c} 4 \mathrm{cc}} \\
{[\mathrm{n}+](\mathrm{Cc} 5 \mathrm{cc}(\mathrm{OC}(\mathrm{F})(\mathrm{F}) \mathrm{F}) \mathrm{c}(\mathrm{F}) \mathrm{cc} 5) \mathrm{c}(\mathrm{cccc} 6) \mathrm{c} 46}\end{array}$ \\
\hline Rnd5_3 & 2.22 & 9.18 & 80.36 & $\begin{array}{l}{[\mathrm{C} @ @] 1([\mathrm{H}])(\mathrm{CC}(\mathrm{CC} 2)[\mathrm{C} @ @ \mathrm{H}](\mathrm{C}=\mathrm{C}) \mathrm{C}[\mathrm{N}+] 12 \mathrm{Cc} 3 \mathrm{cc}(\mathrm{C}(\mathrm{C}(\mathrm{C}(\mathrm{F})(\mathrm{F}) \mathrm{F})(\mathrm{F}) \mathrm{F})(\mathrm{F}) \mathrm{F}) \mathrm{cc}(\mathrm{F}) \mathrm{c} 3)[\mathrm{C} @ \mathrm{H}](\mathrm{O}) \mathrm{c} 4 \mathrm{cc}} \\
{[\mathrm{n}+](\mathrm{Cc} 5 \mathrm{cc}(\mathrm{C}(\mathrm{F})(\mathrm{F}) \mathrm{F}) \mathrm{c}(\mathrm{F}) \mathrm{cc} 5) \mathrm{c}(\mathrm{cccc} 6) \mathrm{c} 46}\end{array}$ \\
\hline Rnd5_4 & 2.21 & 9.14 & 80.27 & $\begin{array}{l}{[\mathrm{C} @ @] 1([\mathrm{H}])(\mathrm{CC}(\mathrm{CC} 2)[\mathrm{C} @ @ \mathrm{H}](\mathrm{C}=\mathrm{C}) \mathrm{C}[\mathrm{N}+] 12 \mathrm{Cc} 3 \mathrm{cc}(\mathrm{C}(\mathrm{C}(\mathrm{C}(\mathrm{F})(\mathrm{F}) \mathrm{F})(\mathrm{F}) \mathrm{F})(\mathrm{F}) \mathrm{F}) \mathrm{cc}(\mathrm{F}) \mathrm{c} 3)[\mathrm{C} @ \mathrm{H}](\mathrm{O}) \mathrm{c} 4 \mathrm{cc}} \\
{[\mathrm{n}+](\mathrm{Cc} 5 \mathrm{cc}(\mathrm{OC}(\mathrm{F})(\mathrm{F}) \mathrm{F}) \mathrm{c}(\mathrm{Cl}) \mathrm{cc} 5) \mathrm{c}(\mathrm{cccc} 6) \mathrm{c} 46}\end{array}$ \\
\hline Rnd5_5 & 2.20 & 9.01 & 80.02 & $\begin{array}{l}{[\mathrm{C} @ @] 1([\mathrm{H}])(\mathrm{CC}(\mathrm{CC} 2)[\mathrm{C} @ @ \mathrm{H}](\mathrm{C}=\mathrm{C}) \mathrm{C}[\mathrm{N}+] 12 \mathrm{Cc} 3 \mathrm{cc}(\mathrm{C}(\mathrm{C}(\mathrm{F})(\mathrm{F}) \mathrm{F})(\mathrm{F}) \mathrm{F}) \mathrm{cc}(\mathrm{F}) \mathrm{c} 3)[\mathrm{C} @ \mathrm{H}](\mathrm{O}) \mathrm{c} 4 \mathrm{cc}[\mathrm{n}+](\mathrm{C}} \\
\mathrm{c5cc}(\mathrm{C}(\mathrm{F})(\mathrm{C}(\mathrm{C}(\mathrm{F})(\mathrm{F}) \mathrm{F})(\mathrm{F}) \mathrm{F}) \mathrm{F}) \mathrm{ccc5}) \mathrm{c}(\mathrm{cccc} 6) \mathrm{c} 46\end{array}$ \\
\hline Rnd5_6 & 2.18 & 8.86 & 79.72 & $\begin{array}{l}{[\mathrm{C} @ @] 1([\mathrm{H}])(\mathrm{CC}(\mathrm{CC} 2)[\mathrm{C} @ @ \mathrm{H}](\mathrm{C}=\mathrm{C}) \mathrm{C}[\mathrm{N}+] 12 \mathrm{Cc} 3 \mathrm{cc}(\mathrm{C}(\mathrm{C}(\mathrm{C}(\mathrm{F})(\mathrm{F}) \mathrm{F})(\mathrm{F}) \mathrm{F})(\mathrm{F}) \mathrm{F}) \mathrm{cc}(\mathrm{F}) \mathrm{c} 3)[\mathrm{C} @ \mathrm{H}](\mathrm{O}) \mathrm{c} 4 \mathrm{cc}} \\
{[\mathrm{n}+](\mathrm{Cc} 5 \mathrm{cc}(\mathrm{C}(\mathrm{F})(\mathrm{F}) \mathrm{F}) \mathrm{ccc} 5) \mathrm{c}(\mathrm{cccc} 6) \mathrm{c} 46}\end{array}$ \\
\hline Rnd5_7 & 2.16 & 8.71 & 79.40 & $\begin{array}{l}{[C @ @] 1([\mathrm{H}])(\mathrm{CC}(\mathrm{CC} 2)[\mathrm{C} @ @ \mathrm{H}](\mathrm{C}=\mathrm{C}) \mathrm{C}[\mathrm{N}+] 12 \mathrm{Cc} 3 \mathrm{cc}(\mathrm{C}(\mathrm{C}(\mathrm{F})(\mathrm{F}) \mathrm{F})(\mathrm{F}) \mathrm{F}) \mathrm{cc}(\mathrm{F}) \mathrm{c} 3)[\mathrm{C} @ \mathrm{H}](\mathrm{O}) \mathrm{c} 4 \mathrm{cc}[\mathrm{n}+](\mathrm{C}} \\
\mathrm{c5cc}(\mathrm{OC}(\mathrm{F})(\mathrm{F}) \mathrm{F}) \mathrm{c}(\mathrm{F}) \mathrm{cc} 5) \mathrm{c}(\mathrm{cccc} 6) \mathrm{c} 46\end{array}$ \\
\hline Rnd5_8 & 2.06 & 7.83 & 77.35 & $\begin{array}{l}{[\mathrm{C} @ @] 1([\mathrm{H}])(\mathrm{CC}(\mathrm{CC} 2)[\mathrm{C} @ @ \mathrm{H}](\mathrm{C}=\mathrm{C}) \mathrm{C}[\mathrm{N}+] 12 \mathrm{Cc} 3 \mathrm{cc}(\mathrm{C}(\mathrm{F})(\mathrm{C}(\mathrm{F})(\mathrm{F}) \mathrm{F}) \mathrm{C}(\mathrm{F})(\mathrm{F}) \mathrm{F}) \mathrm{cc}(\mathrm{F}) \mathrm{c} 3)[\mathrm{C} @ \mathrm{H}](\mathrm{O}) \mathrm{c} 4 \mathrm{cc}} \\
{[\mathrm{n}+](\mathrm{Cc} 5 \mathrm{cc}(\mathrm{OC}(\mathrm{F})(\mathrm{F}) \mathrm{F}) \mathrm{c}(\mathrm{Cl}) \mathrm{cc} 5) \mathrm{c}(\mathrm{cccc} 6) \mathrm{c} 46}\end{array}$ \\
\hline
\end{tabular}




\begin{tabular}{|c|c|c|c|c|}
\hline Rnd5_9 & 2.06 & 7.82 & 77.32 & $\begin{array}{l}{[\mathrm{C} @ @] 1([\mathrm{H}])(\mathrm{CC}(\mathrm{CC} 2)[\mathrm{C} @ @ \mathrm{H}](\mathrm{C}=\mathrm{C}) \mathrm{C}[\mathrm{N}+] 12 \mathrm{Cc} 3 \mathrm{cc}(\mathrm{C}(\mathrm{F})(\mathrm{C}(\mathrm{F})(\mathrm{F}) \mathrm{F}) \mathrm{C}(\mathrm{F})(\mathrm{F}) \mathrm{F}) \mathrm{cc}(\mathrm{F}) \mathrm{c} 3)[\mathrm{C} @ \mathrm{H}](\mathrm{O}) \mathrm{c} 4 \mathrm{cc}} \\
{[\mathrm{n}+](\mathrm{Cc} 5 \mathrm{cc}(\mathrm{OC}(\mathrm{F})(\mathrm{F}) \mathrm{F}) \mathrm{c}(\mathrm{F}) \mathrm{cc} 5) \mathrm{c}(\mathrm{cccc} 6) \mathrm{c} 46}\end{array}$ \\
\hline Rnd5_10 & 2.04 & 7.70 & 77.00 & $\begin{array}{l}{[\mathrm{C} @ @] 1([\mathrm{H}])(\mathrm{CC}(\mathrm{CC} 2)[\mathrm{C} @ @ \mathrm{H}](\mathrm{C}=\mathrm{C}) \mathrm{C}[\mathrm{N}+] 12 \mathrm{Cc} 3 \mathrm{cc}(\mathrm{C}(\mathrm{F})(\mathrm{C}(\mathrm{F})(\mathrm{F}) \mathrm{F}) \mathrm{C}(\mathrm{F})(\mathrm{F}) \mathrm{F}) \mathrm{cc}(\mathrm{F}) \mathrm{c} 3)[\mathrm{C} @ \mathrm{H}](\mathrm{O}) \mathrm{c} 4 \mathrm{cc}} \\
{[\mathrm{n}+](\mathrm{Cc} 5 \mathrm{cc}(\mathrm{C}(\mathrm{F})(\mathrm{F}) \mathrm{F}) \mathrm{ccc} 5) \mathrm{c}(\mathrm{cccc} 6) \mathrm{c} 46}\end{array}$ \\
\hline Rnd5_11 & 2.03 & 7.62 & 76.79 & $\begin{array}{l}{[\mathrm{C} @ @] 1([\mathrm{H}])(\mathrm{CC}(\mathrm{CC} 2)[\mathrm{C} @ @ \mathrm{H}](\mathrm{C}=\mathrm{C}) \mathrm{C}[\mathrm{N}+] 12 \mathrm{Cc} 3 \mathrm{cc}(\mathrm{C}(\mathrm{C}(\mathrm{F})(\mathrm{F}) \mathrm{F})(\mathrm{F}) \mathrm{F}) \mathrm{cc}(\mathrm{F}) \mathrm{c} 3)[\mathrm{C} @ \mathrm{H}](\mathrm{O}) \mathrm{c} 4 \mathrm{cc}[\mathrm{n}+](\mathrm{C}} \\
\mathrm{c5cc}(\mathrm{OC}(\mathrm{F})(\mathrm{F}) \mathrm{F}) \mathrm{ccc} 5) \mathrm{c}(\mathrm{cccc} 6) \mathrm{c} 46\end{array}$ \\
\hline Rnd5_12 & 2.03 & 7.61 & 76.78 & $\begin{array}{l}{[\mathrm{C} @ @] 1([\mathrm{H}])(\mathrm{CC}(\mathrm{CC} 2)[\mathrm{C} @ @ \mathrm{H}](\mathrm{C}=\mathrm{C}) \mathrm{C}[\mathrm{N}+] 12 \mathrm{Cc} 3 \mathrm{cc}(\mathrm{C}(\mathrm{C}(\mathrm{C}(\mathrm{F})(\mathrm{F}) \mathrm{F})(\mathrm{F}) \mathrm{F})(\mathrm{F}) \mathrm{F}) \mathrm{cc}(\mathrm{F}) \mathrm{c} 3)[\mathrm{C} @ \mathrm{H}](\mathrm{O}) \mathrm{c} 4 \mathrm{cc}} \\
{[\mathrm{n}+](\mathrm{Cc} 5 \mathrm{cc}(\mathrm{C}(\mathrm{F})(\mathrm{C}(\mathrm{F})(\mathrm{F}) \mathrm{F}) \mathrm{C}(\mathrm{F})(\mathrm{F}) \mathrm{F}) \mathrm{ccc} 5) \mathrm{c}(\mathrm{cccc} 6) \mathrm{c} 46}\end{array}$ \\
\hline Rnd5_13 & 2.02 & 7.54 & 76.58 & $\begin{array}{l}{[\mathrm{C} @ @] 1([\mathrm{H}])(\mathrm{CC}(\mathrm{CC} 2)[\mathrm{C} @ @ \mathrm{H}](\mathrm{C}=\mathrm{C}) \mathrm{C}[\mathrm{N}+] 12 \mathrm{Cc} 3 \mathrm{cc}(\mathrm{C}(\mathrm{C}(\mathrm{C}(\mathrm{F})(\mathrm{F}) \mathrm{F})(\mathrm{F}) \mathrm{F})(\mathrm{F}) \mathrm{F}) \mathrm{cc}(\mathrm{F}) \mathrm{c} 3)[\mathrm{C} @ \mathrm{H}](\mathrm{O}) \mathrm{c} 4 \mathrm{cc}} \\
{[\mathrm{n}+](\mathrm{Cc} 5 \mathrm{cc}(\mathrm{OC}(\mathrm{F})(\mathrm{F}) \mathrm{F}) \mathrm{ccc} 5) \mathrm{c}(\mathrm{cccc} 6) \mathrm{c} 46}\end{array}$ \\
\hline Rnd5_14 & 2.01 & 7.48 & 76.42 & $\begin{array}{l}{[\mathrm{C} @ @] 1([\mathrm{H}])(\mathrm{CC}(\mathrm{CC} 2)[\mathrm{C} @ @ \mathrm{H}](\mathrm{C}=\mathrm{C}) \mathrm{C}[\mathrm{N}+] 12 \mathrm{Cc} 3 \mathrm{cc}(\mathrm{C}(\mathrm{C}(\mathrm{C}(\mathrm{C}(\mathrm{F})(\mathrm{F}) \mathrm{F})(\mathrm{F}) \mathrm{F})(\mathrm{F}) \mathrm{F})(\mathrm{F}) \mathrm{F}) \mathrm{cc}(\mathrm{F}) \mathrm{c} 3)[\mathrm{C} @ \mathrm{H}](} \\
0) \mathrm{s} 4 \mathrm{cc}[\mathrm{n}+\mathrm{C}](\mathrm{Cc} 5 \mathrm{cc}(\mathrm{C}(\mathrm{F})(\mathrm{C}(\mathrm{F})(\mathrm{F}) \mathrm{F}) \mathrm{F}) \mathrm{ccc} 5) \mathrm{c}(\mathrm{cccc} 6) \mathrm{c} 46\end{array}$ \\
\hline Rnd5_15 & 2.01 & 7.47 & 76.39 & $\begin{array}{l}{[C @ @] 1([\mathrm{H}])(\mathrm{CC}(\mathrm{CC} 2)[\mathrm{C} @ @ \mathrm{H}](\mathrm{C}=\mathrm{C}) \mathrm{C}[\mathrm{N}+] 12 \mathrm{Cc} 3 \mathrm{cc}(\mathrm{C}(\mathrm{C}(\mathrm{F})(\mathrm{F}) \mathrm{F})(\mathrm{F}) \mathrm{F}) \mathrm{cc}(\mathrm{F}) \mathrm{c} 3)[\mathrm{C} @ \mathrm{H}](\mathrm{O}) \mathrm{c} 4 \mathrm{cc}[\mathrm{n}+](\mathrm{C}} \\
\mathrm{c5cc}(\mathrm{C}(\mathrm{F})(\mathrm{F}) \mathrm{C}(\mathrm{C}(\mathrm{C}(\mathrm{F})(\mathrm{F}) \mathrm{F})(\mathrm{F}) \mathrm{F})(\mathrm{F}) \mathrm{F}) \mathrm{ccc} 5) \mathrm{c}(\mathrm{cccc} 6) \mathrm{c} 46\end{array}$ \\
\hline Rnd5_16 & 1.97 & 7.19 & 75.57 & $\begin{array}{l}{[\mathrm{C} @ @] 1([\mathrm{H}])(\mathrm{CC}(\mathrm{CC} 2)[\mathrm{C} @ @ \mathrm{H}](\mathrm{C}=\mathrm{C}) \mathrm{C}[\mathrm{N}+] 12 \mathrm{Cc} 3 \mathrm{cc}(\mathrm{C}(\mathrm{C}(\mathrm{F})(\mathrm{F}) \mathrm{F})(\mathrm{F}) \mathrm{F}) \mathrm{cc}(\mathrm{F}) \mathrm{c} 3)[\mathrm{C} @ \mathrm{H}](\mathrm{O}) \mathrm{c} 4 \mathrm{cc}[\mathrm{n}+](\mathrm{C}} \\
\mathrm{c5ccc}(\mathrm{C}(\mathrm{F})(\mathrm{F}) \mathrm{F}) \mathrm{cc} 5) \mathrm{c}(\mathrm{cccc} 6) \mathrm{c} 46\end{array}$ \\
\hline Rnd5_17 & 1.96 & 7.11 & 75.34 & $\begin{array}{l}{[C @ @] 1([\mathrm{H}])(\mathrm{CC}(\mathrm{CC} 2)[\mathrm{C} @ @ \mathrm{H}](\mathrm{C}=\mathrm{C}) \mathrm{C}[\mathrm{N}+] 12 \mathrm{Cc} 3 \mathrm{cc}(\mathrm{C}(\mathrm{C}(\mathrm{F})(\mathrm{F}) \mathrm{F})(\mathrm{F}) \mathrm{F}) \mathrm{cc}(\mathrm{F}) \mathrm{c} 3)[\mathrm{C} @ \mathrm{H}](\mathrm{O}) \mathrm{c} 4 \mathrm{cc}[\mathrm{n}+](\mathrm{C}} \\
\mathrm{c5cc}(\mathrm{C}(\mathrm{F})(\mathrm{F}) \mathrm{F}) \mathrm{c}(\mathrm{C}(\mathrm{F})(\mathrm{F}) \mathrm{F}) \mathrm{cc} 5) \mathrm{c}(\mathrm{cccc} 6) \mathrm{c} 46\end{array}$ \\
\hline Rnd5_18 & 1.94 & 6.96 & 74.86 & $\begin{array}{l}{[\mathrm{C} @ @] 1([\mathrm{H}])(\mathrm{CC}(\mathrm{CC} 2)[\mathrm{C} @ @ \mathrm{H}](\mathrm{C}=\mathrm{C}) \mathrm{C}[\mathrm{N}+] 12 \mathrm{Cc} 3 \mathrm{cc}(\mathrm{C}(\mathrm{F})(\mathrm{C}(\mathrm{F})(\mathrm{F}) \mathrm{F}) \mathrm{C}(\mathrm{F})(\mathrm{F}) \mathrm{F}) \mathrm{cc}(\mathrm{F}) \mathrm{c} 3)[\mathrm{C} @ \mathrm{H}](\mathrm{O}) \mathrm{c} 4 \mathrm{cc}} \\
{[\mathrm{n}+](\mathrm{Cc} 5 \mathrm{cc}(\mathrm{OC}(\mathrm{F})(\mathrm{F}) \mathrm{F}) \mathrm{ccc5}) \mathrm{c}(\mathrm{cccc} 6) \mathrm{c} 46}\end{array}$ \\
\hline Rnd5_19 & 1.93 & 6.90 & 74.69 & $\begin{array}{l}{[\mathrm{C} @ @] 1([\mathrm{H}])(\mathrm{CC}(\mathrm{CC} 2)[\mathrm{C} @ @ \mathrm{H}](\mathrm{C}=\mathrm{C}) \mathrm{C}[\mathrm{N}+] 12 \mathrm{Cc} 3 \mathrm{cc}(\mathrm{C}(\mathrm{C}(\mathrm{F})(\mathrm{F}) \mathrm{F})(\mathrm{F}) \mathrm{F}) \mathrm{cc}(\mathrm{F}) \mathrm{c} 3)[\mathrm{C} @ \mathrm{H}](\mathrm{O}) \mathrm{c} 4 \mathrm{cc}[\mathrm{n}+](\mathrm{C}} \\
\mathrm{c5cc}(\mathrm{C}(\mathrm{F})(\mathrm{C}(\mathrm{F})(\mathrm{F}) \mathrm{F}) \mathrm{C}(\mathrm{F})(\mathrm{F}) \mathrm{F}) \mathrm{ccc} 5) \mathrm{c}(\mathrm{cccc} 6) \mathrm{c} 46\end{array}$ \\
\hline Rnd5_20 & 1.92 & 6.82 & 74.44 & $\begin{array}{l}{[C @ @] 1([\mathrm{H}])(\mathrm{CC}(\mathrm{CC} 2)[\mathrm{C} @ @ H](\mathrm{C}=\mathrm{C}) \mathrm{C}[\mathrm{N}+] 12 \mathrm{Cc} 3 \mathrm{cc}(\mathrm{C}(\mathrm{C}(\mathrm{C}(\mathrm{C}(\mathrm{F})(\mathrm{F}) \mathrm{F})(\mathrm{F}) \mathrm{F})(\mathrm{F}) \mathrm{F})(\mathrm{F}) \mathrm{F}) \mathrm{cc}(\mathrm{F}) \mathrm{c} 3)[\mathrm{C} @ \mathrm{H}](} \\
\mathrm{O}) \mathrm{c} 4 \mathrm{cc}[\mathrm{n}+](\mathrm{Cc} 5 \mathrm{cc}(\mathrm{C}(\mathrm{F})(\mathrm{C}(\mathrm{F})(\mathrm{F}) \mathrm{F}) \mathrm{C}(\mathrm{F})(\mathrm{F}) \mathrm{F}) \mathrm{ccc5}) \mathrm{c}(\mathrm{cccc} 6) \mathrm{c} 46\end{array}$ \\
\hline
\end{tabular}




\begin{tabular}{|c|c|c|c|c|}
\hline Rnd5_21 & 1.92 & 6.81 & 74.38 & $\begin{array}{l}{[\mathrm{C} @ @] 1([\mathrm{H}])(\mathrm{CC}(\mathrm{CC} 2)[\mathrm{C} @ @ \mathrm{CH}](\mathrm{C}=\mathrm{C}) \mathrm{C}[\mathrm{N}+] 12 \mathrm{Cc} 3 \mathrm{cc}(\mathrm{C}(\mathrm{C}(\mathrm{C}(\mathrm{F})(\mathrm{F}) \mathrm{F})(\mathrm{F}) \mathrm{F})(\mathrm{F}) \mathrm{F}) \mathrm{cc}(\mathrm{F}) \mathrm{c} 3)[\mathrm{C} @ \mathrm{H}](\mathrm{O}) \mathrm{c} 4 \mathrm{cc}} \\
{[\mathrm{n}+](\mathrm{Cc} 5 \mathrm{cc}(\mathrm{C}(\mathrm{F})(\mathrm{F}) \mathrm{C}(\mathrm{C}(\mathrm{C}(\mathrm{F})(\mathrm{F}) \mathrm{F})(\mathrm{F}) \mathrm{F})(\mathrm{F}) \mathrm{F}) \mathrm{ccc} 5) \mathrm{c}(\mathrm{cccc} 6) \mathrm{c} 46}\end{array}$ \\
\hline Rnd5_22 & 1.90 & 6.67 & 73.91 & $\begin{array}{l}{[\mathrm{C} @ @] 1([\mathrm{H}])(\mathrm{CC}(\mathrm{CC} 2)[\mathrm{C} @ @ \mathrm{H}](\mathrm{C}=\mathrm{C}) \mathrm{C}[\mathrm{N}+] 12 \mathrm{Cc} 3 \mathrm{cc}(\mathrm{C}(\mathrm{C}(\mathrm{C}(\mathrm{F})(\mathrm{F}) \mathrm{F})(\mathrm{F}) \mathrm{F})(\mathrm{F}) \mathrm{F}) \mathrm{cc}(\mathrm{F}) \mathrm{c} 3)[\mathrm{C} @ \mathrm{H}](\mathrm{O}) \mathrm{c} 4 \mathrm{cc}} \\
{[\mathrm{n}+](\mathrm{Cc} 5 \mathrm{ccc}(\mathrm{C}(\mathrm{F})(\mathrm{F}) \mathrm{F}) \mathrm{cc} 5) \mathrm{c}(\mathrm{cccc} 6) \mathrm{c} 46}\end{array}$ \\
\hline Rnd5_23 & 1.90 & 6.66 & 73.89 & $\begin{array}{l}{[\mathrm{C} @ @] 1([\mathrm{H}])(\mathrm{CC}(\mathrm{CC} 2)[\mathrm{C} @ @ \mathrm{H}](\mathrm{C}=\mathrm{C}) \mathrm{C}[\mathrm{N}+] 12 \mathrm{Cc} 3 \mathrm{cc}(\mathrm{C}(\mathrm{C}(\mathrm{C}(\mathrm{C}(\mathrm{F})(\mathrm{F}) \mathrm{F})(\mathrm{F}) \mathrm{F})(\mathrm{F}) \mathrm{F})(\mathrm{F}) \mathrm{F}) \mathrm{cc}(\mathrm{F}) \mathrm{c} 3)[\mathrm{C} @ \mathrm{H}](} \\
0) \mathrm{C} 4 \mathrm{cc}[\mathrm{n}+](\mathrm{Cc} 5 \mathrm{cc}(\mathrm{C}(\mathrm{F})(\mathrm{C}(\mathrm{C}(\mathrm{F})(\mathrm{F}) \mathrm{F})(\mathrm{F}) \mathrm{F}) \mathrm{F}) \mathrm{ccc} 5) \mathrm{c}(\mathrm{cccc} 6) \mathrm{c} 46\end{array}$ \\
\hline Rnd5_24 & 1.87 & 6.47 & 73.23 & $\begin{array}{l}{[\mathrm{C} @ @] 1([\mathrm{H}])(\mathrm{CC}(\mathrm{CC} 2)[\mathrm{C} @ @ \mathrm{H}](\mathrm{C}=\mathrm{C}) \mathrm{C}[\mathrm{N}+] 12 \mathrm{Cc} 3 \mathrm{cc}(\mathrm{C}(\mathrm{C}(\mathrm{C}(\mathrm{F})(\mathrm{F}) \mathrm{F})(\mathrm{F}) \mathrm{F})(\mathrm{F}) \mathrm{F}) \mathrm{cc}(\mathrm{F}) \mathrm{c} 3)[\mathrm{C} @ \mathrm{H}](\mathrm{O}) \mathrm{c} 4 \mathrm{cc}} \\
{[\mathrm{n}+](\mathrm{Cc} 5 \mathrm{cc}(\mathrm{SC}(\mathrm{F})(\mathrm{F}) \mathrm{F}) \mathrm{c}(\mathrm{Cl}) \mathrm{cc5}) \mathrm{c}(\mathrm{cccc} 6) \mathrm{c} 46}\end{array}$ \\
\hline Rnd5_25 & 1.87 & 6.46 & 73.19 & $\begin{array}{l}{[\mathrm{C} @ @] 1([\mathrm{H}])(\mathrm{CC}(\mathrm{CC} 2)[\mathrm{C} @ @ \mathrm{H}](\mathrm{C}=\mathrm{C}) \mathrm{C}[\mathrm{N}+] 12 \mathrm{Cc} 3 \mathrm{cc}(\mathrm{C}(\mathrm{C}(\mathrm{C}(\mathrm{F})(\mathrm{F}) \mathrm{F})(\mathrm{F}) \mathrm{F})(\mathrm{F}) \mathrm{F}) \mathrm{cc}(\mathrm{F}) \mathrm{c} 3)[\mathrm{C} @ \mathrm{H}](\mathrm{O}) \mathrm{c} 4 \mathrm{cc}} \\
{[\mathrm{n}+](\mathrm{Cc} 5 \mathrm{cc}(\mathrm{C}(\mathrm{F})(\mathrm{C}(\mathrm{C}(\mathrm{F})(\mathrm{F}) \mathrm{F})(\mathrm{F}) \mathrm{F}) \mathrm{F}) \mathrm{ccc5}) \mathrm{c}(\mathrm{cccc} 6) \mathrm{c} 46}\end{array}$ \\
\hline Rnd5_26 & 1.86 & 6.45 & 73.16 & $\begin{array}{l}{[\mathrm{C} @ @] 1([\mathrm{H}])(\mathrm{CC}(\mathrm{CC} 2)[\mathrm{C} @ @ \mathrm{H}](\mathrm{C}=\mathrm{C}) \mathrm{C}[\mathrm{N}+] 12 \mathrm{Cc} 3 \mathrm{cc}(\mathrm{C}(\mathrm{C}(\mathrm{C}(\mathrm{C}(\mathrm{F})(\mathrm{F}) \mathrm{F})(\mathrm{F}) \mathrm{F})(\mathrm{F}) \mathrm{F})(\mathrm{F}) \mathrm{F}) \mathrm{cc}(\mathrm{F}) \mathrm{c} 3)[\mathrm{C} @ \mathrm{H}](} \\
\mathrm{O}) \mathrm{c} 4 \mathrm{cc}[\mathrm{n}+](\mathrm{Cc} 5 \operatorname{cc}(\mathrm{OC}(\mathrm{F})(\mathrm{F}) \mathrm{F}) \mathrm{c}(\mathrm{F}) \mathrm{cc} 5) \mathrm{c}(\mathrm{cccc} 6) \mathrm{c} 46\end{array}$ \\
\hline Rnd5_27 & 1.83 & 6.22 & 72.31 & $\begin{array}{l}{[\mathrm{C} @ @] 1([\mathrm{H}])(\mathrm{CC}(\mathrm{CC} 2)[\mathrm{C} @ @ \mathrm{H}](\mathrm{C}=\mathrm{C}) \mathrm{C}[\mathrm{N}+] 12 \mathrm{Cc} 3 \mathrm{cc}(\mathrm{C}(\mathrm{C}(\mathrm{C}(\mathrm{C}(\mathrm{F})(\mathrm{F}) \mathrm{F})(\mathrm{F}) \mathrm{F})(\mathrm{F}) \mathrm{F})(\mathrm{F}) \mathrm{F}) \mathrm{cc}(\mathrm{F}) \mathrm{c} 3)[\mathrm{C} @ \mathrm{H}](} \\
\mathrm{O}) \mathrm{c} 4 \mathrm{cc}[\mathrm{n}+](\mathrm{Cc} 5 \mathrm{cc}(\mathrm{C}(\mathrm{F})(\mathrm{F}) \mathrm{F}) \mathrm{ccc} 5) \mathrm{c}(\mathrm{cccc} 6) \mathrm{c} 46\end{array}$ \\
\hline Rnd5_28 & 1.82 & 6.20 & 72.23 & $\begin{array}{l}{[\mathrm{C} @ @] 1([\mathrm{H}])(\mathrm{CC}(\mathrm{CC} 2)[\mathrm{C} @ @ \mathrm{H}](\mathrm{C}=\mathrm{C}) \mathrm{C}[\mathrm{N}+] 12 \mathrm{Cc} 3 \mathrm{cc}(\mathrm{C}(\mathrm{C}(\mathrm{C}(\mathrm{C}(\mathrm{F})(\mathrm{F}) \mathrm{F})(\mathrm{F}) \mathrm{F})(\mathrm{F}) \mathrm{F})(\mathrm{F}) \mathrm{F}) \mathrm{cc}(\mathrm{F}) \mathrm{c} 3)[\mathrm{C} @ \mathrm{H}](} \\
\mathrm{O}) \mathrm{c} 4 \mathrm{cc}[\mathrm{n}+](\mathrm{Cc} 5 \operatorname{cc}(\mathrm{OC}(\mathrm{F})(\mathrm{F}) \mathrm{F}) \operatorname{ccc} 5) \mathrm{c}(\mathrm{cccc} 6) \cos \end{array}$ \\
\hline Rnd5_29 & 1.81 & 6.11 & 71.88 & $\begin{array}{l}{[\mathrm{C} @ @] 1([\mathrm{H}])(\mathrm{CC}(\mathrm{CC} 2)[\mathrm{C} @ @ \mathrm{H}](\mathrm{C}=\mathrm{C}) \mathrm{C}[\mathrm{N}+] 12 \mathrm{Cc} 3 \mathrm{cc}(\mathrm{C}(\mathrm{C}(\mathrm{C}(\mathrm{F})(\mathrm{F}) \mathrm{F})(\mathrm{F}) \mathrm{F})(\mathrm{F}) \mathrm{F}) \mathrm{cc}(\mathrm{F}) \mathrm{c} 3)[\mathrm{C} @ \mathrm{H}](\mathrm{O}) \mathrm{c} 4 \mathrm{cc}} \\
{[\mathrm{n}+](\mathrm{Cc} 5 \mathrm{cc}(\mathrm{C}(\mathrm{F})(\mathrm{F}) \mathrm{F}) \mathrm{c}(\mathrm{C}(\mathrm{F})(\mathrm{F}) \mathrm{F}) \mathrm{cc} 5) \mathrm{c}(\mathrm{cccc} 6) \mathrm{c} 6}\end{array}$ \\
\hline Rnd5_30 & 1.80 & 6.05 & 71.65 & $\begin{array}{l}{[\mathrm{C} @ @] 1((\mathrm{H}])(\mathrm{CC}(\mathrm{CC} 2)[\mathrm{C} @ @ \mathrm{H}](\mathrm{C}=\mathrm{C}) \mathrm{C}[\mathrm{N}+] 12 \mathrm{Cc} 3 \mathrm{cc}(\mathrm{C}(\mathrm{C}(\mathrm{C}(\mathrm{C}(\mathrm{F})(\mathrm{F}) \mathrm{F})(\mathrm{F}) \mathrm{F})(\mathrm{F}) \mathrm{F})(\mathrm{F}) \mathrm{F}) \mathrm{cc}(\mathrm{F}) \mathrm{c} 3)[\mathrm{C} @ \mathrm{H}](} \\
0) \mathrm{l} 4 \mathrm{cc}[\mathrm{n}+](\mathrm{Cc} 5 \mathrm{ccc}(\mathrm{C}(\mathrm{F})(\mathrm{F}) \mathrm{F}) \mathrm{cc5}) \mathrm{c}(\mathrm{cccc} 6) \mathrm{c} 46\end{array}$ \\
\hline Rnd5_31 & 1.79 & 6.01 & 71.46 & $\begin{array}{l}{[\mathrm{C} @ @] 1([\mathrm{H}])(\mathrm{CC}(\mathrm{CC} 2)[\mathrm{C} @ @ \mathrm{H}](\mathrm{C}=\mathrm{C}) \mathrm{C}[\mathrm{N}+] 12 \mathrm{Cc} 3 \mathrm{cc}(\mathrm{C}(\mathrm{F})(\mathrm{C}(\mathrm{F})(\mathrm{F}) \mathrm{F}) \mathrm{C}(\mathrm{F})(\mathrm{F}) \mathrm{F}) \mathrm{cc}(\mathrm{F}) \mathrm{c} 3)[\mathrm{C} @ \mathrm{H}](\mathrm{O}) \mathrm{c} 4 \mathrm{cc}} \\
{[\mathrm{n}+](\mathrm{Cc} 5 \mathrm{cc}(\mathrm{C}(\mathrm{F})(\mathrm{C}(\mathrm{F})(\mathrm{F}) \mathrm{F}) \mathrm{F}) \mathrm{ccc} 5) \mathrm{c}(\mathrm{cccc} 6) \mathrm{c} 46}\end{array}$ \\
\hline Rnd5 32 & 1.79 & 5.96 & 71.28 & $\begin{array}{l}{[\mathrm{C} @ @] 1([\mathrm{H}])(\mathrm{CC}(\mathrm{CC} 2)[\mathrm{C} @ @ \mathrm{H}](\mathrm{C}=\mathrm{C}) \mathrm{C}[\mathrm{N}+] 12 \mathrm{Cc} 3 \mathrm{cc}(\mathrm{C}(\mathrm{F})(\mathrm{C}(\mathrm{F})(\mathrm{F}) \mathrm{F}) \mathrm{C}(\mathrm{F})(\mathrm{F}) \mathrm{F}) \mathrm{cc}(\mathrm{F}) \mathrm{c} 3)[\mathrm{C} @ \mathrm{H}](\mathrm{O}) \mathrm{c} 4 \mathrm{cc}} \\
{[\mathrm{n}+](\mathrm{Cc} 5 \mathrm{cc}(\mathrm{C}(\mathrm{F})(\mathrm{F}) \mathrm{C}(\mathrm{C}(\mathrm{C}(\mathrm{F})(\mathrm{F}) \mathrm{F})(\mathrm{F}) \mathrm{F})(\mathrm{F}) \mathrm{F}) \mathrm{ccc} 5) \mathrm{c}(\mathrm{cccc} 6) \mathrm{c} 46}\end{array}$ \\
\hline
\end{tabular}




\begin{tabular}{|c|c|c|c|c|}
\hline Rnd5_33 & 1.78 & 5.93 & 71.15 & $\begin{array}{l}{[\mathrm{C} @ @] 1([\mathrm{H}])(\mathrm{CC}(\mathrm{CC} 2)[\mathrm{C} @ @ \mathrm{CH}](\mathrm{C}=\mathrm{C}) \mathrm{C}[\mathrm{N}+] 12 \mathrm{Cc} 3 \mathrm{cc}(\mathrm{C}(\mathrm{F})(\mathrm{C}(\mathrm{F})(\mathrm{F}) \mathrm{F}) \mathrm{C}(\mathrm{F})(\mathrm{F}) \mathrm{F}) \mathrm{cc}(\mathrm{F}) \mathrm{c} 3)[\mathrm{C} @ \mathrm{H}](\mathrm{O}) \mathrm{c} 4 \mathrm{cc}} \\
{[\mathrm{n}+](\mathrm{Cc} 5 \mathrm{cc}(\mathrm{C}(\mathrm{F})(\mathrm{F}) \mathrm{F}) \mathrm{c}(\mathrm{F}) \mathrm{cc} 5) \mathrm{c}(\mathrm{cccc} 6) \mathrm{c} 46}\end{array}$ \\
\hline Rnd5_34 & 1.77 & 5.85 & 70.80 & 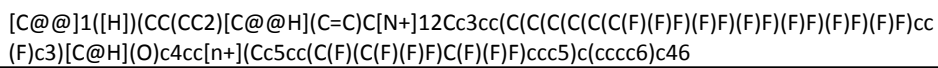 \\
\hline Rnd5_35 & 1.76 & 5.81 & 70.64 & $\begin{array}{l}{[\mathrm{C} @ @] 1([\mathrm{H}])(\mathrm{CC}(\mathrm{CC} 2)[\mathrm{C} @ @ \mathrm{~B}](\mathrm{C}=\mathrm{C}) \mathrm{C}[\mathrm{N}+] 12 \mathrm{Cc} 3 \mathrm{cc}(\mathrm{C}(\mathrm{C}(\mathrm{C}(\mathrm{C}(\mathrm{F})(\mathrm{F}) \mathrm{F})(\mathrm{F}) \mathrm{F})(\mathrm{F}) \mathrm{F})(\mathrm{F}) \mathrm{F}) \mathrm{cc}(\mathrm{F}) \mathrm{c} 3)[\mathrm{C} @ \mathrm{H}](} \\
0) \mathrm{C} 4 \mathrm{cc}[\mathrm{n}+](\mathrm{Cc} 5 \mathrm{cc}(\mathrm{C}(\mathrm{F})(\mathrm{F}) \mathrm{C}(\mathrm{C}(\mathrm{C}(\mathrm{F})(\mathrm{F}) \mathrm{F})(\mathrm{F}) \mathrm{F})(\mathrm{F}) \mathrm{F}) \mathrm{ccc5}) \mathrm{c}(\mathrm{cccc} 6) \mathrm{c} 46\end{array}$ \\
\hline Rnd5_36 & 1.74 & 5.69 & 70.11 & $\begin{array}{l}{[\mathrm{C} @ @] 1([\mathrm{H}])(\mathrm{CC}(\mathrm{CC} 2)[\mathrm{C} @ @ \mathrm{H}](\mathrm{C}=\mathrm{C}) \mathrm{C}[\mathrm{N}+] 12 \mathrm{Cc} 3 \mathrm{cc}(\mathrm{C}(\mathrm{C}(\mathrm{C}(\mathrm{C}(\mathrm{C}(\mathrm{C}(\mathrm{F})(\mathrm{F}) \mathrm{F})(\mathrm{F}) \mathrm{F})(\mathrm{F}) \mathrm{F})(\mathrm{F}) \mathrm{F})(\mathrm{F}) \mathrm{F})(\mathrm{F}) \mathrm{F}) \mathrm{cc}} \\
(\mathrm{F}) \mathrm{c} 3)[\mathrm{C} @ \mathrm{H}](\mathrm{O}) \mathrm{c} 4 \mathrm{cc}[\mathrm{n}+](\mathrm{Cc} 5 \mathrm{cc}(\mathrm{OC}(\mathrm{F})(\mathrm{F}) \mathrm{F}) \mathrm{c}(\mathrm{Cl}) \mathrm{cc} 5) \mathrm{c}(\mathrm{cccc} 6) \mathrm{c} 46\end{array}$ \\
\hline Rnd5_37 & 1.70 & 5.45 & 68.99 & $\begin{array}{l}{[\mathrm{C} @ @] 1([\mathrm{H}])(\mathrm{CC}(\mathrm{CC} 2)[\mathrm{C} @ @ \mathrm{H}](\mathrm{C}=\mathrm{C}) \mathrm{C}[\mathrm{N}+] 12 \mathrm{Cc} 3 \mathrm{cc}(\mathrm{C}(\mathrm{C}(\mathrm{C}(\mathrm{C}(\mathrm{C}(\mathrm{C}(\mathrm{F})(\mathrm{F}) \mathrm{F})(\mathrm{F}) \mathrm{F})(\mathrm{F}) \mathrm{F})(\mathrm{F}) \mathrm{F})(\mathrm{F}) \mathrm{F})(\mathrm{F}) \mathrm{F}) \mathrm{cc}} \\
(\mathrm{F}) \mathrm{c} 3)[\mathrm{C} @ \mathrm{H}](\mathrm{O}) \mathrm{c} 4 \mathrm{cc}[\mathrm{n}+](\mathrm{Cc} 5 \mathrm{cc}(\mathrm{OC}(\mathrm{F})(\mathrm{F}) \mathrm{F}) \mathrm{c}(\mathrm{F}) \mathrm{cc} 5) \mathrm{c}(\mathrm{cccc} 6) \mathrm{c} 46\end{array}$ \\
\hline Rnd5_38 & 1.68 & 5.39 & 68.69 & 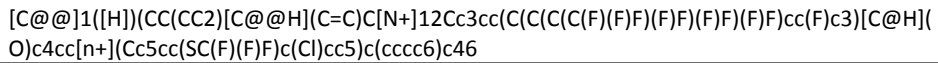 \\
\hline Rnd5_39 & 1.67 & 5.29 & 68.20 & $\begin{array}{l}{[\mathrm{C} @ @] 1([\mathrm{H}])(\mathrm{CC}(\mathrm{CC} 2)[\mathrm{C} @ @ \mathrm{H}](\mathrm{C}=\mathrm{C}) \mathrm{C}[\mathrm{N}+] 12 \mathrm{Cc} 3 \mathrm{cc}(\mathrm{C}(\mathrm{C}(\mathrm{C}(\mathrm{C}(\mathrm{F})(\mathrm{F}) \mathrm{F})(\mathrm{F}) \mathrm{F})(\mathrm{F}) \mathrm{F})(\mathrm{F}) \mathrm{F}) \mathrm{cc}(\mathrm{F}) \mathrm{c} 3)[\mathrm{C} @ \mathrm{H}](} \\
\mathrm{O}) \mathrm{c} 4 \mathrm{cc}[\mathrm{n}+](\mathrm{Cc} 5 \mathrm{cc}(\mathrm{OC}(\mathrm{F})(\mathrm{F}) \mathrm{F}) \mathrm{c}(\mathrm{Cl}) \mathrm{cc} 5) \mathrm{c}(\mathrm{cccc} 6) \mathrm{c} 46\end{array}$ \\
\hline Rnd5_40 & 1.66 & 5.26 & 68.06 & $\begin{array}{l}{[\mathrm{C} @ @] 1([\mathrm{H}])(\mathrm{CC}(\mathrm{CC} 2)[\mathrm{C} @ @ \mathrm{H}](\mathrm{C}=\mathrm{C}) \mathrm{C}[\mathrm{N}+] 12 \mathrm{Cc} 3 \mathrm{cc}(\mathrm{C}(\mathrm{F})(\mathrm{C}(\mathrm{F})(\mathrm{F}) \mathrm{F}) \mathrm{C}(\mathrm{F})(\mathrm{F}) \mathrm{F}) \mathrm{cc}(\mathrm{F}) \mathrm{c} 3)[\mathrm{C} @ \mathrm{H}](\mathrm{O}) \mathrm{c} 4 \mathrm{cc}} \\
{[\mathrm{n}+](\mathrm{Cc} 5 \mathrm{cc}(\mathrm{F}) \mathrm{c}(\mathrm{C}(\mathrm{F})(\mathrm{F}) \mathrm{F}) \mathrm{cc} 5) \mathrm{c}(\mathrm{cccc} 6) \mathrm{c} 46}\end{array}$ \\
\hline Rnd5_41 & 1.66 & 5.25 & 67.98 & $\begin{array}{l}{[\mathrm{C} @ @] 1([\mathrm{H}])(\mathrm{CC}(\mathrm{CC} 2)[\mathrm{C} @ @ \mathrm{H}](\mathrm{C}=\mathrm{C}) \mathrm{C}[\mathrm{N}+] 12 \mathrm{Cc} 3 \mathrm{cc}(\mathrm{C}(\mathrm{C}(\mathrm{C}(\mathrm{C}(\mathrm{C}(\mathrm{C}(\mathrm{F})(\mathrm{F}) \mathrm{F})(\mathrm{F}) \mathrm{F})(\mathrm{F}) \mathrm{F})(\mathrm{F}) \mathrm{F})(\mathrm{F}) \mathrm{F})(\mathrm{F}) \mathrm{F}) \mathrm{cc}} \\
(\mathrm{F}) \mathrm{c} 3)[\mathrm{C} @ \mathrm{H}](\mathrm{O}) \mathrm{c} 4 \mathrm{cc}[\mathrm{n}+](\mathrm{Cc} 5 \mathrm{cc}(\mathrm{C}(\mathrm{F})(\mathrm{C}(\mathrm{F})(\mathrm{F}) \mathrm{F}) \mathrm{F}) \mathrm{ccc} 5) \mathrm{c}(\mathrm{cccc} 6) \mathrm{c} 46\end{array}$ \\
\hline Rnd5_42 & 1.65 & 5.23 & 67.91 & $\begin{array}{l}{[\mathrm{C} @ @] 1([\mathrm{H}])(\mathrm{CC}(\mathrm{CC} 2)[\mathrm{C} @ @ H](\mathrm{C}=\mathrm{C}) \mathrm{C}[\mathrm{N}+] 12 \mathrm{Cc} 3 \mathrm{cc}(\mathrm{C}(\mathrm{C}(\mathrm{F})(\mathrm{F}) \mathrm{F})(\mathrm{F}) \mathrm{F}) \mathrm{cc}(\mathrm{F}) \mathrm{c} 3)[\mathrm{C} @ \mathrm{H}](\mathrm{O}) \mathrm{c} 4 \mathrm{cc}[\mathrm{n}+](\mathrm{C}} \\
\mathrm{c5cc}(\mathrm{F}) \mathrm{c}(\mathrm{C}(\mathrm{F})(\mathrm{F}) \mathrm{F}) \mathrm{cc} 5) \mathrm{c}(\mathrm{cccc} 6) \mathrm{c} 46\end{array}$ \\
\hline Rnd5_43 & 1.65 & 5.23 & 67.89 & $\begin{array}{l}{[\mathrm{C} @ @] 1([\mathrm{H}])(\mathrm{CC}(\mathrm{CC} 2)[\mathrm{C} @ @ \mathrm{H}](\mathrm{C}=\mathrm{C}) \mathrm{C}[\mathrm{N}+] 12 \mathrm{Cc} 3 \mathrm{cc}(\mathrm{C}(\mathrm{F})(\mathrm{C}(\mathrm{F})(\mathrm{F}) \mathrm{F}) \mathrm{C}(\mathrm{F})(\mathrm{F}) \mathrm{F}) \mathrm{cc}(\mathrm{F}) \mathrm{c} 3)[\mathrm{C} @ \mathrm{H}](\mathrm{O}) \mathrm{c} 4 \mathrm{cc}} \\
{[\mathrm{n}+](\mathrm{Cc} 5 \mathrm{ccc}(\mathrm{c6c}(\mathrm{cccc} 7) \mathrm{c} 7 \mathrm{ccc} 6) \mathrm{cc} 5) \mathrm{c}(\mathrm{cccc}) \mathrm{c} 48}\end{array}$ \\
\hline Rnd5 44 & 1.65 & 5.19 & 67.69 & $\begin{array}{l}{[\mathrm{C} @ @] 1([\mathrm{H}])(\mathrm{CC}(\mathrm{CC} 2)[\mathrm{C} @ @ \mathrm{H}](\mathrm{C}=\mathrm{C}) \mathrm{C}[\mathrm{N}+] 12 \mathrm{Cc} 3 \mathrm{cc}(\mathrm{C}(\mathrm{F})(\mathrm{C}(\mathrm{F})(\mathrm{F}) \mathrm{F}) \mathrm{C}(\mathrm{F})(\mathrm{F}) \mathrm{F}) \mathrm{cc}(\mathrm{F}) \mathrm{c} 3)[\mathrm{C} @ \mathrm{H}](\mathrm{O}) \mathrm{c} 4 \mathrm{cc}} \\
{[\mathrm{n}+](\mathrm{Cc} 5 \mathrm{cc}(\mathrm{C}(\mathrm{F})(\mathrm{F}) \mathrm{F}) \mathrm{c}(\mathrm{C}(\mathrm{F})(\mathrm{F}) \mathrm{F}) \mathrm{cc} 5) \mathrm{c}(\mathrm{cccc} 6) \mathrm{c} 46}\end{array}$ \\
\hline
\end{tabular}




\begin{tabular}{|c|c|c|c|c|}
\hline Rnd5_45 & 1.64 & 5.15 & 67.48 & $\begin{array}{l}{[\mathrm{C} @ @] 1([\mathrm{H}])(\mathrm{CC}(\mathrm{CC} 2)[\mathrm{C} @ @ \mathrm{H}](\mathrm{C}=\mathrm{C}) \mathrm{C}[\mathrm{N}+] 12 \mathrm{Cc} 3 \mathrm{cc}(\mathrm{C}(\mathrm{F})(\mathrm{C}(\mathrm{F})(\mathrm{F}) \mathrm{F}) \mathrm{C}(\mathrm{F})(\mathrm{F}) \mathrm{F}) \mathrm{cc}(\mathrm{F}) \mathrm{c} 3)[\mathrm{C} @ \mathrm{H}](\mathrm{O}) \mathrm{c} 4 \mathrm{cc}} \\
{[\mathrm{n}+](\mathrm{Cc} 5 \mathrm{ccc}(\mathrm{C}(\mathrm{F})(\mathrm{F}) \mathrm{F}) \mathrm{cc} 5) \mathrm{c}(\mathrm{cccc} 6) \mathrm{c} 46}\end{array}$ \\
\hline Rnd5_46 & 1.63 & 5.13 & 67.36 & $\begin{array}{l}{[\mathrm{C} @ @] 1((\mathrm{H}])(\mathrm{CC}(\mathrm{CC} 2)[\mathrm{C} @ @ \mathrm{H}](\mathrm{C}=\mathrm{C}) \mathrm{C}[\mathrm{N}+] 12 \mathrm{Cc} 3 \mathrm{cc}(\mathrm{C}(\mathrm{C}(\mathrm{C}(\mathrm{C}(\mathrm{F})(\mathrm{F}) \mathrm{F})(\mathrm{F}) \mathrm{F})(\mathrm{F}) \mathrm{F})(\mathrm{F}) \mathrm{F}) \mathrm{cc}(\mathrm{F}) \mathrm{c} 3)[\mathrm{C} @ \mathrm{H}](} \\
\mathrm{O}) \mathrm{c} 4 \mathrm{cc}[\mathrm{n}+](\mathrm{Cc} 5 \mathrm{cc}(\mathrm{C}(\mathrm{F})(\mathrm{F}) \mathrm{F}) \mathrm{c}(\mathrm{C}(\mathrm{F})(\mathrm{F}) \mathrm{F}) \mathrm{cc} 5) \mathrm{c}(\mathrm{cccc} 6) \mathrm{c} 46\end{array}$ \\
\hline Rnd5_47 & 1.62 & 5.08 & 67.09 & $\begin{array}{l}{[\mathrm{C} @ @] 1([\mathrm{H}])(\mathrm{CC}(\mathrm{CC} 2)[\mathrm{C} @ @ \mathrm{H}](\mathrm{C}=\mathrm{C}) \mathrm{C}[\mathrm{N}+] 12 \mathrm{Cc} 3 \mathrm{cc}(\mathrm{C}(\mathrm{C}(\mathrm{C}(\mathrm{C}(\mathrm{C}(\mathrm{C}(\mathrm{F})(\mathrm{F}) \mathrm{F})(\mathrm{F}) \mathrm{F})(\mathrm{F}) \mathrm{F})(\mathrm{F}) \mathrm{F})(\mathrm{F}) \mathrm{F})(\mathrm{F}) \mathrm{F}) \mathrm{cc}} \\
(\mathrm{F}) \mathrm{c} 3)[\mathrm{C} @ \mathrm{H}](\mathrm{O}) \mathrm{c} 4 \mathrm{cc}[\mathrm{n}+](\mathrm{Cc} 5 \mathrm{cc}(\mathrm{SC}(\mathrm{F})(\mathrm{F}) \mathrm{F}) \mathrm{c}(\mathrm{Cl}) \mathrm{cc} 5) \mathrm{c}(\mathrm{cccc} 6) \mathrm{c} 46\end{array}$ \\
\hline Rnd5_48 & 1.62 & 5.05 & 66.94 & $\begin{array}{l}{[\mathrm{C} @ @] 1([\mathrm{H}])(\mathrm{CC}(\mathrm{CC} 2)[\mathrm{C} @ @ \mathrm{H}](\mathrm{C}=\mathrm{C}) \mathrm{C}[\mathrm{N}+] 12 \mathrm{Cc} 3 \mathrm{cc}(\mathrm{C}(\mathrm{C}(\mathrm{C}(\mathrm{C}(\mathrm{C}(\mathrm{C}(\mathrm{F})(\mathrm{F}) \mathrm{F})(\mathrm{F}) \mathrm{F})(\mathrm{F}) \mathrm{F})(\mathrm{F}) \mathrm{F})(\mathrm{F}) \mathrm{F})(\mathrm{F}) \mathrm{F}) \mathrm{cc}} \\
(\mathrm{F}) \mathrm{c} 3)[\mathrm{C} @ \mathrm{H}](\mathrm{O}) \mathrm{c} 4 \mathrm{cc}[\mathrm{n}+](\mathrm{Cc} 5 \mathrm{cc}(\mathrm{OC}(\mathrm{F})(\mathrm{F}) \mathrm{F}) \mathrm{ccc} 5) \mathrm{c}(\mathrm{cccc} 6) \mathrm{c} 46\end{array}$ \\
\hline Rnd5_49 & 1.62 & 5.04 & 66.91 & $\begin{array}{l}{[\mathrm{C} @ @] 1([\mathrm{H}])(\mathrm{CC}(\mathrm{CC} 2)[\mathrm{C} @ @ \mathrm{H}](\mathrm{C}=\mathrm{C}) \mathrm{C}[\mathrm{N}+] 12 \mathrm{Cc} 3 \mathrm{cc}(\mathrm{C}(\mathrm{C}(\mathrm{C}(\mathrm{C}(\mathrm{F})(\mathrm{F}) \mathrm{F})(\mathrm{F}) \mathrm{F})(\mathrm{F}) \mathrm{F})(\mathrm{F}) \mathrm{F}) \mathrm{cc}(\mathrm{F}) \mathrm{c} 3)[\mathrm{C} @ \mathrm{H}](} \\
\mathrm{O}) \mathrm{c} 4 \mathrm{cc}[\mathrm{n}+](\mathrm{Cc} 5 \mathrm{cc}(\mathrm{C}(\mathrm{F})(\mathrm{F}) \mathrm{F}) \mathrm{c}(\mathrm{F}) \mathrm{cc} 5) \mathrm{c}(\mathrm{cccc} 6) \mathrm{c} 46\end{array}$ \\
\hline Rnd5_50 & 1.62 & 5.03 & 66.83 & $\begin{array}{l}{[\mathrm{C} @ @] 1([\mathrm{H}])(\mathrm{CC}(\mathrm{CC} 2)[\mathrm{C} @ @ \mathrm{H}](\mathrm{C}=\mathrm{C}) \mathrm{C}[\mathrm{N}+] 12 \mathrm{Cc} 3 \mathrm{cc}(\mathrm{C}(\mathrm{C}(\mathrm{F})(\mathrm{F}) \mathrm{F})(\mathrm{F}) \mathrm{F}) \mathrm{cc}(\mathrm{F}) \mathrm{c} 3)[\mathrm{C} @ \mathrm{H}](\mathrm{O}) \mathrm{c} 4 \mathrm{cc}[\mathrm{n}+](\mathrm{C}} \\
\mathrm{c5ccc}(\mathrm{c} 6 \mathrm{c}(\mathrm{cccc} 7) \mathrm{c} 7 \mathrm{ccc} 6) \operatorname{cc5} 5) \mathrm{c}(\mathrm{cccc} 8) \mathrm{c} 48\end{array}$ \\
\hline Rnd5_51 & 1.59 & 4.91 & 66.14 & $\begin{array}{l}{[\mathrm{C} @ @] 1([\mathrm{H}])(\mathrm{CC}(\mathrm{CC} 2)[\mathrm{C} @ @ \mathrm{H}](\mathrm{C}=\mathrm{C}) \mathrm{C}[\mathrm{N}+] 12 \mathrm{Cc} 3 \mathrm{cc}(\mathrm{C}(\mathrm{C}(\mathrm{C}(\mathrm{F})(\mathrm{F}) \mathrm{F})(\mathrm{F}) \mathrm{F})(\mathrm{F}) \mathrm{F}) \mathrm{cc}(\mathrm{F}) \mathrm{c} 3)[\mathrm{C} @ \mathrm{H}](\mathrm{O}) \mathrm{c} 4 \mathrm{cc}} \\
{[\mathrm{n}+](\mathrm{Cc} 5 \mathrm{cc}(\mathrm{F}) \mathrm{c}(\mathrm{C}(\mathrm{F})(\mathrm{F}) \mathrm{F}) \mathrm{cc5}) \mathrm{c}(\mathrm{ccccc}) \mathrm{c} 46}\end{array}$ \\
\hline Rnd5_52 & 1.56 & 4.76 & 65.26 & $\begin{array}{l}{[\mathrm{C} @ @] 1([\mathrm{H}])(\mathrm{CC}(\mathrm{CC} 2)[\mathrm{C} @ @ \mathrm{H}](\mathrm{C}=\mathrm{C}) \mathrm{C}[\mathrm{N}+] 12 \mathrm{Cc} 3 \mathrm{cc}(\mathrm{C}(\mathrm{C}(\mathrm{C}(\mathrm{C}(\mathrm{C}(\mathrm{C}(\mathrm{F})(\mathrm{F}) \mathrm{F})(\mathrm{F}) \mathrm{F})(\mathrm{F}) \mathrm{F})(\mathrm{F}) \mathrm{F})(\mathrm{F}) \mathrm{F})(\mathrm{F}) \mathrm{F}) \mathrm{cc}} \\
(\mathrm{F}) \mathrm{c} 3)[\mathrm{C} @ \mathrm{H}](\mathrm{O}) \mathrm{c} 4 \mathrm{cc}[\mathrm{n}+](\mathrm{Cc} 5 \mathrm{cc}(\mathrm{C}(\mathrm{F})(\mathrm{C}(\mathrm{C}(\mathrm{F})(\mathrm{F}) \mathrm{F})(\mathrm{F}) \mathrm{F}) \mathrm{F}) \mathrm{ccc}(\mathrm{c}) \mathrm{c}(\mathrm{cccc}) \mathrm{c}(\mathrm{c})\end{array}$ \\
\hline Rnd5_53 & 1.53 & 4.64 & 64.52 & $\begin{array}{l}{[\mathrm{C} @ @] 1([\mathrm{H}])(\mathrm{CC}(\mathrm{CC} 2)[\mathrm{C} @ @ \mathrm{H}](\mathrm{C}=\mathrm{C}) \mathrm{C}[\mathrm{N}+] 12 \mathrm{Cc} 3 \mathrm{cc}(\mathrm{C}(\mathrm{F})(\mathrm{C}(\mathrm{F})(\mathrm{F}) \mathrm{F}) \mathrm{C}(\mathrm{F})(\mathrm{F}) \mathrm{F}) \mathrm{cc}(\mathrm{F}) \mathrm{c} 3)[\mathrm{C} @ \mathrm{H}](\mathrm{O}) \mathrm{c} 4 \mathrm{cc}} \\
{[\mathrm{n}+](\mathrm{Cc} 5 \mathrm{cc}(\mathrm{C}(\mathrm{F})(\mathrm{C}(\mathrm{C}(\mathrm{F})(\mathrm{F}) \mathrm{F})(\mathrm{F}) \mathrm{F}) \mathrm{F}) \mathrm{ccc} 5) \mathrm{c}(\mathrm{cccc} 6) \mathrm{c} 46}\end{array}$ \\
\hline Rnd5_54 & 1.52 & 4.59 & 64.22 & $\begin{array}{l}{[\mathrm{C} @ @] 1([\mathrm{H}])(\mathrm{CC}(\mathrm{CC} 2)[\mathrm{C} @ @ \mathrm{H}](\mathrm{C}=\mathrm{C}) \mathrm{C}[\mathrm{N}+] 12 \mathrm{Cc} 3 \mathrm{cc}(\mathrm{C}(\mathrm{C}(\mathrm{C}(\mathrm{C}(\mathrm{C}(\mathrm{C}(\mathrm{C}(\mathrm{C}(\mathrm{F})(\mathrm{F}) \mathrm{F})(\mathrm{F}) \mathrm{F})(\mathrm{F}) \mathrm{F})(\mathrm{F}) \mathrm{F})(\mathrm{F}) \mathrm{F})(\mathrm{F})} \\
\mathrm{F})(\mathrm{F}) \mathrm{F})(\mathrm{F}) \mathrm{F}) \mathrm{cc}(\mathrm{F}) \mathrm{c} 3)[\mathrm{C} @ \mathrm{H}](\mathrm{O}) \mathrm{C} 4 \mathrm{cc}[\mathrm{n}+](\mathrm{Cc} 5 \mathrm{cc}(\mathrm{OC}(\mathrm{F})(\mathrm{F}) \mathrm{F}) \mathrm{C}(\mathrm{Cl}) \mathrm{cc} 5) \mathrm{c}(\mathrm{cccc} 6) \mathrm{c} 46\end{array}$ \\
\hline Rnd5_55 & 1.52 & 4.58 & 64.18 & $\begin{array}{l}{[\mathrm{C} @ @] 1([\mathrm{H}])(\mathrm{CC}(\mathrm{CC} 2)[\mathrm{C} @ @ \mathrm{H}](\mathrm{C}=\mathrm{C}) \mathrm{C}[\mathrm{N}+] 12 \mathrm{Cc} 3 \mathrm{cc}(\mathrm{C}(\mathrm{F})(\mathrm{C}(\mathrm{F})(\mathrm{F}) \mathrm{F}) \mathrm{C}(\mathrm{F})(\mathrm{F}) \mathrm{F}) \mathrm{cc}(\mathrm{F}) \mathrm{c} 3)[\mathrm{C} @ \mathrm{H}](\mathrm{O}) \mathrm{c} 4 \mathrm{cc}} \\
[\mathrm{n}+](\mathrm{Cc} 5 \mathrm{cc}(\mathrm{SC}(\mathrm{F})(\mathrm{F}) \mathrm{F}) \mathrm{c}(\mathrm{Cl}) \mathrm{cc}) \mathrm{c}) \mathrm{c}(\mathrm{cccc} 6) \mathrm{c} 46\end{array}$ \\
\hline Rnd5_56 & 1.50 & 4.50 & 63.62 & $\begin{array}{l}{[\mathrm{C} @ @] 1([\mathrm{H}])(\mathrm{CC}(\mathrm{CC} 2)[\mathrm{C} @ @ \mathrm{H}](\mathrm{C}=\mathrm{C}) \mathrm{C}[\mathrm{N}+] 12 \mathrm{Cc} 3 \mathrm{cc}(\mathrm{C}(\mathrm{C}(\mathrm{C}(\mathrm{C}(\mathrm{C}(\mathrm{C}(\mathrm{F})(\mathrm{F}) \mathrm{F})(\mathrm{F}) \mathrm{F})(\mathrm{F}) \mathrm{F})(\mathrm{F}) \mathrm{F})(\mathrm{F}) \mathrm{F})(\mathrm{F}) \mathrm{F}) \mathrm{cc}} \\
(\mathrm{F}) \mathrm{c} 3)[\mathrm{C} @ \mathrm{H}](\mathrm{O}) \mathrm{c} 4 \mathrm{cc}[\mathrm{n}+](\mathrm{Cc} 5 \mathrm{cc}(\mathrm{C}(\mathrm{F})(\mathrm{F}) \mathrm{F}) \mathrm{c}(\mathrm{F}) \mathrm{cc5}) \mathrm{c}(\mathrm{cccc}) \mathrm{c} 46\end{array}$ \\
\hline
\end{tabular}




\begin{tabular}{|c|c|c|c|c|}
\hline Rnd5_57 & 1.49 & 4.46 & 63.37 & $\begin{array}{l}{[\mathrm{C} @ @] 1([\mathrm{H}])(\mathrm{CC}(\mathrm{CC} 2)[\mathrm{C} @ @ \mathrm{H}](\mathrm{C}=\mathrm{C}) \mathrm{C}[\mathrm{N}+] 12 \mathrm{Cc} 3 \mathrm{cc}(\mathrm{C}(\mathrm{F})(\mathrm{C}(\mathrm{F})(\mathrm{F}) \mathrm{F}) \mathrm{C}(\mathrm{F})(\mathrm{F}) \mathrm{F}) \mathrm{cc}(\mathrm{F}) \mathrm{c} 3)[\mathrm{C} @ \mathrm{H}](\mathrm{O}) \mathrm{c} 4 \mathrm{cc}} \\
{[\mathrm{n}+](\mathrm{Cc} 5 \mathrm{cc}(\mathrm{F}) \mathrm{ccc} 5) \mathrm{c}(\mathrm{cccc} 6) \mathrm{c} 46}\end{array}$ \\
\hline Rnd5_58 & 1.49 & 4.44 & 63.26 & $\begin{array}{l}{[\mathrm{C} @ @] 1([\mathrm{H}])(\mathrm{CC}(\mathrm{CC} 2)[\mathrm{C} @ @ \mathrm{H}](\mathrm{C}=\mathrm{C}) \mathrm{C}[\mathrm{N}+] 12 \mathrm{Cc} 3 \mathrm{cc}(\mathrm{C}(\mathrm{C}(\mathrm{C}(\mathrm{C}(\mathrm{F})(\mathrm{F}) \mathrm{F})(\mathrm{F}) \mathrm{F})(\mathrm{F}) \mathrm{F})(\mathrm{F}) \mathrm{F}) \mathrm{cc}(\mathrm{F}) \mathrm{c} 3)[\mathrm{C} @ \mathrm{H}](} \\
\mathrm{O}) \mathrm{c} 4 \mathrm{cc}[\mathrm{n}+](\mathrm{Cc} 5 \mathrm{ccc}(\mathrm{c} 6 \mathrm{c}(\mathrm{cccc} 7) \mathrm{c} 7 \mathrm{ccc} 6) \mathrm{cc5}) \mathrm{c}(\mathrm{cccc} 8) \mathrm{c} 48\end{array}$ \\
\hline Rnd5_59 & 1.47 & 4.36 & 62.71 & $\begin{array}{l}{[\mathrm{C} @ @] 1([\mathrm{H}])(\mathrm{CC}(\mathrm{CC} 2)[\mathrm{C} @ @ \mathrm{CH}](\mathrm{C}=\mathrm{C}) \mathrm{C}[\mathrm{N}+] 12 \mathrm{Cc} 3 \mathrm{cc}(\mathrm{C}(\mathrm{C}(\mathrm{C}(\mathrm{C}(\mathrm{C}(\mathrm{C}(\mathrm{C}(\mathrm{C}(\mathrm{F})(\mathrm{F}) \mathrm{F})(\mathrm{F}) \mathrm{F})(\mathrm{F}) \mathrm{F})(\mathrm{F}) \mathrm{F})(\mathrm{F}) \mathrm{F})(\mathrm{F})} \\
\mathrm{F})(\mathrm{F}) \mathrm{F})(\mathrm{F}) \mathrm{F}) \mathrm{cc}(\mathrm{F}) \mathrm{F} 3)[\mathrm{C} @ \mathrm{H}](\mathrm{O}) \mathrm{c} 4 \mathrm{cc}[\mathrm{n}+](\mathrm{Cc} 5 \mathrm{cc}(\mathrm{SC}(\mathrm{F})(\mathrm{F}) \mathrm{F}) \mathrm{c}(\mathrm{Cl}) \mathrm{cc}) \mathrm{c}(\mathrm{ccc}) \mathrm{ccc}(\mathrm{c}) \mathrm{c} 46\end{array}$ \\
\hline Rnd5_60 & 1.46 & 4.31 & 62.35 & $\begin{array}{l}{[\mathrm{C} @ @] 1([\mathrm{H}])(\mathrm{CC}(\mathrm{CC} 2)[\mathrm{C} @ @ \mathrm{H}](\mathrm{C}=\mathrm{C}) \mathrm{C}[\mathrm{N}+] 12 \mathrm{Cc} 3 \mathrm{cc}(\mathrm{C}(\mathrm{C}(\mathrm{C}(\mathrm{C}(\mathrm{F})(\mathrm{F}) \mathrm{F})(\mathrm{F}) \mathrm{F})(\mathrm{F}) \mathrm{F})(\mathrm{F}) \mathrm{F}) \mathrm{cc}(\mathrm{F}) \mathrm{c} 3)[\mathrm{C} @ \mathrm{H}](\mathrm{C}} \\
\mathrm{O}) \mathrm{c} 4 \mathrm{cc}[\mathrm{n}+](\mathrm{Cc} 5 \mathrm{cc}(\mathrm{F}) \mathrm{c}(\mathrm{C}(\mathrm{F})(\mathrm{F}) \mathrm{F}) \mathrm{cc} 5) \mathrm{c}(\mathrm{cccc} 6) \mathrm{c} 46\end{array}$ \\
\hline Rnd5_61 & 1.45 & 4.25 & 61.90 & $\begin{array}{l}{[\mathrm{C} @ @] 1([\mathrm{H}])(\mathrm{CC}(\mathrm{CC} 2)[\mathrm{C} @ @ \mathrm{H}](\mathrm{C}=\mathrm{C}) \mathrm{C}[\mathrm{N}+] 12 \mathrm{Cc} 3 \mathrm{cc}(\mathrm{C}(\mathrm{C}(\mathrm{C}(\mathrm{F})(\mathrm{F}) \mathrm{F})(\mathrm{F}) \mathrm{F})(\mathrm{F}) \mathrm{F}) \mathrm{cc}(\mathrm{F}) \mathrm{c} 3)[\mathrm{C} @ \mathrm{H}](\mathrm{O}) \mathrm{c} 4 \mathrm{cc}} \\
{[\mathrm{n}+](\mathrm{Cc} 5 \operatorname{ccc}(\mathrm{c} 6 \mathrm{c}(\mathrm{cccc} 7) \mathrm{c} 7 \mathrm{ccc} 6) \operatorname{cc} 5) \mathrm{c}(\mathrm{cccc} 8) \mathrm{c} 48}\end{array}$ \\
\hline Rnd5_62 & 1.45 & 4.25 & 61.87 & 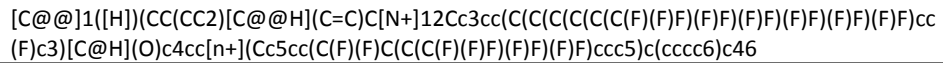 \\
\hline Rnd5_63 & 1.41 & 4.11 & 60.89 & $\begin{array}{l}{[\mathrm{C} @ @] 1([\mathrm{H}])(\mathrm{CC}(\mathrm{CC} 2)[\mathrm{C} @ @ \mathrm{H}](\mathrm{C}=\mathrm{C}) \mathrm{C}[\mathrm{N}+] 12 \mathrm{Cc} 3 \mathrm{cc}(\mathrm{C}(\mathrm{C}(\mathrm{C}(\mathrm{C}(\mathrm{C}(\mathrm{C}(\mathrm{F})(\mathrm{F}) \mathrm{F})(\mathrm{F}) \mathrm{F})(\mathrm{F}) \mathrm{F})(\mathrm{F}) \mathrm{F})(\mathrm{F}) \mathrm{F})(\mathrm{F}) \mathrm{F}) \mathrm{cc}} \\
(\mathrm{F}) \mathrm{c} 3)[\mathrm{C} @ \mathrm{H}](\mathrm{O}) \mathrm{c} 4 \mathrm{cc}[\mathrm{n}+](\mathrm{Cc} 5 \mathrm{cc}(\mathrm{C}(\mathrm{F})(\mathrm{F}) \mathrm{F}) \mathrm{ccc} 5) \mathrm{c}(\mathrm{cccc} 6) \mathrm{c} 46\end{array}$ \\
\hline Rnd5 64 & 1.41 & 4.10 & 60.80 & $\begin{array}{l}{[\mathrm{C} @ @] 1([\mathrm{H}])(\mathrm{CC}(\mathrm{CC} 2)[\mathrm{C} @ @ \mathrm{H}](\mathrm{C}=\mathrm{C}) \mathrm{C}[\mathrm{N}+] 12 \mathrm{Cc} 3 \mathrm{cc}(\mathrm{C}(\mathrm{C}(\mathrm{C}(\mathrm{C}(\mathrm{C}(\mathrm{C}(\mathrm{F})(\mathrm{F}) \mathrm{F})(\mathrm{F}) \mathrm{F})(\mathrm{F}) \mathrm{F})(\mathrm{F}) \mathrm{F})(\mathrm{F}) \mathrm{F})(\mathrm{F}) \mathrm{F}) \mathrm{cc}} \\
(\mathrm{F}) \mathrm{c} 3)[\mathrm{C} @ \mathrm{H}](\mathrm{O}) \mathrm{c} 4 \mathrm{cc}[\mathrm{n}+](\mathrm{Cc} 5 \mathrm{cc}(\mathrm{C}(\mathrm{F})(\mathrm{F}) \mathrm{F}) \mathrm{c}(\mathrm{C}(\mathrm{F})(\mathrm{F}) \mathrm{F}) \mathrm{cc} 5) \mathrm{c}(\mathrm{cccc} 6) \mathrm{c} 46\end{array}$ \\
\hline Rnd5_65 & 1.41 & 4.09 & 60.70 & $\begin{array}{l}{[\mathrm{C} @ @] 1([\mathrm{H}])(\mathrm{CC}(\mathrm{CC} 2)[\mathrm{C} @ @ \mathrm{H}](\mathrm{C}=\mathrm{C}) \mathrm{C}[\mathrm{N}+] 12 \mathrm{Cc} 3 \mathrm{cc}(\mathrm{C}(\mathrm{F})(\mathrm{C}(\mathrm{F})(\mathrm{F}) \mathrm{F}) \mathrm{C}(\mathrm{F})(\mathrm{F}) \mathrm{F}) \mathrm{cc}(\mathrm{F}) \mathrm{c} 3)[\mathrm{C} @ \mathrm{H}](\mathrm{O}) \mathrm{c} 4 \mathrm{cc}} \\
{[\mathrm{n}+](\mathrm{Cc} 5 \mathrm{cc}(\mathrm{C}(\mathrm{F})(\mathrm{C}(\mathrm{F})(\mathrm{F}) \mathrm{F}) \mathrm{C}(\mathrm{F})(\mathrm{F}) \mathrm{F}) \mathrm{ccc} 5) \mathrm{c}(\mathrm{cccc} 6) \mathrm{c} 46}\end{array}$ \\
\hline Rnd5_66 & 1.39 & 4.01 & 60.05 & $\begin{array}{l}{[\mathrm{C} @ @] 1([\mathrm{H}])(\mathrm{CC}(\mathrm{CC} 2)[\mathrm{C} @ @ \mathrm{H}](\mathrm{C}=\mathrm{C}) \mathrm{C}[\mathrm{N}+] 12 \mathrm{Cc} 3 \mathrm{cc}(\mathrm{C}(\mathrm{C}(\mathrm{C}(\mathrm{C}(\mathrm{C}(\mathrm{C}(\mathrm{F})(\mathrm{F}) \mathrm{F})(\mathrm{F}) \mathrm{F})(\mathrm{F}) \mathrm{F})(\mathrm{F}) \mathrm{F})(\mathrm{F}) \mathrm{F})(\mathrm{F}) \mathrm{F}) \mathrm{cc}} \\
(\mathrm{F}) \mathrm{c} 3)[\mathrm{C} @ \mathrm{H}](\mathrm{O}) \mathrm{c} 4 \mathrm{cc}[\mathrm{n}+](\mathrm{Cc} 5 \mathrm{cc}(\mathrm{F}) \mathrm{ccc} 5) \mathrm{c}(\mathrm{cccc} 6) \mathrm{c} 46\end{array}$ \\
\hline Rnd5_67 & 1.34 & 3.83 & 58.59 & $\begin{array}{l}{[\mathrm{C} @ @] 1([\mathrm{H}])(\mathrm{CC}(\mathrm{CC} 2)[\mathrm{C} @ @ \mathrm{CH}](\mathrm{C}=\mathrm{C}) \mathrm{C}[\mathrm{N}+] 12 \mathrm{Cc} 3 \mathrm{cc}(\mathrm{C}(\mathrm{C}(\mathrm{C}(\mathrm{C}(\mathrm{C}(\mathrm{C}(\mathrm{C}(\mathrm{C}(\mathrm{F})(\mathrm{F}) \mathrm{F})(\mathrm{F}) \mathrm{F})(\mathrm{F}) \mathrm{F})(\mathrm{F}) \mathrm{F})(\mathrm{F}) \mathrm{F})(\mathrm{F})} \\
\mathrm{F})(\mathrm{F}) \mathrm{F})(\mathrm{F}) \mathrm{F}) \mathrm{cc}(\mathrm{F}) \mathrm{c} 3)[\mathrm{C} @ \mathrm{H}](\mathrm{O}) \mathrm{c} 4 \mathrm{cc}[\mathrm{n}+](\mathrm{Cc} 5 \mathrm{cc}(\mathrm{OC}(\mathrm{F})(\mathrm{F}) \mathrm{F}) \mathrm{c}(\mathrm{F}) \mathrm{cc} 5) \mathrm{c}(\mathrm{cccc} 6) \mathrm{c} 46\end{array}$ \\
\hline Rnd5 68 & 1.34 & 3.82 & 58.54 & $\begin{array}{l}{[\mathrm{C} @ @] 1([\mathrm{H}])(\mathrm{CC}(\mathrm{CC} 2)[\mathrm{C} @ @ \mathrm{H}](\mathrm{C}=\mathrm{C}) \mathrm{C}[\mathrm{N}+] 12 \mathrm{Cc} 3 \mathrm{cc}(\mathrm{C}(\mathrm{C}(\mathrm{C}(\mathrm{C}(\mathrm{C}(\mathrm{C}(\mathrm{C}(\mathrm{C}(\mathrm{F})(\mathrm{F}) \mathrm{F})(\mathrm{F}) \mathrm{F})(\mathrm{F}) \mathrm{F})(\mathrm{F}) \mathrm{F})(\mathrm{F}) \mathrm{F})(\mathrm{F})} \\
\mathrm{F})(\mathrm{F}) \mathrm{F})(\mathrm{F}) \mathrm{F}) \mathrm{cc}(\mathrm{F}) \mathrm{c} 3)[\mathrm{C} @ \mathrm{H}](\mathrm{O}) \mathrm{c} 4 \mathrm{cc}[\mathrm{n}+](\mathrm{Cc} 5 \mathrm{cc}(\mathrm{C}(\mathrm{F})(\mathrm{F}) \mathrm{F}) \mathrm{c}(\mathrm{C}(\mathrm{F})(\mathrm{F}) \mathrm{F}) \mathrm{cc} 5) \mathrm{c}(\mathrm{cccc} 6)(\mathrm{c}) \mathrm{C}\end{array}$ \\
\hline
\end{tabular}




\begin{tabular}{|c|c|c|c|c|}
\hline Rnd5_69 & 1.32 & 3.73 & 57.69 & $\begin{array}{l}{[\mathrm{C} @ @] 1([\mathrm{H}])(\mathrm{CC}(\mathrm{CC} 2)[\mathrm{C} @ @ \mathrm{CH}](\mathrm{C}=\mathrm{C}) \mathrm{C}[\mathrm{N}+] 12 \mathrm{Cc} 3 \mathrm{cc}(\mathrm{C}(\mathrm{C}(\mathrm{C}(\mathrm{C}(\mathrm{C}(\mathrm{C}(\mathrm{F})(\mathrm{F}) \mathrm{F})(\mathrm{F}) \mathrm{F})(\mathrm{F}) \mathrm{F})(\mathrm{F}) \mathrm{F})(\mathrm{F}) \mathrm{F})(\mathrm{F}) \mathrm{F}) \mathrm{cc}} \\
(\mathrm{F}) \mathrm{c} 3)[\mathrm{C} @ \mathrm{H}](\mathrm{O}) \mathrm{c} 4 \mathrm{cc}[\mathrm{n}+](\mathrm{Cc} 5 \mathrm{ccc}(\mathrm{c} 6 \mathrm{c}(\mathrm{cccc} 7) \mathrm{c} 7 \mathrm{ccc} 6) \mathrm{cc} 5) \mathrm{c}(\mathrm{cccc} 8) \mathrm{c} 48\end{array}$ \\
\hline Rnd5_70 & 1.31 & 3.70 & 57.45 & $\begin{array}{l}{[\mathrm{C} @ @] 1([\mathrm{H}])(\mathrm{CC}(\mathrm{CC} 2)[\mathrm{C} @ @ \mathrm{H}](\mathrm{C}=\mathrm{C}) \mathrm{C}[\mathrm{N}+] 12 \mathrm{Cc} 3 \mathrm{cc}(\mathrm{C}(\mathrm{C}(\mathrm{C}(\mathrm{C}(\mathrm{F})(\mathrm{F}) \mathrm{F})(\mathrm{F}) \mathrm{F})(\mathrm{F}) \mathrm{F})(\mathrm{F}) \mathrm{F}) \mathrm{cc}(\mathrm{F}) \mathrm{c} 3)[\mathrm{C} @ \mathrm{H}](} \\
\mathrm{O}) \mathrm{c} 4 \mathrm{cc}[\mathrm{n}+](\mathrm{Cc} 5 \mathrm{cc}(\mathrm{F}) \mathrm{ccc} 5) \mathrm{c}(\mathrm{cccc} 6) \mathrm{c} 46\end{array}$ \\
\hline Rnd5_71 & 1.26 & 3.54 & 55.94 & $\begin{array}{l}{[\mathrm{C} @ @] 1([\mathrm{H}])(\mathrm{CC}(\mathrm{CC} 2)[\mathrm{C} @ @ \mathrm{H}](\mathrm{C}=\mathrm{C}) \mathrm{C}[\mathrm{N}+] 12 \mathrm{Cc} 3 \mathrm{cc}(\mathrm{C}(\mathrm{C}(\mathrm{C}(\mathrm{C}(\mathrm{C}(\mathrm{C}(\mathrm{F})(\mathrm{F}) \mathrm{F})(\mathrm{F}) \mathrm{F})(\mathrm{F}) \mathrm{F})(\mathrm{F}) \mathrm{F})(\mathrm{F}) \mathrm{F})(\mathrm{F}) \mathrm{F}) \mathrm{cc}} \\
(\mathrm{F}) \mathrm{c} 3)[\mathrm{C} @ \mathrm{H}](\mathrm{O}) \mathrm{c} 4 \mathrm{cc}[\mathrm{n}+](\mathrm{Cc} 5 \mathrm{cc}(\mathrm{F}) \mathrm{c}(\mathrm{C}(\mathrm{F})(\mathrm{F}) \mathrm{F}) \mathrm{cc5}) \mathrm{c}(\mathrm{cccc} 6) \mathrm{c} 46\end{array}$ \\
\hline Rnd5_72 & 1.25 & 3.51 & 55.62 & $\begin{array}{l}{[\mathrm{C} @ @] 1([\mathrm{H}])(\mathrm{CC}(\mathrm{CC} 2)[\mathrm{C} @ @ \mathrm{CH}](\mathrm{C}=\mathrm{C}) \mathrm{C}[\mathrm{N}+] 12 \mathrm{Cc} 3 \mathrm{cc}(\mathrm{C}(\mathrm{C}(\mathrm{C}(\mathrm{C}(\mathrm{C}(\mathrm{C}(\mathrm{C}(\mathrm{C}(\mathrm{F})(\mathrm{F}) \mathrm{F})(\mathrm{F}) \mathrm{F})(\mathrm{F}) \mathrm{F})(\mathrm{F}) \mathrm{F})(\mathrm{F}) \mathrm{F})(\mathrm{F})} \\
\mathrm{F})(\mathrm{F}) \mathrm{F})(\mathrm{F}) \mathrm{F}) \mathrm{cc}(\mathrm{F}) \mathrm{c} 3)[\mathrm{C} @ \mathrm{H}](\mathrm{O}) \mathrm{c} 4 \mathrm{cc}[\mathrm{n}+](\mathrm{Cc} 5 \mathrm{cc}(\mathrm{F}) \mathrm{c}(\mathrm{C}(\mathrm{F})(\mathrm{F}) \mathrm{F}) \mathrm{cc} 5) \mathrm{c}(\mathrm{cccc} 6) \mathrm{c} 6\end{array}$ \\
\hline Rnd5_73 & 1.24 & 3.44 & 54.94 & $\begin{array}{l}{[\mathrm{C} @ @] 1([\mathrm{H}])(\mathrm{CC}(\mathrm{CC} 2)[\mathrm{C} @ @ \mathrm{H}](\mathrm{C}=\mathrm{C}) \mathrm{C}[\mathrm{N}+] 12 \mathrm{Cc} 3 \mathrm{cc}(\mathrm{C}(\mathrm{C}(\mathrm{C}(\mathrm{C}(\mathrm{C}(\mathrm{C}(\mathrm{F})(\mathrm{F}) \mathrm{F})(\mathrm{F}) \mathrm{F})(\mathrm{F}) \mathrm{F})(\mathrm{F}) \mathrm{F})(\mathrm{F}) \mathrm{F})(\mathrm{F}) \mathrm{F}) \mathrm{cc}} \\
(\mathrm{F}) \mathrm{c} 3)[\mathrm{C} @ \mathrm{H}](\mathrm{O}) \mathrm{c} 4 \mathrm{cc}[\mathrm{n}+](\mathrm{Cc} 5 \mathrm{ccc}(\mathrm{C}(\mathrm{F})(\mathrm{F}) \mathrm{F}) \mathrm{cc5}) \mathrm{c}(\mathrm{cccc} 6) \mathrm{c} 46\end{array}$ \\
\hline Rnd5_74 & 1.23 & 3.43 & 54.83 & $\begin{array}{l}{[\mathrm{C} @ @] 1([\mathrm{H}])(\mathrm{CC}(\mathrm{CC} 2)[\mathrm{C} @ @ \mathrm{CH}](\mathrm{C}=\mathrm{C}) \mathrm{C}[\mathrm{N}+] 12 \mathrm{Cc} 3 \mathrm{cc}(\mathrm{C}(\mathrm{C}(\mathrm{C}(\mathrm{C}(\mathrm{C}(\mathrm{C}(\mathrm{C}(\mathrm{C}(\mathrm{F})(\mathrm{F}) \mathrm{F})(\mathrm{F}) \mathrm{F})(\mathrm{F}) \mathrm{F})(\mathrm{F}) \mathrm{F})(\mathrm{F}) \mathrm{F})(\mathrm{F})} \\
\mathrm{F})(\mathrm{F}) \mathrm{F})(\mathrm{F}) \mathrm{F}) \mathrm{cc}(\mathrm{F}) \mathrm{c} 3)[\mathrm{C} @ \mathrm{H}](\mathrm{O}) \mathrm{c} 4 \mathrm{cc}[\mathrm{n}+](\mathrm{Cc} 5 \mathrm{cc}(\mathrm{C}(\mathrm{F})(\mathrm{C}(\mathrm{F})(\mathrm{F}) \mathrm{F}) \mathrm{F}) \mathrm{ccc} 5)(\mathrm{cccc} 6) \mathrm{c} 46\end{array}$ \\
\hline Rnd5_75 & 1.23 & 3.41 & 54.62 & $\begin{array}{l}{[\mathrm{C} @ @] 1([\mathrm{H}])(\mathrm{CC}(\mathrm{CC} 2)[\mathrm{C} @ @ @]](\mathrm{C}=\mathrm{C}) \mathrm{C}[\mathrm{N}+] 12 \mathrm{Cc} 3 \mathrm{cc}(\mathrm{C}(\mathrm{C}(\mathrm{C}(\mathrm{C}(\mathrm{C}(\mathrm{C}(\mathrm{C}(\mathrm{C}(\mathrm{F})(\mathrm{F}) \mathrm{F})(\mathrm{F}) \mathrm{F})(\mathrm{F}) \mathrm{F})(\mathrm{F}) \mathrm{F})(\mathrm{F}) \mathrm{F})(\mathrm{F})} \\
\mathrm{F})(\mathrm{F}) \mathrm{F})(\mathrm{F}) \mathrm{F}) \mathrm{cc}(\mathrm{F}) \mathrm{F} 3)[\mathrm{C} @ \mathrm{H}](\mathrm{O}) \mathrm{C} 4 \mathrm{cc}[\mathrm{n}+](\mathrm{Cc} 5 \mathrm{cc}(\mathrm{OC}(\mathrm{F})(\mathrm{F}) \mathrm{F}) \mathrm{ccc} 5) \mathrm{c}(\mathrm{cccc}) \mathrm{C}) \mathrm{c} 46\end{array}$ \\
\hline Rnd5_76 & 1.22 & 3.40 & 54.53 & $\begin{array}{l}{[\mathrm{C} @ @] 1([\mathrm{H}])(\mathrm{CC}(\mathrm{CC} 2)[\mathrm{C} @ @ \mathrm{H}](\mathrm{C}=\mathrm{C}) \mathrm{C}[\mathrm{N}+] 12 \mathrm{Cc} 3 \mathrm{cc}(\mathrm{C}(\mathrm{C}(\mathrm{C}(\mathrm{C}(\mathrm{C}(\mathrm{C}(\mathrm{C}(\mathrm{C}(\mathrm{F})(\mathrm{F}) \mathrm{F})(\mathrm{F}) \mathrm{F})(\mathrm{F}) \mathrm{F})(\mathrm{F}) \mathrm{F})(\mathrm{F}) \mathrm{F})(\mathrm{F})} \\
\mathrm{F})(\mathrm{F}) \mathrm{F})(\mathrm{F}) \mathrm{F}) \mathrm{cc}(\mathrm{F}) \mathrm{c} 3)[\mathrm{C} @ \mathrm{H}](\mathrm{O}) \mathrm{c} 4 \mathrm{cc}[\mathrm{n}+](\mathrm{Cc} 5 \mathrm{cc}(\mathrm{C}(\mathrm{F})(\mathrm{F}) \mathrm{F}) \mathrm{ccc} 5) \mathrm{c}(\mathrm{cccc} C 6) \mathrm{c} 46\end{array}$ \\
\hline Rnd5_77 & 1.22 & 3.39 & 54.42 & $\begin{array}{l}{[\mathrm{C} @ @] 1([\mathrm{H}])(\mathrm{CC}(\mathrm{CC} 2)[\mathrm{C} @ @ \mathrm{H}](\mathrm{C}=\mathrm{C}) \mathrm{C}[\mathrm{N}+] 12 \mathrm{Cc} 3 \mathrm{cc}(\mathrm{C}(\mathrm{C}(\mathrm{C}(\mathrm{C}(\mathrm{C}(\mathrm{C}(\mathrm{C}(\mathrm{C}(\mathrm{F})(\mathrm{F}) \mathrm{F})(\mathrm{F}) \mathrm{F})(\mathrm{F}) \mathrm{F})(\mathrm{F}) \mathrm{F})(\mathrm{F}) \mathrm{F})(\mathrm{F})} \\
\mathrm{F})(\mathrm{F}) \mathrm{F})(\mathrm{F}) \mathrm{F}) \mathrm{cc}(\mathrm{F}) \mathrm{c} 3)[\mathrm{C} @ \mathrm{H}](\mathrm{O}) \mathrm{c} 4 \mathrm{cc}[\mathrm{n}+](\mathrm{Cc} 5 \mathrm{cc}(\mathrm{C}(\mathrm{F})(\mathrm{F}) \mathrm{F}) \mathrm{F}(\mathrm{F}) \mathrm{cc} 5) \mathrm{c}(\mathrm{ccccc}) \mathrm{c}(46\end{array}$ \\
\hline Rnd5_78 & 1.17 & 3.21 & 52.48 & $\begin{array}{l}{[\mathrm{C} @ @] 1([\mathrm{H}])(\mathrm{CC}(\mathrm{CC} 2)[\mathrm{C} @ @ \mathrm{CH}](\mathrm{C}=\mathrm{C}) \mathrm{C}[\mathrm{N}+] 12 \mathrm{Cc} 3 \mathrm{cc}(\mathrm{C}(\mathrm{C}(\mathrm{C}(\mathrm{C}(\mathrm{C}(\mathrm{C}(\mathrm{C}(\mathrm{C}(\mathrm{F})(\mathrm{F}) \mathrm{F})(\mathrm{F}) \mathrm{F})(\mathrm{F}) \mathrm{F})(\mathrm{F}) \mathrm{F})(\mathrm{F}) \mathrm{F})(\mathrm{F})} \\
\mathrm{F})(\mathrm{F}) \mathrm{F})(\mathrm{F}) \mathrm{F}) \mathrm{cc}(\mathrm{F}) \mathrm{c} 3)[\mathrm{C} @ \mathrm{H}](\mathrm{O}) \mathrm{c} 4 \mathrm{cc}[\mathrm{n}+](\mathrm{Cc} 5 \mathrm{cc}(\mathrm{C}(\mathrm{F})(\mathrm{C}(\mathrm{F})(\mathrm{F}) \mathrm{F}) \mathrm{C}(\mathrm{F})(\mathrm{F}) \mathrm{F}) \mathrm{ccc}) \mathrm{c}(\mathrm{ccc}) \mathrm{ccc}) \mathrm{c} 46\end{array}$ \\
\hline Rnd5_79 & 1.07 & 2.92 & 48.96 & $\begin{array}{l}{[\mathrm{C} @ @] 1([\mathrm{H}])(\mathrm{CC}(\mathrm{CC} 2)[\mathrm{C} @ @ \mathrm{CH}](\mathrm{C}=\mathrm{C}) \mathrm{C}[\mathrm{N}+] 12 \mathrm{Cc} 3 \mathrm{cc}(\mathrm{C}(\mathrm{C}(\mathrm{C}(\mathrm{C}(\mathrm{C}(\mathrm{C}(\mathrm{C}(\mathrm{C}(\mathrm{F})(\mathrm{F}) \mathrm{F})(\mathrm{F}) \mathrm{F})(\mathrm{F}) \mathrm{F})(\mathrm{F}) \mathrm{F})(\mathrm{F}) \mathrm{F})(\mathrm{F})} \\
\mathrm{F})(\mathrm{F}) \mathrm{F})(\mathrm{F}) \mathrm{F}) \mathrm{cc}(\mathrm{F}) \mathrm{c} 3)[\mathrm{C} @ \mathrm{H}](\mathrm{O}) \mathrm{c} 4 \mathrm{cc}[\mathrm{n}+](\mathrm{Cc} 5 \mathrm{cc}(\mathrm{C}(\mathrm{F})(\mathrm{F}) \mathrm{C}(\mathrm{C}(\mathrm{C}(\mathrm{F})(\mathrm{F}) \mathrm{F})(\mathrm{F}) \mathrm{F})(\mathrm{F}) \mathrm{F}) \mathrm{ccc} 5) \mathrm{c}(\mathrm{cccc} 6) \mathrm{c} 46\end{array}$ \\
\hline Rnd5_80 & 1.06 & 2.88 & 48.42 & $\begin{array}{l}{[\mathrm{C} @ @] 1([\mathrm{H}])(\mathrm{CC}(\mathrm{CC} 2)[\mathrm{C} @ @ \mathrm{H}](\mathrm{C}=\mathrm{C}) \mathrm{C}[\mathrm{N}+] 12 \mathrm{Cc} 3 \mathrm{cc}(\mathrm{C}(\mathrm{C}(\mathrm{C}(\mathrm{C}(\mathrm{C}(\mathrm{C}(\mathrm{C}(\mathrm{C}(\mathrm{F})(\mathrm{F}) \mathrm{F})(\mathrm{F}) \mathrm{F})(\mathrm{F}) \mathrm{F})(\mathrm{F}) \mathrm{F})(\mathrm{F}) \mathrm{F})(\mathrm{F})} \\
\mathrm{F})(\mathrm{F}) \mathrm{F})(\mathrm{F}) \mathrm{F}) \mathrm{cc}(\mathrm{F}) \mathrm{F} 3)[\mathrm{C} @ \mathrm{H}](\mathrm{O}) \mathrm{c} 4 \mathrm{cc}[\mathrm{n}+](\mathrm{Cc} 5 \mathrm{cc}(\mathrm{C}(\mathrm{F})(\mathrm{C}(\mathrm{C}(\mathrm{F})(\mathrm{F}) \mathrm{F})(\mathrm{F}) \mathrm{F}) \mathrm{F})(\mathrm{ccc} 5) \mathrm{c}(\mathrm{cccc} 6)(46\end{array}$ \\
\hline
\end{tabular}




\begin{tabular}{|c|c|c|c|c|}
\hline Rnd5_81 & 1.05 & 2.85 & 48.10 & $\begin{array}{l}{[\mathrm{C} @ @] 1([\mathrm{H}])(\mathrm{CC}(\mathrm{CC} 2)[\mathrm{C} @ @ \mathrm{H}](\mathrm{C}=\mathrm{C}) \mathrm{C}[\mathrm{N}+] 12 \mathrm{Cc} 3 \mathrm{cc}(\mathrm{C}(\mathrm{C}(\mathrm{C}(\mathrm{F})(\mathrm{F}) \mathrm{F})(\mathrm{F}) \mathrm{F})(\mathrm{F}) \mathrm{F}) \mathrm{cc}(\mathrm{F}) \mathrm{c} 3)[\mathrm{C} @ \mathrm{H}](\mathrm{O}) \mathrm{c} 4 \mathrm{cc}} \\
{[\mathrm{n}+](\mathrm{Cc} 5 \mathrm{cc}(\mathrm{F}) \mathrm{ccc} 5) \mathrm{c}(\mathrm{cccc} 6) \mathrm{c} 46}\end{array}$ \\
\hline Rnd5_82 & 1.02 & 2.78 & 47.15 & $\begin{array}{l}{[\mathrm{C} @ @] 1([\mathrm{H}])(\mathrm{CC}(\mathrm{CC} 2)[\mathrm{C} @ @ \mathrm{H}](\mathrm{C}=\mathrm{C}) \mathrm{C}[\mathrm{N}+] 12 \mathrm{Cc} 3 \mathrm{cc}(\mathrm{C}(\mathrm{C}(\mathrm{C}(\mathrm{C}(\mathrm{C}(\mathrm{C}(\mathrm{C}(\mathrm{C}(\mathrm{F})(\mathrm{F}) \mathrm{F})(\mathrm{F}) \mathrm{F})(\mathrm{F}) \mathrm{F})(\mathrm{F}) \mathrm{F})(\mathrm{F}) \mathrm{F})(\mathrm{F})} \\
\mathrm{F})(\mathrm{F}) \mathrm{F})(\mathrm{F}) \mathrm{F}) \mathrm{cc}(\mathrm{F}) \mathrm{c} 3)[\mathrm{C} @ \mathrm{H}](\mathrm{O}) \mathrm{c} 4 \mathrm{cc}[\mathrm{n}+](\mathrm{Cc} 5 \mathrm{ccc}(\mathrm{c} 6 \mathrm{c}(\mathrm{cccc} 7) \mathrm{c} 7 \mathrm{ccc} 6) \operatorname{cc} 5) \mathrm{c}(\mathrm{cccc}) \mathrm{c} 48\end{array}$ \\
\hline Rnd5_83 & 1.01 & 2.74 & 46.47 & $\begin{array}{l}{[\mathrm{C} @ @] 1([\mathrm{H}])(\mathrm{CC}(\mathrm{CC} 2)[\mathrm{C} @ @ \mathrm{H}](\mathrm{C}=\mathrm{C}) \mathrm{C}[\mathrm{N}+] 12 \mathrm{Cc} 3 \mathrm{cc}(\mathrm{C}(\mathrm{C}(\mathrm{C}(\mathrm{C}(\mathrm{C}(\mathrm{C}(\mathrm{C}(\mathrm{C}(\mathrm{F})(\mathrm{F}) \mathrm{F})(\mathrm{F}) \mathrm{F})(\mathrm{F}) \mathrm{F})(\mathrm{F}) \mathrm{F})(\mathrm{F}) \mathrm{F})(\mathrm{F})} \\
\mathrm{F})(\mathrm{F}) \mathrm{F})(\mathrm{F}) \mathrm{F}) \mathrm{cc}(\mathrm{F}) \mathrm{c} 3)[\mathrm{C} @ \mathrm{H}](\mathrm{O}) \mathrm{c} 4 \mathrm{cc}[\mathrm{n}+](\mathrm{Cc} 5 \mathrm{ccc}(\mathrm{C}(\mathrm{F})(\mathrm{F}) \mathrm{F}) \mathrm{cc} 5) \mathrm{c}(\mathrm{cccc} 6) \mathrm{c} 46\end{array}$ \\
\hline Rnd5_84 & 0.99 & 2.68 & 45.69 & $\begin{array}{l}{[C @ @] 1([\mathrm{H}])(\mathrm{CC}(\mathrm{CC} 2)[\mathrm{C} @ @ \mathrm{H}](\mathrm{C}=\mathrm{C}) \mathrm{C}[\mathrm{N}+] 12 \mathrm{Cc} 3 \mathrm{cc}(\mathrm{C}(\mathrm{C}(\mathrm{F})(\mathrm{F}) \mathrm{F})(\mathrm{F}) \mathrm{F}) \mathrm{cc}(\mathrm{F}) \mathrm{c} 3)[\mathrm{C} @ \mathrm{H}](\mathrm{O}) \mathrm{c} 4 \mathrm{cc}[\mathrm{n}+](\mathrm{C}} \\
\mathrm{c5} 5 \mathrm{cc}(\mathrm{F}) \mathrm{ccc} 5) \mathrm{c}(\mathrm{cccc} 6) \mathrm{c} 46\end{array}$ \\
\hline Rnd5_85 & 0.77 & 2.17 & 36.85 & $\begin{array}{l}{[\mathrm{C} @ @] 1([\mathrm{H}])(\mathrm{CC}(\mathrm{CC} 2)[\mathrm{C} @ @ \mathrm{H}](\mathrm{C}=\mathrm{C}) \mathrm{C}[\mathrm{N}+] 12 \mathrm{Cc} 3 \mathrm{cc}(\mathrm{C}(\mathrm{C}(\mathrm{C}(\mathrm{C}(\mathrm{C}(\mathrm{C}(\mathrm{C}(\mathrm{C}(\mathrm{F})(\mathrm{F}) \mathrm{F})(\mathrm{F}) \mathrm{F})(\mathrm{F}) \mathrm{F})(\mathrm{F}) \mathrm{F})(\mathrm{F}) \mathrm{F})(\mathrm{F})} \\
\mathrm{F})(\mathrm{F}) \mathrm{F})(\mathrm{F}) \mathrm{F}) \mathrm{cc}(\mathrm{F}) \mathrm{c} 3)[\mathrm{C} @ \mathrm{H}](\mathrm{O}) \mathrm{c} 4 \mathrm{cc}[\mathrm{n}+](\mathrm{Cc} 5 \mathrm{cc}(\mathrm{F}) \mathrm{ccc} 5) \mathrm{c}(\mathrm{cccc} 6) \mathrm{c} 46\end{array}$ \\
\hline
\end{tabular}


3.6 Table of all molecular structures

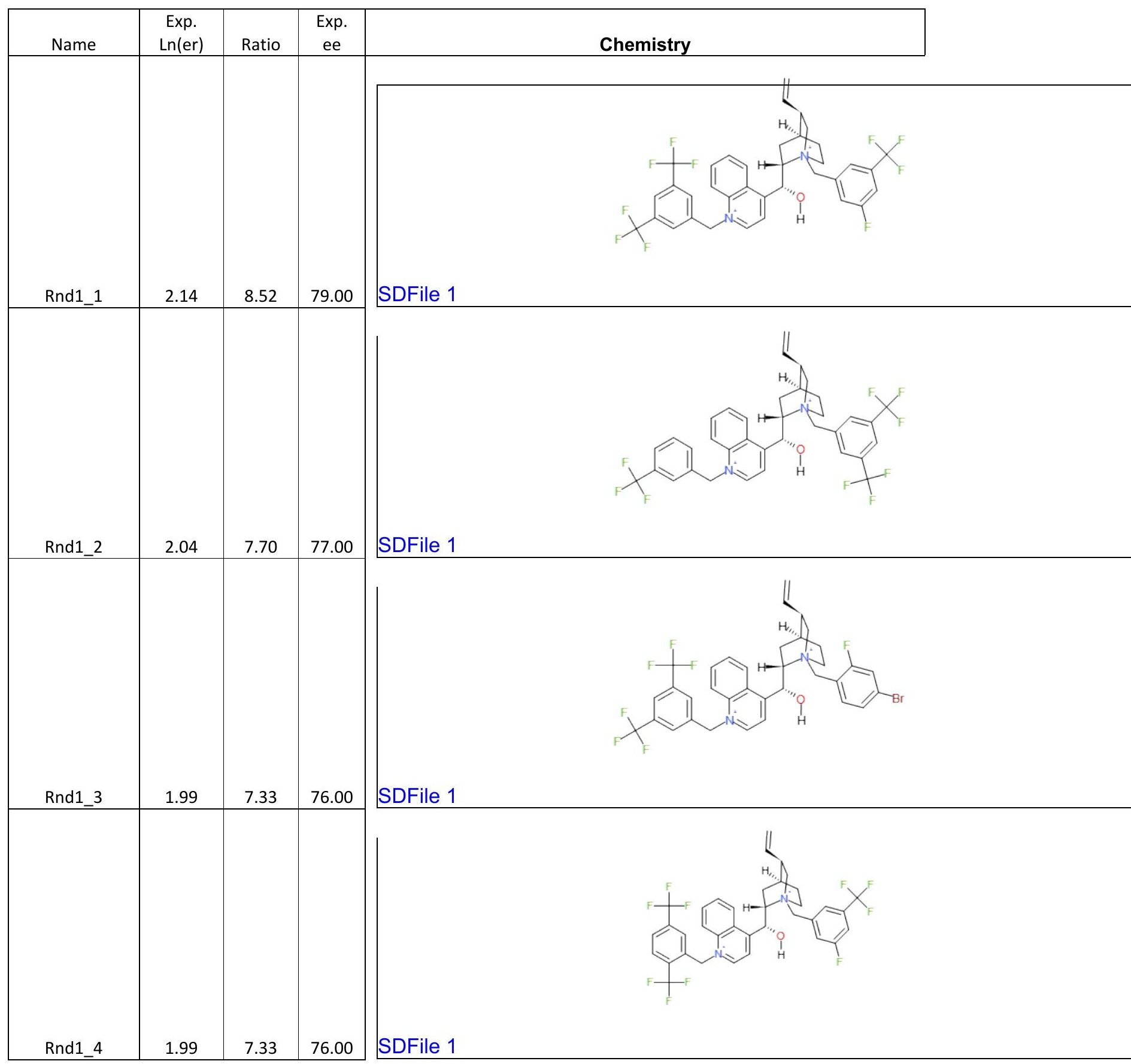

69 


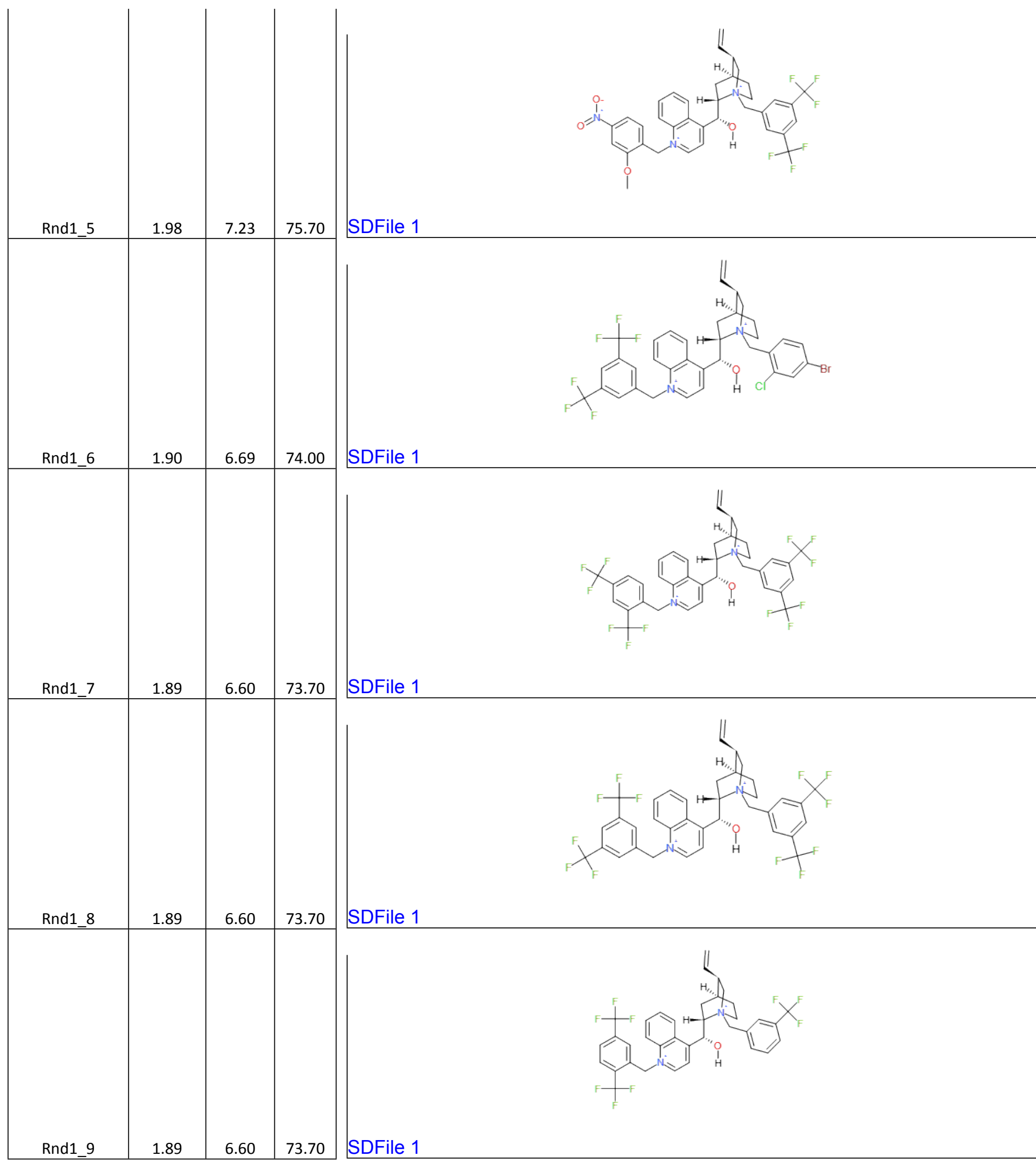

70 


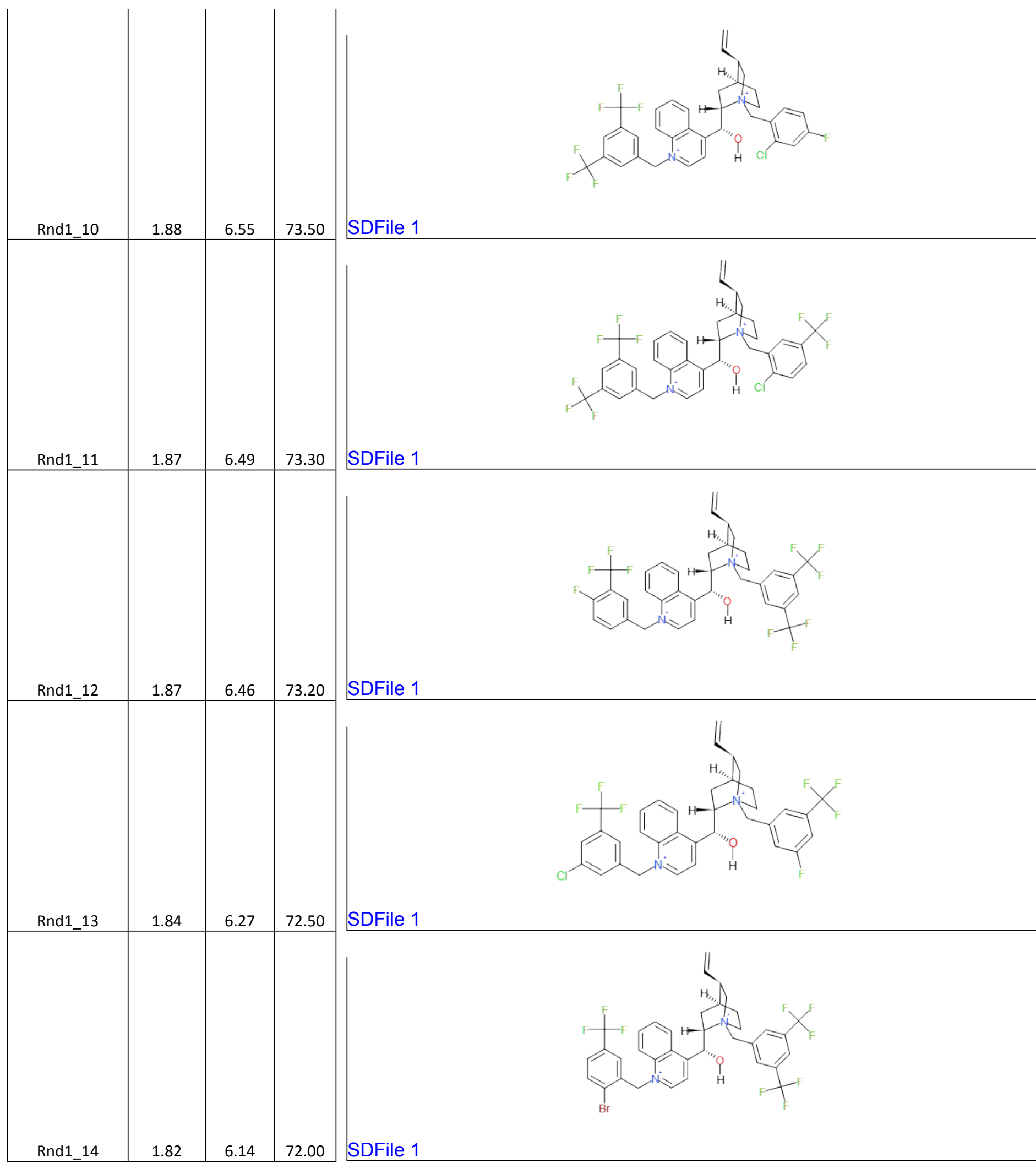

71 


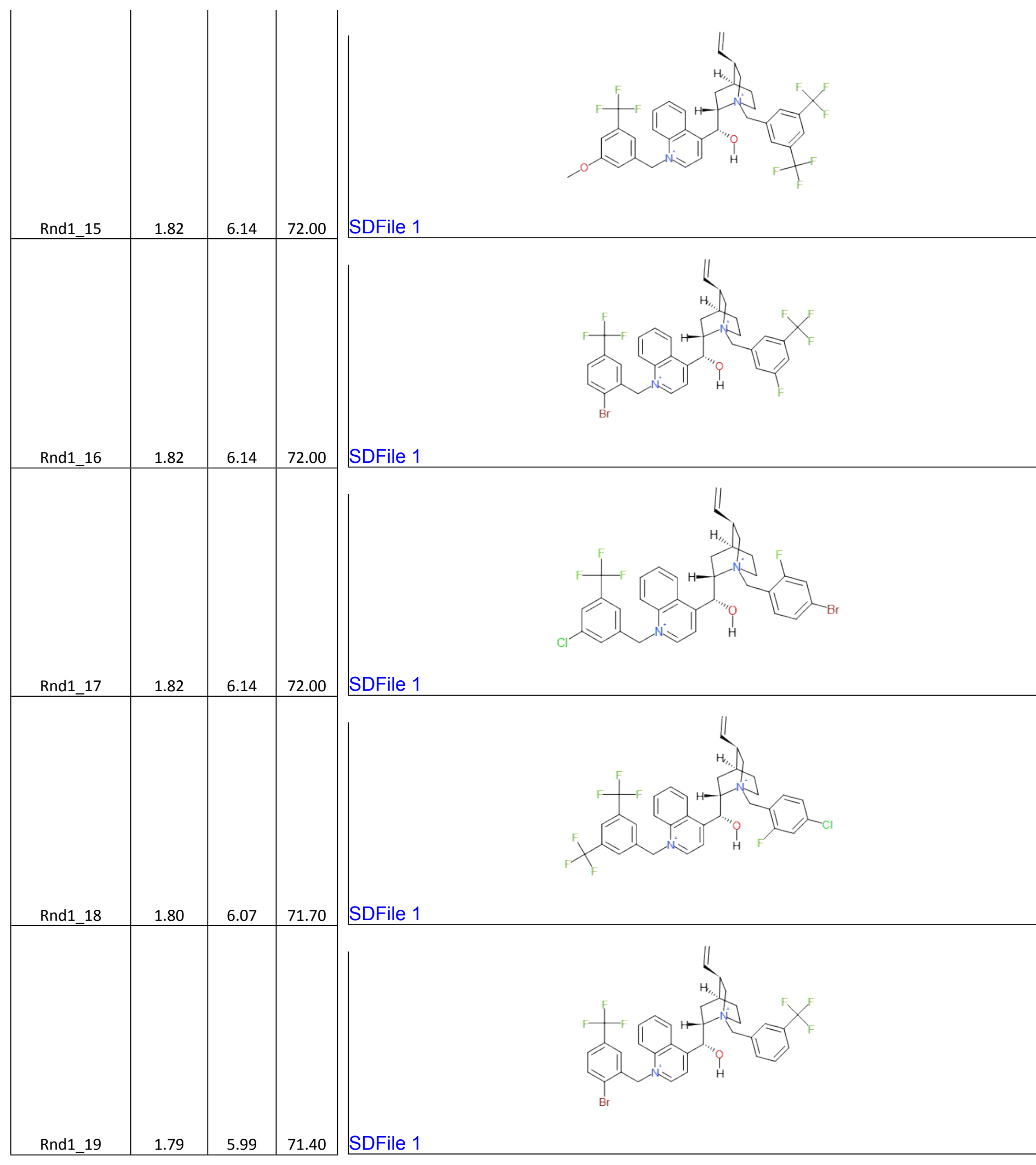




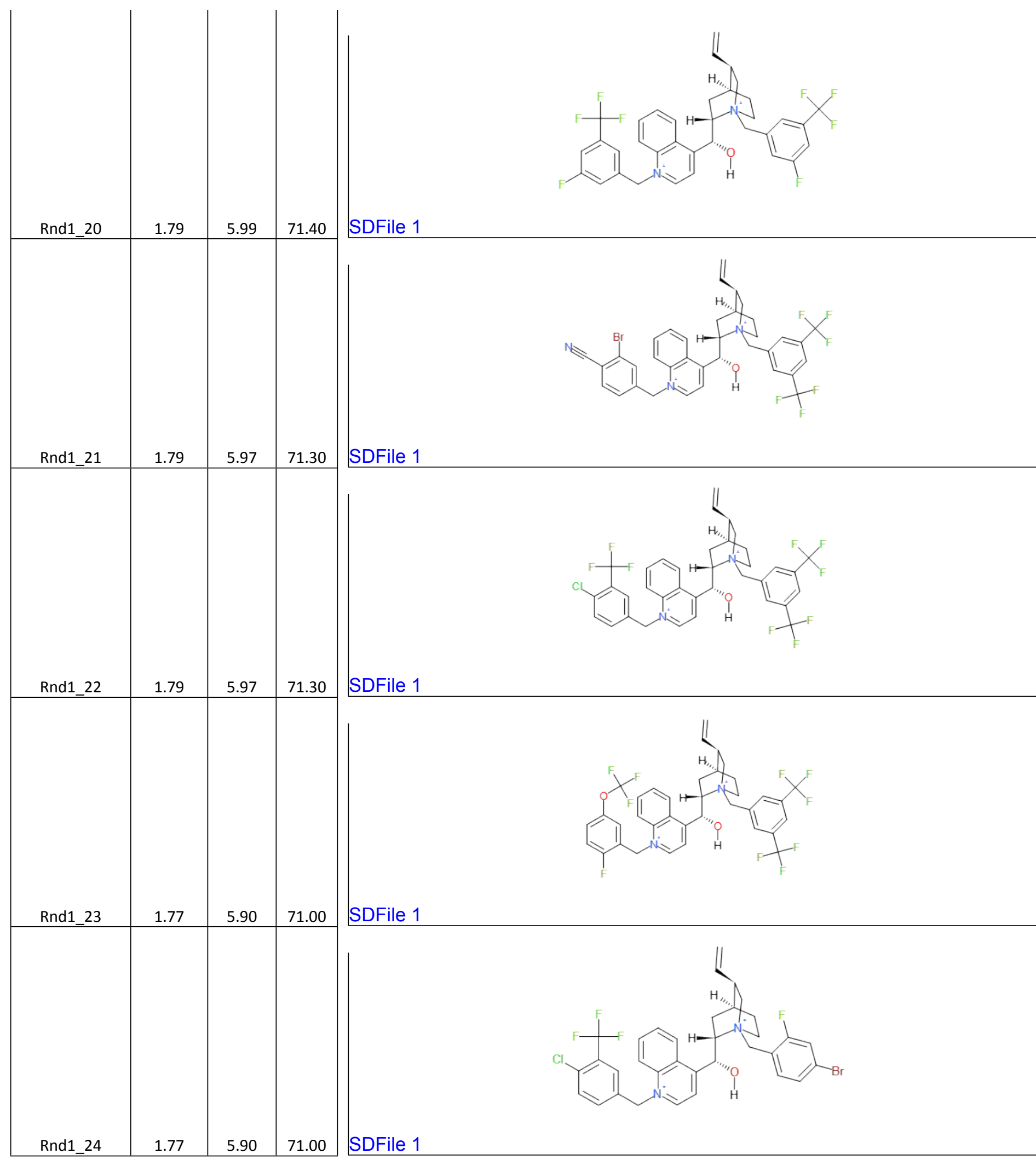




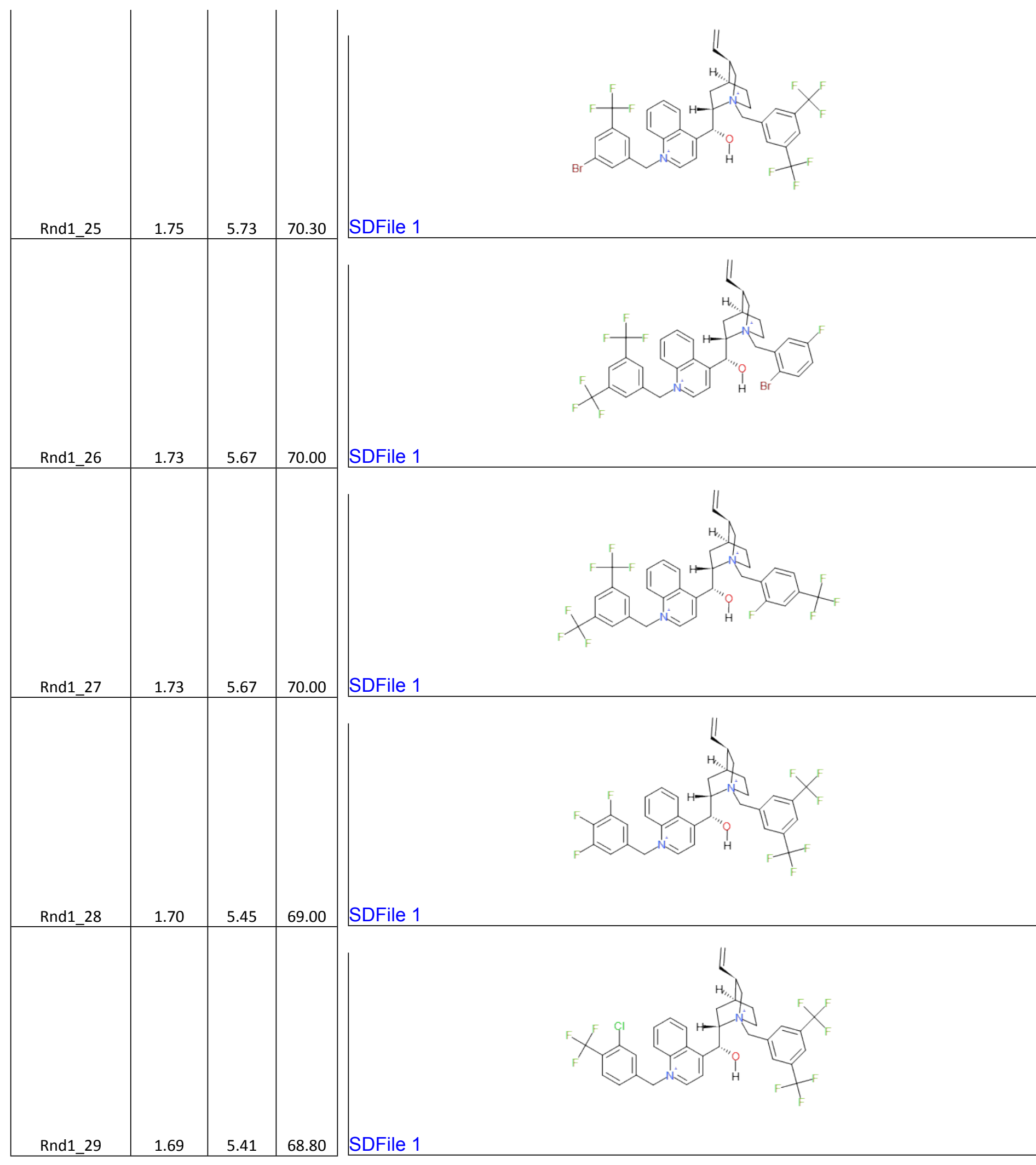




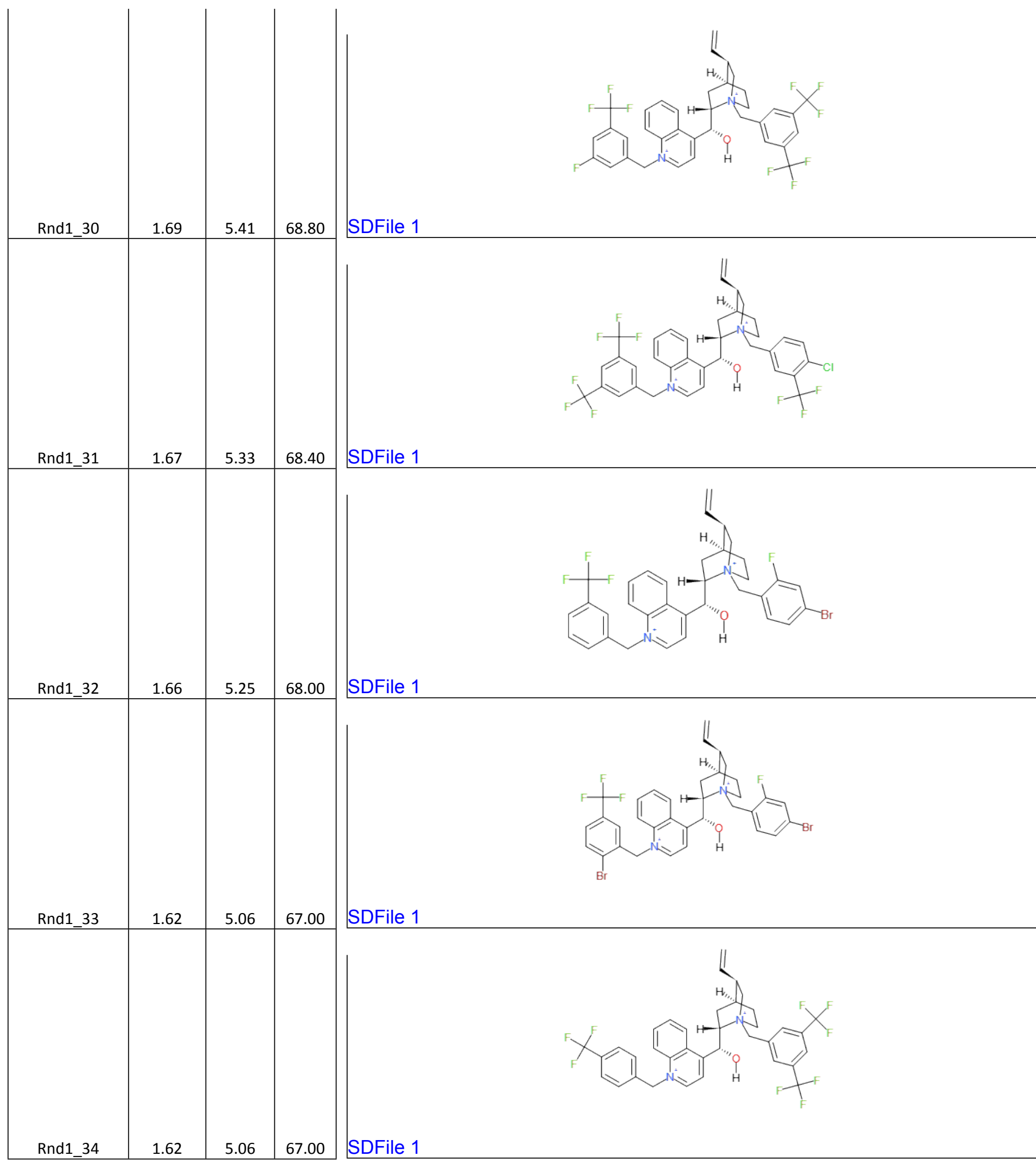

75 


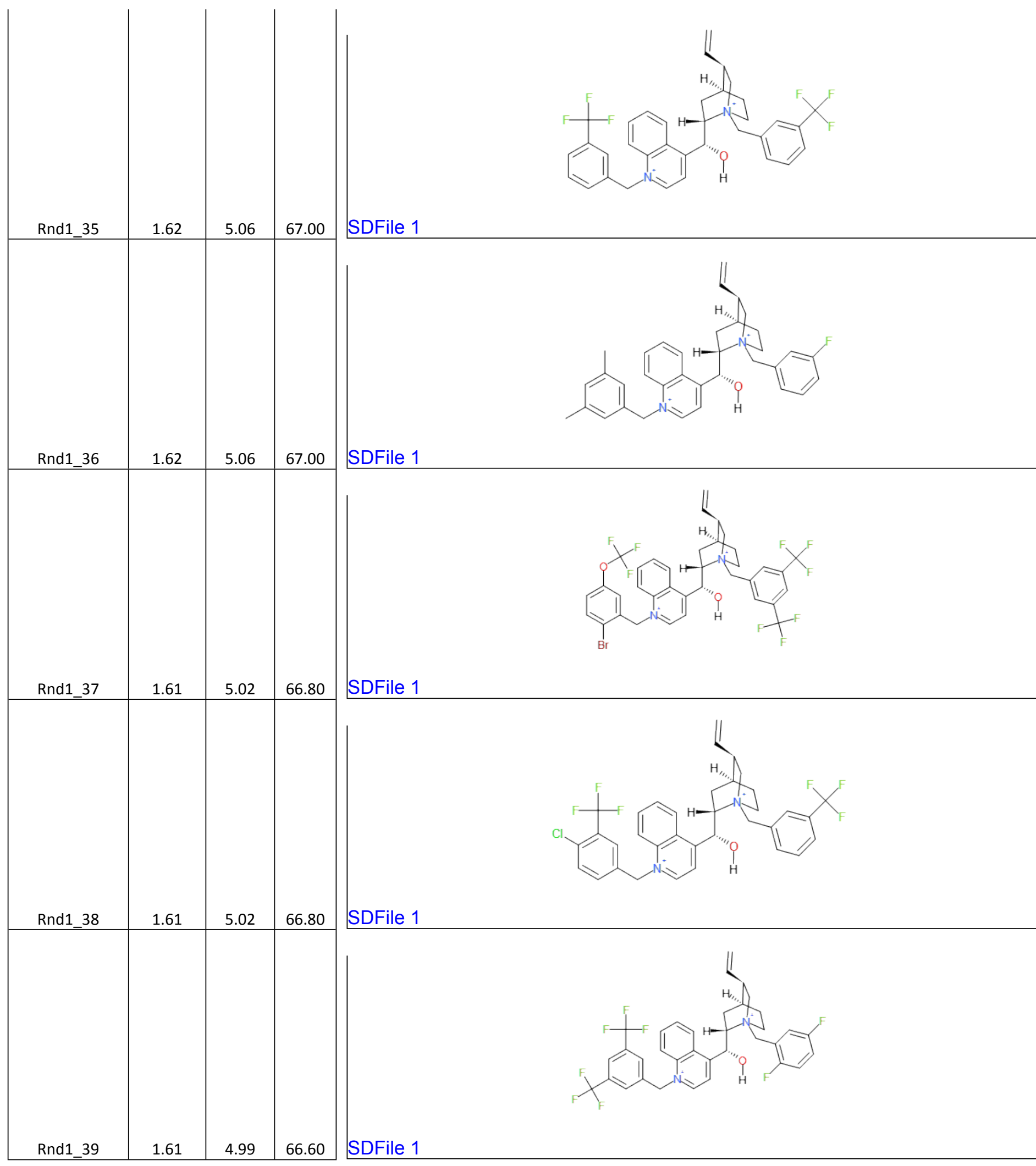

76 


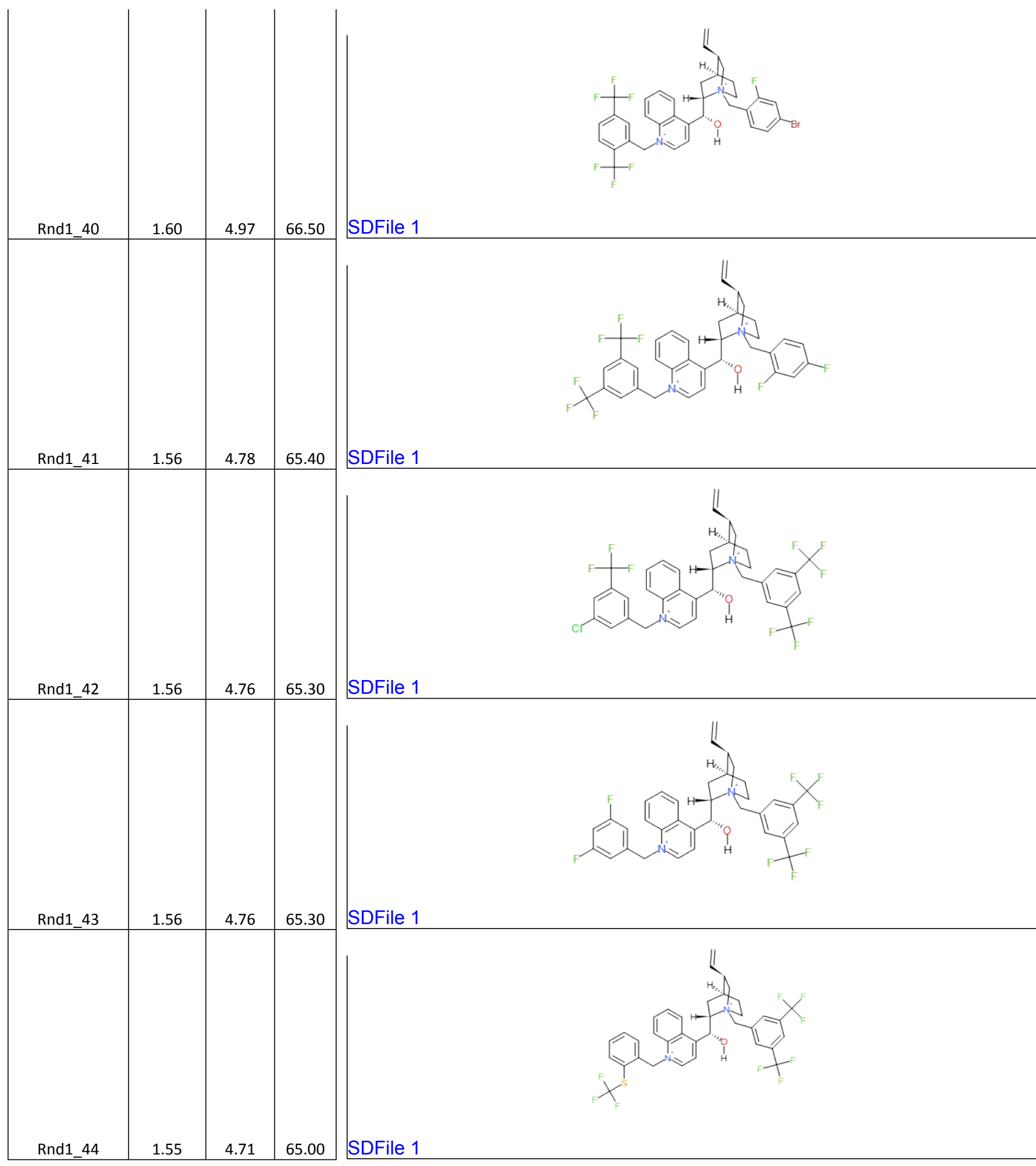

77 


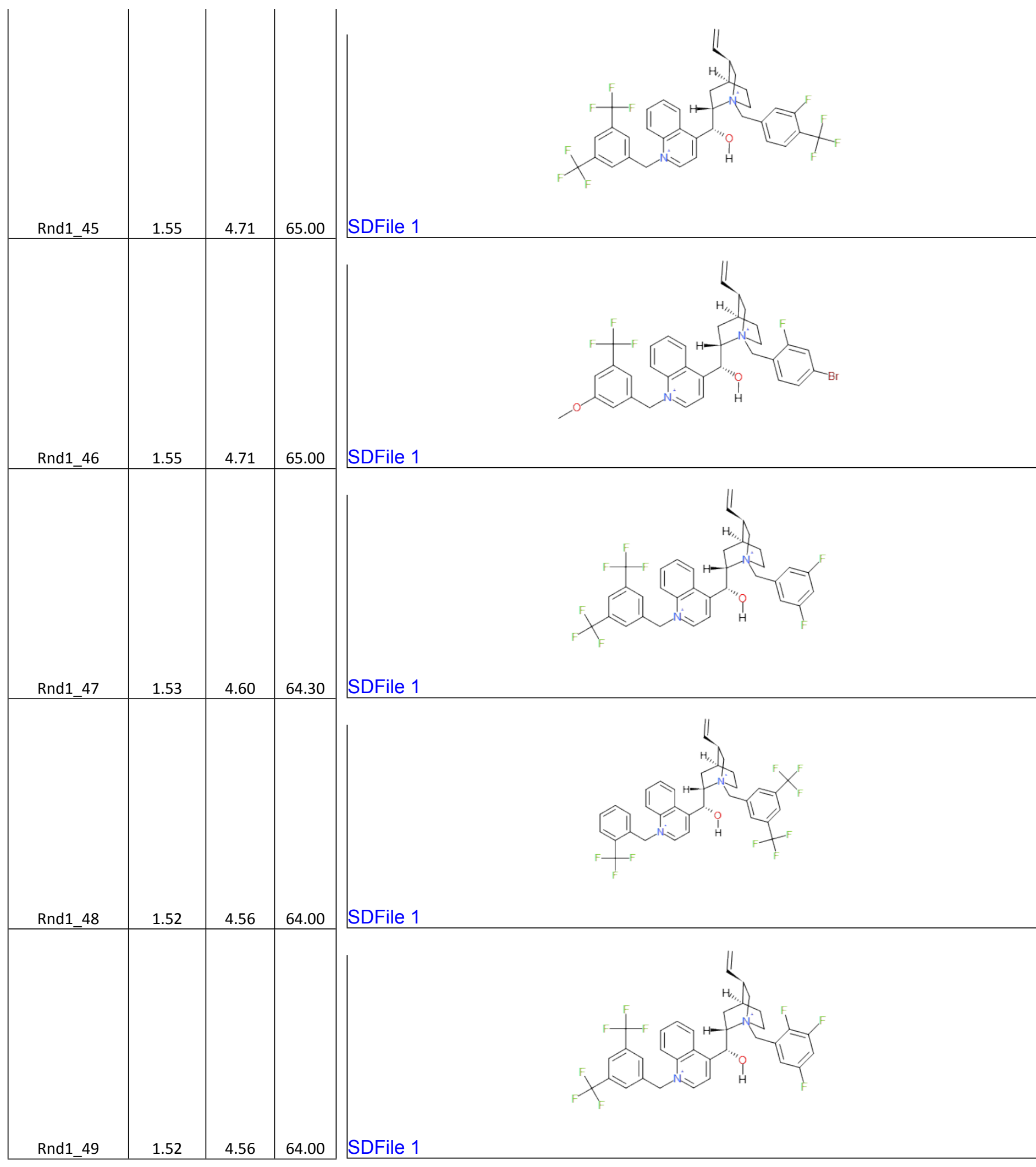

78 


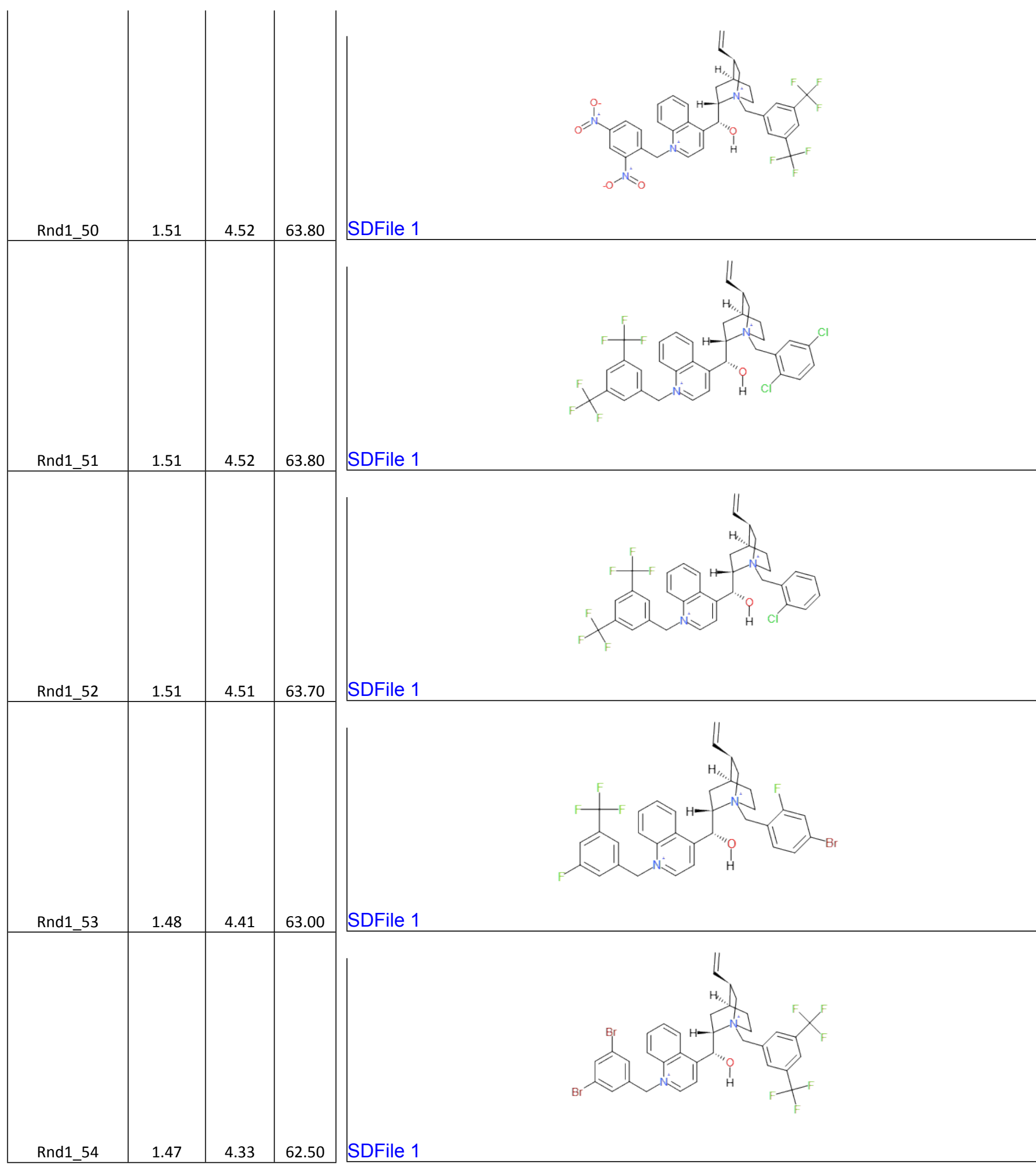

79 


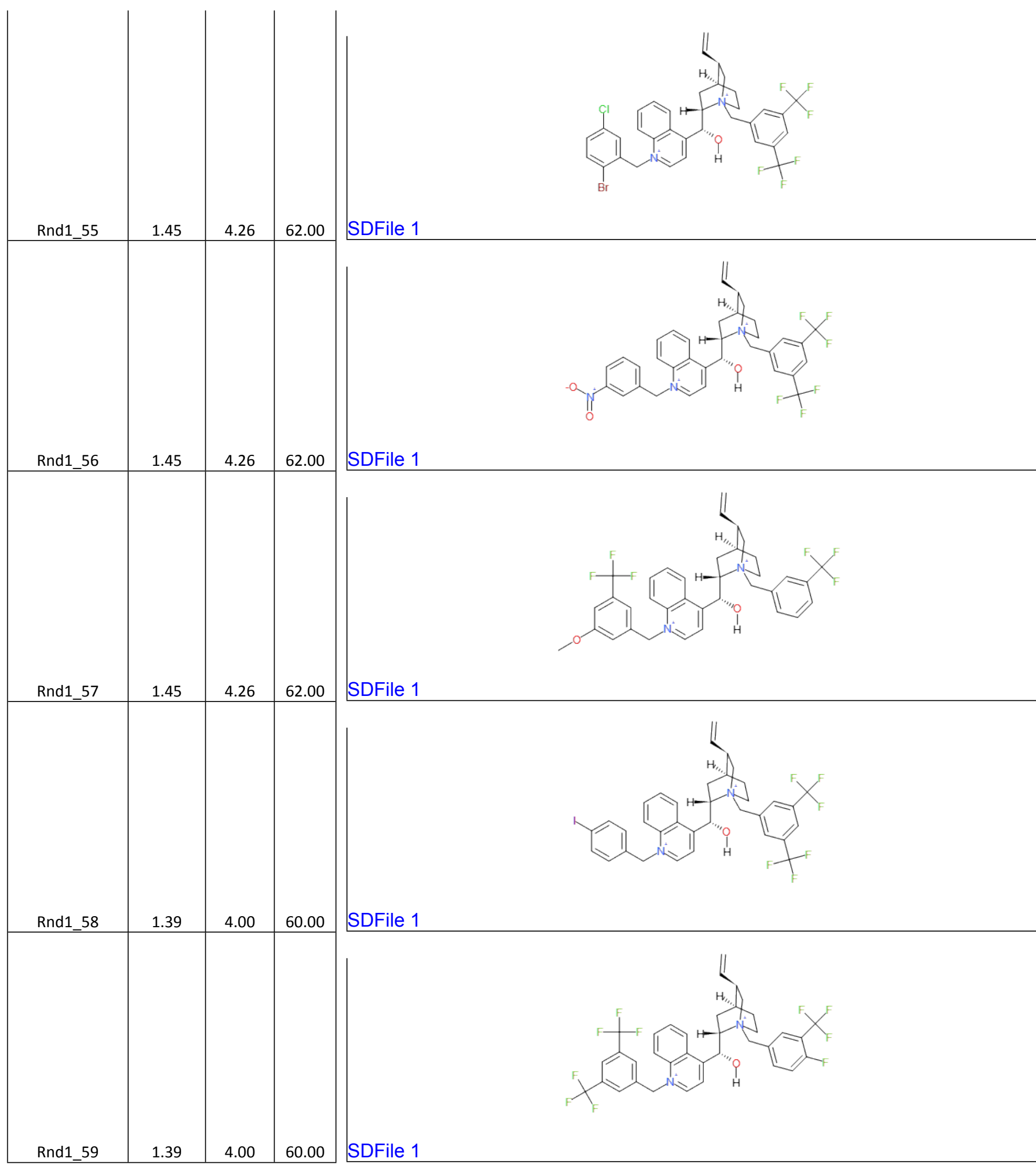

80 


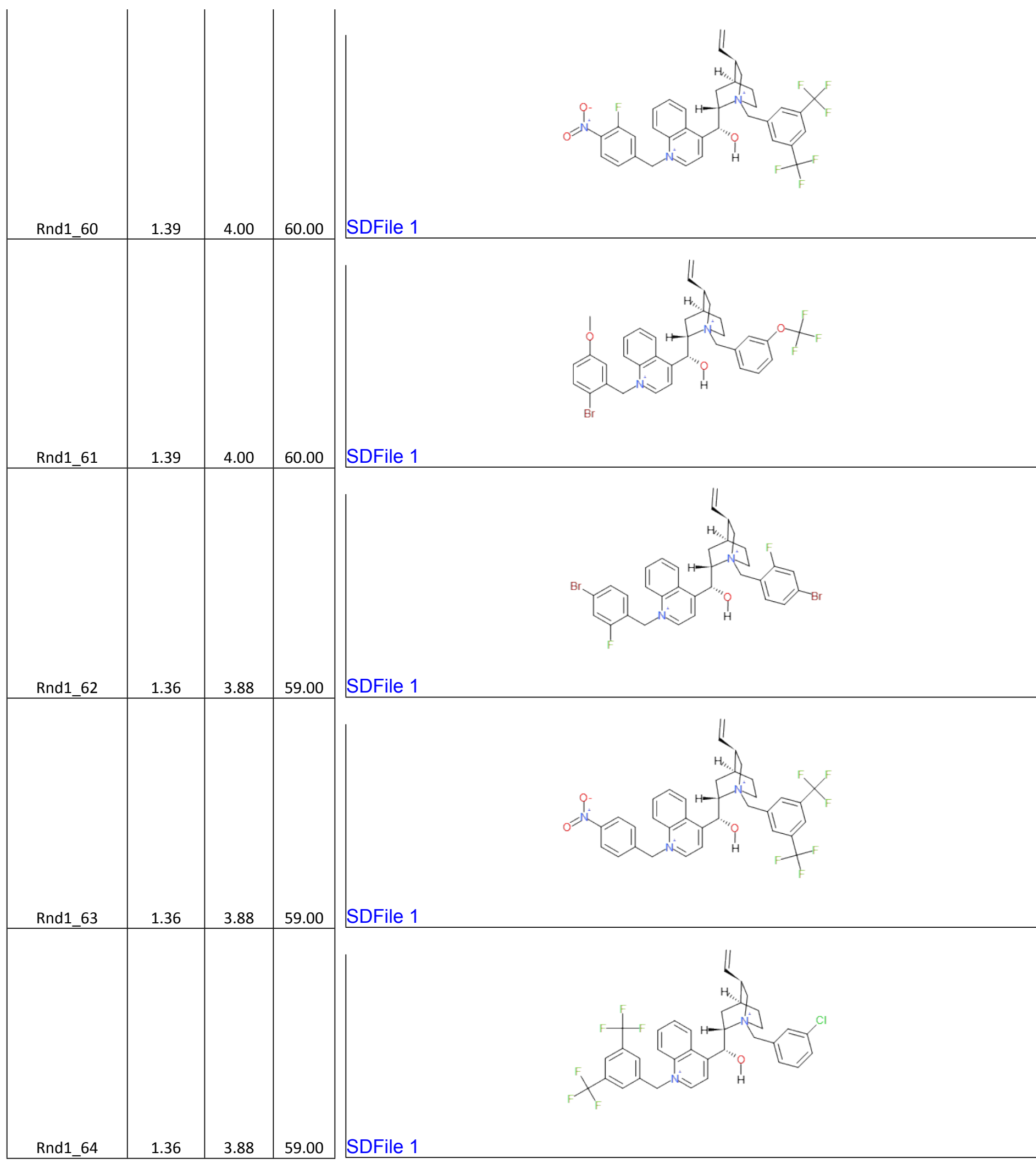




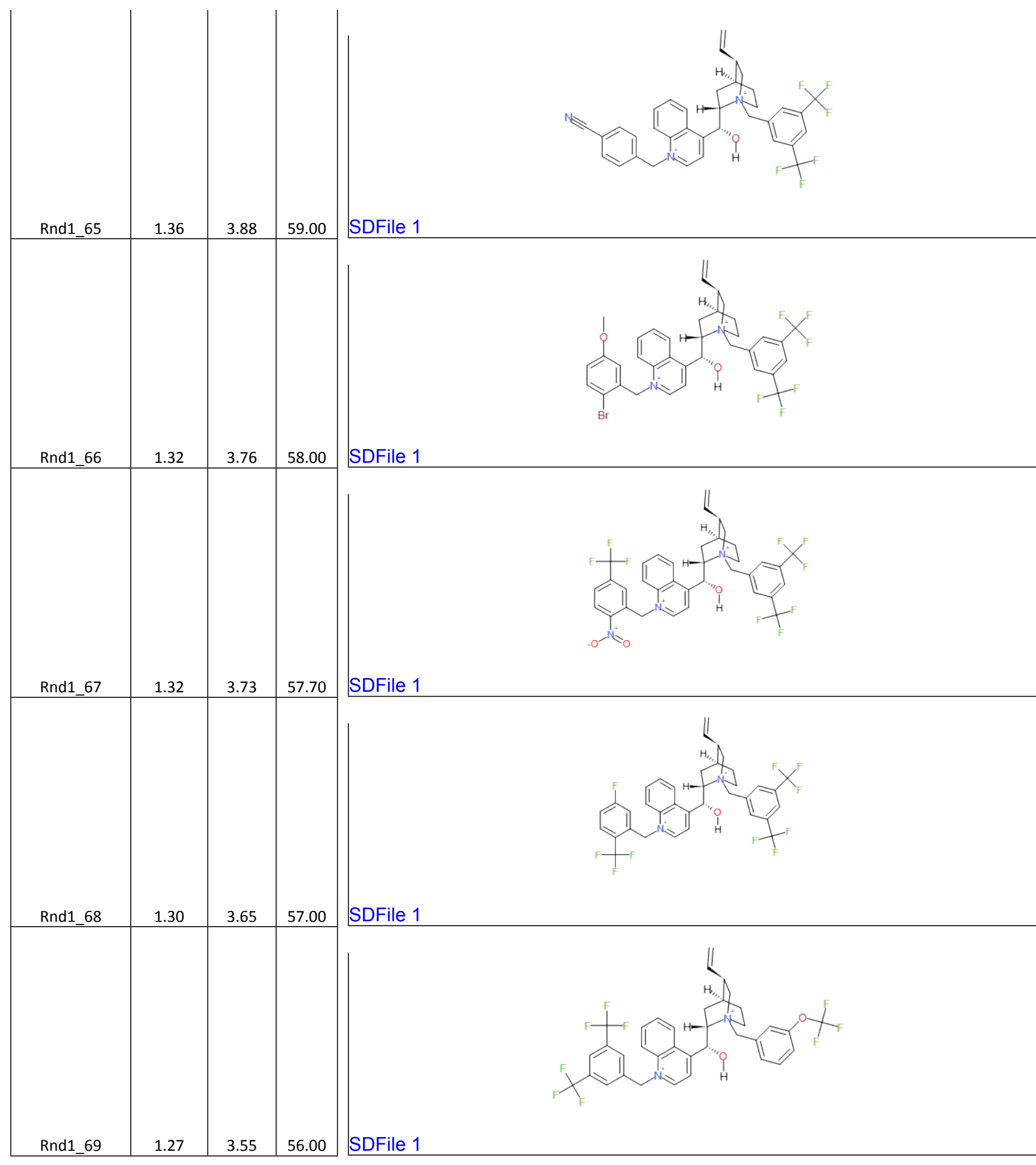




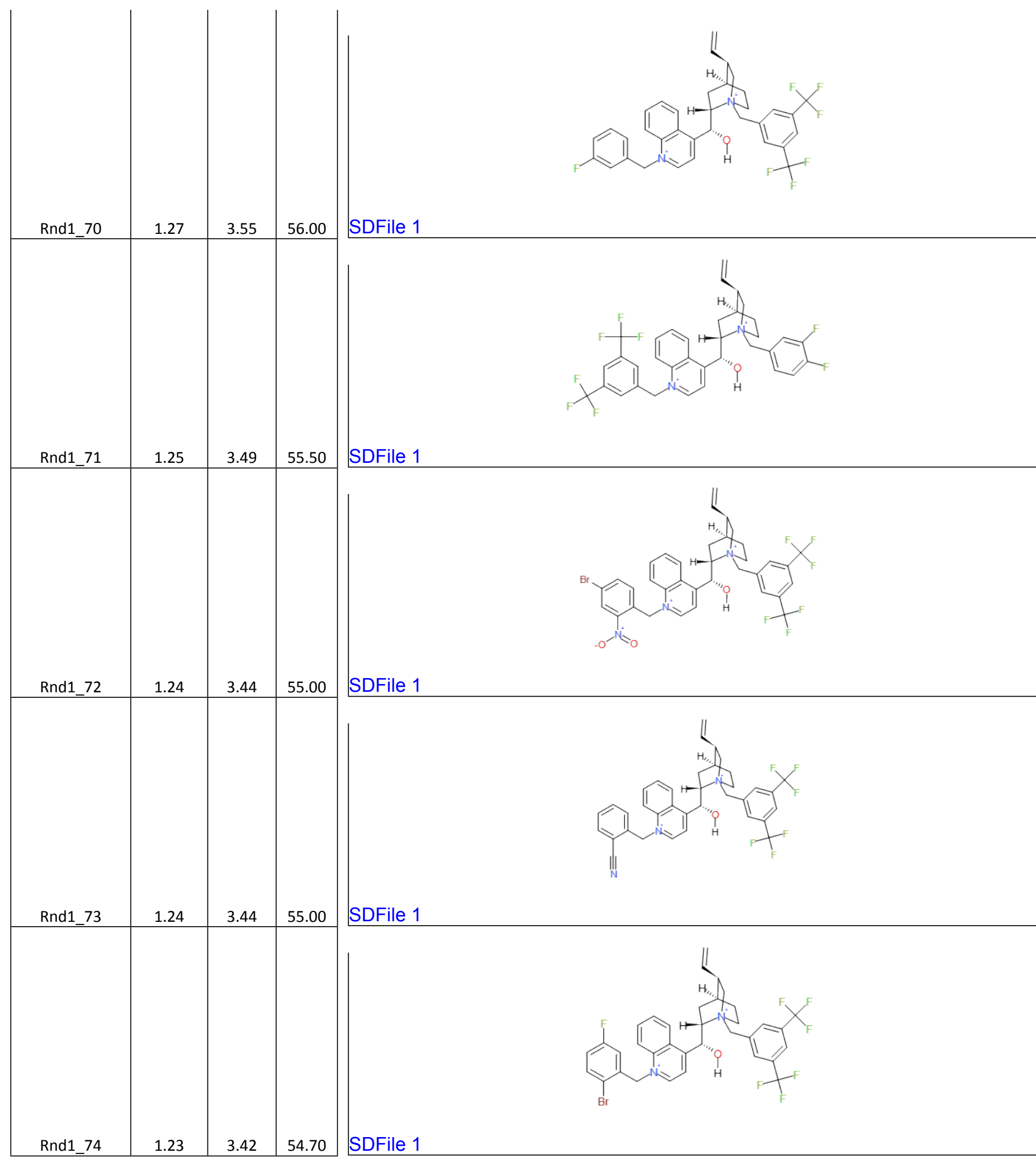




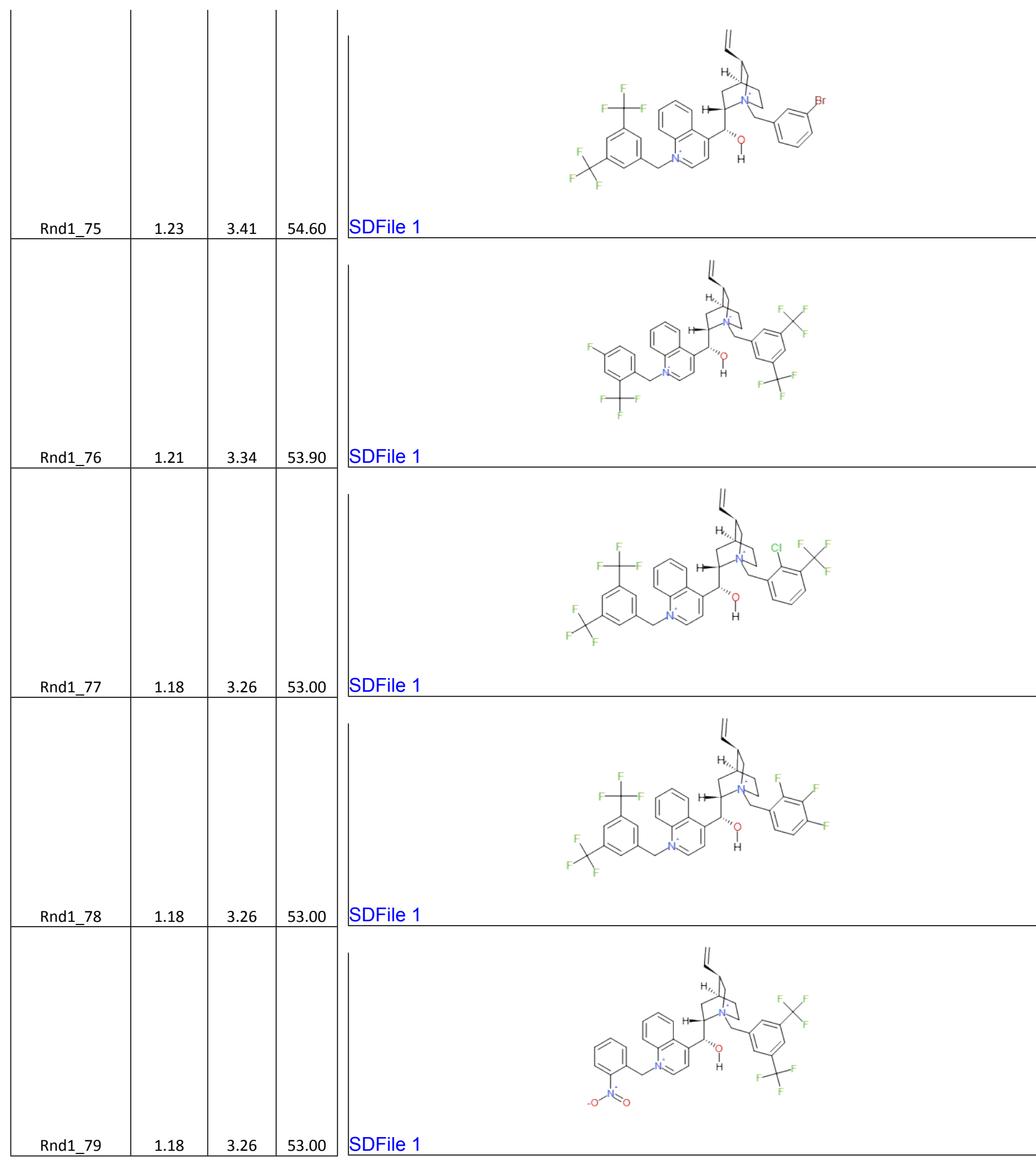




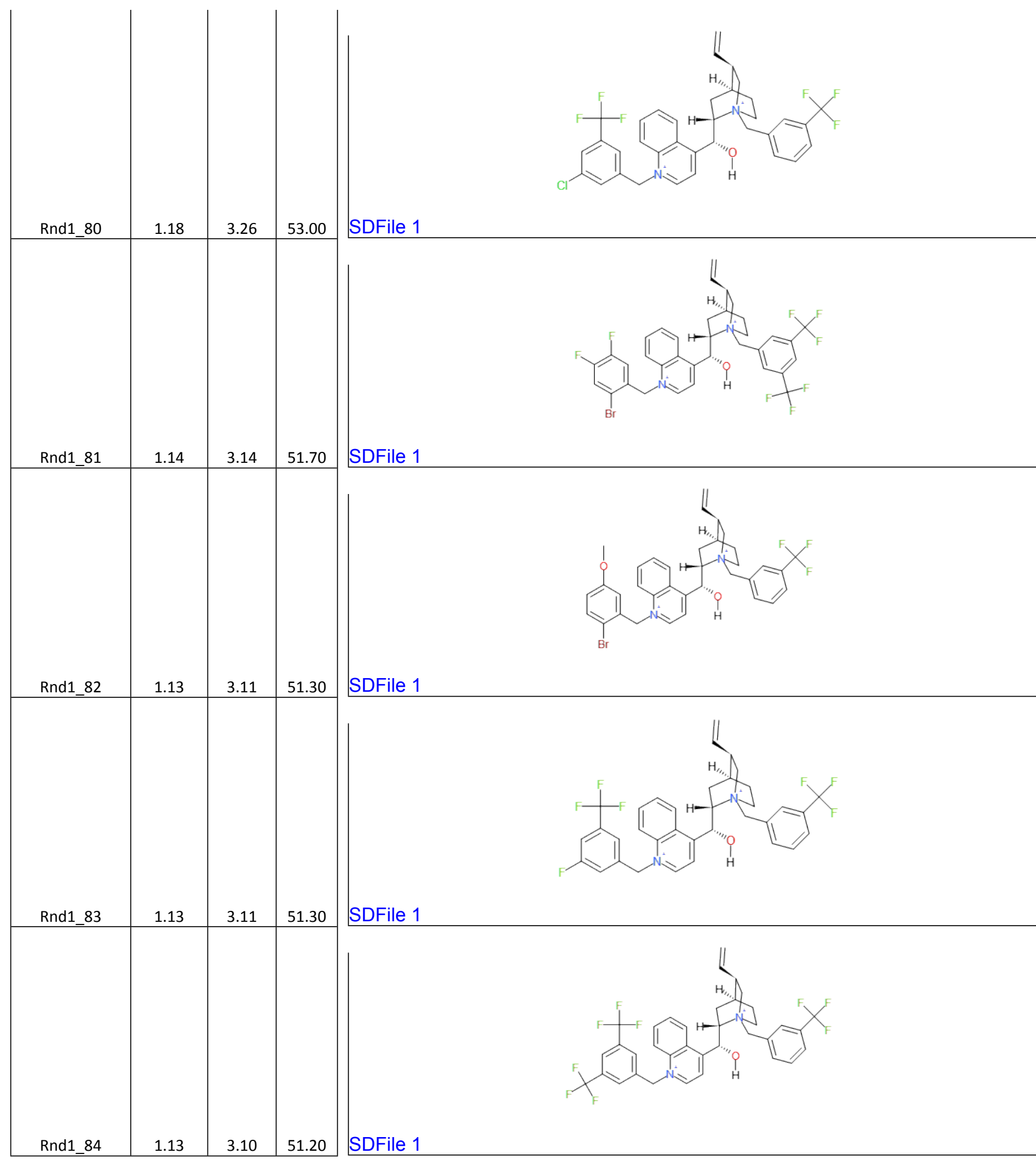




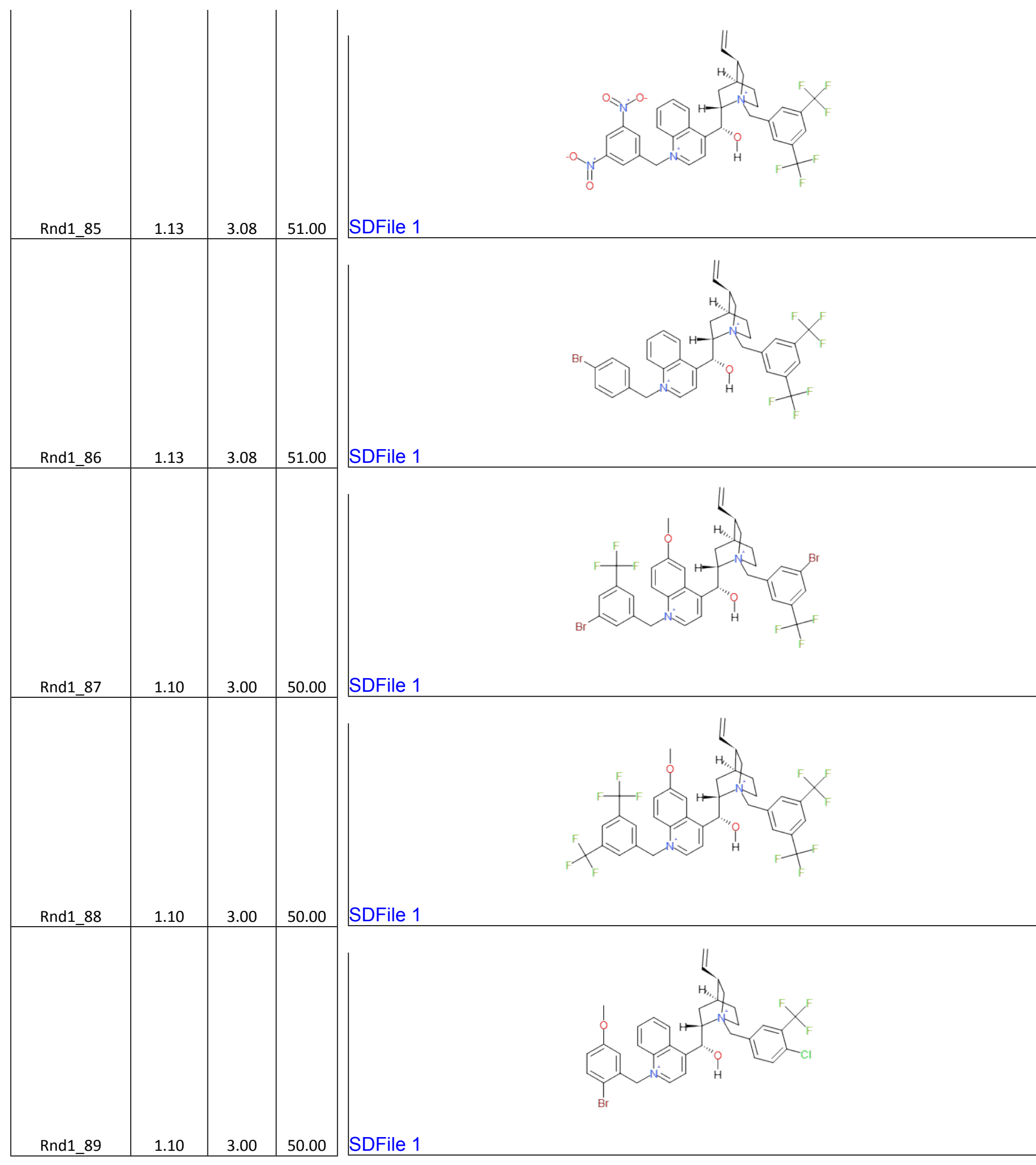




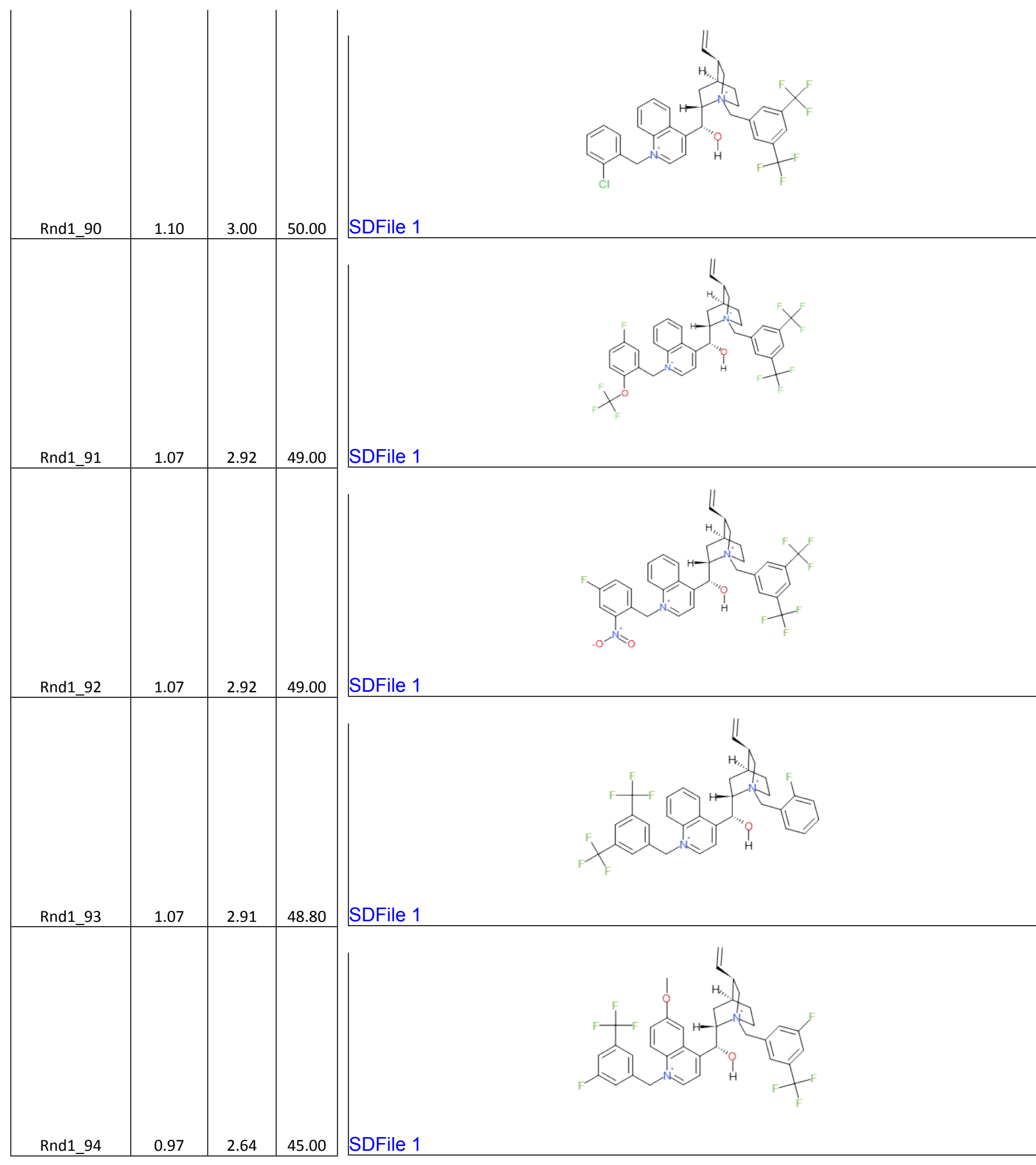




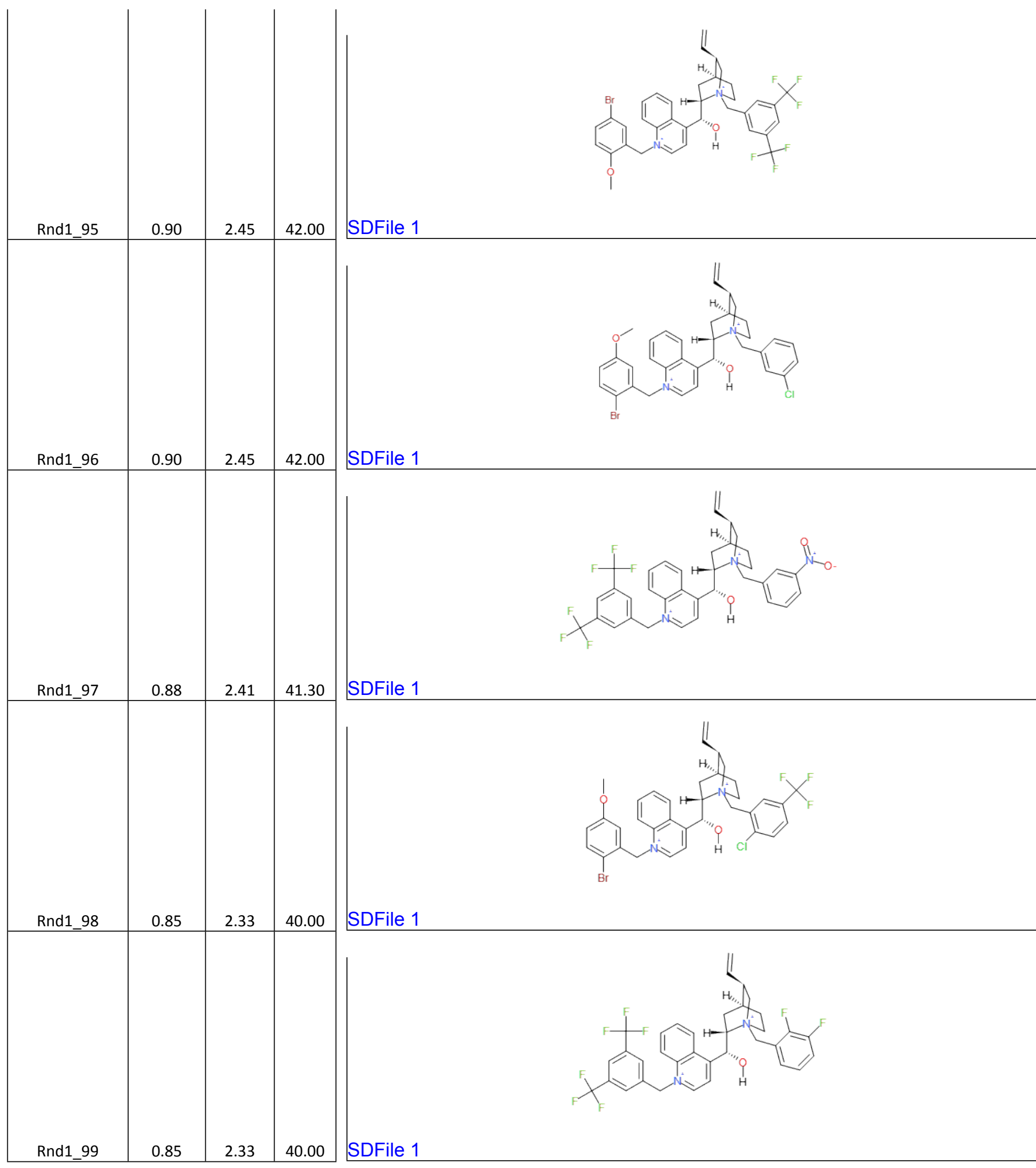




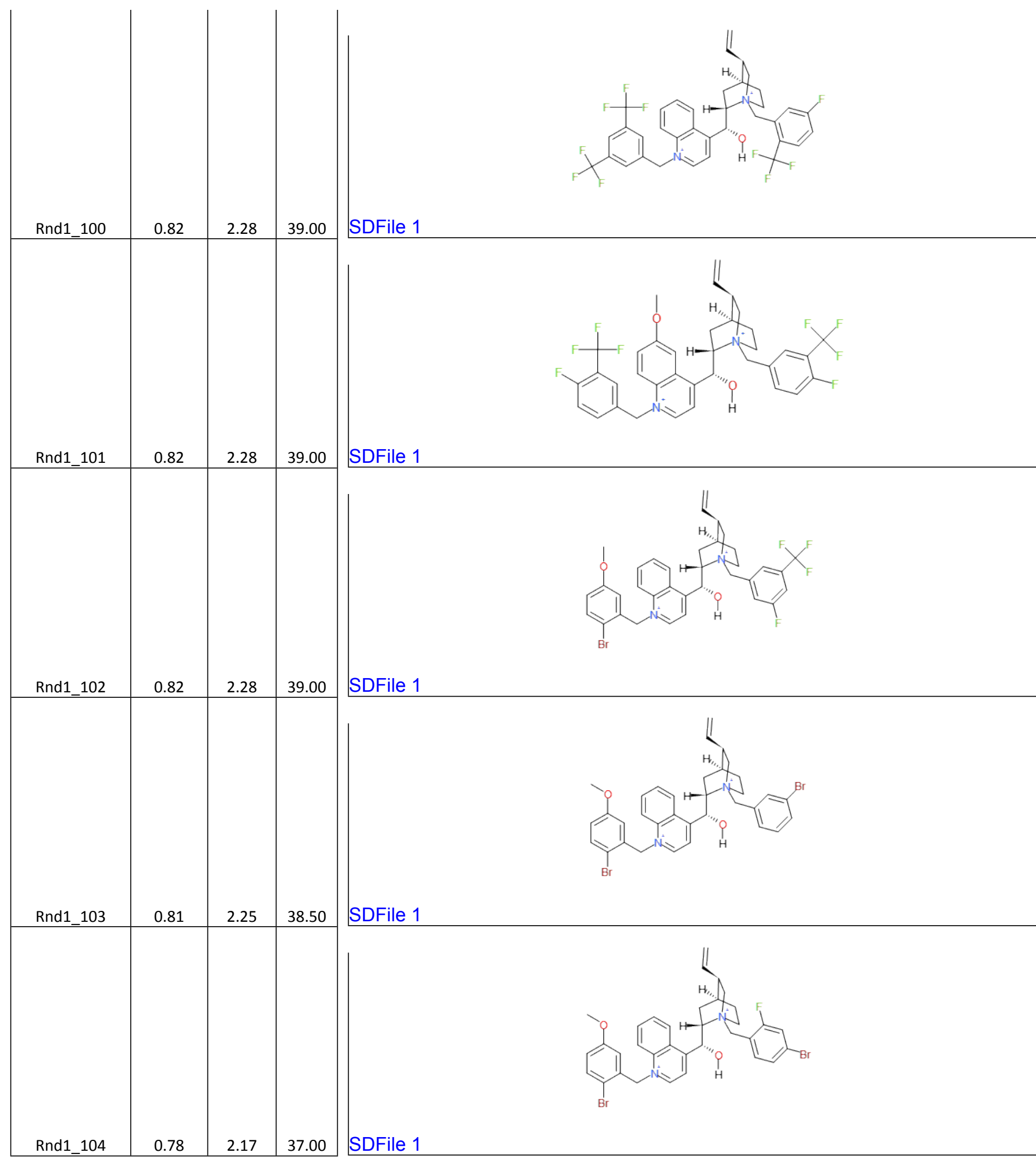




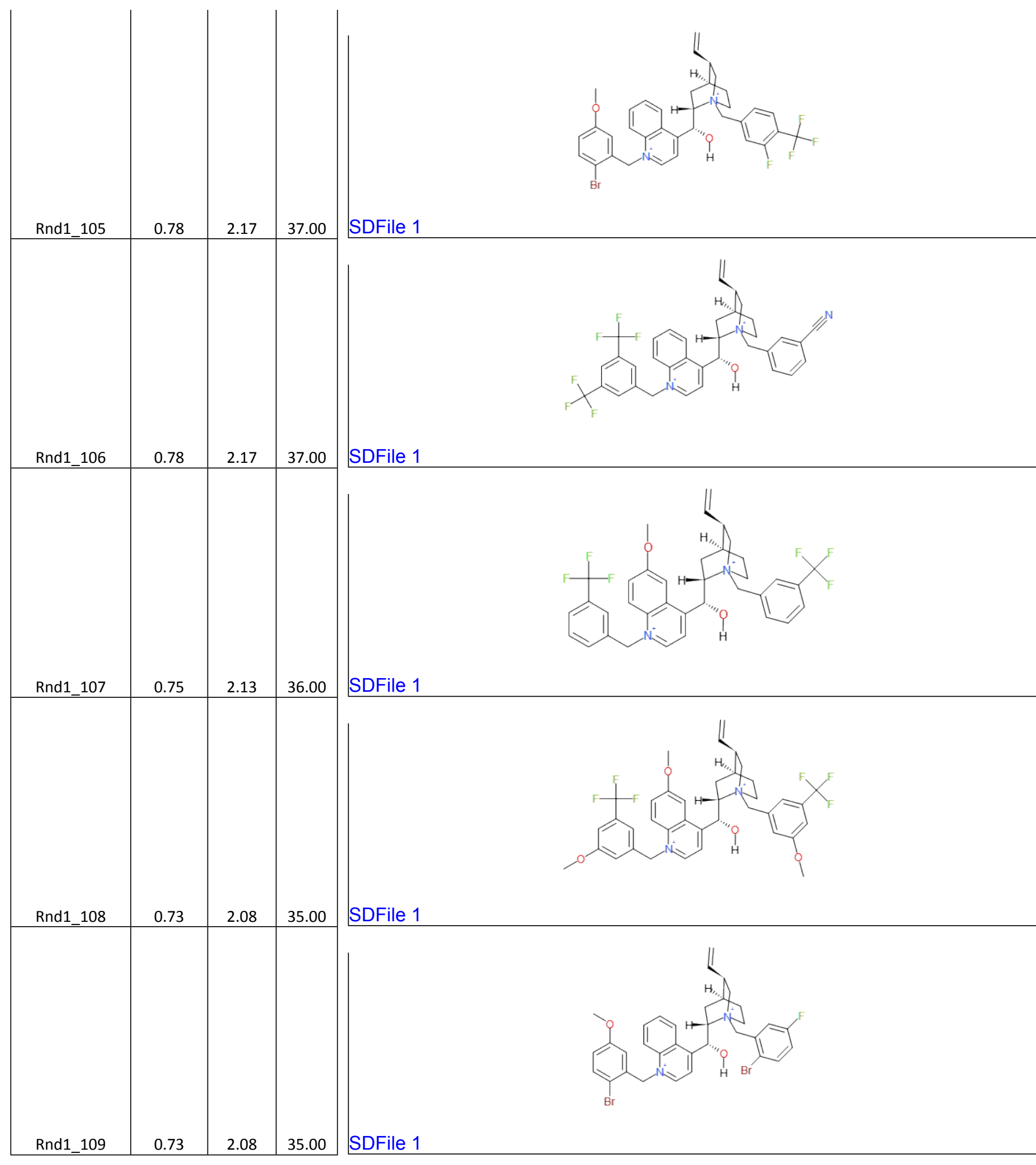




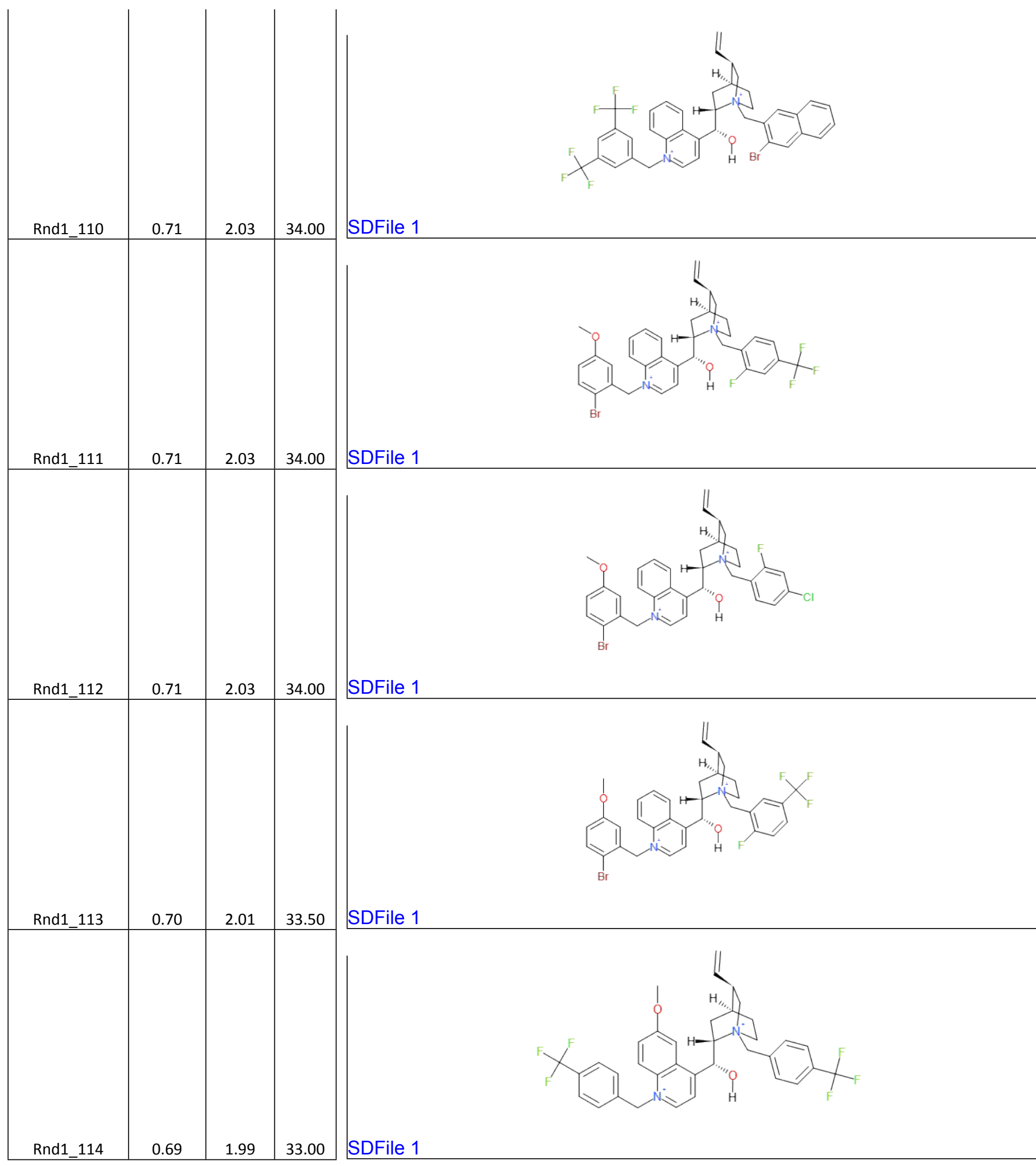

91 


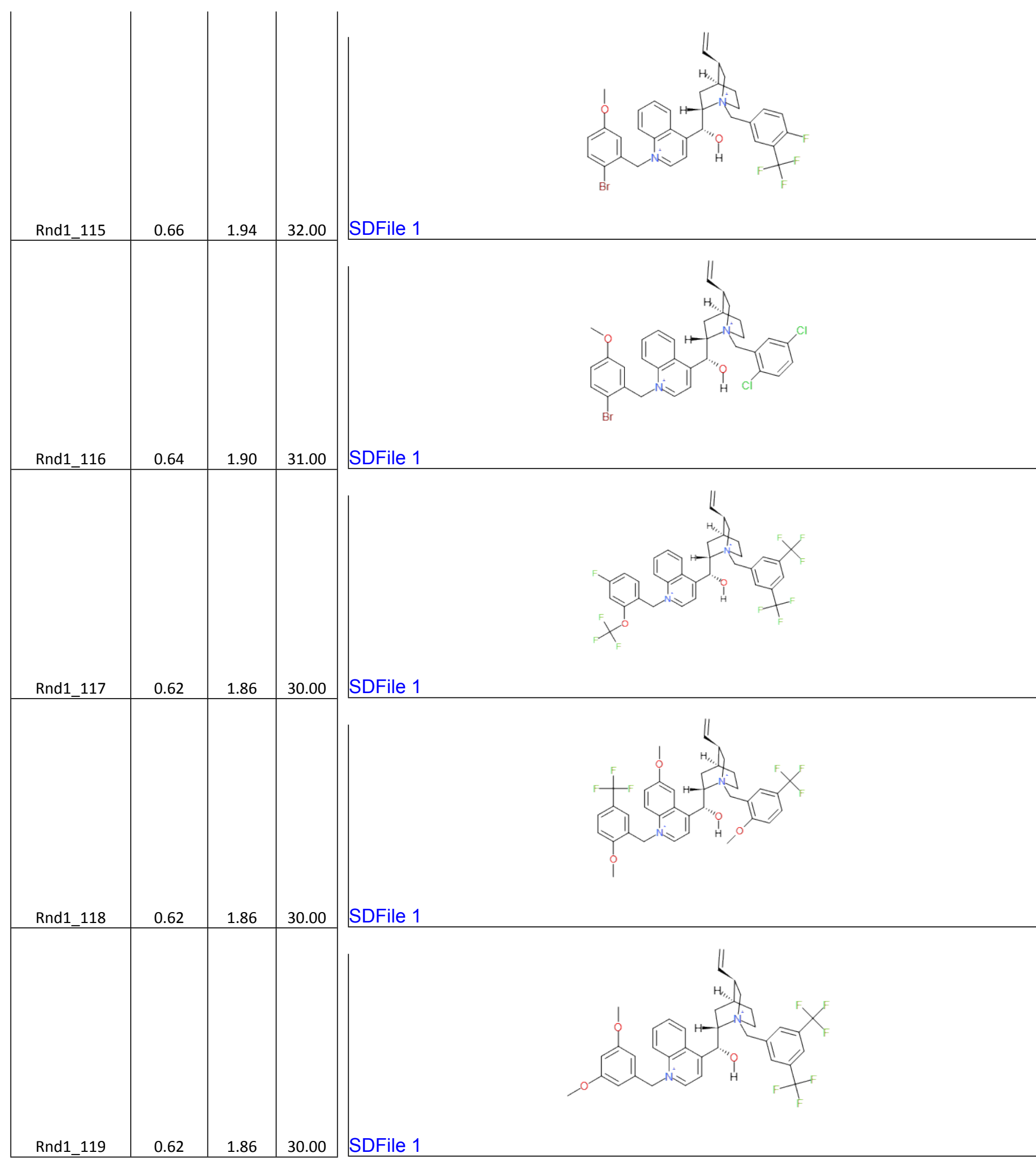




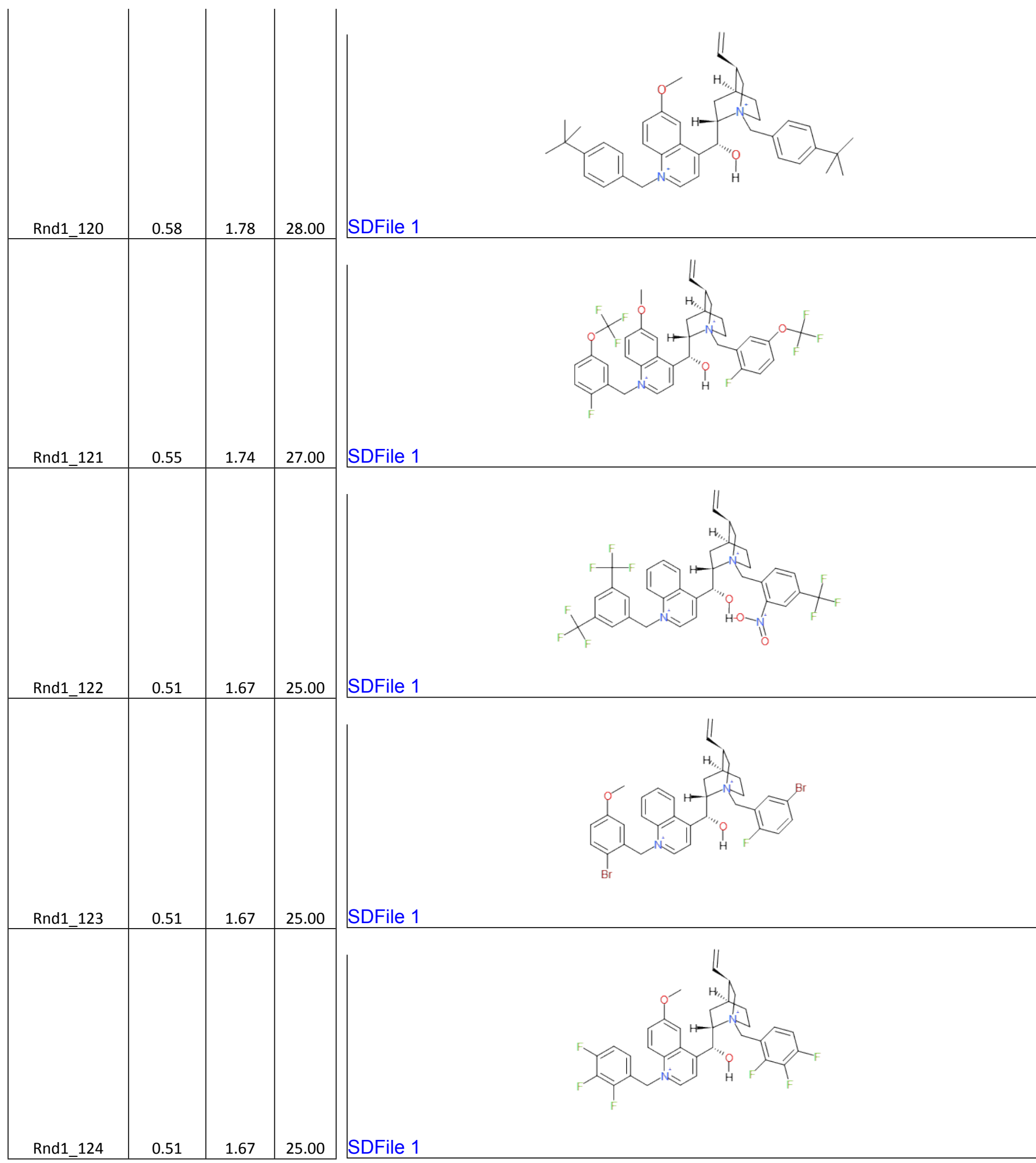

93 


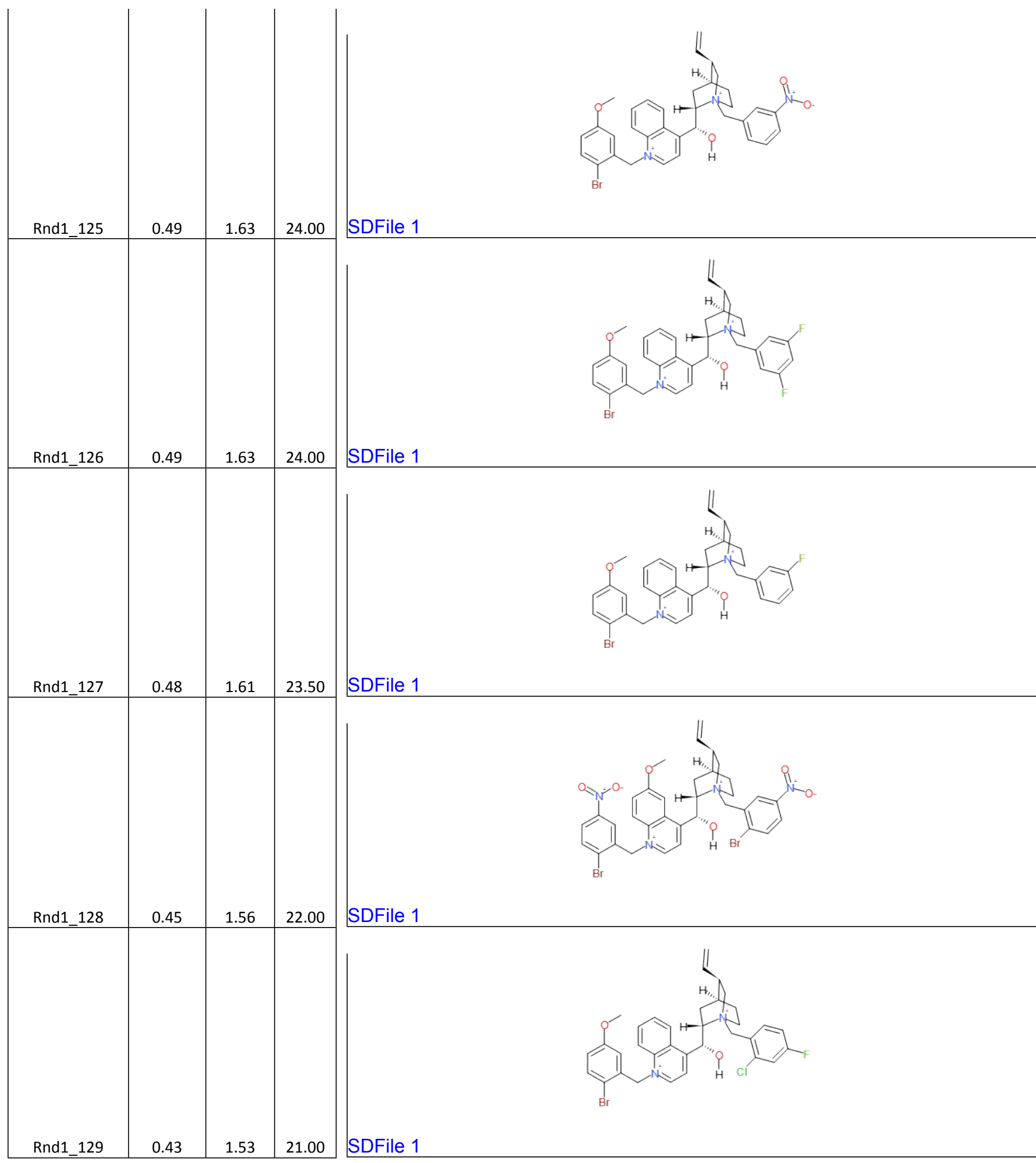




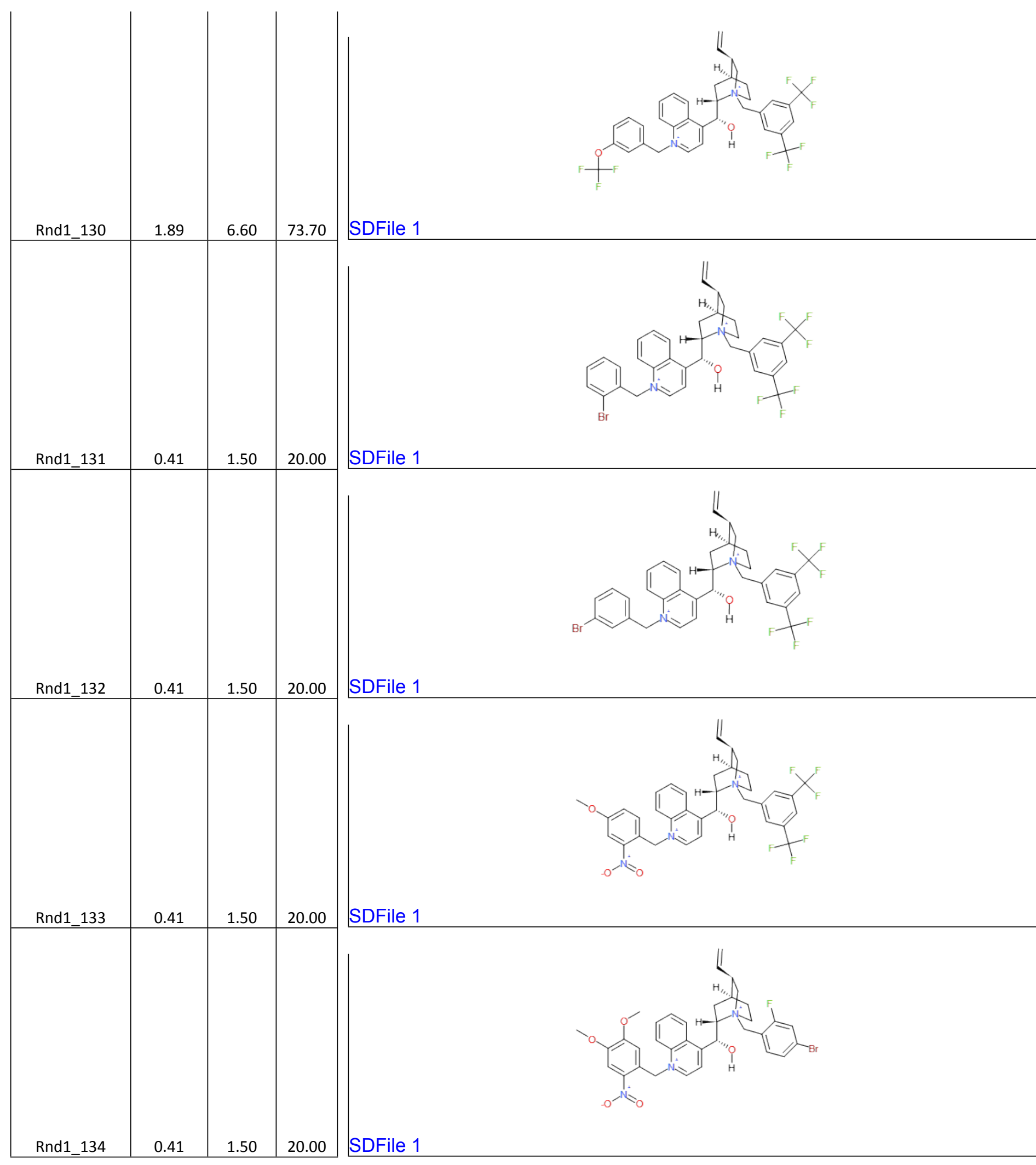




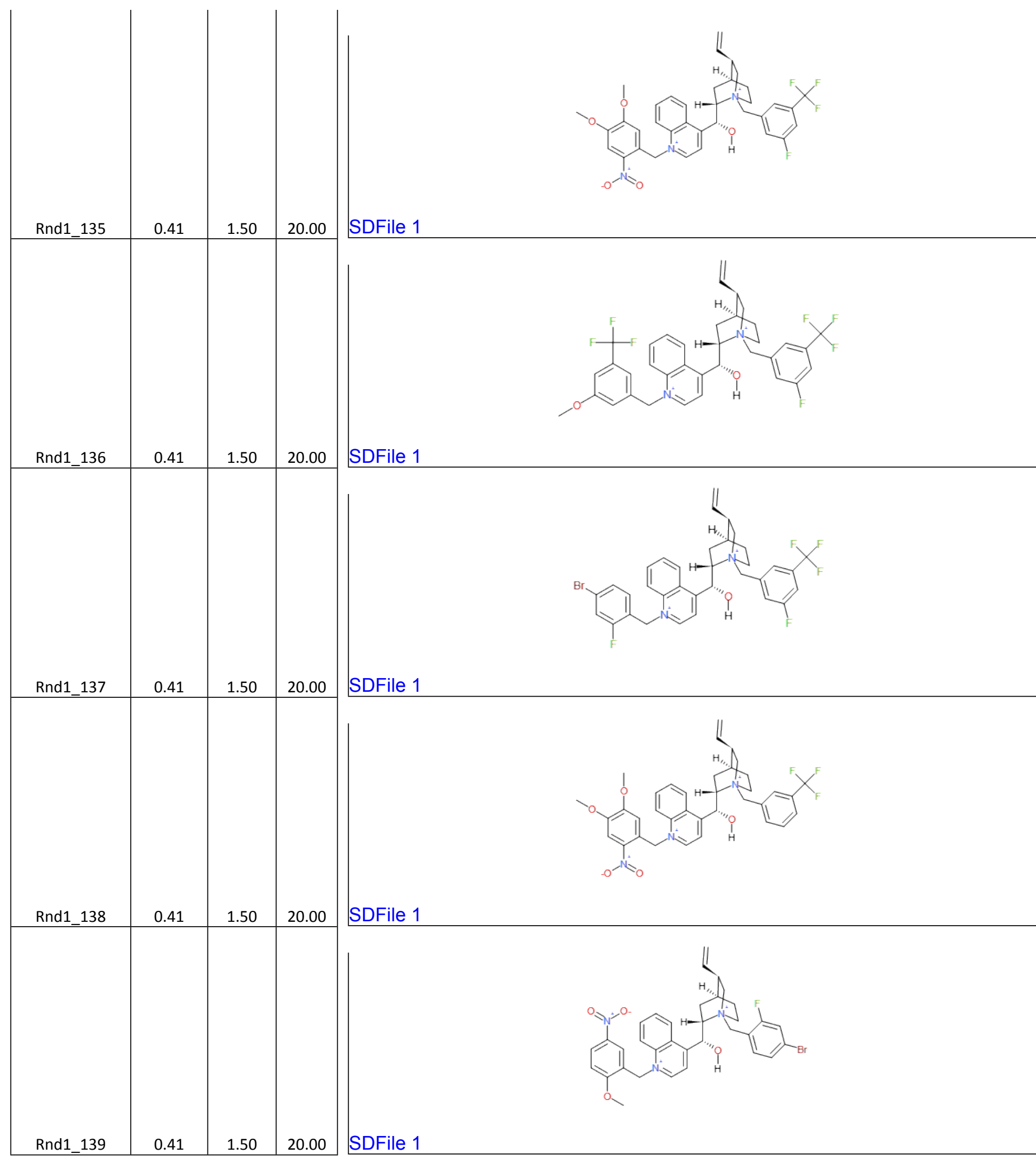




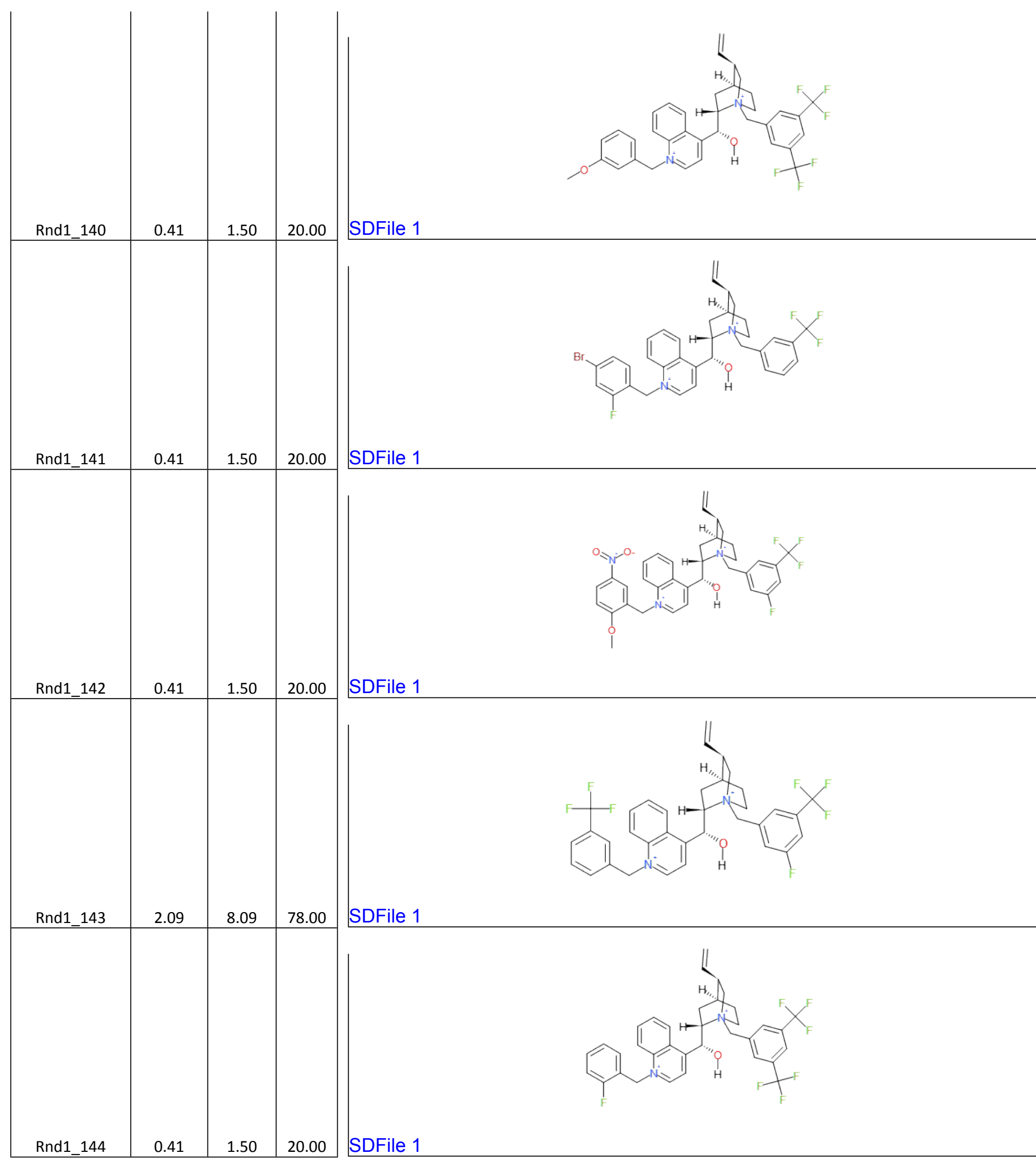




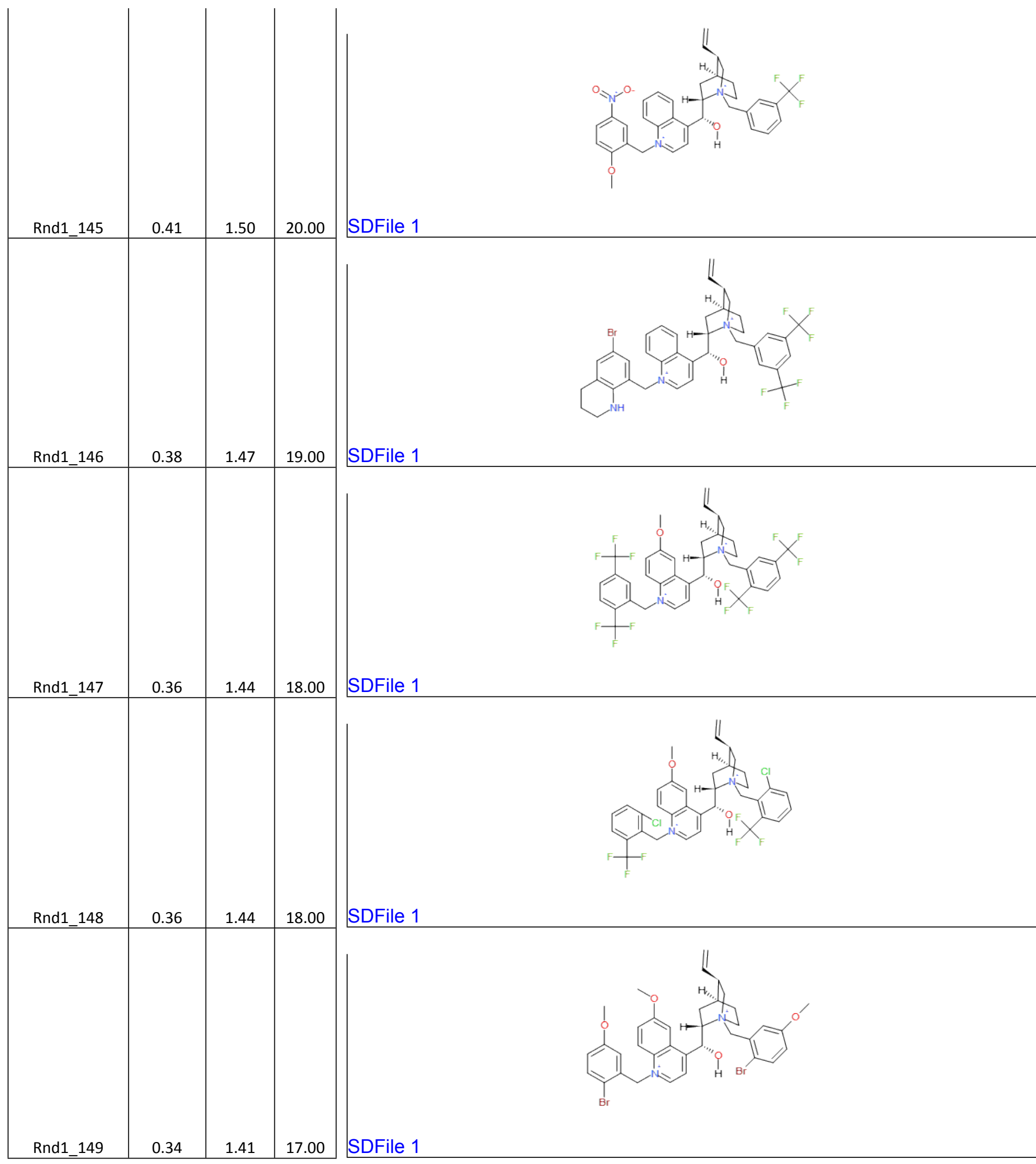




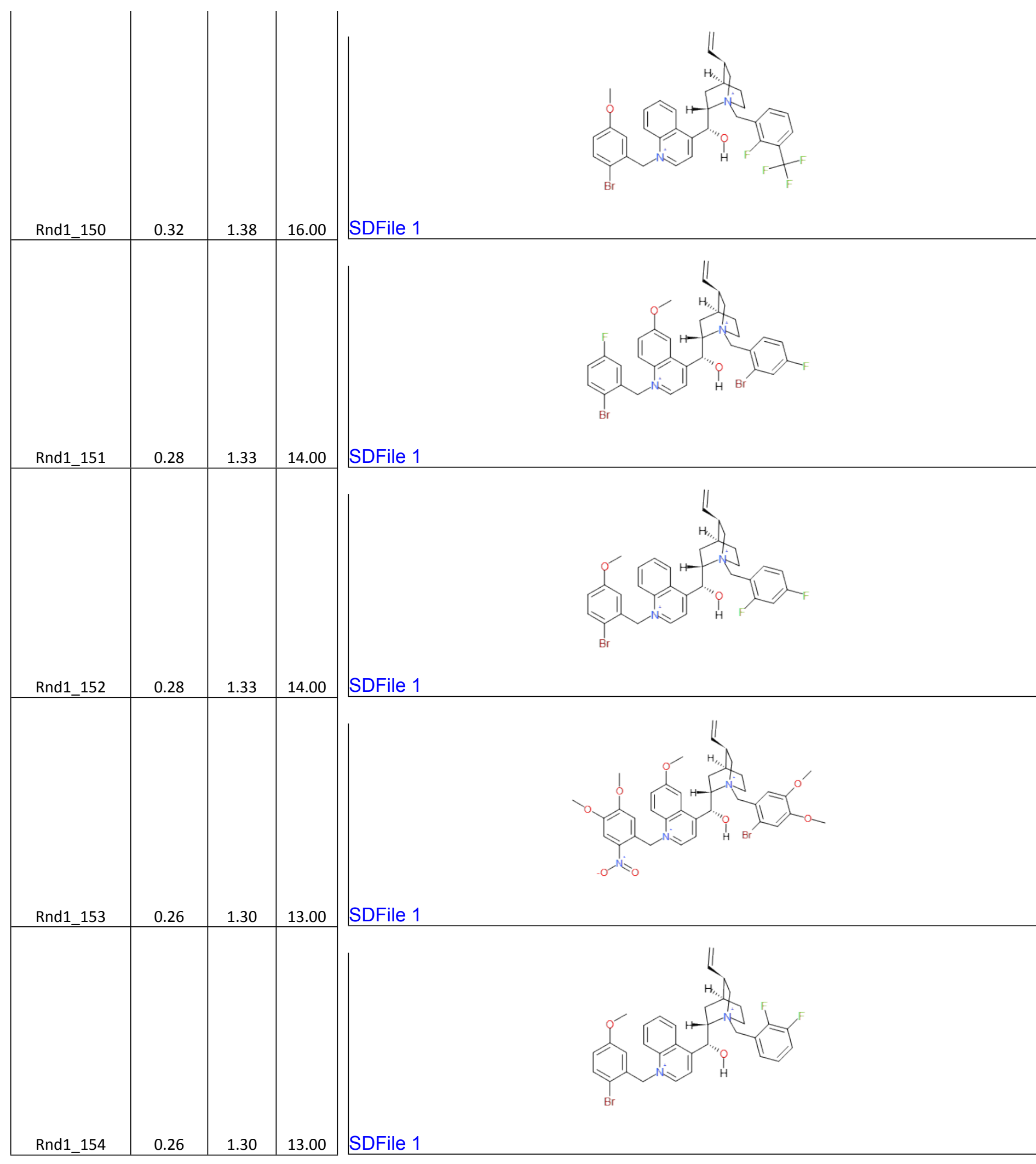




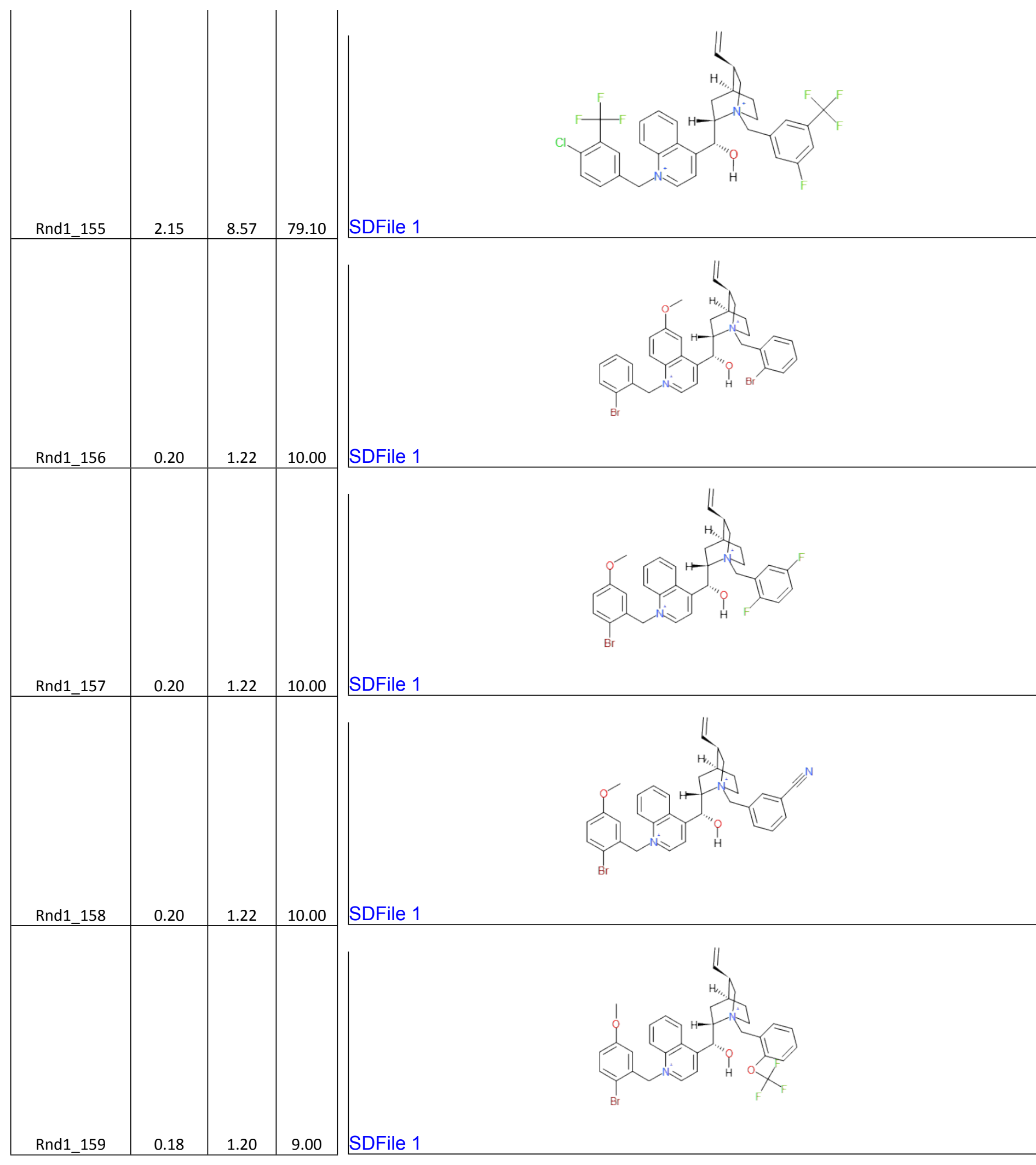




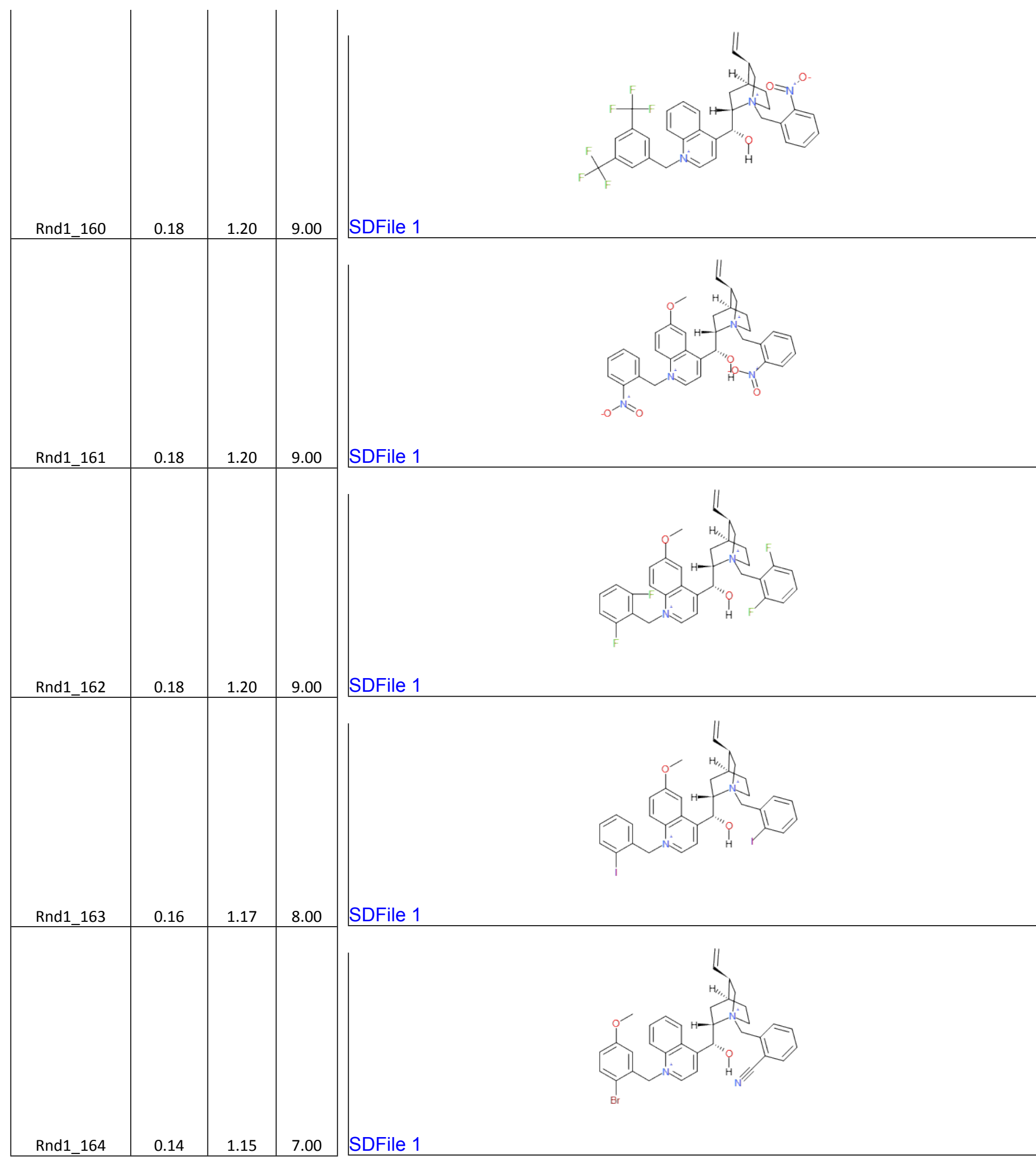




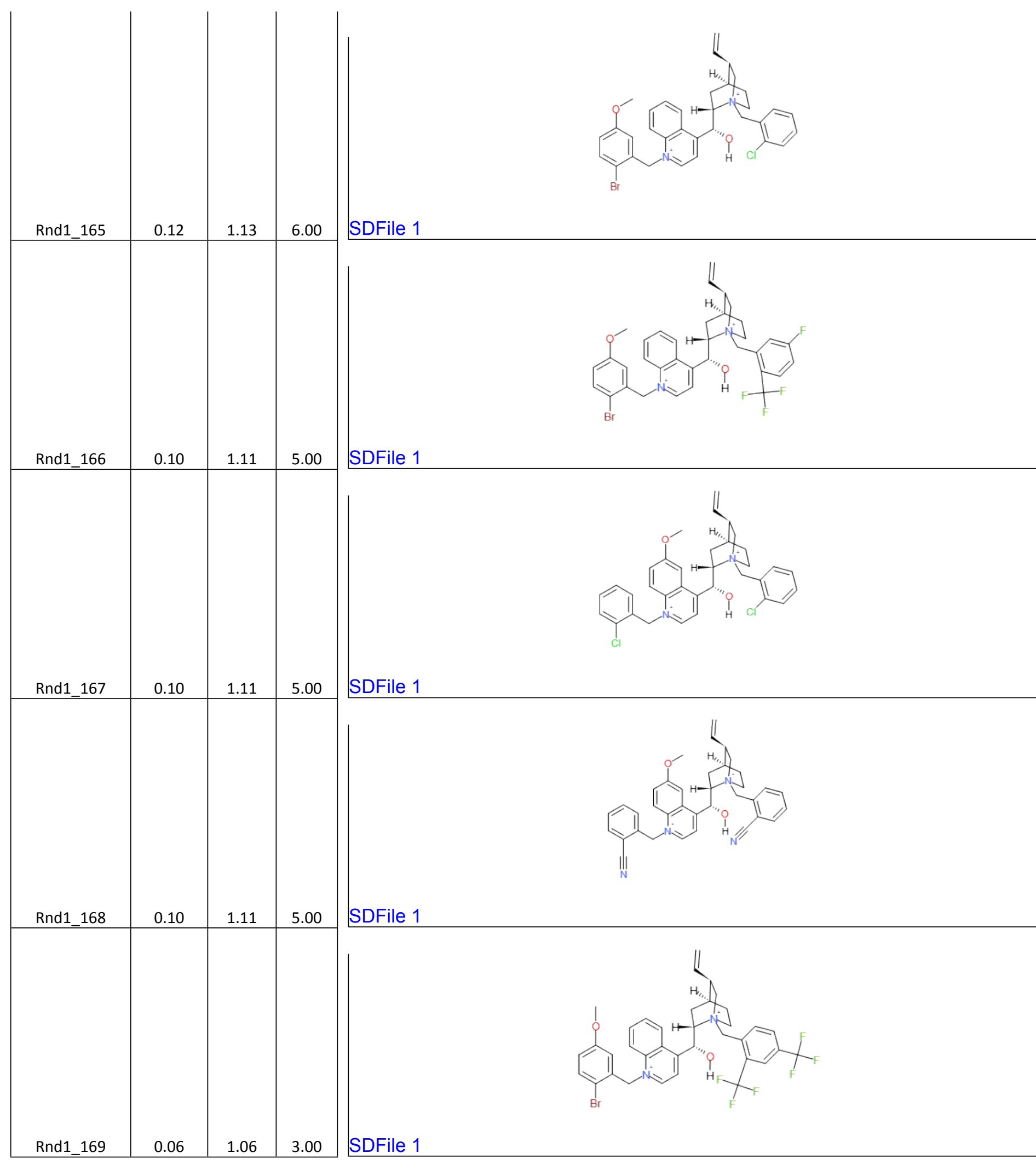




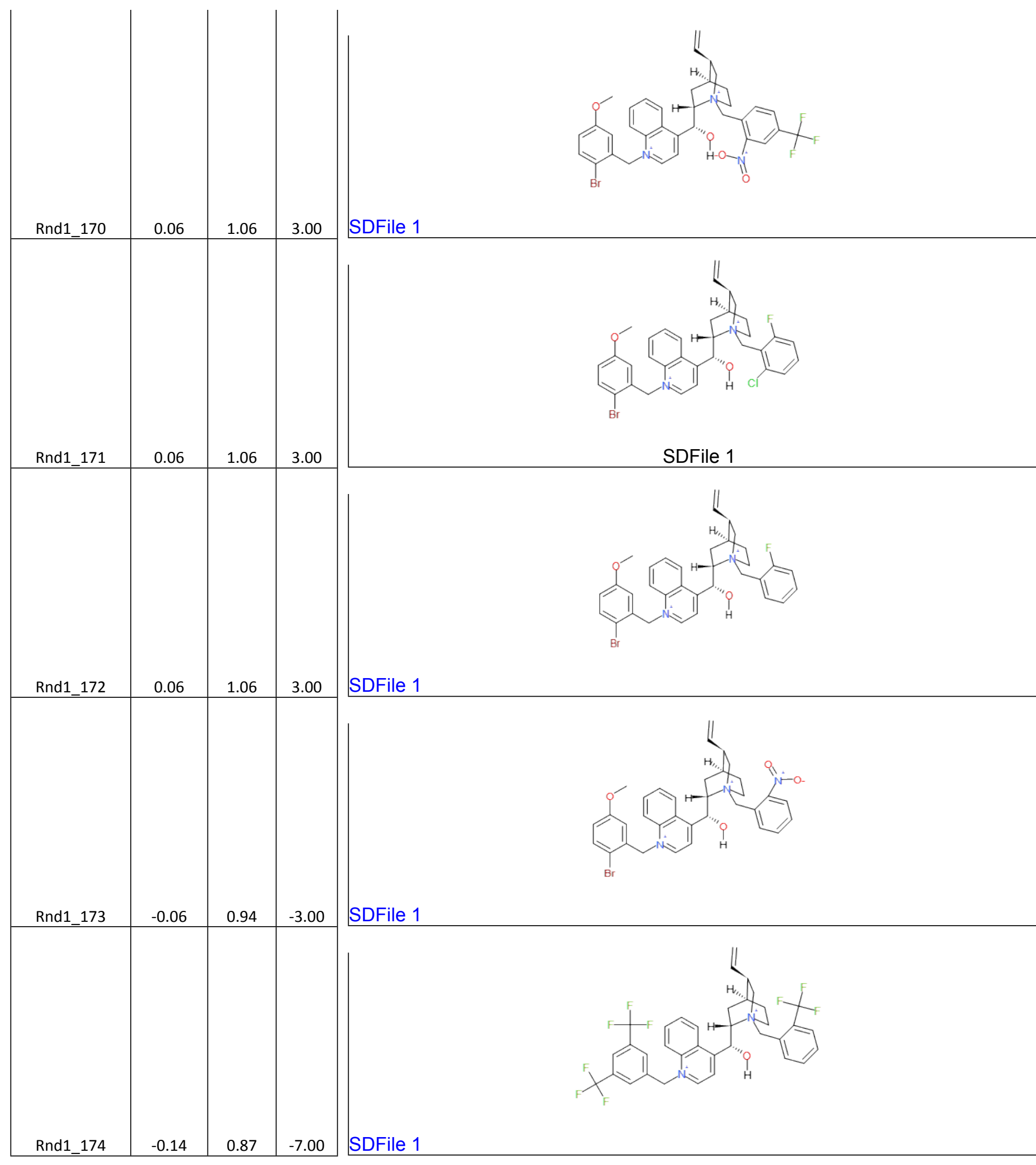




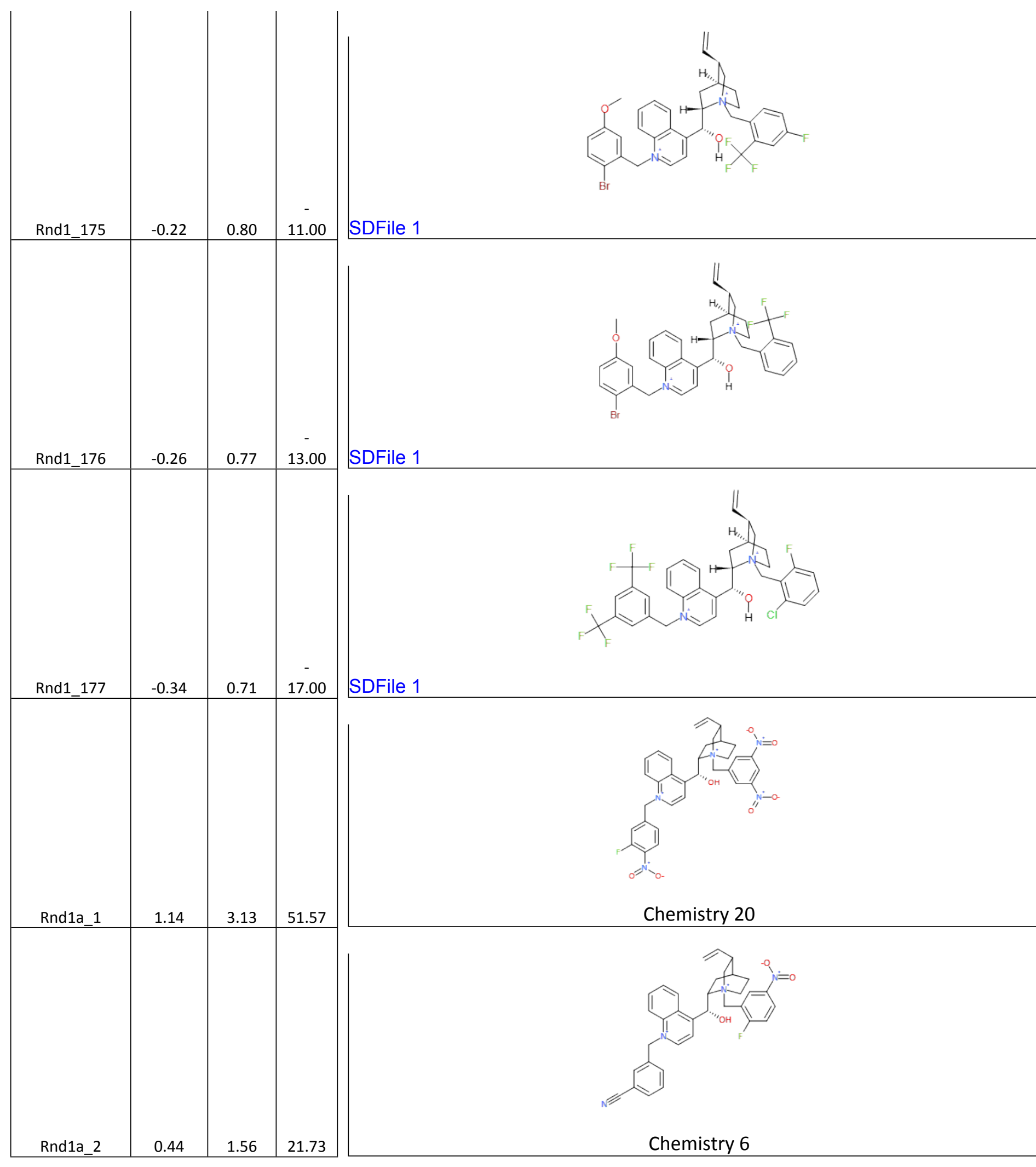




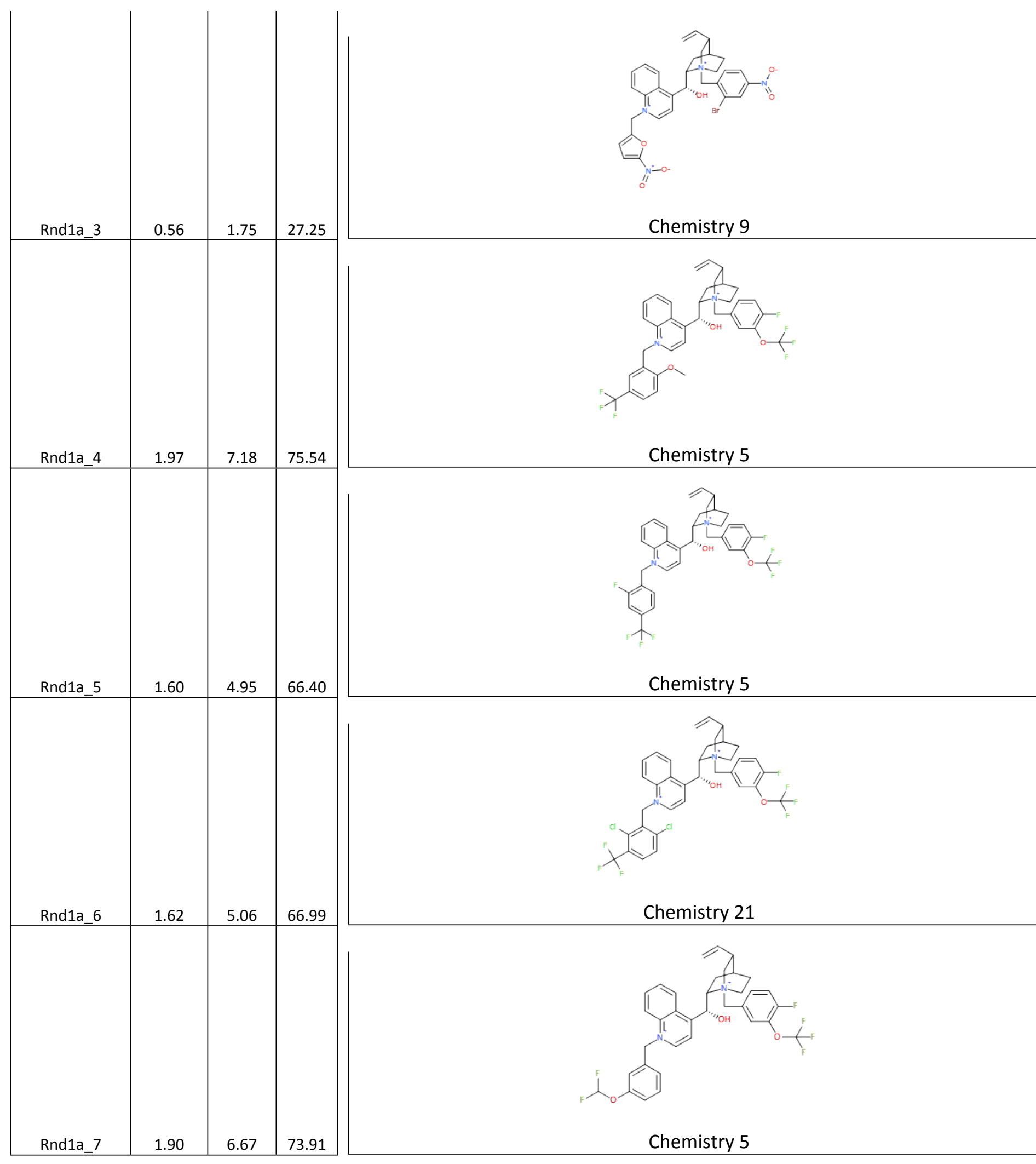




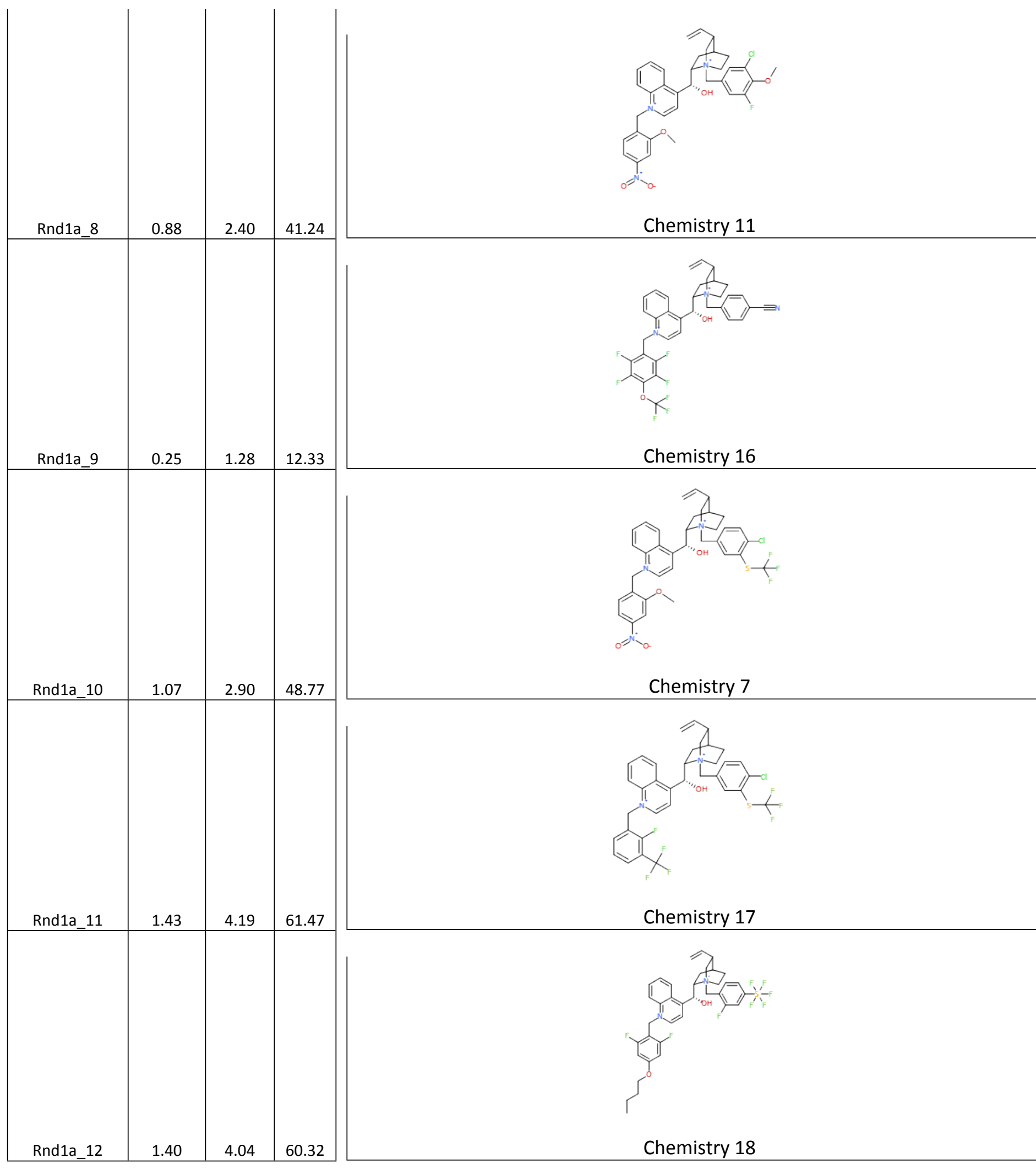

106 


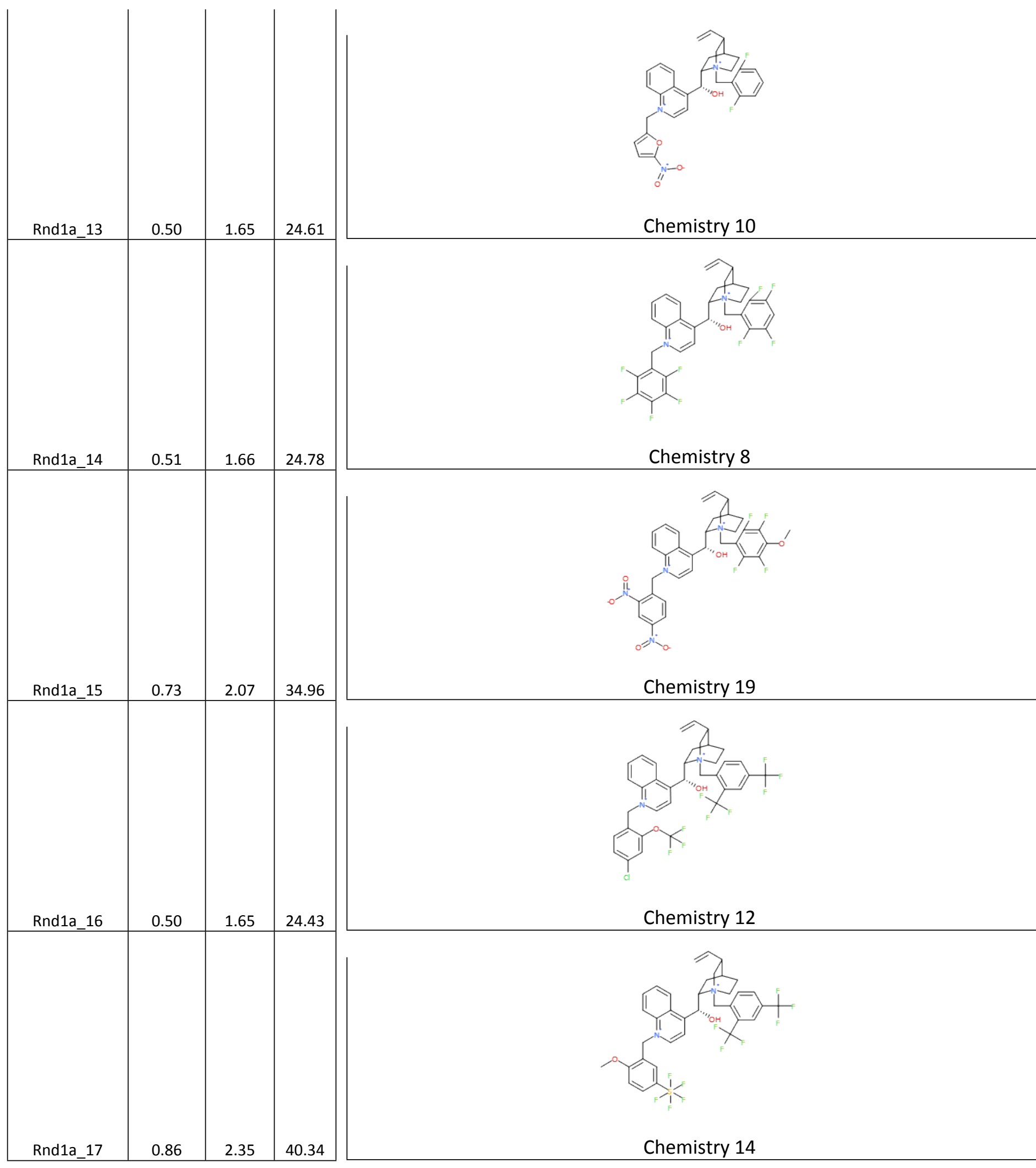

107 


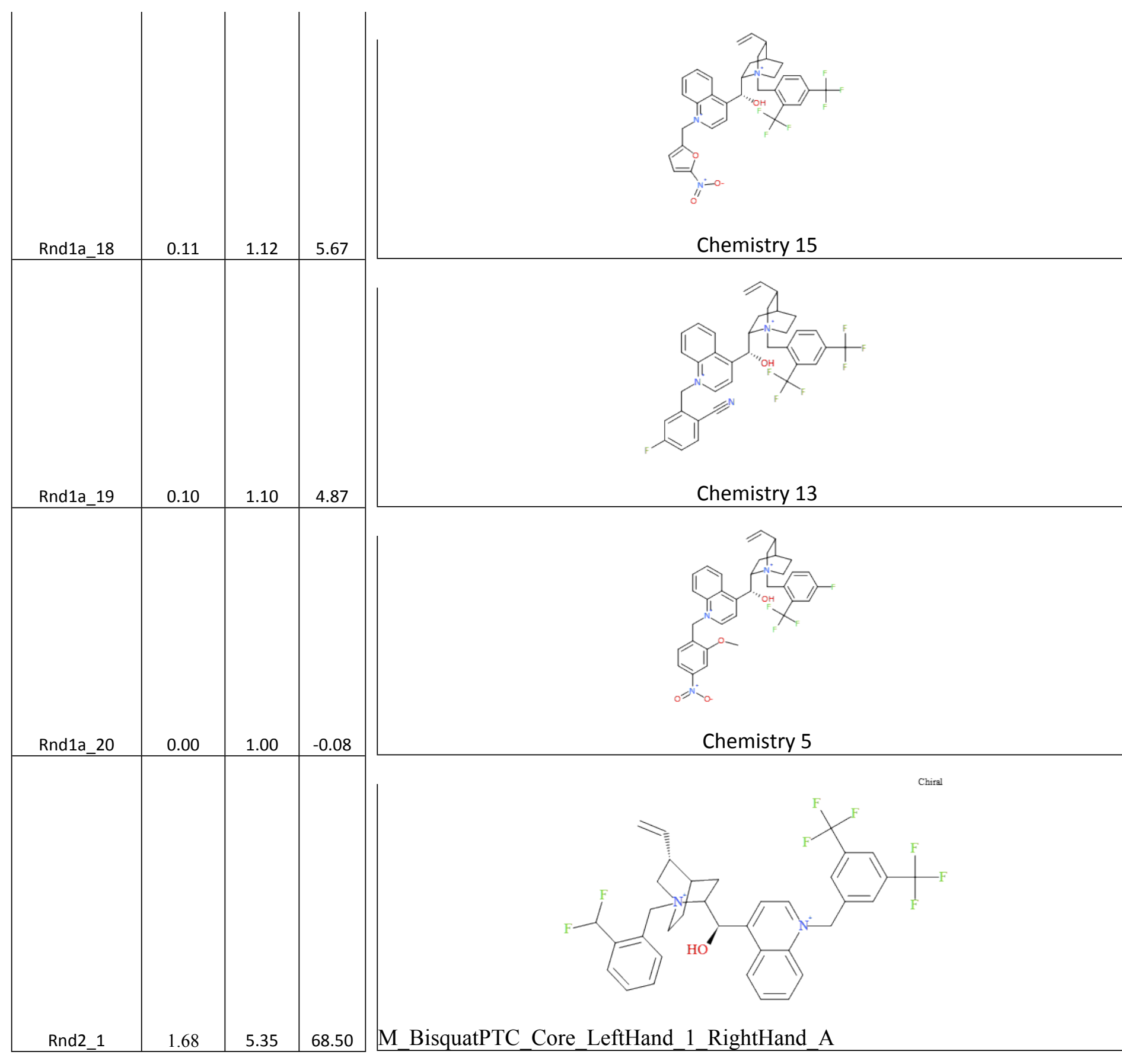




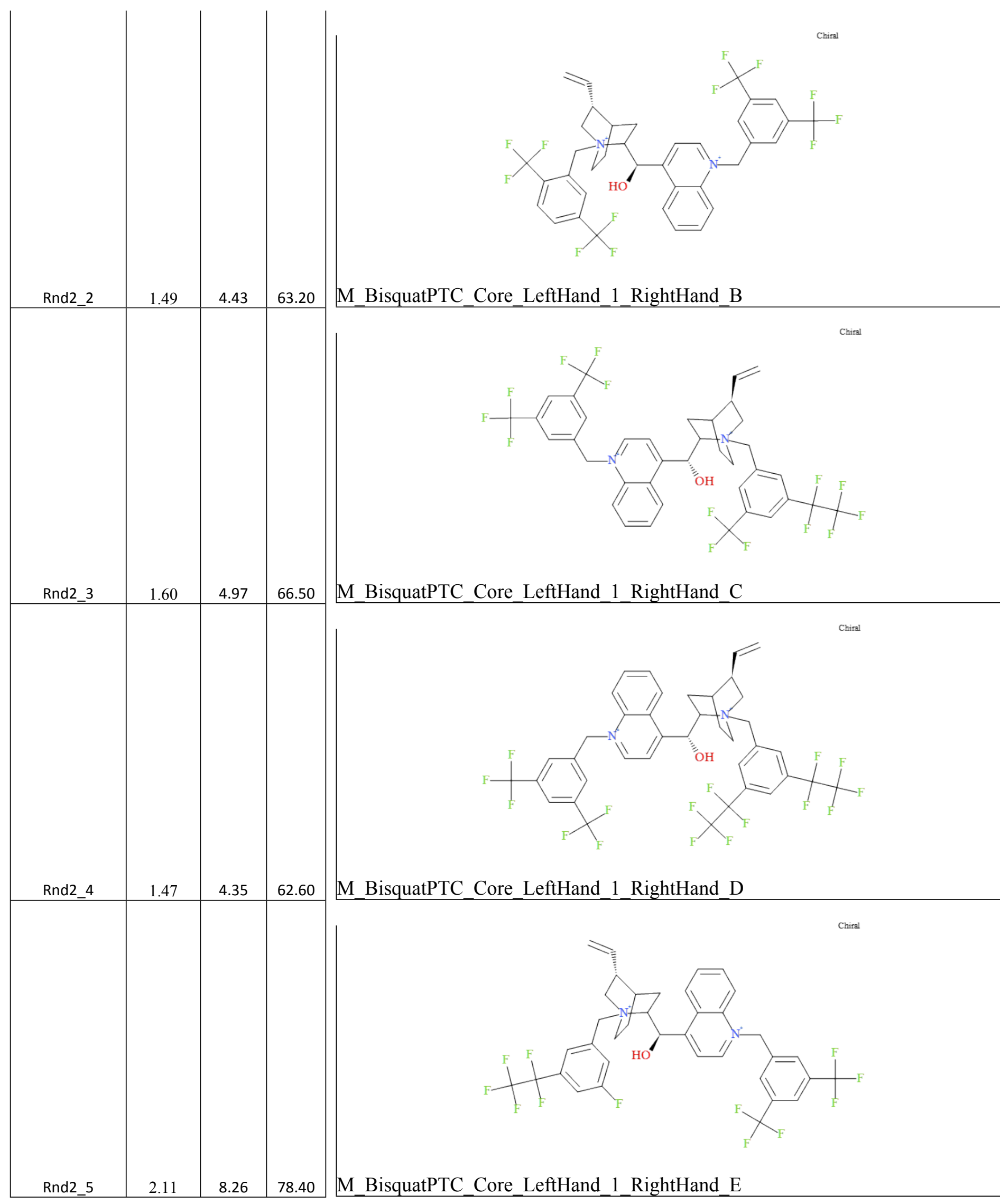




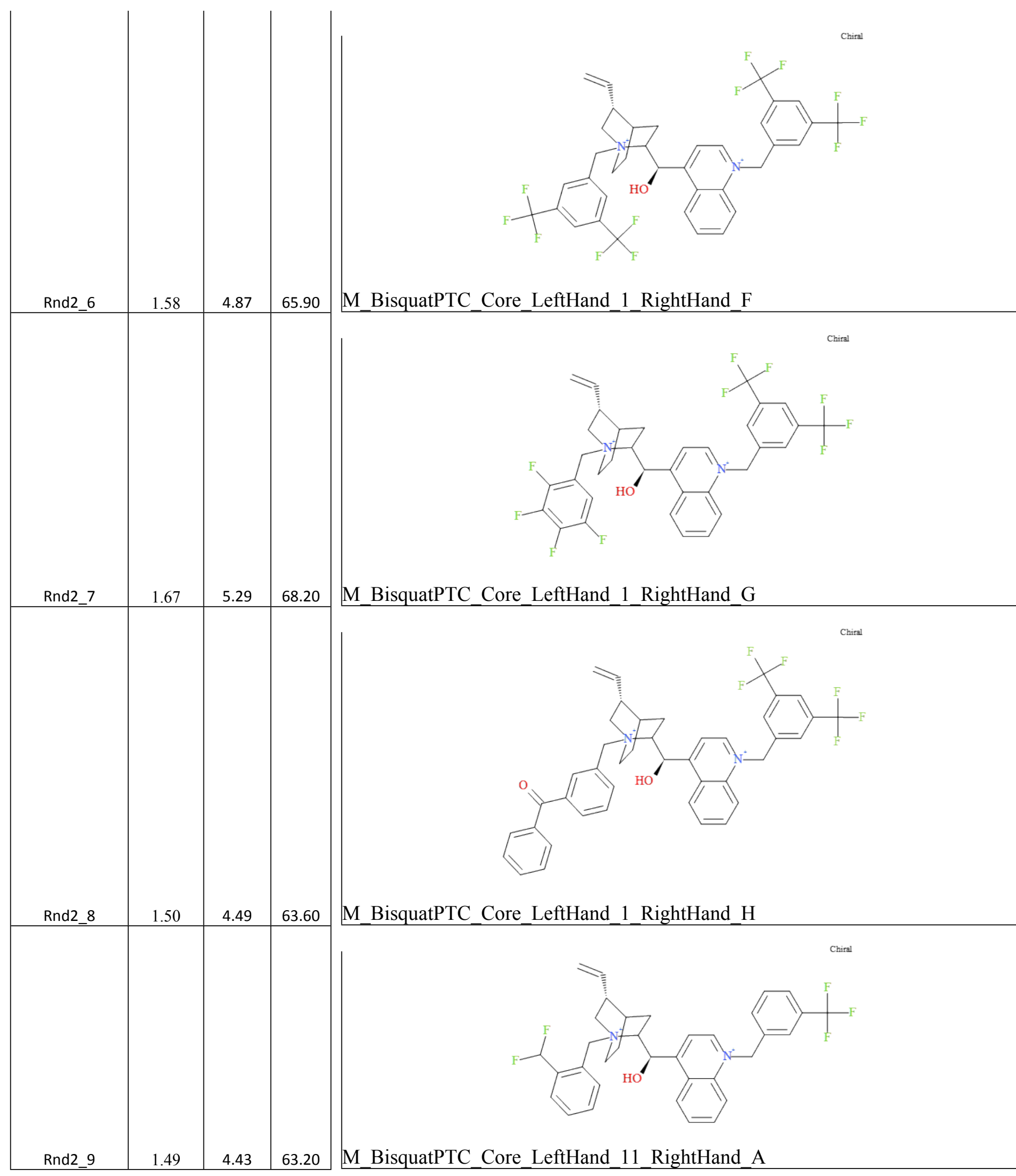




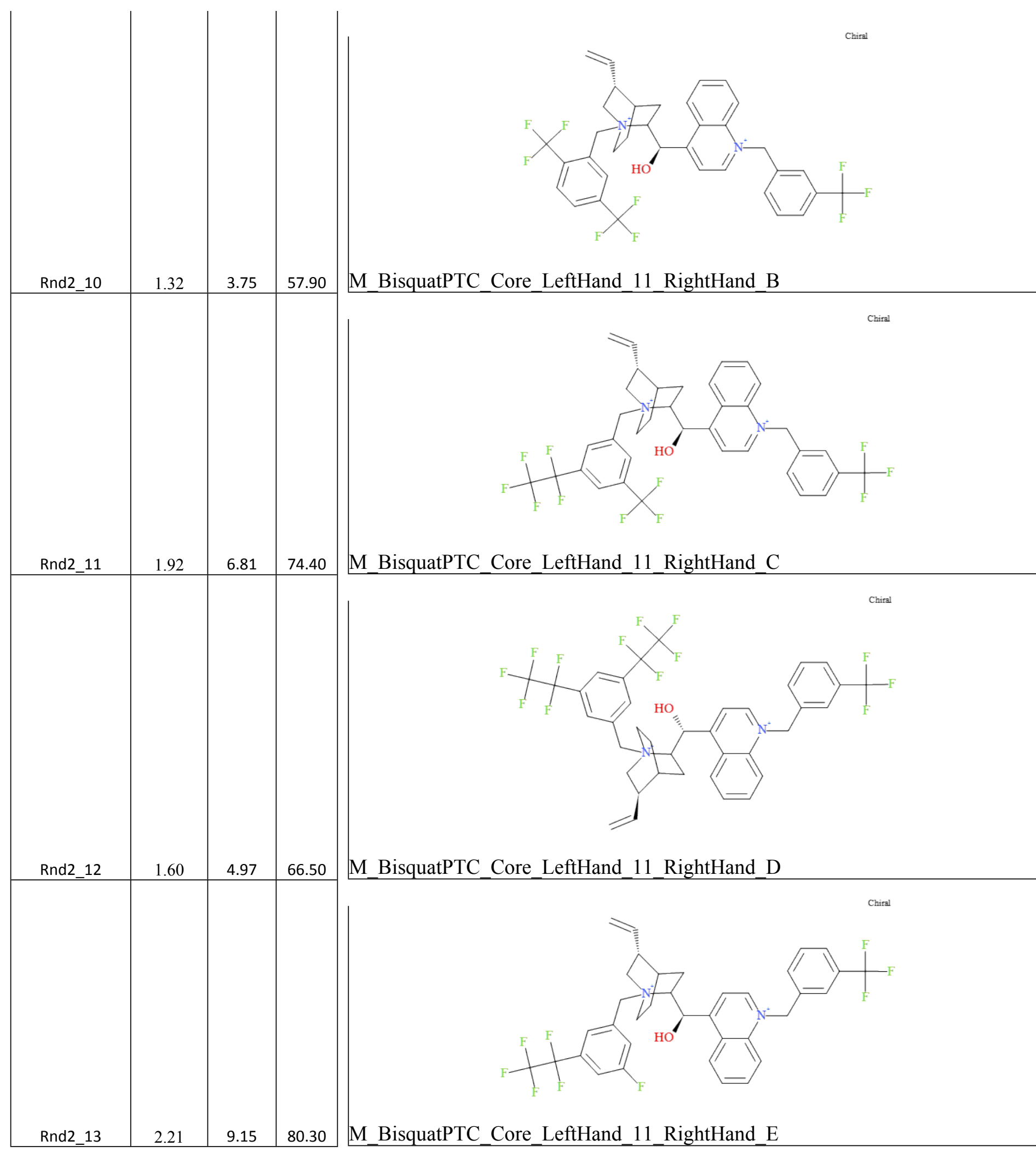




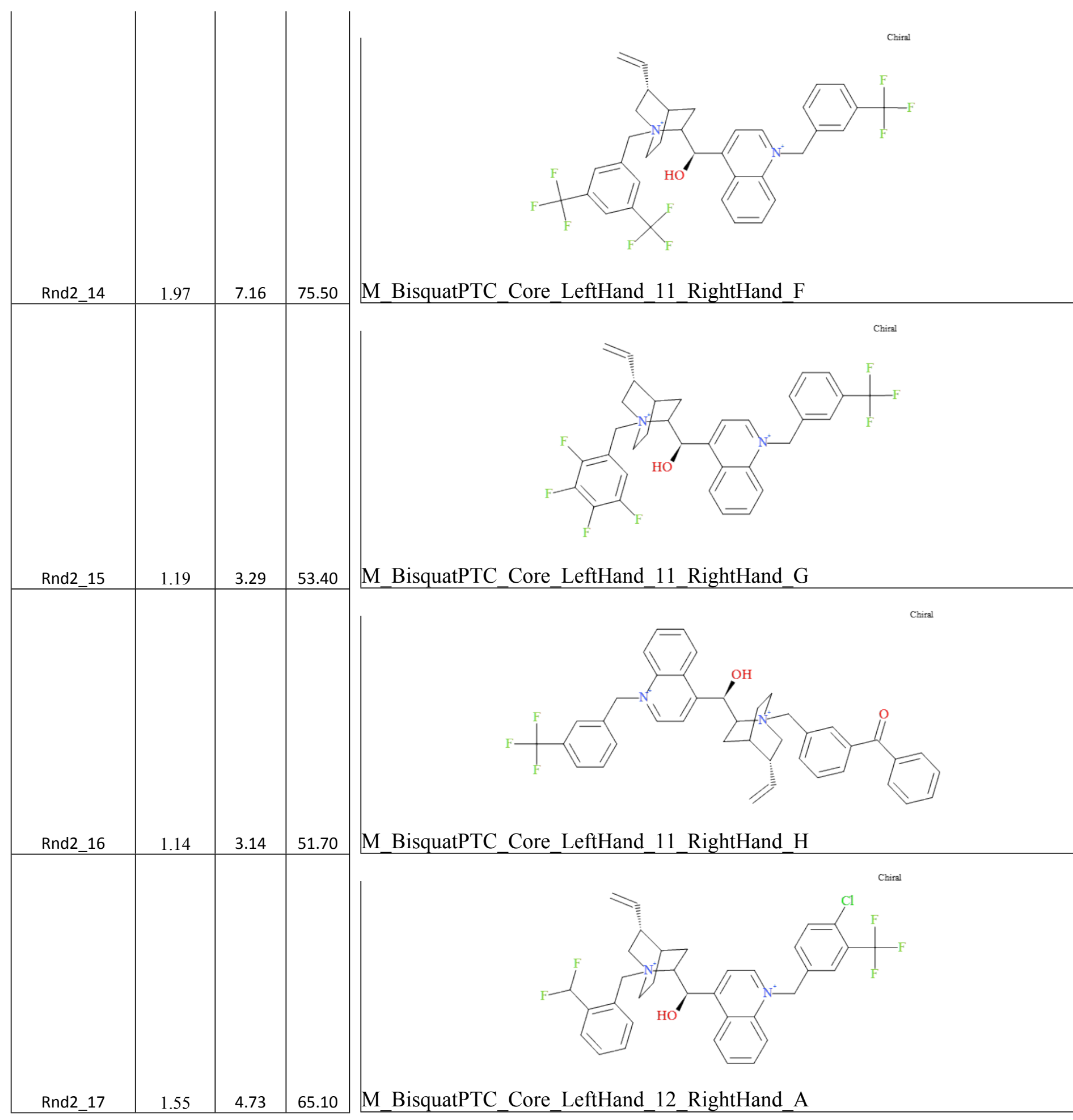




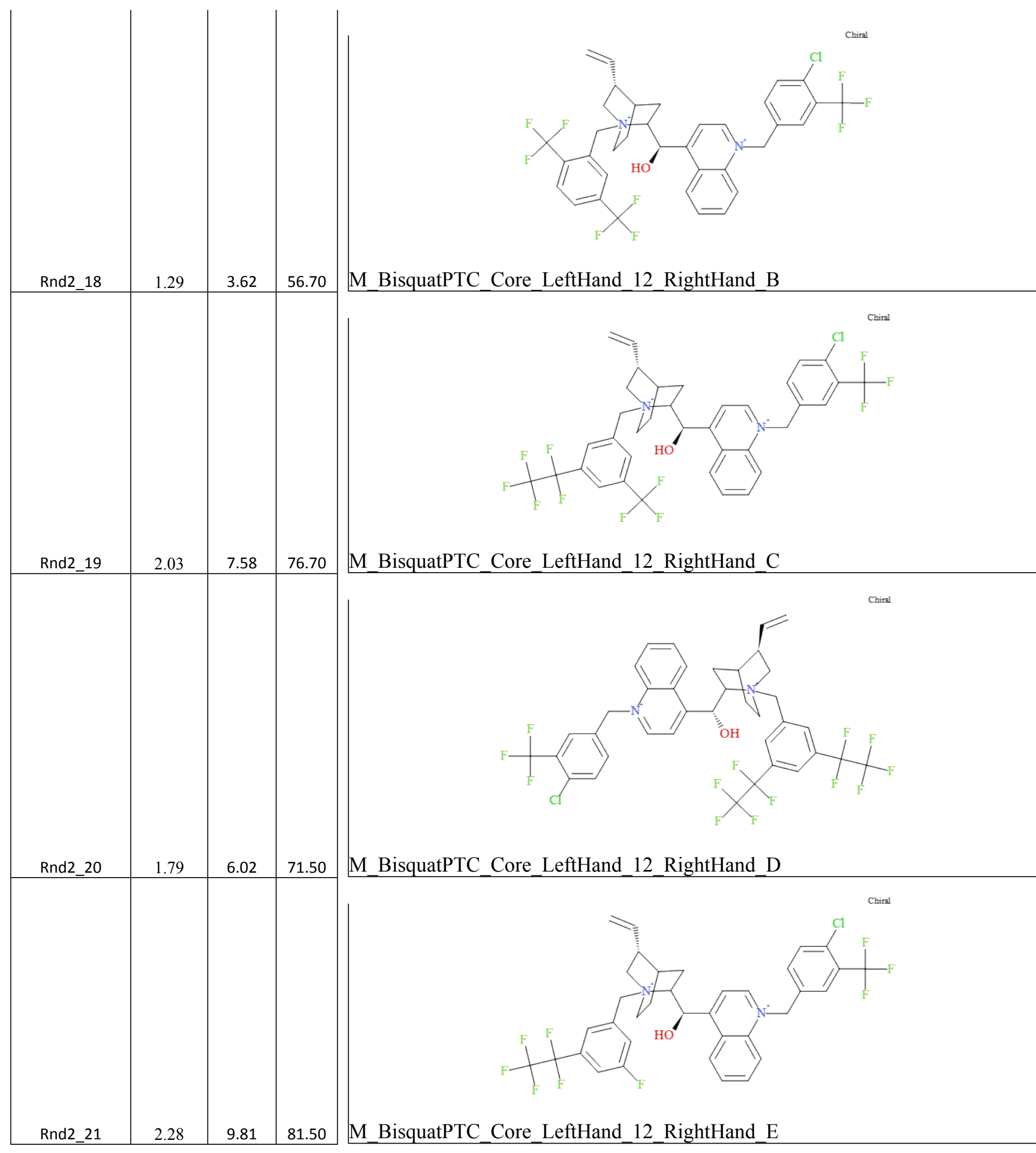




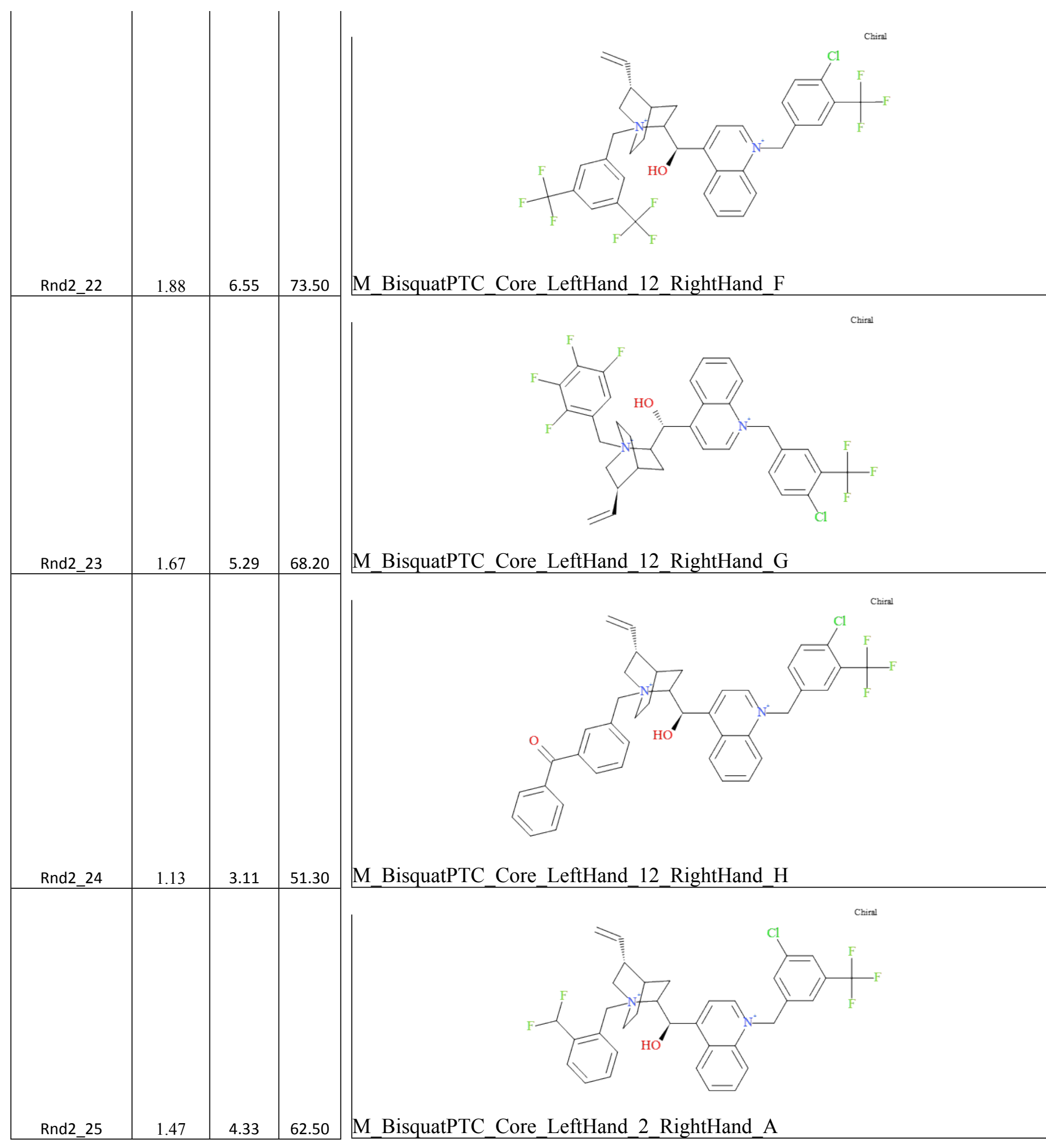




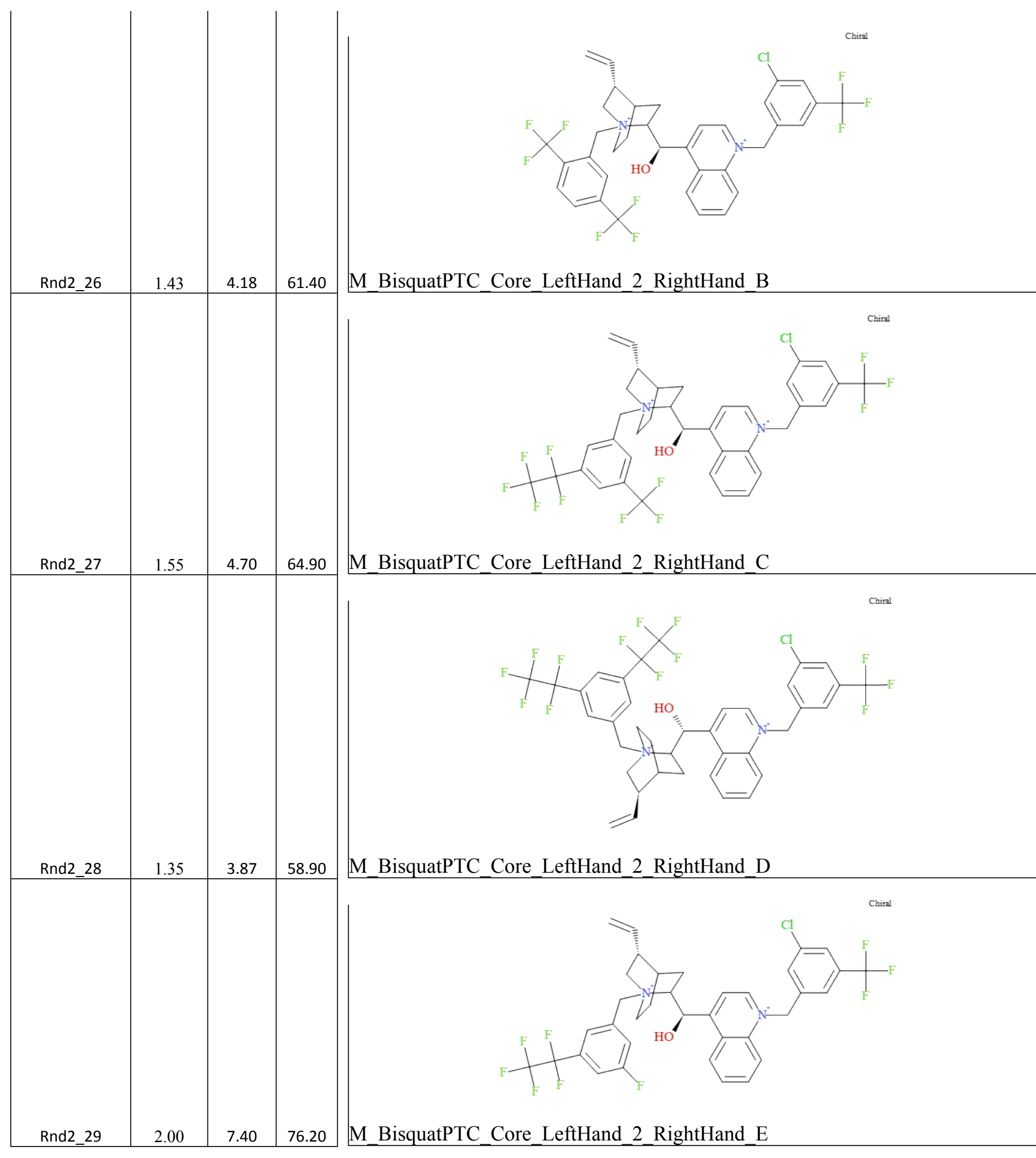




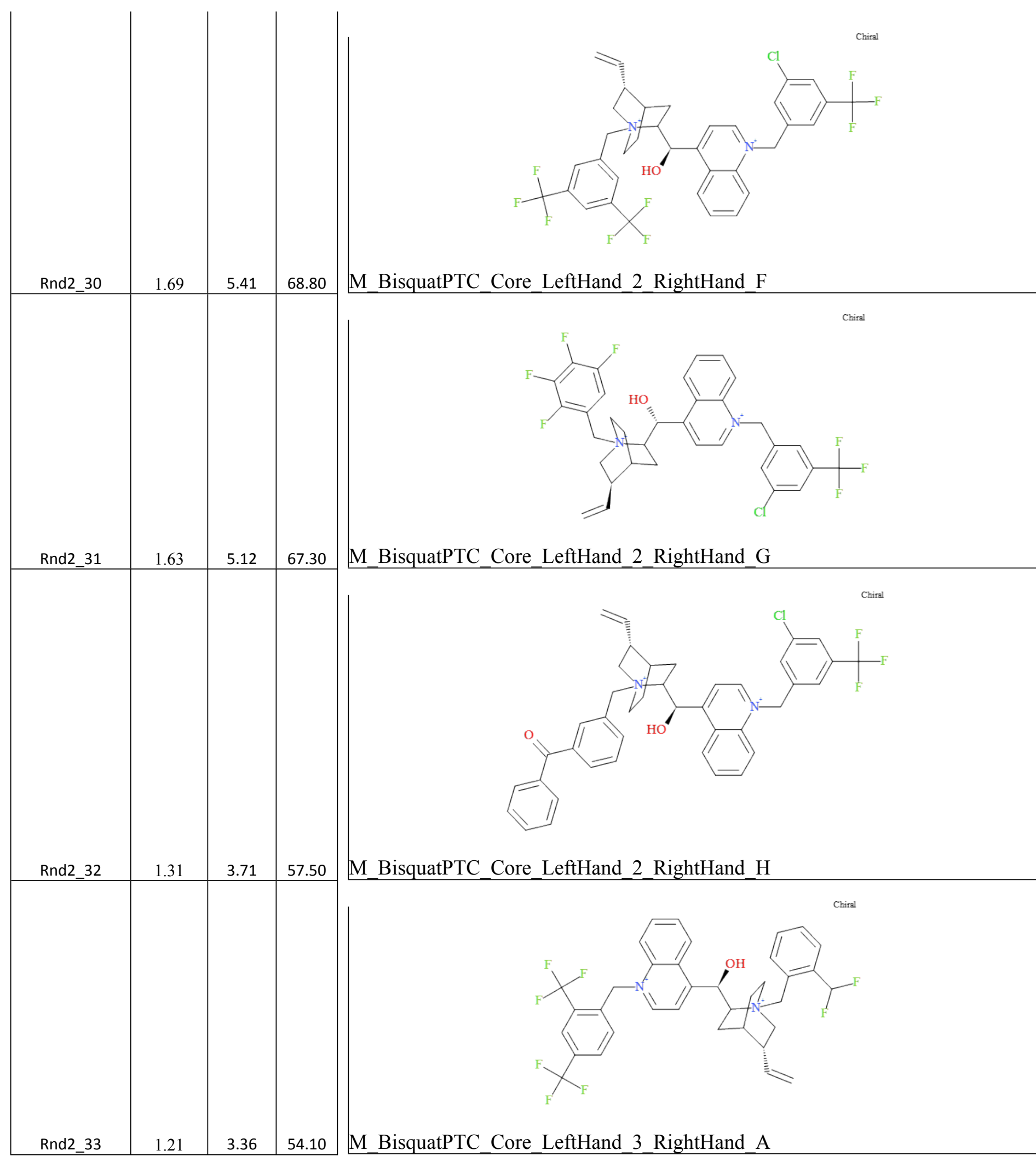




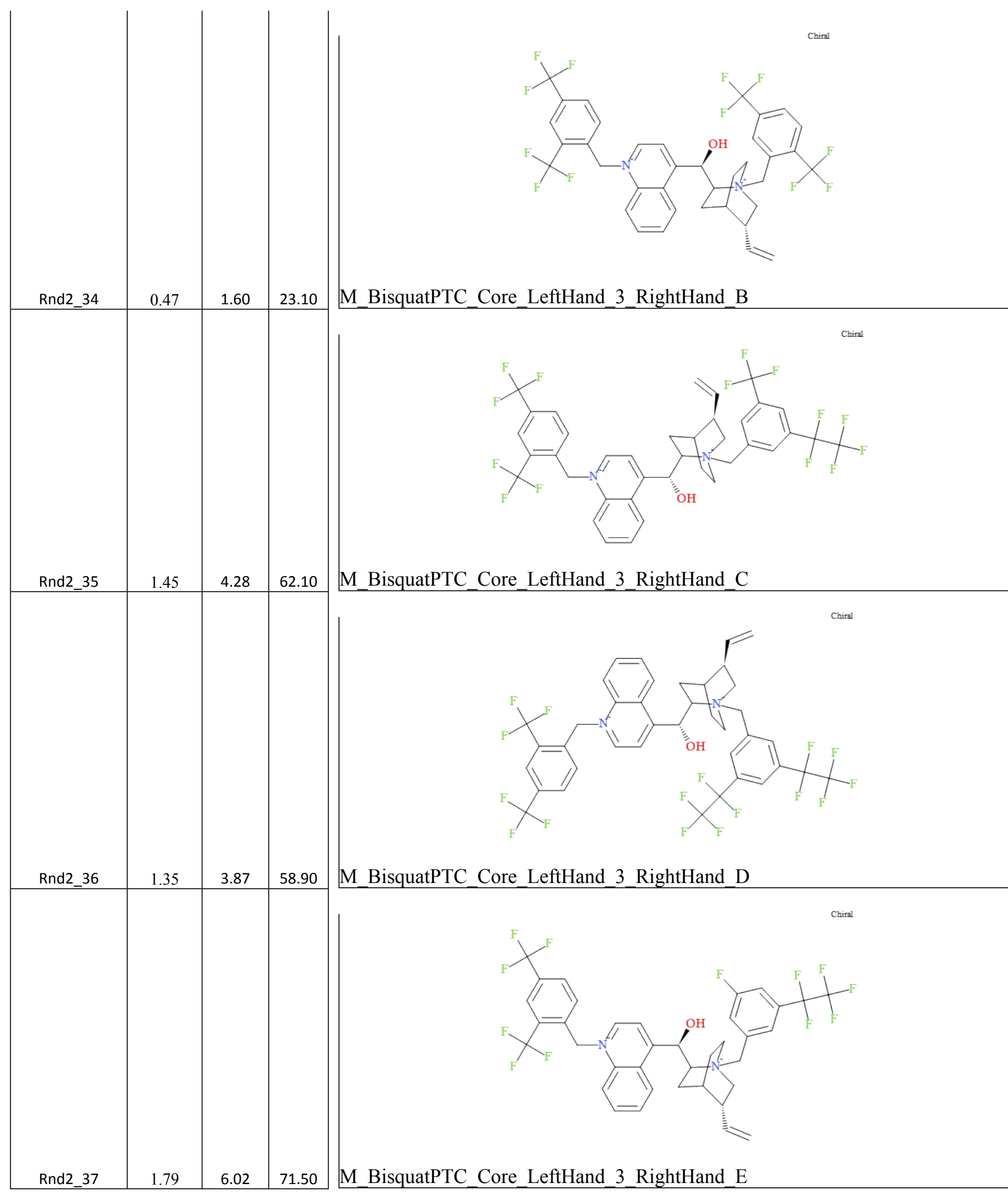




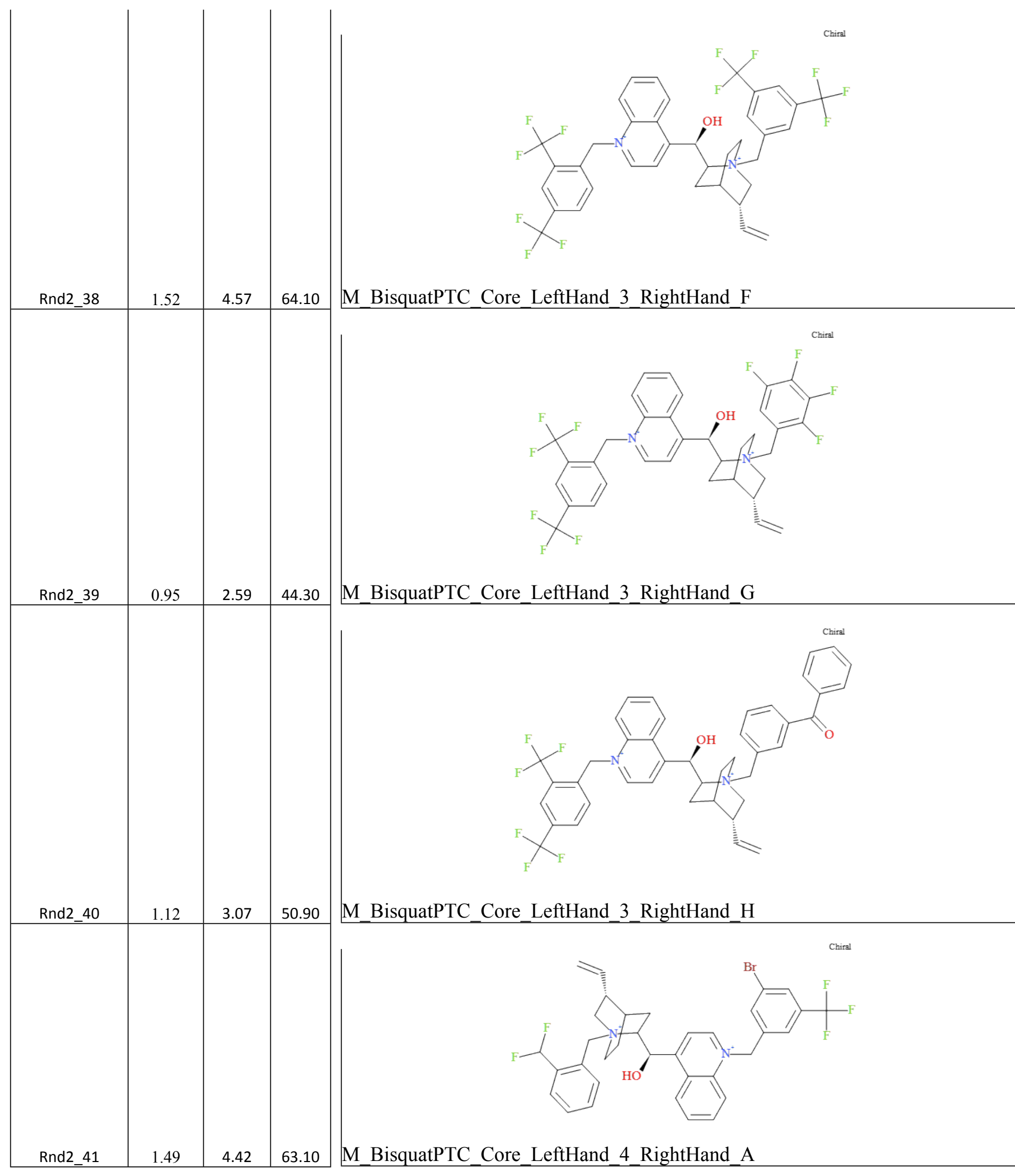




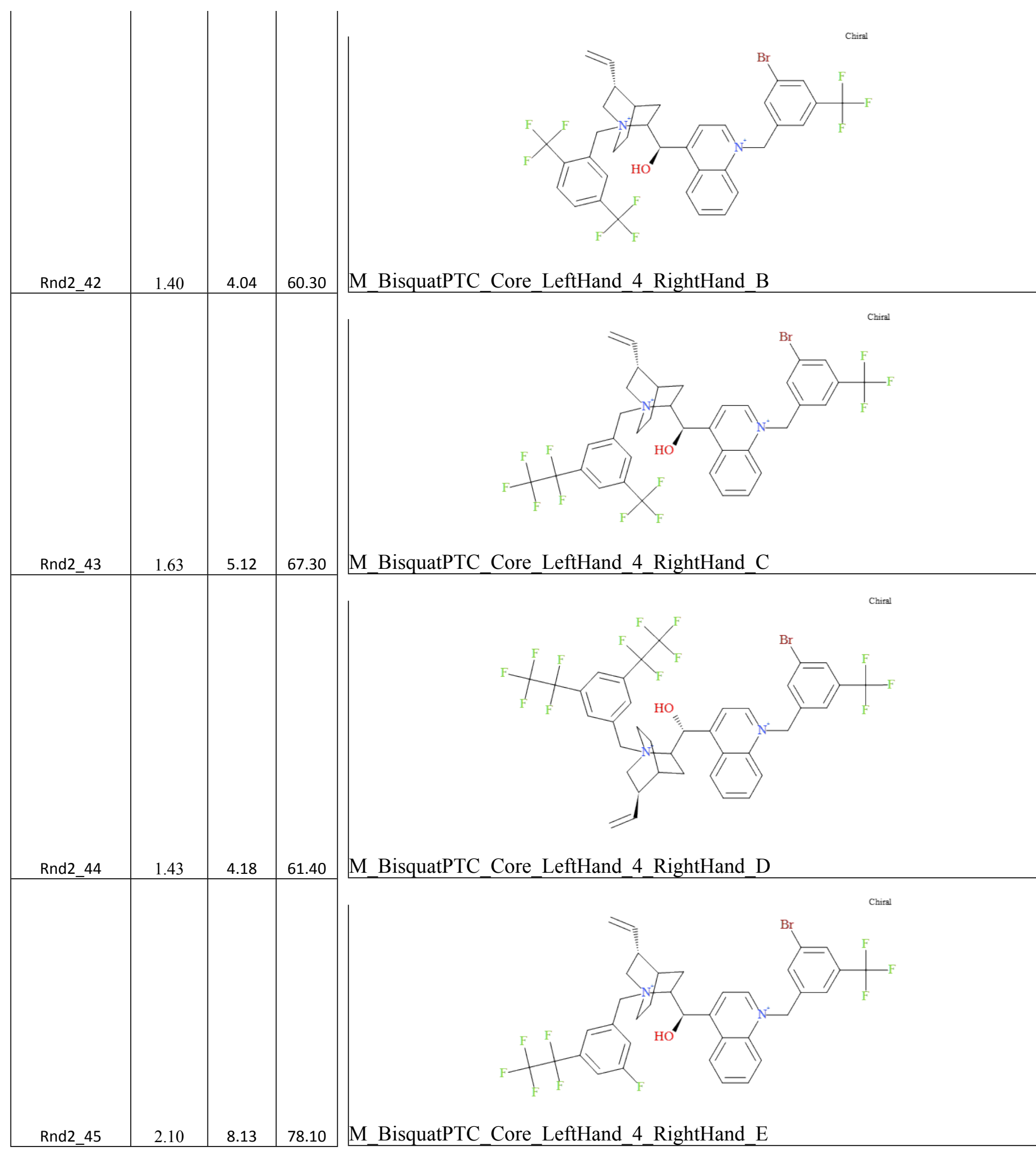




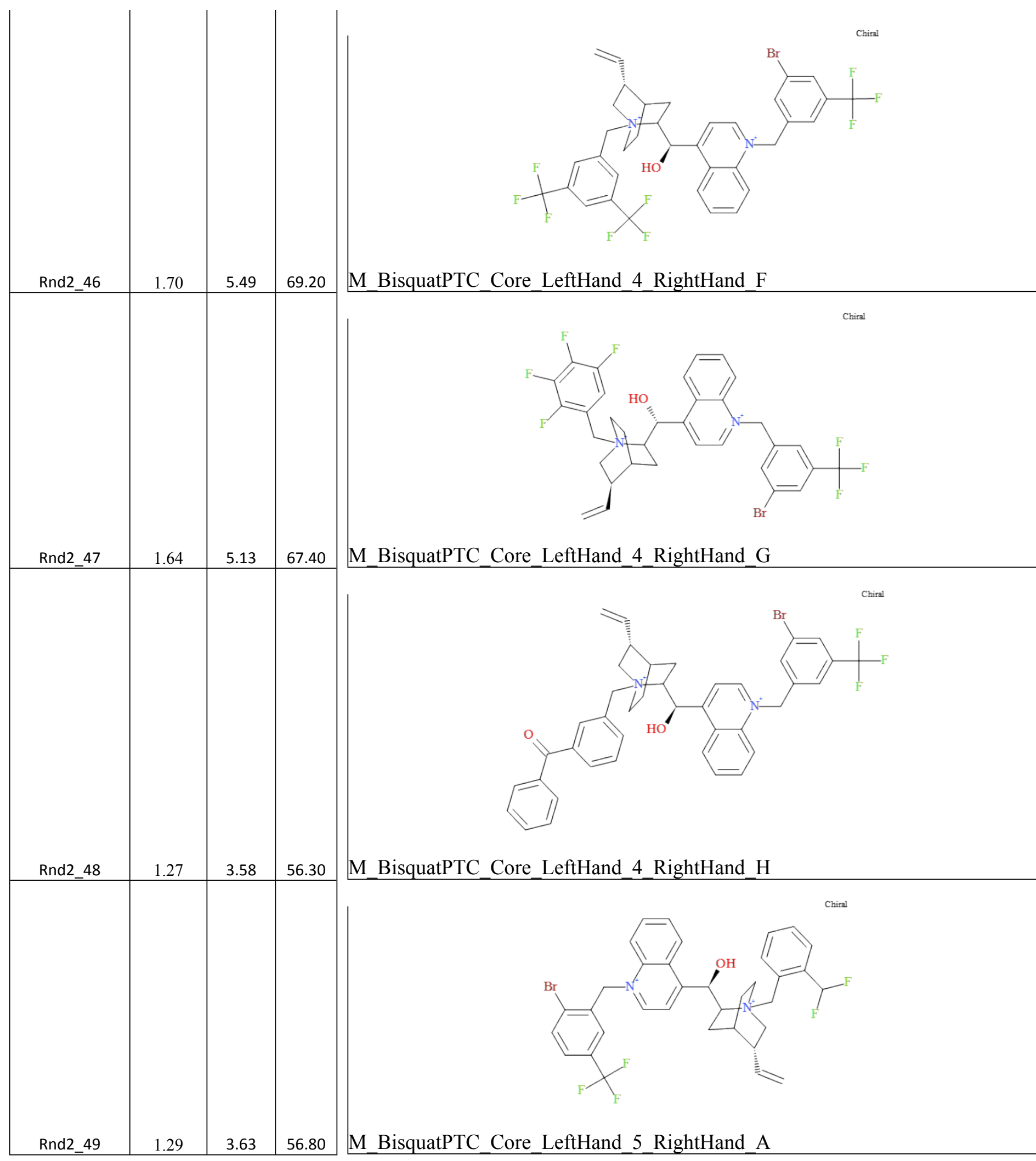




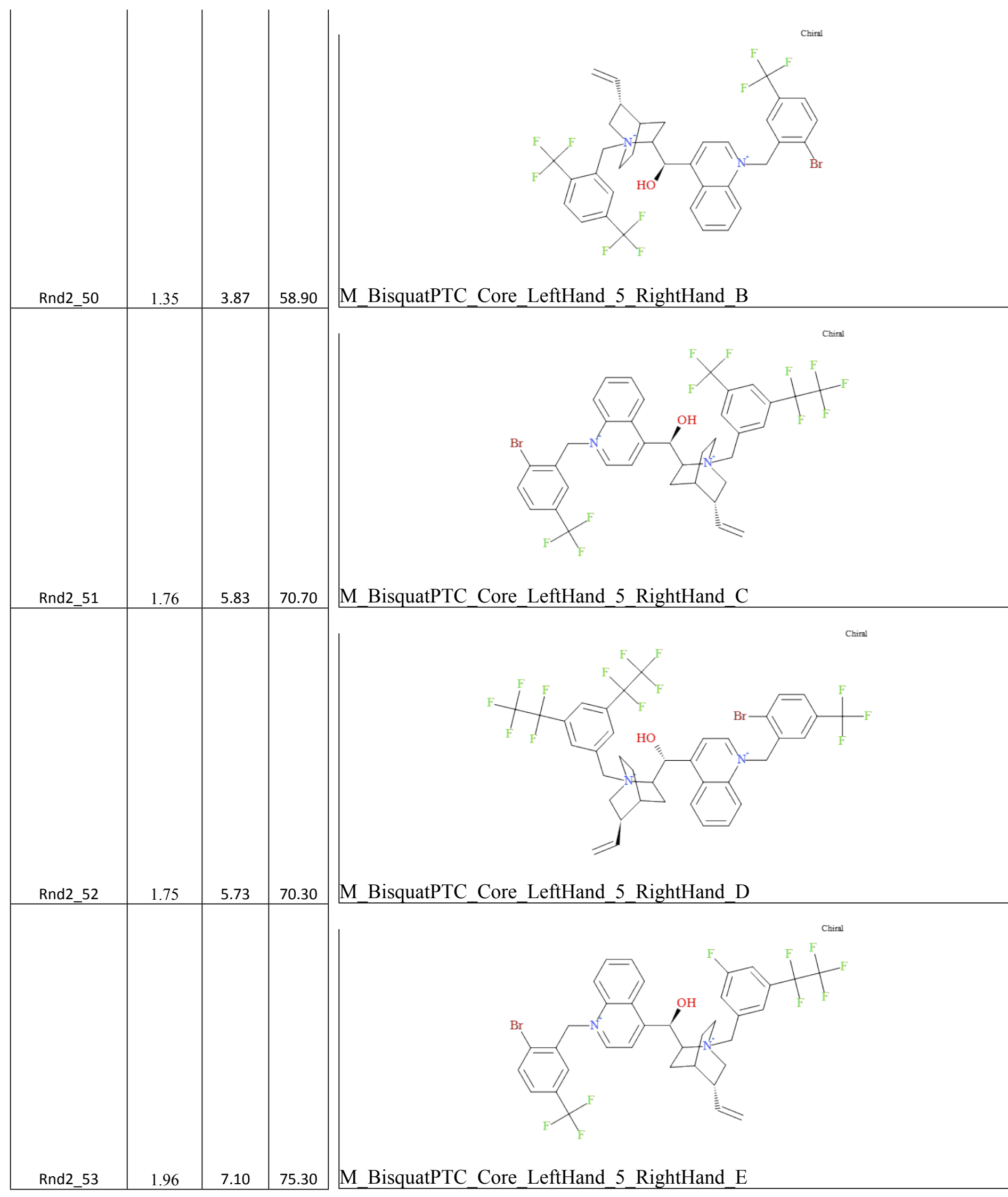




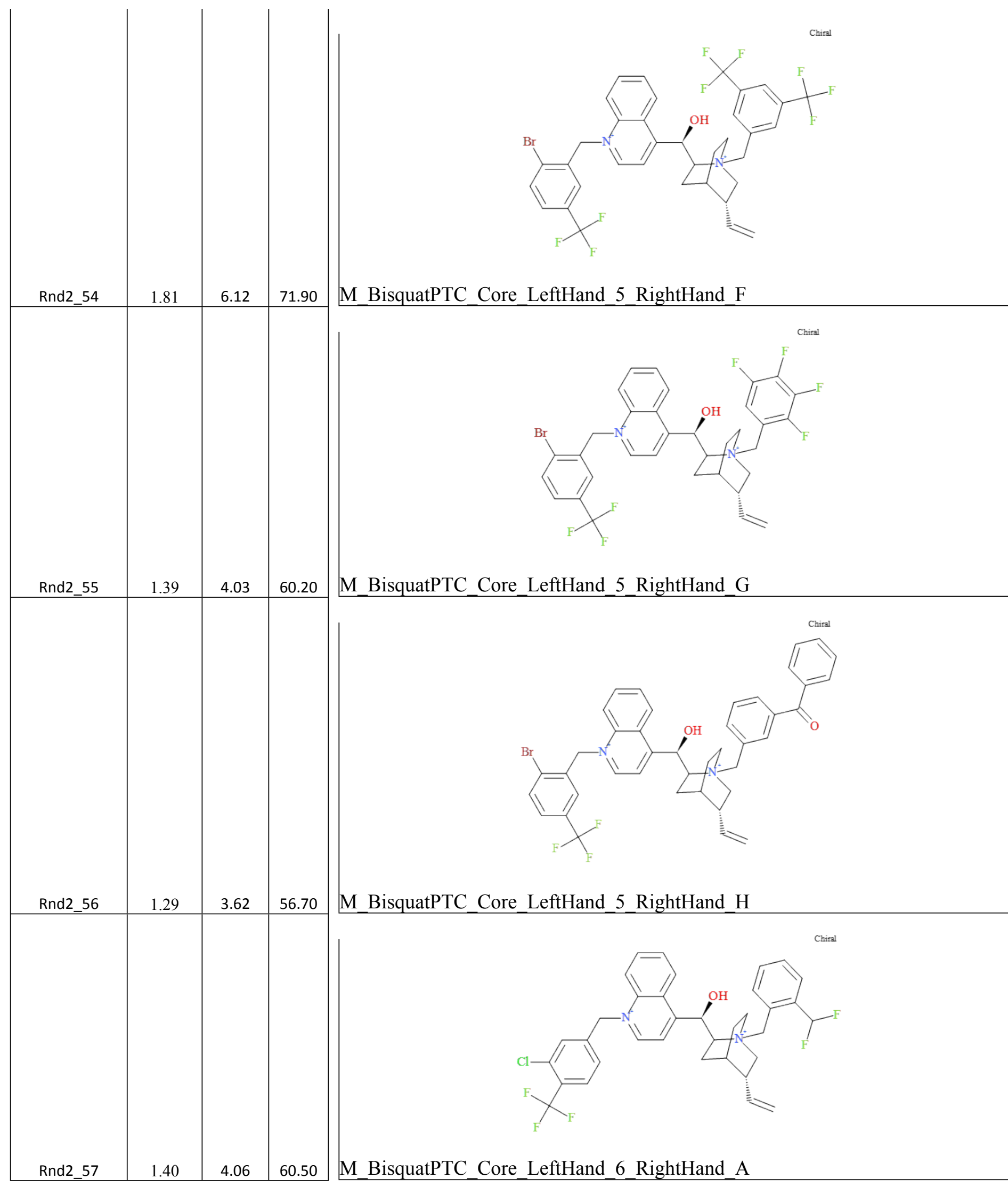




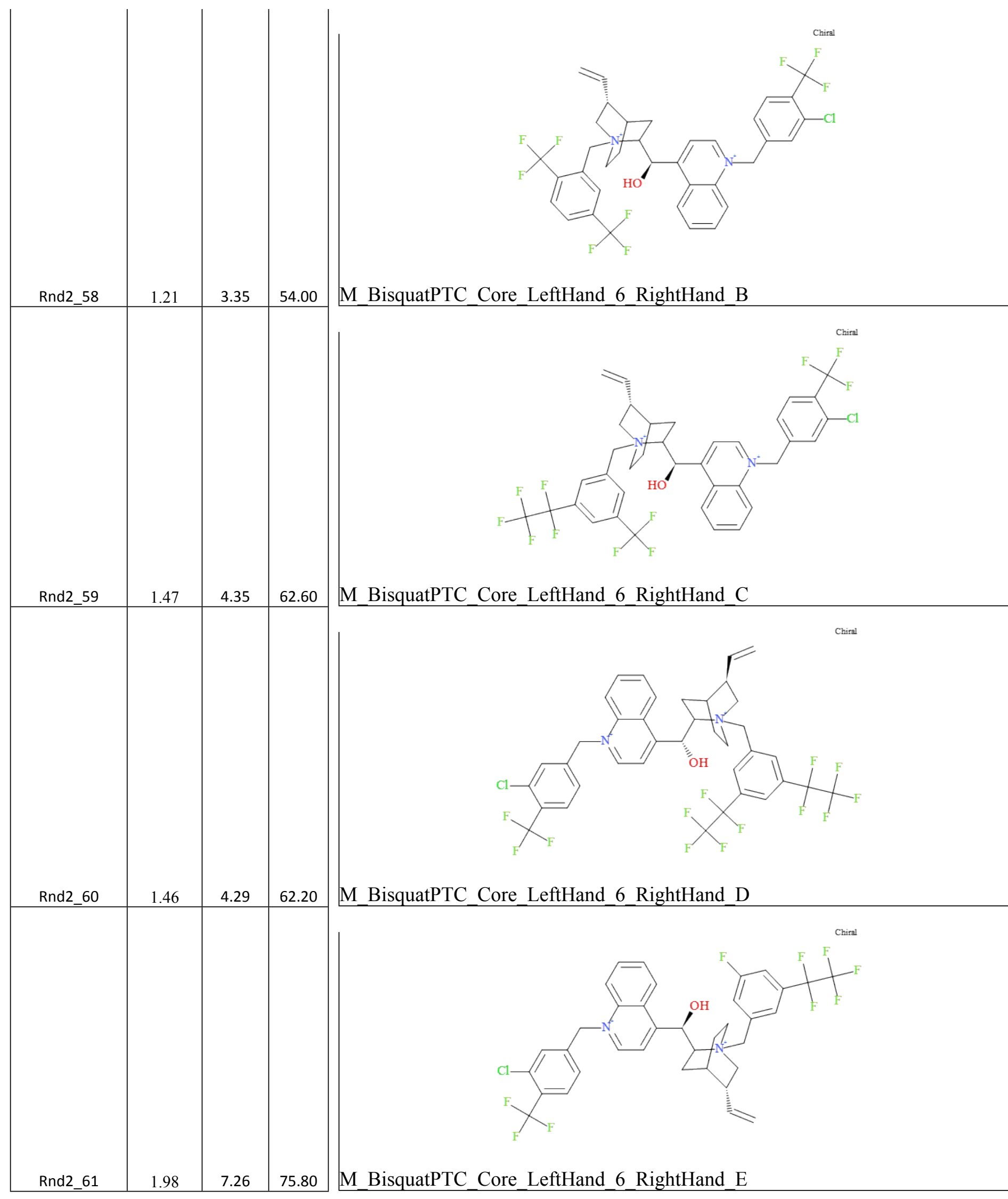




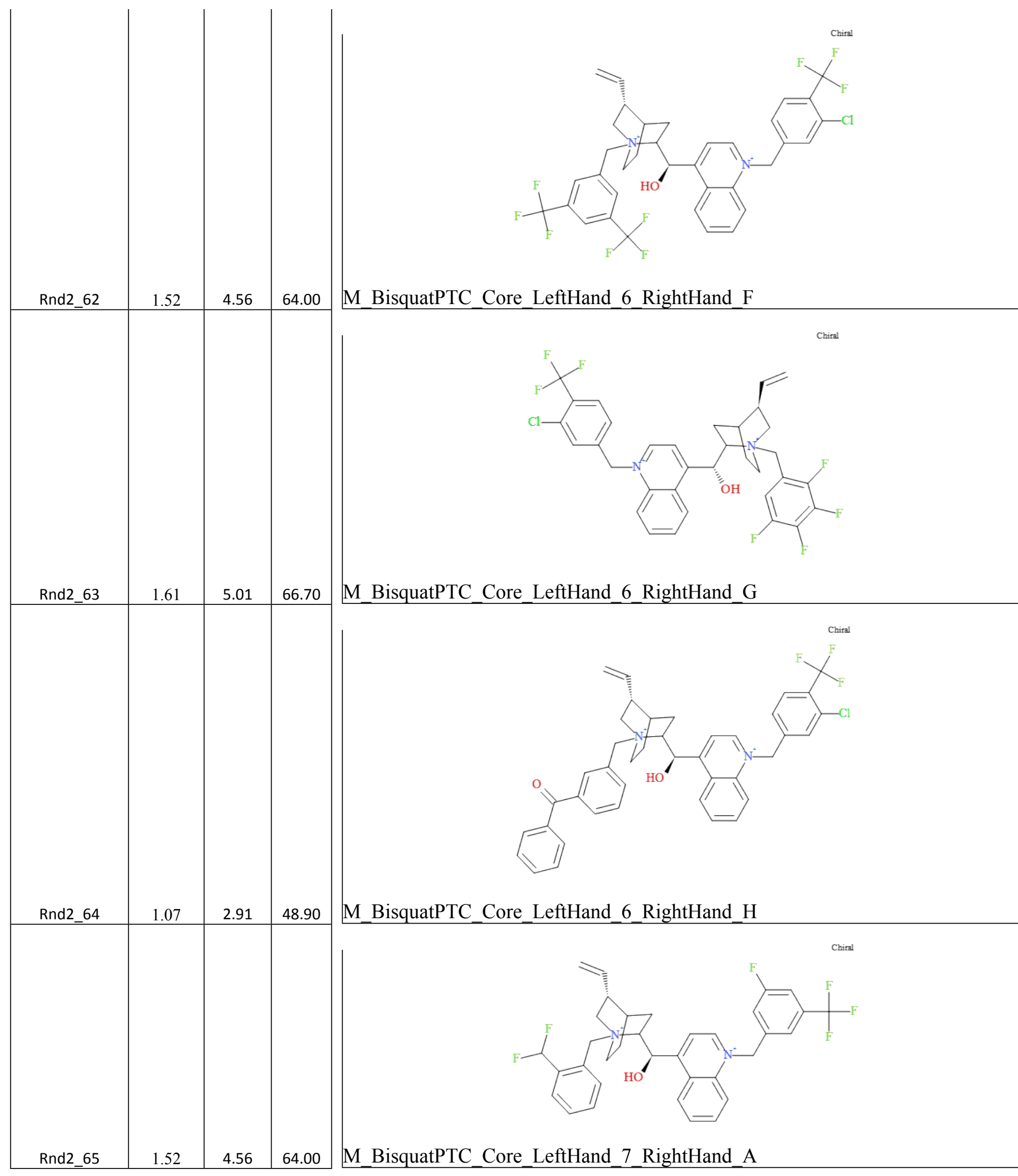




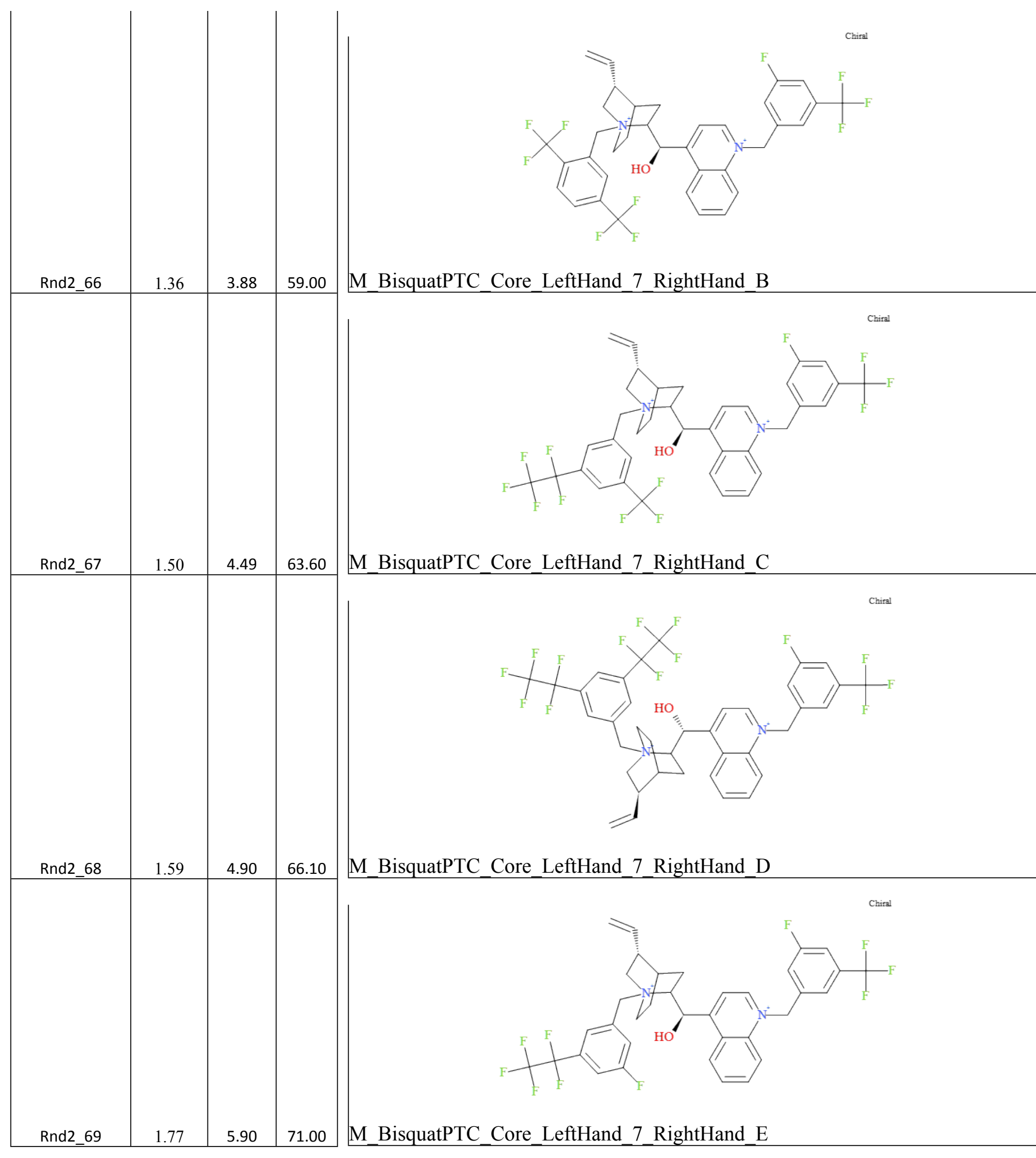




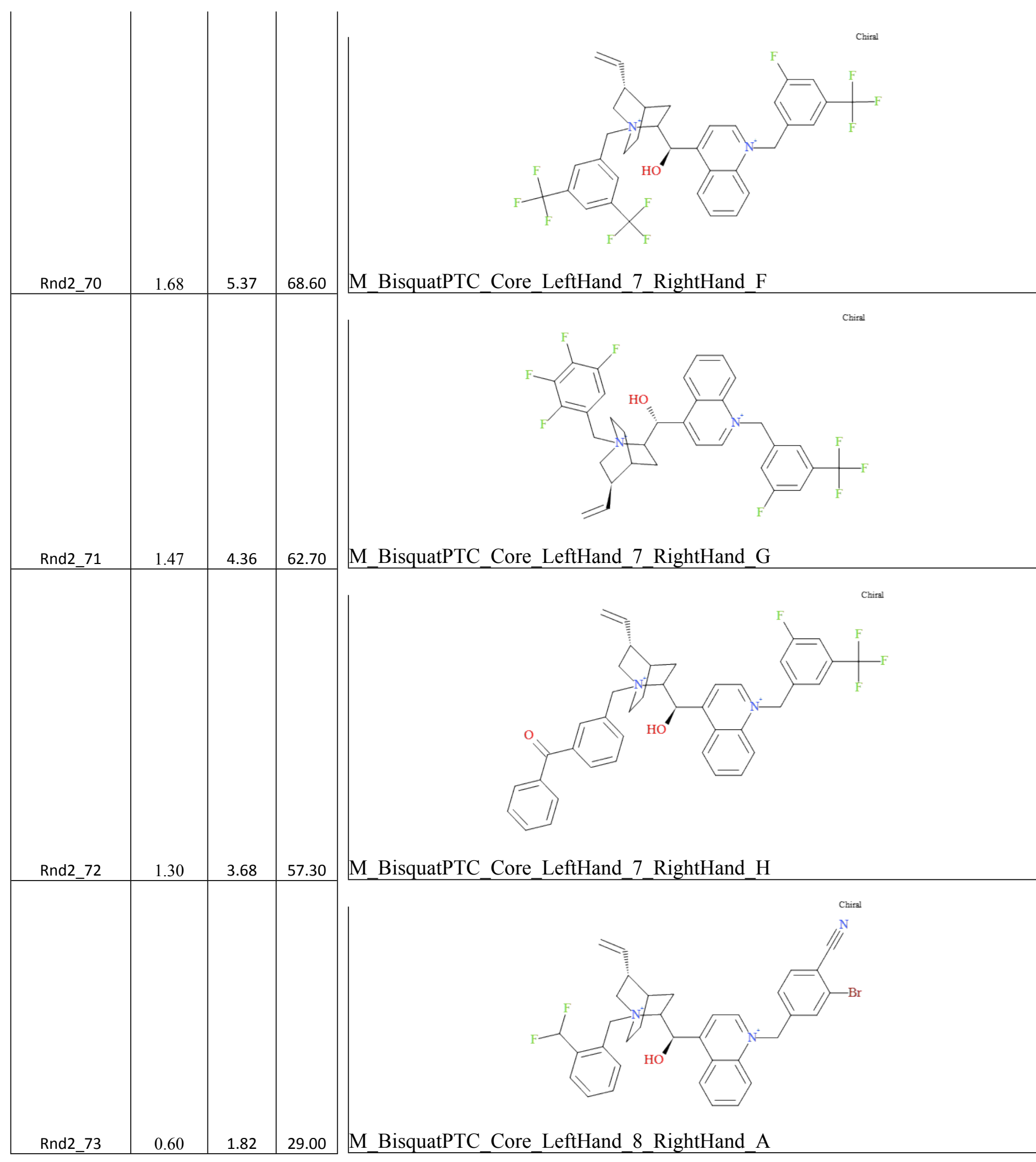




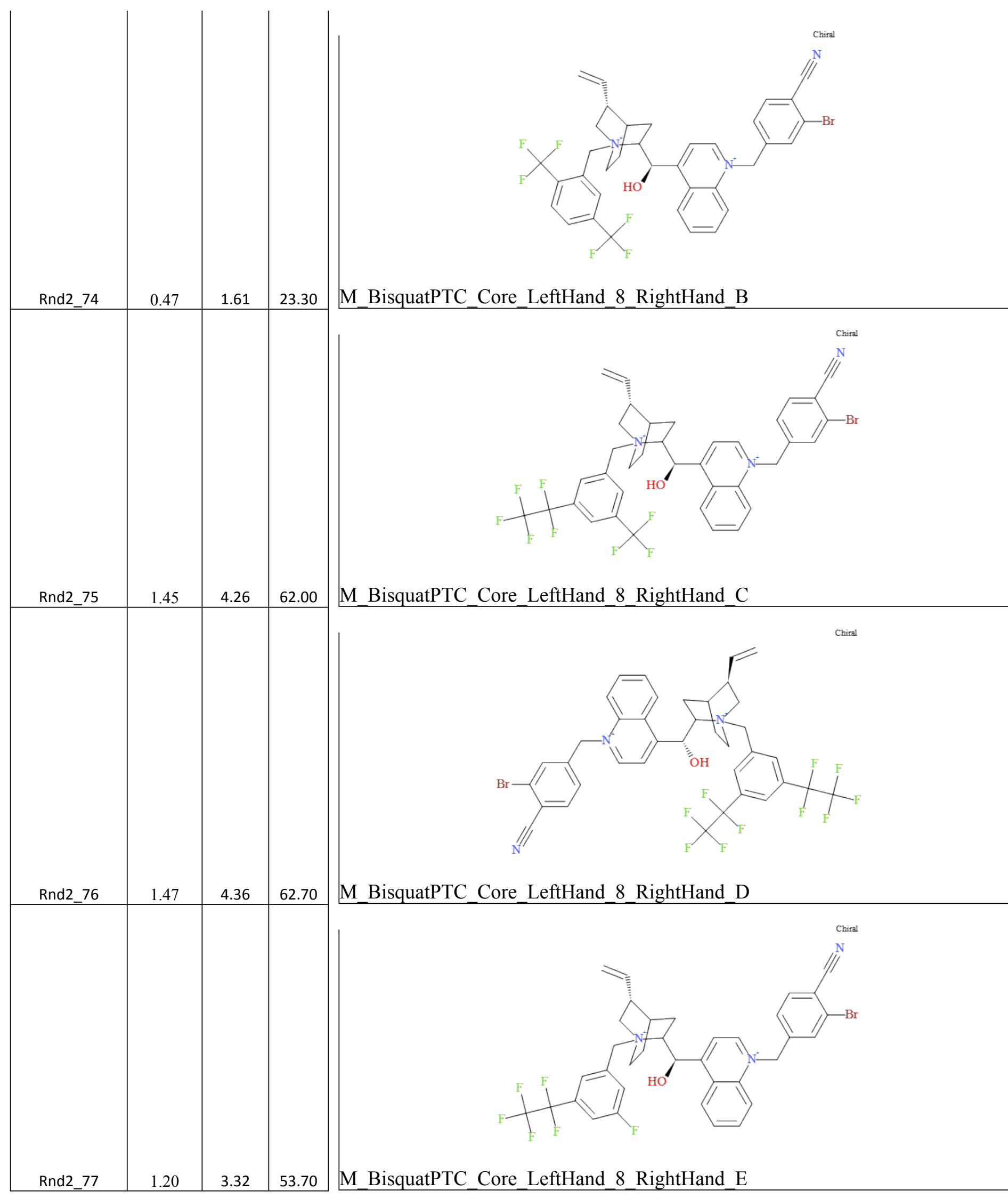




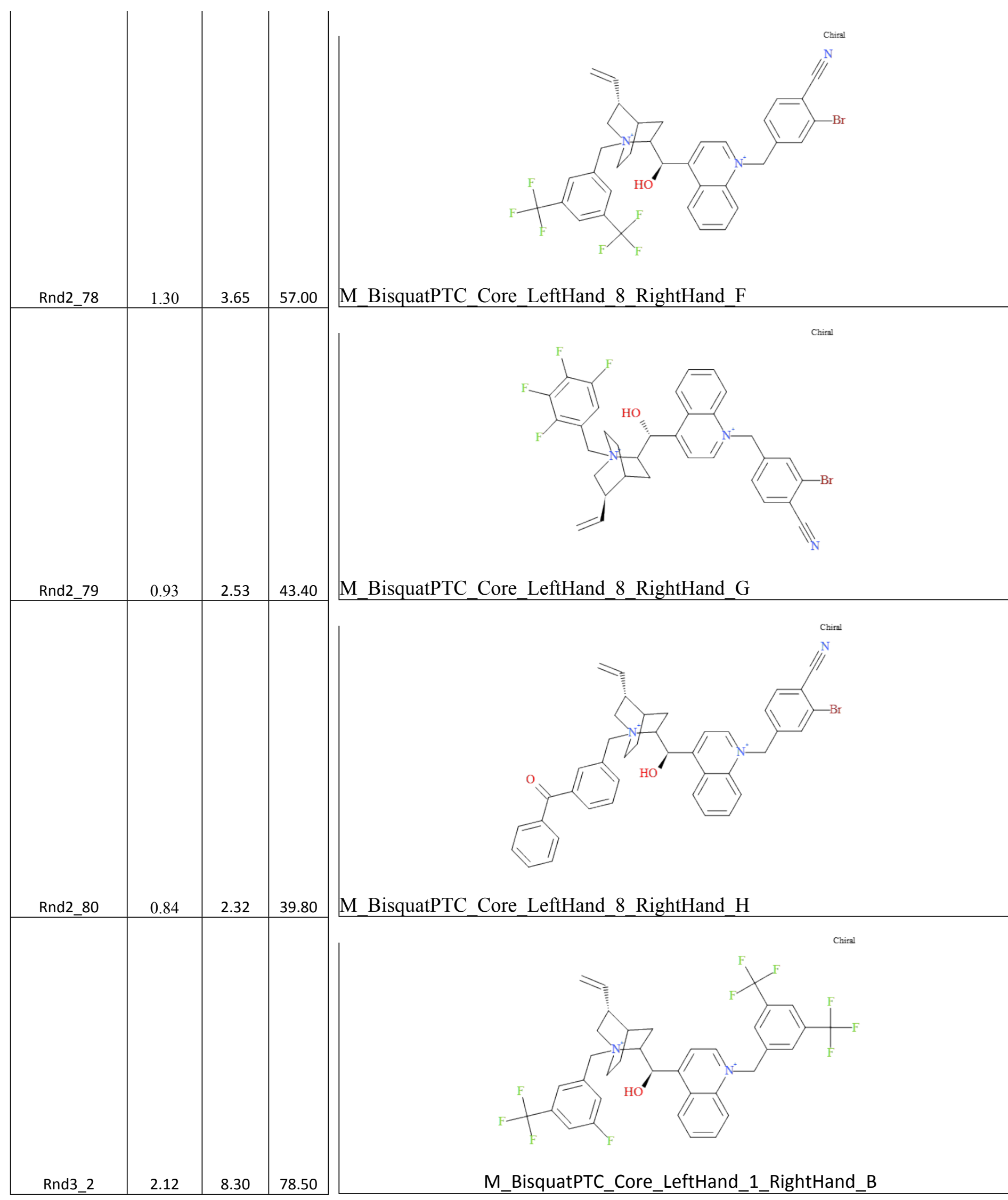




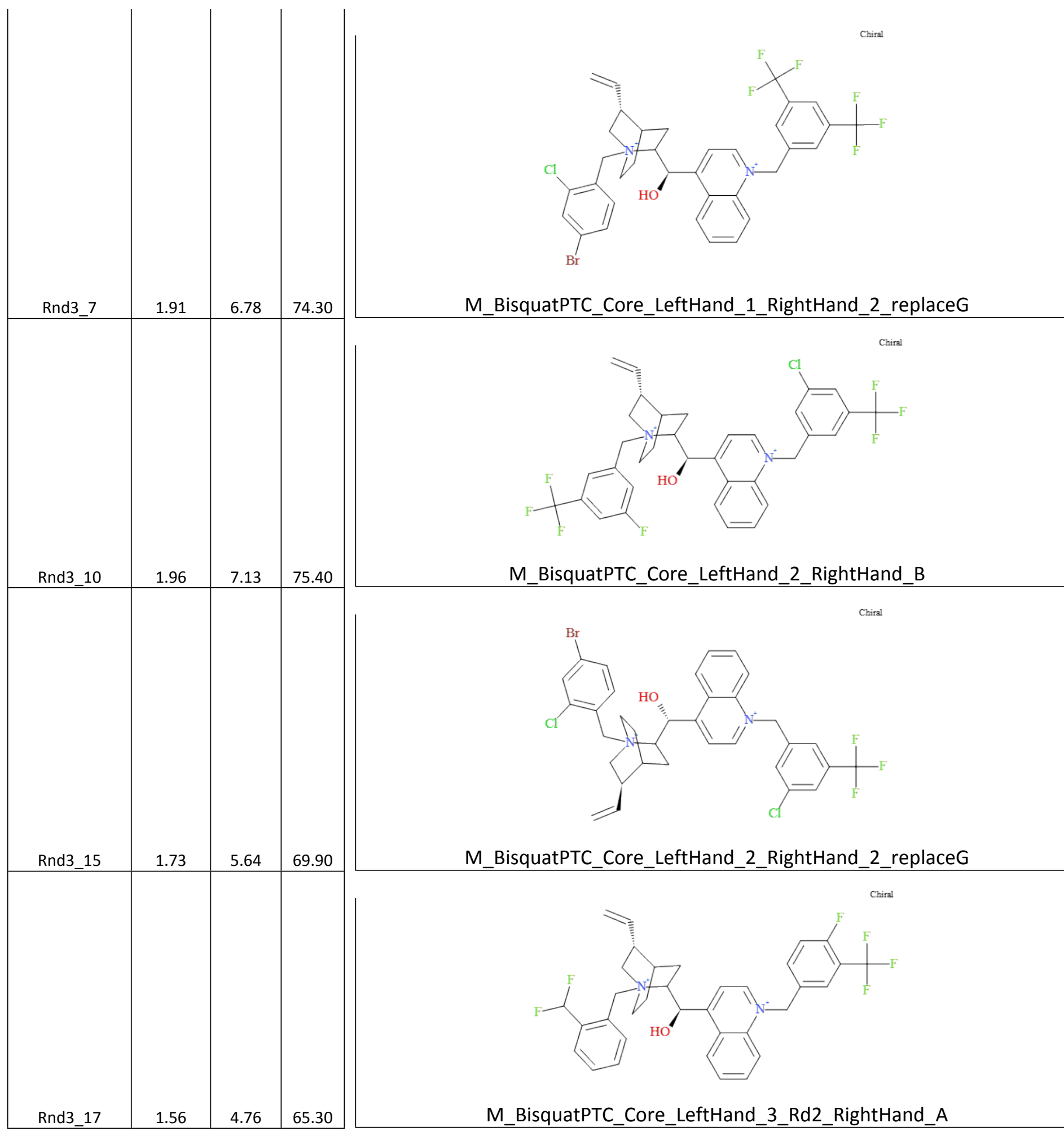




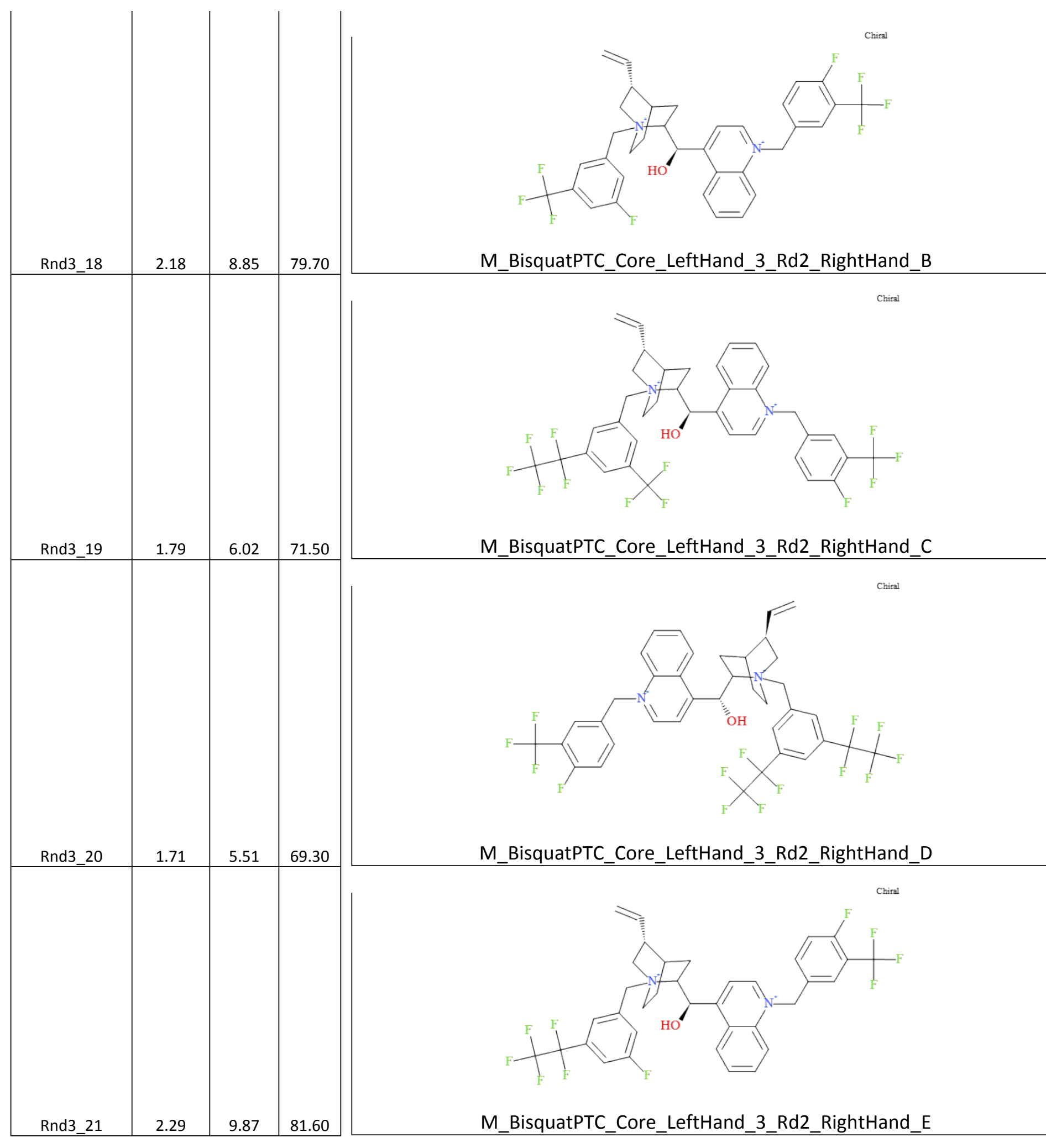




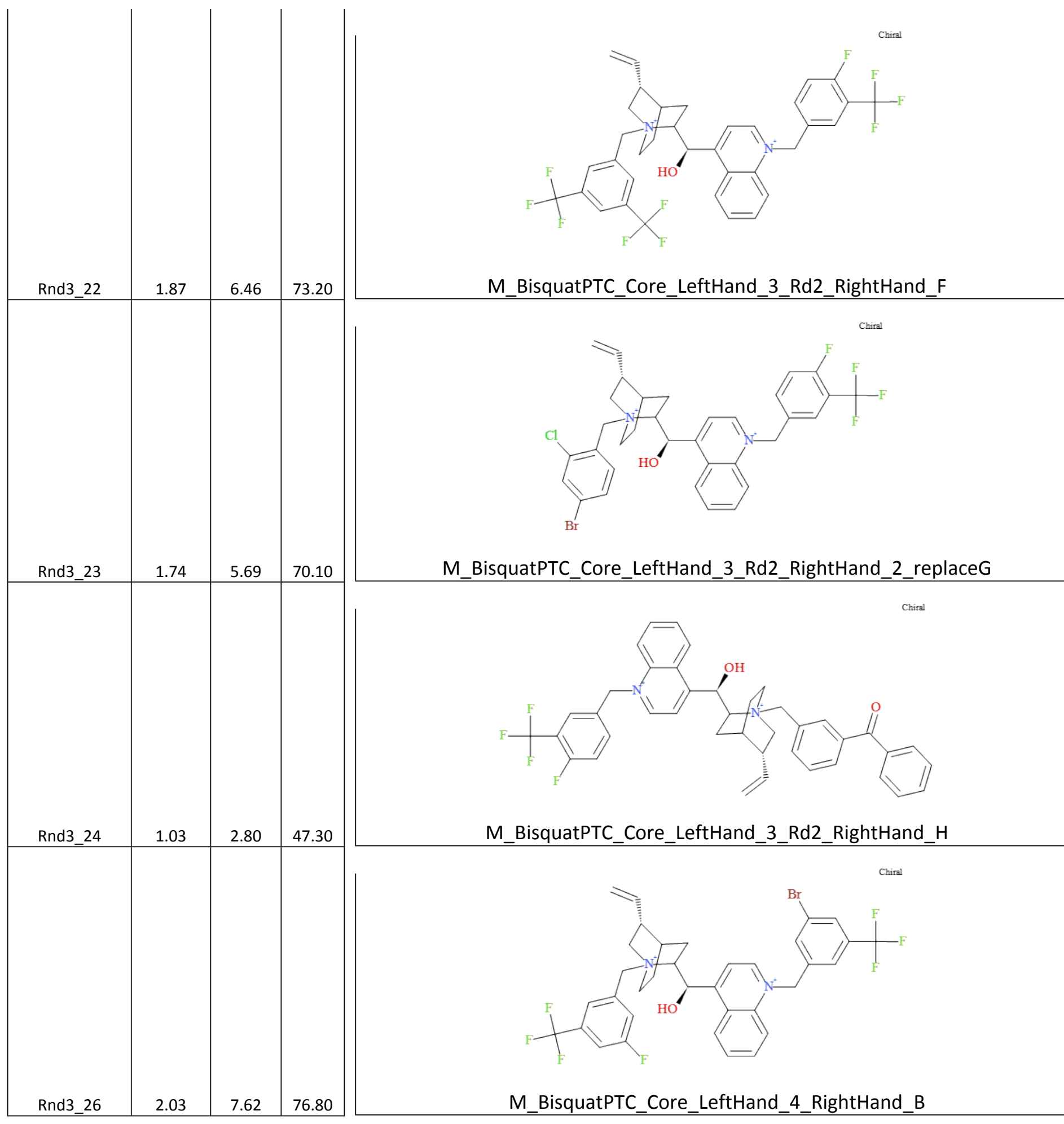




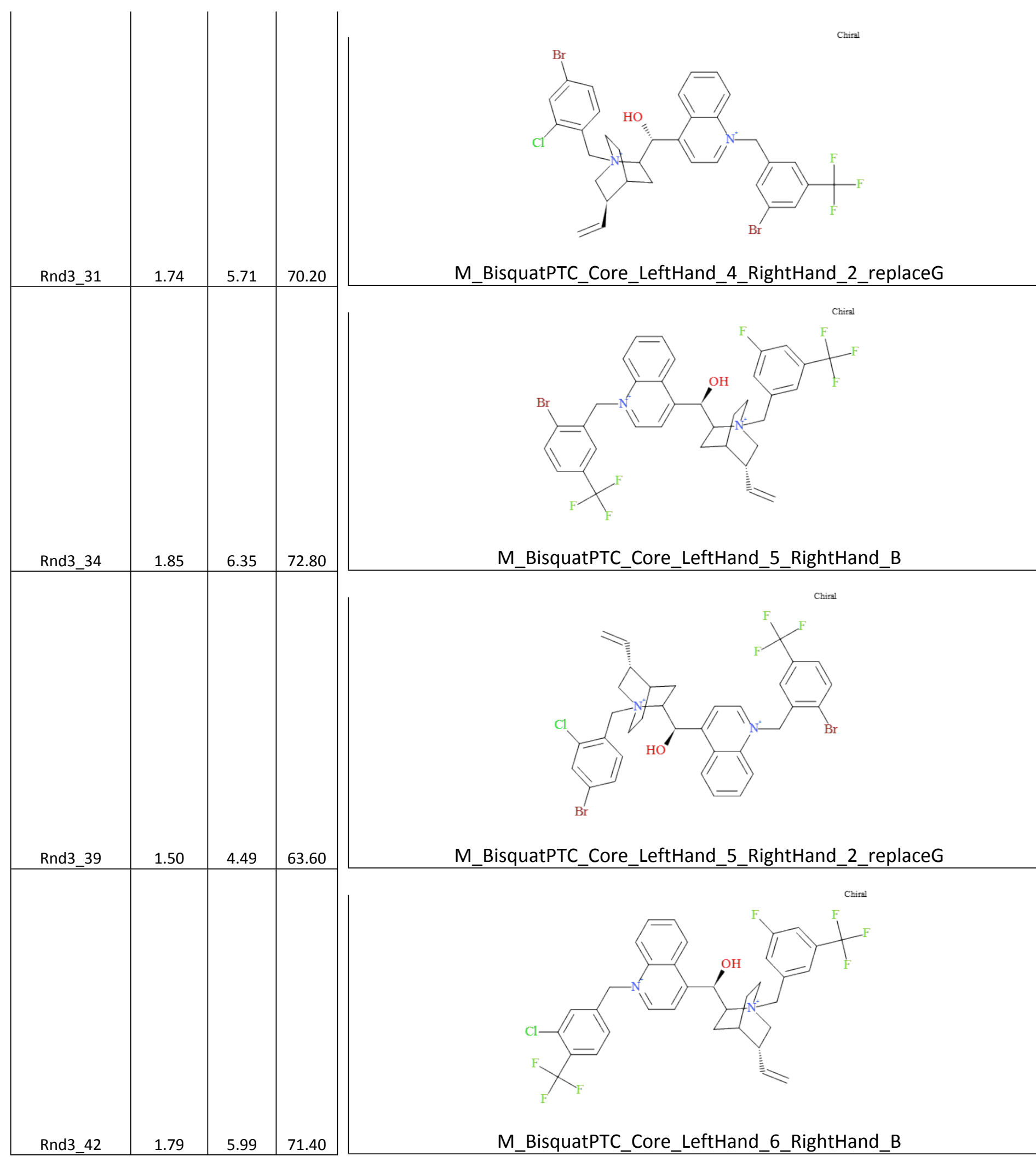




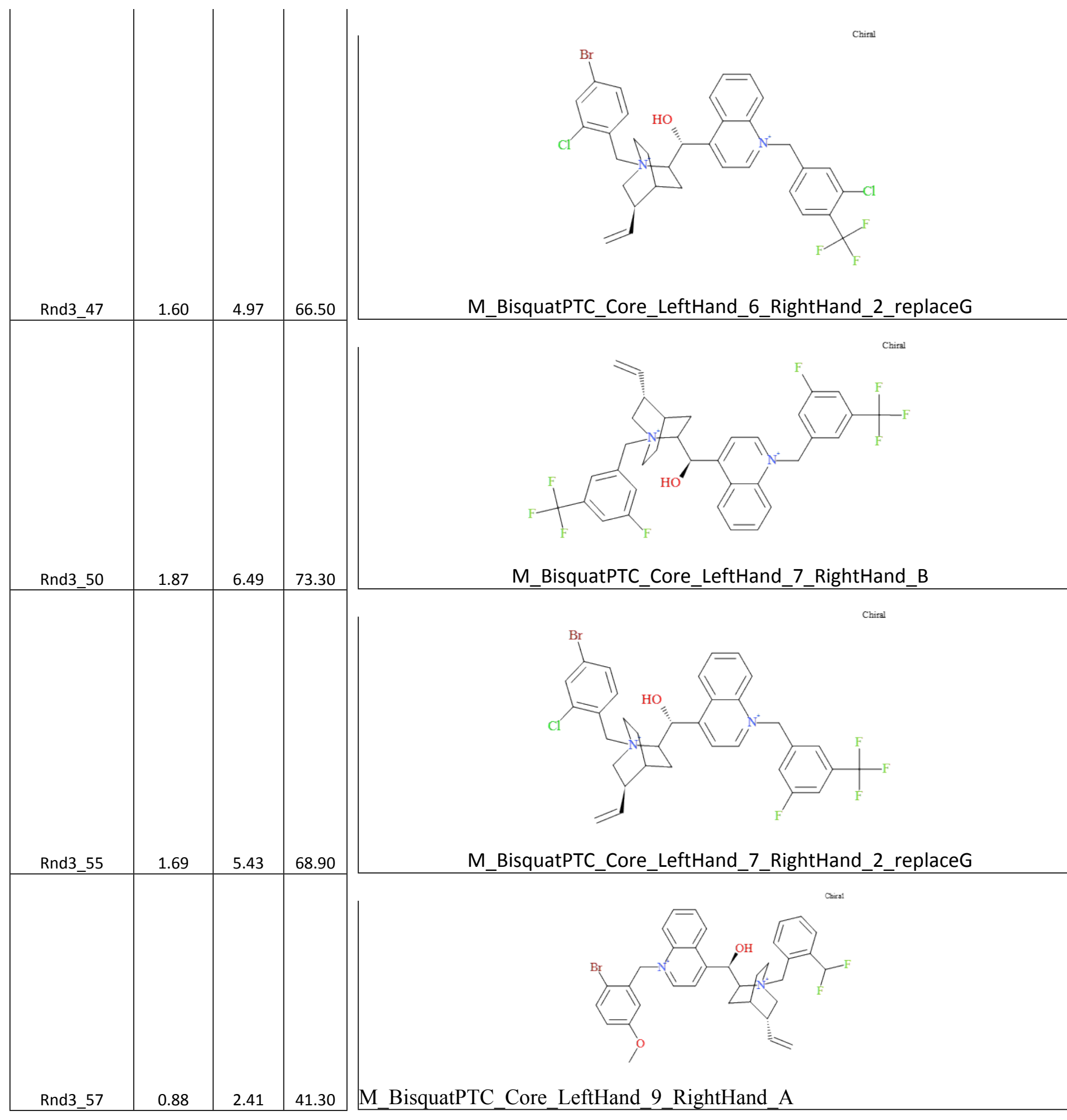




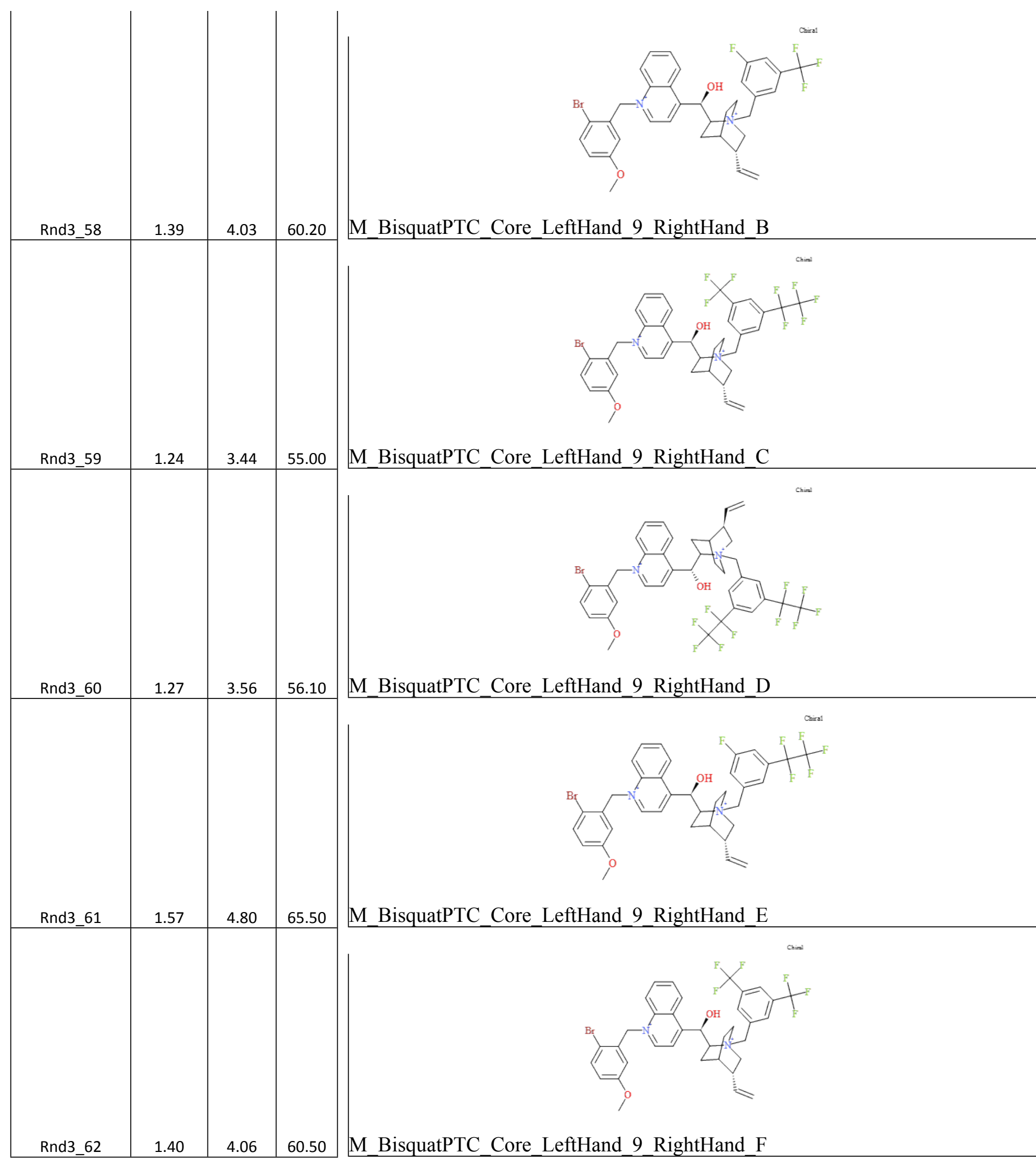




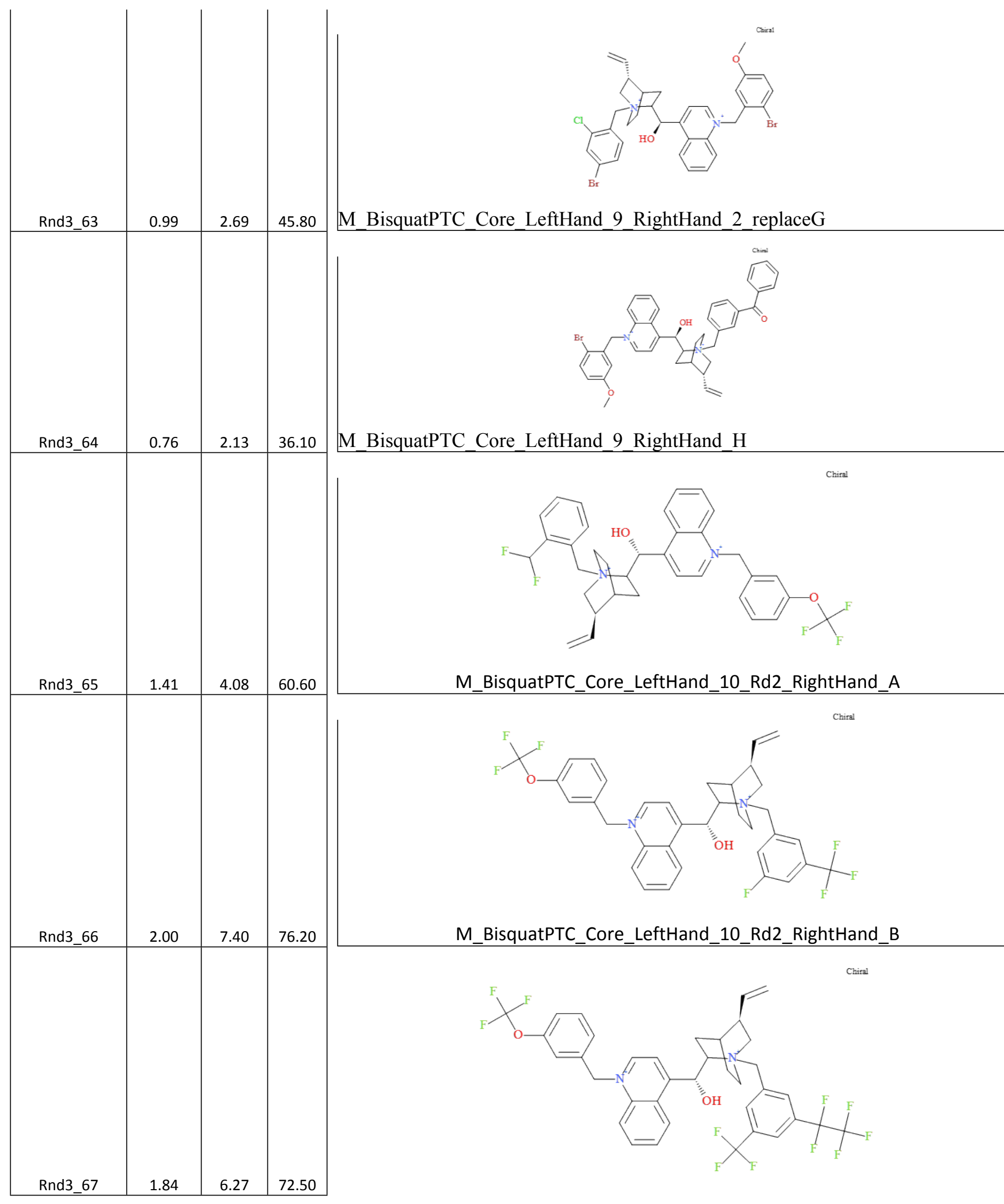




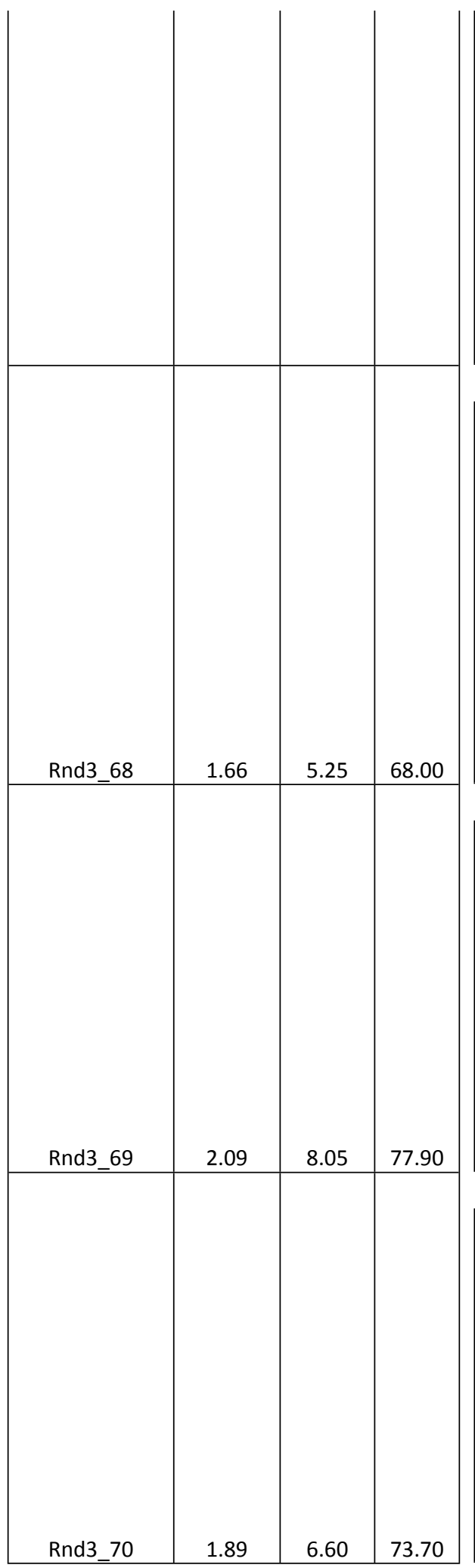

M_BisquatPTC_Core_LeftHand_10_Rd2_RightHand_C

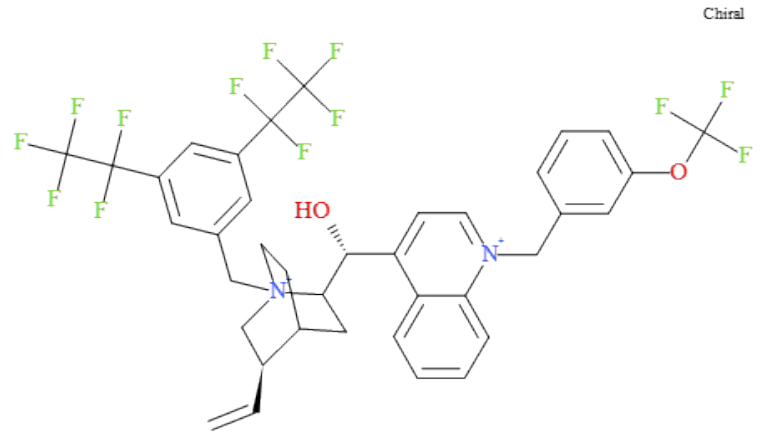

M_BisquatPTC_Core_LeftHand_10_Rd2_RightHand_D

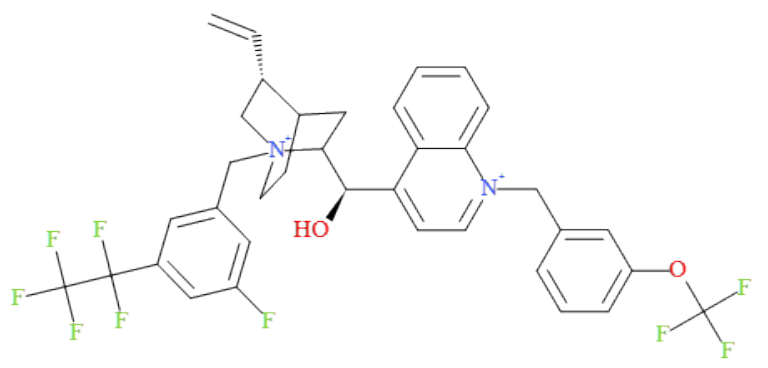

M_BisquatPTC_Core_LeftHand_10_Rd2_RightHand_E

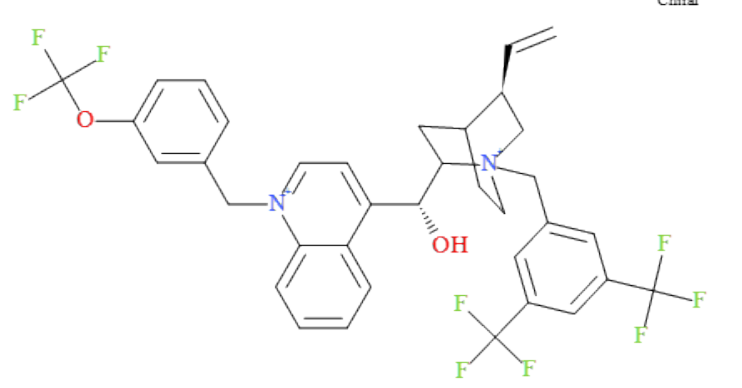

M_BisquatPTC_Core_LeftHand_10_Rd2_RightHand_F 


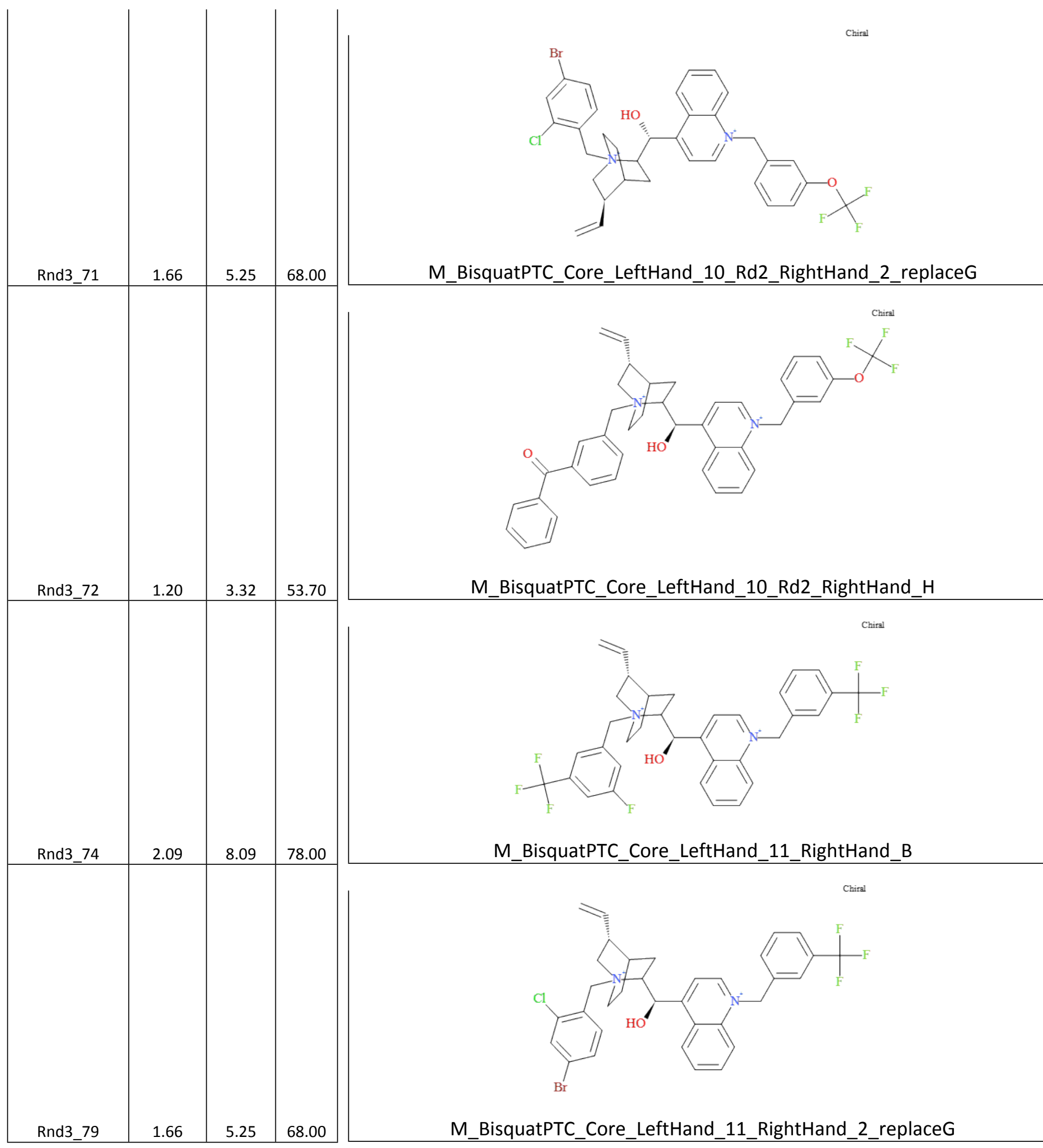




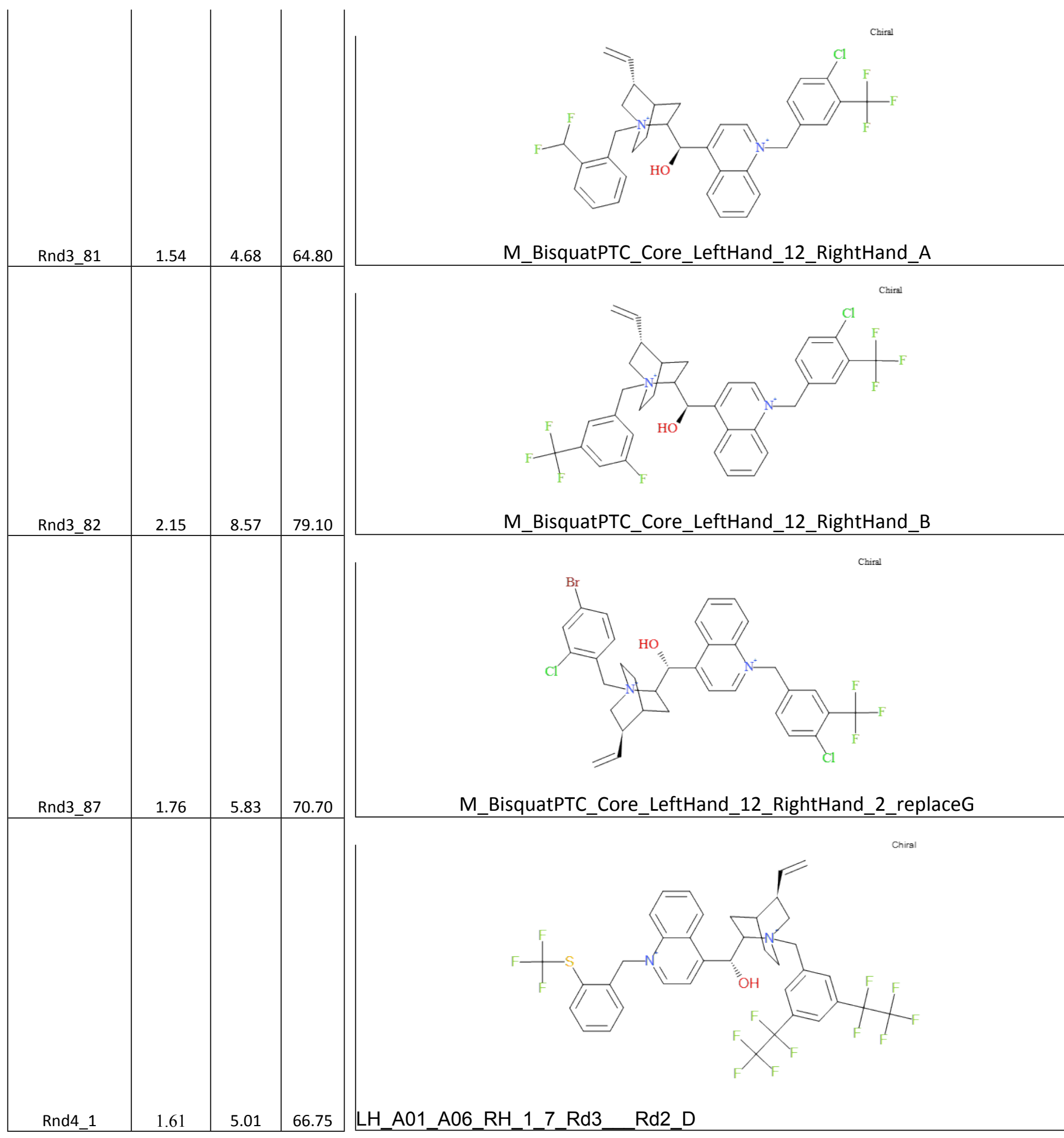




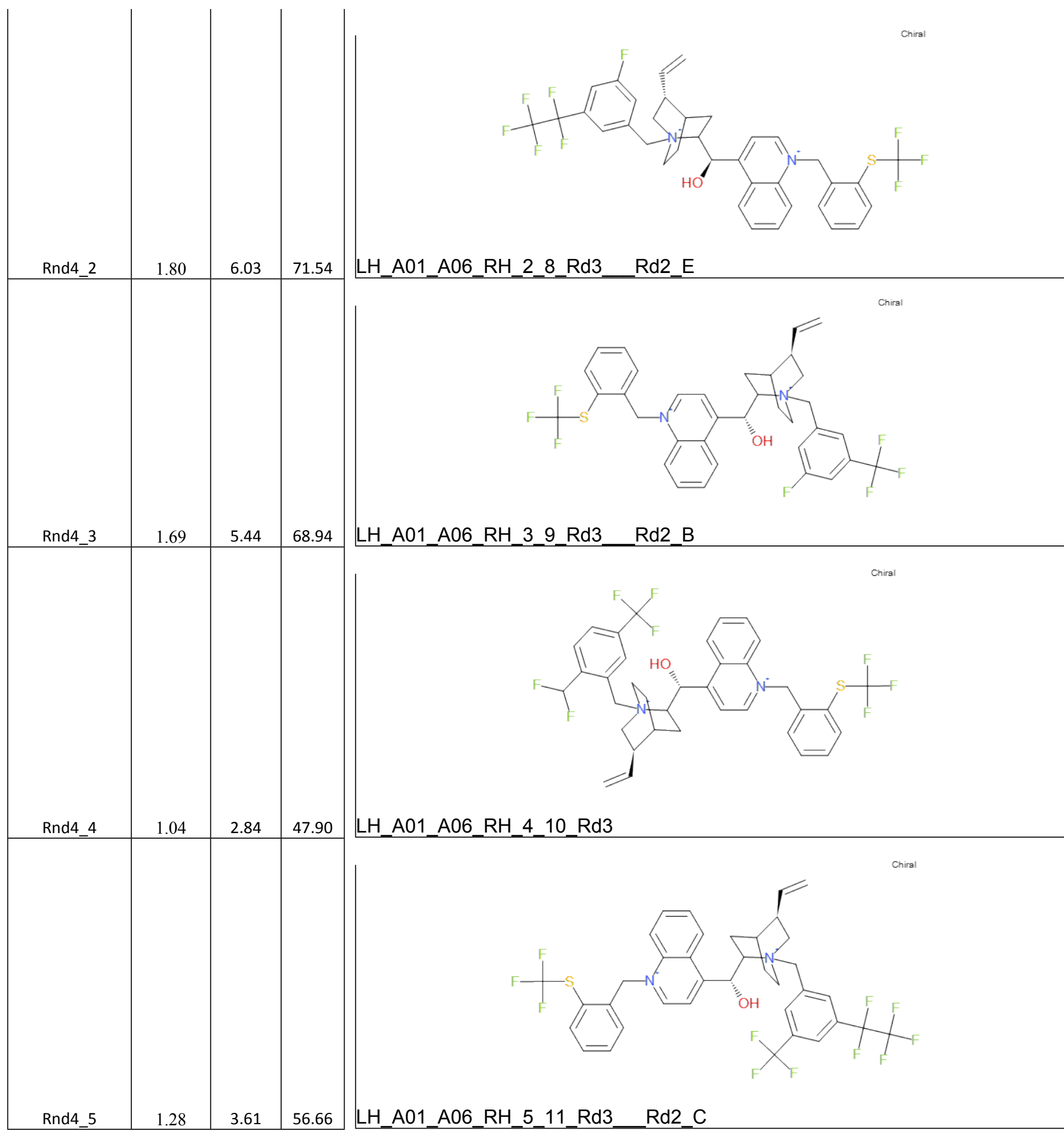




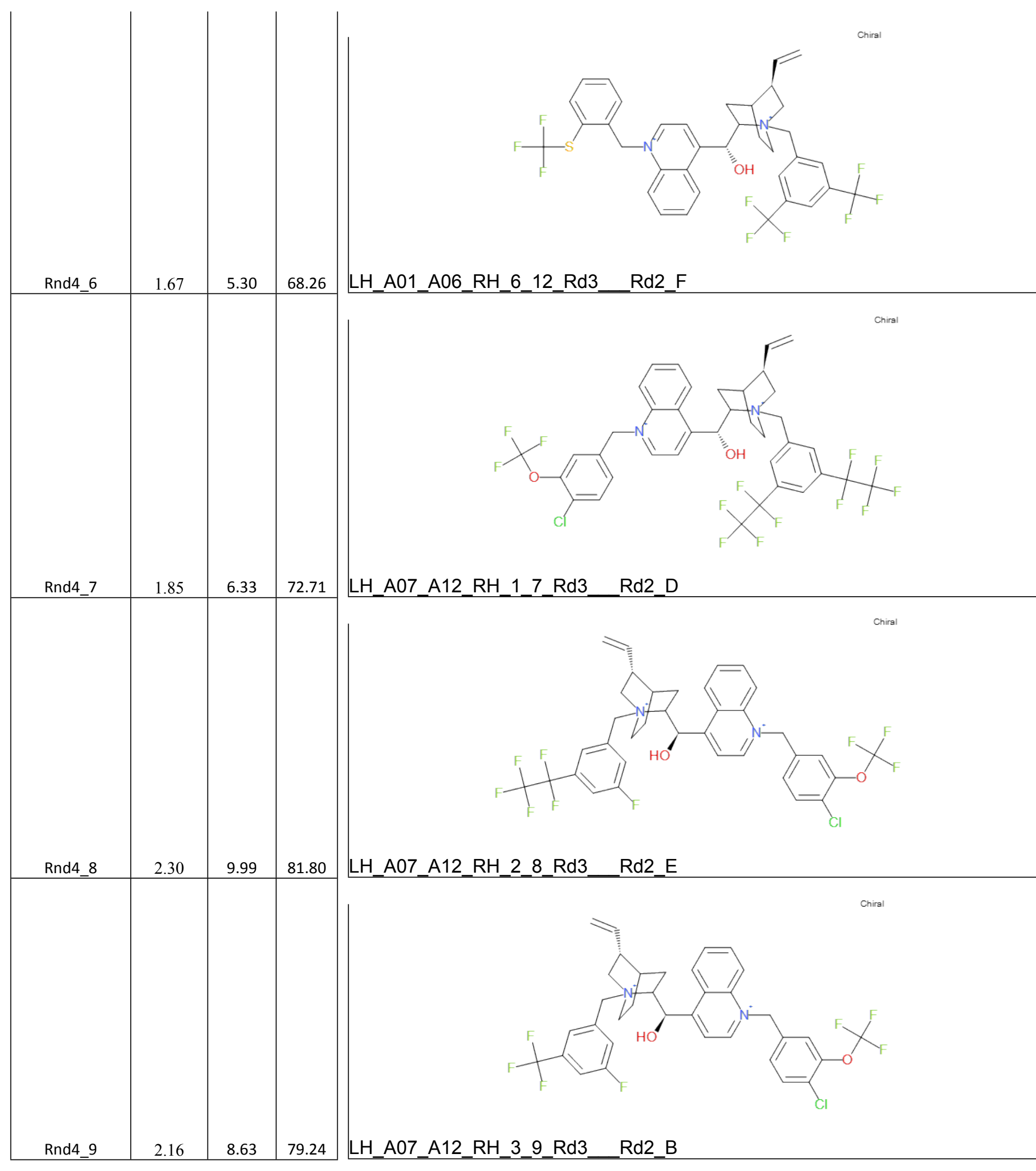




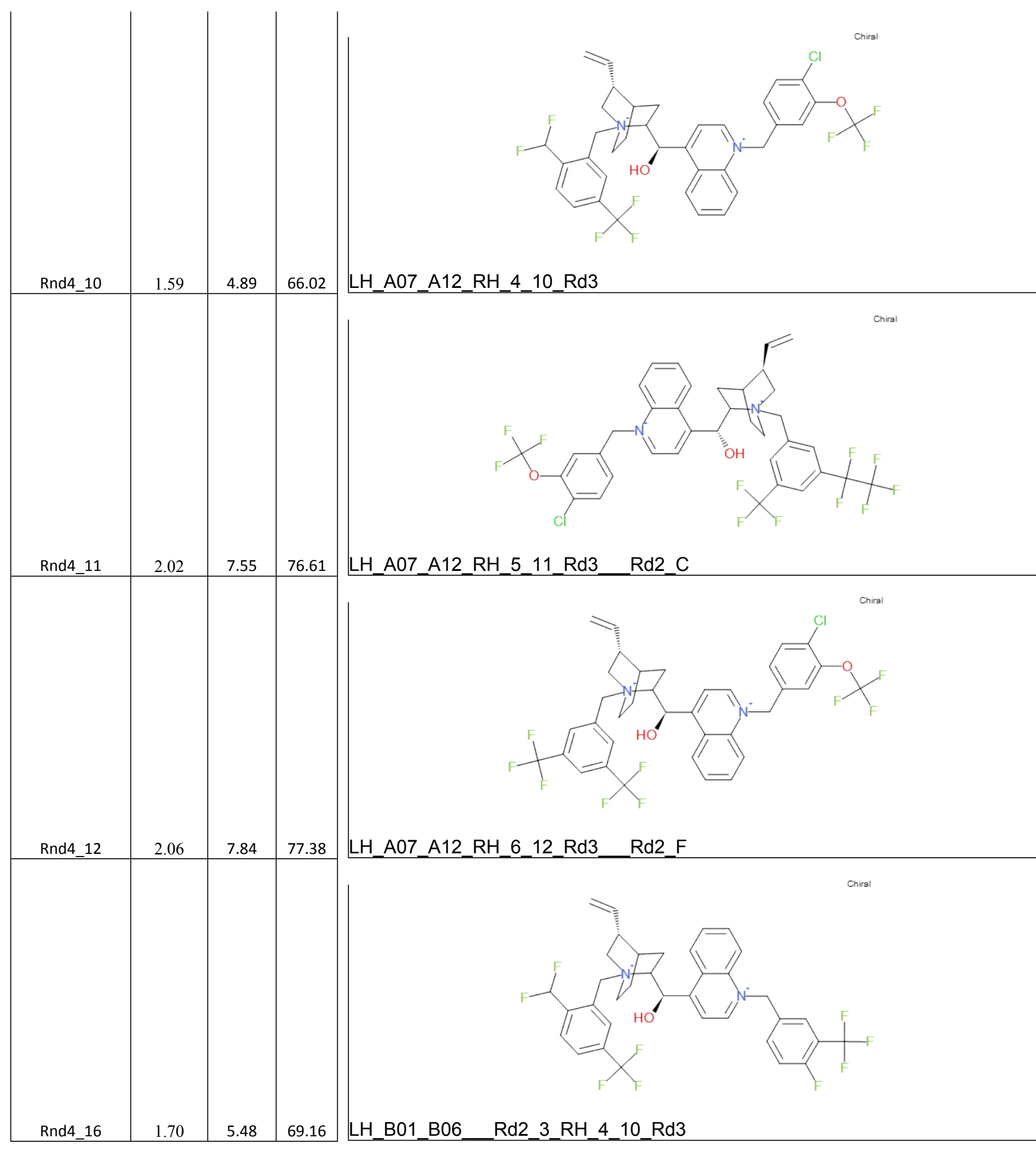




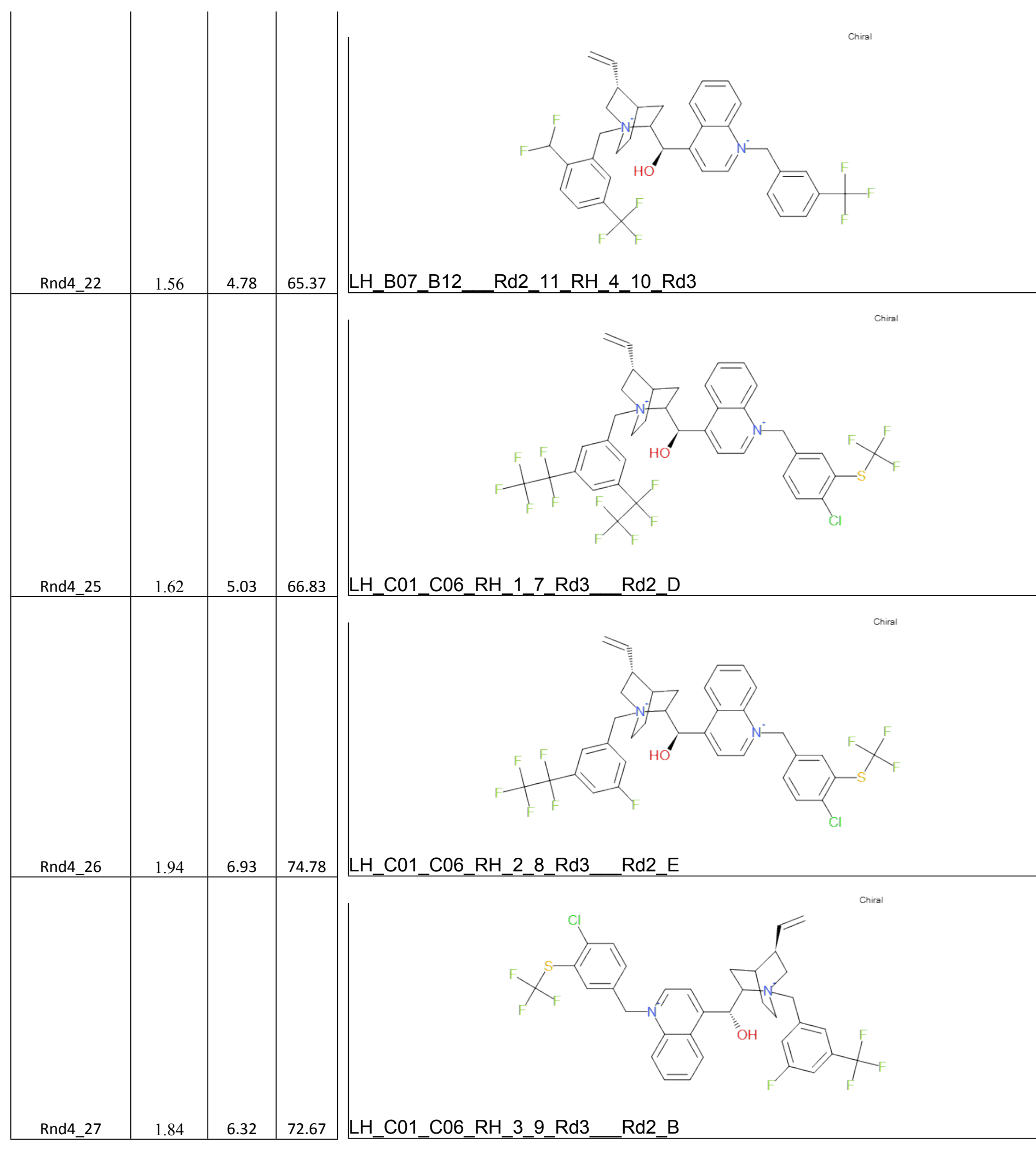




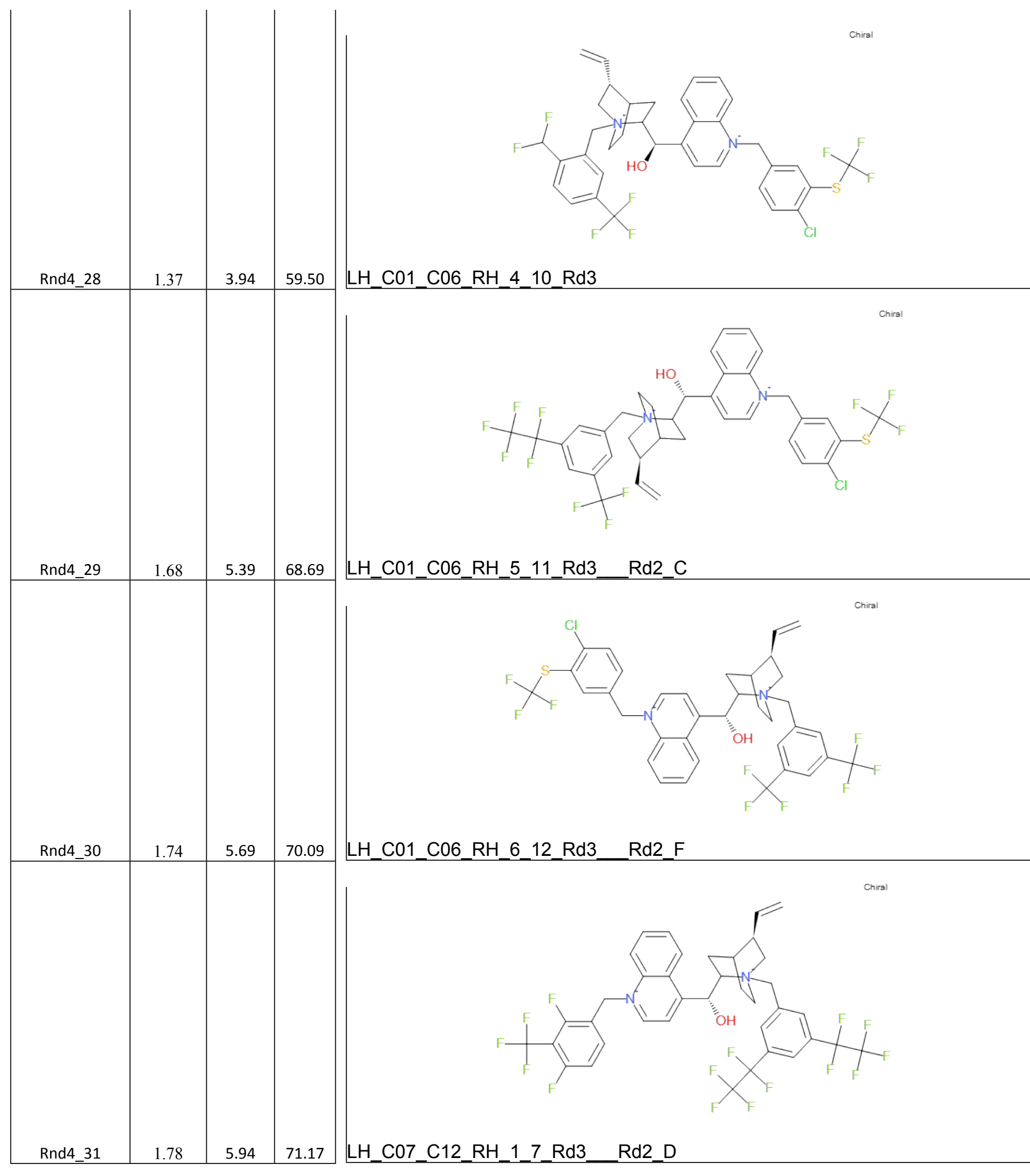




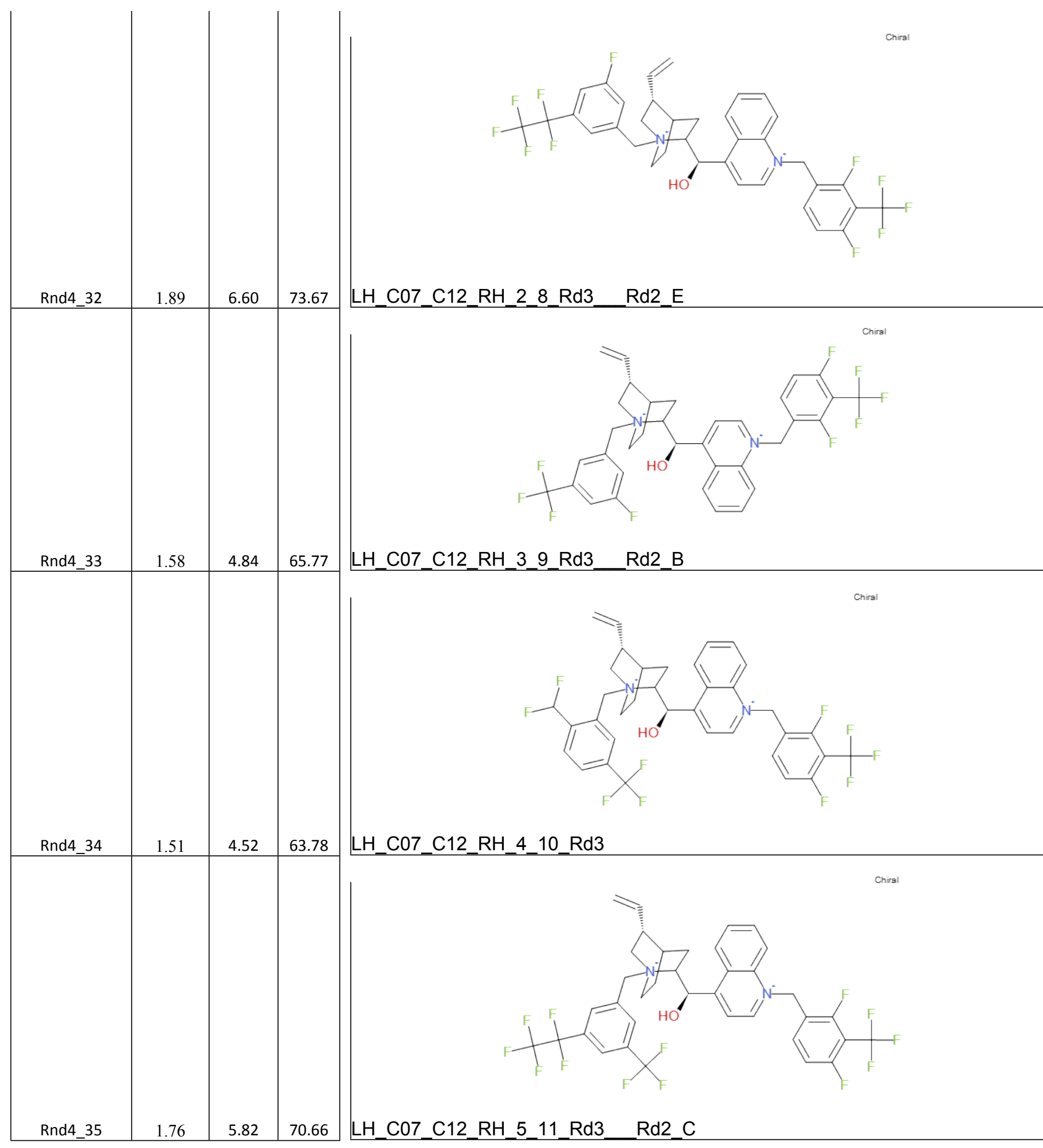




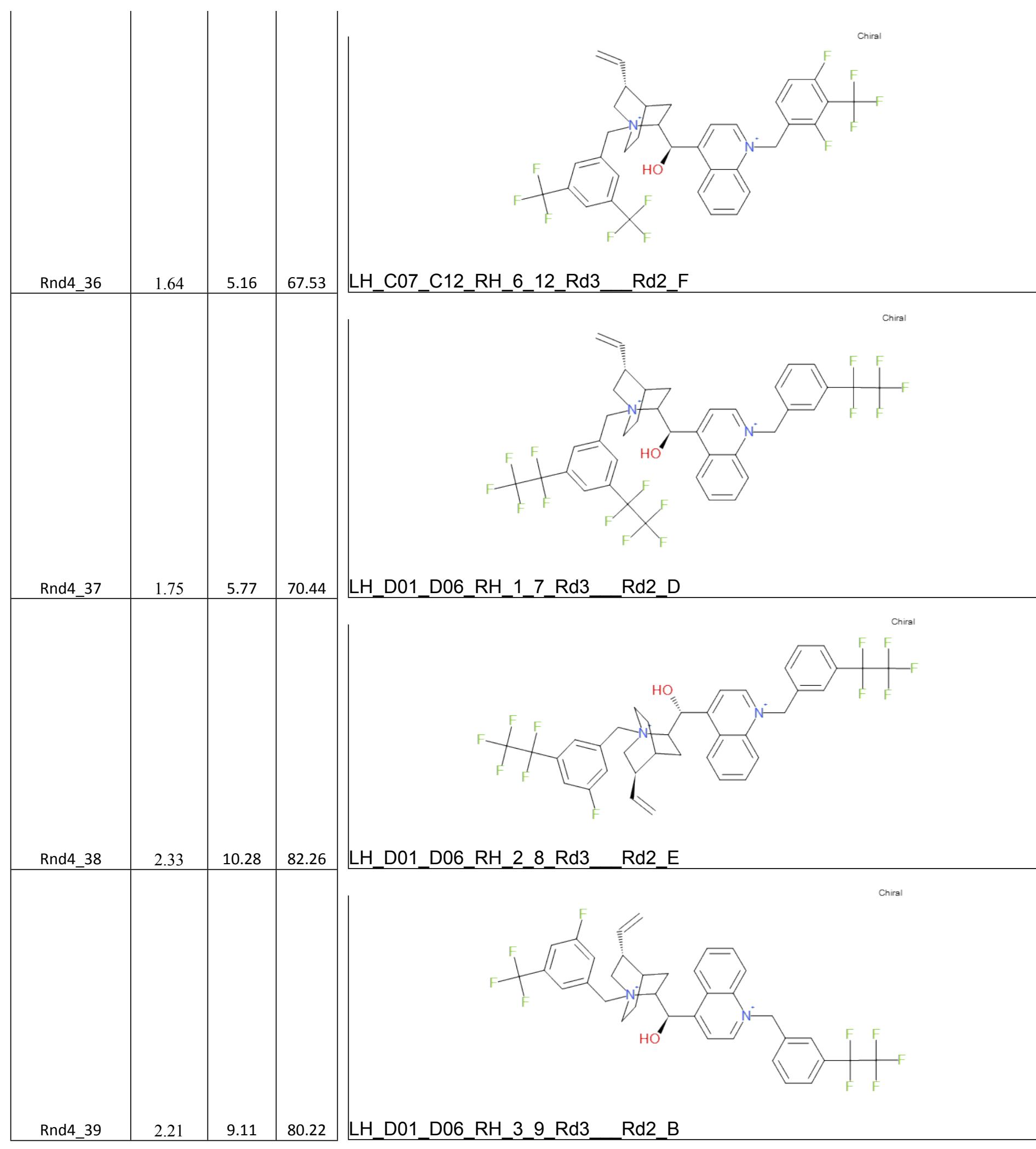




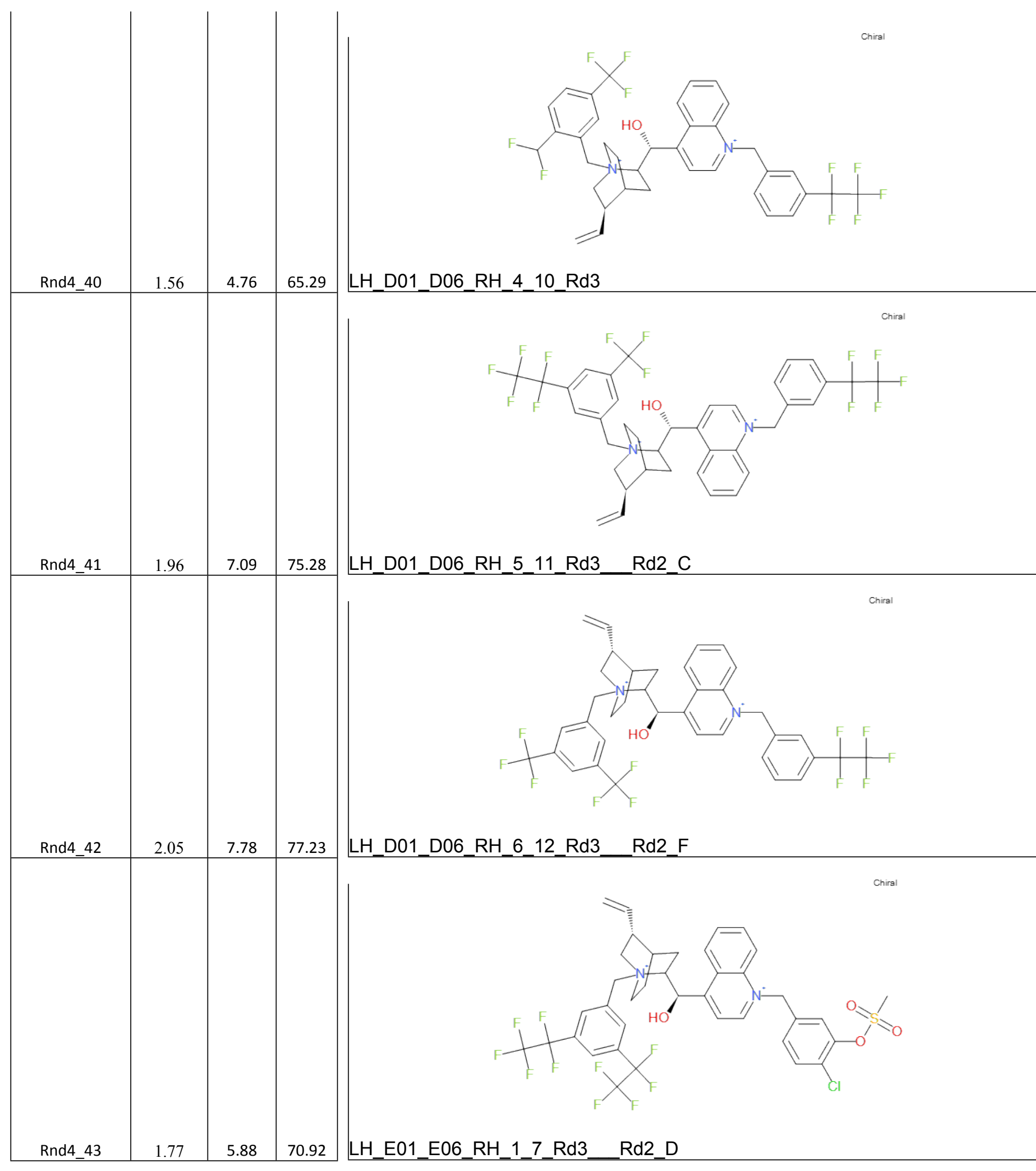




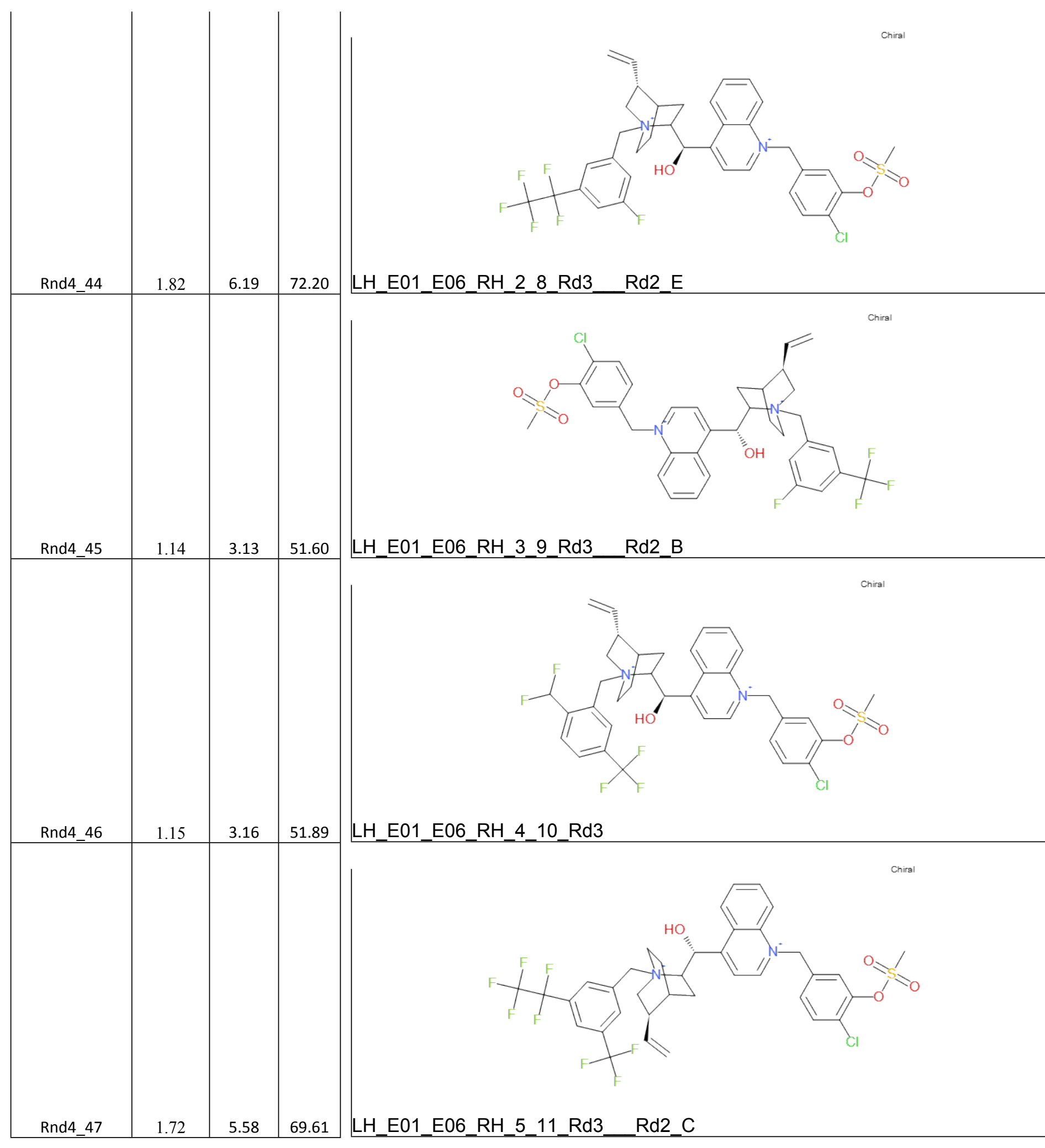




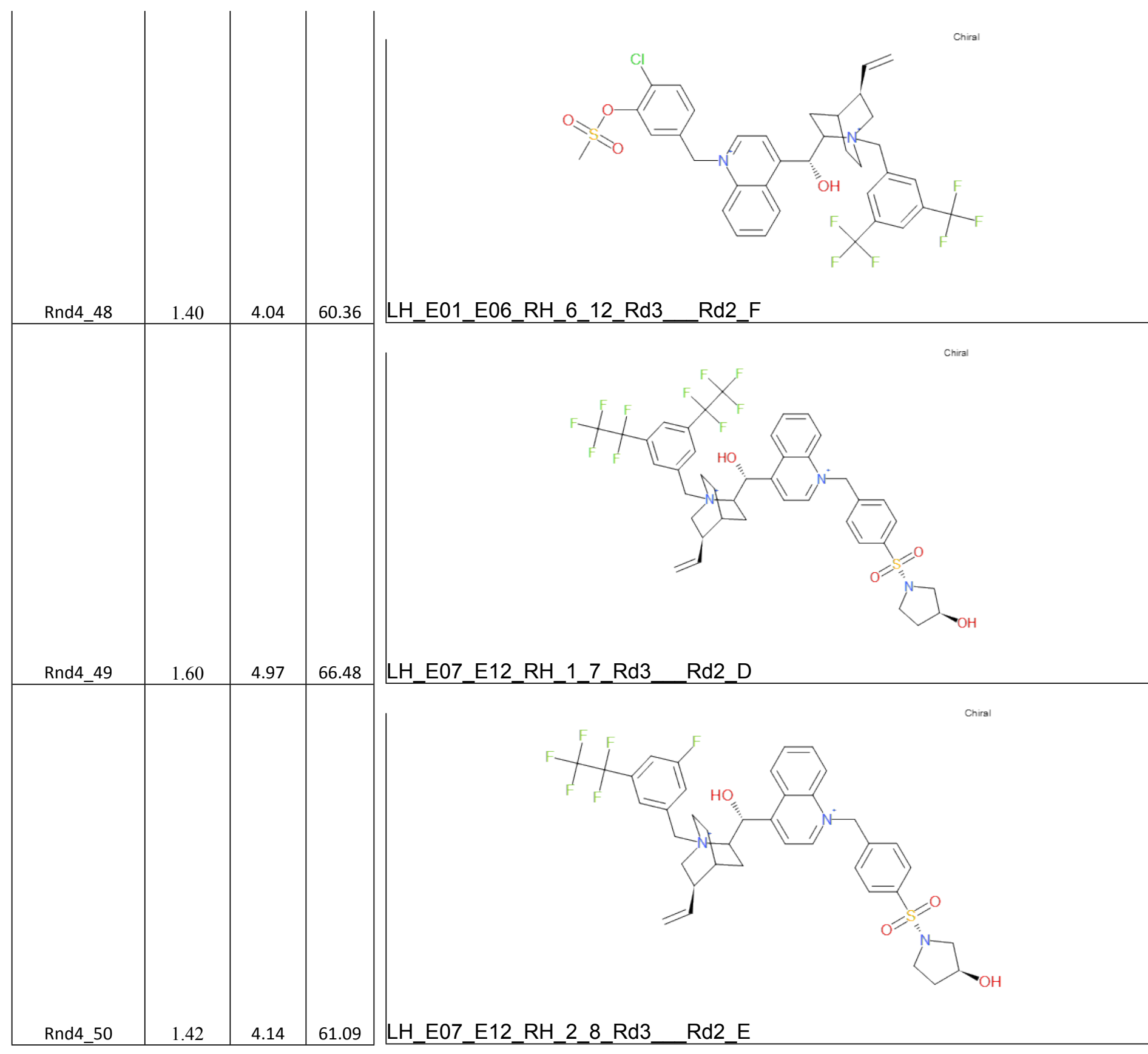




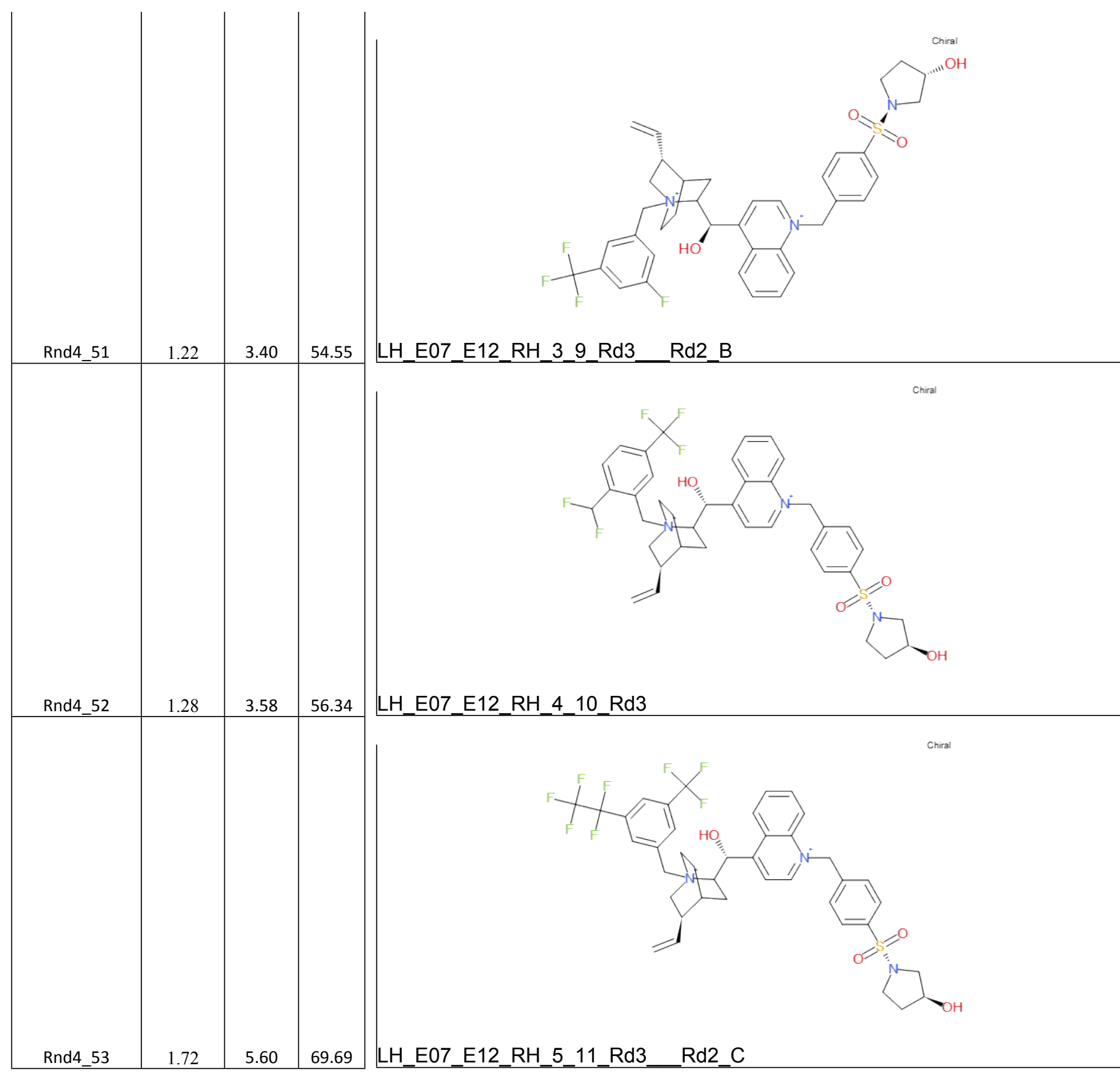




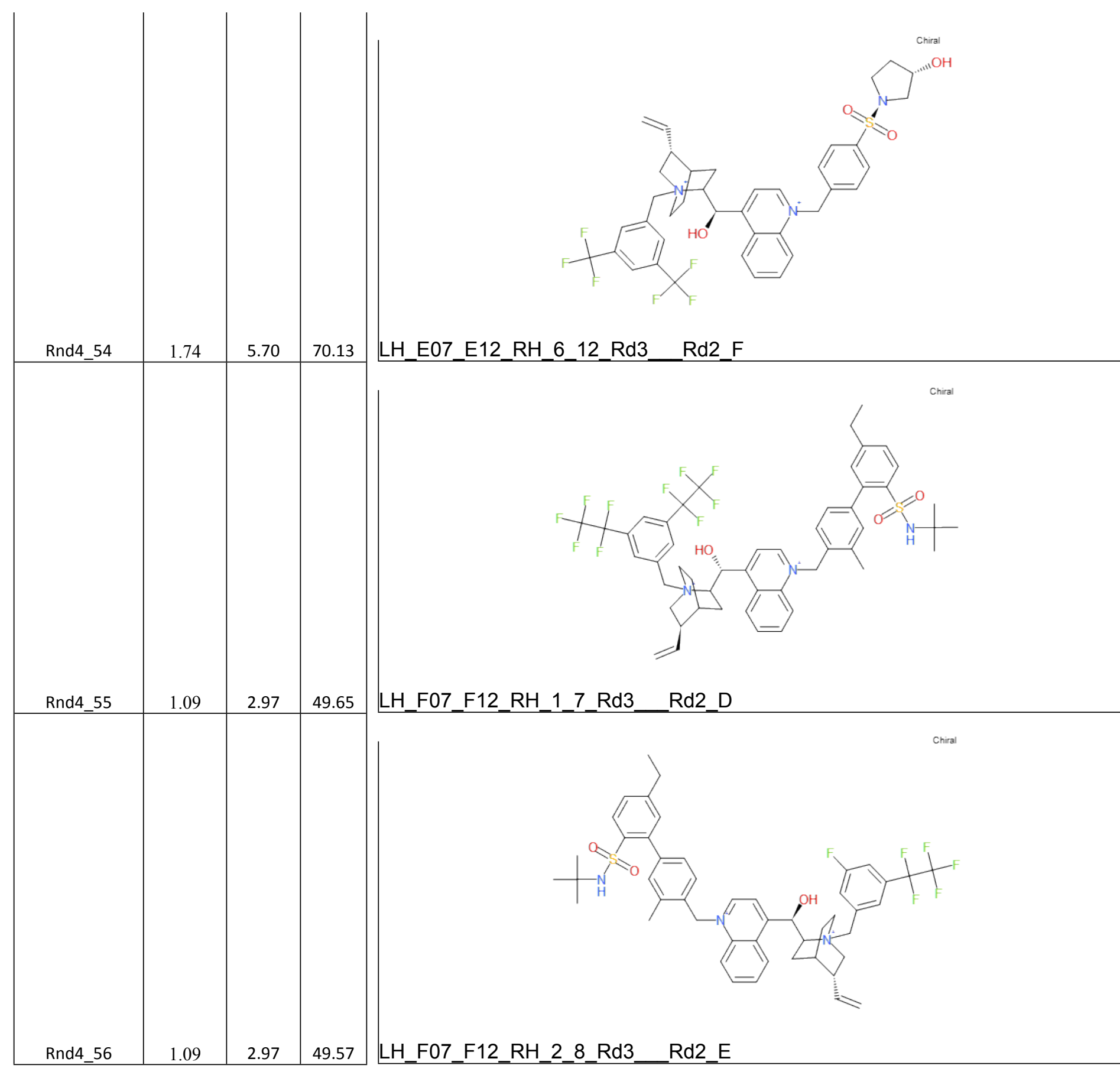




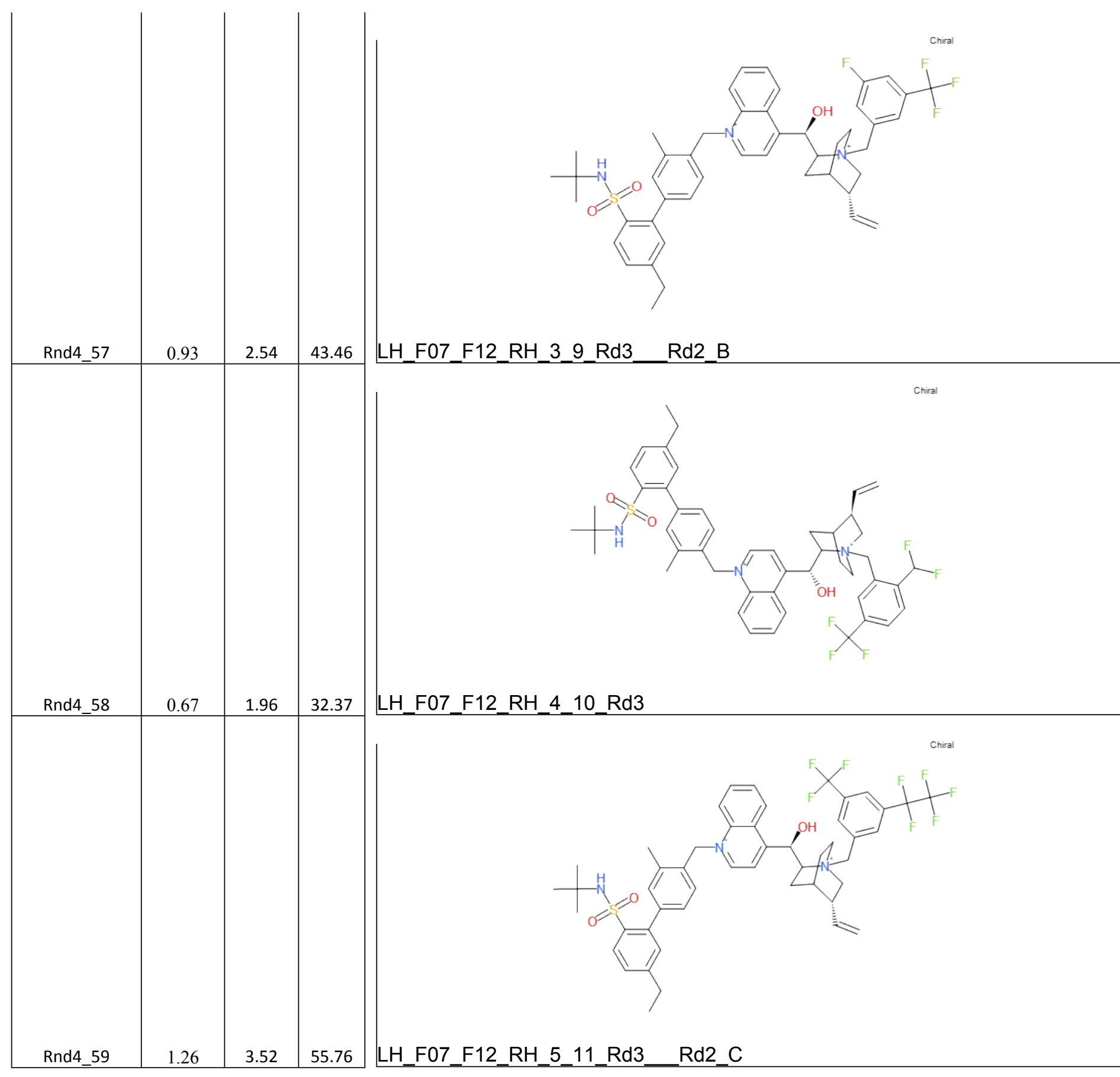




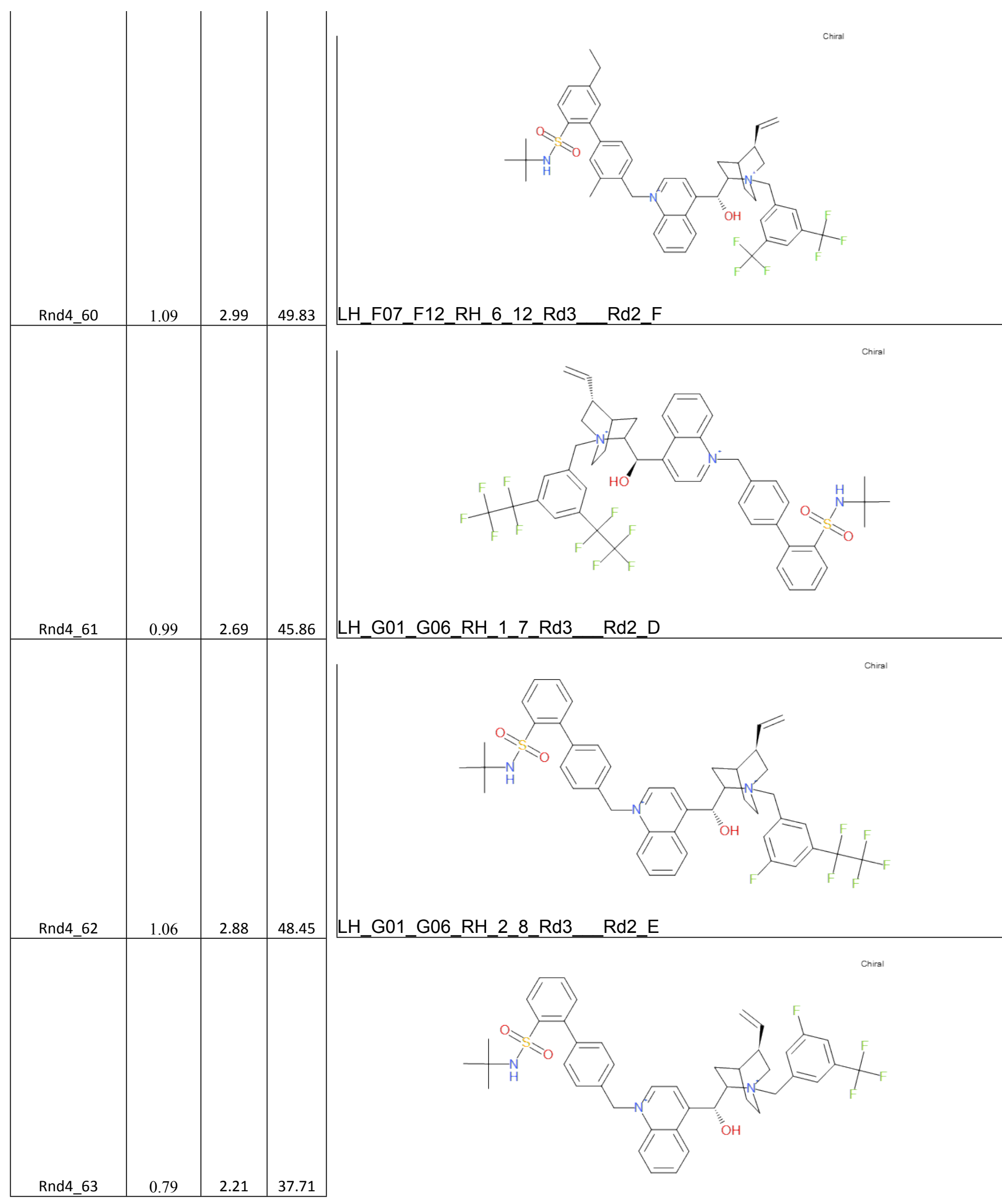




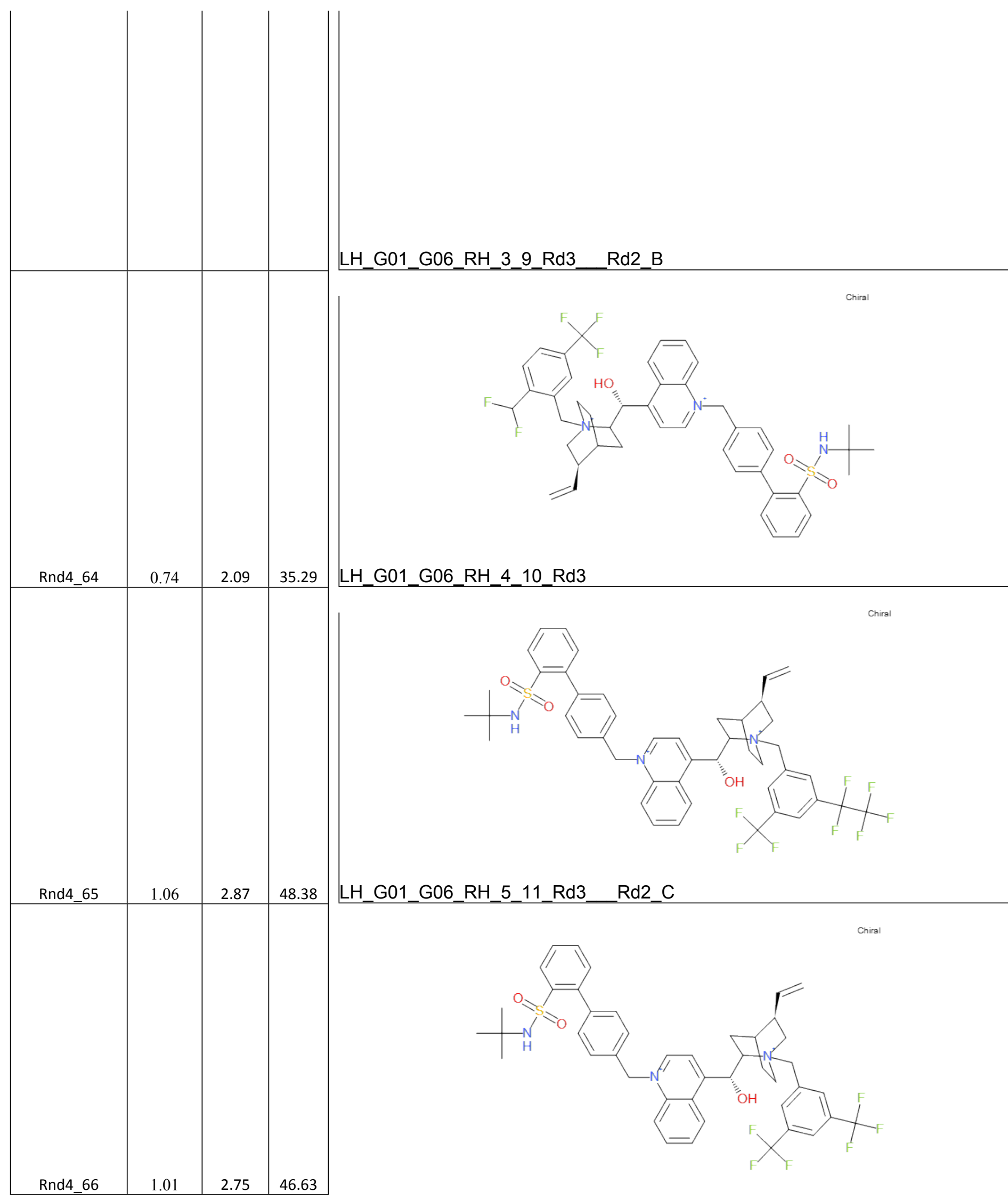




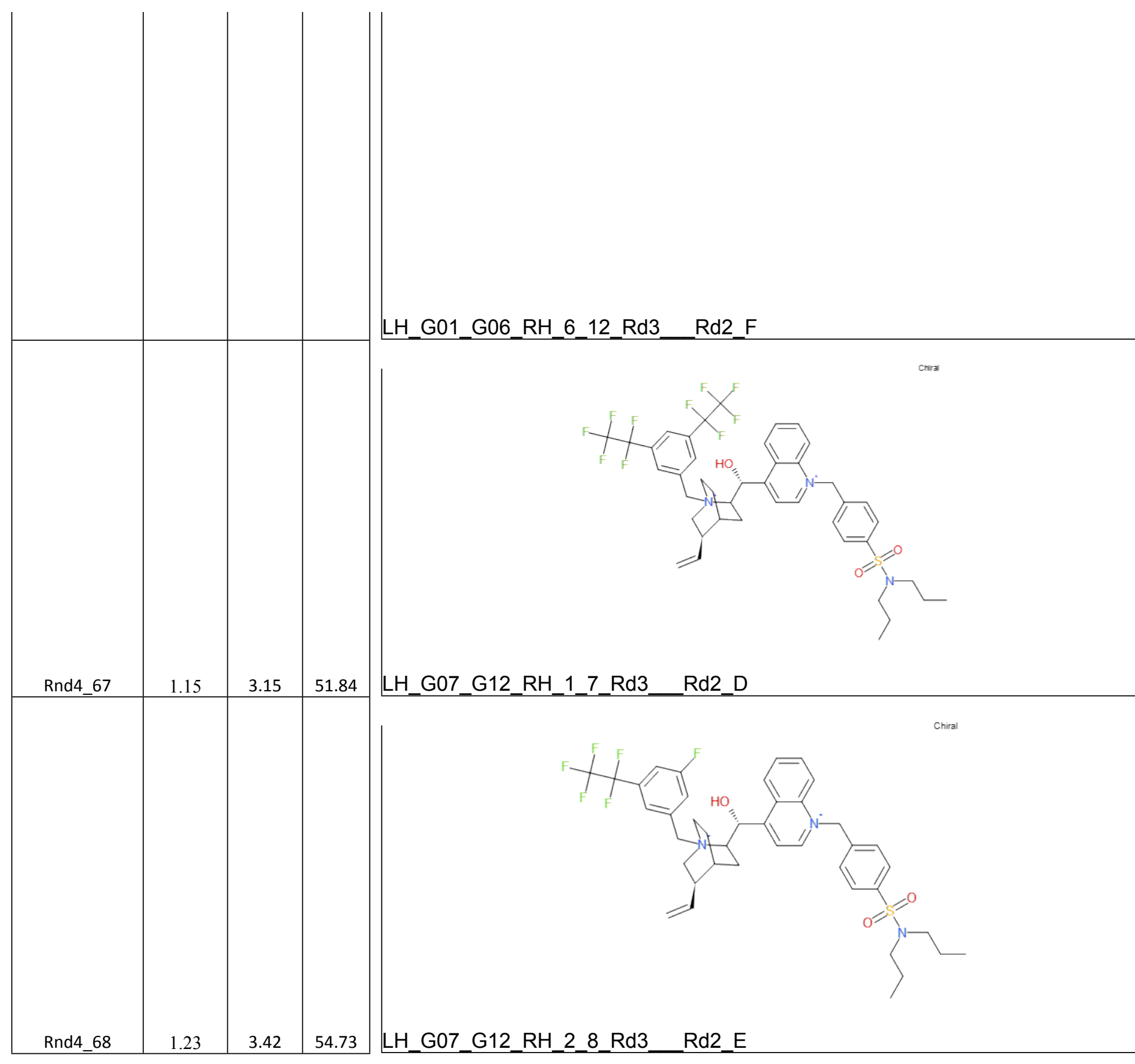




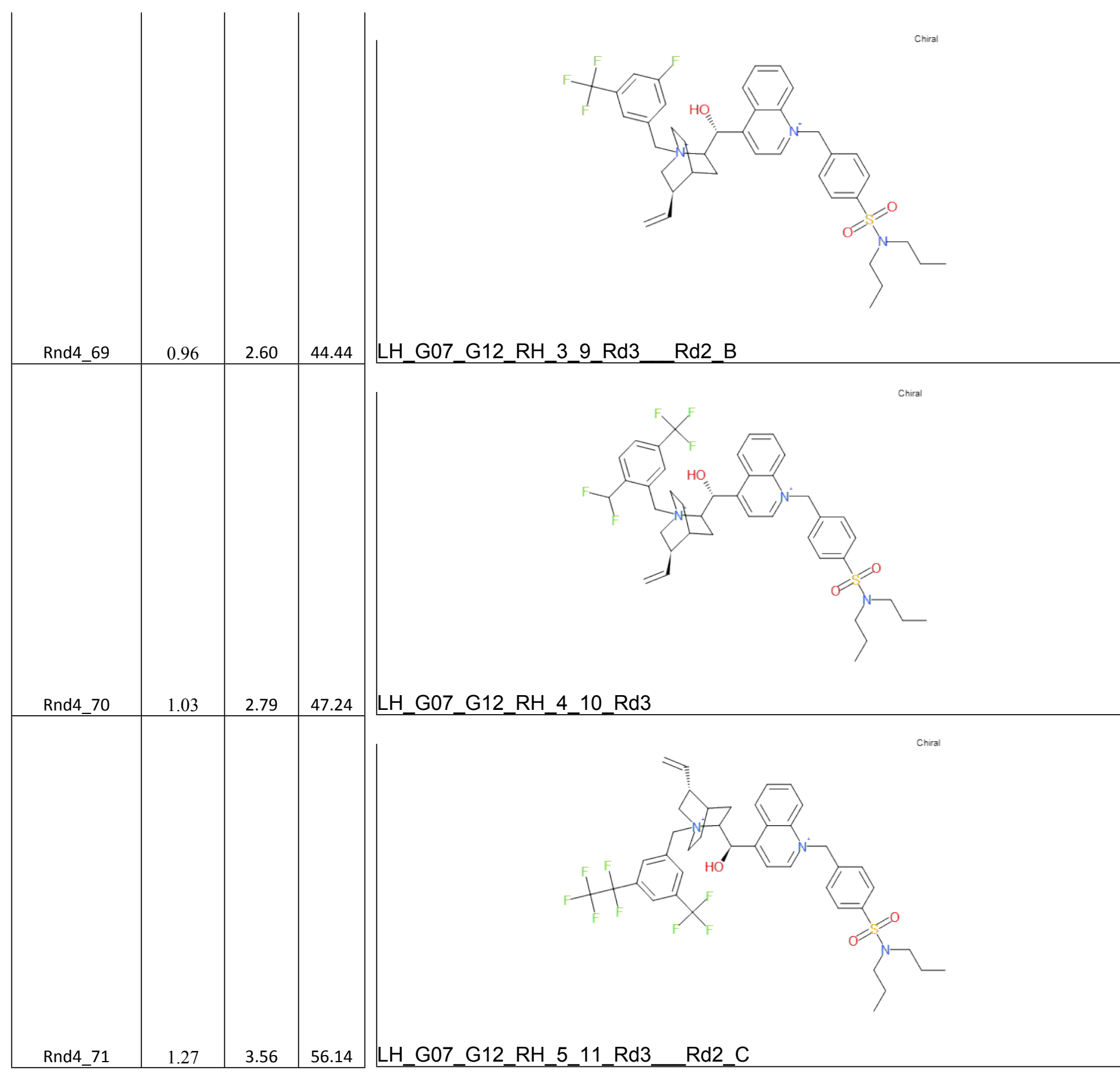




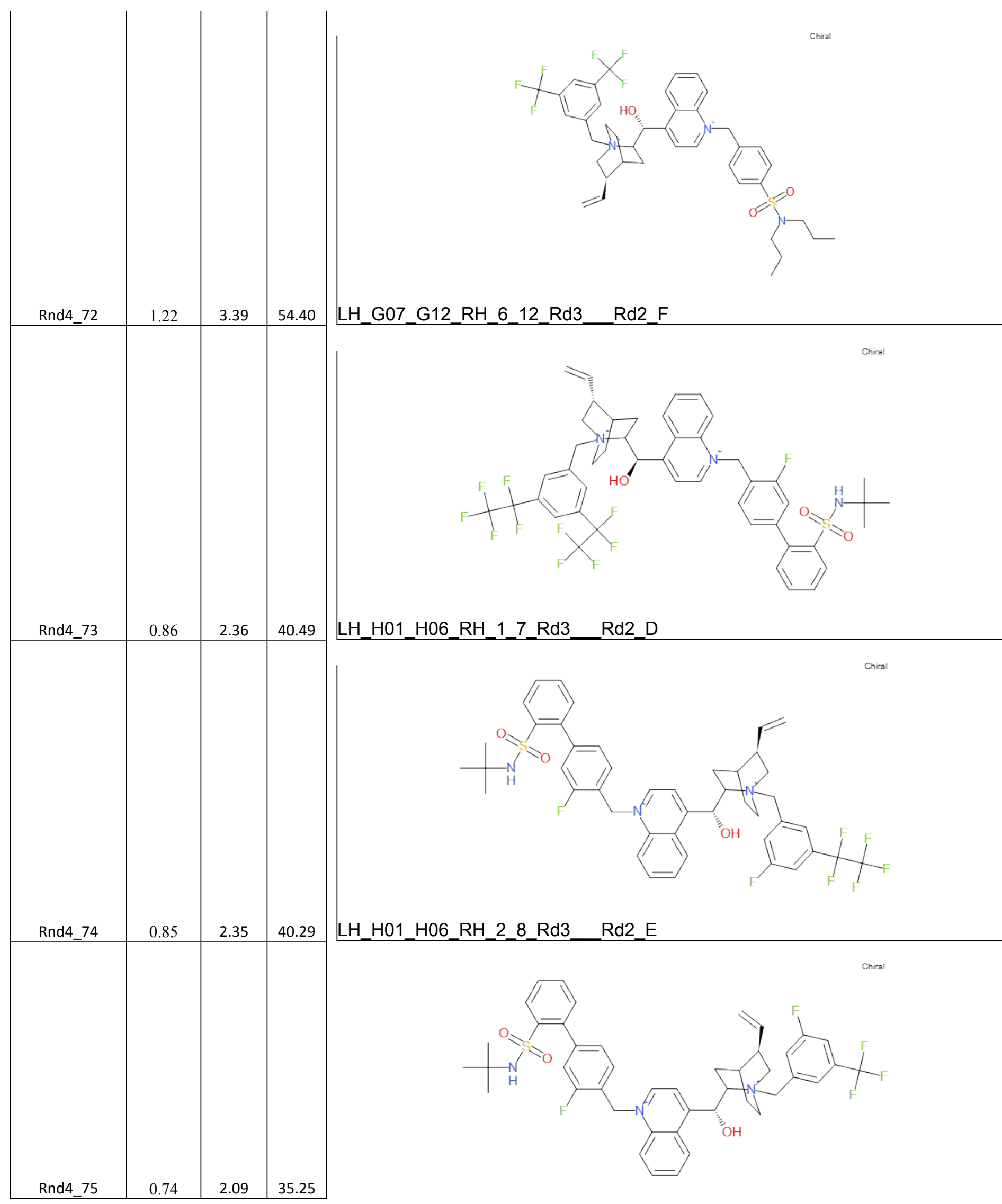




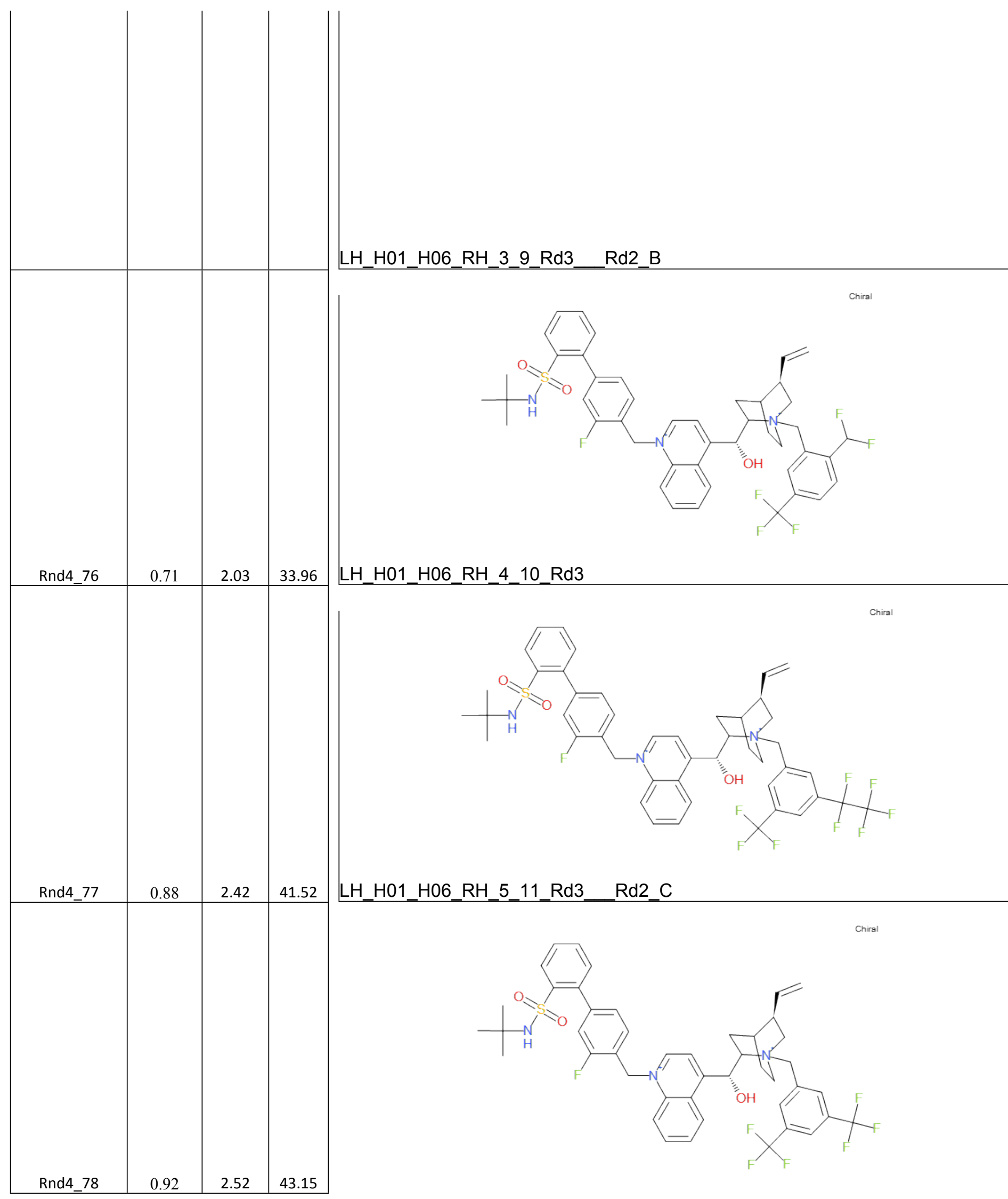




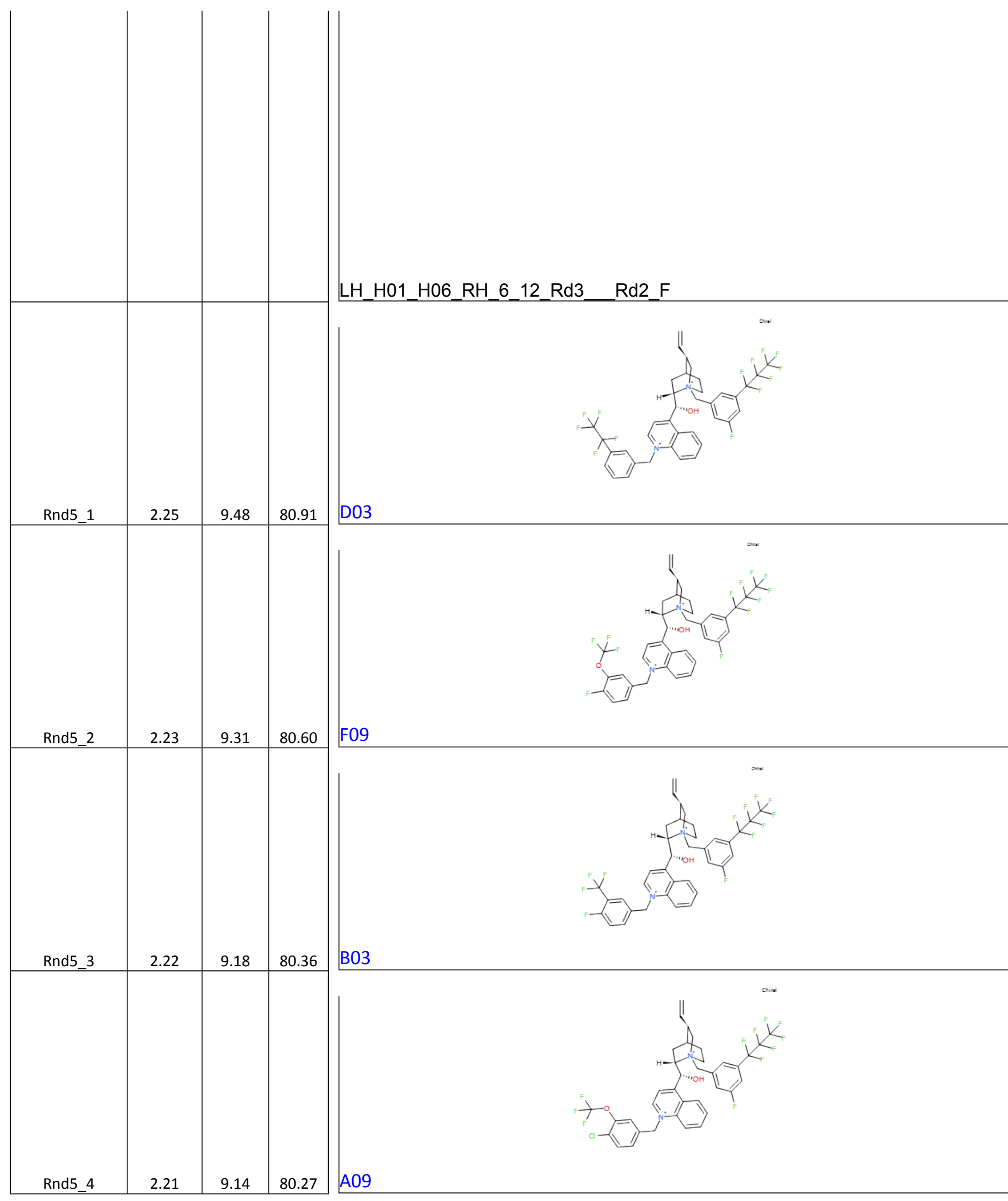




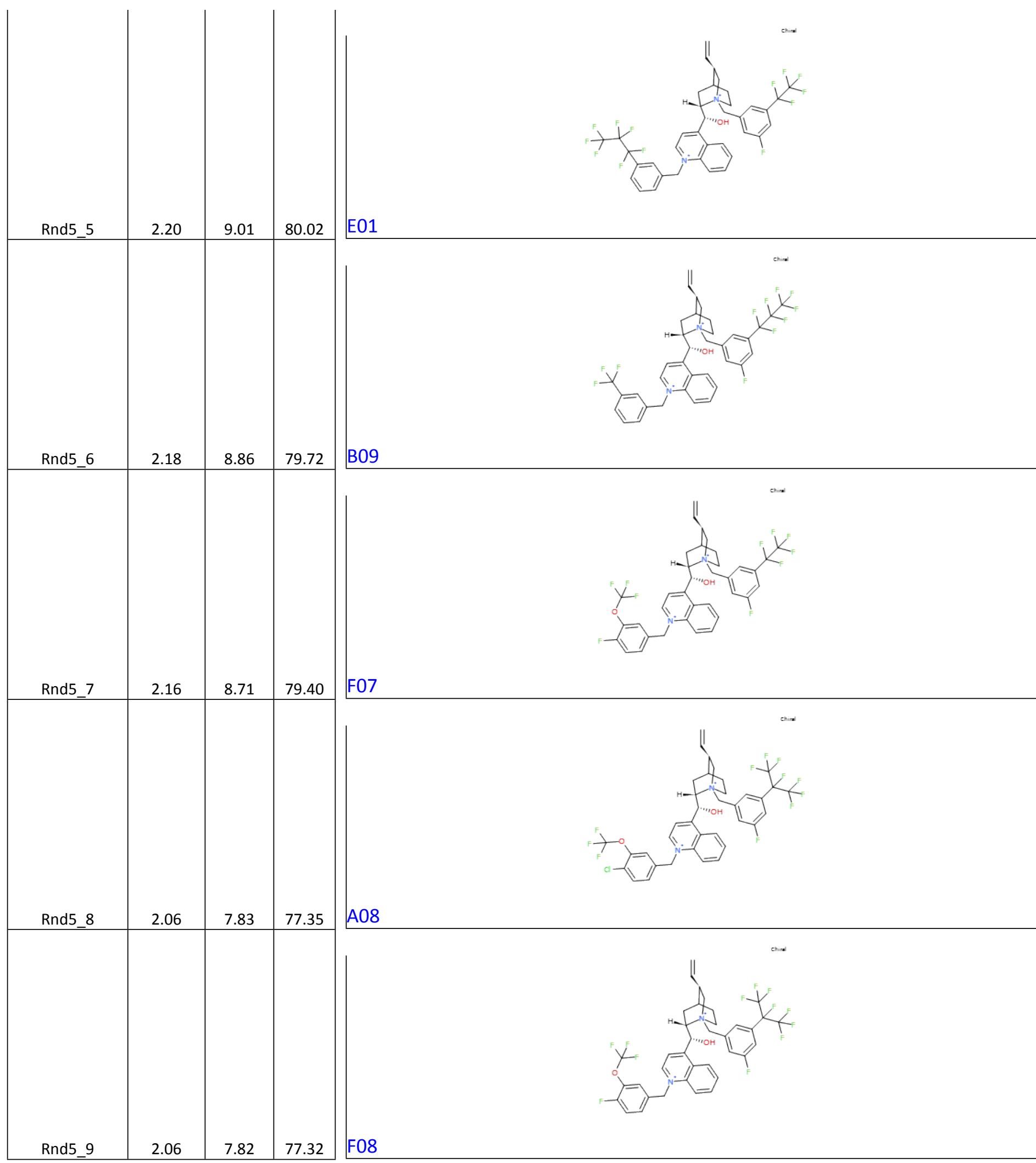




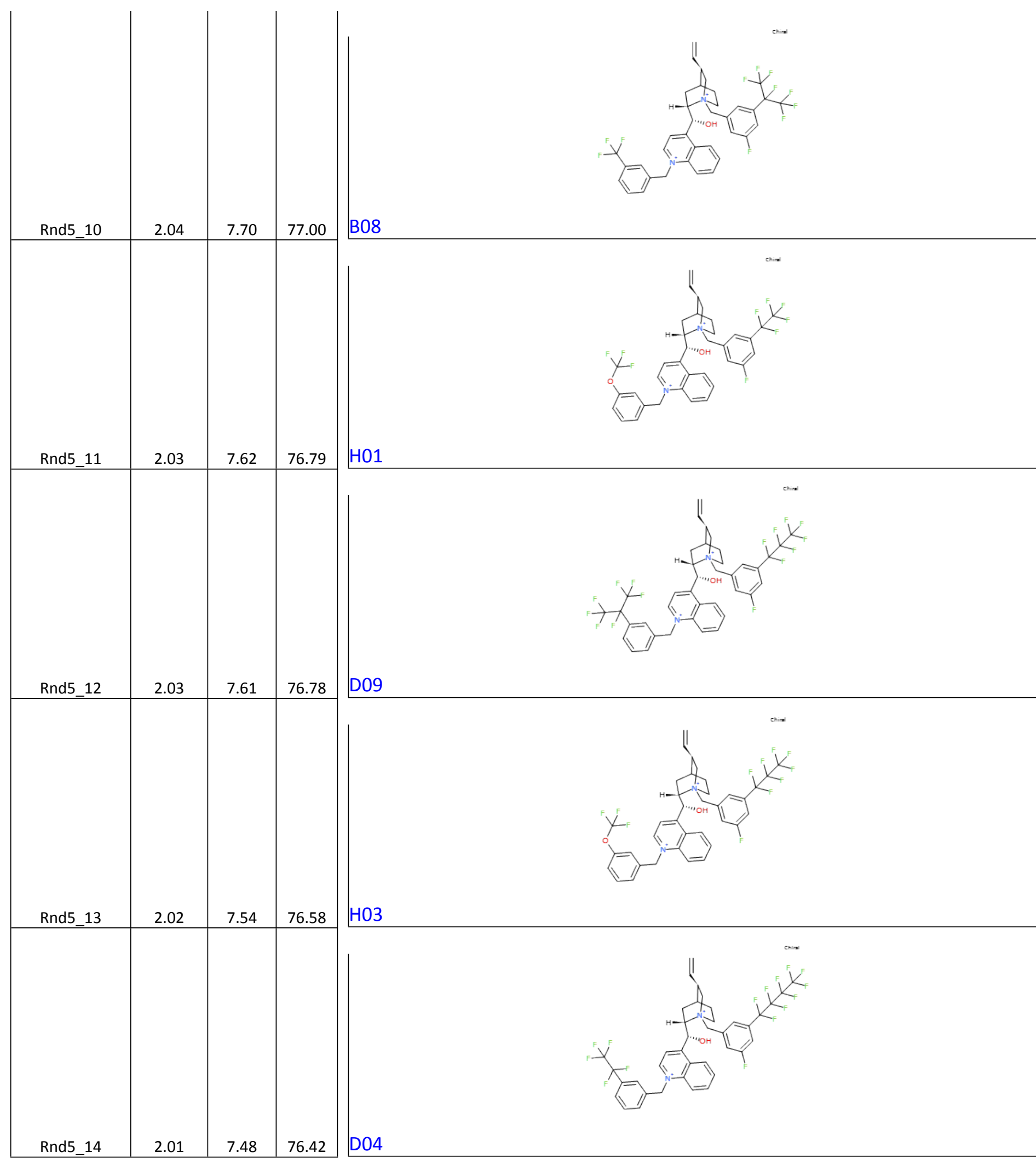




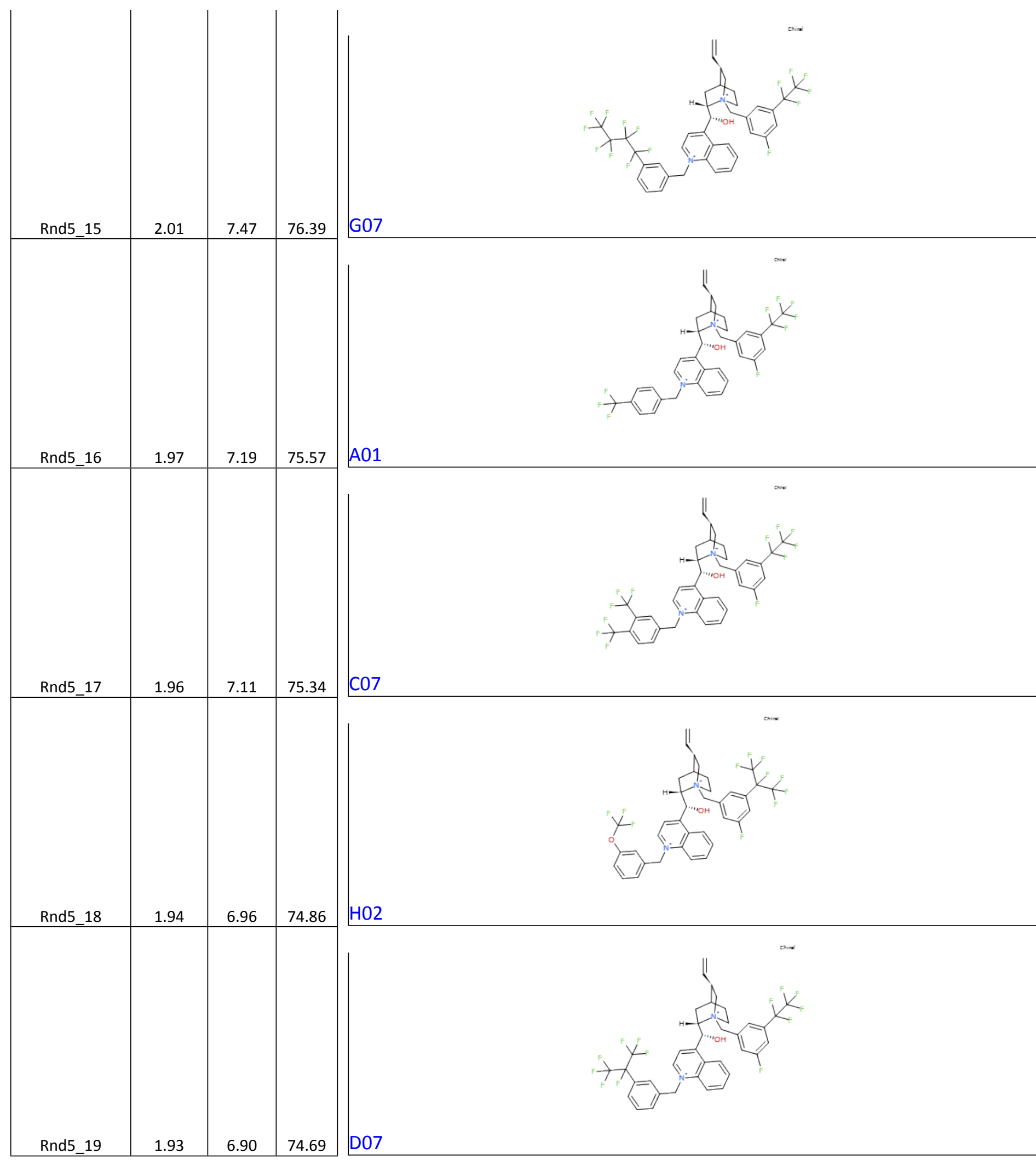




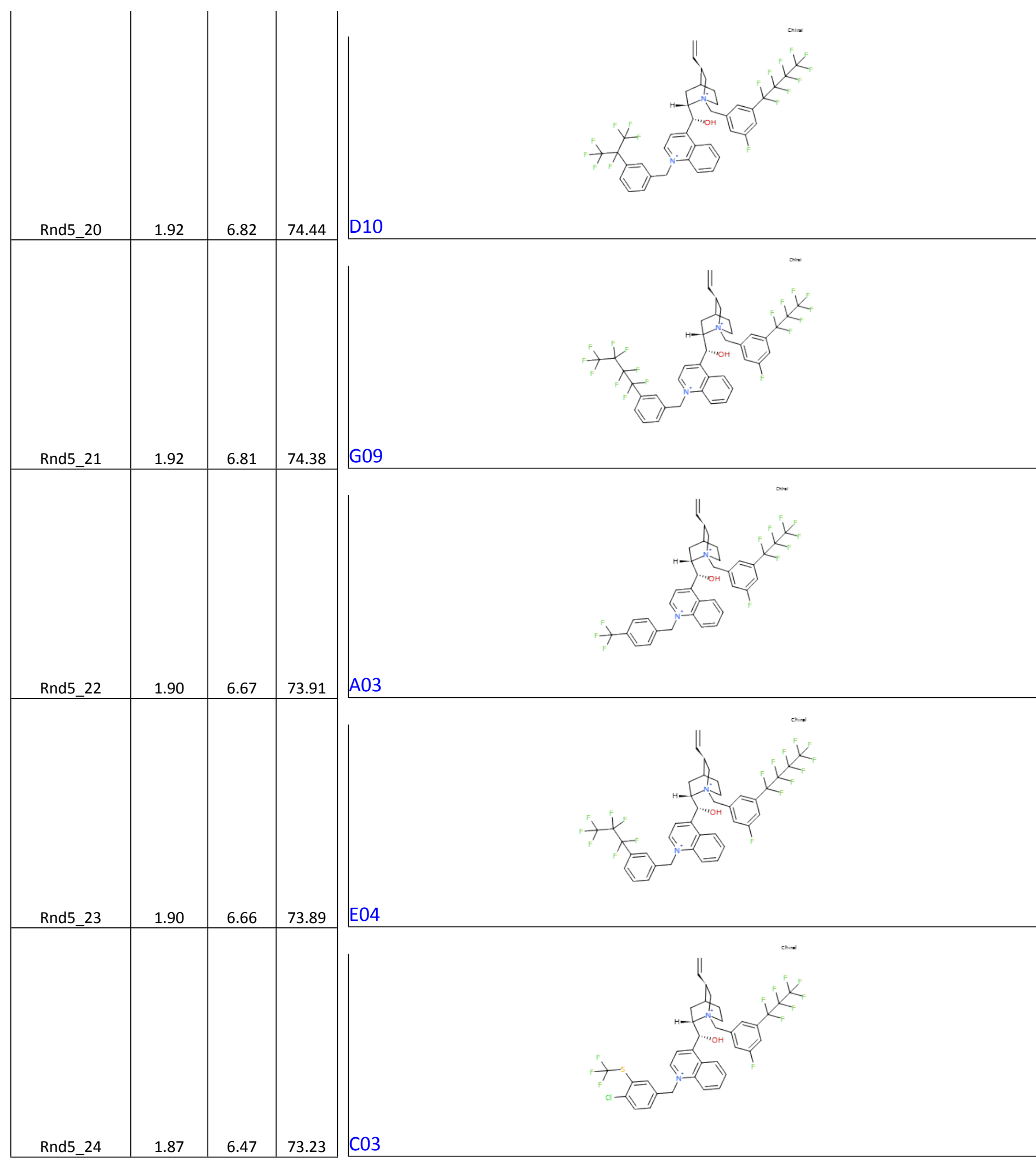




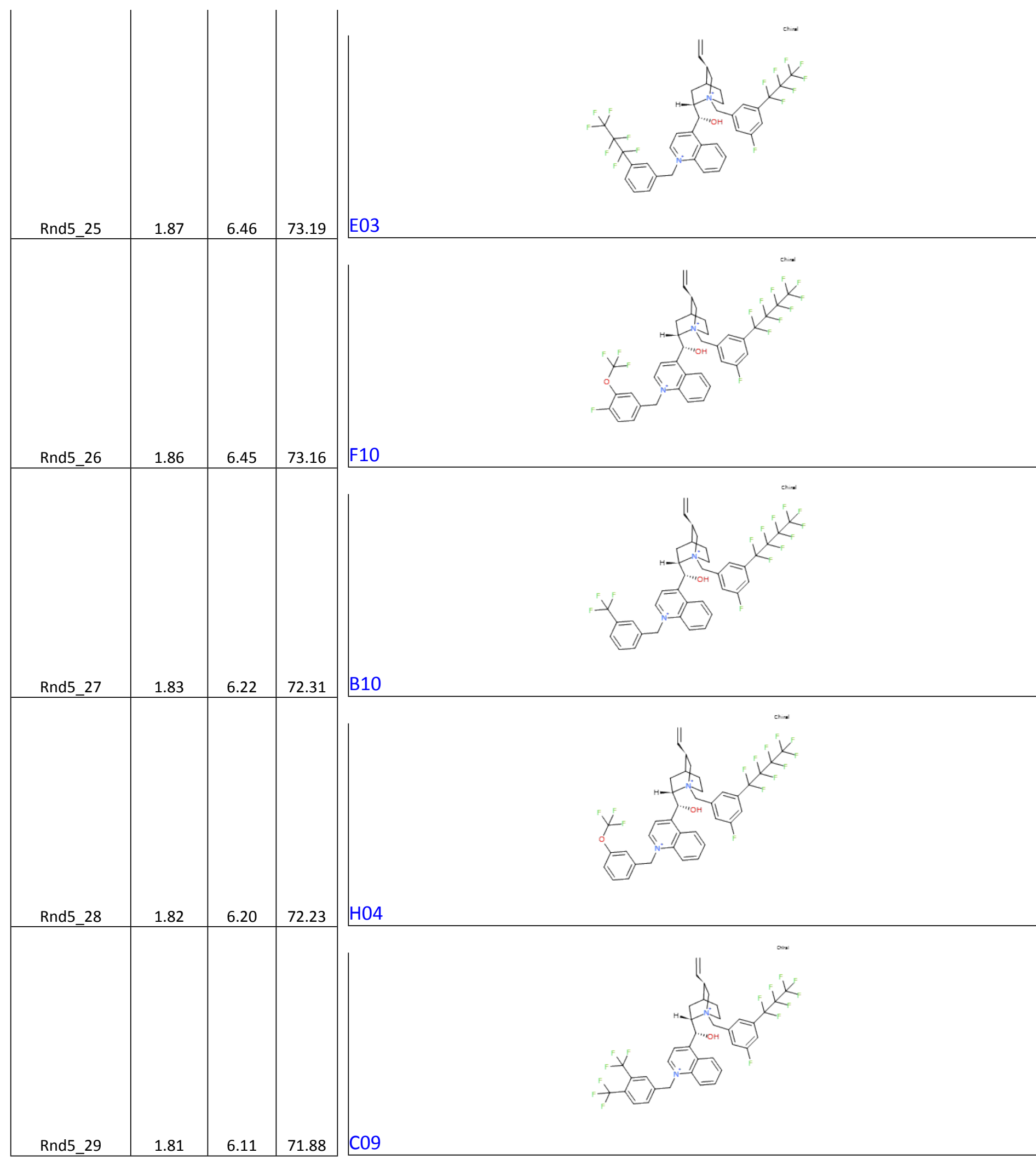




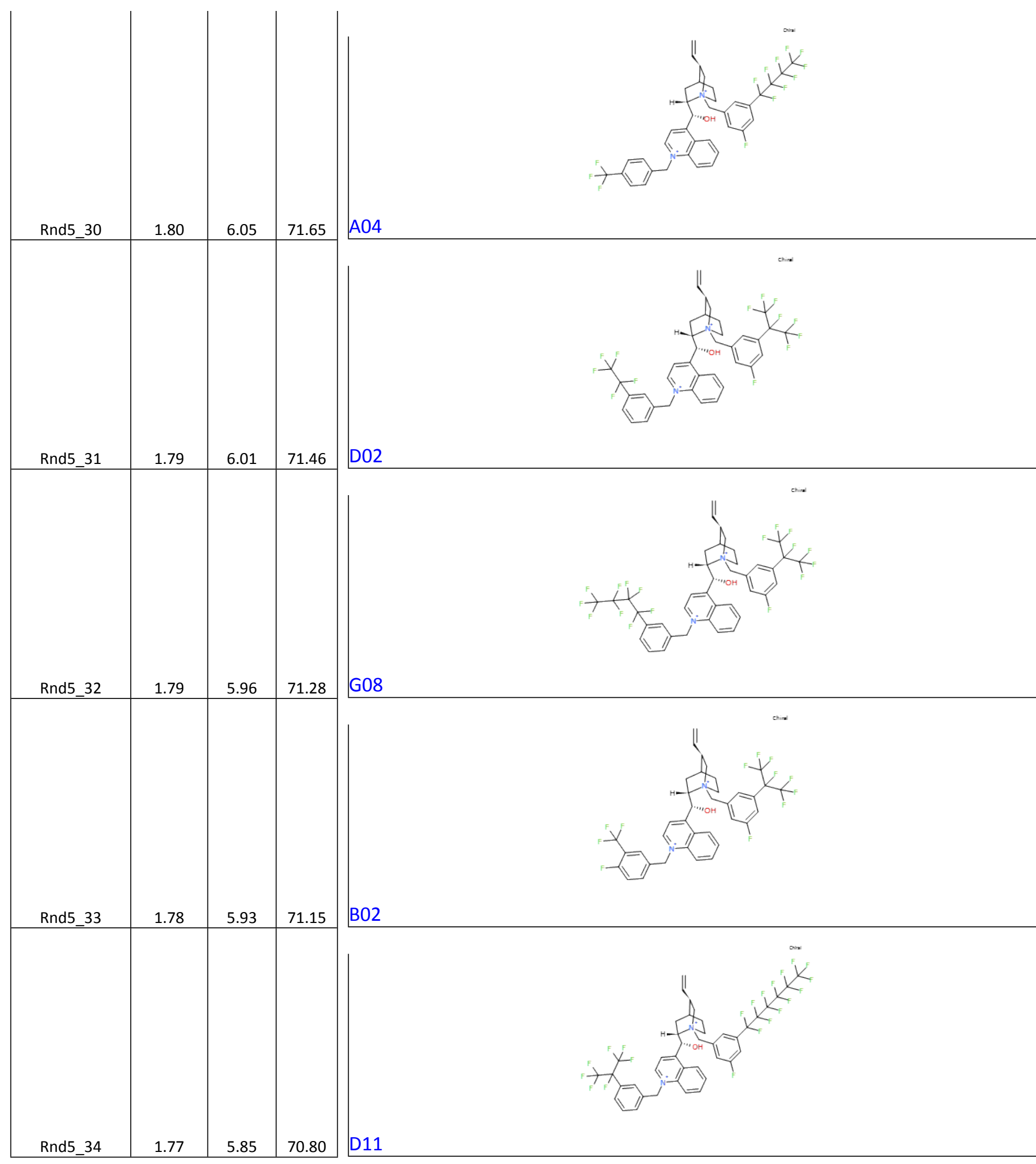




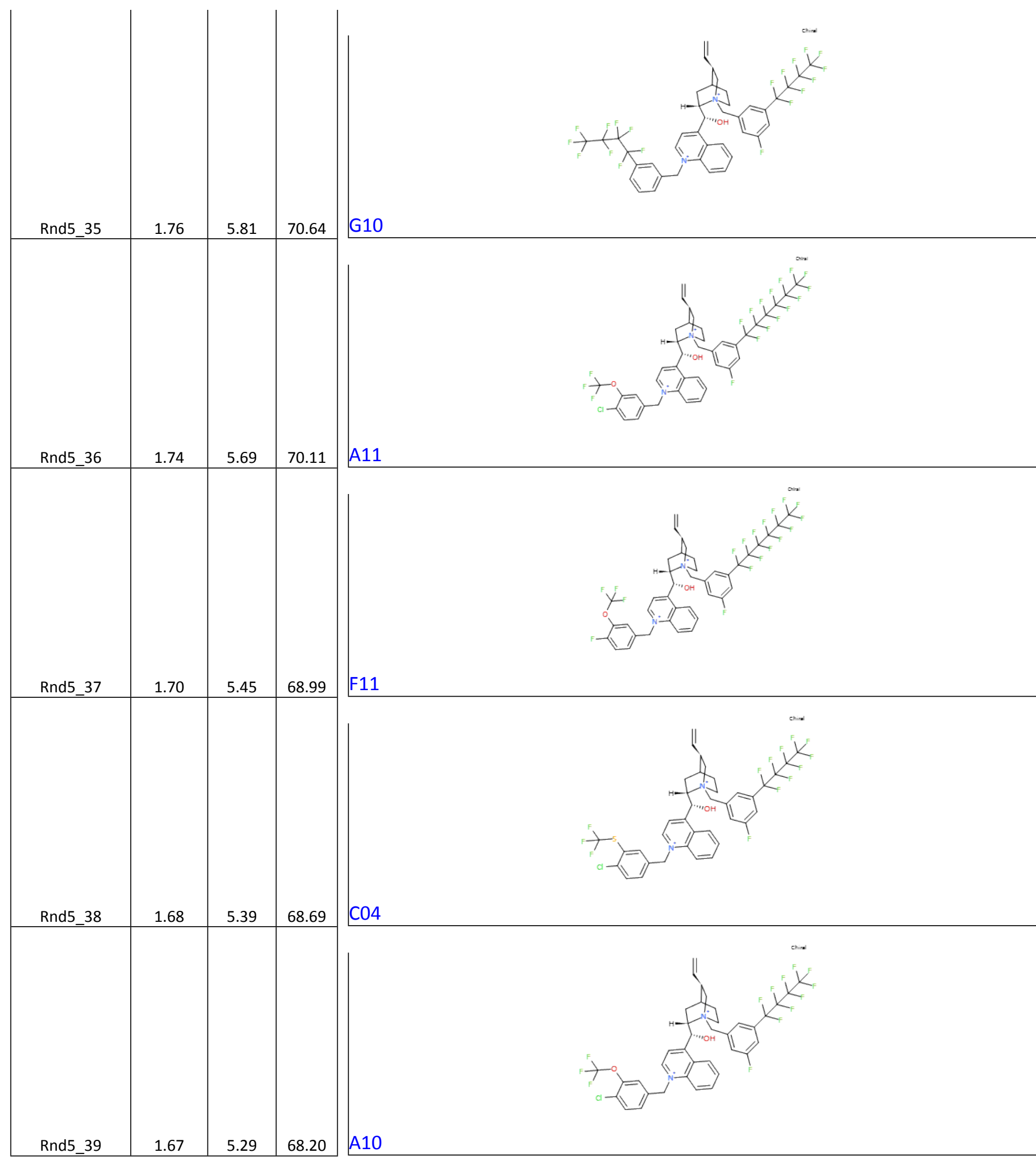




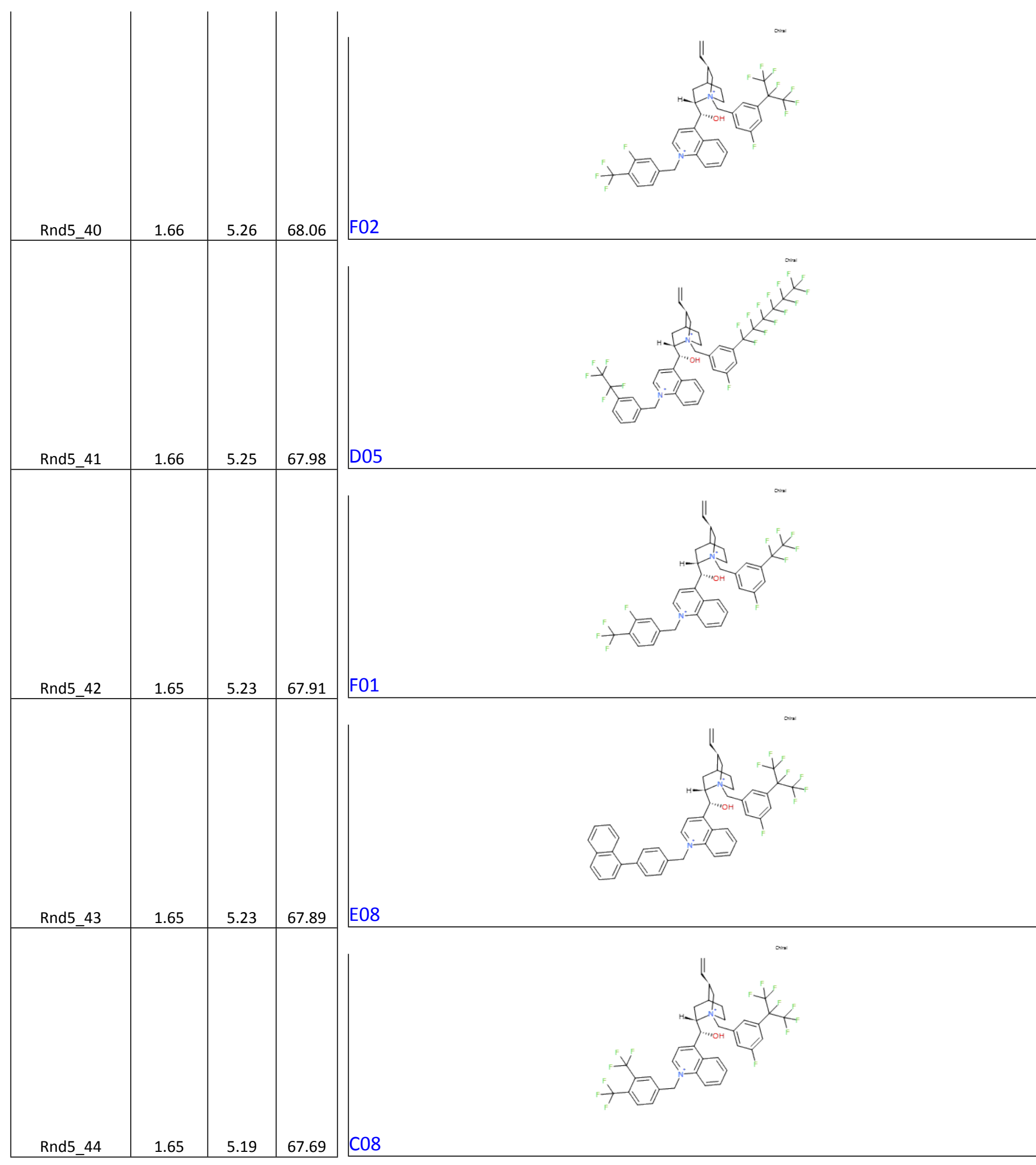




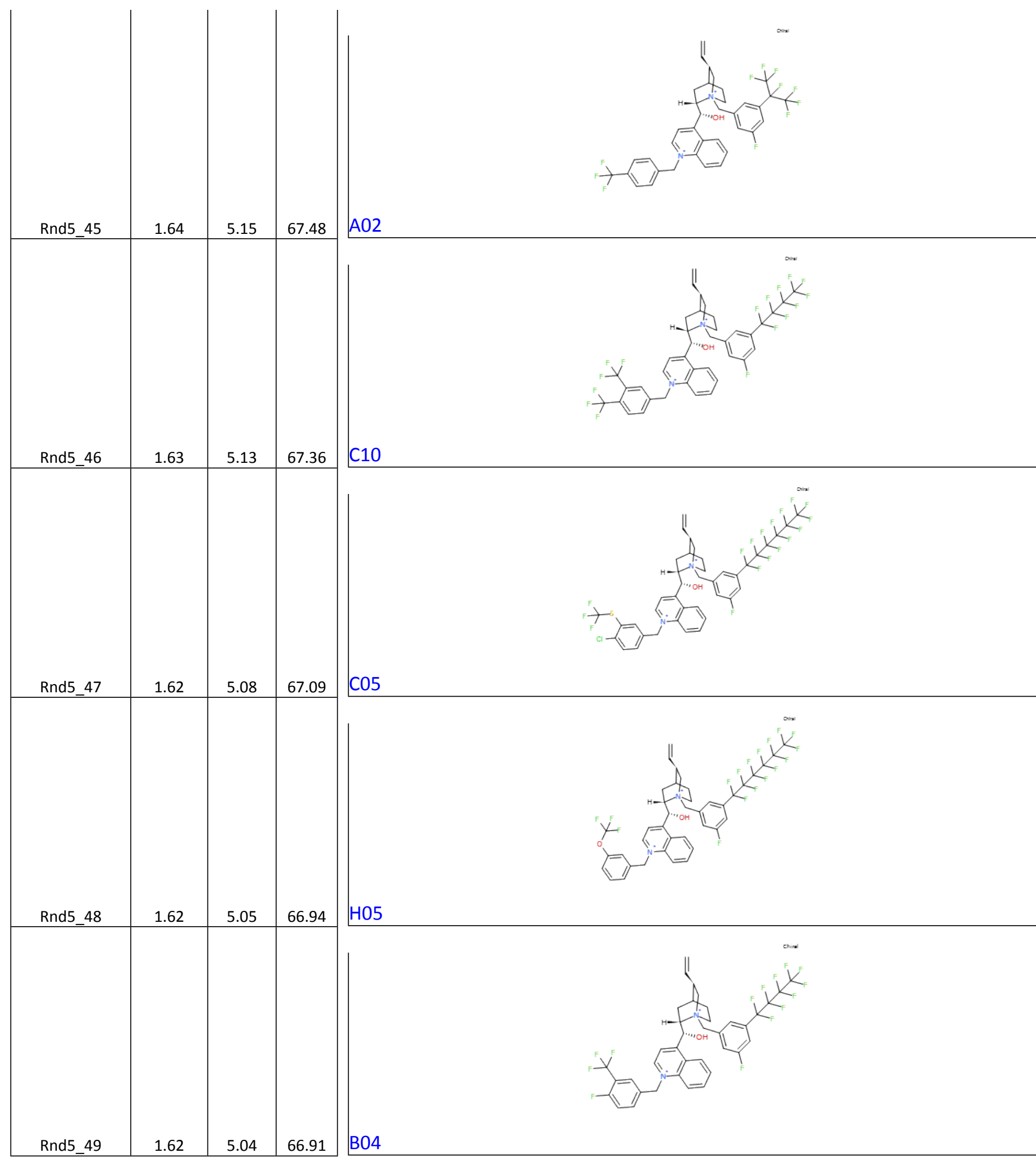




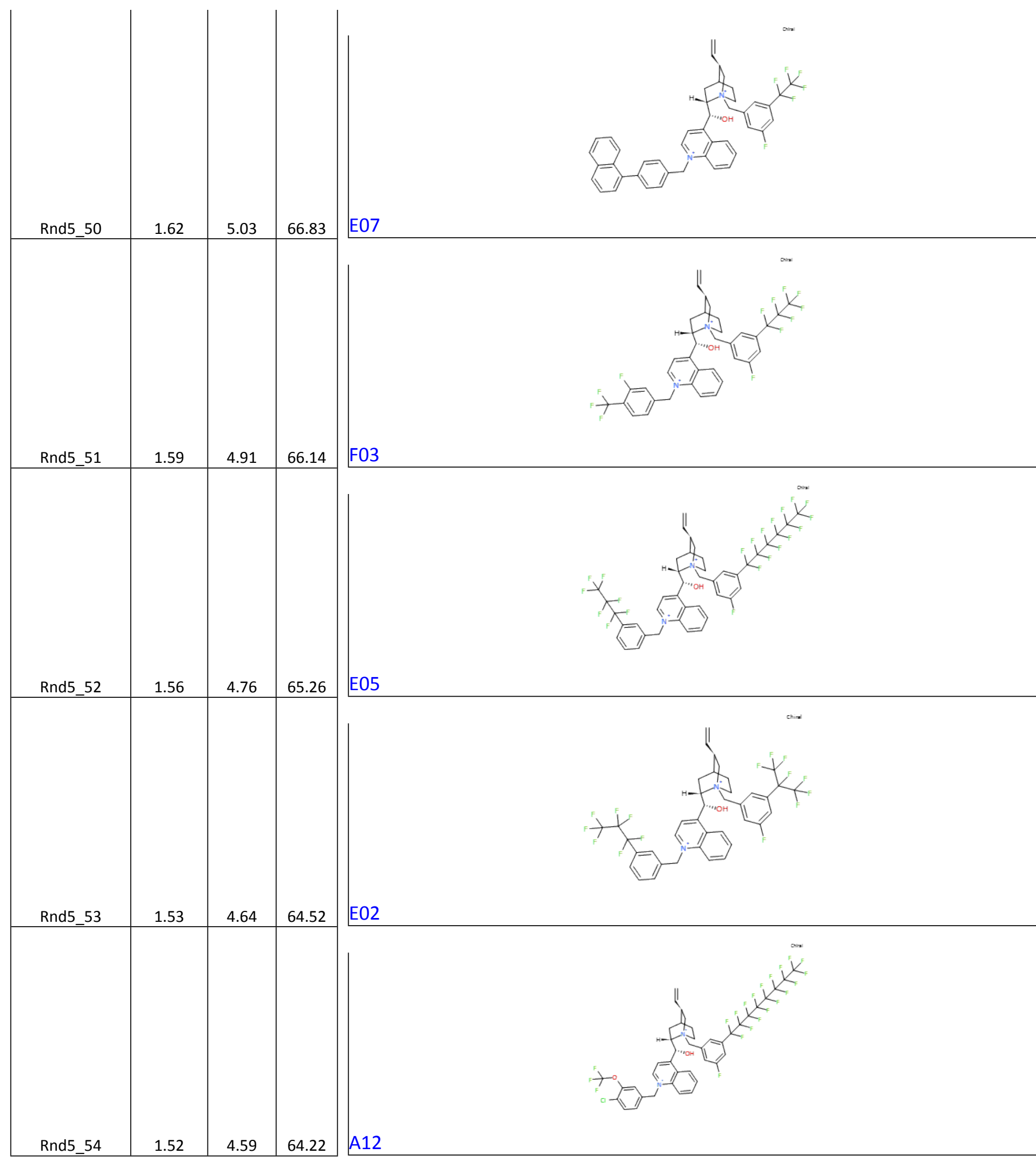




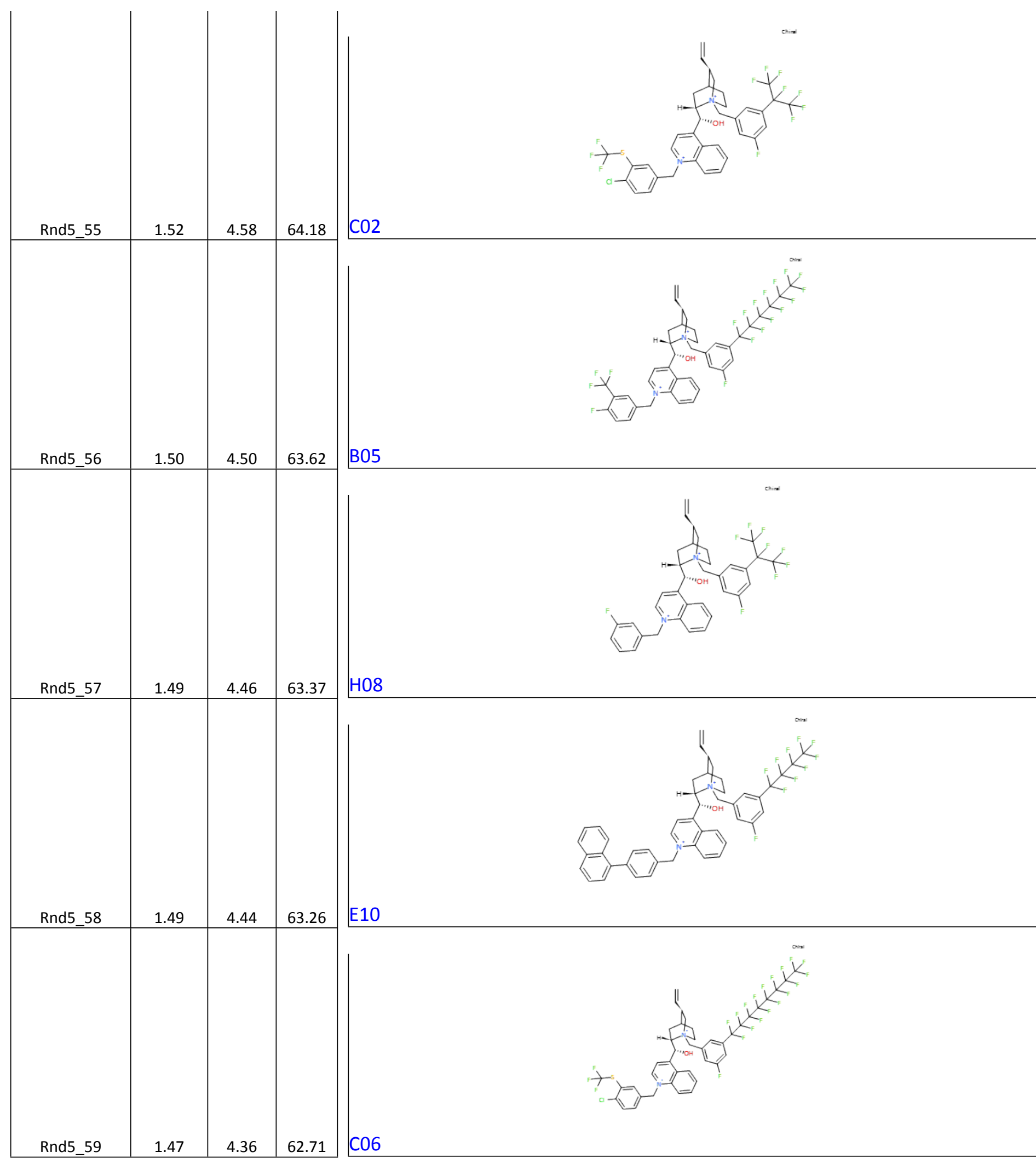




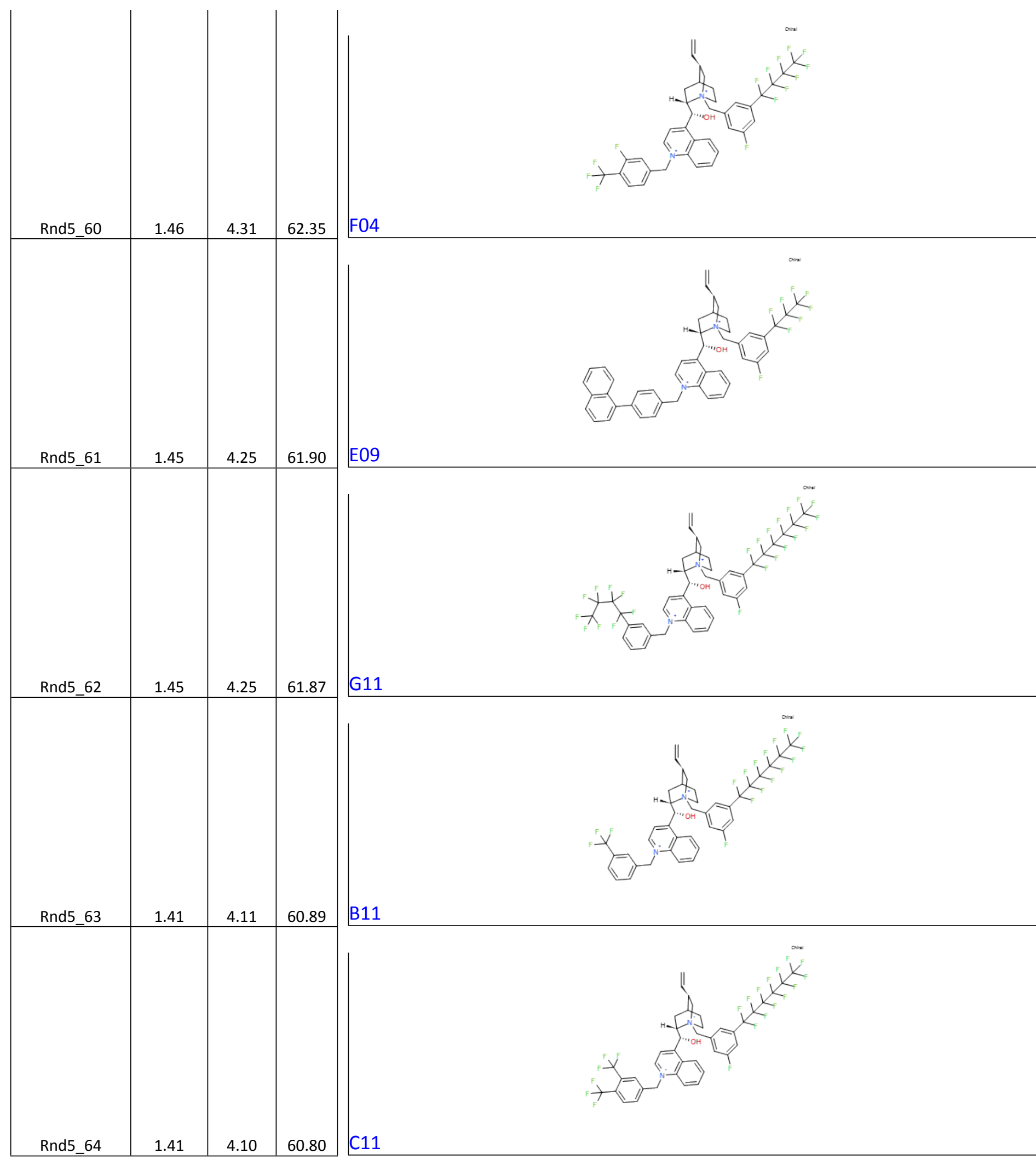




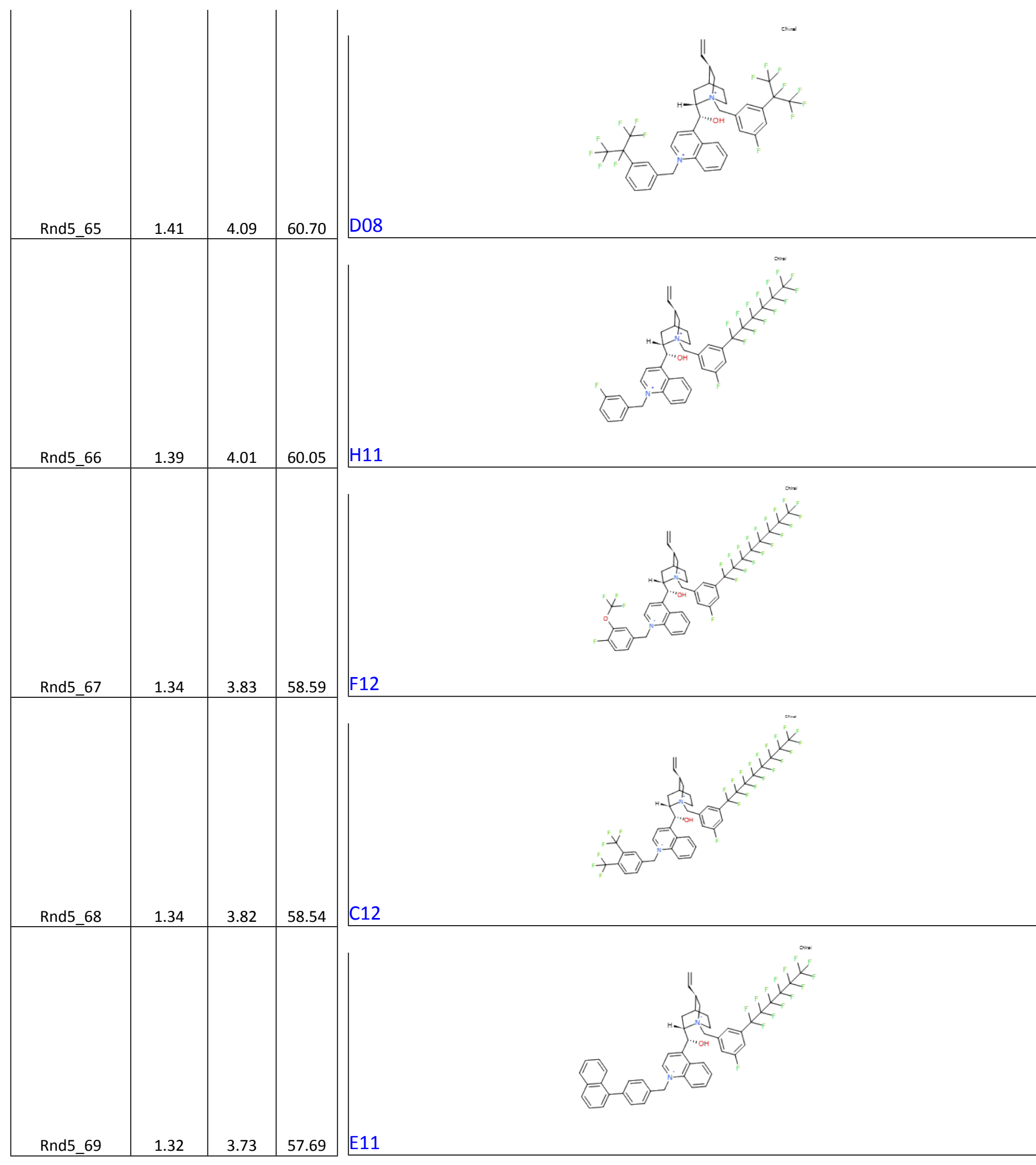




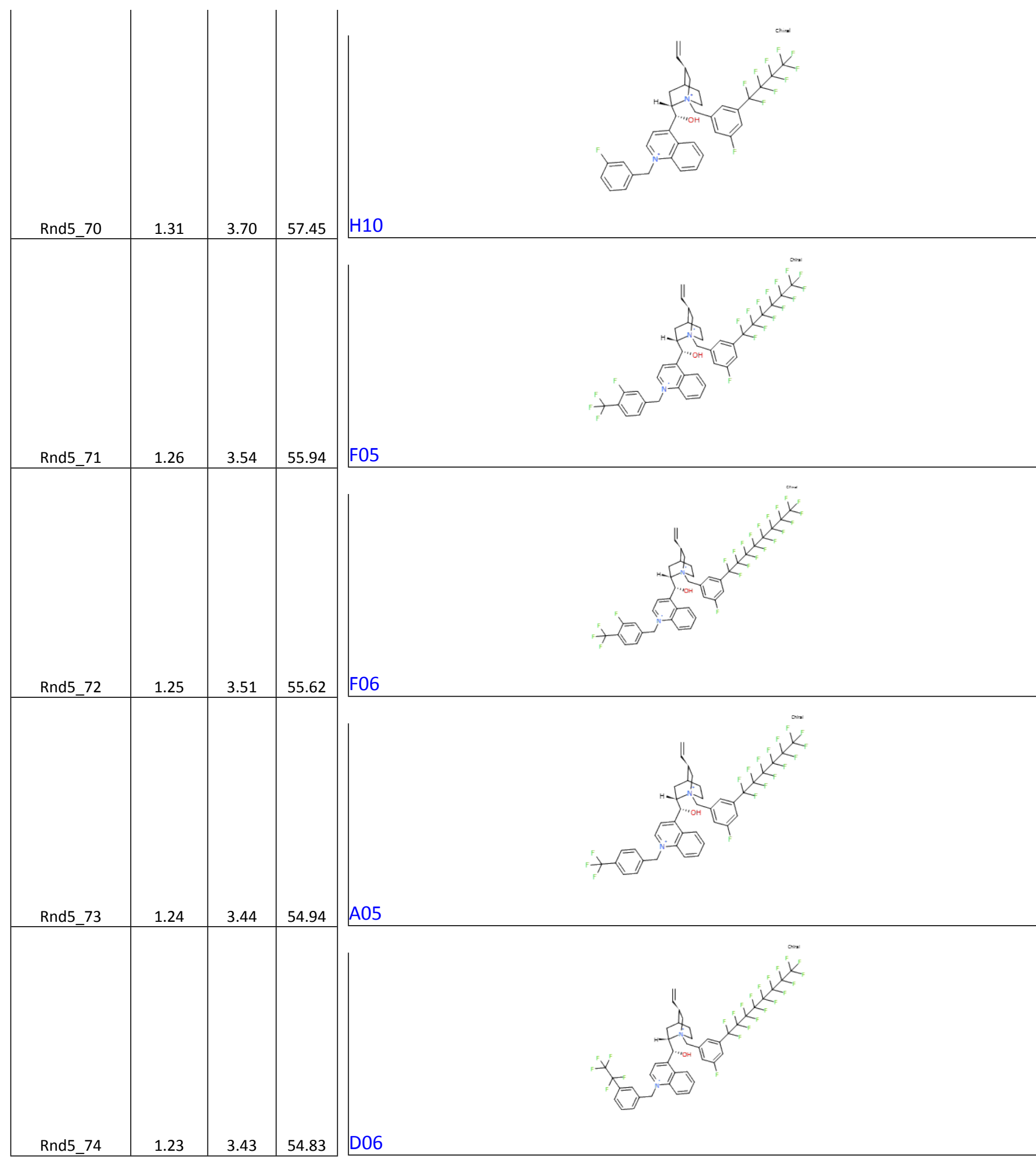




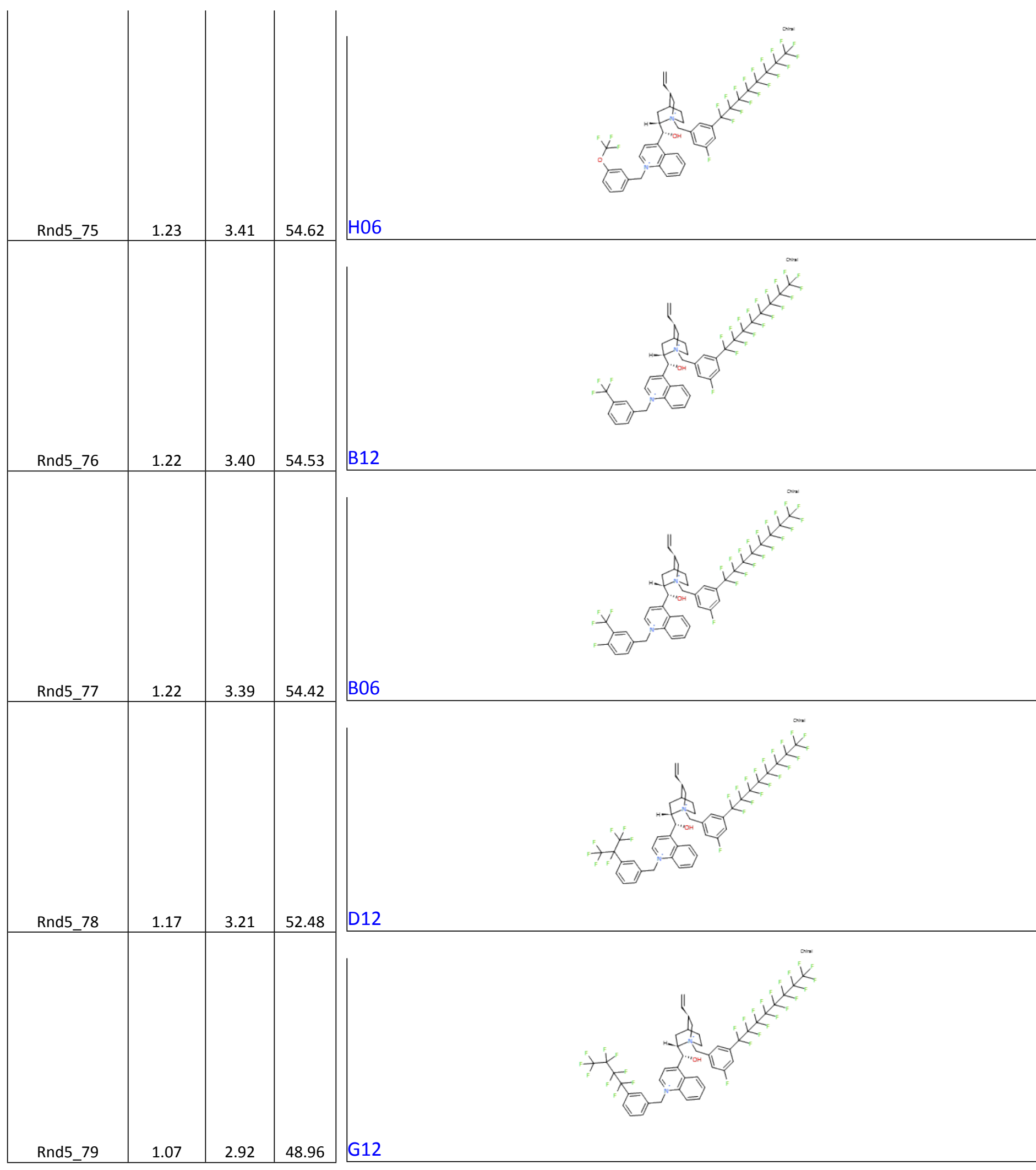

173 


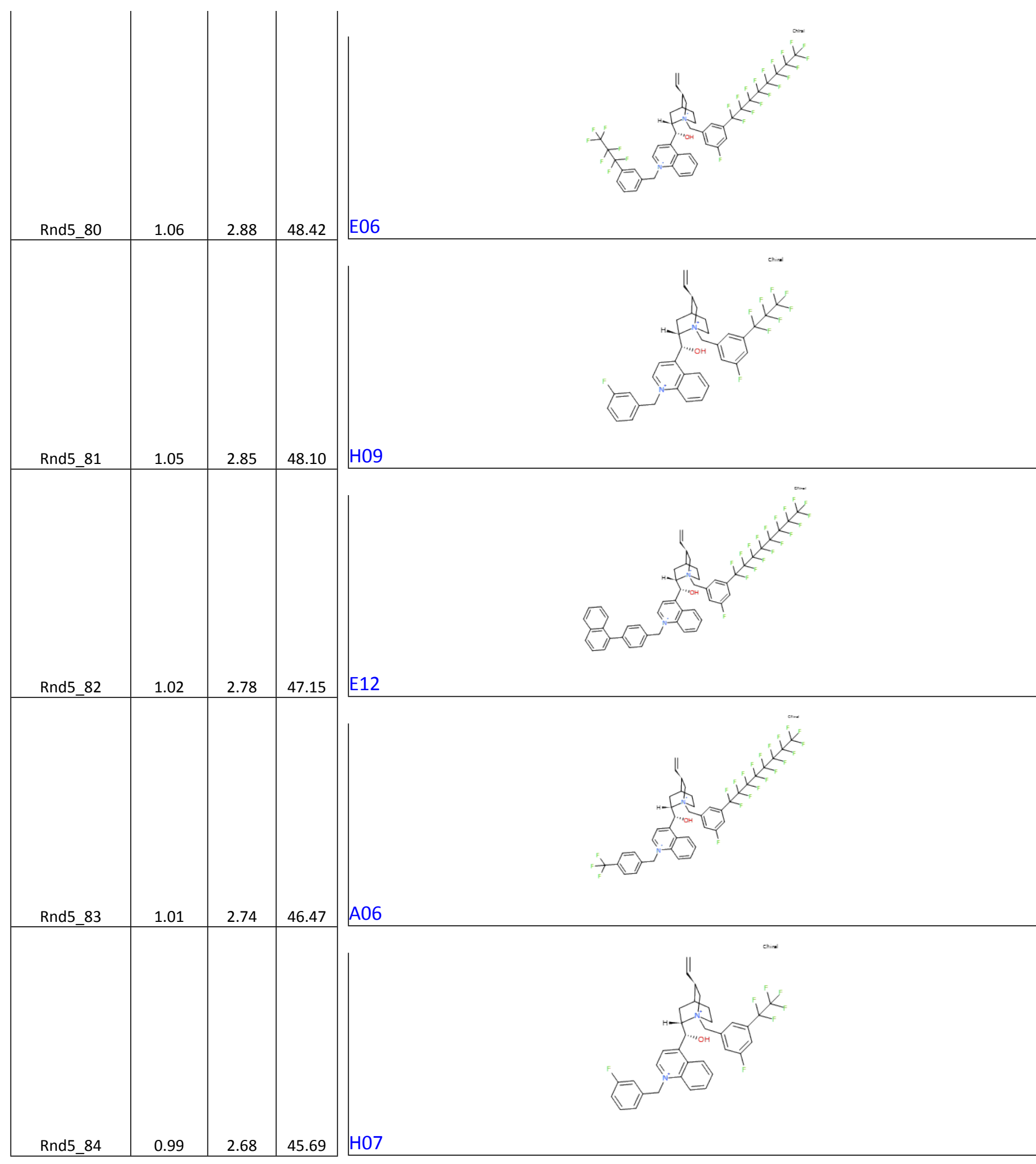



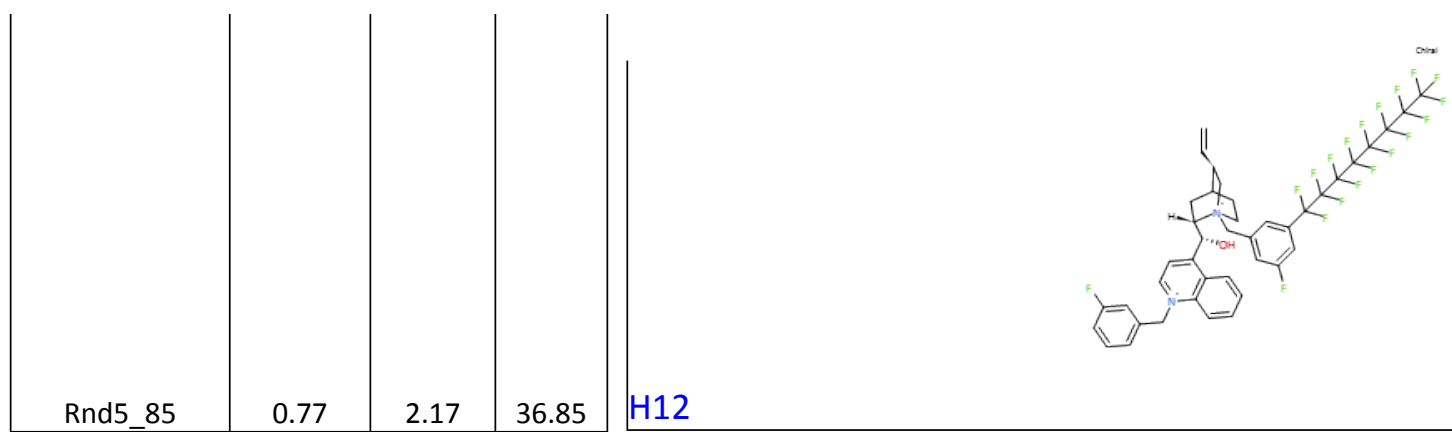

$\mathrm{H} 12$

\section{References}

1. Humphrey, G. R.; Dalby, S. M.; Andreani, T.; Xiang, B. P.; Luzung, M. R.; Song, Z. J.; Shevlin, M.; Christensen, M.; Belyk, K. M.; Tschaen, D. M., Asymmetric Synthesis of Letermovir Using a Novel Phase-Transfer Catalyzed Aza-Michael Reaction. Org. Process Res. Dev. 2016, 20 (6), 1097-1103.

2. $\quad$ Belyk, K. M.; Xiang, B. P.; Bulger, P. G.; Leonard, W. R.; Balsells, J.; Yin, J. J.; Chen, C. Y., Enantioselective Synthesis of (1R,2S)-1-Amino-2vinylcyclopropanecarboxylic Acid Ethyl Ester (Vinyl-ACCA-0Et) by Asymmetric PhaseTransfer Catalyzed Cyclopropanation of (E)-N-Phenylmethyleneglycine Ethyl Ester. Org. Process Res. Dev. 2010, 14 (3), 692-700.

3. Krska, S. W.; DiRocco, D. A.; Dreher, S. D.; Shevlin, M., The Evolution of Chemical High-Throughput Experimentation To Address Challenging Problems in Pharmaceutical Synthesis. Accounts of Chemical Research 2017, 50 (12), 2976-2985.

4. Xiang, B.; Belyk, K. M.; Reamer, R. A.; Yasuda, N., Discovery and application of doubly quaternized cinchona-alkaloid-based phase-transfer catalysts. Angew. Chem. Int. Ed. Engl. 2014, 53 (32), 8375-8.

5. Daponte, J. A.; Guo, Y. J.; Ruck, R. T.; Hein, J. E., Using an Automated Monitoring Platform for Investigations of Biphasic Reactions. ACS Catal. 2019, 9 (12), 1148411491.

6. Carhart, R. E.; Smith, D. H.; Venkataraghavan, R., Atom Pairs as Molecular-Features in Structure Activity Studies - Definition and Applications. J. Chem. Inf. Comp. Sci. 1985, 25 (2), 64-73. 
7. Molecular Operating Environment (MOE), 1010 Sherbrooke St. West, Suite \#910, Montreal, QC, Canada, H3A 2R7, 2012.

8. Harper, K. C.; Bess, E. N.; Sigman, M. S., Multidimensional steric parameters in the analysis of asymmetric catalytic reactions. Nat. Chem. 2012, 4 (5), 366-374.

9. Harper, K. C.; Sigman, M. S., Predicting and optimizing asymmetric catalyst performance using the principles of experimental design and steric parameters. Proc. Natl. Acad .Sci. U.S.A. 2011, 108 (6), 2179-2183.

10. Milo, A.; Bess, E. N.; Sigman, M. S., Interrogating selectivity in catalysis using molecular vibrations. Nature 2014, 507 (7491), 210-214.

11. Milo, A.; Neel, A. J.; Toste, F. D.; Sigman, M. S., Organic chemistry. A data-intensive approach to mechanistic elucidation applied to chiral anion catalysis. Science 2015, 347 (6223), 737-743.

12. Reid, J. P.; Sigman, M. S., Holistic prediction of enantioselectivity in asymmetric catalysis. Nature 2019, 571 (7765), 343-348.

13. Sigman, M. S.; Werner, E. W., Imparting catalyst control upon classical palladiumcatalyzed alkenyl C-H bond functionalization reactions. Acc. Chem. Res. 2012, 45 (6), 874-884.

14. Schrödinger Release 2021-3, Schrödinger, LLC: New York, NY, 2021.

15. Kearsley, S. K.; Sallamack, S.; Fluder, E. M.; Andose, J. D.; Mosley, R. T.; Sheridan, R. P., Chemical similarity using physiochemical property descriptors. J. Chem. Inf. Comp. Sci. 1996, 36 (1), 118-127.

16. Denmark, S. E.; Gould, N. D.; Wolf, L. M., A systematic investigation of quaternary ammonium ions as asymmetric phase-transfer catalysts. Application of quantitative structure activity/selectivity relationships. J. Org. Chem. 2011, 76 (11), 4337-4357.

17. Sciabola, S.; Alex, A.; Higginson, P. D.; Mitchelll, J. C.; Snowden, M. J.; Morao, I., Theoretical prediction of the enantiomeric excess in asymmetric catalysis. An alignmentindependent molecular interaction field based approach. J. Org. Chem. 2005, 70 (22), 9025-9027.

18. Pastor, M.; Cruciani, G.; McLay, I.; Pickett, S.; Clementi, S., GRid-INdependent descriptors (GRIND): A novel class of alignment-independent three-dimensional molecular descriptors. J. Med. Chem. 2000, 43 (17), 3233-3243.

19. Zahrt, A. F.; Henle, J. J.; Rose, B. T.; Wang, Y.; Darrow, W. T.; Denmark, S. E., Prediction of higher-selectivity catalysts by computer-driven workflow and machine learning. Science 2019, 363 (6424), 247-258.

20. Hastie, T. a. F., The Elements of Statistical Learning. Springer-Verlag: 2009.

21. SYBL-X, Certara: 2011. 